\title{
Abstracts from the 6th international conference on prevention \& infection control (ICPIC 2021)
}

Switzerland. 14-17 September 2021

Published: 3 September 2021

\section{ICPIC 2021 Innovation Academy}

\section{1}

Evaluating the acoustical performance of elastomeric half mask respirators, surgical masks, and single use N95 using measurements of speech test intelligibility

E. Rodgers ${ }^{1, *}$ on behalf of David Larson, Graeme Fridlay, Heather Smart, Trevor Penner

${ }^{1}$ Precision Medical, Winnipeg, Canada

Correspondence: E. Rodgers

Antimicrobial Resistance \& Infection Control 10(1): 101

Introduction: With the use of elastomeric-half mask respirators in healthcare settings, it has been reported that the verbal communication is decreased or compromised when wearing the masks (Palmiero, Symons, Morgan and Schaffer, 2016). This study examines the communication effectiveness of this innovation (elastomeric reusable respirator) and others in the industry, using speech intelligibility objective scoring, and qualitative research.

Objectives: This research measured the acoustical performance, speech transmission index (STI) (Palmeiro, et al., 2016) on an innovative elastomeric respirator, and others utilized in health care, according to IEC 60268-16 Objective rating of speech intelligibility standard.

Methods: STI measurements were obtained in a semi-anechoic acoustic test chamber/quiet room with background noise levels of less than $15 \mathrm{dBA}$. Then higher levels of background noise $(57.6 \mathrm{dBA}$ and $72 \mathrm{dBA}$, Zunn and Downey, 2005) was added to the test room and additional STI tests will be conducted in the presence of these elevated background noise levels.

The "voice" signal was emitted by the artificial voice of an acoustic head and torso simulator (HATS) and was one of two types of sound: the STI test waveform or the the Harvard sentences sound waveform (phonetically balanced and very clearly spoken human speech). The background noise portion, when used, was added separately by a high fidelity loudspeaker. The speech and STI waveform sounds were produced inside the test room at the sound level of $60 \mathrm{dBA}(1 \mathrm{~m}$ microphone distance).
Results: In an environment with no background noise, the innovation of the re-usable elastomeric respirator, yielded the highest STI rating compared to other elastomeric respirators $(0.90-0.91)$ or excellent rating. Other elastomeric respirators tested, showing fair, to low excellent range. The re-usable elastomeric respirator innovation had a 0.03 less in speech intelligibility than, single use N95 STI rating.

Conclusion: Speech intelligibility is complex, and incorporates subjective (listener) criteria, objective speech intelligibility, and background noise, as well as the environment. Additional subjective testing, with the recorded sound files, with a randomized control clinical trial would benefit this research.

Disclosure of Interest: E. Rodgers Consultant for: Precision Medical.

\section{2}

Proximity, frequency and duration of close patient contacts among nurses in a COVID-19 intensive care unit: an electronic measurement

M. M. Neuwirth ${ }^{1, *}$, T. Pommeranz ${ }^{2}$, F. Mattner ${ }^{1}$, R. Otchwemah ${ }^{1}$

${ }^{1}$ Institute for Hygiene, ${ }^{2}$ Lung Clinic, Cologne Merheim Medical Centre, University Hospital Witten/Herdecke, Cologne, Germany

Correspondence: M. M. Neuwirth

Antimicrobial Resistance \& Infection Control 10(1): 102

Introduction: SARS-CoV-2 is mainly transmitted via respiratory ingestion of virus-containing particles. In principle, the likelihood of exposure to infectious particles of any size is increased within a distance of $1-2 \mathrm{~m}$ to an infected person. Nurses are particularly at risk of contracting COVID-19 from patients due to the close patient contact and the number of intensive contacts.

Objectives: It has not yet been investigated, how long and often nurses are exposed to a close patient contact due to the intensive care during their service. The study aimed to determine the duration and frequency of close patient contacts between nurses and COVID-19 patients during nursing activities.

Methods: In the period from 14.12 .2020 to $28.02 .2021,12$ beds of a COVID-19 pulmonary intensive care unit were equipped with

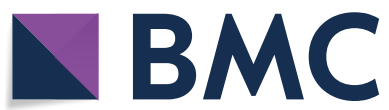

(c) The Author(s) 2021. Open Access This article is licensed under a Creative Commons Attribution 4.0 International License, which permits use, sharing, adaptation, distribution and reproduction in any medium or format, as long as you give appropriate credit to the original author(s) and the source, provide a link to the Creative Commons licence, and indicate if changes were made. The images or other third party material in this article are included in the article's Creative Commons licence, unless indicated otherwise in a credit line to the material. If material is not included in the article's Creative Commons licence and your intended use is not permitted by statutory regulation or exceeds the permitted use, you will need to obtain permission directly from the copyright holder. To view a copy of this licence, visit http://creativecommons.org/licenses/by/4.0/. The Creative Commons Public Domain Dedication waiver (http://creativecommons.org/publicdomain/zero/1.0/) applies to the data made available in this article, unless otherwise stated in a credit line to the data. 
Bluetooth Low Energy (BLE) transmitters and 12 nurses were equipped with BLE wearables (Clinaris $\mathrm{GmbH}$, Germany) in order to record the frequency and duration of patient contact as well as the distance of nurses to the headboard (face) of the patient bed. For the data evaluation, only the measurement data with at least two directly consecutive (chained) measurement contacts longer than $1 \mathrm{~s}$. and a maximum distance of $1.5 \mathrm{~m}$ from the patient's head were considered as relevant. Results: Per eight-hour shift a nurse had an average of 73.6 relevant patient contacts with a mean length of stay of $45.7 \mathrm{~s}$. per contact. The most frequent and longest contacts occurred during the early shift (6:00-14:00 o'clock) with an average number of 87.7 contacts and an average duration of $47.9 \mathrm{~s}$. This was followed by the late shift (14:0022:00; 85.1 contacts; duration $44.1 \mathrm{~s}$.) and the night shift (22:00-6:00; 42.6 contacts; $44.6 \mathrm{~s}$.). A nurse was closer than $1.5 \mathrm{~m}$ to patients' heads for an average of $60.29 \mathrm{~min}$. per shift.

Conclusion: The results show for the first time how many relevant contacts take place when caring for Covid-19 intensive care patients and provide initial indications for assessing the risk of infection in the daily work of intensive care nurses. In general, nurses work approx. 1/8 of their working time closer than $1.5 \mathrm{~m}$ to the head area of COVID-19 infected patients and are, directly exposed to SARSCoV-2 within the typical droplet distance.

Disclosure of Interest: None declared.

\section{3}

Targeting TMPRSS2 and ACE2 through AR degradation inhibits SARS-COV-2 pseudotype viral entry

S.-D. Yeh ${ }^{1, *}$

${ }^{1}$ Department of Urology and Cancer Center, Taipei Medical University Hospital, Taipei, Taiwan, Province of China

Correspondence: S.-D. Yeh

Antimicrobial Resistance \& Infection Control 10(1): 103

Introduction: TMPRSS2 and ACE2 are critical surface proteins enabling cell infection by SARS-CoV-2 and some respiratory viruses. Androgen receptor regulates TMPRSS2 and ACE2 expression. Male gender is one of the risk factors in the increasing mortality and severe COVID-19 infection. Targeting androgen receptor degradation is a proposed strategy for therapy and prevention of SARS-CoV-2 infections.

Objectives: We study the expression of AR, TMPRSS2 and ACE2 by treating with small molecule AR degradation enhancers, then examine the effect of supression SARS-CoV-2 pseudotyped viral particles entry after inducing AR degradation.

Methods: Small molecule AR degradation enhencers Asc-JM-17 and Asc-JM-176 were obtained from ALLIANZ PHARMASCIENCE LTD. (Taipei, TWN). The expression of AR, TMPRSS2 and ACE2 were studied by western blotting. Small hairpin RNA lentivirus against AR and control lentivirus were tranfected to knockdown AR in A549, H1299 and CL1-5 cell lines. SARS-CoV-2 spike fusion protein pseudotyped viral particles were purchased from RNAi Core Lab in Academia Sinica. Peudotyped viral assay was performed in HEK293T/17 ACE2-pBsd cells. DHT, hydroxyflutamide, enzalutamide(MDV3100), Asc-JM-17, and AscJM-176 were applied to A549, H1299, CL1-5, HEK293T/17 ACE2-pBsd cells.

Results: Asc-JM-17 and Asc-JM-176 rather than hydroxyflutamide and enzalutamide inhibits AR, TMPRSS2 and ACE2 in a dose- and timedependent manner. The suppression effect of Asc-JM-17 and AscJM-176 on TMPRSS2 expression was partially reversed by knockdown $A R$ with shAR lentivirus infection. Asc-JM-17 and Asc-JM-176 rather then hydroxyflutamid and enzalutamide robustly prevent the pseudotyped viral particles in entry of HEK293T/17 ACE2-pBsd cells with EC50 at $1.2 \mu \mathrm{M}$ and $3.3 \mu \mathrm{M}$ respectively. Finally Asc-JM-17 and Asc-JM-176 were safely administered in mice for 35 days without obvious toxicity.

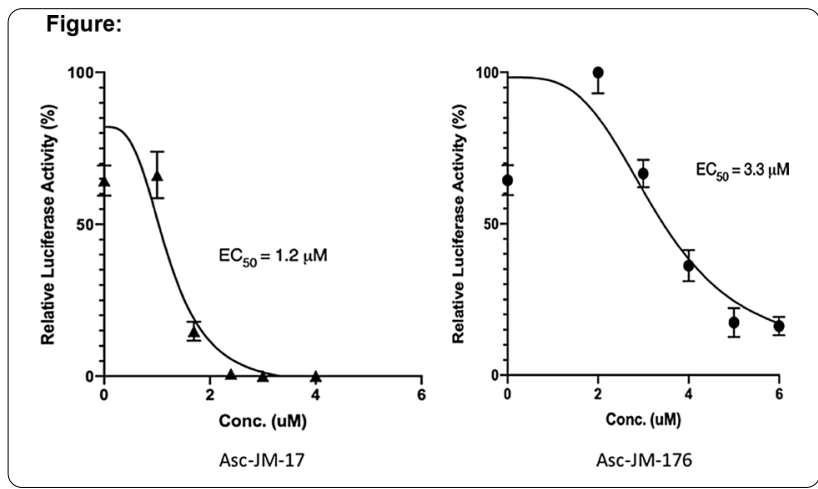

Conclusion: Take together, our results support that application of Asc-JM-17 and Asc-JM-176 through targeting AR degradation is a novel strategy for therapy and prevention of respiratoy viral infection such as SARS-CoV-2. Suppression of SARS-CoV-2 viral culture and animal model with AR degradation enhancers merit further study.

Disclosure of Interest: None declared.

104

Antimicrobial photodynamic disinfection of SARS-COV-2 in a thin-film intranasal model

C. Romo ${ }^{1}$, C. Cross ${ }^{1}$, R. Andersen ${ }^{1}$, N. Loebel ${ }^{1, *}$

${ }^{1}$ Ondine Research Labs, Bothell, United States

Correspondence: N. Loebel

Antimicrobial Resistance \& Infection Control 10(1): 104

Introduction: SARS-CoV-2 infection initiates in the upper respiratory tract with viral shedding prior to onset of symptoms. Asymptomatic carriers are associated with the majority of COVID-19 transmissions, complicating prophylaxis. Up to half of all clinical cases of COVID-19 develop secondary bacterial infections, primarily secondary bacterial pneumonia which may prove lethal. Effective intranasal antiviral treatment options for SARS-CoV-2 carriers are limited.

Objectives: Higher viral titer is associated with increased severity of symptoms and mortality risk. Targeting SARS-CoV-2 colonization sites in the upper airway with a potent, safe, antiviral modality may mitigate the severity of lower tract respiratory disease as well as reduce hospitalization and mortality rates. Intranasal APDT has been deployed for 10 years in Canadian hospitals as an effective, broad-spectrum nasal antimicrobial for reduction of post-surgical infections. The objective of this work was to evaluate effectiveness of APDT against SARS-CoV-2 in vitro.

Methods: Studies were carried out using wild-type virus (SARSRelated Coronavirus 2, Isolate USA-WA1/2020, Cat\# NR-52286, heat-inactivated, US Centers for Disease Control and Prevention/BEI Resources, NIAID, NIH). APDT was carried out by exposing the viral culture to a photosensitizer formulation containing 320 uM methylene blue in an aqueous carrier, immediately followed by $36 \mathrm{~J} / \mathrm{cm}^{2}$ of non-thermal laser light at $664 \mathrm{~nm}$. Illumination was conducted in a custom thin-film cell replicating the anterior nasal architecture. Studies were repeated at $72 \mathrm{~J} / \mathrm{cm}^{2}$ for comparison purposes. RNA quantitation was carried out using RT-qPCR with N1/N2 primers and probes and bacteriophage controls. All experiments were repeated in triplicate.

Results: Results demonstrated APDT was capable of completely eliminating the RNA signature of SARS-CoV-2. Reduction of threshold count $\Delta \mathrm{C}_{\mathrm{t}}=22$ was accomplished in a linear light-dose-dependent manner (starting $\mathrm{Ct}=23$, ending $\mathrm{Ct}>45$ nondetectable) with light doses up to $8 \min (\mathrm{p}<0.001)$.

Conclusion: Using standard clinical parameters for intranasal pathogen reduction, APDT proved capable of eliminating the RNA signature of wild-type SARS-CoV-2 in vitro. This work supports further 
development of APDT as a potent, antiviral modality for inhibition of SARS-CoV-2 transmission.

Disclosure of Interest: C. Romo Employee of: Ondine Research Labs, C. Cross Employee of: Ondine Research Labs, R. Andersen Employee of: Ondine Research Labs, N. Loebel Employee of: Ondine Research Labs.

105

Virucidal efficacy of an ozone-generating system for automated room disinfection

F. H. H. Brill1,*, T. Burkard ${ }^{2}$, B. Becker ${ }^{1}$, D. Paulmann ${ }^{1}$, D. Todt ${ }^{2}$, B. Bischoff ${ }^{1}$, E. Steinmann ${ }^{2}$, J. Steinmann ${ }^{1}$

${ }^{1}$ DR. BRILL + PARTNER GMBH INSTIUTE FOR HYGIENE AND MICROBIOL-

OGY, Bremen, ${ }^{2}$ Institute for Medical and Molecular Virology, Ruhr-Univer-

sity, Bochum, Germany

Correspondence: F. H. H. Brill

Antimicrobial Resistance \& Infection Control 10(1): 105

Introduction: Besides conventional prevention measures, no-touch technologies based on gaseous systems have been introduced in hospital hygiene for room disinfection. These results will help to establish realistic conditions for virus inactivation and assessment of efficacy of ozone technology against non-enveloped and enveloped viruses.

Objectives: The aim of the study was evaluate the activity of ozon for automated disinfection of surfaces in a room.

Methods: A whole-room disinfectant device which creates ozone as biocidal agent, was tested for its virucidal efficacy based on the NF T 72-281:2014 AFNOR Standard against modified Vacciniavirus strain Ankara murine Norovirus, simian virus 40 and Adenovirus in a standardized room on practice relevant surfaces.

Results: All test virus titers were reduced after 150 and $300 \mathrm{~min}$ of decontamination with mean reduction factors (RF) ranging from 2.63 (murine norovirus) to 3.94 (simian virus 40).

Conclusion: These results will help to establish realistic conditions for virus inactivation and assessment of efficacy of ozone technology against non-enveloped and enveloped viruses.

Disclosure of Interest: None declared.

106

Efficiency of a dry-fog based decontamination method

for the reduction of bacterial contamination

S. Buhl ${ }^{1, *}$, A. Stich ${ }^{1}$, C. Bulitta ${ }^{1}$

${ }^{1}$ medical engineering, Ostbayerische Technische Hochschule

Amberg-Weiden, Weiden in der Oberpfalz, Germany

Correspondence: S. Buhl

Antimicrobial Resistance \& Infection Control 10(1): 106

Introduction: Dry fog disinfection, which has been a topic in the food industry for a long time, has the potential to be an alternative to common disinfection methods. The possibility of the technology to decontaminate the whole room in one disinfection cycle has also led to serious exposure to this technology during the pandemic.

Objectives: In this work, the disinfecting potential of the TBT dry fogging technology (TBT Desinfektion GmbH \& Co. KG, Germany) and the corresponding disinfectant Defeat AR (Biofluid $\mathrm{GmbH}$, Germany) with regard to microbiological contamination will be examined.

Methods: Defeat AR is a HOCl-based active ingredient and the principle disinfection potential of the disinfectant is tested by means of the standardized 4-field test (EN 16615) in a first step. Then TBT dry fog method for decontaminating microbiological contamination is tested in an experimental setup. Ceramic tiles are soiled with a suspension of Staphylococcus aureus (ATCC 6538) and distributed at predetermined points in the room. An active air sampling is also performed before and after the decontamination cycle to determine the air decontamination properties.

Results: The examination of the efficacy in accordance with the EN 16615 standard consistently showed a strong efficacy of the tested disinfectant. In the airborne germ measurement, this effect was a reduction of approx. $66 \%$ of the microbiological room contamination. This germ-reducing effect could be shown at all measuring points. Similarly, decontamination of the surfaces was shown to be effective at all measuring points in the test setup we selected. Based on the calculated maximum contamination of $5 \times 105 \mathrm{CFU}$, the fogging achieved an efficiency of $>3 \log$ on some of these surfaces. However, this decontaminating effect depends on the accessibility of the surfaces.

Conclusion: Our results could confirm the general antibacterial efficacy of the fogging technology. However, there were strong differences in efficacy depending on the accessibility of the test samples. The test procedure must also be refined in order to be able to accurately determine the actual germ reduction by means of dilution levels. Further tests are needed to determine the potential of the technology more precisely.

Disclosure of Interest: None declared.

107

Evaluation of an UVC robot for terminal disinfection of hospital rooms

J. E. Sierra Garcia 1, ${ }^{1}$, F. Guillen-Grima ${ }^{2}$, M. P. Garcia-Garcia ${ }^{3}$, L. Cascajar ${ }^{4}$, F. Rodriguez-Merino ${ }^{5}$

${ }^{1}$ Electromechanical Engineering, University of Burgos, Burgos, ${ }^{2}$ Preventive Medicine, ${ }^{3}$ Preventive Medicine, Clinica Universidad de Navarra,

Pamplona, ${ }^{4}$ ASTI Mobile Robotics, Burgos, ${ }^{5}$ Boos Technical Lighting,

Valladolid, Spain

Correspondence:

Antimicrobial Resistance \& Infection Control 10(1): 107

Introduction: The dramatic spread of COVID-19 has disrupted lives, communities and businesses worldwide. A new autonomous robot, equipped with UVC light to perform fast and reliable disinfections while it is on movement, has been developed to contribute to the reduction of the impact on public health.

Objectives: In this work, we have scientifically evaluated the ability of this robot to disinfect hospital rooms.

Methods: We selected empty rooms just after patient discharge and before terminal cleaning. We took 94 microbiological samples of highly touched surfaces inside the hospital patient's rooms using Rodac plates, before introducing the robot in the room. After the robot operation, samples were taken in the same places. The plates were incubated and read after 5 days. We performed descriptive statistics, and non-parametric Wilcoxon test for repeated measures, using IBM SPSS and OPENEPI. Results are presented in colony-forming units per plate $(\mathrm{cfu} / \mathrm{p})$ per plate. Patients are considered to be at risk when the bacterial count on hospital surfaces exceeds $120 \mathrm{FCU} /$ plate $(5 \mathrm{fcu} / \mathrm{cm} 2)$.

Results: The initial situation was that in $4 \%$ of the locations there was a risk for the patients (more than 120 cfu per plaque). After applying this robot there were no locations with risk for the patients. The table shows that the mean colony reduction is $81.96 \%$ and the median experiments a reduction of $94.8 \%$. In any case, both levels mean and median are below the threshold for disinfection that is $10 \mathrm{fcu} /$ plate. The Wilcoxon test for related samples indicates that the differences are statistically significant $(P<0.0001)$. The normal procedure is to clean the room using a Chloramine-based disinfectant after the UVC, so the cumulated effect will be even higher. 


\begin{tabular}{|l|l|l|}
\hline & PRE & POST \\
\hline Mean & $30.71^{*}$ & $5.54^{* *}$ \\
\hline SD & 34.6 & 9.5 \\
\hline CI 95\% & $23.6-37.8$ & $3.60-7.49$ \\
\hline Median & 17.5 & 1 \\
\hline Quartile deviation & 21 & 3 \\
\hline
\end{tabular}

* FCU per plate

** Disinfection treshold < $10 \mathrm{FCU}$ per Plate

Table 1: Reduction of the colony-forming units.

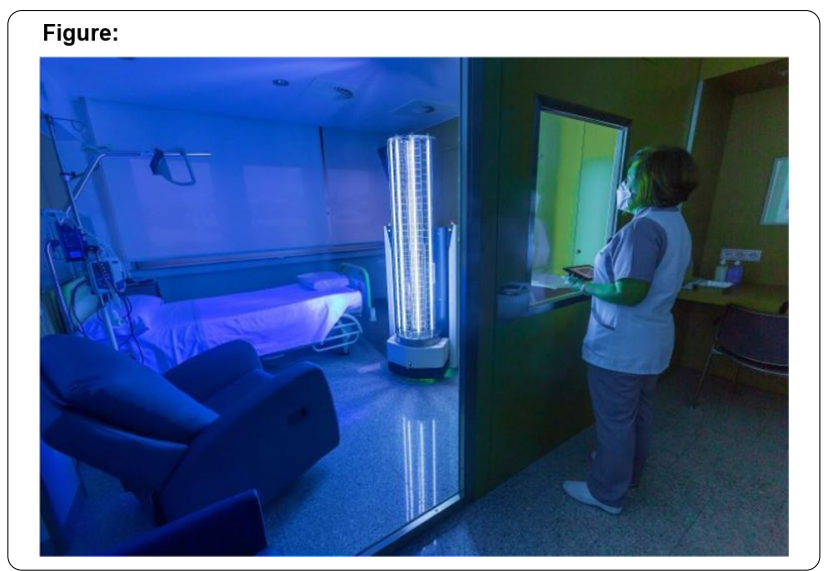

Conclusion: The use of this robot allowed to reduce the colony-forming units below the risk threshold. Therefore it is a valid technique for terminal disinfection of hospital rooms.

Disclosure of Interest: None declared.

108

Use of robotic process automation (RPA) for rapid analysis and interpretation of multidrug resistant organisms and COVID-19 results

J. Jerry, ${ }^{1, *}$, D. A. O'malley ${ }^{1}$

${ }^{1}$ Infection Prevention and Control, Mater Misericordiae University Hospital, Dublin 7, Ireland

Correspondence: J. Jerry

Antimicrobial Resistance \& Infection Control 10(1): 108

Introduction: The IPC (Infection Prevention \& Control) nurses workload had trebled in the last 5 years due to increased number of patients diagnosed with known Multi Drug Resistant Organisms (MRDOs). IPC nurse resources were saturated with downloading and processing essential microbiology results from an old laboratory system. The potential for human error whilst collating the microbiology results from the laboratory system was moderate as some parts of the process required items to be transcribed.

Objectives: 1 . To employ Robotic Process Automation (RPA) to lift the burden of repetitive tasks from IPC team and reduce the significant amount of time spent on daily processing of lab results and administrative work. 2. Redirect the specialist nurses' time saved by using RPA towards quality improvement initiatives and patient care. 3. Improve job satisfaction and reduce turnover of staff in the IPC team. 4. Eliminate human error.

Methods: IPC team identified RPA as the best solution to lift the administrative burden. A process check was undertaken by UiPath Software Team to identify tasks which could be automated. The RPA was developed to navigate through electronic medical records and lab systems. Buy-in was sought from all relevant stakeholders including Hospital management, Data protection, Information Technology, Procurement, Laboratory, Infection Prevention \& Control and UiPath. A solution design document was developed and agreed along with controls and risk assessment. Three key processes were assigned to RPA: Microbiology Results for MDROs, COVID-19 \& MDROs Surveillance and Nurse Task Admin Handover.

Results: At present, Uipath robot works 24/7, 365 days a year with no break requirements and eliminates human error. RPA reduced administration time by $3 \mathrm{~h}$ a day, which equates to $18 \mathrm{~h}$ per week or $936 \mathrm{~h}$ a year. In addition, RPA saved substantial amount of time required for processing COVID-19 results and surveillance. In fact, RPA facilitated more efficient work flow. RPA liberated resources to deliver essential IPC services such as surgical site infection surveillance, PPE training and education, guideline development, COVID-19 and CPE contact tracing.

\begin{tabular}{|c|c|c|c|}
\hline \multicolumn{4}{|l|}{ Figure: } \\
\hline \multicolumn{4}{|c|}{ Time Saved with Automation } \\
\hline & March 2020 & April 2020 & May 2020 \\
\hline $\begin{array}{l}\text { Download microbiology results and } \\
\text { updating Surveillance }\end{array}$ & 93.0 & 90.0 & 93.0 \\
\hline Create Covid-19 nurse referral & 27.2 & 48.0 & 14.8 \\
\hline Complete Covid-19 surveillance data & 54.3 & 96.0 & 29.7 \\
\hline Hours saved /month & 174.5 & 234.0 & 137.5 \\
\hline $\begin{array}{c}\text { Total time saved in three months } \\
\text { period }\end{array}$ & \multicolumn{3}{|c|}{546.0 hours } \\
\hline
\end{tabular}

Conclusion: RPA could be implemented across the wider healthcare environment to optimise use of Infection Prevention and Control Nurses time.

Disclosure of Interest: None declared.

109

Decolonization of human skin by application

of a photodynamically active hydrogel

D. B. Eckl ${ }^{1, *}$, A. Hoffmann ${ }^{1}$, N. Landgraf ${ }^{1}$, A. Eichner ${ }^{2}$, H. Huber ${ }^{1}$, W.

Bäumler ${ }^{2}$

${ }^{1}$ Department of Microbiology, University Regensburg, ${ }^{2}$ Department

of Dermatology, University Hospital Regensburg, Regensburg, Germany

Correspondence: D. B. Eckl

Antimicrobial Resistance \& Infection Control 10(1): 109

Introduction: Skin colonization with pathogenic, even multi-resistant bacteria is a potential source of infections. Photodynamic inactivation (PDI) of bacteria is a promising, new approach for the decolonization of the human skin. PDI needs a dye molecule (photosensitizer), which generates mainly singlet oxygen upon light exposure. Thereby bacteria are oxidative destroyed regardless their type and resistance profile. Preliminary PDI experiments already provided a decontamination efficacy on human ex vivo skin of $99.9 \%$ using an aqueous photosensitizer solution.

Objectives: Based on findings mentioned before, we aimed to improve the decolonization efficacy by applying a photodynamically active hydrogel in a follow-up study.

Methods: Fresh ex vivo human skin was inoculated with bacterial suspensions of methicillin-resistant Staphylococcus aureus (MRSA) After visible drying of bacteria, different hydrogels containing different photosensitizers were added to the inoculated skin surface. Subsequently, the skin surface were irradiated with visible blue light at different radiant exposures $\left(\mathrm{J} / \mathrm{cm}^{2}\right)$. Then, bacteria were recovered from skin with a swap and plated via drop-plate method on Mueller Hinton Agar. Colony count unfold reduction efficacy when referenced 
to internal controls. After recovery, skin biopsies were taken and nitro blue tetrazolium (NBT) staining was applied to check viability of skin cells via mitochondrial activity. TUNEL stain revealed the presence of apoptotic skin cells.

Results: The results showed that PDI created an improved MRSA reduction on skin of around $99.99 \%$. Staining of the tissue revealed no noteworthy apoptosis or lack in mitochondrial activity of the human cells due to the application of the photodynamically active hydrogel. Conclusion: PDI should represent an easy, quick and efficient method to decolonize human skin from MRSA. The results of the study are remarkable, as substances on skin like inorganic ions or small organic molecules such as histidine are capable to inhibit the photodynamic efficacy. Since PDI is known to eradicate a large set of pathogens independent of their antibiotic resistance profiles, this new technology should be tested in clinical studies in the near future.

Disclosure of Interest: None declared.

110

Development of the first washable, fluorescent alcohol-based hand rubbing training solution

P. G. Szeremy ${ }^{1, *}$, V. Sari $^{1}$, P. Vezse ${ }^{1}$, T. Haidegger ${ }^{2}$

${ }^{1}$ HandlnScan Zrt., ${ }^{2}$ Research, Innovation and Service Center (EKIK), Óbuda University, Budapest, Hungary

Correspondence: P. G. Szeremy

Antimicrobial Resistance \& Infection Control 10(1): 110

Introduction: Fluorescent, alcohol-based hand hygiene training solutions combined with a UV lamp are a well-established tool to teach and survey hand rubbing performance. One of their main drawbacks is that these training rubs stain the hand, and remain detectable for $24 \mathrm{~h}$ on the skin. As a consequence these are only applicable once a day, and prohibited from many applications. Washable non-liquid (i.e., gel and lotion consistency) fluorescent training products are known, but they are creamy, not alcohol based, therefore inadequate to properly train the act of hand rubbing.

Objectives: To develop and assess a novel fluorescent-labeled hand hygiene training product which is completely removable and rinsible by soap and water, thus providing repeatable and universal hand hygiene training.

Methods: A new compound was selected to meet the strict health and safety regulations in the food, pharma and healthcare industry. All fluorophore candidates were tested from the narrow cross section of the aforementioned industries. The washability, detectability, visual staining and skin protection was optimized. Instead of the traditional UV-A light, a completely harmless blue light was picked. It was further field tested for two weeks in a food processing plant, supporting a hand hygiene training intervention.

Results: A first in class training solution was successfully experimented to support cross-industry hand hygiene training. The solution was registered as a cosmetic product in the EU according to the EU Regulation (EC) No 1223/2009 (Article 13).

During the performance assessment, 654 hand hygiene actions were performed by 75 users. No skin complaints or other usability issues were detected. The intervention resulted an increase of 13 percentage point from a $86 \%$ hand coverage baseline to a $99 \%$ final result.

Conclusion: The usability and performance tests of a new, washable hand hygiene training solution was performed, with identical outcomes to the traditional UV fluorescent training. The chosen labeling compound is a common food and drug additive, without known allergic reactions. The complete removal of the fluorescent label by hand washing makes the new solution optimal for objective, sustained hand rubbing and hand washing training, assessment and quality assurance.

Disclosure of Interest: P. Szeremy Employee of: HandInScan Zrt., V. Sari Employee of: HandInScan Zrt., P. Vezse Employee of: HandInScan Zrt., T. Haidegger Employee of: HandlnScan Zrt.
Slide session: Epidemiology and spread of MDRO in hospital and community

001

Colonization pressure as a risk factor for acquisiton

of ESBL-producing enterobacterales

F. Maechler ${ }^{1, *}$, F. Schwab ${ }^{1}$, S. Hansen ${ }^{1}$, P. Gastmeier ${ }^{1}$ on behalf of R-Gnosis WP5 Study Group

${ }^{1}$ Institute of Hygiene, Charité Universitätsmedizin, Berlin, Germany

Correspondence: F. Maechler

Antimicrobial Resistance \& Infection Control 10(1): 001

Introduction: The so-called colonization pressure, which is the proportion of patients colonized with certain microorganisms, affects the spread of these microorganisms in hospitals.

Objectives: The aim of this project was to analyze the impact of colonization pressure on the acquisition of ESBL-producing Enterobacterales in adult non-ICU wards.

Methods: The project was a subanalysis of a trial that compared different infection control strategies (Maechler et. al; LID 2020;20:575-84). Patients admitted to 20 adult medical and surgical non-ICUs in four European countries were screened for ESBL-E carriage on admission, weekly thereafter and on discharge. We defined colonization pressure as the number of ESBL-E days per 100 patient days during the previous 7 days. For each calendar day and ward, we associated the number of ESBL-E acquisitions and the colonization pressure. The multivariate analysis adjusted for clustering, season, infection control strategy, ward type, and unscreened patient days (days to first screening per 100 patient days).

Results: Between January 2014 and August 2016, 50,870 patients were admitted to the participating wards, accounting for 356,298 patient days. Of those, 32,254 patients had a length of stay $\geq 3$ days and $\geq 1$ screening culture, accounting for 296,298 patient days with information on ESBLE carriage. The median time to first sample was 2 days (IQR 1-3); the median number of samples per screened patient was 2 (IQR 1-2); ESBL-E admission prevalence was $11 \%(95 \% \mathrm{Cl} 9.3-10.2)$, and the incidence density of ward-acquired ESBL-E per 1000 patient days was 4.0 (95\%Cl 3.74.3). Across all wards, the median 7 day-colonization pressure was $13.5 \%$ (IQR 8.2-20.5), and the median percentage of unscreened patient days was $22.7 \%$ (IQR 13.2-34.2). The table shows the results of the multivariate analysis.

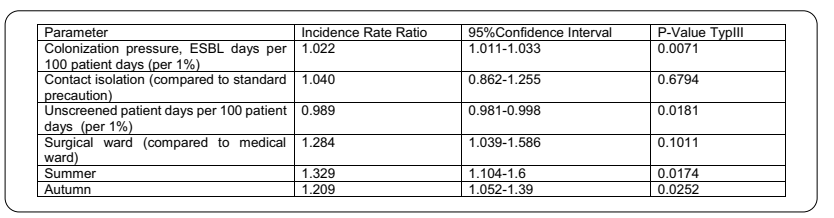

Conclusion: Per $1 \%$ increase of colonization pressure, the adjusted risk of ESBL-E acquisition increased by $2.2 \%$.

Disclosure of Interest: None declared.

002

An interventional study to evaluate the impact of a rapid screening strategy in improving nosocomial ESBL and CPE control in critically ill patients

R. Martischang 1,*, P. François ${ }^{2}$, A. Cherkaoui ${ }^{3}$, E.-J. Bonetti ${ }^{2}$, G. Renzi ${ }^{3}$, J.

Schrenzel ${ }^{3}$, J. Pugin ${ }^{4}$, S. Harbarth ${ }^{1}$

${ }^{1}$ Infection Prevention and Control, ${ }^{2}$ Genomic Research Laboratory, ${ }^{3} \mathrm{Bac}-$

teriology Laboratory, ${ }^{4}$ Intensive Care Units, HUG, Geneva, Switzerland

Correspondence: R. Martischang

Antimicrobial Resistance \& Infection Control 10(1): 002

Introduction: Current phenotypic screening methods may delay timely infection control measures for carriers of Extended-Spectrum Beta-Lactamase Producing Enterobacterales (ESBL-PE) and Carbapenemase Producing Enterobacterales (CPE). 
Objectives: We compared a rapid molecular assay against culturebased method to discontinue preemptive contact precautions (CP) for negative patients at-risk, or to accelerate implementation of CP for newly positive patients.

Methods: This interventional quasi-experimental cohort study compared the diagnostic performance of the Loop-Mediated Isothermal Amplification (LAMP) Eazyplex SuperBug CRE assay (AxonLab, Switzerland) during a $1^{\text {st }}$ period (Apr 2019-Mar 2020) with culture-based method implemented during a $2^{\text {nd }}$ period (May-Oct 2020) to detect ESBL-PE and CPE carriage among critically ill patients at Geneva University Hospitals. Survival analysis assessed time to discontinue unnecessary preemptive $\mathrm{CP}$ in patients at-risk, and time to implement $\mathrm{CP}$ for newly positive patients.

Results: We included 1'044 patients, 147 (14\%) patients at risk screened at admission, $813(78 \%)$ patients with either weekly or fortuitous screenings, and $84(8 \%)$ patients with both screenings. Prevalence was $16 \%$ and $0.8 \%$ for ESBL-PE and CPE. The LAMP assay for CPE detection yielded a positive predictive value (PPV) of $44.0 \%$ [95\%Cl 24.5-63.5], and negative predictive value (NPV) of $99.9 \%$ [95\%Cl 99.7-100.0], and for ESBL-PE a PPV of $88.5 \%$ [95\%Cl 82.3-94.6], and NPV of $95.0 \%$ [95\%Cl 93.6-96.3]. Respectively, 92 and 47 patients at-risk were negative during the interventional and control period. Median time to de-implement unnecessary CP was respectively 88.3 versus $80.5 \mathrm{~h}(p=0.47)$. Due to poor PPV, CP were not implemented based on positive LAMP results.

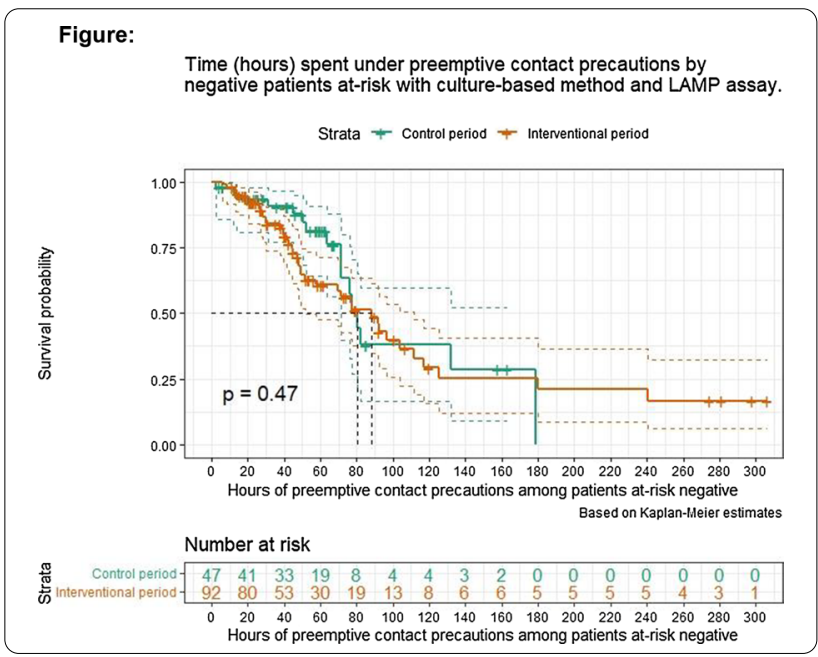

Conclusion: LAMP directly performed on rectal swabs has an excellent NPV to exclude ESBL and CPE carriage, but did not significantly accelerate discontinuation of preemptive CP. Implementation of ESBL and CPE control measures based on this rapid test was however impossible due to poor positive predictive values.

Disclosure of Interest: R. Martischang Grant/Research support from: AxonLab provided the device, and a discount on LAMP reagents, $P$. François: None declared, A. Cherkaoui: None declared, E.-J. Bonetti: None declared, G. Renzi: None declared, J. Schrenzel: None declared, J. Pugin: None declared, S. Harbarth: None declared.

\section{3}

\section{Characterizing the bioburden of ESBL-producing}

enterobacteriales in a neonatal unit in Botswana: a feasible and efficient environmental sampling method using chromogenic culture media

M. Vurayai ${ }^{1, *}$, J. Strysko 2,3 , C. McGann ${ }^{3}$, O. Bayani ${ }^{2}$ on behalf of Neonatal Infections \& Microbiome Working Group, S. Coffin ${ }^{3}$

${ }^{1}$ Biological Sciences, ${ }^{2}$ Paediatric \& Adolescent Health, University of Botswana, Gaborone, Botswana, ${ }^{3}$ Global Health Center, Children's Hospital of Philadelphia, Philadelphia, United States

Correspondence: M. Vurayai

\section{Antimicrobial Resistance \& Infection Control 10(1): 003}

Introduction: Infections due to extended spectrum beta-lactamase producing Enterobacteriales (ESBL-PE) are the leading causes of sepsis among hospitalized neonates in Botswana, yet ESBL-PE transmission dynamics are not well-characterized in the neonatal intensive care unit (NICU) environment.

Objectives: This study aimed to assess the efficiency and feasibility of a chromogenic-culture-media-based environmental sampling strategy characterizing the ESBL-PE bioburden within a NICU.

Methods: The study took place at a 36-bed NICU in a tertiary care hospital in Botswana from January-April 2021. Samples were collected on 3 occasions under semi-sterile technique using 1) flocked swabs \& templates (flat surfaces); 2 ) sterile syringe \& tubing (water aspiration); 3) structured swabbing techniques (hands \& equipment). Swabs were transported in physiological saline-containing tubes, vortexed, and $10 \mu \mathrm{L}$ was inoculated onto chromogenic-agar (CHROMagarTM ESBL, Paris, France; selective/differential for ESBL$\mathrm{PE})$, streaking plates to isolate individual colonies. Bacterial colonies were quantified and phenotypically characterized using biochemical identification tests.

Results: In total, 393 samples were collected. Dense and consistent ESBL-PE contamination was detected in and around sinks and certain high-touch surfaces, while transient contamination was demonstrated on medical equipment, caregivers/healthcare worker hands, insects, and feeding stations (including formula powder) (Figure 1). Results were available within $24-72 \mathrm{~h}$ of collection. To collect, plate, and analyse 50 samples, we estimated a total expenditure of \$269.40 USD and 13.5 cumulative work hours.

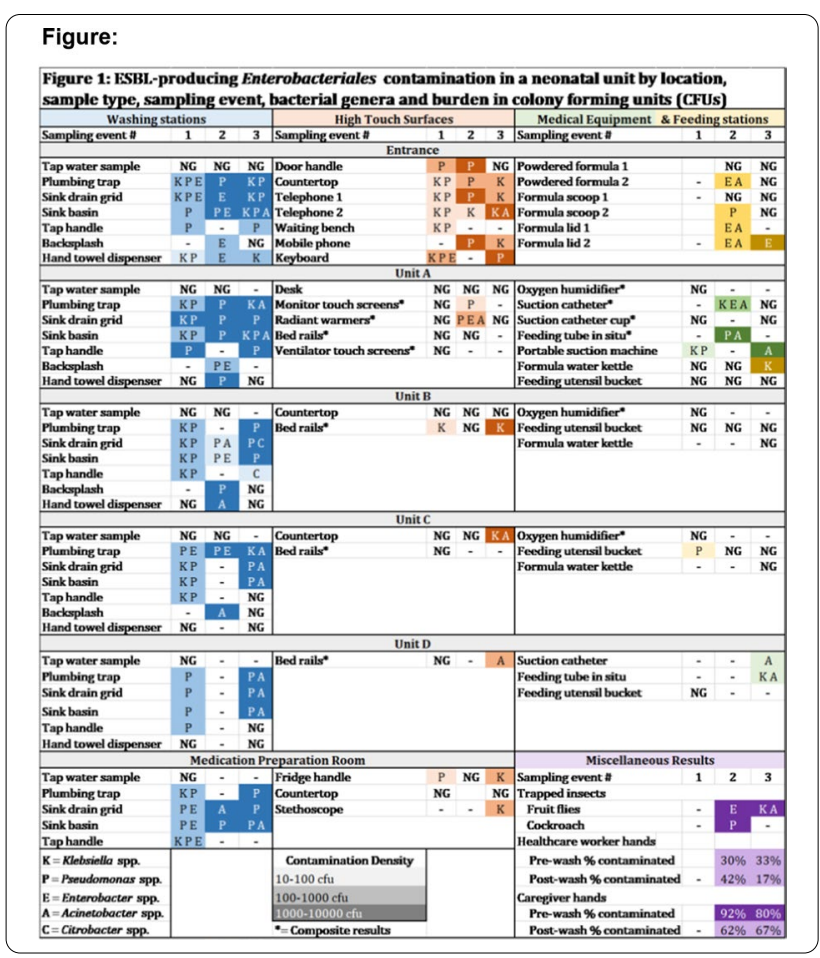

Conclusion: Using basic environmental sampling and laboratory techniques aided by chromogenic culture media, we identified ESBL$P E$ reservoirs (sinks) and plausible transmission vehicles (medical equipment, infant formula, hands of caregivers/healthcare workers, \& insects) in this NICU environment. This strategy was a simple and costefficient method to assess ESBL-PE bioburden and may be feasible for use in other settings to support ongoing infection control assessments and outbreak investigations.

Disclosure of Interest: None declared. 
004

The transmission risk of multidrug-resistant organisms between pets and humans - preliminary results of an exploratory case control study

C. Hackmann 1, ${ }^{1 *}$, P. Gastmeier ${ }^{1}$, S. Schwarz ${ }^{2}$, A. Lübke-Becker ${ }^{2}$, R. Leistner ${ }^{1}$

${ }^{1}$ Institute of Hygiene and Environmental Medicine, Charité-Universitätsmedizin Berlin, ${ }^{2}$ Department of Veterinary Medicine, Freie Universität

Berlin, Berlin, Germany

Correspondence: C. Hackmann

Antimicrobial Resistance \& Infection Control 10(1): 004

Introduction: Up to $60 \%$ of German residents own at least one pet, who live in close contact to their owners. Colonization with multidrugresistant organisms (MDROs) occurs in humans and pets. However, it is still unclear if pets play a significant role as a reservoir for MDROs.

Objectives: This project aims to assess the relevance of pet husbandry in the colonization of MDROs of hospital patients. The project focusses on the most common MDROs in pet owners, methicillin-resistant Staphylococcus aureus (MRSA), vancomycin-resistant enterococci (VRE), $3^{\text {rd }}$ generation cephalosporin-resistant Enterobacterales (3GCRE) and carbapenem-resistant Enterobacterales (CRE).

Methods: We perform an exploratory, unmatched case-controlstudy. Among questions about well-known risk factors, study participants are queried regarding their contact to dogs and cats. To assess the genetic relatedness of the human and pet MDROs, we collect nasal and rectal swabs of participants and their pets to test them for MDROs. Phenotypically matching MDROs in the samples of participants and their pets will be tested for genetic relatedness using whole genome sequencing (WGS). The study is currently being performed at the Charité Universitätsmedizin Berlin. The sample size will comprise about 4,000 human participants and aims at 1,000 animal samples. The study is funded by the Federal Department of Health (BMG).

Results: Among 3,285 participants so far, $25 \%(820 / 3,285)$ tested positive for MDROs. $21 \%$ of the participants $(701 / 3,285)$ stated to own at least one pet dog or cat (range 1-10). Among the first 345 returned and analyzed pet samples, $12 \%$ (42/345) were positive for MDROs. In three cases MDROs of pet and owner were phenotypically matching. The matching pathogens were in two cases VRE and in the other case 3GCRE. WGS analyses revealed that two of the pairs were also genotypically matching.

Conclusion: The investigation of pet husbandry as a risk factor for colonization or infection with MDROs in this study creates an opportunity to identify patients at risk and develop potential prevention strategies. Although it is known that transmission between humans and pets ccurs, the likelihood of this transmission is still unclear and the effect size needs to be investigated.

Disclosure of Interest: None declared.

\section{5}

Epidemiology of ESBL-producing Escherichia coli

(ESBL-EC) from repeated prevalence studies over 11 years in a long-term-care facility (LTCF)

R. Martischang ${ }^{1, *}$, P. François ${ }^{2}$, A. Cherkaoui ${ }^{3}$, E.-J. Bonetti ${ }^{2}$, N. Gaïa ${ }^{2}$, G. Renzi ${ }^{3}$, M. Perez ${ }^{1}$, C. Graf ${ }^{4}$, S. Harbarth ${ }^{1}$

${ }^{1}$ Infection Prevention and Control, ${ }^{2}$ Genomic Research Laboratory, ${ }^{3} \mathrm{Bac}-$ teriology Laboratory, ${ }^{4}$ Department of Rehabilitation and Geriatrics, HUG, Geneva, Switzerland

Correspondence: R. Martischang

Antimicrobial Resistance \& Infection Control 10(1): 005

Introduction: Escherichia coli Sequence type 131-H30 are emerging multidrug resistant subclones, known to spread and cause outbreaks in LTCFs.

Objectives: We aim to assess temporal trends in the prevalence and epidemic potential of ESBL-EC subclones, and to evaluate a potential rebound effect following discontinuation of contact precautions for ESBL-EC carriers in January 2019.
Methods: From 2010 through 2020, we performed 11 prevalence studies (Jan/Feb of each year) with screening for digestive carriage of ESBL-EC among residents in a university-affiliated LCTF. All available isolates were typed for $\mathrm{ST} 131-\mathrm{H} 30$ and a selection was sequenced.

Results: We included 2'403 LTCF residents, with 252 (10.5\%) positive for ESBL-EC. Among the $238 \mathrm{ESBL}-\mathrm{EC}$ isolates available for typing, 58.0\% belonged to the ST131 lineage, including 94/138 (68.1\%) ST131 H30 isolates. An increasing yearly prevalence was observed for ESBL-EC ( $4.6 \%$ to $9.4 \% ; p=0.106$ for linear trend), but not for the ST131 H30 subclone, which peaked in 2015 and declined thereafter. Since 2017, we noted the clonal expansion of a ST131 non-H30 subclone harboring CTX-M-14 and CTX-M-24. Multiple silent ESBLEC outbreaks occurred, mostly before the withdrawal of contact precautions for ESBL-EC carriers in 2019. No rebound effect was observed in ESBL-EC prevalence nor in the different subclones following discontinuation of contact precautions.

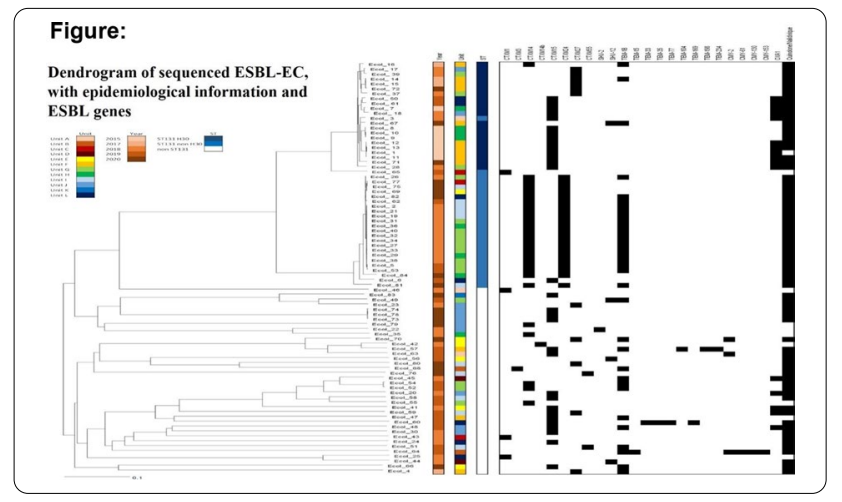

Conclusion: Clonal fluctuation was observed for ST131-H30 ESBL-EC with a current decline in prevalence. Surveillance should include the evolution of ST131 non-H30 subclones, which may spread in LTCFs. Discontinuation of contact precautions for ESBL-EC carriers in LTCFs may be safely implemented, in support of European recommendations to limit ESBL-producing Enterobacteriaceae control measures in endemic settings to non-E.coli.

Disclosure of Interest: None declared.

006

Vancomycin-resistant enterococcus-a health economic

evaluation of a healthcare outbreak in Switzerland

T. Berkes ${ }^{1, *}$, O. Endrich ${ }^{2}$, M. Fiedler ${ }^{2}$, J. Marschall ${ }^{2}$

${ }^{1}$ University Bayreuth, Murrhardt, Germany, ${ }^{2}$ Inselspital Bern, Bern, Swit-

zerland

Correspondence: T. Berkes

Antimicrobial Resistance \& Infection Control 10(1): 006

Introduction: Nosocomial infections caused by Vancomycin-resistant Enterococcus (VRE) are on the rise worldwide. A few studies estimated the economic impact of nosocomial outbreaks and revealed that nosocomial infections caused by VRE are associated with prolonged hospital stays and higher hospital costs.

Objectives: The research was performed to evaluate the economic impact of nosocomial pathogens (here VRE) on hospitals.

Methods: We implemented a retrospective cost analysis on infected or colonised patients between January 2018 and June 2019 in three hospitals: Bern, Tiefenau and Münsingen. We used a clinical process modelling for patients who might be or are affected by nosocomial pathogens (here VRE). Data was collected on true hospital costs (in CHF) and closed beds with regard to clinical process indicators and clinical risk factors. We used cost unit accounting (REKOLE) to identify, assign and differentiate the collected costs. This process made it possible to calculate extra costs per VRE case, a comparison between 
colonised/infected vs. non colonised patients and the calculation of the total outbreak costs.

Results: Overall we identified 500 cases of Vancomycin-resistant Enterococcus caused mostly by E. faecium of sequence type ST 796. Multivariable analyses revealed that several factors increased the risk of being colonised with VRE: age, pre-existing condition, stay in ICU, exposure to antibiotics, number of involved healthcare workers, exposure to specific rooms and to contaminated medical devices. They led to prolonged hospital stays and higher economical impact. The overall hospital costs were significantly higher in VRE cases as compared to non colonised patients (22'908 CHF vs. 21'048 CHF). The additional costs due to extra microbiological diagnostics and extra materials were about 1 ' 860 CHF per VRE case. The total costs generated by the nosocomial outbreak were about 7.2 Mio. CHF.

Conclusion: Nosocomial transmissions caused by multidrug-resistant pathogens are associated with an increase of marginal costs by around $10 \%$. These costs are avoidable. Cost drivers are closed hospital beds, diagnostics, and infection preventions activities (including personnel costs). Prevention is the most important measure to avoid nosocomial outbreaks and is likely to be cost-effective.

Disclosure of Interest: None declared.

\section{7}

Whole genome sequencing sustains cross-transmission of pseudomonas aeruginosa in a neonatal intensive care unit during an outbreak

D. S. Blanc ${ }^{1, *}$, L. Senn ${ }^{1}$, I. Federli ${ }^{1}$, E. Giannoni ${ }^{1}$, F. Legault ${ }^{1}$, M. Roth-Kleiner ${ }^{1}$, B. Grandbastien ${ }^{1}$

${ }^{1}$ Centre Hospitalier Universitaire Vaudois, Lausanne, Switzerland Correspondence: D. S. Blanc

Antimicrobial Resistance \& Infection Control 10(1): 007

Introduction: Pseudomonas aeruginosa is a rare pathogen in neonatal intensive care unit (NICU). Following $P$. aeruginosa infections in three NICU patients in June 2020, we performed an epidemiological investigation.

Objectives: To find possible sources and routes of transmission of $P$. aeruginosa infections in NICU patients using standard and genomic typing methods.

Methods: Retrospective analysis of $P$. aeruginosa isolates from patients hospitalized in NICU was performed and we initiated a prospective weekly screening of all patients for $P$. aeruginosa (which included naso-pharyngeal and rectal swabs). Based on previous investigation, environmental samples targeting sink traps were performed to identify $P$. aeruginosa. Isolates were first typed by double locus sequence typing (www.dlst.org/Paeruginosa) and all isolates identical to the outbreak isolates, were further analysed by whole genome Multi Locus Sequence Typing (wgMLST, BioNumerics v.7.6.3).

Results: The three patients harboured isolates from the same DLST6256 genotype. Retrospective analysis of isolates from patients hospitalized in the NICU revealed five other patients with this genotype from April to July 2020. Weekly screening implemented in August revealed eight new patients with $P$. aeruginosa, of whom only three with the epidemic genotype. No isolate from the environment belonged to this genotype. Whole genome sequencing revealed that all DLST62-56 isolates belonged to the MLST ST667. wgMLST of NICU isolates from this genotype revealed they clustered all in the same group with 0 to 6 loci differences between isolates.

Conclusion: The absence of the epidemic DLST in the environment favoured the hypothesis of cross-transmission. This was corroborated by the fact that the outbreak stopped when weekly screenings were implemented and additional contact measures were taken with positive patients. Molecular typing with DLST in the first approach allowed a rapid identification of a putative problem and selecting isolates for finer typing with whole genome sequencing. wgMLST confirmed the close genetic relatedness between these isolates, sustaining the patient-to-patient chain of transmission.

Disclosure of Interest: None declared.
Slide session: Other healthcare-associated infections

\section{8}

Peripheral venous catheters (PVCS) inserted in the hand are associated with lower PVC-related bloodstream infection (BSI) N. Buetti ${ }^{1}{ }^{*}$, M. Abbas ${ }^{1}$, D. Pittet ${ }^{1}$, M.-N. Chraiti ${ }^{1}$, V. Sauvan ${ }^{1}$, M. de Kraker ${ }^{1}$, M. Boisson ${ }^{1}$, D. Teixeira', W. Zingg ${ }^{2}$, S. Harbarth ${ }^{1}$

${ }^{1}$ Geneva University Hospitals, Geneve, ${ }^{2}$ Department of Infectious

Diseases and Hospital Epidemiology, University Hospital Zurich, Zurich, Switzerland

Correspondence: N. Buetti

Antimicrobial Resistance \& Infection Control 10(1): 008

Introduction: Little is known about the risk of bloodstream infection (BSI) associated with peripheral venous catheters (PVCs) and no large study has previously investigated the insertion site-related risk for PVC-BSI.

Objectives: The objective of this study was to investigate the association between anatomical PVC insertion site and risk of BSI using a large prospective database.

Methods: We included all patients hospitalized in the University of Geneva Hospitals between 1st January 2016 and 29th February 2020 with at least one PVC insertion on the upper extremity. We used univariable and multivariable marginal Cox regression models for clustered data to evaluate the risk of PVC-BSI according to the insertion site (hand versus proximal sites in the upper extremities).

Results: 403'206 upper extremity PVCs with documented insertion site were included. There were $73^{\prime} 325$ females (54\%); median age was $49 y$ (IQR 32-69). The median time from hospital admission to catheter insertion was 1 day (IQR 1-4). 109'686 (27\%) PVCs were inserted in the hand. During the study period, 61 PVC-BSIs were documented, 6 originating from PVCs in the hand, and 55 from PVCs at other insertion sites. Hand insertion was associated with statistically significant lower risk of PVC-BSI than proximal insertion in both univariable models (HR $0.42,95 \% \mathrm{Cl} 0.18-0.99, \mathrm{p}=0.046$ ) and after adjusting for gender, age and time from admission to catheter insertion (adjusted HR 0.42, 95\% $\mathrm{Cl} 0.18-0.98, \mathrm{p}=0.046$ ). In a sensitivity analysis for PVCs with $\geq 3$ days of catheter maintenance, we confirmed a decreased PVC-BSI risk after hand insertion (HR 0.37, 95\% Cl 0.15-0.93, $\mathrm{p}=0.035$ ).

Conclusion: Hand insertion showed a decreased daily risk of PVC-BSI, particularly for catheters in place for $\geq 3$ days.

Disclosure of Interest: N. Buetti Grant/Research support from: Post. doc Mobility grant from the Swiss National Science Foundation (grant number: P4P4PM_194449), M. Abbas: None declared, D. Pittet: None declared, M.-N. Chraiti: None declared, V. Sauvan: None declared, M. de Kraker: None declared, M. Boisson: None declared, D. Teixeira: None declared, W. Zingg: None declared, S. Harbarth: None declared.

009

First COVID-19 wave in Dutch hospitals: effects on central venous catheter care and distribution of bacteremia pathogens

T. van der Koo i ${ }^{1, *}$, W. Altorf-van der Kuil ${ }^{1}$, A. Schoffelen ${ }^{1}$, S. van Rooden ${ }^{1}$, S. de Greeff ${ }^{1}$ on behalf of the ISIS-AR study group

${ }^{1}$ Healthcare-associated infections and Antimicrobial resistance, National Institute for Public Health and the Environment (RIVM), Bilthoven, Netherlands

Correspondence: $T$. van der Kooi

Antimicrobial Resistance \& Infection Control 10(1): 009

Introduction: Due to COVID-19, care capacity has been put to the test. Objectives: We investigated how the first wave affected the frequency of bloodstream infection (BSI), the distribution of associated pathogens and the care for patients with a CVC.

Methods: Hospitals can monitor CVC-related (CR)BSI in the national PREZIES surveillance. All 24 hospitals participating in 2020 received a survey on changes in care for patients with CVCs on COVID-19 ICU/wards.

The frequency and distribution of pathogens were studied in data on blood isolates from patients sampled before (October 2019 to February 
2020), during (March to May 2020), and after (June to September 2020) the first COVID-19 wave from the Dutch Infectious Disease Surveillance System-Antimicrobial Resistance (ISIS-AR) database.

Results: CRBSI data entry is delayed by COVID-19, but increased rates were observed. Nine hospitals responded to the survey. During the wave bed capacity increased ( 9 ICU; 3 wards) and external staff assisted (ICU 9; wards 8). Hospitals reported less frequent oral care and/or washing (5), dressing (2), and CVC set changes in the ICU (1), due to the prone position of the patient and/or work pressure.

The total number of patients with a positive diagnostic blood sample during the first wave remained similar to before, but the mean monthly number of patients with a positive sample in the ICU increased from $268(10 \%[95 \% \mathrm{Cl}=10-11])$ to $409(15 \%[14-16])$, and decreased again to 246 (9\% [9-10]) after the first wave. In the ICU the proportion of several pathogens changed during the wave (table). All values normalized afterwards.

\begin{tabular}{|l|l|l|}
\hline Pathogens in ICU & $\begin{array}{l}\text { Before } \\
\text { wave } \\
\%(\mathbf{9 5} \% \mathbf{C l})\end{array}$ & $\begin{array}{l}\text { During } \\
\text { wave } \\
\%(\mathbf{9 5} \% \mathbf{~ C l})\end{array}$ \\
\hline Coagulase negative Staphylococcus spp. (CNS) & $45(42-47)$ & $56(53-58)$ \\
\hline Enterococcus faecium & $9(8-11)$ & $18(16-19)$ \\
\hline E. faecalis & $3(2-4)$ & $7(6-9)$ \\
\hline Escherichia coli & $10(9-12)$ & $4(3-5)$ \\
\hline Klebsiella pneumoniae & $3(2-4)$ & $1(0-1)$ \\
\hline S. aureus & $9(7-10)$ & $5(4-6)$ \\
\hline
\end{tabular}

Conclusion: All hospitals reported increased demand of care, support of external staff and in some downscaled or hampered CVC and patient care. This may partly explain the more frequently sampled CNS in blood during the first COVID-19 wave.

Disclosure of Interest: None declared.

\section{0}

The effectiveness of introducing a multimodal strategy to improve the CVC care bundle and reduce the rates of CLABSI in a tertiary teaching hospital, Malaysia

S. Ponnampalavanar ${ }^{1,2, *}$, A. Rajandra ${ }^{2}$, I. N. Ismail ${ }^{2}$, S. Z. Mohamad Razali ${ }^{2}$, S. Basri ${ }^{3}$, S. F. Syed Omar' ${ }^{1}$ A. Kukreja ${ }^{3}$, H. Zainol' ${ }^{2}$, S. Saaibon², N. R. Hassan² S. N.Zainon@Md Ali

${ }^{1}$ Medicine, University of Malaya, ${ }^{2}$ Infection Control, ${ }^{3}$ Infectious Disease, University Malaya Medical Centre, Kuala Lumpur, Malaysia

Correspondence: S. Ponnampalavanar

Antimicrobial Resistance \& Infection Control 10(1): 010

Introduction: A Central venous catheters (CVC) care bundle was introduced in October 2016 to prevent Central Line Associated Bloodstream Infection (CLABSI) in University Malaya Medical Centre, a tertiary teaching hospital in Malaysia. However, compliance to interventions are usually low and effective implementation strategies are needed for its success.

Objectives: The aim of this study was to assess the impact of an existing CVC Care Bundle in reducing the rates of CLABSI after implementation using multimodal improvement strategy.

Methods: This quasi-experimental study was conducted in all adult medical wards from July 2018 till December 2020. The World Health Organization (WHO) multimodal improvement strategy was used to implement the existing CVC in January 2019. The pre intervention period was from July 2018 to December 2018 and post intervention was from January 2019 to December 2020 which was further categorized into 4 periods of 6 months duration each (P1, P2, P3 and P4) to reflect the various implementation strategies. Data on adherence to CVC Care bundle compliance and incidence rates of CLABSI were collected by the infection control nurse using a standardized checklist. Data was analyzed using SPSS version 23.0.

Results: The total central line analyzed in the pre-intervention was 93 and in the P1, P2, P3 and P4 periods was 261, 398,181 and
467 respectively. The rate of CLABSI reduced from 12.63/1000 line days (pre-intervention) to $5.91 / 1000$ line days (P1), 3.55/1000 I line days (P2), 7.94/ 1000 line days (P3) and 5.70/ 1000line days (P4). The compliance rate of the CVC care bundle in pre- intervention phase was $80.65 \%$ and post intervention was $85.65 \%$ (P1), $85.43 \%$ (P2), $90.61 \%(\mathrm{P} 3)$ and $92.51 \%(\mathrm{P} 4)$.

Conclusion: Implementation of the WHO multimodal strategy improved the compliance to CVC care bundle and showed a decrease in the CLABSI rate. Thus, to ensure the success of a CVC care bundle, it is important to use a well-designed multimodal implementation strategy.

Disclosure of Interest: None declared.

\section{1}

Epidemiology, risk factors and clinical outcomes

of healthcare-associated bloodstream infections due to multidrug-resistant microorganisms in a single-center cohort in northwestern China

F. Liu ${ }^{1,2}$, K. Lei ${ }^{3}$, H. Wang ${ }^{2}$, Y. Wu ${ }^{2}$, J. Wang ${ }^{4}$, D. Pittet ${ }^{5}$, W. Zingg ${ }^{6, *}$ on behalf of Institute of Global Health, Faculty of Medicine, University of Geneva ${ }^{1}$ Institute of Global Health, Faculty of Medicine, University of Geneva,

Geneva, Switzerland, ${ }^{2}$ Department of Nosocomial Infection Management,

${ }^{3}$ Clinical Microbiology Laboratory, The Second Affiliated Hospital, Xi'an

Jiaotong University, Xi'an, China, ${ }^{4}$ Institute of Social Medicine and Health Systems Research, Medical Faculty, Otto von Guericke University,

Magdeburg, Germany, Infection Control Program and WHO Collaborat-

ing Centre on Patient Safety, University of Geneva Hospitals and Faculty

of Medicine, Geneva, ${ }^{6}$ Department of Infectious Diseases and Hospital

Epidemiology, University Hospital Zurich, Zurich, Switzerland

Correspondence: W. Zingg

Antimicrobial Resistance \& Infection Control 10(1): 0011

Introduction: Healthcare-associated BSI (HABSI) due to multidrugresistant microorganisms (MDROs) is a serious concern worldwide, associated with high morbidity and mortality. Unfortunately, epidemiological burden, attributable risk factors and clinical outcome remain unknown in China.

Objectives: This study aimed to investigate the burden of HABSI due to MDRO in terms of both morbidity and mortality among inpatients in Shaanxi.

Methods: Prospective surveillance of HABSI due to MDRO was conducted in a tertiary-teaching hospital in Xi'an, Shaanxi, from 2013 to 2017. We analyzed first HABSI episodes due to MDRO from clinical samples.

Results: 141 inpatients were diagnosed with bloodstream infections due to MDROs for an overall incidence of 39 per 100,000 inpatients $(141 / 361,715)$. Of these, HABSI was responsible for $73.1 \%$. The incidence of HABSI due to MDRO was 28 per 100,000 inpatients. Median length of stay for inpatients with HABSI due to MDRO was 24 days. Among inpatients suffering from HABSI due to $\mathrm{MDRO}$, most $(75.7 \%)$ were secondary bloodstream infections. Lower respiratory tract $(41.7 \%)$ was the most frequent primary infection site, followed by peritoneal cavity and abdominal organs $(14.1 \%)$, and skin and soft tissues (9.0\%). The mortality rates of HABSI due to MDRO within 7 and 28 days were $11.7 \%$ and $69.9 \%$, respectively $(P=0.001)$. Overall, gram-negative pathogens were identified in a large number of HABSI episodes $(N=58$ strains, 56.3\%). Importantly, 28 day-mortality associated with Gram-negative MDROs was significantly higher (40.6\%) compared with Grampositive MRDOs (13.9\%; $P<0.001)$. Septic shock, central venous catheterization, age $\geq 60$ years, HABSI due to Acinetobacter baumannii, and a Pitt Bacteremia Score $\geq 6$ were independent risk factors for death at 28 days by multivariable regression analysis.

Conclusion: This study offers detailed epidemiology of HABSI due to MDRO among inpatients in Shaanxi. Prospective surveillance and infection control measures to prevent cross-transmission and infection 
are needed to reduce the burden of HABSI due to MDRO and its associated mortality.

Disclosure of Interest: None declared.

\section{2}

Impact of a dental care intervention on the hospital mortality of critically ill patients: a quasi-experimental study

I. Ribeiro' ${ }^{1}$, W. Bellissimo-Rodrigues ${ }^{1}$, L. Innocentini ${ }^{1}$, L. Macedo ${ }^{1}$, F.

Barbosa-Júnior ${ }^{1}$, A. P. Pereira ${ }^{1,2}$, H. Souza $^{1}$, W. Lovato ${ }^{1}$, M. Menegueti ${ }^{1,2}, \mathrm{G}$. Gaspar', A. Schmidt', C. Miranda', M. Auxiliadora-Martins' ${ }^{1}$, A. Basile-Filho', F. Bellissimo-Rodrigues ${ }^{1, *}$

${ }^{1}$ Ribeirão Preto Medical School, University of São Paulo, ${ }^{2}$ Ribeirão Preto

Nursing School, University of São Paulo, Ribeirão Preto, Brazil

Correspondence: F. Bellissimo-Rodrigues

Antimicrobial Resistance \& Infection Control 10(1): 012

Introduction: It has been previously demonstrated that providing dental care to critically ill patients improves the prevention of respiratory tract infections. However, no studies have explored the impact of this intervention on hospital mortality.

Objectives: To evaluate the impact of providing regular dental care on the risk of in-hospital death and ventilator-associated pneumonia (VAP) among critically ill patients admitted to intensive care units (ICU).

Methods: A quasi-experimental study through an interrupted time series analysis (2016-2019) was conducted in two ICU, one medical ICU and one specialized in emergency care. The intervention began in January 1st 2019, and consisted of implementing routine dental care three times a week for patients admitted to the study units. Dental care was focused on oral hygiene and subgingival instrumentation. On the pre-intervention period, dentists provided punctual care to patients, focusing more on dental extraction and abscess drainage, as necessary. The primary outcome of the study was the mortality rate, evaluated at the end of the ICU stay. Secondary study outcome was VAP rate. Data was analyzed using the ARIMA (autoregressive integrated moving average) time series model in $\mathrm{R}$ software (version 4.03).

Results: During the intervention period, 5,147 dental procedures were performed among 355 adult patients. The time series showed that the ICU death rates were $36.11 \%, 32.71 \%$, and $32.30 \%$ within the three years before the intervention, and $28.71 \%$ during the intervention period $(p=0.016)$, as demonstrated in Figure 1. VAP rates were $5.21 \%$, $5.02 \%$ and $4.19 \%$ within the three years before the intervention, and $3.59 \%$ during the intervention period $(p=0.072)$.

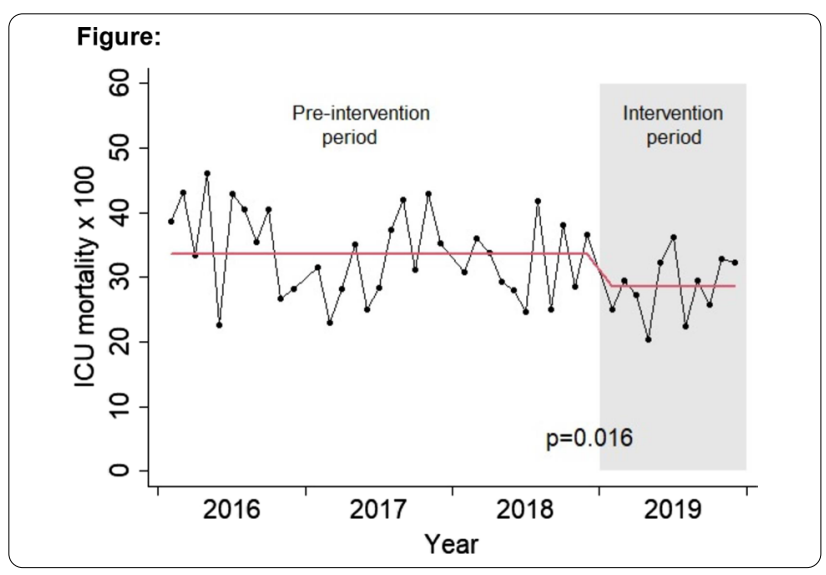

Conclusion: A dental care intervention focused on oral hygiene and subgingival instrumentation provided by dentists to critically ill patients significantly decreases their risk of dying in the ICU.

Funding: FAPESP (process 2017/19166-7, 2018/17531-2) and FAEPA.

Trial registration: $\mathrm{RBR}-4 \mathrm{jmz} 36$.

Disclosure of Interest: None declared.
Slide session: hand hygiene

013

Hand hygiene implementation strongly associated

with availability of funds: results from the WHO hand hygiene self-assessment framework global survey 2019

M. De Kraker ${ }^{1,2,}{ }^{*}$, E. Tartari ${ }^{2,3}$, S. Tomczyk ${ }^{4}$, A. Twymann ${ }^{4}$, L. Francioli ${ }^{5}$, A. Cassini ${ }^{4}$, B. Allegranzi ${ }^{4}$, D. Pittet ${ }^{2,6}$

${ }^{1}$ Infection Control Program, Geneva University Hospitals, ${ }^{2}$ WHO Collaborating Centre on Patient Safety, Geneva, Switzerland, ${ }^{3}$ Infection Control Unit, Mater Dei Hospital, Msida, Malta, ${ }^{4}$ Infection Prevention and Control Technical and Clinical Hub, Department of Integrated Health Services, WHO, Geneva, Switzerland, ${ }^{5}$ Program in Medical and Population Genetics, Broad Institute of MIT and Harvard, Cambridge, United States, ${ }^{6}$ Infection

Control Program, HUG, Geneva, Switzerland

Correspondence: M. De Kraker

Antimicrobial Resistance \& Infection Control 10(1): 013

Introduction: Healthcare-associated infections (HAls) impact the quality of healthcare services, jeopardize patient safety and increase healthcare costs. Improvement in hand hygiene compliance has been highlighted as the most effective measure to lower the incidence of HAl.

Objectives: Ten years after the development of the World Health Organization (WHO) Multimodal Hand Hygiene Improvement Strategy, a global survey was conducted, using the Hand Hygiene Self Assesment Framework (HHSAF), to ascertain the level of hand hygiene implementation and its drivers in healthcare facilities (HCF) worldwide.

Methods: In 2019, IPC professionals were invited through email/ campaigns to complete the online HHSAF. A geospatial clustering algorithm selected unique HCF responses, surveys from countries with low response rates were excluded, and post-stratification weighting was applied to improve representativeness. Weighted, median HHSAF scores and interquartile ranges (IQR) were reported. Drivers of the HHSAF score were determined through a generalized estimation equation, considering country clustering and weights.

Results: In total, 3206 unique responses from 90 countries (46\% of WHO member states) were included. The weighted, median HHSAF score indicated an intermediate hand hygiene level (350 points, IQR 248-430), which was positively associated with country income level and HCF funding structure. Overall, System Change had the highest score (85 points, IQR 55-100), and Institutional Safety Climate the lowest (55 points, IQR 35-75). In low-income countries, Evaluation and Feedback was the weakest element (34 points, IQR 15-65). From 2015 to 2019, the median HHSAF score stagnated.

Conclusion: Most HCFs had an intermediate level of hand hygiene implementation or higher, for which HCF funding and country income level were important drivers. Availability of resources, leadership and organizational support are key elements to further improve quality of care and provide access to safe care for all.

Disclosure of Interest: None declared.

014

Strengthening infection prevention and control programme in Turkey according to WHO's multimodal strategy

E. Alp ${ }^{1,}$, D. Altun ${ }^{1}$, C. H. Hekimoglu ${ }^{1}$, E. Batir ${ }^{1}$, E. Yildirim Gozel ${ }^{1}$, F.

Timurkaynak², M.-L. McLaws ${ }^{3}$

${ }^{1}$ Ministry of Health, Ankara, Turkey, ${ }^{2}$ University of Geneva, Geneva, Switzerland, ${ }^{3}$ UNSW, Sydney, Australia

Correspondence: E. Alp

Antimicrobial Resistance \& Infection Control 10(1): 014

Introduction: Implementation of WHO's multimodal (MM) strategy is essential for strengthening infection prevention and control programme (IPC) in a country. 
Objectives: To share the experience of Turkey about the strengthening infection prevention and control programme.

Methods: In Turkey, structured IPC (infection control committees, training, surveillance, hand hygiene activities) has been put into practice since 2006 by MoH. However, after $2018 \mathrm{MoH}$ focused on IPC core components for strengthening IPC. Electronic surveillance was strengthened. Turkey signed the pledge of hand hygiene and Train the Trainers (TTT) programme was organised for standardized approach based on the WHO MM Hand Hygiene Improvement Strategy. In TTT programme, 34 IPC professionals from 18 hospitals were trained for multimodal $\mathrm{HH}$ improvement strategy and a short TTT programme was organized with 32 participants in the largest teaching hospital in Turkey. After Covid-19 pandemic, videos about multimodal hand hygiene strategy were used for e-learning by $\mathrm{MoH}$. To this e-learning programme, 1845 IPC professionals registered and 1287 of them completed. National Infection Control Committee was established in 2018 and National Infection Control Programme and Action Plan was introduced in 2019. The Hand Hygiene Self-Assessment Framework (HHSAF) was first used nationally in 2019 for the documentation of $\mathrm{HH}$ situation and also focus on the future plans and challenges. HHSAF was translated in Turkish to increase participation of facilities. Results: HHSAF was sent to 216 hospitals and 125 (58\%) submitted their HHSAF. Of these hospitals, 92 (74\%) were state hospital, $20(16 \%)$ were university hospital and $13(10 \%)$ were private hospital. Responses to HHSAF questions related to key indicators of the WHO improvement strategy implementation were shown in Figure 1.

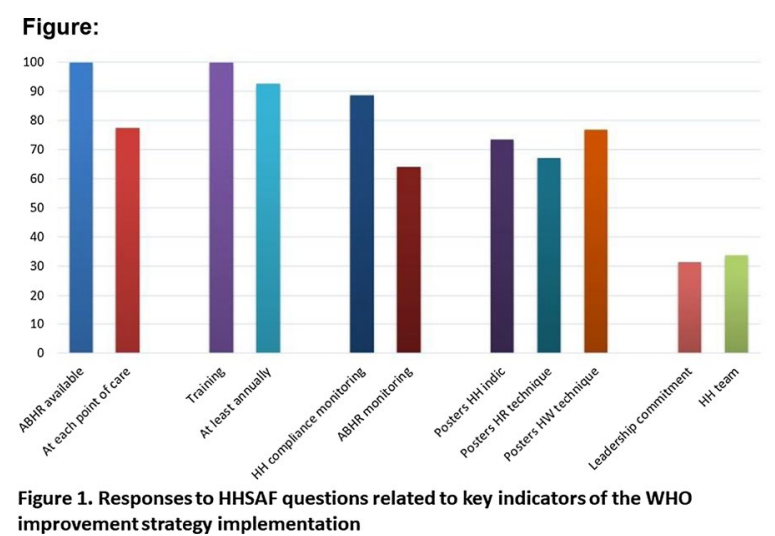

Conclusion: The survey shows that improvement was achieved on having alcohol-based handrubs available, undertaking staff training, evaluation and feedback and displaying posters on hand hygiene around their hospital. However, a dedicated budget in infection control and improvements in institutional safety climate are gaps that should be focused on.

Disclosure of Interest: None declared.

\section{5}

Effectiveness and sustainability of the WHO multimodal hand hygiene improvement strategy in the university hospital Bouaké, Republic of Côte D'ivoire in the context of the COVID-19 pandemic S. Müller 1,*, M. N'Guessann², R. Wood ${ }^{1}$, L. Landsmannn', C. Rocha' ', B. J. Kouame $^{2}$, D. Djadji2 ${ }^{2}$ K. Abrokwa' ${ }^{1}$ S. Tomczyk' , M. Arvand ${ }^{1}$, B. Diané2, M. Borchert $^{1}$

${ }^{1}$ Robert Koch Institute, Berlin, Germany, ${ }^{2} \mathrm{CHU}$, Bouaké, Côte d'Ivoire Correspondence: S. Müller

Antimicrobial Resistance \& Infection Control 10(1): 015

Introduction: Healthcare worker adherence to proper Hand Hygiene $(\mathrm{HH})$ practices and access to alcohol-based hand-rub (ABHR) remains limited in many low-resource settings. An effective $\mathrm{HH}$ improvement strategy is a critical element of infection prevention and control (IPC) programmes, as seen in the ongoing COVID-19 pandemic. The project
PASQUALE aimed to establish the WHO multimodal $\mathrm{HH}$ strategy at the University Hospital Bouaké, Côte d'Ivoire.

Objectives: The project PASQUALE aimed to establish the WHO multimodal HH strategy at the University Hospital Bouaké, Côte d'Ivoire.

Methods: The improvement of $\mathrm{HH}$ knowledge and $\mathrm{HH}$ compliance was assessed in a before-and-after intervention study. The intervention consisted of a $\mathrm{HH}$ training for staff of all departments regardless of their participation in the initial assessment and the introduction of local production of ABHR. Assessment of $\mathrm{HH}$ knowledge and compliance was performed 12 months before (06/2018), right after the intervention (10/2019) and at a ten months interval (08/2020), whereby knowledge was assessed through a self-administered questionnaire and $\mathrm{HH}$ compliance through direct observations.

Results: Baseline knowledge score was 14/25, increased significantly to $17 / 25$ upon the first and decreased to $13 / 25$ in the second assessment. $\mathrm{HH}$ compliance showed a significant increase from $12.7 \%$ to $36.8 \%(p<0.001)$ in first and remained at $36.4 \%$ in second follow-up. An average of $74.4 \mathrm{~L}$ ABHR per month were produced from 07/2019 until 03/2020, when the first COVID-19 infection was confirmed in Côte d'Ivoire, and 138L per month from 04/2020 onwards. Monthly consumption of ABHR in the same time increased from 76 to $125 \mathrm{~L}$.

Conclusion: The implementation of the WHO HH improvement strategy led to sustained improvements in $\mathrm{HH}$ compliance without relying on costly interventions. This study emphasized that the implementation of the strategy is feasible and has effectively contributed to the IPC response during the COVID-19 pandemic.

Disclosure of Interest: None declared.

016

Knowledge and perception on hand hygiene and correlation with IPC practices and structures in health facilities in Uganda H. Kasujja 1,", J. P. Waswa' ${ }^{1}$, R. Kiggundu' ${ }^{1}$, M. Murungi ${ }^{1}$, A. Zagorski², D. Tjipura'2, M. Josh², T. Hafner², F. Alombah²

${ }^{1}$ USAID, MTaPS, MSH, Kampala, Uganda, ${ }^{2}$ USAID, MTaPS, MSH, Washington, United States

Correspondence: $\mathrm{H}$. Kasujja

Antimicrobial Resistance \& Infection Control 10(1): 016

Introduction: IPC is an evidence-based approach to improve patient safety and contain AMR. This reqiures HR capacity building and ensuring system change for IPC programs.

Objectives: To assess knowledge and perception of hand hygiene $(\mathrm{HH})$ among HCWs in Uganda.

To assess the correlation between $\mathrm{HH}$ knowlegde \& perception (KaP) with IPC practices and structues in Ugandan facilities.

Methods: We conducted a cross-sectional study in March 2021. Included a random sample of $140 \mathrm{HCW}$ and 28 senior managers (SMs) from 14 hospitals selected by cluster sampling. We assessed $\mathrm{HH}$ KaP using the WHO HH Knowledge Questionnaire and Perception Surveys for HCWs \& SMs. Facility HH and IPC practices and structures were assessed using the WHO HHSAF and IPCAF tools. Data was collected concurently and analyzed using STATA to compute means, standard deviations (SD) and correlations.

Results: $140 \mathrm{HCWs} ; 4$ (52.86\%) female and 66 (47.14\%) male, and 28 senior managers; 13 (46.43\%) female and $15(53.57 \%)$ male were sampled. The average score of HH knowledge was $42.41 \%$ (SD 10.93), a closely similar study in Ugandan medical students revealed $49.4 \%$.

The HCWs believe that $\mathrm{HH}$ is $84.39 \%$ (SD 5.52) effective in reducing hospital acquired infections (HAls). The SMs believe that the HH program proposed by WHO is $78.09 \%$ effective (SD 9.93) in increasing $\mathrm{HH}$ compliance. The average IPCAF score was 453.61(SD 130.02) corresponding to intermediate level. The mean HHSAF score was 226.69 (SD 49.22) which means a basic hand hygiene level. Knowledge on $\mathrm{HH}$ postively correlated with IPCAF (0.395078) yet negatively correlated with HHSAF (-0.13303). There was a strong correlation (1) between perception of SMs on IPC and knowledge of HCWs.

Conclusion: The results indicate need for increasing knowledge and behavioral interventions on IPC, particularly HH among HCWs. The moderate scores on IPCAF and HHSAF reflect efforts being made in building the national IPC program. The correlation between 
knowledge and perception between senior managers and HCWs suggest that educating and supporting managers could be a good way to increase knowledge among other HCWs. We intend to use the results in addressing key knowledge gaps.

Disclosure of Interest: None declared.

\section{7}

Reduction of multidrug resistant pathogens and clostioides difficile after implementation of an individualised behavior monitoring system to increase hand hygiene compliance

C. Alefelder ${ }^{1, *}$, R. Brinks ${ }^{2}$

${ }^{1}$ Hosptial Hygiene and Infection prevention, Helios University Hospital Wuppertal, Wuppertal, ${ }^{2}$ Chair for Medical Biometry and Epidemiology, University Witten-Herdecke, Witten, Germany

Correspondence: C. Alefelder

Antimicrobial Resistance \& Infection Control 10(1): 017

Introduction: Hospital aquired infections with multidrugresistant pathogens (MDRO) and Clostridioides difficile associated diarrhea (CDAD) are a major thread to patients. Improving hand hygiene compliance among health care professionals (HCPs) is the most effective way to reduce nosocomial infections (NI).

Objectives: Aim of this pilot study was to assess efficiacy of a personal technological behaviour monitor system to increase the hand hygiene compliance and reduce the incidence of MDRO and CDAD.

Methods: We conducted a prospective, pre- post study over two years on two university non intensive-care hospital wards. Every HCP had to wear a smart badge with an alcohol vapour sensor. This device collects data and gives direct feedback about hand hygiene related behavior via light, vibration and display information. Furthermore, summary data is presented automatically to all HCPs on the ward. We calculated the incidence density per 100.000 patients days (pd) for infections with MDRO (MRSA, MRGN, VRE) and CDAD at each ward according to the protocol of the National Reference Center (NRZ) for NI in Germany. A Poisson model was used to estimate the effect of the intervention in terms of $\mathrm{NI}$ incidence density reduction (IDR) with $95 \%$ confidence interval (CI). To evaluate the hand hygiene compliance and consumption of hand disinfectant, we used a linear regression for the logit-transformed compliance and a linear regression over the two years, respectively.

Results: The incidence density of NI with MDRO and CDAD for ward A was in the pre period $66.4(\mathrm{Cl}: 65.2-67.7)$ and in the intervention period 40.0 (Cl: $39.3-40.8$ ) (per 100,000 pds). Ward B could reduce the incidence density from 75.7 (Cl:74.7-76.8) to 32.5 (Cl:32.0-33.0). IDR was $51.5 \%$ ( $\mathrm{Cl}: 39.0-61.4, \mathrm{p}=0.0251$ ). Due to the intervention, the hand hygiene compliance rate increased by factor 1.293 (Cl:0.437-2.148, $\mathrm{p}=0.098)$ and the consumption about $4.285 \mathrm{ml}$ per pd (Cl:1.863$6.707, p=0.074$ ).

Conclusion: The implementation of a personal behavior monitoring system increased the hand hygiene compliance and hand desinfection consumption and reduced the rate of MDRO and CDAD. Due to different electronic monitoring systems and the question of sustainability, there is a need for further studies.

Disclosure of Interest: None declared.

\section{8}

Development of practice-simulation in vivo efficacy test method for alcohol-based hand rubs based on EN 1500 and ASTM E2755 F. H. H. Brilll, ${ }^{1}$, R. Leslie R. ${ }^{2}$, T. Neal ${ }^{2}$, S. Pahll ${ }^{1}$, M. Suchomel ${ }^{3}$, D. R. Macinga ${ }^{2}$ ${ }^{1}$ DR. BRILL + PARTNER GMBH INSTIUTE FOR HYGIENE AND MICROBIOLOGY, Hamburg, Germany, ${ }^{2}$ GOJO Industries, Akron, Ohio, United States, ${ }^{3}$ Medical University Vienna, Wien, Austria

Correspondence: F. H. H. Brill

Antimicrobial Resistance \& Infection Control 10(1): 018
Introduction: The WHO and US CDC have called for the development of improved methods for evaluating efficacy of hand hygiene products, which more closely reflect typical clinical use.

Objectives: The aim of the study was to select test organisms with high environmental stability known for their potential to be spread by contaminated hands. In addition, the application of contamination and preparations volume should simulate practical conditions. Individual conditions such as hand size were considered.

Methods: Methodology was based on EN 1500:2017. Test organisms were Enterococcus faecalis (ATCC 47,077) or Escherichia coli K12 (NCTC 10,538). Hand contamination was performed either via the immersion method from EN 1500 or via spreading of $0.5 \mathrm{~mL}$ of a high titer virus suspension over all surfaces of both hands ("low volume contamination") according to ASTM E2755. 50 subjects were categorized among 3 groups of either small, medium, or large hand sizes and 1,2, or $3 \mathrm{~mL}$ volumes of $60 \% \mathrm{v} / \mathrm{v} 2$-propanol were applied and rubbed in until dry, respectively.

Results: Mean log pre-values for $E$. faecalis contamination by immersion were $6.56 \pm 0.27$ and $6.29 \pm 0.60$ and mean log reduction factors (RF) were $5.68 \pm 0.92$ and $4.63 \pm 0.26$, at the respective labs. Mean log pre-value for $E$. coli contamination by immersion was $5.38 \pm 0.55$ and mean log RF was $4.61 \pm 0.74$. Mean log pre-values for $E$. faecalis contamination by low volume contamination were $5.78 \pm 0.40$ and $6.82 \pm 0.09$ and mean log RFs were $5.03 \pm 0.91$ and $5.16 \pm 0.56$, at the respective labs. Mean log pre-value for $E$. coli contamination by rubbing in was $4.76 \pm 0.52$ and mean log RF was $3.86 \pm 0.95$. E. faecalis yielded pre-values that exceeded those of $E$. coli by approximately 1-2 log units using either mode of contamination, suggesting greater survival of $E$. faecalis during the drying procedure. The activity is depending on application volume and hand size.

Conclusion: E. faecalis demonstrates strong potential as a test organism representing a pathogen with high environmental stability known to be spread by contaminated hands in healthcare settings. Additionally, the low-volume contamination procedure simulates the condition of hands when ABHR use is indicated in clinical settings more closely. Disclosure of Interest: None declared.

019

Hand sampling: what's the best method?

H. Soule ${ }^{1, *}$, R. Martischang ${ }^{1}$, N. Akoum ${ }^{1}$, C. Fankhauser ${ }^{1}$, J. Sauser ${ }^{1}$, D. Pittet $^{1}$

${ }^{1}$ Infection Control Programme and WHO Collaborating Centre on Patient Safety, Geneva University Hospitals, Geneva 4, Switzerland

Correspondence: $\mathrm{H}$. Soule

Antimicrobial Resistance \& Infection Control 10(1): 019

Introduction: Hands are often sampled when assessing hand hygiene programs or studying microorganisms' transmission and the hand microbiome. The most common methods used are agar print or rubbing with a swab. But the sensitivity of these methods is poorly understood.

Objectives: In a laboratory experiment, we compared 4 methods for sampling artificially contaminated hands.

Methods: Ten volunteers randomly performed 6 tests. In each test, the following sequence was repeated 4 times: hand hygiene with isopropanol $60 \%$, contamination of the fingertips of one hand with a known amount of bacteria, drying for $3 \mathrm{~min}$, and sampling with one of the 4 tested methods in random order. Fingertips were contaminated with E coli NCTC 10,538 in 3 tests (with about $10^{2}$ or $10^{4}$ or $10^{6}$ bacteria) and with S aureus NC10788 in the 3 other tests. The 4 methods tested were: $M 1 /$ fingerprint on agar plate, $M 2$ /fingertips rubbing with a swab which was vigorously shaken in $10 \mathrm{ml}$ nutritive broth, M3/ fingertips rubbing in a plate containing $10 \mathrm{ml}$ nutritive broth and M4/ massage of fingertips in a sterile bag containing $10 \mathrm{ml}$ nutritive broth. For the 3 last methods, both dilutions and filtration of the recovering 
solution were inoculated on agar. All agar plates were incubated for $48 \mathrm{~h}$. Colonies number was compared to the baseline inoculum. A linear mixed model was used with the inoculum, the species and the sampling method as fixed effect.

Results: Pairwise comparisons between the 4 methods demonstrate that $M 3$ and $M 4$ were not significantly different but superior to $M 1$ and $M 2$. The ratio of geometric means of bacteria recovered with $M 3$ vs $M 1$ and vs $M 2$ were respectively 23.91 and 4.78 , and with $M 4$ vs $M 1$ and vs $M 2$, it was respectively 40.62 and $8.12(p<0.001)$. The amount of bacteria recovered was different between the 2 species for each method: the numbers of bacteria recovered with $\mathrm{M} 3$ and $\mathrm{M} 4$ were respectively 29 and 64 times higher for $S$ aureus than for $E$ coli $(p<0.001)$. With $E$ coli, less than $1 \%$ of the inoculum was recovered with $M 4$, whereas for Saureus, it was $37 \%(p<0.001)$.

Conclusion: Many more bacteria were collected when fingers were sampled in a liquid, either by rubbing or by massage, in relation to the agar print or swab. Regardless of the inoculum, the dose recovered was significantly higher for $S$ aureus compared to $E$ coli.

Disclosure of Interest: None declared.

\section{Slide session: Outbreak}

\section{0}

Role of the environmental reservoir in carbapenem-resistant enterobacteriaceae (CPE) outbreaks

C. Dananché ${ }^{1,2, *}$, S. Gardes ${ }^{1}$, O. Dauwalder ${ }^{3}$, P. Ceruse ${ }^{4}$, S. Couraud ${ }^{5}$, G. Devouassoux $x^{6}$, L. Dortet ${ }^{7}$, P. Cassier ${ }^{2,8}$, P. Vanhems $s^{1,2}$

${ }^{1}$ Infection control unit, Hospices Civils de Lyon, ${ }^{2}$ Centre International de recherche en infectiologie, Université Claude Bernard Lyon 1, ${ }^{3}$ Microbiology laboratory, Hospices Civils de Lyon, Lyon, ${ }^{4}$ ENT unit, Hospices Civils de Lyon, France, ${ }^{5}$ Pneumology Unit, Hospices Civils de Lyon Groupement Sud, ${ }^{6}$ Pneumology unit, Hospices Civils de Lyon Groupement Nord, Lyon, ${ }^{7}$ CNR Résistance aux Antibiotiques, Assistance Publique Hôpitaux de Paris, Paris, ${ }^{8}$ Environmental Microbiology Laboratory, Hospices Civils de Lyon, Lyon, France

Correspondence: C. Dananché

Antimicrobial Resistance \& Infection Control 10(1): 020

Introduction: Since 2019, four outbreaks of carbapenemase-producing Enterobacteriaceae (CPE) digestive colonizations, involving 73 patients, occurred in 4 wards of a multi-site universitary-affilated hospital. Citrobacter freundii OXA-48 was the most frequently isolated bacteria in patient samples.

Objectives: The aim of this study was to assess the role of environment (and particularly toilet bowls) during CPE outbreaks and the efficiency of enhanced cleaning of the toilet bowls.

Methods: Visual observations and environmental sampling of bathrooms were performed between $03 / 2019$ and $01 / 2020$. Patients with CPE digestive colonization were not hospitalized in rooms when samples were collected. During one outbreak, OXA-48-producing $C$. freundii isolates were sequenced using whole genome sequencing. Enhanced cleaning of toilet bowls was performed using steam and hydrochloric acid.

Results: The presence of crevices and dead space (rim, water supply line) in toilets complicated the cleaning and disinfection with a toilet brush. 234 environmental samples were collected in bathrooms of the wards. CPE (mainly C. freundii OXA-48) were isolated in $37 \%$ to $59 \%$ of the sampled toilets bowls and 11 to $29 \%$ of the close environment of the toilet (Table).

In one unit, a specific clone of $C$. freundii OXA-48 was isolated from the toilet bowl of a room on 01/07/2019. A patient without CPE digestive colonization at admission was hospitalized in the same room between $01 / 10 / 2019$ and 30/10/2019, and acquired the same $C$. freundii OXA48 clone on 30/10/2019. After discharge of the patient and cleaning of the toilet bowl, the same clone was found again on 31/10/2019. Enhanced cleaning showed a mild efficacy.

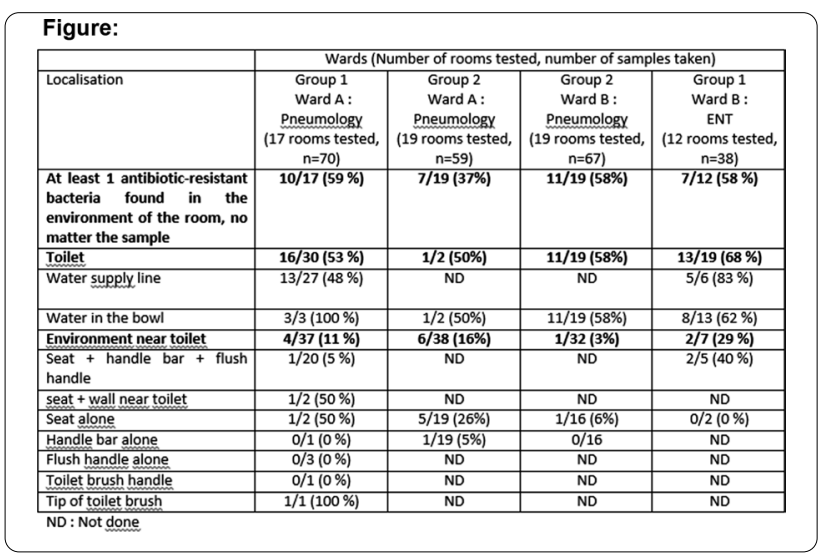

Conclusion: This study showed that the environment plays a role in CPE transmission. Targeted measures as the installation of easy-toclean toilets with limited dead spaces (rim-free), enhanced daily cleaning, the education of patients regarding hand hygiene might help to control these CPE outbreaks.

Disclosure of Interest: None declared.

021

An azole-resistant C. parapsilosis outbreak in a Spanish hospital: an emerging challenge during the COVID-19 pandemic

M. Puig-Asensio 1,*, E. Jiménez', M. Aguilar ${ }^{1}$, J. Ayats ${ }^{1}$, O. Zaragoza ${ }^{2}$, L. Gavaldà', C. Gutiérrez'1 , L. Alcázar-Fuoli², C. Ardanuy', A. Padullés', J. Carratalà', M. Pujol ${ }^{1}$

${ }^{1}$ Bellvitge University Hospital, Barcelona, ${ }^{2}$ Spanish National Centre for Microbiology, Madrid, Spain

Correspondence: M. Puig-Asensio

Antimicrobial Resistance \& Infection Control 10(1): 021

Introduction: Candida parapsilosis is the second leading cause of candidemia and fluconazole is the treatment of choice. In July 2020, an outbreak caused by azole-resistant C. parapsilosis (ARCP) strains was detected in the Intensive Care Unit (ICU) of a tertiary-care center in Spain.

Objectives: This study aims to describe an ongoing ARCP outbreak that started during the COVID-19 pandemic.

Methods: We implemented a package of interventions: 1) collection of surveillance cultures (throat, axilla, and groin) on patient admission and weekly thereafter; 2 ) daily chlorhexidine bathing of patients; 3) change of daily routine cleaning from $1 \%$ sodium hypochlorite to hydrogen peroxide. The presence of ERG11 mutations and the genotyping of 17 random ARCP isolates were performed by microsatellite analysis. Changes in annual defined daily doses (DDD) of antifungals/100 patient-days from 2019 to 2020 were reported to assess antifungal overuse. Cultures from the environment and gowns were obtained with a sterile gauze soaked in saline solution.

Results: Between April 2020-May 2021, 239 patients (2 retrospectively identified) were found to be colonized or infected with an ARCP. Patients were initially identified in a COVID-19 ICU ward, but other units were affected after transferring colonized patients. Microbiological analysis: $15 / 17(88.2 \%)$ strains had the $\mathrm{Y} 132 \mathrm{~F}$ mutation in the ERG11 gene, 1 had the K143R mutation, and 1 did not have any mutations. Most ARCP isolates belonged to the same genotype III. The use of antifungals increased 1.4-fold from 2019 to 2020 ( 2.7 to 3.7 DDD/100 patient-days). Environmental cultures obtained before daily routine cleaning were positive for ARCP strains in 34/88 (38.6\%). Patient care equipment, surfaces in close contact to the patient, and high-touch surfaces were frequently contaminated. Two out of 3 reusable gowns ( $99 \%$ polyester), cultured after patient care, and 1 sink were contaminated with ARCP.

Conclusion: The frequent contamination of the environment coupled with suboptimal infection prevention practices during the COVID-19 
pandemic (shared gowns between patients) have been important challenges to control an ARCP outbreak.

Disclosure of Interest: None declared.

\section{2}

Outbreak of wildtype klebsiella pneumoniae in a neonatal

\section{intensive care unit}

G. Catho ${ }^{1, *}$, V. Sauvan ${ }^{2}$, F. Rosa Mangeret ${ }^{3}$, O. Baud ${ }^{3}$, G. Renzi ${ }^{4}$, J.

Schrenzel ${ }^{4}$, S. Harbarth ${ }^{2}$

${ }^{1}$ Infection Prevention Control, Geneva University Hospitals and Fac-

ulty of Medicine, Switzerland, ${ }^{2}$ Infection Prevention Control, ${ }^{3}$ Division

of Neonatology and Pediatric Intensive Care, ${ }^{4}$ Bacteriology Laboratory,

Division of Laboratory Medicine ", Geneva University Hospitals and Faculty

of Medicine, Geneva, Switzerland

Correspondence: G. Catho

Antimicrobial Resistance \& Infection Control 10(1): 022

Introduction: Outbreaks of multidrug resistant (MDR) Klebsiella pneumoniae in neonatal intensive care units (NICU) have largely been reported but wildtype $K$. pneumoniae outbreaks have rarely been described.

Objectives: We describe the investigation of a $K$. pneumoniae outbreak in a NICU and the control measures implemented.

Methods: Three consecutive point prevalence surveys (PPS) searching for intestinal or cutaneous colonisation by K. pneumoniae among neonates were performed between November 2020 and March 2021. We also conducted environmental sampling of incubators, ultrasound gel and sinks. All K. pneumoniae isolates were investigated by whole genome sequencing (WGS) using RidomSeqSphere v.5.1.0.

Results: Between October and November 2020, 7 neonates hospitalised in the NICU of a university-affiliated tertiary care centre in Switzerland, were found to be infected ( 3 bloodstream infection, 1 intra-abdominal infection) or colonised at a catheter insertion site (3) by a wildtype, multi-susceptible $K$. pneumoniae strain. Eight additional neonates were found colonised by a wildtype $K$. pneumoniae strain during the first PPS, 1 during the second PPS and none during the third PPS, leading to a total number of 13 neonates infected or colonised. WGS identified two distinct clusters of respectively 2 and 10 cases (ST282 and ST 20) and one strain (ST-3132) was only found in one patient (Figure 1). All environmental samples were negative. Audits of best practices were performed and a working group with IPC practitioners and NICU staff members was implemented to reinforce hand hygiene and environmental cleaning, provide feedback and re-assess infection control procedures. No further invasive infection due to $K$. pneumoniae occurred over the 6 months following the outbreak detection.

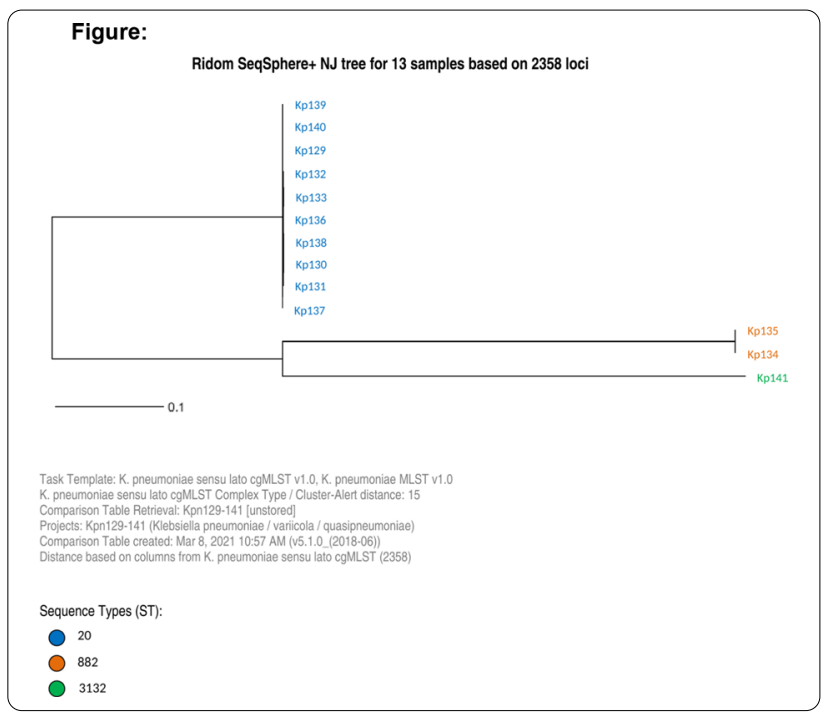

Conclusion: WGS can highlight in-hospital transmission events with multi-susceptible strains that could go undetected. Reinforced infection control measures, particularly hand hygiene, proved effective in terminating the outbreak.

Disclosure of Interest: None declared.

Slide session: COVID-19 in long-term care facilities

023

Hospital-level work organization drives the spread of SARS-COV-2 within hospitals: insights from a multi-ward model

A. Oodally ${ }^{1,2,3, *}$, P. Hammami ${ }^{1,2,3,4}$, A. Reilhac ${ }^{5}$, G. Guérineau de Lamérie ${ }^{5}$ L. Opatowski ${ }^{1,2}$, L. Temime ${ }^{3,6}$

${ }^{1}$ Institut Pasteur, Epidemiology and Modelling of Antibiotic Evasion (EMAE), Paris, ${ }^{2}$ Université Paris-Saclay, UVSQ, Inserm, CESP, Anti-infective evasion and pharmacoepidemiology team, Montigny-Le-Bretonneux,

${ }^{3}$ Modélisation, épidémiologie et surveillance des risques sanitaires (MESURS), Conservatoire national des arts et métiers, Paris, ${ }^{4}$ ANSES, French Agency for Food, Environmental and Occupational Health \& Safety Epidemiology, Health and Welfare Research Unit, Ploufragan-Plouzané-Niort Laboratory, Ploufragan, ${ }^{5}$ Département d'information médicale, Centre hospitalier Guillaume Régnier, Rennes, ${ }^{6}$ PACRI unit, Institut Pasteur, Conservatoire national des arts et métiers, Paris, France

Correspondence: A. Oodally

Antimicrobial Resistance \& Infection Control 10(1): 023

Introduction: Despite extensive protective measures, SARS-CoV-2 extensively circulates within health care facilities, threatening those essential to the fight against the pandemic while putting vulnerable patients at risk. Several control strategies have been proposed; however, the global efficacy of local measures implemented at the ward level may depend on hospital-level organizational factors.

Objectives: We aimed at better understanding the role of betweenward interactions on nosocomial outbreaks and their control in a multi-ward Long Term Care Facility (LTCF).

Methods: We built a stochastic compartmental transmission model of SARS-CoV-2 in a 24-ward LTCF, accounting for the various infection states among patients and staff and between-ward connections resulting from staff sharing. The model was specifically parametrized to mimic detailed data from a psychiatric hospital in Western France. We first evaluated the potential of hospital-wide diffusion of local outbreaks. We then assessed control strategies, including a screening area upon patient admission, an isolation ward for COVID-positive patients and changes in staff schedules to limit between-ward mixing.

Results: Much larger and more frequent outbreaks occurred when the index case originated in one of the most connected hospital wards with up to four times more transmissions when compared to the more isolated wards. The number of wards affected was decreased by $62 \%$ on average after limiting the number of healthcare workers shared between wards while the reverse led to a $75 \%$ decrease. In addition, we found that setting up an isolation ward for all COVID-positive patients reduced the number of transmissions by up to $60 \%$, while adding a screening area before admission seemed ineffective. 

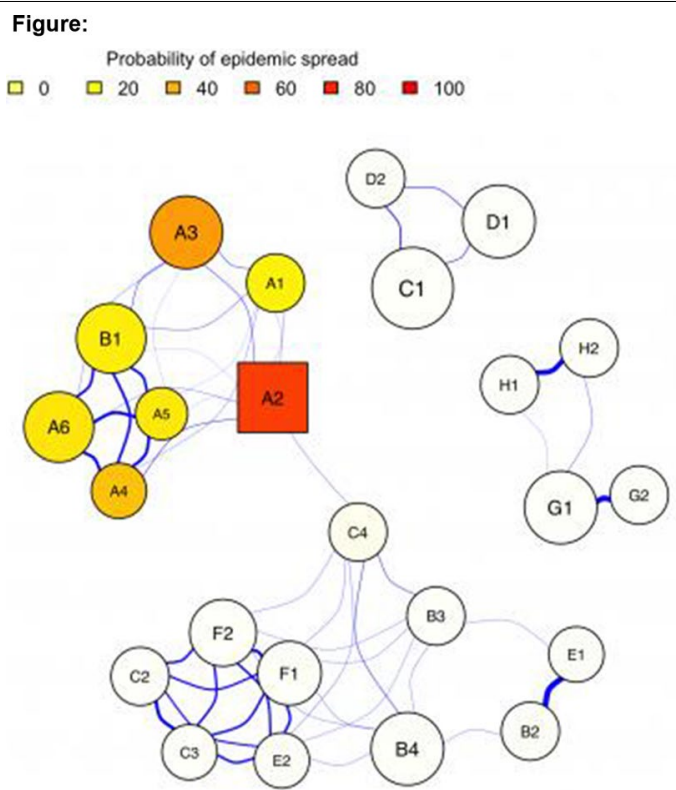

Epidemic spread following the introduction of a non-contagious incubating patient in ward A2 based on 500 stochastic simulations

Conclusion: Our findings highlight the importance of diligent monitoring of COVID-19 transmission in LTCFs and hospitals, which host fragile patients. In particular, hospital-level work organization plays a significant role in driving the spread of infection and should not be overlooked when designing surveillance and control strategies. Disclosure of Interest: None declared.

\section{4}

Mass rapid diagnostic testing as a public health response to SARS-COV-2 outbreaks in long-term care settings D. Smith 1,2,3,4,*, A. Duval 1,2,3, L. Temime ${ }^{4}$, L. Opatowski ${ }^{1,2,3}$

${ }^{1}$ Institut Pasteur, ${ }^{2}$ UVSQ, ${ }^{3}$ Inserm, ${ }^{4}$ Conservatoire National des Arts et Métiers, Paris, France

Correspondence: D. Smith

Antimicrobial Resistance \& Infection Control 10(1): 024

Introduction: Antigen rapid diagnostic testing (Ag-RDT) is more affordable and less logistically demanding than RT-PCR for SARSCoV-2 surveillance, but less diagnostically sensitive. This trade-off has fuelled debate about whether Ag-RDT convenience outweighs elevated risk of false negative diagnoses.

Objectives: To evaluate the public health performance of reactive population screening using Ag-RDT in response to emerging nosocomial SARS-CoV-2 outbreaks.

Methods: We simulated SARS-CoV-2 outbreaks across a range of long-term care facilities (LTCFs) with different Covid-19 interventions in place (social distancing, face masks, vaccination). Assuming that individuals with true positive test results were isolated and no longer transmitted, we evaluated performance of routine RT-PCR testing (tests for all individuals with Covid-19 symptoms and all new hospital admissions) and reactive mass screening using $\mathrm{Ag}-\mathrm{RDT}$ (population-wide testing upon outbreak detection). Imperfect and time-varying diagnostic sensitivity was accounted for. We reported efficacy as the proportion of nosocomial infections averted in the two weeks following outbreak detection, and efficiency as the number of nosocomial infections averted per 1,000 tests used.
Results: Across LTCFs, routine RT-PCR testing prevented a mean $40-47 \%$ of nosocomial infections. With the addition of a single round of reactive Ag-RDT screening, 58-63\% of infections were averted. This increased to up to $69-75 \%$ when conducting a further second round of screening, with greatest performance $4-5$ days after the first round. In addition to routine RT-PCR, a single round of well-timed Ag-RDT screening prevented a mean 15.2 infections/1000 Ag-RDT tests in a high-risk LTCF, but in a low-risk LTCF just 0.5 infections/ 1000 test.

Figure:

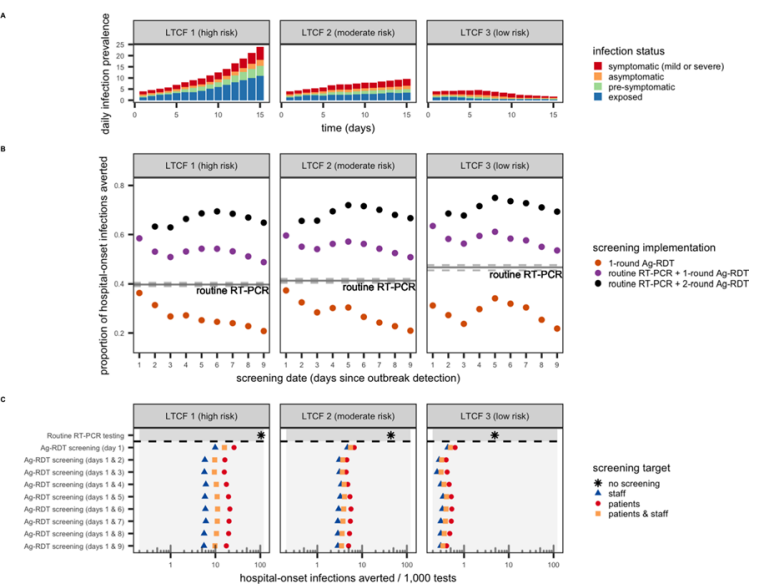

Conclusion: For LTCFs alerted to potential SARS-CoV-2 outbreaks, reactive mass Ag-RDT screening is an effective means to detect asymptomatic and pre-symptomatic infections and limit subsequent nosocomial transmission. A well-timed second round of follow-up screening can help overcome limited and time-varying nature of Ag-RDT sensitivity. Health-economic gains of Ag-RDT screening scale with outbreak risk.

Disclosure of Interest: None declared.

025

Explosive nosocomial outbreak of SARS-COV-2 in a rehabilitation clinic: the limits of genomics for outbreak reconstruction

M. Abbas ${ }^{1, *},{ }^{\prime}$,. Robalo Nunes ${ }^{1}$, A. Cori ${ }^{2}$, S. Cordey ${ }^{1}$, F. Laubscher ${ }^{1}$, S. Baggio $^{1}$, T. Jombart ${ }^{3}$, A. Iten ${ }^{1}$, L. Vieux ${ }^{1}$, D. Teixeira ${ }^{1}$, M. Perez ${ }^{1}$, D. Pittet ${ }^{1}$, E. Frangos ${ }^{1}$, C. Graf ${ }^{1}$, W. Zingg ${ }^{1}$, S. Harbarth

${ }^{1}$ Geneva University Hospitals, Geneva, Switzerland, ${ }^{2}$ Imperial College London, ${ }^{3}$ London School of Hygiene and Tropical Medicine, London, United Kingdom

Correspondence: M. Abbas

Antimicrobial Resistance \& Infection Control 10(1): 025

Introduction: Nosocomial outbreaks of SARS-CoV-2 are frequent despite implementation of conventional infection control measures.

Objectives: To use advanced genomic and statistical techniques to reconstruct transmission chains and assess the role of healthcare workers (HCWs) in SARS-CoV-2 transmission.

Methods: We investigated a nosocomial SARS-CoV-2 outbreak in a university-affiliated rehabilitation clinic, involving patients and HCWs, with high coverage of pathogen whole genome sequences. We estimated the time-varying reproduction number $\left(R_{t}\right)$ and produced a maximum likelihood phylogenetic tree. We combined genomic and epidemiological data into a Bayesian framework to model directionality of transmission. We performed a case-control study to investigate risk factors for nosocomial SARS-CoV-2 acquisition in patients.

Results: The outbreak spanned from 14.03-12.04.2020 and involved 37 patients and 39 employees ( $37 \mathrm{HCWs).} \mathrm{We} \mathrm{estimated} \mathrm{a} \mathrm{peak} R_{t}$ between 2.2-3.6. The phylogenetic tree showed showed genetically almost identical isolates, as $97 \%$ of isolates formed one large cluster. Despite uncertainty in reconstructed transmission events, our 
analyses suggest that HCWs (one of whom was the index case) had a significantly larger fraction of infections $(p<2.2 e-16)$ attributable to HCWs $(71 \%)$ than expected given the number of HCW cases $(47 \%)$. The excess of transmission from HCWs was larger when considering infection of patients $(79 \% ; 95 \% \mathrm{Cl} 78.5 \%>79.5 \%)$, and especially frail patients (Clinical Frailty Scale > 5: 82\%; 95\%Cl 81.8\% > 83.4\%). Furthermore, frail patients were found to be at higher risk for nosocomial COVID-19 than other patients (adjusted OR 6.9; 95\% Cl 2.1-22.5).

Conclusion: This outbreak report highlights the essential role of HCWs in SARS-CoV-2 transmission dynamics in healthcare settings. Limited genetic diversity in pathogen genomes hampered the reconstruction of individual transmission events, resulting in substantial uncertainty in who infected whom. However, our study shows that despite such uncertainty, significant transmission patterns can be observed. Disclosure of Interest: None declared.

\section{6}

\section{Is COVID-19 in hospital employees automatically} an occupational disease? SARS-COV-2 acquisition modes and rates among employees in a long-term care facility (LTCF) during the first pandemic wave

L. Lenggenhager ${ }^{1, *}$, R. Martischang ${ }^{1}$, J. Sauser ${ }^{1}$, M. Perez $^{1}$, L. Vieux ${ }^{2}, C$ Graf $^{3}$, S. Cordey ${ }^{4,5}$, F. Laubscher ${ }^{4}$, T. Robalo Nunes ${ }^{1}$, W. Zingg ${ }^{1}$, A. Cori ${ }^{6}$, M. Abbas $^{1,7}$, S. Harbarth ${ }^{1,5}$

${ }^{1}$ Infection Control Program, ${ }^{2}$ Occupational Health Service, ${ }^{3}$ Department of Rehabilitation and Geriatrics, ${ }^{4}$ Laboratory of Virology, HUG, ${ }^{5}$ Faculty of Medicine, UniGE, GE, Switzerland, ${ }^{6}$ MRC GIDA, ${ }^{7} \mathrm{HPRU}$, Imperial College London, London, United Kingdom.

Correspondence: L. Lenggenhager

Antimicrobial Resistance \& Infection Control 10(1): 026

Introduction: LTCFs are at risk of COVID-19 outbreaks but evidence regarding SARS-CoV-2 acquisition and transmission routes among their employees remains weak.

Objectives: We investigated the relative contribution of occupational (vs. community) exposure for COVID-19 acquisition among employees of a university affiliated LTCF in Switzerland, from March to June 2020.

Methods: This is a prospective cohort study with a nested analysis of a COVID-19 seroprevalence study among LTCF staff. We performed Poisson regression to determine risk factors for seropositivity and to measure the influence of community vs. nosocomial exposure to COVID-19 on SARS-CoV-2 seropositivity using adjusted prevalence ratios (aPR). In addition, we conducted a COVID-19 outbreak investigation in a LTCF ward using both epidemiological and genetic sequencing data. We constructed a maximum likelihood phylogenetic tree and evaluated strain relatedness to discriminate between community- vs. hospital-acquired infections among employees.

Results: Among 285 LTCF employees, we included 176 participants in the seroprevalence study, of whom $30(17 \%)$ became seropositive for SARS-CoV-2. The majority $(141 / 176,80 \%)$ were healthcare workers and had $\geq 1$ symptom compatible with COVID-19 (127/167, 76\%). Risk factors for seropositivity included exposure to a COVID19 patient in the LTCF (aPR 2.6; 95\% Cl 0.9-8.1) and exposure to a SARS-CoV-2 positive person in the community (aPR $1.7 ; 95 \% \mathrm{Cl} 0.8-$ 3.5). Among 18 employees included in the outbreak investigation, phylogenetic analysis suggests that $8(44 \%)$ acquired their infection in the community.

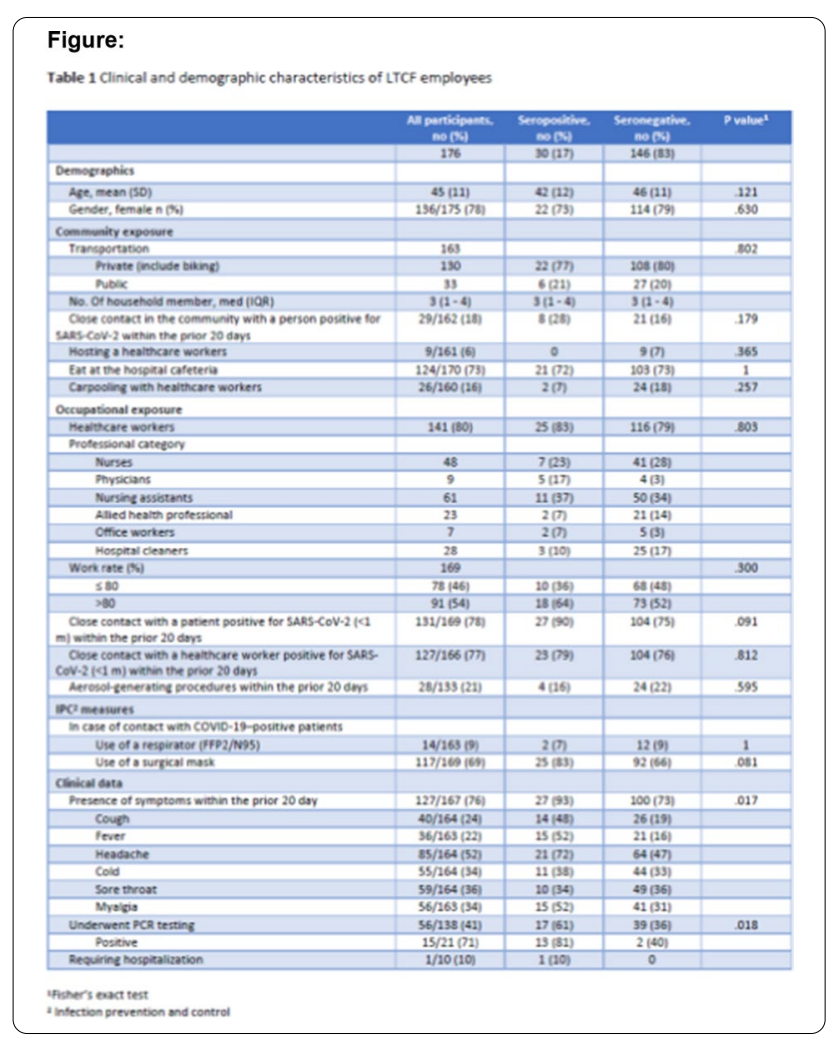

Conclusion: During the first pandemic wave, there was a high burden of COVID-19 among LTCF employees. Both occupational and community exposures contributed to seropositivity and infection risk. These data may allow to better assess occupational health hazards and related legal implications during the COVID-19 pandemic.

Disclosure of Interest: None declared.

\section{7}

Estimating SARS-COV-2 transmission and efficacy of preventive measures in a long-term care facility

G. Shirreff ${ }^{1,2,3, *}$, J.-R. Zahar ${ }^{4}$, L. Temime ${ }^{1,5}$, L. Opatowski ${ }^{2,3}$

${ }^{1}$ Laboratoire MESuRS, Conservatoire National des Arts et Métiers, ${ }^{2}$ Epidemiology and Modelling of Antibiotic Evasion unit, Institut Pasteur, Paris,

${ }^{3}$ Laboratoire UMR1018, University of Versailles Saint-Quentin-en-Yvelines, Montigny-le-Bretonneux, ${ }^{4}$ Infection Control Unit, Hôpital Avicenne, AP-HP, ${ }^{5}$ PACRI Unit, Institut Pasteur, Conservatoire national des arts et métiers, Paris, France

Correspondence: G. Shirreff

Antimicrobial Resistance \& Infection Control 10(1): 027

Introduction: Hospitals and nursing homes are particularly at risk of nosocomial dissemination of SARS-CoV-2 everywhere in the world, and understanding this transmission is key to implementing efficient control measures. However, estimates of nosocomial transmission rates are missing, and are challenging due to stochasticity, heterogeneity of the environments, and lack of appropriate test data.

Objectives: Mechanistic modelling applied to detailed data were used to quantify nosocomial transmission rates.

Methods: We developed a stochastic compartmental model of SARSCoV-2 transmission in hospitalized patients and fit it to data from an outbreak in a 350-bed long-term care facility (LTCF) in Paris during the first wave of COVID-19. Reported cases occurred in March and April 2020 , during which $312 / 459$ patients present received a PCR test, and 152 tested positive, many being asymptomatic. The model captures 
LTCF specificities regarding distribution of tests, admission and discharge. We also assessed the change in transmission risk after the implementation of contact precautions.

Results: We found that a model considering two separate time periods characterized by their own transmission rate, was better supported by the data. In the first period the rate was estimated at 1.3 (95\% confidence interval 0.8-2.4) infections per patient per day in symptomatic infection, corresponding to an $\mathrm{R}_{0}$ of $8.7(5.1-16.3)$, and $0.19(0.10-0.30)$ in the second phase corresponding to an $R_{0}$ of $1.3(0.7-2.0)$. These results are consistent with a short period of high transmission, during which hospital members were not aware of ongoing transmission, followed by a moderate transmission rate following implementation of obligatory mask-wearing. Under this assumption, the contact precautions introduced had $85 \%$ effectiveness in reducing transmission. We also found substantial heterogeneity in transmission among individual wards.

Conclusion: These results provide estimates for nosocomial transmission rates within a healthcare setting containing vulnerable patients, and indicate the efficacy of preventive measures. The model can be readily extended to other datasets.

Disclosure of Interest: None declared.

\section{8}

Exploring behavioral factors influencing COVID19 specific infection prevention and control measures in Finland-a mixed methods study

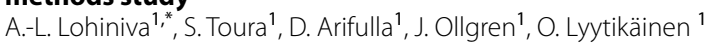

${ }^{1}$ Finnish Institute for Health and Welfare, Helsinki, Finland

Correspondence: A.-L. Lohiniva

Antimicrobial Resistance \& Infection Control 10(1): 028

Introduction: Healthcare worker (HCW) compliance with infection prevention and control (IPC) measures plays a critical role in preventing COVID-19 transmission in healthcare settings. To ensure optimal adherence to COVID-19-specific IPC practices, there is a need to understand the factors that influencing them.

Objectives: To identify and explain factors that influence the compliance to COVID-19 specific IPC measures among HCWs in LTCFs in Finland.

Methods: The study included a web-based survey and a qualitative research study based on the Theoretical Domains Framework (TDF). The questions in the survey covered background information of the respondent and LTCF as well as behaviour factors. The link to the anonymous survey was distributed to the LTCFs through regional IPC experts and authorities. The outcome was modeled using both ordinary logistic regression and penalized ridge logistic regression using regrouped explanatory variables and original more correlated set of explanatory variables, respectively. The in-depth interviews were conducted by using a semi-structured guide. The subjects were recruited from those who participate in the survey. The interviews were conducted between January and February 2021. Data was analysed thematically using NVIVO12.

Results: A total of $422 \mathrm{HCWs}$ from 17/20 regions responded to the survey. The study findings suggested that three TDF domains influence the compliance with COVID19 specific IPC measures: environmental factors and resources (inadequate staffing), beliefs about capabilities and reinforcement (follow up and feedback of the management). The qualitative study included $20 \mathrm{HCWs}$ consisting of nurses and assistant nurses who provided care in LTCFs during the pandemic. The study identified two themes that explain environmental factors and resources; lack of planning for emergency; changing work responsibilities. Beliefs in capabilities were linked with knowledge, resources and responsibilities, and reinforcement was linked with management absence and culture.

Conclusion: This study provides insights into TDF domains that can be used to develop evidence-based behaviour change interventions to support HCW compliance with pandemic-specific IPC measures in LTCFs during future epidemics.

Disclosure of Interest: None declared.
Slide session: Surgical site infections

029

PVP iodine vs chlorhexidine in alcohol for preparation

of the surgical site: a cluster-randomized multicenter cross-over trial

A. F. Widmer ${ }^{1,}$, A. Atkinson ${ }^{2}$, H. Sax ${ }^{3}$, P. Jent ${ }^{4}$, S. Kuster $^{5}$, R. Sommerstein ${ }^{6}$, J.

Marschall ${ }^{6}$, A. Schweiger ${ }^{1}$

${ }^{1}$ Hospital Epidemiology, University Hospital Basel, Basel, ${ }^{2}$ Infectious disease, Bern University Hospital, Inselspital, University o, Bern, ${ }^{3} \mathrm{Hospi}$ tal Epidemiology, Universitätsspital, Zürich, ${ }^{4}$ Hospitabl Epidemiology, Inselspital bern, bern, ${ }^{5}$ Hospital Epidemiology, Universitätsspital Zürich, Zürich, ${ }^{6}$ Hospital Epidemiology, Inselspital bern, bern, Switzerland

Correspondence: A. F. Widmer

Antimicrobial Resistance \& Infection Control 10(1): 029

Introduction: Preoperative skin antisepsis is an established infection control procedure to prevent surgical site infections (SSIs) The choice of antiseptic agent-chlorhexidine $(\mathrm{CHX})$ or iodine $(\mathrm{PI})$ remains matter of debate:

Objectives: To compare rates of surgical site infections after preparation of the skin with alcoholic soluttion with either PVP iodine or Chlorhexidine.

Methods: In this multicenter, cluster-randomized, triple -blinded, randomized, controlled trial, we compared the rate of SSIs after preparation of the skin with alcoholic solutions with either $\mathrm{CHX}$ or PI. The primary outcome was SSIs within 30 days after abdominal, and one year after cardiac surgery, using CDC definitions.

Results: From September 2017 through June 2020, a total of 3321 patients from three university hospitals were enrolled: 1155 to PI vs 1011 assigned to $\mathrm{CHX}$. SSIs were diagnosed overall in 80 (5.1\%) patients in the $\mathrm{PI}$ group vs $97(5.5 \%)$ in the $\mathrm{CHX}$ group (relative risk $\left[\mathrm{Cl}_{95} 0.92,0.69\right.$ 1.23], $P=0.50)$. $P I$ was superior to $C H X$ in abdominal surgeries $(P=0.06)$, but slightly less active to prevent SSIs in cardiac surgery $(P=0.29)$ [Table].

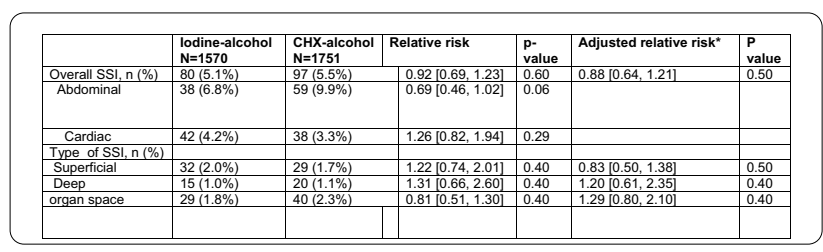

Conclusion: This largest cluster randomized trial demonstrated minuscule difference in the rates of SSIs, independent of the choice of PI or $\mathrm{CHX}$ to prepare the surgical site. Discordant results of other studies might be related to the type of intervention studied, the high free available content of PI tested, and the different products under investigation. Disclosure of Interest: A. Widmer Grant/Research support from: Funded by the Swiss National Science Foundation \# 33IC30_173534. Clinicaltrials.org identifier: NCT03685604 and Swiss National Clinical Trials Portal \# NCT03685604, A. Atkinson: None declared, H. Sax: None declared, P. Jent: None declared, S. Kuster: None declared, R. Sommerstein: None declared, J. Marschall: None declared, A. Schweiger: None declared.

\section{0}

SSI-Preventive compliance after tailored interventions

for infection prevention and control teams: results of the cluster-randomized controlled wach-trial in six non-university centres

T. von Lengerke ${ }^{1, *}$, I. Hartlep ${ }^{2}$, P. Schipper ${ }^{2}$, M. Stolz ${ }^{3}$, I. Tomsic ${ }^{4}$, C. Krauth ${ }^{5}$, I. F. Chaberny ${ }^{2}$

${ }^{1}$ Department of Medical Psychology, Hannover Medical School, Centre for Public Health and Healthcare, Hannover, ${ }^{2}$ Institute of Hygiene, Hospital Epidemiology and Environmental Medicine, Leipzig University Hospital, Centre for Infection Medicine, Leipzig, ${ }^{3}$ Institute of Epidemiology, Social Medicine and Health Systems Research, ${ }^{4}$ Department of Medical Psychology, Hannover Medical School, Centre of Public Health and Healthcare, ${ }^{5}$ Institute of Epidemiology, Social Medicine and Health Systems Research, Hannover Medical School, Centre for Public Health 
and Healthcare, Hannover, Germany

Correspondence: T. von Lengerke

Antimicrobial Resistance \& Infection Control 10(1): O30

Introduction: Preventing surgical site infections (SSI) implies compliance with numerous measures. Few randomized studies have addressed compliance promotion in this context.

Objectives: The cluster-randomized trial WACH ("SSI and Antibiotics Use in Surgery", 2018-20; German Clinical Trials Register-ID: DRKS00015502) aimed at improving SSI-preventive compliance by adapting the psychological tailoring approach of the PSYGIENE-trial (DRKS00010960) from interventions addressing clinical staff to infection prevention and control (IPC) teams and other stakeholders in non-university hospitals.

Methods: After briefing, in each of $\mathrm{N}=6$ hospitals in-house staff observed compliance for 14 measures in $\mathrm{N}=1034$ ward rounds and $\mathrm{N}=905$ surgical procedures (general/visceral and/or orthopedic/trauma surgery) before and after a 3-4 months intervention ("tailoring"-arm) or "usual practice" period. Interventions consisted of written reports and 2-day IPC-workshops incl. coaching, and were tailored based on appraisals of compliance and its determinants (COM-B [1]) using mixed methods. Data analysis (cluster-level) were performed using OpenEpi 3.01 for compliance estimations, chi-square and Breslow-Day tests.

Results: In the "tailoring"-arm, a compliance increase was observed for all measures (mean: $11 \%$ ), with $p<0.05$ in 11 cases. However, for 7 measures baseline rates were lower than in the "usual practice"-arm, in which 1 in- and 3 decreases were significant. Given similar baseline, "tailoring" was superior for hand hygiene during ward rounds $(+12 \%$ vs. $+3 \%$; interaction: $p=0.007)$ and wearing surgical cap $(+22 \%$ vs. $-13 \% ; p<0.001)$. For perioperative antibiotic prophylaxis, the interaction was significant $(+5$ vs. $-5 ; p=0.038)$

Conclusion: Adapting psychological tailoring to IPC teams in non-university hospitals to promote SSI-preventive compliance showed mixed results partly due to lower baselines in the "tailoring"-arm. Also, hand hygiene tended to be emphasized in the interventions and by their addressees, possibly explaining the positive result. This suggests stricter use of bundles within multimodal strategies.

Ref. 1. https://doi.org/10.1186/s13756-021-00932-9

Disclosure of Interest: None declared.

\section{1}

Acne cream reduces the deep cutibacterium acnes tissue load before elective open shoulder surgery: a randomized-controlled pilot trial

I. Uçkay ${ }^{1, *}$, I. Unterfrauner ${ }^{1}$, K. Wieser ${ }^{1}$, S. Bouaicha ${ }^{1}$

${ }^{1}$ Balgrist University Hospital, Zürich, Switzerland

Correspondence: I. Uçkay

Antimicrobial Resistance \& Infection Control 10(1): 031

Introduction: Cutibacterium acnes is the hallmark pathogen of healthcare-associated deep surgical site infections (SSI) after shoulder surgery.

Objectives: We investigate a topical acne cream to reduce the subcutaneous Cutibacterium acnes in open elective shoulder surgery. The Benzoyl Peroxide-Miconazole Nitrate-cream for acne vulgaris reduces the superficial C. acnes burden, which is the main pathogen of shoulder infections. However, the preventive potential of the cream against SSI in shoulder surgery remains unknown.

Methods: We performed a prospective-randomized pilot trial allocating 60 adult patients (1:1) between a 7-days-preoperative application of a commercial acne cream (Benzoyl Peroxide; Miconazole Nitrate) on the pre-operative skin (intervention group) versus no cream (controls). We sampled the superficial skin at enrolment, before incision, and performed deep subcutaneous and capsular samples during shoulder surgery.

Registration: Switzerland BASEC 2018-01510; International NCT03949751.
Results: We included 60 patients (mean age 59 years, 55\% females) with primary open shoulder surgery (17 Latarjet procedures, 43 arthroplasties) in the study. At baseline, both randomized groups revealed the presence of $C$. acnes on the skin to $60 \%(18 / 30$ in the intervention group; $19 / 30$ in the controls; $p=0.79$ ). In the patients with $C$. acnes skin colonization, the intervention resulted in a significant reduction of the number of positive intraoperative samples compared to the control group ( $8 / 18$ vs. $16 / 19 ; p=0.01)$; including regarding the capsular samples $(0 / 18$ vs. $4 / 19 ; p=0.04)$. There were no surgical site infection (SSI) in either group after a mean clinical follow-up of eleven months. The cream was well tolerated.

Conclusion: The topical 7 days-preoperative skin application of a commercial acne cream (Benzoyl Peroxide and Miconazole Nitrate) was well tolerated and substantially reduced the intraoperative $C$. acnes-load in $56 \%$ of the patients of the intervention group. Our findings represent a strong rationale for its use as a prevention against SSIs in shoulder surgery. Confirmatory clinical experience and further research are needed. Disclosure of Interest: None declared.

032

Economic evaluation of preoperative shower with antiseptic shower to prevent surgical site infections

J. Ory ${ }^{1, *}$, R. Moreau ${ }^{2}$, M. Masseti ${ }^{2}$

${ }^{1}$ Univesity hospital, NImes, ${ }^{2}$ Public Health Expertise, Paris, France

Correspondence: $\lrcorner$. Ory

Antimicrobial Resistance \& Infection Control 10(1): 032

Introduction: A preoperative shower is recommended before surgery to prevent surgical site infection (SSI).

Objectives: To characterize the benefit of SSI prevention associated with antimicrobial soap (AS), we modelled the use of AS in a University Hospital (UH) in orderto characterize its impact in terms of SSI and its cost compared to shower with a placebo.

Methods: The occurrence of SSI and the potentials saving made by patients undergoing AS shower before surgery at a UH was modelled using a decision tree approach. SSI rates, by type and Altemeier classification, were based on healthcare infection database. The impact of AS shower on the risk of SSI was based on a multivariate meta-analysis comparing antiseptic shower vs. shower with a placebo.

Baseline mortality for patients undergoing procedures, additional risk of death in patients with SSI and cost of SSI management, including additional care and length of stay (LoS) were obtained from two prospective studies realized at a UH. Costs of AS shower were based on the price of povidone iodine (Betadine scrub $4 \%$ ), assuming that $10 \%$ of patients showered before surgery, while AS performed at home by patients did not incur costs to the hospital. Results were extrapolated to the average annual number of surgeries. Univariate deterministic sensitivity analyses and scenario analyses were performed to assess uncertainty.

Results: AS was associated with the prevention of 209 SSI, leading to potential savings of $€ 632,210$ compared to the absence of AS. The most interessant impact of the preoperative shower before surgery was mainly on the clean surgeries.

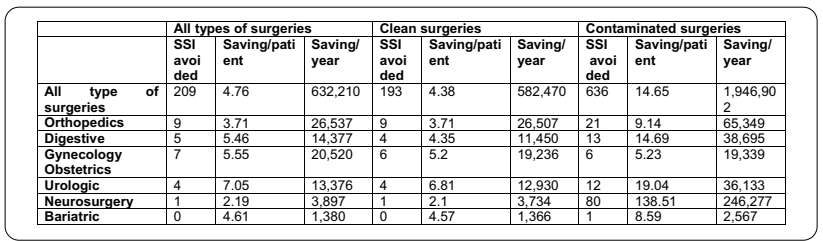

Conclusion: AS shower before surgery is a simple procedure that could lead to significant savings through SSI prevention, avoiding additional care and LoS.

Disclosure of Interest: None declared. 


\section{3}

Evaluating a management team training by measuring

the adherence to a surgical site infection (SSI)-bundle

M. van Dijk ${ }^{1, *}$, E. van Beeck ${ }^{2}$, A. Huis ${ }^{3}$, B. van der Gun ${ }^{4}$, S. Polinder ${ }^{2}$, A. van Eijsden ${ }^{2}$, A. Burdorf ${ }^{2}$, M. Vos ${ }^{1}$, V. Erasmus ${ }^{2}$

${ }^{1}$ Medical Microbiology and Infectious Diseases, ${ }^{2}$ Public Health, Erasmus MC University Medical Centre Rotterdam, Rotterdam, ${ }^{3} \mathrm{IQ}$ healthcare,

Radboud University Nijmegen Medical Centre, Nijmegen, ${ }^{4}$ Medical Microbiology, University Medical Centre Groningen, Groningen, Netherlands

Correspondence: M. van Dijk

Antimicrobial Resistance \& Infection Control 10(1): 033

Introduction: Hospitals trying to reduce Surgical Site Infections (SSI) encounter difficulties since implementing a SSI-bundle requires cooperation between different management levels.

Objectives: We aimed to evaluate a Quality Improvement(QI) teamtraining intervention focussed on improving cooperation between the Executive Board, medical specialists, quality advisors and infection control specialists. The QI-teamtraining was executed by implementing a SSI bundle.

Methods: This was a multicentre before-after study, in 4 hospitals in the Netherlands. The intervention consisted of 4 sessions and stimulated participants to set culture norms and targets, identify barriers, take leadership, empower employees and formulate management activities to improve patient safety. SSI-bundle adherence was measured by observing standard operating procedures(SOP) and hand hygiene $(\mathrm{HH})$ at baseline and post-intervention. To analyse change in compliance, generalized random effects modelling was used.

Results: The main difference between the 4 hospitals was involvement of the Executive Board, but also involvement of medical specialists and quality advisors differed per hospital. In hospitals where mainly quality advisors were involved, only local actions in the OR were executed. In 1 hospital, with 3 actively involved medical specialists, also actions at manager level were executed. The effect of the Ql-teamtraining was evaluated by determining adherence to a SSI-bundle at baseline(B)(296 operations) and post-intervention(PI)(387 operations). The average door movements decreased (B:11,PI:10), timely antibiotic prophylaxis administration improved (B:58\%,Pl:68\%), preoperative shaving scores were maintained (B:94\%,PI:96\%) and adherence to normotherapy guidelines improved (B:38\%,Pl:50\%). Adherence to all 4 SOP (B:1\%,Pl:19\%) and $\mathrm{HH}$ compliance (B:5\%,Pl:10\%) improved, but stayed low.

Conclusion: This study showed that including different management levels in discussions on Q1-interventions could increase the adherence. It also showed that modest involvement of managers will result in moderate effect. Thus, involvement of all management levels is essential. Disclosure of Interest: None declared.

Slide session: Nosocomial COVID-19: The chicken or the egg

\section{4}

Seroconversion for SARS-COV-2 in health care workers after the first and the second wave of COVID-19 in the canton of Grisons in Switzerland

K. Szajek ${ }^{1}$, S. Güsewell ${ }^{2}$, S. Hutter ${ }^{3}$, T. Bechmann ${ }^{4}$, M. Risch ${ }^{5}$, F. Fleisich ${ }^{6}$, V. Luyckx $^{7}$, C. Hirzel ${ }^{8}$, A. Cusini ${ }^{*}$,

${ }^{1}$ Internal Medicine, Cantonal Hospital Grisons, Chur, ${ }^{2} \mathrm{CTU}$, Cantonal Hospital St.GAllen, St.Gallen, ${ }^{3}$ Laboratory, ${ }^{4} \mathrm{ICT},{ }^{5}$ Labortory, Cantonal Hospital Grisons, ${ }^{6}$ Infectious Diseases, Cantonal Hospital, ${ }^{7}$ Cantonal Hospital Grisons, Chur, ${ }^{8}$ Infectious Diseases, University Hospital Bern, Bern, ${ }^{9}$ Infectious Diseases, Cantonal Hospital Grisons, Chur, Switzerland

Correspondence: A. Cusini

Antimicrobial Resistance \& Infection Control 10(1): 034

Introduction: Health care workers (HCW) are exposed to SARS-CoV-2 through patient contact.

Objectives: We aimed to assess the seroprevalence for SARS-CoV-2 among HCW in the canton Grisons and analyze risk factors associated with seroconversion.

Methods: HCW from 13 health care institutions were recruited. Sera were analyzed for SARS-CoV-2 antibodies using an electro-chemiluminescence immunoassay of Roche Diagnostics in mid-2020 (survey I) and early 2021 (survey II). Participants reported risk factors for COVID-19 in a questionnaire.Odds ratios (OR) and $95 \%$ confidence intervals $(95 \% \mathrm{Cl})$ for the association of seropositivity with each risk factor were determined by logistic regression.

Results: SARS-CoV2 serology was positive in 99 of 2794 (3.5\%) participants in survey I and in 376 of 2315 (13.5\%) participants in survey II. By survey II, 86 of $88(97.7 \%)$ initially seropositive participants remained seropositive. In multivariable analysis of both surveys, the strongest association for SARS-CoV-2 seroconversion was contact with a household member with COVID-19 (adjusted OR: 21.2, 95\% Cl: 8.5-51.4, $\mathrm{p}<0.001$, survey l; aOR: $8.5,95 \% \mathrm{Cl}: 6.0-12.1, \mathrm{p}<0.001$, survey II). Significant occupational risk factors included contact with patients with COVID-19 in both surveys (aOR: $2.7,95 \% \mathrm{Cl}: 1.4-5.4, \mathrm{p}<0.001$, survey I and aOR: $1.4,95 \% \mathrm{Cl}: 1.0-2.2, \mathrm{p}=0.046$, survey II). Contact to a SARSCoV-2 positive co-worker was a significant risk factor only in survey I, whereas private contact with SARS-CoV-2 positive persons and the COVID-19 incidence in the region of the $\mathrm{HCl}$ were associated with seroconversion only in survey II.

Conclusion: Contact with patients with COVID-19 was an important risk factor for seroconversion, although the risk was higher for household contacts. These findings highlight the need to optimize preventive measures for SARS-CoV2 infection among HCW.

Disclosure of Interest: None declared.

035

Use of respirator vs. surgical masks in healthcare personnel and its impact on SARS-COV-2 acquisition-a prospective multicentre cohort study

S. Haller', S. Güsewell2, T. Egger ${ }^{1}$, G. Scanferla', R. Thoma' ${ }^{1}$, O. Leal-Neto ${ }^{3}$, D. Flury ${ }^{1}$, A. Brucher ${ }^{4}$, E. Lemmenmeier ${ }^{5}$, C. Möller ${ }^{6}$, P. Rieder ${ }^{7}$, M. Rütti ${ }^{8}$, R. Stocker ${ }^{7}$, D. Vuichard-Gysin ${ }^{9}$, B. Wiggli10, U. Besold ${ }^{11}$, S. Kuster ${ }^{12}$, A. McGeer ${ }^{13}$, L. Risch ${ }^{14}$, M. Schlegel ${ }^{1}$, A. Friedl ${ }^{10}$, P. Vernazza', C. Kahlert ${ }^{1}$, P. Kohler ${ }^{1, *}$

${ }^{1}$ Division of Infectious Diseases and Hospital Epidemiology, ${ }^{2}$ Clinical Trials Unit, Cantonal Hospital St Gallen, St. Gallen, Switzerland, ${ }^{3}$ Epitrack, Recife, Brazil, ${ }^{4}$ Psychiatry Services of the Canton of St. Gallen (South), St. Gallen,

${ }^{5}$ Clienia Littenheid AG, Littenheid, ${ }^{6}$ Center for Neurological Rehabilitation, Zihlschlacht, ${ }^{7}$ Hirslanden Clinic, Zurich, ${ }^{8}$ Hospital Region Fürstenland Toggenburg, Wil, ${ }^{9}$ Division of Infectious Diseases and Hospital Epidemiology, Thurgau Hospital Group, Muensterlingen, ${ }^{10}$ Division of Infectious Diseases and Hospital Epidemiology, Cantonal Hospital Baden, Baden, ${ }^{11}$ Geriatric Clinic St. Gallen, St. Gallen, ${ }^{12}$ Division of Infectious Diseases and Hospital Epidemiology, University Hospital and University of Zurich, Zurich, Switzerland, ${ }^{13}$ Sinai Health System, Toronto, Canada, ${ }^{14}$ Labormedizinisches Zentrum Dr Risch Ostschweiz AG, Buchs, Switzerland Correspondence: P. Kohler

Antimicrobial Resistance \& Infection Control 10(1): 035

Introduction: There is insufficient evidence regarding the role of respirators in the prevention of SARS-CoV-2 infection.

Objectives: We analysed the impact of filtering facepiece class 2 (FFP2) vs. surgical masks on the risk of SARS-CoV-2 acquisition in Swiss healthcare workers (HCW).

Methods: Our prospective multicentre cohort enrolled HCW from June to August 2020, who were asked about COVID-19 risk exposures/behaviours, including preferred mask type when caring for COVID-19 patients outside of aerosol-generating procedures (AGP). HCW performing AGP were also asked about universal FFP2 use (i.e. irrespective of patients' COVID-19 status). We assessed the impact of FFP2 on i) self-reported SARS-CoV2-positive nasopharyngeal PCR/rapid antigen tests (weekly surveys), and ii) SARS-CoV-2 seroconversion (baseline to January/February 2021).

Results: We enrolled 3'259 participants from nine healthcare institutions, whereof 716 (22\%) preferentially used FFP2 respirators. Among these, $81 / 716(11 \%)$ reported a SARS-CoV-2-positive swab, compared to $352 / 2543$ (14\%) surgical mask users (median follow-up 242 days); seroconversion was documented in 85/656 (13\%) FFP2 and 426/2255 (19\%) surgical mask users. Adjusted for baseline characteristics, COVID-19 exposure, and risk behaviour, FFP2 use was marginally associated with a decreased risk for SARS-CoV-2-positive swab (aHR 0.8, 
$\mathrm{p}=0.052$ ) and seroconversion ( $a \mathrm{OR} 0.7, \mathrm{p}=0.053)$; household exposure was the strongest risk factor (aHR for positive swab 10.1, $p<0.001$; aOR for seroconversion 5.0, $\mathrm{p}<0.001)$. In subgroup analysis, FFP2 use was clearly protective among HCW with frequent ( $>20$ patients) COVID-19 exposure (aHR 0.7, $\mathrm{p}<0.001 ; \mathrm{aOR} 0.6, \mathrm{p}=0.036$ ). Universal FFP2 use during AGP showed no additional protective effect (aHR 1.1, $\mathrm{p}=0.7 ; \mathrm{aOR} 0.9, \mathrm{p}=0.53$ ).

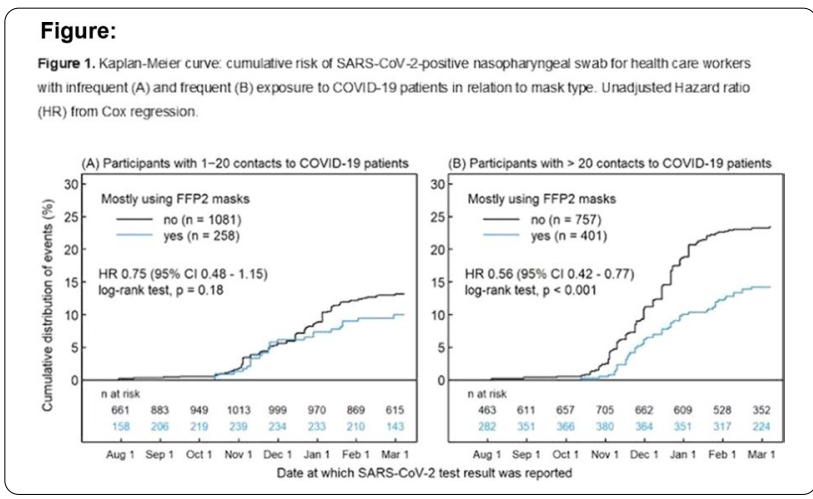

Conclusion: FFP2 compared to surgical masks may convey additional protection from SARS-CoV-2 for HCW with frequent exposure to COVID-19 patients.

Disclosure of Interest: None declared.

\section{6}

Transmission of SARS-COV-2 in the hospital setting: who is infecting whom?

Y. Mo ${ }^{1}$, D. Eyre, ${ }^{1, *}$, S. Lumley ${ }^{1}$, T. Walker ${ }^{1}$, R. Shaw ${ }^{2}$, D. O'Donnell ${ }^{2}$, L. Butcher $^{2}$, K. Jeffery ${ }^{2}$, C. Donnelly ${ }^{1}$, B. S. Cooper ${ }^{1}$ on behalf of Oxford COVID infection review team

${ }^{1}$ University of Oxford, ${ }^{2}$ Oxford University Hospitals, Oxford, United Kingdom

Correspondence: D. Eyre

Antimicrobial Resistance \& Infection Control 10(1): 036

Introduction: A better understanding of the relative importance of different transmission pathways of SARS-CoV-2 in hospital settings has the potential to help improve targeting of control measures aimed at reducing nosocomial spread.

Objectives: To quantify the associations between risks of nosocomial SARS-CoV-2 infection and exposure on the same ward to infected healthcare workers (HCWs), to patients likely to have been infected nosocomially, and to patients with community onset COVID-19.

Methods: Ward-level data were collected from four teaching hospitals in Oxfordshire, UK, over an 8 month period in 2020. SARS-CoV-2 infections were identified using both PCR results from symptomatic and asymptomatic testing and serological data coupled with symptom recall. A series of statistical models were used to quantify associations between exposures and probable hospital transmission events.

Results: Risk to patients of probable nosocomial acquisition was most strongly associated with exposure to other patients with hospitalacquired SARS-CoV-2 (aOR, 1.76, 95\%Cl 1.51, 2.04), followed by the presence of an infected $\mathrm{HCW}$ on the same ward $(\mathrm{aOR} 1.45,95 \% \mathrm{Cl}$
$1 \cdot 22,1 \cdot 71)$. The association with patients with community onset COVID19 was weaker (aOR $1 \cdot 12,95 \% \mathrm{Cl} 0 \cdot 96,1 \cdot 26)$.

Transmission to HCWs was associated with exposure to other infectious HCWs and patients with hospital-acquired SARS-CoV-2 (aOR $1 \cdot 66,95 \% \mathrm{Cl} 1 \cdot 55,1 \cdot 78$ and aOR $1 \cdot 45,95 \% \mathrm{Cl} 1 \cdot 32,1 \cdot 58$ respectively). The introduction of more stringent infection prevention and control measures which included testing all patients for SARS-CoV-2 by PCR on admission and at weekly intervals was associated with substantial reduction in transmission risk to both patients (adjusted odds ratio, aOR $0.25,95 \% \mathrm{Cl} 0.14,0.42$ ) and HCWs (aOR 0.43 , 95\% Cl 0.34, 0.53).

Conclusion: Patients who acquired SARS-CoV-2 in the hospital and, to a lesser degree, infectious HCWs likely working prior to the onset of symptoms, were the most strongly associated with increased risk of SARSCoV-2 transmission. In contrast, exposure to patients who had acquired SARS-CoV-2 in the community was associated with, at most, modest increases in the daily risk of infection for both healthcare staff and the other patients.

Disclosure of Interest: None declared.

037

Nosocomial COVID-19 infections in Belgian hospitals:

a longitudinal study

J. Claes ${ }^{1}$, K. Blot ${ }^{2}$, B. Serrien ${ }^{2}$, K. Mertens ${ }^{3}$, B. Catry ${ }^{3,4, *}$

${ }^{1}$ Ghent University, Ghent, ${ }^{2}$ Sciensano, Brussels, Belgium, ${ }^{3}$ Healthcare associated infections \& Antimicrobial resistance (NSIH), Sciensano, ${ }^{4}$ Faculty

of Medicine, Université libre de Bruxelles, Brussels, Belgium

Correspondence: B. Catry

Antimicrobial Resistance \& Infection Control 10(1): 037

Introduction: COVID-19 has posed an immense burden on healthcare systems.

Objectives: The objective of this study was to estimate the proportion of nosocomial infections in the Belgian hospitals since the start of the pandemic, as well as the main characteristics of patients involved.

Methods: Surveillance data were extracted from the Clinical Hospital Surveillance established by Sciensano (ca 66\% coverage, March 2020-March 2021, $n=51,293$ ). European Centres for Disease Control and Prevention (ECDC) definitions for nosocomial COVID19 were applied; out-of-hospital: $<=\mathrm{d} 2$; indeterminate: $\mathrm{d} 3$ to $\mathrm{d} 7$, probably nosocomial: $\mathrm{d} 8-\mathrm{d} 14$ or definitely nosocomial: $>$ d14 after admission. Logistic regression was used to identify risk factors for mortality.

Results: Inclusion criteria (dates of admission, diagnosis and/or sympton onset) were met for 49,623 patients (median age 72, IQR 57-83). 22,445 observations $(45.23 \%$ ) were tested due to symptoms indicative of a COVID-19 infection, $7868(15.86 \%)$ due to systematic screening 1042 (2.1\%) for reasons described as 'other', and for 18,268 (36.81\%) as 'unknown'. 7076 observations (14.26\%) were considered asymptomatic 23,533 (47.42\%) had fever-related symptoms, 3819 (7.7\%) upper respiratory, 30,782 (62.03\%) lower respiratory symptoms, 2656 (5.35\%) had anosmia, 11,147 (22.46\%) gastro-intestinal symptoms, and 11,147 (22.46\%) symptoms associated with a viral syndrome. Of all COVID infections $(n=5486,11.0 \%), 1270(2.6 \%), 1563(3.1 \%)$, and $2653(5.3 \%)$ were identified as indeterminate, probably nosocomial, and definitely nosocomial, respectively (Fig). The odds ratio (OR) of in-hospital mortality, comparing (probably + definitely) nosocomial to community acquired (out-of-hospital + indeterminate) COVID, for the entire studied population was 1.62 $(95 \% \mathrm{Cl} 1.50-1.75)$. Significant increase in risk was seen only for age categories $40-59(3.13 ; 2.18-4.50)$ and $60-79(1.5 ; 1.32-1.71)$. 


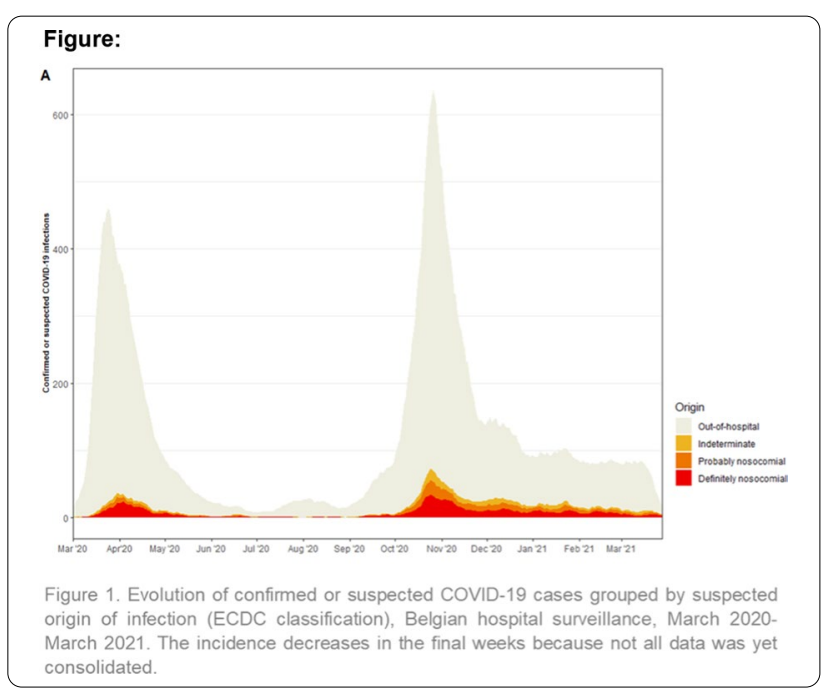

Conclusion: Determining origin infection due to the ambiguousness around incubation periods, asymptomatic transmission and viral load over time needs further research. The proportion of nosocomial cases after the second wave emphasizes the need to further investigate the compliance of infection prevention measures over long periods.

Disclosure of Interest: None declared.

\section{8}

Evaluation of ward-level risk factors for nosocomial COVID-19 outbreaks: a matched case-control study

R. Thoma 1,", P. Kohler ${ }^{1}$, S. Haller ${ }^{1}$, J. Maenner ${ }^{1}$, M. Schlegel ${ }^{1}$, D. Flury ${ }^{1}$

${ }^{1}$ Division of Infectious diseases and hospital epidemiology, Cantonal Hospital St. Gallen, St. Gallen, Switzerland

Correspondence: R. Thoma

Antimicrobial Resistance \& Infection Control 10(1): 038

Introduction: The prevention of SARS-CoV-2 outbreaks in acute care hospitals is a major challenge. In the second wave of the pandemic, we observed several wards with outbreaks whereas others were spared.

Objectives: We aimed to investigate risk factors for nosocomial COVID-19 outbreaks on a ward level.

Methods: We conducted a matched case-control study in our tertiary care centre with over 700 beds by defining outbreak ( $\geq 2$ nosocomial patients infected within a 14-day period) and control wards. Nosocomial infection was defined if a patient tested positive for COVID-19 on day 5 or later of hospitalisation. Matching was done 1:1 for approximate number of beds $( \pm 10)$ and the time of the outbreak. The beginning of the outbreak was defined as the day of the first positively tested nosocomial COVID-19 case on the ward. Intensive care units and designated COVID19 wards were excluded. Presumed ward-, patient- and staff-specific variables were investigated. Paired Wilcoxon signed-rank test was used to compare variables between outbreak- and control wards.

Results: From July to December 2020, we observed 9 outbreak wards (surgical and medical, range of beds $17-31$, range nurses $19-41$ per ward) with a total of 40 patients infected (range 2-7 per ward). The percentage of healthcare workers (HCW) tested positive within a period of 14 days prior until 2 days after the start of the outbreak was the only significant risk factor $(9.7 \%$ vs $2.7 \%, p=0.04)$. No difference in the percentage of infected HCW was observed in a time period further preceding the start of the outbreak ( 3 months to 2 weeks). Outbreak wards trended towards a higher number of beds per room (2.22 vs $1.97, p=0.09)$ and a younger HCW age (33.3 vs 36.2 years, $p=0.17$ ) compared to control wards. No association was found for factors reflecting work-load, patient turnover, or work experience of HCW (Table).

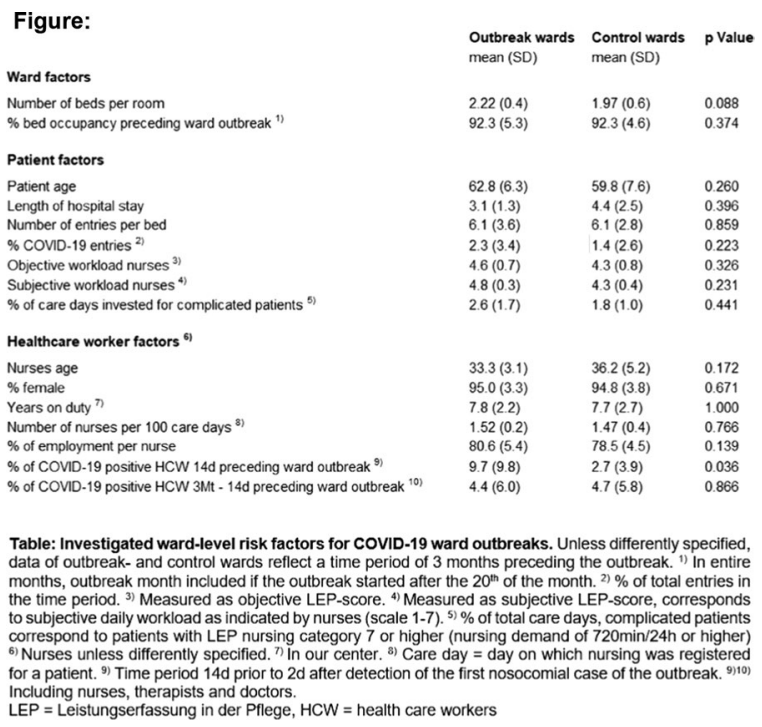

Conclusion: Increased numbers of infected HCW shortly before the outbreak seem to be a risk factor for nosocomial SARS-CoV-2 outbreaks. This supports the notion that infected HCW are an important source of nosocomial COVID-19 and underscores the importance of adequate infection control- and prevention measures of HCW in- and outside the hospital. Disclosure of Interest: None declared.

039

Value of SARS-COV-2 genomic characterization in elucidating within-hospital transmission

L. Cadenau" ${ }^{1, *}$, M. Welkers ${ }^{1}$, M. Jonges ${ }^{1}$, J. Schinkel ${ }^{1}$, I. Spijkerman ${ }^{1}$, M. de Jong ${ }^{1}$, R. van Mansfeld ${ }^{1}$

${ }^{1}$ Department of Medical Microbiology and Infection Prevention, Amsterdam UMC, University of Amsterdam, Amsterdam, Netherlands

Correspondence: L. Cadenau

Antimicrobial Resistance \& Infection Control 10(1): 039

Introduction: Identifying probable transmission routes of SARS-CoV-2 in the hospital setting is needed to direct implementation of infection prevention measures to stop transmission.

Objectives: We investigated probable transmission routes of clusters of SARS-CoV-2 using both epidemiological data and Next generation sequencing (NGS), and assessed congruence.

Methods: All positive SARS-CoV-2 PCR test performed in our hospital from both patients and healthcare workers (HCW) between September and December 2020 were evaluated. When more than two HCW or patients from the same ward tested positive within 14 days (a potential cluster), the ward was contacted to identify transmission routes. Suspected transmission was categorized based on epidemiological data (e.g. attendance, contacts in and outside the hospital). All available samples within clusters with sufficient viral load $(C T$ value $<30$ ) were analysed using NGS. A team of molecular biologists, infection prevention specialists and virologists discussed the NGS results and called possible, probable or unlikely clusters based on phylogenetic reconstruction, background prevalence and the epidemiological data. Results: From 14 possible clusters NGS was performed on $98 \mathrm{sam}$ ples (Table 1). Congruence between epidemiological data and expert conclusion was high for probable transmission (89\%) and unlikely transmission (76\%). In case of possible transmission NGS guided the definite conclusion in $85 \%$ towards probable. Four of the five cases in which no transmission was suspected based on epidemiological data, but were identical in NGS analysis, occurred in one cluster. Other disconcordances were dispersed in different clusters. 


\begin{tabular}{|l|l|l|}
\hline & $\begin{array}{l}\text { Expert } \\
\text { interpret } \\
\text { ation } \\
\text { (with } \\
\\
\end{array}$ \\
& NGS) \\
\hline Epidemiological data & Ye & N \\
& S & 0 \\
& $(73$ & $(25$ \\
& ) & ) \\
\hline Yes (56) & 50 & 6 \\
\hline Possible (21) & 18 & 3 \\
\hline No (21) & 5 & 16 \\
\hline
\end{tabular}

Conclusion: In $33 \%$ NGS changed the conclusion on transmission events. NGS analysis enables confirmation that transmission has ended and thereby helps evaluate outbreak management(OM). Besides, concerns of HCW can be managed by definite results on specific transmission events. One of fourteen clusters was only detected by NGS. Epidemiological data alone can give direction to OM, however NGS analysis gives essential feedback on OM and specific transmission events.

Disclosure of Interest: None declared.

\section{0}

Management of a COVID outbreak on a hematology-oncology ward

W. Van Der Zwett ${ }^{1,}$, A. Demandt ${ }^{2}$, E. Klomp¹ ${ }^{1}$, J. Dingemans ${ }^{1}$, L. van Alphen ${ }^{1}$, P. Savelkoul ${ }^{1}$

${ }^{1}$ Medical Microbiology, ${ }^{2}$ Hematology, MUMC + , Maastricht, Netherlands Correspondence: W. Van Der Zwet

Antimicrobial Resistance \& Infection Control 10(1): 040

Introduction: Within one week, 11 members of the nursing staff and one patient of the hematology-oncology tested positive for COVID-19. Transmission of SARS-COV-2 on the ward was suspected.

Objectives: Description of measures to end this outbreak

Methods: Additional to standard COVID-19 precautions in our hospital, all admitted asymptomatic patients were investigated by PCRscreening on a regular basis and positive specimens were investigated via Whole Genome Sequencing (WGS). The following measures were installed: 1) Temporary closure of the ward for new admissions; 2) Restriction of number of visitors to a minimum; 3) Cohorting of patients and healthcare workers (HCW); 4) Nursing personnel was required to change their FFP-2 mask every hour; 5) Transfer of positive patients to the specialized COVID-ward, if medically safely possible.

Additional measures were employed when new cases occurred despite measures: a) Pre-admission PCR-screening of new admissions; b) Critical appraisal of all non-patient-related activities of HCW and improvement where possible; c) Expansion of use of FFP-2 masks in care of asymptomatic non-COVID patients to some long-lasting non-aerosol forming close-contact procedures; d) Avoidance of the use of multiple person rooms for the non-COVID cohort as much as possible; e) Expansion of a working ban for COVID-19 positive HCW with CT-values $<30$ to any positive result, as it became evident that early detected patients/HCW frequently had a high initial CT-value which decreased dramatically in follow-up samples.

Results: WGS results proved the transmission of a particular SARSCOV-2 variant (B.1.221 lineage, unique mutation A26513G) on the ward. New cases occurred for 3 weeks. After installing extra measures, the incidence of new cases declined gradually.

The total outbreak eventually comprised 19 patients and $33 \mathrm{HCW}$. An important part of COVID-19 positive healthcare workers had long-lasting complaints of the infection and remained incapacitated far longer than the period that their CT-value required them to be banned from work. Therefore, extra personnel had to be recruited.

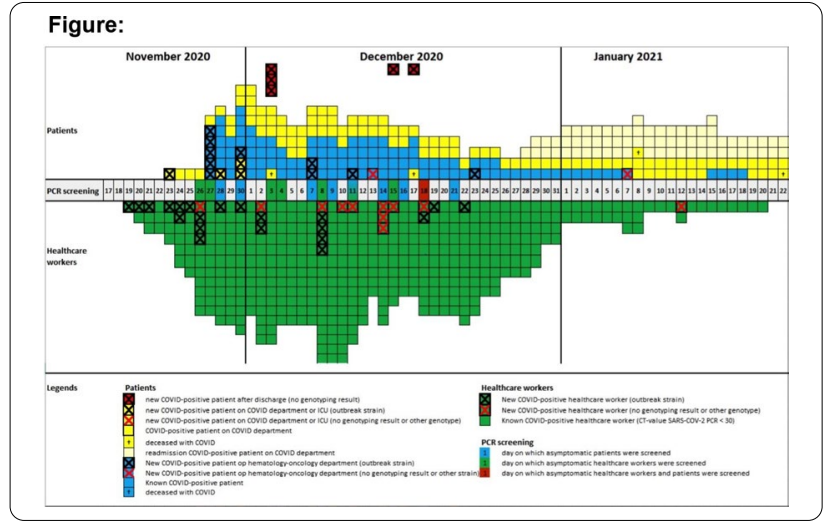

Conclusion: A bundle of preventive measures was effective in controlling this outbreak. The screening of asymptomatic patients and HCW was very helpful in ending it.

Disclosure of Interest: None declared.

Slide session: Global infection control

041

Current status of the initiatives related to infection prevention and control programs in the region of the Americas

J. Toledo ${ }^{1, *}$, E. Chapman ${ }^{1}$, S. Aldighieri ${ }^{1}$

${ }^{1}$ Pan American Health Organization (PAHO), Washington, United States

Correspondence: J. Toledo

Antimicrobial Resistance \& Infection Control 10(1): 041

Introduction: Implementation of Infection Prevention and Control (IPC) Programmes is a fundamental strategy to reduce the burden of healthcare-associated infections (HAI) and to contain the spread of emerging and re-emerging pathogens in community and health facilities settings. The World Health Organization IPC Core Components $(\mathrm{CC})^{1}$ is a framework with evidence-based recommendations comprising of eight areas for the implementation of IPC Programmes at the national and sub-national levels.

Objectives: This study aims to map and characterize initiatives related to four of the CC in the Region of the Americas.

Methods: Descriptive study of mapping valid and current national documents of IPC related with regulatory frameworks, programs, policies (CC1), evidence-based guidelines (CC2), policies on education and training of health workers (CC3), and documents on HAI surveillance (CC4).We conducted a search for documents from August/December 2021 using various sources of information (Ministries of Health official webpages, legal records, official communications, and technical documents). We presented the results in frequencies, percentages, and ranges.

Results: We included a total of 295 documents from 30/41 (73\%) Member States in the analysis, with an overall range from 1 to 23 documents per Member State. Due to the variability of the findings regarding the contents, the final classification of the documents was dichotomized as "general" or "specific", considering the overlap of the domains with other areas. We found that CC4 (39,5\%) and CC1 (34,3\%) had more "general" documents compared to CC2/CC3. There was no "general" policy on education programs in IPC for CC3. In regard to CC2, $83 \%$ of the guidelines were "specific" and $65 \%(80 / 123)$ of these related to COVID-19 and IPC. Figure 1 presents the frequencies for each one of the $\mathrm{CC}$ according to the subregions of the Americas. 
Figure:

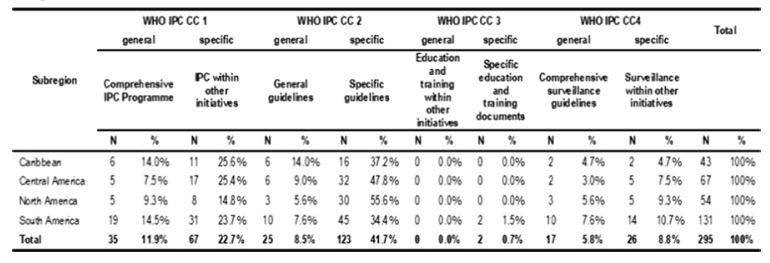

Conclusion: This study provides an overview of the current status of the initiatives related to IPC Programs in the Region of the Americas and an understanding of its evolution during the COVID-19 pandemic. However, it remains as priorities (i) improvement of health education and (ii) monitoring and evaluation strategies of the IPC practices at the health facilities level.

(REF: ${ }^{1}$ - https://www.who.int/gpsc/ipc-components/en/).

Disclosure of Interest: None declared.

\section{2}

Building a network of infection prevention and control model hospitals: the turn Nigeria orange strategy

M. Gadanya', B. Okoro', A. Ogunniyi', A.-B. A. Sambo ${ }^{1}$, T. Okwor 1,*

${ }^{1}$ Nigeria Centre for Disease Control, Abuja, Nigeria

Correspondence: T. Okwor

Antimicrobial Resistance \& Infection Control 10(1):042.

Introduction: The Nigeria Centre for Disease Control coordinates the National IPC programme and implements other IPC core components, aimed at supporting implementation of IHR 2005 and the Nigeria AMR-NAP. Nigeria has signed on to the WHO Global Patient Safety Initiative.

Objectives: We describe our experience in building the National IPC programme using a stepwise implementation strategy called "Turn Nigeria Orange". Launched in 2019, the TNO supports a network of health facilities (Orange Network) across Nigeria, in setting up functional IPC programs; in line with WHO recommendations.

Methods: Situational assessment conducted in 33/58 tertiary health facilities. Dedicated personnel to IPC were appointed and trained. IPC committees were set up with clear roles. Facilities identified into the Orange Network using WHO recommended criteria. Baseline IPC evaluation at national and facility levels, using structured close-ended questionnaires (IPCAT2 and IPCAF) with a scoring system, was conducted from July to September 2020. Data was analyzed, workplans developed and ratified by IPC team and leadership. Advocacy visits to relevant stakeholders conducted.

Results: At National level, baseline assessments revealed following scores for IPC programs (64\%), IPC guidelines (36\%), IPC education and training $(61 \%)$, HAI surveillance (24\%), multimodal strategies (71\%), monitoring/audit of IPC practices, feedback and control activities (25\%). The median score from the facilities was 433 (IQR: 178-637), with a mean of 416. Using the 4 IPCAF sub-categories classified thus: none was Inadequate, while 17, 13 and 2 were in Basic, Intermediate and Advanced categories respectively.

Conclusion: The scores placed TNO facilities largely in basic \& intermediate category. IPC program implementation requires strengthening HAI surveillance, monitoring/audit of IPC practices using multimodal strategy. At national level, IPC guidelines development, monitoring/adherence and training of end-users is required. Midaction review of the TNO strategy will be done in 2022 and impact evaluation is planned for 2024.
Key words: IPC, IPCAF, IPCAT2, IPC program implementation, WHO IPC core components, Orange Network.

Disclosure of Interest: None declared.

043

Infection prevention and control multi-level capacity building

for health workers-a cascade training and mentorship approach M. S. Kesande 1,*, D. Akankwatsa1 , P. Kabagambe ${ }^{1}$, R. Ayebare ${ }^{1}$, E. Katwesigye $^{2}$, D. Bulwadda' ${ }^{1}$ S. Nakato' ${ }^{1}$ S. Okware², E. Ayebazibwe', J. Nanyondo ${ }^{1}$, R. Walwema ${ }^{1}$, M. Lamorde ${ }^{1}$ on behalf of $n / a$

${ }^{1}$ Global Health Security, ${ }^{2}$ Resolve to Save Lives, Infectious Diseases Insti-

tute, Makerere University, Kampala, Uganda

Correspondence: M. S. Kesande

Antimicrobial Resistance \& Infection Control 10(1): 043

Introduction: Structures established during outbreak preparedness tend to diminish post-outbreak requiring rapid re-activation, re-training and re-orientation at the detection of a new outbreak. Sustainable Infection Prevention and Control (IPC) structures are required to offset rapid preparedness activities. Implementation of a district led IPC capacity building approach during the 2018 Ebola outbreak in Kasese, Uganda registered improved IPC compliance for majority of the health facilities. The same approach was scaled up to national level to enhance preparedness activities for COVID-19.

Objectives: a) To build and strengthen IPC capacity at regional, district and health facility levels. b) To build and strengthen IPC structures through establishment of IPC committees at district and facility levels. Methods: Didactic, practical and video IPC training materials were developed. The approach was piloted in 3 regions; each regional team underwent a training of trainers (TOT). At district level, the TOTs conducted a 3-day training of the district IPC committee and 10-20 selected mentors per district. The mentors were supported to conduct facility mentorship visits. A handbook endorsed by the director general of health services was developed to guide the mentorship sessions at facility level.

Results: 114 TOTs from 3 regions were trained, cascading the training to 29 districts. 557 district IPC committee members and district mentors attended the district level training and were attached to 1-3 facilities. 976 health facilities were reached and a total of 13,455 health workers were mentored. The mentors continue with the sustainability phase visiting a facility once a month.

Conclusion: The sustainability of IPC practices among health workers has an aspect of attitude and behavioural change and therefore cannot be achieved with a one-off training. This strategy provides suggestions for the Ministries of Health to maintain a functional and robust IPC structure at national, regional, district and health facility levels. Impact evaluation assessments are recommended periodically.

Disclosure of Interest: None declared.

\section{4}

Institutional safety climate: is it important for infection prevention and control nurses?

C. H. Hekimoglu' ${ }^{1}$, E. Batır ${ }^{1}$, E. Yildirim Gozel ${ }^{1}$, D. Altun ${ }^{1}$, M.-L. McLaws ${ }^{2}$, E. Alp $p^{1, *}$

${ }^{1}$ Ministry of Health, Ankara, Turkey, ${ }^{2}$ UNSW, Sydney, Australia

Correspondence: $\mathrm{E}$. Alp

Antimicrobial Resistance \& Infection Control 10(1): 044

Introduction: Infection prevention and control (IPC) programme in Turkey has been carried out at the national and hospitals level with the publication of "Inpatient Treatment Institutions Infection Control Regulation" in 2006. According to the regulation, the IPC committees were established and IPC doctors and nurses were assigned in all hospitals with $>200$ beds. IPC nurse training, consisting of theoretical and 
practical parts, has been introduced since 2007. Many IPC programme activities (surveillance, training of healthcare workers, hand hygiene observation, etc.) are carried out by IPC nurses.

Objectives: This study was conducted in order to evaluate institutional safety climate for IPC nurses at the $15^{\text {th }}$ year of national IPC programme of $\mathrm{MoH}$.

Methods: IPC nurses completed the self-questionnaire on a voluntary basis, via the online link, between 09/12/2019 and 31/12/2019. Minimum sample size was calculated to be 749 , assuming the number of IPC nurses 2500 , expected prevalence $50 \%$, absolute certainty \pm 3 and confidence level 0.05 . The analysis was done institutionally and individually according to the variables. In institutional evaluations, if there is more than one IPC nurses participated in an institution, one of them was chosen as "random".

Results: A total of 879 nurses from 693 hospitals participated in the study. It was determined that administrators actually fully attended national IPC nurse training programme in $75.0 \%$, partially attended in $22.7 \%$ and not attended in $2.3 \%$ of the hospitals. The ratio of working with an IPC doctor is $80.5 \%$ and have a regular IPC team meeting is $69.6 \%$. A microbiologist routinely attends IPC team meetings in $43.3 \%$ of facilities. The facility leadership made a clear commitment to support IPC decisions in $66.4 \%$ of the hospitals, partially in $32.9 \%$ and not in $2.5 \%$. All the IPC nurses are full time assigned according to the regulation, however $59.0 \%$ are commissioned other than IPC activities. IPC nurses stated that they mostly need training programme on new epidemiological criteria and new diagnostic criteria, and $24.2 \%$ stated that they need a full re-training about IPC.

Conclusion: The survey results indicate the importance of institutional support and continuous training in IPC for infection control nurses after national regulations.

Disclosure of Interest: None declared.

Poster Session: Antimicrobial resistance-Surveillance

\section{P001}

Healthcare associated multidrug resistant organism (HA-MDRO) incidence in Malaysia public hospitals from January to December 2019

N. F. Bakhtiar ${ }^{1}$ on behalf of Infection control unit, S. Amir Husin ${ }^{1, *}$ ${ }^{1}$ Infection Control Unit, Ministry of Health Malaysia, Putrajaya, Malaysia

Correspondence: $\mathrm{S}$. Amir Husin

Antimicrobial Resistance \& Infection Control 10(1): P001

Introduction: Healthcare associated infections due to Multidrug Resistant Organism (MDRO) present a growing threat to global health. Objectives: This study aimed to determine the incidence of specified MDROs in Malaysia hospitals using standardized laboratory and clinical criteria.

Methods: The surveillance program is a laboratory based which involved $28 \mathrm{MOH}$ and 3 university hospitals. It is a continuous active monitoring of the incidence of specified organisms of clinical interests. Results: A total of 13,966 clinical isolates of 6 alert organisms and other groups were reported from January to December 2019. Out of these isolates, $75 \%(10,438)$ were HA-MDRO. The incidence for each MDROs is shown in Table 1. MDROs rate was highest in Medical department (32.4\%) followed by Surgical (15.4\%) and Anaesthesia department (15.1\%). Majority of the HA-MDRO infection were bloodstream infection $(38.1 \%)$, followed by pneumonia $(14.8 \%)$, ventilatorassociated pneumonia (12.4\%) and surgical site infection (11.1\%). The top 3 commonest antibiotics prescribed within the last 3 months among MDRO patients were Cephalosporins, Beta lactamases and Carbapenems.

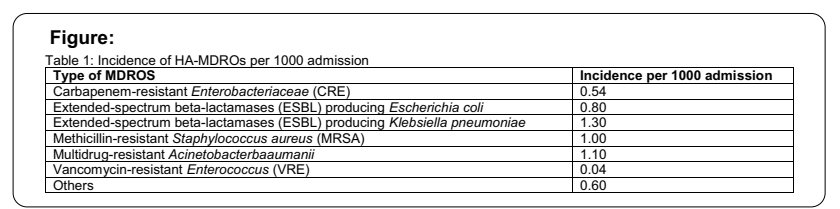

Conclusion: MDRO surveillance program is crucial in order to monitor the trend of MDRO and to detect newly emerging pathogen or outbreak. Besides that, it is used to monitor the effectiveness of infection prevention and control interventions and to identify areas for improvement.

Disclosure of Interest: N. F. Bakhtiar Employee of: Ministry of Health Malaysia, S. Amir Husin Employee of: Ministry of Health Malaysia.

P002

High adherence to national IPC guidelines as key to sustainable VRE control in Swiss hospitals?

D. Vuichard-Gysin 1,2,*, R. Sommerstein ${ }^{3}$, N. Buetti ${ }^{4}$, A. Kronenberg ${ }^{5}$, C. Gardiol $^{6}$, S. Harbarth $^{4}$, A. Widmer ${ }^{7}$ on behalf of Swissnoso

${ }^{1}$ Swiss Center for Infection Prevention, Swissnoso, Bern, ${ }^{2}$ Infectious Diseases, Thurgau Hospital Group, Muensterlingen, Infectious Diseases, Hirslanden Central Switzerland, Lucerne, ${ }^{4}$ Infection Control Programme, University of Geneva Hospitals, Geneva, ${ }^{5}$ Infectious Diseases, University of Bern, ${ }^{6}$ Federal Office of Public Health, Bern, ${ }^{7}$ Infectious Diseases, University of Basel Hospitals, Basel, Switzerland

Correspondence: D. Vuichard-Gysin

Antimicrobial Resistance \& Infection Control 10(1): P002

Introduction: In 2018, the national center for infection prevention (Swissnoso) elaborated guidelines to prevent nosocomial outbreaks with vancomycin resistant enterococci (VRE).

Objectives: To evaluate the level of adherence to this new guideline and its impact on the VRE epidemiology in Swiss acute hospitals.

Methods: In a survey, we asked Swiss acute care hospitals about changes in infection prevention and control (IPC) measures, details on VRE surveillance and outbreaks in the years 2018 and 2019.

Results: Overall, 97/146 (66\%) hospitals participated and 72/81 (88\%) have adopted the Swissnoso guideline. Since 2018, 57 hospitals have changed their IPC measures: $89.5 \%$ introduced admission screening, $56 \%$ screening and $58 \%$ preventive contact precaution (CP) for suspected VRE cases. Hospitals with VRE cases were significantly more likely to have changed their IPC strategies (38/51) than those hospitals without VRE cases $(19 / 38)(p=0.015) .52$ hospitals reported 569 new VRE cases ( 14 bacteremia) in 2018, and 472 new cases ( 10 bacteremia) in 2019. The 10 largest outbreaks accounted for 671 (64\%) VRE cases, $93 \%$ were colonization events, $4 \%$ infections and $2 \%$ bacteremia. Of 85 respondents, $13(15.3 \%)$ reported difficulties in implementing all recommendations. 9 reservations were related to implementation of CP for VRE cases $(2 / 84,2.1 \%)$, adoption of preventive CP for VRE contacts (3/84, 3.1\%) and admission screening (4/85, 4.1\%).

Conclusion: Acceptance and adherence with the new national VRE control guideline among Swiss hospitals seem high. However, whether this has a sustained effect on VRE control at the national level remains to be seen.

Disclosure of Interest: None declared.

P003

Proposal of a national definition of multidrug-resistant invasive gram-negative organisms by ANRESIS

O. Friedli $i^{1, *}$, A. Kronenberg ${ }^{1}$

${ }^{1}$ Institute for Infectious Diseases (IFID), University of Bern, Bern, Switzerland

Correspondence: O. Friedli

Antimicrobial Resistance \& Infection Control 10(1):P003. 
Introduction: Surveillance of multidrug-resistant (MDR) microorganisms is key in national AMR programs, however there is no universally accepted MDR definition in Switzerland so far.

Objectives: In this study, we aimed to develop a national definition of MDR-Enterobacterales (MDR-E), adapting international definitions to local testing algorithms and surveillance data available in ANRESIS.

Methods: Using interpreted qualitative susceptibility data (SIR) from 2019-2020 from the ANRESIS database, we analyzed the test algorithms applied by swiss laboratories and examined the crossresistance within antibiotic groups. Based on these analyses, two different MDR-E definitions depending on the intended use, a broader one for epidemiological surveillance ("ANRESIS-EPI") and a more restrictive one for infection control ("ANRESIS-IPC") were specified. Using these algorithms, the rates of invasive MDR-E identified in our national dataset were compared with international and national definitions, namely the ECDC-definition, the KRINKO-definition and the definition proposed by the University Hospital Zurich. Results: Aminoglycosides, Piperacillin-Tazobactam, $3^{\text {rd }} / 4^{\text {th }}$ gen. cephalosporins, carbapenems and fluoroquinolones were used for our MDR-E definition. Major differences in testing algorithms between different laboratories were observed. The analysis of crossresistance within an antibiotic group revealed, that the substance most likely to be effective against a specific gram-negative bacterium, is not tested preferentially (e.g. amikacin). For all bacterial species tested, the highest rates of multidrug-resistant isolates were found using the "ECDC-MDR" definition $(N=3664)$. The number of MDR-E identified by using the ANRESIS-IPC $(N=277)$ definition was comparable to those detected with the KRINKO $(\mathrm{N}=267)$ respectively UHZ definition $(N=193)$. However, the isolates classified as MDR-E by KRINKO, UHZ and ANRESIS-IPC $(\mathrm{N}=387)$ differed significantly. Only 101 of the isolates (26.1\%) were uniformly classified as MDR-E according to the KRINKO, UHZ and ANRESIS-IPC definitions.

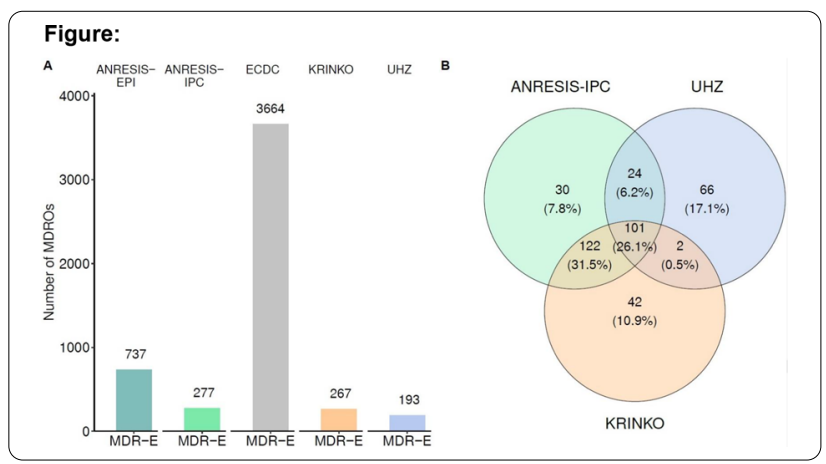

Conclusion: The application of different MDR definitions leads not only to considerable variations in the rates of MDR-E, but also in the isolates that are finally classified as MDR-E. Different testing algorithms complicate an uniform MDRO definition.

Disclosure of Interest: None declared.

P004

Hospital outbreaks of multidrug-resistant bacteria-ICU-based surveillance data indicate underreporting to health authorities C. Schroeder ${ }^{1}$, M. Behnke', P. Gastmeier ${ }^{1}$, F. Maechler ${ }^{1, *}$

${ }^{1}$ Institute of Hygiene, Charité Universitätsmedizin, Berlin, Germany

Correspondence: F. Maechler

Antimicrobial Resistance \& Infection Control 10(1): P004

Introduction: In Germany, $\geq 2$ hospital acquired infections with an epidemiological link and subsequent colonisations with the same organism are subject to reporting obligations to the public health authorities.

Objectives: We aimed to calculate the number of clusters which met the reporting criteria from ICU-based multidrug resistant organisms
(MDRO) surveillance data within the Hospital Infection Surveillance System (KISS), and to compare them with published outbreak data from the health authorities.

Methods: We used surveillance data on Methicillin-resistant Staphylococcus aureus (MRSA), Vancomycin-resistant Enterococci (VRE), multidrug-resistant Escherichia coli (MDR-Ec) and Klebsiella pneumoniae (MDR-Kp) from the ICU-based MDRO surveillance within KISS. The patient-based data comprise the day of admission, and the days of MDRO acquisition and infection, if applicable. ICU clusters were defined as $\geq 2$ ICU-acquired MDRO infections within 14 days, and all subsequent ICU-acquired MDRO infections or colonisations in corresponding 14 day-periods.

Results: Between 2015-2019, 608 ICUs from 428 hospitals participated, representing $22 \%$ of German hospitals. 223 MDRO clusters that met reporting criteria were identified. The table presents the description of cases and clusters per MDRO. Published data from the health authorities reported hospital outbreaks with Enterococcus spp. $(\mathrm{N}=68)$, Staphylococcus spp. $(\mathrm{N}=93)$, Escherichia coli $(\mathrm{N}=12)$ and Klebsiella spp. (63) during the same time, without specified antimicrobial phenotype and without restriction to ICUs.

\begin{tabular}{|l|l|l|l|l|}
\hline & VRE & MRSA & MDR-EC & MDR-Kp \\
\hline ICUs N & 571 & 580 & 575 & 575 \\
\hline Patients N & $1,900,128$ & $1,936,421$ & $1,925,967$ & $1,925,967$ \\
\hline MDRO cases N & & & & \\
\hline Colonisations/lnfections & 24,490 & 23,915 & 25,705 & 6,589 \\
\hline Acquired N & $21,246 / 3,24$ & $19,516 / 4,39$ & $20,211 / 5,49$ & $4750 / 183$ \\
\hline Clusters N & 4,789 & 2,452 & 4,207 & 9 \\
\hline Median clusters/lCU N (max) & 91 & 68 & 53 & 28 \\
\hline Cases in clusters N (\% acquired cases) & $1(8)$ & $1(6)$ & $1(3)$ & $1(2)$ \\
\hline Median cases/cluster N (min, max) & $298(4 \%)$ & $176(7 \%)$ & $137(4 \%)$ & $77(6 \%)$ \\
\hline
\end{tabular}

Conclusion: MDRO outbreaks from ICU-based KISS surveillance alone would account for 223 of 260 (86\%) of all published hospital outbreaks. Compared to hospital-wide outbreak data from the health authorities which include more bacterial species and phenotypes, our data indicate underreporting of hospital outbreaks.

Disclosure of Interest: None declared.

P005

Bacteriological profile and trends of antimicrobial resistance in healthcare-associated pathogens in Tunisian teaching hospital

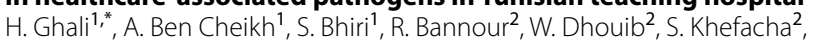
H. Said Latiri ${ }^{1}$, M. Ben Rejeb ${ }^{1}$

${ }^{1}$ Department of Prevention and Security of Care, Sahloul university hospital, Faculty of Medicine of Sousse, University of Sousse, ${ }^{2}$ Department of Prevention and Security of Care, Sahloul university hospital, Sousse, Tunisia

Correspondence: $\mathrm{H}$. Ghali

Antimicrobial Resistance \& Infection Control 10(1): P005

Introduction: Antimicrobial resistance in health care-associated pathogens is a growing concern for health care and for public health. Thus, studying temporal changes in resistant pathogens causing healthcare-associated infections (HAls) is crucial in improving local antimicrobial and infection control practices.

Objectives: The objective was to describe nine-year trends of resistance in pathogens causing HAls in a tertiary care setting in Tunisia.

Methods: Annual prevalence surveys are part of a quality improvement program promoted by our department in Sahloul university hospital for the prevention and control of HAls in order to ensure patient safety. The current study focused on data collected from 2012 to 2020. Definitions and methodology of HAls and antimicrobial resistance were based on CDC. Data collection was carried out using NosoTun plug.

Results: Results for microbiological investigation were available for 165 HAls $(55.7 \%)$. The proportion of HAls that was microbiologically confirmed decreased from $58.1 \%$ in 2012 to $56.5 \%$ in 2020 $(p=0.1)$. A total of 234 pathogens causing $297 \mathrm{HAl}$ events were included. Gram negative pathogens (GNP) were the most common 
pathogens. A decreasing trend was notified for Klebsiella spp, Pseudomonas aeruginosa, and Staphylococcus aureus. However, this decrease was not statistically significant. Antimicrobial resistance was found in $32.1 \%$ of cases.

Significant increasing trend in antimicrobial resistance was noticed (from $15.6 \%$ in 2012 to $21 \%$ in $2020 ; p<10-3$ ).

Conclusion: There is a high burden of drug resistance to common antibiotics in Sahloul university hospital. These findings may highlight the need for effective antimicrobial stewardship programs, including monitoring and feedback on antimicrobial use and resistance.

Disclosure of Interest: None declared.

\section{P006}

Survival analysis of multidrug-resistant organisms infection among patients with different catheterization in ICU J. Yang ${ }^{1, *}$, Y. Tan ${ }^{2}$, W. Wu ${ }^{3}, \mathrm{~F} . \mathrm{Hu}^{4}$, Y. Wang ${ }^{5}$

${ }^{1}$ Tongji Medical College, Huazhong University of Science and Technology, ${ }^{2}$ Department of Nosocomial Infection Management, Zhongnan Hospital of Wuhan University, Wuhan, ${ }^{3}$ Hubei University of Medicine, Shiyan, ${ }^{4}$ Department of critical care medicine, Zhongnan Hospital of Wuhan University, ${ }^{5}$ Zhongnan Hospital of Wuhan University, Wuhan, China Correspondence: J. Yang

Antimicrobial Resistance \& Infection Control 10(1): P006

Introduction: The incidence of nosocomial infection in ICU is significantly higher than that in general wards, and the detection rate of MDRO is much higher than the overall level of the hospital, making ICU the key department of MDRO infection and the high-risk Department of nosocomial infection outbreak. It is reported that ICU patients widely use "three tubes", namely ventilator, catheter and central venous catheter, with application rates of $10.8 \%, 53.5 \%$ and $47.2 \%$ respectively. However, in the whole process of catheter insertion, pipeline maintenance and extubating, there are many risk points of MDRO infection. It can be seen that the prevention and control of "three tube" infection is the top priority in the prevention and control of nosocomial infection in ICU.

Objectives: To reveal the infection time and risk factors of multidrugresistant organisms (MDROs) in different kinds of catheter patients in intensive care unit (ICU), so as to provide a reference time risk point for the prevention and control of MDRO in ICU, and to supplement reasonable evidence of prevention and control time for existing prevention and control measures.

Methods: Retrospectively analyzed the basic situation of MDRO infection patients with three different tube placements in a general ICU from January 1, 2019 to December 31, 2019 in a third-class A hospital. The median time, time difference and risk factors of infection in patients with different catheterization were revealed by survival analysis.

Results: 5138 cases were included. Age, operation or not, three tube use are high risk factors of MDRO infection $(p<0.05)$. The median survival time (Infection time) of patients with ventilator, catheter and central vein intubation was $\left(M=1.00 ; P_{25}=0.00 ; P_{75}=5.00\right) d ;(M=1.00$; $\left.P_{25}=0.00 ; P_{75}=5.00\right) d ;\left(M=1.00 ; P_{25}=0.00 ; P_{75}=6.00\right) d$. Ventilator and catheter were independent risk factors.

Conclusion: It is an important measure to reduce MDRO infection in ICU by reducing the use days of ventilator and catheter.

Disclosure of Interest: None declared.

\section{P007}

Resistance features in bacterial strains isolated from the hospital environment before and during COVID-19

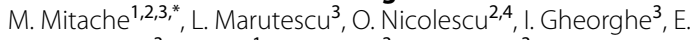
Hassanzadeh $^{3}$, E. Rusu', C. Curutiu ${ }^{3}$, C. Chifiriuc ${ }^{3}$

${ }^{1}$ Faculty of Medicine,University Titu Maiorescu Bucharest, ${ }^{2}$ Public Health Directorate Bucharest Romania, ${ }^{3}$ Faculty of Biology University of Bucharest, ${ }^{4}$ Faculty of Medicine, Carol Davila University, Bucuresti, Romania Correspondence: M. Mitache

Antimicrobial Resistance \& Infection Control 10(1): P007
Introduction: The coronavirus disease 2019 (COVID-19) pandemic has imposed the use of increased amounts of disinfectants and antibiotics, that will probably influence the features of microbial strains colonizing the hospital surface.

Objectives: The purpose was to assess the impact of disinfectants and antibiotics used during the COVID-19 pandemic in hospitals with patients coronavirus positive, by the comparative analysis of the antibiotic resistance and biocides tolerance profiles of bacterial strains isolated before and during COVID-19.

Methods: A number of 103 bacterial strains: 61 from 2018 and 42 from 2021 were isolated from the hospitals and identified by MALDITOF. The antibiotic resistance phenotypes were established by disk diffusion and the minimal inhibitory concentrations (MIC) for five disinfectants were determined by broth microdilution method.

Results: The analyzed strains were mainly E. coli, A.baumannii, S.aureus before and S. haemolyticus, P. aeruginosa and Enterobacter sp. after COVID-19. If before pandemic the resistance rates of Gram-negatives to 3rd and 4th generation cephalosporins reached $73.3 \%$ and $70-85 \%$ for quinolones and tetracyclines, after the pandemic the resistance rates $3 \mathrm{rd}$ generation cephalosporins remained high, i.e. $87.5 \%$, while for the other antibiotics the rates were $25-50 \%$. The MIC for the disinfectants varied between $1.56-25 \%$ for Ethanol, Propan-2-ol, 0.78-12.5\% for Ethanol/Denatured isopropano and Ethanol, $0.0019-0.125 \%$ for menthol, ethyl alcohol/hydrogen peroxide, peracetic acid.

Conclusion: By using high amounts of disinfectants and antibiotics for containment of COVID-19 the diversity and resistance features of microbial strains from the hospital environment were modified, an increased microbial diversity after COVID-19, decreased resistance rates to non-beta-lactam antibiotics and higher tolerance to ethanol containing disinfectants.

Disclosure of Interest: None declared.

\section{P008}

Implementing an antimicrobial resistance surveillance tool in a low-income country: the TSARA project in Madagascar C. Elias 1,", M. Raad ${ }^{2}$, Y. Mallet ${ }^{2}$, L. Rakotoarisoa ${ }^{3}$, L. Raskine ${ }^{2}$, F.-X. Babin², M. Randria ${ }^{4}$

${ }^{1}$ Lyon University Hospital, ${ }^{2}$ Fondation Mérieux, Lyon, France, ${ }^{3}$ Fondation

Mérieux, ${ }^{4}$ HUJR Befelatanana, Antananarivo, Madagascar

Correspondence: C. Elias

Antimicrobial Resistance \& Infection Control 10(1): P008

Introduction: Antimicrobial stewarship programs(ASP) in hospitals are a vital component of national action plans for antimicrobial resistance(AMR), and have been shown to significantly reduce AMR, in particular in a low-income country such as Madagascar. As part of an ASP, AMR surveillance provides essential information to guide medical practice. We developed an AMR surveillance tool called TSARA with the support of Fondation Mérieux, with the objective of combining patient data with laboratory and epidemiological surveillance data to provide a better understanding of the scope of AMR in Madagascar.

Objectives: To describe how the TSARA project was developed in Malagasy hospitals and its outcomes.

Methods: Based on the Oxford GRAM protocol, TSARA is a standardized data collection tool deployed since 2018 in five Malagasy hospitals participating to the RESAMAD laboratory network, monitoring microbiological, demographic and clinical data (Figure). Any hospitalized patient with a positive bacterial sample was included.

Results: Individual isolate-level data and antimicrobial susceptibility information on bacteria were collected. These data were compiled with clinical, demographic, and epidemiological information (including current antibiotic prescription) retrieved daily from face-to-face interviews with the patient and completed by medical records where necessary. Data were then entered in an online platform. Results were secondarily discussed with clinicians to provide guidance on the antibiotic prescription adapted to local resistance patterns. As an example, 2018 data showed that $57.8 \%$ of empiric antibiotic prescriptions were eligible for de-escalation after reception of antimicrobial susceptibility tests. Finally, 
results of aggregated data were disseminated to relevant stakeholders to improve awareness among prescribers and hospital gouvernance.

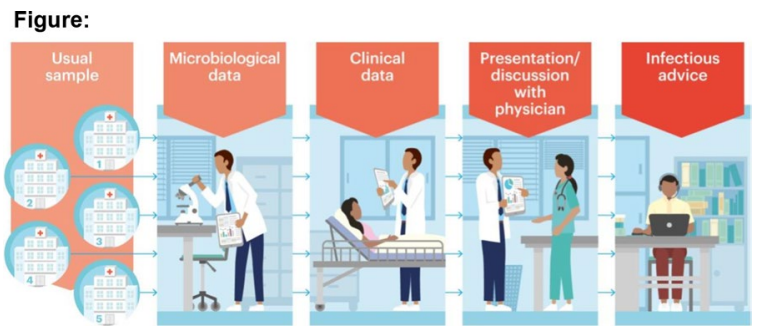

Conclusion: Thanks to TSARA, regular analysis and feedback on surveillance data to healthcare workers helped to optimize patient care. Treatment guidelines were produced by Malagasy experts according to type of patient and type of infection, with consideration of circulating pathogens and local resistance levels.

Disclosure of Interest: None declared.

Poster Session: Antimicrobial resistance in paediatrics

\section{P009}

Antimicrobial resistance in enterococcus faecalis and enterococcus faecium in children hospitals in China: a meta-analysis

J. Wang ${ }^{1, *}$, M. Zhou ${ }^{2}$, F. Liu ${ }^{3}$, Y. F. Lee ${ }^{4}$

${ }^{1}$ Institute of Social Medicine and Health Systems Research, Medical Faculty, Otto von Guericke University, Magdeburg, Germany, ${ }^{2}$ Department of Infection Control, Dongguan Tung Wah Hospital, Dongguan city, China, ${ }^{3}$ Institute of Global Health, Faculty of Medicine, University of Geneva, Geneva, Switzerland, ${ }^{4}$ Sarawak General Hospital, Kuching, Malaysia

Correspondence: J. Wang

Antimicrobial Resistance \& Infection Control 10(1): P009

Introduction: Enterococci is one of the leading cause of highly antibiotic-resistance infections, especially healthcare-associated infections. However, few epidemiological data estimated the burden of antimicrobial resistance in Enterococci in children healthcare settings.

Objectives: This study aimed to assess the incidence proportions of antimicrobial resistance profiles in Enterococcus faecalis and Enterococcus faecium in children hospitals in China, using data published in both English and Chinese literatures.

Methods: We searched PubMed, the China National Knowledge Infrastructure for antimicrobial resistance surveillance reports in the children hospitals in China from January 2019 until May 2021.

Results: Seven studies were eligible for final analysis. Most of studies were conducted in higher socioeconomic regions in China (e.g. Beijing, Shanghai, and Guangdong). Among E. faecalis of 1608 strains from all samples, the weighted incidence proportion of vancomycin-resistant $E$. faecalis, nitrofurantoin-resistant E. faecalis, linezolid-resistant $E$. faecalis, ampicillin-resistant $E$. faecalis, levofloxacin-resistant $E$. faecalis, and gentamicin-resistant $E$. faecalis were $0.2 \%$, $4.4 \%, 3.2 \%, 11.7 \%, 12.2 \%$, and $40.3 \%$, respectively. Among E. faecium of 2872 strains from all samples, the weighted incidence proportion of vancomycin-resistant $E$. faecium, nitrofurantoin-resistant $E$. faecium, linezolid-resistant $E$. faecium, ampicillin-resistant $E$. faecium, levofloxacin-resistant $E$. faecium, and gentamicin-resistant $E$. faecium were $0.8 \%$, $59.2 \%, 0.9 \%, 92.5 \%, 78.9 \%$, and $48.7 \%$, respectively.

Conclusion: This is the first review estimating about epidemiological burden of antimicrobial resistance in E. faecalis and E. faecium in the children hospitals in China. The identified burden of vancomycinresistant E. faecium was similar to the 2017 CHINET report for general hospitals, but lower compared to 2019 ECDC AMR report for general hospitals.

Disclosure of Interest: None declared.
P010

Implementation of a bundle to control of MDR-gram negative bacteria spreading and clinical impact in a pediatric intensive care unit

L. M. Parra Ramírez', E. Doñate ${ }^{1, *}$, M. Nieto ${ }^{2}$, B. Hernandez ${ }^{3}$ on behalf

of Infection Prevention and Control Unit

${ }^{1}$ Preventive Medicine Department, ${ }^{2}$ Intensive care unit, ${ }^{3}$ Department

of Microbiology, Niño Jesús University Hospital, Madrid, Spain

Correspondence: E. Doñate

Antimicrobial Resistance \& Infection Control 10(1): P010

Introduction: During October and November 2020, we observed a significance increase of MDR-GNB in clinical specimens from patients cared in a pediatric intensive care unit (PICU).

Objectives: We conducted an epidemiological investigation.

Methods: In addition to clinical samples, all hospitalized patients in PICU were screened on admission and weekly thereafter for MDR-GNB in samples from the nose, trachea and rectum. Environmental sampling was performed from surfaces (sinks, faucets) air and water.

Identification of the species isolated from both clinical and environmental samples was performed by Vitek ${ }^{\circledR} 2$ Compact, bioMérieux, Inc., Durham, North Carolina, USA. Samples with positive isolation were sent to the national reference laboratory of microbiology (ISCIII) for genotypic study by sequencing (PFGE).

We performed a spatiotemporal analysis of cases and sequential analyses of the measures taken to determine the effectiveness in cluster.

Results: From October 12 through early November 2020, 25 specimens were identified, corresponding to 7 patients positive for MDR-GNB.

The attack rate (colonization) was $8.6 \%(7 / 81)$. Four of patients were colonized with extended-spectrum ß-lactamase (ESBL) and the others with carbapenemase-producing Enterobacteriaceae (CPE).Three of the patients (42.9\%) had polymicrobial colonization.

Of 30 environmental samples, five were positive for CPE (sinks- 100\% $K$. pneumoniae VIM). None of the water and air samples tested were positive.Genotypic sequencing by PFGE was performed on the 4 clinical samples (all patient colonized with CPE) and five environmental samples. Different PFGE profiles were found between patients, but environmental samples had similar and indistinguishable PFGE pattern.The initial measures taken were contact isolation of patients, removal of atomizers, and closing the sinks.

After implementation of bundle (contact precautions, hand hygiene re-education, waterless cleaning of patient, anti-splash screens, routine cleaning of sinks, routine environmental screening) no cases appeared.

Conclusion: Implementation of a focused bundle on patient hygiene and regular environmental cleaning in PICU reduced the MDR-GNB colonization rate.

Disclosure of Interest: None declared.

P011

"Neonatal sepsis" - gram positive \& negative bacteria's and their antibiotic susceptibility in NICU

J. Thankachan ${ }^{1, *}$

${ }^{1}$ King Saud Medical City, Riyadh, Saudi Arabia

Correspondence: J. Thankachan

Antimicrobial Resistance \& Infection Control 10(1): P011

Introduction: Neonatal sepsis is one of the most common causes of Neonatal Mortality and Morbidity in developing countries. Its causative bacteria's and their sensitive patterns are different in each hospitals and regions. The objective of the study was to determine the causation bacteria's and their pattern of susceptibility to antibiotic $s$ in NICU of a tertiary care center.

Objectives: Gram Positive \& Negative Bacteria's and to identify their Antibiotic Susceptibility.

Methods: The prospective study was carried out at a tertiary care hospital during the period from January 2020 to January 2021. A total of 1712 blood cultures sent from different sites of the patients in NICU 
during this period. Out of which 1011 cultures came with positive bacterial growth.

(Includes gram + ve \& -ve, Cons and other Recognized pathogens). All cultures were screened by using a micro ESR \& Culture and Sensitivity methods.

Results: A total of 1011 cultures were found to be positive out of 1712 cultures sent. Out of 1011 positive cultures $450+$ are of gram positive organisms and remaining are of positive results with growth.

The most common organisms isolated was Staph Epidermidis (68\%), followed by Staph Heamolyticus (10\%), Staph Hominis(7\%), Enterococcus Fecalis(5\%). Other Organisms where much less in number which includes Pathogenic Streptococcus, Enterococcus Feacium, Staph Capitis, Bacillus Species etc.

There was Incidence of Gram Negative Organisms like E. coli, Proteus Mirabilis, Serratia Marcescens were also got isolated from Cultures.

The Gram Positive Organisms except Streptococcus displayed a high degree of resistance to most Penicillin and Ciprofloxacin and were most time sensitive to Vancomycin, Amikacin and Cefepime. In some cases high incidence of resistance noted with Ampicillin, Gentamicin amongst most Gram Negative Organisms, where in Cefepime, Amikacin and Meropenem were effective in most cases.

Conclusion: There is an increasing trend of antibiotic resistance to the commonly used front line drugs. Continuo's surveillance for antibiotic susceptibility is needed to ensure proper empirical therapy.

Disclosure of Interest: None declared.

\section{P012}

Microbial spectra and antibiotic susceptibility of acute respiratory infections among children under 5 years of age in a developing country

M. M. Bullo1,*

${ }^{1}$ Ministry Of Health Islamabad, Federal General Hospital Islamabad,

Islamabad, Pakistan

Correspondence: M. M. Bullo

Antimicrobial Resistance \& Infection Control 10(1): P012

Introduction: Estimated ARI mortality is 4 to 5 million children annually in developing countries. Around 15 million episodes of ARI occur in Pakistan every year among children.

Objectives: The study aimed to examine microbial spectra and antimicrobial susceptibility of bacterial isolates of acute respiratory infections among under-fives.

Methods: An observational study was conducted among under 5 children in a public sector hospital of Islamabad Pakistan from Oct 2018 to January 2019. Overall 350 nasal swabs were collected (300 from symptomatic and 50 from asymptomatic children). RT-qPCR was used for screening viral pathogens. Bacteriological culture methodology was used for bacterial pathogens and CLSI guidelines were adapted for antimicrobial susceptibility.

Results: Among symptomatic cases (300), 54\% were females and median age was 25 months ( $R=59-1$ months). Most affected age group was 12 months and below. Overall positive samples for microbes were $83 \%(n=249)$. Of 249 infected cases, single bacterial pathogen was detected among $6 \%(n=18)$ of cases, while single viral pathogen was detected among $30 \%(n=90)$ of cases. Remaining $64 \%(n=192)$ had co-infections. Predominantly detected viruses were RSV (32\%), human rhinovirus (30\%), HMPV (14\%), HPIV-3 (11\%), $\mathrm{HBoV}(9 \%)$, and adenovirus (4\%). Frequently isolated bacteria's were Klebsiella pneumaniae (15\%), Streptococcus pneumonia, Enterobacter agglomerans, and Haemophilus influenzae were $13 \%, 10 \%$ and $7 \%$, respectively. HPIV-3, Human rhinovirus, Adenovirus, Klebsiella pneumaniae and Streptococcus pneumonia found significantly involved in co-infections with P values of $0.01,0.03,0.01,0.01$ and 0.01 . Of 17 multidrug-resistant bacteria, isolate of Enterobacter agglomerans and Klebsiella pneumonia were identified as resistant bacteria against more than 5 different antibiotics. Pathogen detection rate (14\%) was significantly lower in asymptomatic group than the symptomatic $(P<0.01)$.

Conclusion: Under five children are at high risk of ARI and prone to AMR. Regular surveillance of the type of isolates and their AMR patterns should be considered along with proper infection control measures.

Key words: Microbial spectra, Antibiotic susceptibility, ARI, Pakistan.

Disclosure of Interest: None declared.

P013

Microbiology patterns of otorrhea at the university hospital of Cocody, Abidjan

E. Ngoran ${ }^{1, *}$, A. I. Yapi ${ }^{2}$, J. A. Bahan², M. A. NGuiachi' ${ }^{1}$ E. Angora ${ }^{1}$, B. TEA ${ }^{3}$, A. KACOU N'DOUBA ${ }^{4}$

${ }^{1}$ Parasitology Mycology, ${ }^{2}$ Microbiology, University Hospital Center of Angre, ${ }^{3}$ OtoRhinoLaryngology, Faculty of medicine, University Felix Houphouet Boigny, ${ }^{4}$ Medical Biology, University Hospital Center of Angre, Abidjan, Côte d'Ivoire

Correspondence: E. Ngoran

Antimicrobial Resistance \& Infection Control 10(1): P013

Introduction: Otorrhea is a frequent symptom in routine clinical practice and a source of complications. The treatment is often empirical by prescribing topical antibiotics. The interest based on etiological diagnosis in order to improve the management of otorrhea.

Objectives: This study aimed to characterize the microbiology etiology of otorrhea.

Methods: This was a descriptive cross-sectional study extending from 2009 to 2019. We enrolled patients with otorrhea who presented to ear nose and throat (ENT) consultation of the Cocody University Hospital. Sociodemographic, clinical and therapeutic data were collected in patient records. Microbiology data were recorded at Pasteur Institute of Côte dlvoire where ear fluid samples were analyzed.

Results: The global prevalence of otorrhea was 3.27\% (555/16921). Children aged 0 to 15 years predominated with 40.2\% (223/555). Female patients accounted for 53\% (298/555).

The performance of microbiological analysis was low $13.7 \%(76 / 555)$. Bacterial etiology was observed in $11.2 \%(62 / 555)$ versus $2.9 \%(16 / 555)$ of mycosis. The association of germs was found in 10 cases ie $1.8 \%$. The main bacteria were Pseudomonas aeruginosa $4.3 \%$ (24/555) and Staphylococcus aureus 3.1\% (17/555). The isolation rate of yeast were Candida albicans 1.1\% (6/555), Candida sp 0,9\% (5/555) and Aspergillus niger $0,5 \%(3 / 555)$. Antibiotic and antifungal susceptibility test was performed respectively in $6.8 \%(38 / 555)$ and $0.7 \%(4 / 555)$.

Conclusion: This study showed that the frequency of the germs isolated in otorrhea remains low in Côte d'Ivoire. Knowledge of microbiological etiology of otorrhea may improve their management.

Disclosure of Interest: None declared.

P014

Effect of mutation on ampc promoter in multidrug resistant

isolates of diarrheagenic Escherichia coli in children

T. Singh ${ }^{1, *}$, S. Das ${ }^{1}$

${ }^{1}$ Microbiology, University of Delhi, Delhi, India

Correspondence: T. Singh

Antimicrobial Resistance \& Infection Control 10(1): P014

Introduction: Drug resistance among gram-negative bacteria is mainly conferred by Beta ( $\beta$ )-lactamase enzymes. AmpC beinga $\beta$-lactamase facilitate resistance to a widerangeof antibiotics. $\beta$ lactamases are diverse in nature due to existence of frequent mutations. There are multiple ways for the hyper production of $\beta$-lactamases in gram-negative bacteria but mutations in the promoter region is the most common mechanism.

Objectives: The main objective of the study is to find out the role of promoters in drug resistance.

Methods: Analysis of m-RNA expression of three genes of AmpC $\beta$-lactamases $(A B L)$ was carried out in fifteen drug-resistant and fifteen sensitive $E$. coli isolates in children up to five years of age. Three $A m p C \beta$-lactamase genes were selected (ACT, DHA and CMY). In order to know the effect of mutation on $\mathrm{m}$-RNA expression level in,sequence analysis of the PCR amplicons ( $A m p C$ promoter region) was performed. The promoter sequences were compared with E. coli ATCC 25922, 
using multiple sequence alignment tool (BioEdit version 7.0.5).and sequences were submitted to NCBI for obtaining accession numbers.

Results: Analysis of m-RNA expression of three genes of $A m p C$ $\beta$-lactamases (ABL) was carried out in fifteen drug-resistant and fifteen sensitive $E$. coli isolates in children up to five years of age. Three $A m p C \beta$-lactamase genes were selected ( $A C T, D H A$ and $C M Y$ ). In order to know the effect of mutation on $\mathrm{m}$-RNA expression level in,sequence analysis of the PCR amplicons ( $A m p C$ promoter region) was performed. The promoter sequences were compared with E. coli ATCC 25922, using multiple sequence alignment tool (BioEdit version 7.0.5).and sequences were submitted to NCBI for obtaining accession numbers.

Conclusion: $A m p C$ genes showed many folds increase in the expression levels in resistant isolates due to mutation in their promoter region, indicating their potential role in disease transmission. Strength of expression level of $A m p C \beta$-lactamase genes depends upon the positions of mutation in the promoter region. Hence, strict antibiotic policy has to be implemented in symptomatic and asymptomatic individuals to bring down the rising threat of drug resistance in the community.

Disclosure of Interest: None declared.

Poster Session: Antimicrobial resistance in the community

P015

Treatment fidelity of drug-resistant tuberculosis treatment delivery in primary healthcare in Surabaya city, East Java

Province, Indonesia: an implementary research

A. E. Noveyani $i^{1, *}$, A. N. Probandari ${ }^{2}$, Y. Mahendradhata ${ }^{3}$

${ }^{1}$ Epidemiology, Faculty of Public Health, University of Jember, Jember,

${ }^{2}$ Faculty of Medicine, University of Sebelas Maret, Surakarta, ${ }^{3}$ Faculty

of Medicine, Public Health, and Nursing, University of Gadjah Mada,

Yogyakarta, Indonesia

Correspondence: A. E. Noveyani

Antimicrobial Resistance \& Infection Control 10(1): P015

Introduction: The nearest service for Multidrug-resistant tuberculosis (MDR-TB) may increase treatment adherence. Since 2014, MDR-TB patients are able to get treatment from the nearest Primary Healthcare (PHC). However, the patients' loss of follow-up remains high (20\%) in 2016.

Objectives: This study aimed to assess the fidelity of the treatment process of MDR-TB at the Primary Healthcare level.

Methods: The study applied a mixed-method design in all PHCs in Surabaya, Indonesia from March to June 2017. The study population was all MDR-TB patients and staffs who responsible for the MDR-TB treatment service. A survey and in-depth interview were conducted. The quantitative data were analyzed descriptively and the qualitative data were analyzed by content analysis.

Results: 23 out of 30 PHCs adhered to the National MDR-TB treatment guideline regarding the treatment delivery components, the counseling and psychosocial support components, and the adverse drug effect management components. However, from the interview, we found that there is no Standard Operating Procedure (SOP) specific for MDR TB treatment at the PHC level, and it was a key factor for staffs did not conduct some core components of the MDR-TB guideline. Those were the TB staffs did not give comprehensive information support at the beginning of MDR TB treatment; The staff did not monitor the patient during the treatment, and even rarely gave counseling to the MDR TB patient.
Conclusion: MDR-TB treatment delivery at PHCs showed high fidelity, but it missed in some core points: giving complete informational support, monitoring the patient taking drugs, and counseling the patient. Insufficient mandate due to MDR TB treatment resulted an inadequate patient-centred care. Developing SOP for MDR-TB treatment in Primary Healthcare is needed for improving the compliance of the staffs and the quality of patient care.

Disclosure of Interest: None declared.

\section{P016}

Status of drug resistant (DR) tuberculosis (TB) infection control in Nepal

P. Shrestha,*

${ }^{1}$ Young Professional Development Society Nepal (YPDSN), Kathmandu, Nepal

Correspondence: P. Shrestha

Antimicrobial Resistance \& Infection Control 10(1): P016

Introduction: Infection control involves combination of measures that minimizes risk of TB transmission. Infection control is a major component of END TB Strategy. In Nepal, infection control is a key component of TB preventive services. The National Strategic Plan (NSP) 2016-2021 envisions TB infection control as a part of national infection prevention and control policy throughout the country.

Objectives: To identify the status of DR TB infection control in Nepal.

Methods: Desk review was conducted. Different literatures, reports and policy documents related to infection control were reviewed and descriptive analysis was conducted.

Results: National Tuberculosis Program (NTP) Nepal identifies three levels of infection control measures: administrative, environmental and personal protection. NSP identifies lack of infection control policy, program and mechanisms at treatment centers and lack of awareness on infection control as a major challenge for management of DR TB and emphasizes appointment of focal person for infection control. The major intervention includes improving infection control at all DR TB centres and subcentres based on proposed infection control policy. However, till date national infection control policy is still not in place and focal person has not been appointed.

As part of infection control, DR treatment centers were provided with exhaust fans, Ultraviolet Germicidal Irradiation (UVGI), N95 masks and surgical masks. Sessions on infection prevention were incorporated in all TB training. NTP also initiated FAST (Finding Actively, Separate temporarily and Treat effectively) approach in 15 major hospitals to actively find people with cough, testing through rapid molecular diagnostics and enabling prompt treatment, decreasing TB transmission. NTP has also focused on infection control at TB consultation room, DR TB patient isolation room and infection control at home. NTP also recommends health workers to follow all infection control procedures and decrease their risk to TB disease.

Conclusion: NTP requires significant efforts for DR TB infection control. The national policy and guidelines on infection control should be in place. Infection control measures should be strengthened with gradual expansion to all centers. A designated focal person should be assigned. In addition, awareness on infection control among all health workers, patients and community members is required.

Disclosure of Interest: None declared. 
P017

Burden of drug resistant (DR) tuberculosis (TB) in Nepal P. Shrestha ${ }^{1, *}$

${ }^{1}$ Young Professional Development Society Nepal (YPDSN), Kathmandu, Nepal

Correspondence: $P$. Shrestha

Antimicrobial Resistance \& Infection Control 10(1): P017

Introduction: Tuberculosis (TB) is a major cause of ill health, one of the top 10 causes of death worldwide and leading cause of death from single infectious agent. Drug Resistant (DR) TB continues to be a public health threat. TB is a major public health problem and one of the top 10 causes of deaths in Nepal. However, DR TB is not as high as the regional or global burden.

Objectives: The objective of the study was to review and assess the burden of DR TB in Nepal.

Methods: Desk review was conducted. Various literatures, reports, policy documents and guidelines related to DR TB in Nepal were reviewed. Descriptive analysis was conducted to present the findings. Results: DR TB services in Nepal are provided through 21 DR TB treatment centers and 81 sub-centers. Although services are ambulatory, facility-based services are being provided through referral centres, DR hostels and DR home. Diagnosis for DR TB is provided through Gene Xpert, culture and DST (Drug Susceptibility Testing) and LPA (Line Probe Assay). DR TB diagnosis is offered to presumptive patients remaining smear-positive on any follow up including failures of first-line treatment and those at high-risk. Responses to treatment is monitored by culture. In 2019, WHO estimated a total of 1,400 DR TB cases for Nepal but only $45.4 \%$ (635) were notified and among them only 392 cases (62\%) were enrolled for treatment, with $38 \%$ primary loss to follow up. Data from previous years also show huge gaps between estimated and notified cases. The achievement for treatment enrollment is below the level of global achievement of $84 \%$ notified cases posing high risk of transmission, presenting that progress in detection is outstripping the capacity to provide treatment and follow-up services. The major barriers to adequate access to treatment are centralized services and reliant on hospital-based care model.

\section{Figure:}

DR TB Case Notification in Nepal, 2017-2019

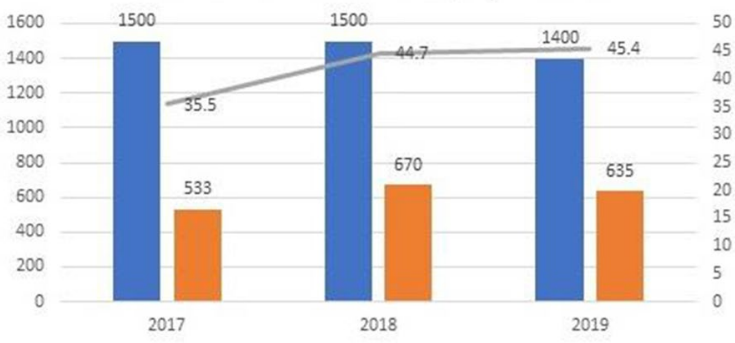

Estimated DR TB Cases Notified DR TB Cases —Percent Notfied

DR TB Case Notification and Enrollment in Treatement, 2017-2019

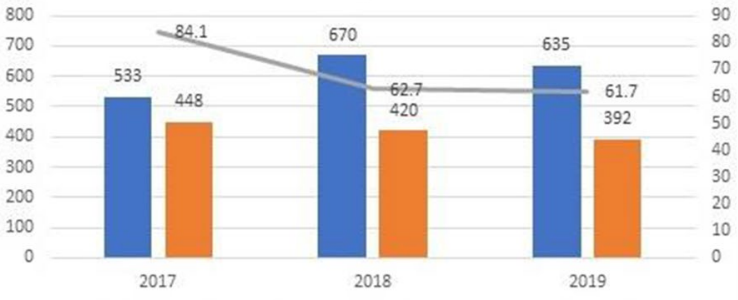

Notified DR TB Cases Enrolled in Treatement —Percent Enrolled
Conclusion: Significant efforts are required to identify and notify the missing cases and enroll the notified cases into treatment without any loss to follow up. Decentralization of diagnosis and treatment services, expansion of ambulatory model of care, quality service delivery and patient centered care can improve DR services.

Disclosure of Interest: None declared.

P018

Barriers and enablers of tuberculosis infection prevention and control activities in selected hospitals in Nepal

S. Paudel $l^{1,2, *}$, R. S. Padmawati ${ }^{3}$, A. Ghimire ${ }^{2}$, C. Lama Yonzon ${ }^{2}$, Y.

Mahendradhata ${ }^{4}$

${ }^{1}$ Public Health Youth Society of Nepal, Pokhara, Nepal, ${ }^{2}$ Faculty of Medicine, Public Health and Nursing, ${ }^{3}$ Department of Health Behavior, Environment, and Social Medicine, ${ }^{4}$ Department of Health Policy and Management, Universitas Gadjah Mada, Yogyakarta, Indonesia

Correspondence: S. Paudel

Antimicrobial Resistance \& Infection Control 10(1): P018

Introduction: Tuberculosis (TB) is a major public health problem in Nepal. Prevention of the spread of TB infection from the hospital \& healthcare setting is one of the important interventions to reduce the burden of disease. The government of Nepal, Nepal TB Control Center, realized the importance of hospital-based intervention to prevent TB infection in a clinical setting and initiated an intervention in major hospitals in high TB burden districts.

Objectives: This study was conducted to identify the barriers, enablers and associated factors of tuberculosis infection prevention activities in selected hospitals in Nepal.

Methods: A qualitative study was conducted from April to August 2019 in four hospitals in Nepal. Focused group discussion, key informant interviews, and client exit interviews were conducted with 27 purposively sampled health professionals and patients. The transcripts were coded, categorized, and analyzed by using thematic analysis.

Results: The study identified that unavailability of a separate room for screening and counseling for TB suspected patients, long waiting period for laboratory report due to limited capacity of GeneXpert machine were commonly reported barriers for infection prevention measures. Similarly, it was found that free TB service, equipment supplies from Government are major enabling factors, regular monitoring, and onsite coaching help to create an enabling environment.The use of wallpapers and the distribution of brochures to the patients plays an important role to increate awareness. It was found that private medical colleges and community hospitals were well managed whereas very limited measures were adopted at Government hospitals for infection prevention.

Conclusion: This study uncovers the barriers and enablers which help to formulate necessary actions and planning for effectively implementation of IPC measures. A separate waiting room, sputum collection booth, dedicated health worker for TB infection prevention activities, and well coordination between departments of the hospitals is a step forward to prevent the spread of TB infection in a hospital setting.

Disclosure of Interest: None declared.

P019

Nutritionals factors of childhood tuberculosis in a developing country: a case control study

M. M. Bullo ${ }^{1, *}$

${ }^{1}$ Ministry of Health Islamabad, Federal General Hospital Islamabad, Islamabad, Pakistan

Correspondence: M. M. Bullo

Antimicrobial Resistance \& Infection Control 10(1): P019

Introduction: Tuberculosis has emerged as a public health issue especially in developing countries like Pakistan and undernutrition is one of the important predictor of Childhood TB. Tuberculosis is considered as significant cause of mortality and morbidity among young children. An adequate and baseline epidemiological information on Childhood TB is critical for its effective control and prevention. 
Objectives: The purpose of this study was to evaluate the nutritional risk factors of Childhood TB at Federal General Hospital Islamabad Pakistan.

Methods: A case control study was conducted at Federal General Hospital (FGH) Islamabad. A case was defined as "any culture confirmed patient of Pulmonary TB under 12 years of age registered in DOTS centre of FGH from $1^{\text {st }}$ January to $31^{\text {st }}$ July 2019. Against each case a healthy age and sex matched control was selected. Structured questionnaire was used for data collection and odds ratios were calculated with $95 \%$ confidence interval.

Results: Total 184 children under the age of 12 years with Pulmonary TB were recruited (92 cases and 92 controls), mean age of the cases were 7 years ( $R=12-4$ years) and $53 \%$ cases were males while rest were females. Most affected age group was below 10 years. Significant nutritional factors were undernutrition (OR 8.11, Cl 4-16.2), stunting (OR 6.63, Cl 3.5-12.5), unhygienic feeding practices (OR 5.3, Cl 2.9-9.6), wasting (OR 4.3, $\mathrm{Cl}$ 2.4-7.5), use of unpasteurised milk (OR 4.1, Cl 2.37.2), underweight (OR 3.3, $\mathrm{Cl} 2-5.5)$, inadequate breast feeding (OR $1.9, \mathrm{Cl} 1.32-2.9)$. While normal weight, overweight and obesity found insignificant with childhood tuberculosis.

Conclusion: Nutritional factors have significant impact on childhood TB. Nutritional education and counselling is required at institutional and community level for prevention of childhood TB. On our recommendation awareness campaign was started at FGH regarding importance of nutritional factors and prevention of childhood TB.

Key words: Nutritional Factors, Childhood Tuberculosis, Developing Country, Pakistan.

Disclosure of Interest: None declared.

\section{P020}

The use of 4-hexylresorcinol as antibiotic adjuvant against mycobacteria

A. Tutelyan 1,*, Y. Nikolaev², O. Manzenyk ${ }^{3}$, G. El'Registan² , V. Akimkin ${ }^{1}$

${ }^{1}$ Central Researche Institute of epidemiology, ${ }^{2}$ the federal research centre fundamentals of biotechnology" of the Russian Academy of Sciences, Moscow, ${ }^{3}$ State research center for applied microbiology and biotechnology of federal service for surveillance on consumer rights protection and human wellbeing, Obolensk, Russian Federation

Correspondence: A. Tutelyan

Antimicrobial Resistance \& Infection Control 10(1): P020

Introduction: Development of antimicrobial agents that are effective against mycobacteria is of great interest. One of promising approaches is to combine known antibiotics and adjuvants chosen from already known compounds ("off-label usage", "repurposed drugs"). Previously we have shown that 4-Hexylresorcinol is one of effective such adjuvants.

Objectives: To study effect of 4-Hexylresorcinol (HR), approved for use in medical practice, as adjuvant to fight mycobacteria in the in vivo experiments.

Methods: Test object was Mycobacterium smegmatis, a non-pathogenic relative of $M$. tuberculosis; bacteria were injected to tail venous $\left(0.3 \times 10^{8} \mathrm{CFU}\right.$, one time). Studied drugs: isoniazide (INI), anti-tuberculosis drug - $0.3 \mathrm{mg} / \mathrm{mice} /$ day; 4-hexylresorcinol (HR) (as adjuvant of INI) $-3 \mathrm{mg} / \mathrm{mice} /$ day; drugs were administrated with water for 4 days. Effect of HR was studied in in vivo experiments with the use of white mice of BALB/c line. Amount of M.smegmatis cells was determined in mice spleen at $4^{\text {th }}$ day by seeding of spleen gomogetate to Sotton solid medium.

Results: Amount of M.smegmatis cells in spleen without treatment was $1.2 \times 10^{4} \mathrm{CFU} / \mathrm{g}$. INI or HR were not effective against this bacterium (bacteria content were $1.9 \times 10^{4}$ and $0.8 \times 10^{4} \mathrm{CFU} / \mathrm{g}$, respectively). When both drugs were administrated, bacteria concentration dropped 20 -fold (cells content was $0.06 \times 10^{4} \mathrm{CFU} / \mathrm{g}$ ).

Conclusion: The use of 4-hexylresorcinol increases anti-mycobacterial activity of antibiotic in one order of magnitude.

As 4-hexylresorcinol is allowed to medical use, therefore it is highly possible to use it as a part of combined antimicrobial drugs.

Disclosure of Interest: None declared.
P021

Comparative analysis of foodborne multi-drug resistant

salmonella enterica isolates from Russia and Belarus

A. Egorova ${ }^{1, *}$, A. Shelenkov ${ }^{1}$, A. Chernyshkov ${ }^{1}$, N. Kulikova ${ }^{1}$, Y. Mikhaylova ${ }^{1}$, V. Akimkin ${ }^{1}$

${ }^{1}$ Central Research Institute of Epidemiology, Moscow, Russian Federation Correspondence: A. Egorova

Antimicrobial Resistance \& Infection Control 10(1): P021

Introduction: Salmonella enterica remains one of the most important global food origin pathogens and may play an important role in the dissemination of antimicrobial resistance (AMR) and virulence.

Objectives: The goal of this work is to perform comparative genomic epidemiology analysis of foodborne multi-drug resistant $S$. enterica isolates from Russia (SER) and Belarus (SEB) based on whole-genome sequencing (WGS).

Methods: WGS was performed on the Illumina HiSeq platform for 65 SEB and 310 SER isolates in the period 2017-2020. The susceptibility to antimicrobial compounds was determined both by the boundary concentration method and disc-diffusion test. Genome assemblies and comparisons were performed using SPAdes, roary, dnadiff, and custom software.

Results: Most of the analyzed S. enterica isolates belonged to ST32 and 07 serotype, but ST11 and O9 were prevailing for Belarus. SEB and SER isolates were characterized by 4 and 6 similar profiles of antimicrobial susceptibility, respectively. The data correspond well to the revealed AMR determinants according to WGS results. Both SEB and SER isolates had common genes to aminoglycosides, beta-lactam, and chloramphenicol. Additionally, Russian samples had tetracycline and quinolones resistance genes. In silico search for plasmids showed that some of the isolates were characterized by the presence of several types of plasmids simultaneously. The most common plasmids for SER were IncFIB and IncX1, while IncFIB and IncFIl prevailed for SEB. The set of virulence factors in isolates studied was similar and quite extensive (up to 108) and consisted of structural components of the cell, host invasion factors, phage-related compounds, and plasmid genes. Phylogenetic analysis showed high similarity groups consisted of ST11 strains isolated from the same country. However, there was no connection found for ST32 SEB and SER clades.

Conclusion: Thus, applying WGS technology enabled us to reveal unique genome features of $S$. enterica isolates from Russia and Belarus. Such characteristics are important for monitoring of AMR and virulence genes of the pathogens studied in terms of food risk safety and analysis evolutionary changes.

Disclosure of Interest: None declared.

P022

Genetic diversity of Russian antibiotic resistant L. monocytogenes isolates of food origin

N. Sukharicheva ${ }^{1, *}$, A. Shelenkov ${ }^{1}$, A. Egorova ${ }^{1}$, A. Chernyshkov ${ }^{1}$, N.

Kulikova', Y. Mikhaylova ${ }^{1}$, V. Akimkin ${ }^{1}$

${ }^{1}$ Central research institute of epidemiology, Moscow, Russia, Moscow,

Russian Federation

Correspondence: N. Sukharicheva

Antimicrobial Resistance \& Infection Control 10(1): P022

Introduction: Listeria monocytogenes is a Gram-positive bacterial pathogen. The food products contaminated by L. monocytogenes, especially ready-to-eat food, may cause different clinical syndromes and is dangerous for immunocompromised people and pregnant women.

Objectives: The aim of this work is the molecular-genetic analysis of antibiotic resistant $L$. monocytogenes isolates of food origin using whole genome sequencing (WGS).

Methods: WGS was performed on Illumina HiSeq platform for 35 isolates collected from meat, fish and ready-to-eat food from different geographical regions of Russia. The susceptibility was determined by the boundary concentration method on VITEK2Compact30 analyzer. Genome assemblies and genome comparisons were performed using SPAdes, roary, dnadiff and custom software. 
Results: L. monocytogenes has maintained a high rate of susceptibility to the antibiotics. All isolates under investigation were resistant to two antibiotics only of beta-lactam and sulfanilamide groups, but most of the isolates were susceptible to these antimicrobial compounds and macrolides, additionally. WGS analysis showed that all isolates were associated with two evolutionary lineages (II, IV) and can be divided into 10 sequence types (ST) with the prevalence of ST37 (II), ST121 (II) and ST9 (II). ST121 and ST9 were identified in isolates from meat products and from fish. These STs belong to hypovirulent clones. We also identified in our collection hypervirulent ST2 isolates that were obtained from meat while usually such clones are associated with dairy products. All isolates carried gene fosA responsible for resistance to fosfomycin. Some isolates had additional genes of resistance to chloramphenicol and tetracyclin. All isolates under investigation carried 33 common virulence genes, except isolates of ST121 and ST2 that characterized by the absence of actA, in/F, inlJ and ami, aut, inlJ genes, respectively.

Conclusion: Thus, WGS-based analysis allowed us to reveal genetic diversity and identify unique genome features of different genetic lines of antibiotic resistant $L$. monocytogenes isolates. The data obtained will greatly facilitate further investigations in genome epidemiology of food-born pathogens.

Disclosure of Interest: None declared.

\section{P023}

Antibiotic susceptibility patterns of listeria monocytogenes recovered from common South African ready-to-eat food A. K. J. Kayode ${ }^{1, *}$, A. Okoh ${ }^{1}$

${ }^{1}$ Department of Biochemistry and Microbiology, University of Fort Hare, Alice, South Africa, University of Fort Hare, Alice, South Africa

Correspondence: A. K. J. Kayode

Antimicrobial Resistance \& Infection Control 10(1): P023

Introduction: Antimicrobial resistance is gradually becoming a serious challenge to public health with increasing reports of microbial resistance including Listeria monocytogenes $(\mathrm{Lm})$ against useful antimicrobial therapeutic agents.

Objectives: The aim of the study was to investigate the antibiogram fingerprint of the Listeria monocytogenes isolates recoverved from common South African ready-to-eat food.

Methods: This study investigated the antibiogram profile of $\mathrm{Lm}$ isolates $(n=194)$ recovered from common South African ready-to-eat (RTE) food against a panel of 22 antibiotics using the disc diffusion techniques and to screen for resistance determinants in the isolates using PCR assay.

Results: Antimicrobial resistance $(>50 \%)$ against trimethoprim $(56.19 \%)$, sulfamethoxazole $(61.86 \%)$, streptomycin, ceftriaxone $(53.09 \%)$, cefotetan $(59.28 \%)$, vancomycin and oxytetracyclines $(62.89 \%)$ were observed. Thirty of the isolates $(15.46 \%)$ resistant to one or two antibiotics, while 162 (83.51\%) exhibited phenotypic multidrug resistance to the antibiotics tested. Only two $(n=2,1.03 \%)$ of the isolates did not exhibit phenotypic resistance to any antibiotics. Multiple antibiotic phenotypes (MARPs) revealed high resistance patterns and the Multiple antibiotic indexes were greater than the Krumperman permissible (>0.2) benchmark. Twenty-two (22) antimicrobial resistance genes were detected in RTE food isolates out of the 44 genes screened including resistance determinants that encodes sulfonamides $(n=125,64.43 \%)$, beta-lactams $(n=86,44.33 \%)$, phenicols $(n=25,12.89)$, aminoglycosides $(n=93,47.94 \%)$ resistance.

Conclusion: This suggests that the consumption of such RTE food in this study could constitute a health risk. Furthermore, the presence of antimicrobial-resistant Listeria monocytogenes in RTE food could compromise the safety of human consumption.

Disclosure of Interest: None declared.
P024

Antibiotic resistance profiles of vibrio cholerae 01 strains

of human origin isolated in Benin from 2012 to 2016

B. Boya ${ }^{1, *}$, P. F. Djidjoho Fonton ${ }^{2}$, T. A. AHOYO ${ }^{2}$

${ }^{1}$ Laboratory of Biology and Molecular Typing in Microbiology, ${ }^{2}$ Ecole

Polytechnique d'Abomey-Calavi, Ecole Polytechnique d'Abomey-Calavi, Cotonou, Benin

Correspondence: B. Boya

Antimicrobial Resistance \& Infection Control 10(1): P024

Introduction: The use of antibiotics during cholera epidemics in both patients and contacts has contributed significantly to the emergence of resistant bacteria.

Objectives: To determine the antibiotic resistance profile of Vibrio cholerae 01 strains isolated during the 2012-2016 cholera epidemics in Benin.

Methods: Strains isolated from stools of sick subjects from different regions of Benin were used for susceptibility testing. These strains were stored at the National Laboratory of the Ministry of Health (LNSP) of Benin. Strain identification was done according to standard bacteriology methods. Antibiotic susceptibility testing was performed using the agar diffusion technique.

Results: Over a four-year epidemic period, our results showed that the proportion of doxycline (DOX)-resistant strains was $66.67 \%, 66.67 \%$, $75 \%$, and $92.59 \%$ in $2012,2013,2014$, and 2016 , respectively, with $p<0.05$. The proportion of Vibrio cholerae 01 resistant to Erythromycin (E) was $66.67 \%, 100 \%, 100 \%$, and $100 \%$ respectively in 2012,2013 , 2014, and 2016 with $p<0.001$. Trimethoprim/sulfamethoxazole resistance rate was $70 \%$ for the tested strains.

Discussion: The resistance to doxycycline of Vibrio cholerae 01 strains could be the result of the current protocol proposing selective chemoprophylaxis with doxycycline administered to all accompanying persons and health personnel, for whom no contraindication existed in Benin [1]. Resistance to trimethoprim/sulfamethoxazole shows that the use of this drug would contribute to the selection and dissemination of emerging resistant strains in the population [2].

Conclusion: The high percentage of resistance to doxycycline and trimethoprim-sulfamethoxazole insists on reviewing the management protocol.

\section{Référence}

1. Makoutode M. F, Diallo V., Mongbo E., Guevart E, Bazira L, 2010. La riposte à l'épidémie de choléra de 2008 à Cotonou. Santé publique. 22; 435-425. 2. Campos LC, Zahner V, Avelar KE, Alves RM, Pereira DS, Vital BJ, Freitas FS, Salles CA, Karaolis DK. Genetic diversity and antibiotic resistance of clinical and environmental Vibrio Cholerae suggests that many serogroups are reservoirs of resistance. Epidemiol Infect. 2004; 132(5):985-92.

Disclosure of Interest: None declared.

Poster Session: Antimicrobial stewardship

P025

Knowledge of community pharmacy dispensers on providing antibiotics in Khartoum State, Sudan

A. H. S. Saad ${ }^{1, *}$

${ }^{1}$ University of Medical Sciences and Technology, Khartoum, Sudan

Correspondence: A. H. S. Saad

Antimicrobial Resistance \& Infection Control 10(1): P025

Introduction: The irrational use of antibiotics has become very alarming because of the major effects that it inflicts on healthcare. Of these effects is the antimicrobial resistance. As resistance to older antibiotics is increasing, development of newer agents has stalled.

Objectives: To asses knowledge of community pharmacy dispensers towards providing antibiotics. 
Methods: A cross-sectional study was conducted during January 2016 in Jabra area. Self-administered questionnaires were distributed among respondents, each consisted of items assessing the dispenser's academic qualification (diploma, bachelor or master's degree), years of experience, and knowledge about when to dispense antibiotics in relation to presence of prescriptions, different settings and complains that include (dysuria, diarrhea, ear pain and discharge, sore throat, viral infections and fever), these items were measured on a Likert scale ranging from strongly agree to strongly disagree.

Results: A total of 40 pharmacies were visited with a $100 \%$ response rate $(n=40)$, of whom, 22 were males, 35 had a Bachelor degree, and 18 had 1 to 5 years of working experience. Results showed that 32 of respondents indicated that antibiotics should be dispensed in presence or absence of prescriptions. 36 agreed that a dispenser should ask about the reason for requesting antibiotics without prescription. Our respondents disagreed that all clients complaining of diarrhea, ear pain or sore throat should be given an antibiotic $(72.5 \%, 55 \%, 55.5 \%)$, respectively. Majority disagreed with giving antibiotics on client's insistence and that their dispensing habits will not be affected by a monthly required pay up to the pharmacy owner.

Conclusion: Our study concluded that despite good knowledge, antibiotics could be obtained in Jabra area without a medical prescription, this warrants further investigations for causes of such practice to reach a better antibiotic stewardship.

Disclosure of Interest: None declared.

\section{P026}

Knowledge of antimicrobial resistance and antimicrobial prescription among dental practitioners in Chennai city-a cross-sectional study

S. $S^{1, *}$, R. $C^{1}, M . P^{1}$, S. gadde ${ }^{2}$

${ }^{1}$ public health dentistry, ${ }^{2}$ Periodontics, Ragas Dental College and Hospi-

tal, Chennai, India

Correspondence: S. S

Antimicrobial Resistance \& Infection Control 10(1): P026

Introduction: The use of antimicrobials in recent years has become more aggressive and more common. The inappropriate use, to be more precise, the abuse of these prescriptions, is the root cause for increasing anti microbial resistance(AMR).

Objectives: to assess the knowledge of antimicrobial resistance and antimicrobial prescription among dental practitioners in Chennai city.

Methods: A total of 110 registered dental practitioners participated in this study. A pre-validated and self-administered questionnaire was prepared and distributed to the dental practitioners in Chennai city to collect the demographic details and the information regarding knowledge and practice towards antimicrobial resistance and an antimicrobial prescription for the most common oral conditions was obtained.

Results: A total of 110 registered dental practitioners participated of which $64.66 \%$ were males and $35.4 \%$ were females, the respondents were $46.36 \%$ MDS (had Master degree in Dental Specialty) and $54.8 \%$ BDS (Bachelor of Dental surgery). Majority of dentists were found to prescribe antibiotics for managing oral diseases except for orthodontic treatment. MDS practitioners of $92.16 \%$ and BDS of $79.96 \%$ recommended amoxicillin as their first choice antibiotic drug, followed by the combination of amoxicillin with clavulanic acid. When comparisons were made between BDS and MDS practitioners, majority of the questions were found to be not significant except for conditions like tooth sensitivity $(P=0.00)$, dentoalveolar abscess $(P=0.009)$, tooth fracture $(P=0.029)$, halitosis $(P=0.003)$ and periodontal abscess $(P=0.001)$.With regard to the duration of antibiotic prescription, $42.37 \%$ BDS practitioners prescribed antibiotics as a 3-day course and $45.76 \%$ resorted to a 5 -day course. $45.09 \%$ MDS practitioners prescribed antibiotics as a 3 -day course and $49.01 \%$ resorted to a 5 -day course which was not statistically significant. There was a general lack of awareness with regard to the guidelines for antibiotic prescribing in both the groups.

Conclusion: Dental practitioners relied on drug therapy in many orofacial and general conditions where it was not necessary. Based on this cross-sectional survey the overall knowledge of antimicrobial prescription and antimicrobial resistance among dental practitioners was lower.

Disclosure of Interest: None declared.

P027

Attitudes and behavior of Algerian hospital doctors on antibiotic use and resistance

T. Morghad ${ }^{1, *}$, H. Hassaine ${ }^{1}$

'Laboratoire De Microbiologie Appliquée à L'Agroalimentaire Au Bio-

médical et à L'Environnement (LAMAABE), Tlemcen university ( Algeria),

Tlemcen, Algeria

Correspondence: T. Morghad

Antimicrobial Resistance \& Infection Control 10(1): P027

Introduction: Each day, the real prescription is also dependent on non-pharmacological determinants in relation to the behavior of prescribers, hence the objective of this work.

Objectives: Knowledge of the attitudes and behavior of hospital physicians on antibiotics use and resistance.

Methods: Knowledge of the attitudes and behavior of hospital physicians on antibiotics use and resistance.

Results: The response rates were $49 \%$. The majority of the physicians audited were aware of the importance of the antibiotic resistance problem. Physicians were confident in most scenarios, but abstaining to prescribe an antibiotic was the main case where physicians had a significant degree of uncertainty. When prescribing antibiotics, doctors rely mainly on two factors: experience and consultation of practice guidelines. The measure identified as most useful to improve the quality of antibiotic therapy was the organization of training on antibiotic prescribing. The causes of bacterial resistance most often identified in the audited physicians were: too much antibiotic prescription, too much broad-spectrum antibiotic.

Conclusion: In order to deal with the problem of resistance and to improve the rigour of antibiotic prescription, it is necessary to know the attitudes and behaviors of doctors.

Disclosure of Interest: None declared.

\section{P028}

Swiss interprofessional guidance of good practice of acute and complicated diabetic foot infections and syndromes-a national project

I. Uçkay ${ }^{1, *}$, A. Czock ${ }^{2}$, B. Peter-Riesch ${ }^{3}$ on behalf of Interdisciplinary Expert Group for the Diabetic Foot of Swiss Society of Endocrinology and Diabetology

${ }^{1}$ Balgrist University Hospital, Zürich, ${ }^{2}$ QualiCCare, Baden, ${ }^{3}$ General Beaul-

ieu, Geneva, Switzerland

Correspondence: I. Uçkay

Antimicrobial Resistance \& Infection Control 10(1): P028

Introduction: Diabetic foot infections (DFI) and syndromes (DFS) have multiple negative outcomes in a patient population marked with comorbidities and frailty. Their management requires a quality of care consisting of evidence-based, practical guidance tools for primary care providers together with the implementation of an interprofessional care system including nationally standardized diagnose-relevant best practice recommendations.

Objectives: We implement a national best practice guidance for timely and qualitative care of patients with DFI and DFS, including for antibiotic stewardship in DFls.

Methods: Under the umbrella of the national non-profit association QualicCare, an interprofessional and comprehensive Swiss practical guidance for the timely and standardized quality of care management of acute DFI, DFS and diabetic foot ulcers (DFU) was elaborated in a multi-stakeholder approach including all relevant professions. Before the nation-wide implementation of the primary care triage and treatment guidance and indication-specific recommendations for interprofessional networks and footcare centers, three Swiss regions will pilot 
the implementation in different possible primary care settings in collaboration with the respective interprofessional footcare centers.

Results: Twenty experienced stakeholders from twelve different professions issued four protocols for various aspects of DFS between March 2018 and January 2020 and defined criteria for the triage and treatment in primary care as well as the timely referral of patients with DFS and DFU to multidisciplinary footcare centers. All invited professional societies agreed to work together and have a representative in the working group. The resulting guidance were endorsed by all national professional societies.

We propose a framework for specialized footcare networks as well as multidisciplinary footcare centers. The piloting of the proposed concepts is under way.

Conclusion: We provide evidence-based tools regarding antibiotic stewardship and other streamlined approaches for Swiss primary care providers and specialists while increasing the accessibility for patients to specialized care of DFS, DFU and DFI.

Disclosure of Interest: None declared.

\section{P029}

Assesing of antibiotic prescribing in patients hospitalized with confirmed coronavirus disease 2019 (COVID-19)—results of a point prevalence survey

N. Ion Nedelcu, ${ }^{1, *}$, S.-A. Florescu ${ }^{2}$

${ }^{1}$ Infection Control, ${ }^{2}$ Infectious and Tropical Diseases Hospital "Dr Victor Babes", Bucharest, Romania

Correspondence: $\mathrm{N}$. Ion Nedelcu

Antimicrobial Resistance \& Infection Control 10(1): P029

Introduction: Recent reports have drawn attention to the high antibiotic use in patients hospitalised for coronavirus disease 2019 (COVID19), resulting in concerns of increasing antimicrobial resistance with increase antibiotic use in this pandemic. Point prevalence survey (PPS) can be a quick tool to provide antibiotic prescribing information to aid antimicrobial stewardship (AMS) activities.

Objectives: To describe the magnitude and structure of antibiotic utilization in COVID-19 patients using PPS.

Methods: Adapting Global-PPS on antimicrobial use, the survey was conducted in a 490 beds COVID-19 hospital in Bucharest on 18 March 2021 at $0800 \mathrm{~h}$. Patients on systemic antibiotics were included and the prevalence of systemic (J01) antimicrobials used was calculated by WHO ATC classes.

Results: Three hundred and ninety-three patients were screened. The mean age of patients was 58.9 years (IQR: 47-71), the prevalence of female gender was $55.8 \%$ ( $95 \% \mathrm{Cl}: 49.7 \%, 61.7 \%)$, and the mean duration of hospital stay was 14.4 days (IQR: $10-19)$. A total of $89.6 \%$ of patients were on antibiotics on the survey day. Three hundred and ninety-eight doses of antibiotics were prescribed in these patients. Overall, antimicrobials included in class ATC J01D (other betalactam antibacterials: Cephalosporins and Carbapenems) represented 76.8\% from all amtimicrobial prescribed.

Conclusion: Althouh the appropriatednes of prescription of antibiotics was not the goal of this study, intensive use of broader spectrum antimicrobials is concerning and suggestive that AMS interventions such as preauthorization and/or audit and feedback, interventions performed in our hospital in pre-pandemic era, could be the solution to improve the empiric prescription of antimicrobials for patients hospitalised for coronavirus disease 2019.

Disclosure of Interest: None declared.

\section{P030}

Different antimicrobial therapies in the treatment of patients with SARS-COV-2 in the ICU worsens the outcome K. Ugo ${ }^{1, *}$, E. Mikulic ${ }^{1}$, Y. Lamberto ${ }^{1}$ on behalf of Residents of the ICU at $\mathrm{HMC}$

${ }^{1}$ critical care unit, CENTRAL MILITARY HOSPITAL, Buenos Aires, Argentina Correspondence: K. Ugo

Antimicrobial Resistance \& Infection Control 10(1): P030
Introduction: The high rate escalation antimicrobial therapies worsens outcome of patients with SARS-CoV- 2 .

Objectives: Correlate the outcome of SARS-CoV-2 patients and the prescription of antimicrobial therapies within ICU.

Methods: A prospective study of 98 patients with SARS-CoV-2 in ICU from april 2020 till february 2021.Age. risk factors, days in the $I C U$, requirement of invasive mechanical Ventilation (MV), days with MV, SOFA at the admission in the ICU, mean PAFI, complications, ventilator-associated-pneumonia, requirement of one empirical antimicrobial therapy, carbapenen, colistin or vancomycin prescribed antibiotics or multidrug-resistance Gram negative organisms treatment amd outcome, all were tested with linear regression and $p$ value less than 0.01 was significant.

Results: Among the 98 patients enrolled: $39 \%$ required one empirical antimicrobial therapy during the stay in the ICU, 75\% had PAFI less than 210 and $52 \%$ of these group died. $25.5 \%$ suffered from infections and received carbapenen, vancomycin or colistin therapy, and $60 \%$ of them died. $35.7 \%$ patients received 3 or more antibiotics and/or antifungal and $48.6 \%$ were dead. Those with one empirical antimicrobial therapy $37 \%$ suffered from respiratory complications, $42 \%$ had MOF (renal, cardiovascular, respiratory, hematologic complications) and $23 \%$ develop ventilator-associated pneumonia (VAP). In the group with carbapenens, colistin, vancomycin $28 \%$ had respiratory complications, $60 \% \mathrm{MOF}$ and $40 \%$ had VAP. Those who had multi-resistant gram negative organisms treatment $17 \%$ had respiratory complications, $71 \%$ had MOF and 57 suffered from VAP.

Conclusion: The prescribtion of multidrig-resistant Gram negative organisms therapy increases the risk of MOF,decreases the PAFI and increases VAP and worsens the outcome.

Disclosure of Interest: None declared.

\section{P031}

Antimicrobial use in long-term care facilities in the Netherlands

K. Halonen ${ }^{1, *}$, A. Haenen ${ }^{1}$, M. Biesheuvel ${ }^{1}$, S. de Greeff ${ }^{1}$

${ }^{1}$ RIVM, Bilthoven, Netherlands

Correspondence: $\mathrm{K}$. Halonen

Antimicrobial Resistance \& Infection Control 10(1): P031

Introduction: Residents of long-term care facilities are at increased risk of infection and thus of being treated with an antimicrobial. Inappropriate use may lead to the development of resistance. In the Netherlands, the national surveillance network (SNIV) is used to monitor infectious diseases and antimicrobial use in long-term care facilities (LCTFs). We studied trends in antimicrobial use in these Dutch LTCFs.

Objectives: To monitor trend in antimicrobial use in LTCFs in order to identify where the risk of resistance is potentially increasing.

Methods: In 2017-2019, twice a year in April and/or November a point prevalence study was performed. On the registration day, prescribed antimicrobials with a maximum of three antibiotics or antimycotics per resident, and the indication (infection or prophylactic) was registered. Data were collected by elderly care physicians, nurses or infection control practitioners in participating LCTFs. Palliative care was not included in this study. Data were reported to SNIV through a password protected web-based surveillance system.

Results: Over the past three years 136 unique LTCFs [range 23-77 per year] participated with 12,509 residents. The use of antimicrobial agents increased from $6.7 \%$ in 2017 to $9.4 \%$ in 2019 . The percentage of antibiotics remained roughly the same (4.9-5.6\%) however the use of antimycotics increased from $2.3 \%$ in 2017 to $4.8 \%$ in 2019.

In 2017,267 out of $448(60 \%)$ antimicrobials were prescribed to residents with an infection, in 2018 it was 153/240 (64\%) and in 2019 163/247 $(66 \%)$. The rest of the residents received antimicrobials as prophylaxis. In $2017,70 \%$ of these antimicrobials which were prescribed for treatment of an infection, were antibiotics and 30\% antimycotics; in 2019, the percentage of antimycotics has increased to over $50 \%$. The highest increase in antimycotics is seen in the use of ketaconazole. In 2017, ketaconazole was selected in $49 \%$ of the cases and in 2019 this has increased to $65 \%$. In 2017 ketaconazole was mostly used as prophylaxis (67\% in 2017), while in in $201969 \%$ was for the treatment of infections. 
Conclusion: Prescription of antimycotics in LCTFs increased in the past three years. More research is needed to find out the clinical significance of this increasing antimycotic use.

Disclosure of Interest: None declared.

\section{P032}

Impact of antibiotic stewardship and implementation of infection control on prevalence of surgical site infections in a large tertiary-care hospital in Haiphong city

J. Ory ${ }^{1, *}$, H. Phan Tien², V. Vu Hai ${ }^{2}$, C. Elodie ${ }^{1}$, L. M. Quang ${ }^{2}$, L. Didier ${ }^{1}$, J. P. Lavigne $^{1}$, A. Sotto ${ }^{1}$

${ }^{1} \mathrm{CHU}$, Nîmes, France, ${ }^{2} \mathrm{CHU}$ Viet Tiep, Haiphong, Viet Nam

Correspondence: $\mathrm{J}$. Ory

Antimicrobial Resistance \& Infection Control 10(1): P032

Introduction: In low- and middle-income country, the prevalence of SSIs is ranged from $4.5 \%$ to $14.3 \%$. Few points prevalence surveys (PPS) have been conducted in Vietnam on SSIs or on antimicrobial use in surgical patient.

Objectives: The objective of this study was to estimate the PPS of SSI before and after the implementation of antibiotic stewardship programs (ASP) and the implementation of infection control (IC) in orthopedic department in a large tertiary-care hospital in Haiphong city, Vietnam.

Methods: Between 2016 and 2019, ASP and IC practices were implemented in operating rooms and in the orthopedic department including antibiotic training, skin preparation, hand hygiene, gloves and sterile instruments. ASP were implemented in according to WHO guidelines including training and the risk factor of SSIs. In January 2016, before implementation of ASP and IC practices, a 1st PPS of SSIs and antimicrobial use was performed. The 2nd PPS was performed in December 2019. The PPS was performed in according to ECDC methods. Some characteristics were recorded as surgical, antibiotic prophylaxis, microorganisms and risk factors associated with SSIs (low serum albumin concentration, older age, obesity, smoking and diabetes mellitus).

Results: The prevalence of SSI was 7.8\% $(n=4 / 51)$ and $5.4 \%(n=2 / 37)$ in 2016 and in 2019 respectively ( $p>0.05$; Student test). The most common microorganisms was $S$. aureus. Significate differences were determined on diabetes mellitus, NNIS score, scheduled surgery and prophylactic antibiotics ( $>48 \mathrm{~h}$ ). For antibiotic prophylaxis, a 3rd-generation cephalosporin was prescribed more than $48 \mathrm{~h}$, in the majority of cases for the two periods. Between 2016 and 2019, the skin preparation has improved during several informal surveys.

Conclusion: Our finding suggest that IC practices and antibiotic prophylaxis have not become routine in participating facilities. The decrease of prevalence of SSI, even non-statistically significant, lead us to continue this program. It is necessary for hospitals in Vietnam to provide comprehensive education programs, addressing basic IC issues, such as standard precautions and establish more effective institutional IC policies.

Disclosure of Interest: None declared.

\section{P033}

Evaluation of empirical antibiotic treatment in uncomplicated and complicated pyelonephritis cases and determination of risk factors for infection with esbl-producing gram-negative pathogen B. Kayaaslan ${ }^{1}$, Z. Oktay ${ }^{1}$, I. Hasanoglu' ${ }^{1}$, A. K. Kalem ${ }^{1}$, F. Eser ${ }^{1}$, M. Ayhan ${ }^{1}$, R. Guner ${ }^{1}$, E. Alp ${ }^{2, *}$

${ }^{1}$ Ankara City Hospital, ${ }^{2}$ Ministry of Health, Ankara, Turkey

\section{Correspondence: E. Alp}

Antimicrobial Resistance \& Infection Control 10(1): P033

Introduction: Acute pyelonephritis is one of the most common infectious diseases among outpatients and inpatients. ESBL-producing gram-negative pathogens are increasingly responsible for both community and hospital acquired infection. Appropriate and early antibiotic therapy improves clinical outcomes and reduces mortality but rising antibiotic resistance rates make empirical therapy increasingly difficult due to limited treatment options.
Objectives: We aimed to investigate the demographic, epidemiological and clinical characteristics of patients hospitalized with the diagnosis of uncomplicated and complicated pyelonephritis acquired in community and hospital, and to evaluate appropriateness of empirical treatment.

Methods: The study was conducted in patients hospitalized with the diagnosis of uncomplicated or complicated pyelonephritis acquired from the community or hospital between January 1, 2016, and June 30, 2018. Appropriateness of empirical treatment at the 48th-72nd hours was evaluated based on culture results and treatment modification (as escalation/de-escalation/continue) were evaluated.

Results: A total of 369 uncomplicated (94) and complicated (275) episodes of pylenonephritis (in 339 patients) were evaluated. Causative pathogen was obtained in $69.4 \%$ of baseline cultures. The most common agents were E.coli (71.0) and Klebsiella spp (17.7), and ESBLproduction rate was $64.4 \%$. Ceftriaxone, ertapenem and piperacilintazobactam were used in $26.3 \%, 53.7 \%$, and $15.7 \%$ of the episodes, respectively. When the appropriateness of empirical treatment was evaluated at $48-72$ nd hours, ceftriaxone treatment had should be escalated in $40.6 \%$ of all episodes while ertapenem and piperacillintazobactam had been escalated in only $8.8 \%$ and $9.5 \%$ of pyelonephritis episodes, respectively.

Conclusion: E.coli and Klebsiella spp. are responsible for majority of pyelonephritis. ESBL-pruducing pathogens are guite high even if community-acquired infections. Considering the effect of early treatment on mortality, more extended therapy covering ESBL-producing pathogen at the beginning of the treatment appears a better and life-saving choice.

Disclosure of Interest: None declared.

\section{P034}

Evaluation of antibiotic prescription among pregnant women in a Tunisian hospital

N. Fathallah'1, I. Bannour ${ }^{2}$, G. Derouich ${ }^{1}$, R. Bannour ${ }^{2,}{ }^{,}$, B. Ouni ${ }^{1}$, H. Kheiri ${ }^{2}$

${ }^{1}$ Department of Pharmacovigilance, Faculty of Medicine of Sousse,

${ }^{2}$ Departement of Obstetrics and Gynecology, university hospital Farhat

Hached Sousse, Sousse, Tunisia

Correspondence: R. Bannour

Antimicrobial Resistance \& Infection Control 10(1): P034

Introduction: The safety of antibiotics prescription during pregnancy has been the center of an intensive controversy in recent decades due to problem of drug risks for the fetus and the growing problem of the emergence of bacterial resistance.

Objectives: he aim of this study is to evaluate the protocol of antibiotic therapy used in the obstetrics gynecology department of Farhat Hached and to compare it with international recommendations.

Methods: A cross-sectional study was conducted among women hospitalized in the peri-partum during their third trimester of pregnancy and the postpartum sector in the service of gynecology obstetrics of Farhat Hached Hospital in Sousse who received antibiotic therapy during the period from 01/01/2020 until 03/31/2020.

Data was collected using a pre tested grid treating epidemiological, socio-professional, clinical data, prescribed antibiotic therapy the indication, dosage, duration, course, tolerance, and the antibiogram.

Results: A total of 153 women were enrolled in our study with a mean age of $30 \pm 6$ years.

For first-line antibiotic therapy, the most prescribed family of was Beta-lactams (71.8\%), as monotherapy or in association and Amoxicillin (36.3\%) was the most prescribed antibiotic. The most used association was beta-lactam, amino glycoside and imidazole (22.2\%).

A switch of antibiotic adapted to the antibiogram was done only in 23 cases. The average of prescription length was 6 days with favorable outcome mostly. The leading cause of antibiotic prescription was premature rupture of membranes (31.3\%) in third trimester of pregnancy and the suspicion of postpartum chorioamnionitis (27.5\%) after childbirth. The most frequent isolated germ was Eshirechia coli.

Overall, the practice of antibiotic prescription was in accordance with international recommendations of proper use of antibiotic. 
Conclusion: The prescription of an antibiotic during pregnancy should take into account both the maternal prognosis and the safety for the fetus. Collaboration between gynecologists, microbiologists, infectilogists and pharmacologists is necessary in order to establish an updated antibitherapy protocol.

Disclosure of Interest: None declared.

\section{P035}

Antibiotic prescription assessment in public hospitals in Abidjan, Côte D'ivoire: a global point prevalence survey

I. A. D. Yapi ${ }^{1, *}$, A. A. Kacou ${ }^{2}$, A. Versporten ${ }^{3}$, I. pauwel ${ }^{4}$, Y. M.

coulibaly-diallo' ${ }^{1}$, G. A. J. bahan ${ }^{1}$, A. kacou n'douba ${ }^{5}$

${ }^{1}$ biology, ${ }^{2}$ pharmacy, chu angre, abidjan, Côte d'Ivoire, ${ }^{3}$ microbiol-

ogy, vaccine and infectious diseasesinstitute, ${ }^{4}$ microbiology, vaccine and infectious diseases institute, antwerpen, Belgium, ${ }^{5}$ microbiology, chu angre, abidjan, Côte d'Ivoire

Correspondence: I. A. D. Yapi

Antimicrobial Resistance \& Infection Control 10(1): P035

Introduction: Antimicrobial resistance is an emerging global challenge. It is correlated with the consumption of antibiotics. Inappropriate use of antibiotics has been fuelled by lack of surveillance and diagnostic capabilities and lack of treatment guidelines. The government of Côte d'Ivoire in 2019 launched the national action plan on antimicrobial resistance.

Objectives: Our objective was to describe the prescription patterns of antibiotics among hospitalised patients.

Methods: The standardized Global Point Prevalence Survey (Global PPS) method assessed antimicrobial prescribing in 11 hospitals in Abidjan, including 7 secondary level hospitals and 4 tertiary level hospitals from August to December 2020. Data on antimicrobial agents and their indications have been collected.

Results: Out of 740 inpatients, 511 (69\%) received an antimicrobial treatment and 491 (66,35\%) antimicrobial prescription being antibiotics. This prevalence was respectively $64.49 \%$ and $74.56 \%$ for tertiary and secondary level hospitals. Therapeutic prescribing accounted for $44 \%$ (tertiary level) and $43 \%$ (secondary level). For global prophylaxis, it was $52 \%$ (tertiary level) and 54\% (secondary level). The main antibiotics used in tertiary and secondary level hospitals were ceftriaxone ( $27 \%$ vs $44 \%)$, metronidazole (13\% vs $24 \%$ ) and amoxicillin (12\% vs $15 \%)$. In both therapeutic and prophylaxis, ceftriaxone (54\%) was used the most in any hospital. In tertiary hospitals; central nervous system and lower respiratory infections were the most common indications. Sepsis predominated in secondary hospitals. Globally, the duration of prophylaxis was $36,40 \%$ for single dose, $37,71 \%$ for one day, and $25.87 \%$ for more than one day.

Conclusion: Antimicrobial prescription mainly antibiotics is high in Côte d'Ivoire. This situation may be improve by stewardship activities. Key words: Prescription, Antibiotics, Point Prevalence Survey, Hospital, Abidjan.

Disclosure of Interest: None declared.

\section{P036}

Impact of weekly clinical audits and feedback on consumption of protected anti-gram negative antibiotics in 8 hospitals of the French-speaking part of Switzerland

E. Moulin ${ }^{1, *}$, C. Plüss Suard ${ }^{2}$ on behalf of contributed equally as submitting author, C. Bellini ${ }^{3}$, L. Christin ${ }^{4}$, C. Chuard ${ }^{5}$, O. Clerc ${ }^{6}$, A. Cometta ${ }^{7}$, V. Erard ${ }^{5}$, O. Marchetti ${ }^{8}$, N. Troillet ${ }^{3}$, C. Voide ${ }^{3}$, G. Zanetti ${ }^{9}$, L. Senn ${ }^{1}$

${ }^{1}$ Centre Hospitalier Universitaire Vaudois, Lausanne, ${ }^{2}$ Institute for Infectious Diseases, University of Bern, Swiss Centre for Antibiotic Resistance (ANRESIS), Berne, ${ }^{3}$ Institut Central des Hôpitaux, Sion, ${ }^{4}$ Groupement Hospitalier de I'Ouest lémanique, Nyon, ${ }^{5}$ Hôpital de Fribourg, Fribourg,

${ }^{6}$ Hôpital de Pourtalès, Neuchâtel, ${ }^{7}$ Etablissement hospitalier du nord vaudois, Yverdon-les-Bains, ${ }^{8}$ Etablissement Hospitalier de la Côte, Morges,

${ }^{9}$ Université de Lausanne, Lausanne, Switzerland

Correspondence: E. Moulin

Antimicrobial Resistance \& Infection Control 10(1): P036

Introduction: Multiple antibiotic stewardship proactive interventions have been shown to improve antibacterials prescribing practices in hospitals. Nevertheless such interventions are so far limited in Switzerland.

Objectives: to evaluate the impact of weekly clinical audits conducted by an external infectious diseases specialist and an internal senior physician and multifaceted feedback strategies on reducing the use of protected anti-Gram negative antibiotics, including fluoroquinolones $(F Q), 3^{\text {rd }}$ and $4^{\text {th }}$ generation cephalosporins $(G 3 C, G 4 C)$, piperacillintazobactam $(\mathrm{P} / \mathrm{T})$ and carbapenems.

Methods: The study included internal medicine, general surgery and intensive care wards of 8 Swiss acute care hospitals. Wards were allocated to either intervention for 6 months $(n=14)$ or a control group $(n=10)$. Linear regression models of an interrupted time series was performed to assess the impact of the intervention on the monthly use of protected antibiotics.

Results: During the intervention, 9715 in-patients were screened, of whom 1684 (17.3\%) received a "targeted antibiotic". The auditing team proposed a modification of the antibiotic therapy in $24 \%$ of patients, mainly stops, followed by de-escalation and switch to oral route. The rate of inappropriateness varied from $8 \%$ in ICU to $32 \%$ in surgical wards, and from $15 \%$ for carbapenems to $38 \%$ for FQ. We observed a statistically significant decrease use of $F Q, G 4 C$ and $P / T$ in 5,3 and 2 intervention wards, respectively, and a significant decrease in the use of 2 antibiotics in 2 wards. The use of G3C and carbapenems showed no significant change.

Conclusion: Our pragmatic multicenter study showed a statistically significant impact on consumption of FQ, GC4 and P/T in 8/14 intervention units. It allowed a better overview of the appropriateness of antimicrobial prescriptions. Our study offered the opportunity to raise awareness about antibacterial resistances and encourage best daily practices among prescribers.

Disclosure of Interest: None declared.

\section{P037}

Organ dysfunction markers may predict aminoglycoside pharmacokinetics in critically ill patients with abdominal sepsis B. Shahrami ${ }^{1, *}$, A. Sefidani Forough ${ }^{2}$, F. Najmeddin ${ }^{1}$, A. A. Arabzadeh ${ }^{3}$, M. Mojtahedzadeh ${ }^{1}$

'Department of Clinical Pharmacy, Tehran University of Medical Sciences, Tehran, Iran, Islamic Republic Of, ${ }^{2}$ Department of Clinical Pharmacy,

Queensland University of Technology, Brisbane, Australia, ${ }^{3}$ Department of Surgery, Ardabil University of Medical Sciences, Ardabil, Iran, Islamic Republic Of

Correspondence: B. Shahrami

Antimicrobial Resistance \& Infection Control 10(1): P037

Introduction: Pathophysiological changes and organ dysfunction following sepsis alter antibiotic pharmacokinetics. In the absence of pharmacokinetic data at the time of empirical treatment initiation, available biomarkers could play an important role in predicting the pharmacokinetic parameters.

Objectives: This study aimed to evaluate the relationship between the amikacin pharmacokinetics and the biomarkers associated with organ dysfunction in critically ill patients with intra-abdominal sepsis.

Methods: A case series involving critically ill patients with intraabdominal sepsis who received an amikacin loading dose of 20-25 mg/kg intravenous infusion, was studied. The 1-, 2-, 4-, and 6-h amikacin serum concentrations were measured to calculate the pharmacokinetic parameters. The available biomarkers associated with organ dysfunction were recorded. A linear regression analysis was performed to examine the relationship between the amikacin pharmacokinetics and the biological parameters.

Results: Twenty-one patients were studied. A significant correlation was found between the volume of distribution and ESR $(p<0.05$, $r=0.844$ ). Moreover, drug clearance had a significant inverse correlation with serum lactate $(p<0.05, r=-0.603)$. No other significant correlations were found.

Conclusion: ESR and serum lactate were identified as useful predictors of amikacin pharmacokinetics in critically ill patients with intraabdominal sepsis and may help guide the selection of appropriate 
empirical dosing. Further studies are required to confirm these findings.

Disclosure of Interest: None declared.

Poster Session: Nosocomial Candida infections

\section{P038}

An outbreak of candida parapsilosis bloodstream infections in pediatric oncohemathology unit

R. Vatcheva-Dobrevska ${ }^{1, *}$, P. Stefanova ${ }^{1}$, V. Dicheva ${ }^{1}$, I. Philipova ${ }^{2}$, T. Kantardjiev ${ }^{3}$

${ }^{1}$ Microbiology, University Hospital "Queen Joanna" Sofia, ${ }^{2}$ National Reference Lab Fungal and STI, ${ }^{3}$ Director, National Center Infectiouse and Parasitic Diseases, Sofia, Bulgaria

Correspondence: R. Vatcheva-Dobrevska

Antimicrobial Resistance \& Infection Control 10(1): P038

Introduction: The Candida spp is one of the most common causes of bloodstream infections (BSI) in immunocompromised hosts. Their ability of biofilm formation on different implants facilitate the catheterassociated infections.

Objectives: The aim of this study is to investigate an outbreak of bloodstream infections in pediatric hematology-oncology patients with totally implantable venous access port systems.

Methods: A total of 36 episodes of port system-related bloodstream infections were registered and investigated in 11 patients between January and May, 2021. Blood cultures, needles, saline solutions, i.v. plastic lines,bags, staff hands samples sent to microbiology lab. The Candida spp. isolation on SDA. The isolates identification by VITEK 2, BioMerieux and api20CAUX, BioMerieux, France. Re-identified by MALDI-TOF (MALDI-Biotyper, Bruker. Whole genome sequencing was prepared in the National Reference Lab.

Results: The data from patients notes shows that all patients were neutropenic and with fever at the time of blood cultures and candidemia diagnosis Six of all patient had one sepsis episode each. Five of them had three or four sepsis episodes, mostly in March and May, 2021. The all blood cultures isolates as well as needles' isolates were identified as Candida parapsilosis. In the cases of five patients with persistent candidemia and ongoing fever, additionally to antifungal therapy, the ports were removed, based on the international guidelines and manufacturer's instructions. Various gaps in the Infection Prevention and control practices were found: poor hand hygiene compliance, shortage of staff and incidents of using a common saline solution bottle etc.

Conclusion: The totally implantable port systems represent a high risk of bloodstream infections complications in pediatric onco-hematology patients. The healthcare workers hands can be a risk factor for contamination with Candida parapsilosis. Further work need to create a special bundle and develop strategic measures for infection prevention and control of infections in case of totally implantable venous access port systems as well as a medical staff training programme. Disclosure of Interest: None declared.

\section{P039}

Infection control in two COVID-19 patients with evidence for Candida Auris, Germany

M. Wiese-Posseltt, ${ }^{1,}$, C. Hinrichs ${ }^{2}$, B. Weikert ${ }^{1}$, B. Graf ${ }^{3}$, P. Enghard ${ }^{2}$, R. Körner $^{2}$, A. Schrauder ${ }^{3}$, A. Knaust ${ }^{3}$, C. Geffers ${ }^{1}$, O. Kurzai ${ }^{4,5}$, K.-U. Eckardt ${ }^{2}$, P. Gastmeier ${ }^{1}$.

${ }^{1}$ Institute of Hygiene and Environmental Medicine, ${ }^{2}$ Department of Nephrology and Medical Intensive Care, Charité - Universitätsmedizin Berlin corporate member of Freie Universität Berlin, Humboldt-Universität zu Berlin, ${ }^{3}$ Labor Berlin - Charité Vivantes GmbH, Berlin, Berlin, ${ }^{4}$ University of Würzburg, Institute of Hygiene and Microbiology, Würzburg, ${ }^{5}$ National Reference Center for Invasive Fungal Infections NRZMyk, Leibniz Institut für Naturstoff-Forschung und Infektionsbiologie - Hans-Knöll-Institut, Jena, Germany.

Correspondence: M. Wiese-Posselt

Antimicrobial Resistance \& Infection Control 10(1): P039
Introduction: Candida auris is an emerging pathogen in hospital infections that can present multi-resistance to antifungals and causes outbreaks.

Objectives: The aim is to describe the infection prevention and control for C. auris.

Methods: Identification of yeast isolates was performed by MALDITOF and confirmed by ITS sequencing. Infection control measures were decided by a multi-disciplinary ad hoc outbreak panel. Patient screening once or twice a week and extensive environmental testing for $C$. auris was conducted.

Results: C. auris was isolated from a urine sample of a COVID-19 patient who had been transferred from an Egyptian hospital to our COVID-19 intensive care unit (ICU). Immediately, disinfection routine was changed, because $C$. auris is insensitive to quaternary ammonium compounds. The patient had already been isolated from admission due to evidence of 4MRGN Klebsiella pneumoniae. Six days after confirmation of $C$. auris in the index patient, a second COVID-19 patient was identified with $C$. auris. Both patients were isolated in a separated area of the ICU. Strict hygiene and infection control measures were implemented promptly. In the nine weeks from initial confirmation of $C$. auris and discharge of the two affected patients, $C$. auris was repeatedly identified in clinical samples of them. However, it was not detected in any other patient on the ICU $(n=7)$ or discharged from it $(n=13)$ nor in any environmental sample $(n=129)$. The two $C$. auris patients had been intubated using the same video laryngoscope seven days apart. Although the equipment and the spatulas had been manually reprocessed using chlorine dioxide-soaked wipes they might serve as transmission vehicle. Therefore, it was recommended to use disposable spatulas.

Conclusion: A rapid confirmation of a C. auris in the lab and the immediate implementation of adequate hygiene measures at the ward are crucial in order to prevent transmission of $C$. auris to other patients.

Disclosure of Interest: None declared.

P040

Prospective evaluation of Candida colonization index in intensive care unit patients, in Tunisia

M. Ben Brahim 1,*, N. Haddad², S. Boughattas ${ }^{1}$, A. Farah', L. Tilouche ${ }^{1}$, W. Naija $^{3}$, H. Hmouda ${ }^{4}$, A. Trabelsi' ${ }^{1}$, S. Ketata ${ }^{1}$

${ }^{1}$ Laboratory of microbiology, University Hospital Sahloul, ${ }^{2}$ Department of preventive and community medicine, Faculty of Medicine of Sousse,

${ }^{3}$ : Surgical intensive care unit, ${ }^{4}$ Medical intensive care unit, University Hospital Sahloul, Sousse, Tunisia

Correspondence: M. Ben Brahim

Antimicrobial Resistance \& Infection Control 10(1): P040

Introduction: Multiple-site colonization with Candida spp. is commonly recognized as a risk factor for Invasive Candidiasis (IC) among intensive care unit (ICU) patients. Up to $80 \%$ of those patients are colonized one week after their admission.

Objectives: Therefore, this study was carried out to assess the relationship between IC and the determination of Candida colonization index (CI) in patients addmitted to an ICU.

Methods: A prospective observational study was conducted in 2020 during two months in three intensive care units (ICUs) of the university hospital Sahloul in Sousse, Tunisia.

All patients admitted at least for one week in the ICU were included in the study.

After 7 days from the admission in the ICU and every week, five of the following samples were collected for mycological screening: tracheal secretions, rectal swab, armpit skin swab, mouth swab, nasal swab and urine. According to Pittet's definitions, the Candida colonization index (Cl) was calculated as the proportion of the number of distinct body sites (other than blood culture) colonized with Candida spp. over the total number of sites cultured. Patients with $\mathrm{Cl}>0.5$ were considered heavily colonized. Results: Out of 46 studied patients, a total of 21 patients have had an IC based on both biological and clinical evidences. Using a $\mathrm{Cl} \geq 0.5$, the sensitivity was the highest at the third week from the admission $(84,6 \%)$ and the positive predictive value (PPV) was excellent from the second week 
$(92,3 \%)$. The $\mathrm{Cl}$ showed also a good specificity $(92 \%)$ from the first week of the patient's hospitalization.

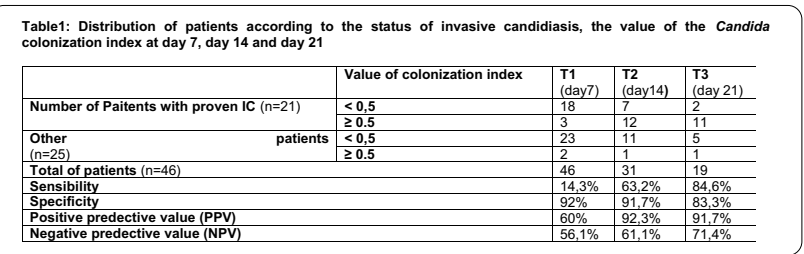

Conclusion: Our study suggested that monitoring Candida colonization index after two weeks of hospitalization could be helpful to identify patients at risk for $\mathrm{IC}(\mathrm{Cl} \geq 0.5)$ allowing therefore the initiation of empirical antifungal therapy. On the other hand, its specificity could be used to exclude the presence of $\mathrm{IC}$ in patients with $\mathrm{Cl}<0.5$.

Disclosure of Interest: None declared.

\section{P041}

Evaluation of "Candida score» in intensive care unit patients: a prospective observational study in Tunisia

M. Ben Brahim ${ }^{1, *}$, N. Haddad ${ }^{2}$, L. Tilouche ${ }^{1}$, S. Boughattas ${ }^{1}$, F. Azouzi ${ }^{1}$, W. Naija $^{3}$, H. hmouda ${ }^{4}$, A. trabelsi ${ }^{1}$, S. Ketata ${ }^{1}$

${ }^{1}$ Laboratory of microbiology, University Hospital Sahloul, ${ }^{2}$ : Department

of preventive and community medicine., Faculty of Medicine of Sousse,

${ }^{3}$ Surgical intensive care unit, ${ }^{4}$ Medical intensive care unit, University

Hospital Sahloul, sousse, Tunisia

Correspondence: M. Ben Brahim

Antimicrobial Resistance \& Infection Control 10(1): P041

Introduction: Key challenges to the management of Invasive candidiasis (IC) include early diagnosis and rapid as well as appropriate antifungal therapy.

Objectives: Hence, this study aimed to evaluate the performance of Candida Score (CS) for early diagnosis of IC in non-neutropenic critically ill patients.

Methods: A prospective observational study was conducted from February to September 2020 in three intensive care units (ICU) of the university hospital Sahloul in Sousse, Tunisia. All patients admitted at least for one week in the ICU were included in the study. The components of «Candida Score» were severe sepsis, total parenteral nutrition, surgery and multifocal Candida colonization. Sepsis was given a score of 2 if present and 0 if absent. The other variables were given a score of 1 if present and 0 if absent. A score more than 2.5 was considered significant.

Results: Out of 112 studied patients a total of 30 patients have had an IC, i.e.an overall incidence of 11.7 IC per 1000 patient days. Candida albicans was the most common Candida species (40\%). Using a CS $>3$, the sensitivity was $40 \%$, and the specificity was $95,1 \%$. The positive predictive value (PPV) was $75 \%$, and the negative predictive value (NPV) was $81,2 \%$. The rate of IC in patients with CS $>3$ was $40 \%$ $(p<0.0001)$ and the mortality rate was $66,6 \%$.

Conclusion: Unlikely to the recommended CS $>2.5$ established by León et al., in our study,

a CS $>3$ was found to be a useful tool to predict IC in critically ill patients, allowing therefore the initiation of antifungal therapy. With a $\mathrm{CS}=2$ or 3 , we must remain vigilant and do more clinical and biological investigations. In fact, with a CS $\leq 2$, an IC is highly improbable and empirical antifungal treatment is not recommended in the absence of positive culture.

Disclosure of Interest: None declared.
Poster session: control of carbapenem-resistant enterobacteriaceae (CRE) and acinetobacter baumannii (CRAB)

P042

Factors related to carbapenemase producing

carbapenem-resistant enterobacteriaceae colonization

I. S. Jeong ${ }^{1, *}$, J. Y. Song ${ }^{2}$, J. S. Jeong ${ }^{3}$

${ }^{1}$ Pusan National University, ${ }^{2}$ Pusan National University Yangsan Hospital,

Yangsan, ${ }^{3}$ Ulsan University, Seoul, Korea, Republic Of

Correspondence: I. S. Jeong

Antimicrobial Resistance \& Infection Control 10(1): P042

Introduction: Carbapenem-resistant Enterobacteriaceae (CRE) is regarded as one of the most urgent threats because of broad resistance to B-lactam antibiotics including carbapenem and a high mortality rate from infection. CP-CRE is big threat as it has spread rapidly, and is associated with high mortality rates, but few studies on risk factors for CP-CRE have been reported.

Objectives: This study was aimed to identify the risk factors of carbapenemase producing carbapenem-resistant Enterobacteriaceae (CP-CRE).

Methods: This retrospective cohort study included 137 CRE colonized patients from previous cohort to develop and assess the external validity of a CRE colonization risk prediction model. The subjects were patients hospitalized in intensive care units of a high-level general hospital located in city $Y$ of Gyeongsang Province, South Korea from October 1, 2016 to May 31, 2018. Every patient admitted these units have perirectal swab to check CRE colonization within 7 days of hospitalization, and weekly thereafter until 7 days after discharge. Patients with confirmatory findings of CRE colonization were included. CRE colonization was confirmed by carbapenem antimicrobial susceptibility testing (imipenem, ertapenem) by disk diffusion method. The collected data were analyzed using SPSS version 21.0 (IBM Corp., Armonk, NY, USA). A two-tailed test was performed with a significance level (a) of 0.05 . The identification of risk factors were done by chi-squared test. Results: Among 137 CRE colonized patients, 24 (17.5\%) patients had CP-CRE. The factors related with CP-CRE were length of stay in intensive care units $(p=0.025)$, use of urinary catheterization $(p=0.012)$ use of mechanical ventilation $(p=0.021)$, and use of nasogastric tube $(p=0.004)$ for $1-14$ days.

Conclusion: Short-term invasive instrumentation of less than two weeks is related to CP-CRE. Therefore, more frequent monitoring of the occurrence of CP-CRE is necessary when invasive procedures are required among the $C R E$ colonized.

Disclosure of Interest: None declared.

P043

Carbapenem resistant enterobacteriaceae (CRE) acquisition rate and risk factors in adult patients, general intensive care unit, hospital pulau Pinang: an observational, cohort, and prospective study

L. Kok Soon 1,*, L. Wei Chyuan², W. Peng Shyan², L. Kar Nim², C. Ting Soo²

${ }^{1}$ Infectious Disease, Hospital Sultanah Aminah, Johor Bahru, ${ }^{2}$ Infectious Disease, Hospital Pulau Pinang, Pulau Pinang, Malaysia

Correspondence: L. Kok Soon

Antimicrobial Resistance \& Infection Control 10(1): P043

Introduction: Carbapenem-resistant Enterobacteriacaea (CRE) is a multidrug-resistant bacterium.Critically ill patients in Intensive Care Units have a high burden of CRE.We conducted an observational study to establish baseline CRE acquisition rate in the General Intensive Care Unit(GICU) of Hospital Pulau Pinang.

Objectives: To determine the CRE acquisition rate,demographic characteristic,risk factors, and mortality rate of CRE infected patients.

Methods: The study was conducted using active surveillance of rectal swabs for CRE from February to June 2020. Adult subjects ( $>18$ years old) had a baseline screening swab done upon admission.Baseline positive swab or known colonizer were excluded.Weekly rectal swabs were performed.Followed up was done until transfer out.Subjects 
who acquired CRE were monitored for CRE related infections. Those who expired or were transferred out from GICU within 7 days were excluded from study.

Results: From 237 admissions to GICU,50 subjects had more than 1 week stay in GICU and were monitored for CRE acquisition.Twelve subjects tested positive for CRE,resulting CRE acquisition rate $\mathbf{2 4 \%}$.

CRE isolated were Klebsiella pneumoniae (91.7\%) and Escherichia coli (8.3\%). No polymycin resistance were detected.Four patients acquired CRE at week 2, 6 patients at week 3, and 1 patient each at week 5 and week 6 respectively.

From $33 \%$ deaths among the subjects who acquired CRE,half was

\begin{tabular}{|c|c|c|}
\hline & CRE Acquired(\%) (n=12) & Non-CRE Acquired(\%)(n=38) \\
\hline Age (mean) & 45 & 46 \\
\hline Days of admission(mean) & 24 & 14.5 \\
\hline Gender:Male & 25 & 71 \\
\hline Female & 75 & \\
\hline Comorbidities & 75 & 75 \\
\hline $\begin{array}{l}\text { History of antibiotic } \\
<3 \text { days } \\
3 \text { to } 7 \text { days } \\
>7 \text { days }\end{array}$ & $\begin{array}{l}0 \\
33.3 \\
66.7\end{array}$ & $\begin{array}{l}0 \\
2.6 \\
97.4\end{array}$ \\
\hline History of Carbapenem & 91.7 & 47 \\
\hline History of Healthcare Contact & 25 & 18.4 \\
\hline History of CRE Contact & 8.3 & \\
\hline History of Ventilation & 100 & 89.4 \\
\hline History of Urinary Catheter & 100 & 97.3 \\
\hline History of Central Venous Line & 91.7 & 81.6 \\
\hline History of Dialysis Catheter & 25 & 39.5 \\
\hline $\begin{array}{l}\text { Clinical Outcome } \\
\text { Expired } \\
\text { Survived }\end{array}$ & $\begin{array}{l}33.3 \\
667\end{array}$ & $\begin{array}{l}39.5 \\
60.5\end{array}$ \\
\hline Survived & 66.7 & \\
\hline
\end{tabular}

attributed to CRE-related infections.

Conclusion: The high acquisition rate shows that CRE is a major issue to be addressed.Early modification of risk factors are paramount in reducing CRE acquisition. Usage of active surveillance screening should be considered as may prevent CRE transmission.

Disclosure of Interest: None declared.

\section{P044}

Enterobacteriaceae-a clinical and environmental longitudinal study in a long-term healthcare unit

C. Santos-Marques ${ }^{1,2, *},{ }^{2}$. Amorim ${ }^{1,3}$, H. Ferreira ${ }^{2}$, S. Gonçalves Pereira ${ }^{1}$

${ }^{1}$ Center for Innovative Care and Health Technology, Polytechnic of Leiria,

Leiria, ${ }^{2}$ UCIBIO/Microbiology, Faculty of Pharmacy of University of Porto,

Porto, ${ }^{3}$ School of Tourism and Maritime Technology, Polytechnic of Leiria,

Peniche, Portugal

Correspondence: C. Santos-Marques

Antimicrobial Resistance \& Infection Control 10(1): P044

Introduction: Antimicrobial resistance (AMR) is a global problem that has long spilled over from hospitals to the community. Long-term healthcare units (LTHU) represent a transition setting by accommodating patients with compromised immunity, previous hospital admissions, invasive devices and wounds, thus more prone to colonization and/or infection by Enterobacteriaceae, some with multidrug resistance (MDR) features.

Objectives: This study scopes for Enterobacteriaceae incidence and related AMR, in a Portuguese LTHU through a longitudinal analysis of its inpatients and environment, to demonstrate the role LTHU have in AMR spread.

Methods: During 6 months, clinical (anus, mouth, hands) and environmental samples (bedside table, bed rail, handbell, door handle) were biweekly collected in 2 LTHU rooms. Samples were culture-based processed and antimicrobial susceptibility profiling (ASP) was conducted in all presumptive Enterobacteriaceae (PE), according to EUCAST methodology, testing amoxicillin + clavulanic acid (AMC), cefotaxime (CTX), cefoxitin (FOX), ceftazidime (CAZ), imipenem (IMP), aztreonam (ATM), meropenem (MEM), ciprofloxacin (CIP) and amikacin (AMK).

Results: A total of 615 samples were collected (129 clinical; 486 environmental) returning a total of $138 \mathrm{PE}$ isolates. Of those, 117 were clinical (92: anus, 20: mouth; 5: hands) and 21 environmental (6: bedside table; 5 : bed rail; 5 : handbell; 5 : door handle).

The best antimicrobial agents against clinical isolates $(\mathrm{Cl})$ were carbapenems, MEM (100\%) and IMP (97.4\%), and the worst AMC (17,9\%). Regarding environmental isolates (EI), results were similar: MEM and IMP $(100 \%$, both) as the best agents, AMC $(19,0 \%)$ the worst.
Cephalosporin susceptibility rates ranged from $91.5 \%$ to $90.6 \%$ in $\mathrm{Cl}$ and $81.0 \%$ to $76.2 \%$ in EI. CIP, a fluoroquinolone, showed $93.2 \% / 95.2 \%$ susceptibility rates and AMK, an aminoglycoside, $70.1 \% / 90.5 \%$ susceptibility rates in $\mathrm{Cl} / \mathrm{El}$, respectively. More importantly, $16(13.7 \%) \mathrm{Cl}$ and 2 (7.0\%) El had a MDR profile.

Conclusion: PE and MDR isolates detected in LTHU patients may be spreading to the environment. Surveillance studies are important to characterize and help fight AMR development and spread in LTHU, by adjusting IPC programs to this new reality in LTHU settings.

Disclosure of Interest: None declared.

\section{P045}

The effect of terminal disinfection on environmental contamination in cabapenem-resistant enterobacteriaceae-carrying patient rooms

J. Kim ${ }^{1, *}$, J. S. Jeong ${ }^{1}$, E. S. Park ${ }^{2}$, M.-N. Kim ${ }^{3}$

${ }^{1}$ Department of Clinical Nursing, University of Ulsan, ${ }^{2}$ Infection Control Team, Yonsei University Severance Hospital, ${ }^{3}$ Department of Laboratory Medicine, Asan Medical Center University of Ulsan College of Medicine,

Seoul, Korea, Republic Of

Correspondence: J. Kim

Antimicrobial Resistance \& Infection Control 10(1): P045

Introduction: CRE can survive not only in a humid environment but also in a dry environment for a long time, is more closely related to hospital environment contamination than other intestinal bacteria, and causes transmission, which is a problem in infection control.

Objectives: This study was aimed to compare the extent of cabapenem-resistant Enterobacteriaceae (CRE) contamination of the environmental surfaces before and after disinfection by using both Adenosin Triphosphate (ATP) measurement and microbial culture tests.

Methods: From April to May 2021, 10 hospital rooms of patients $48 \mathrm{~h}$ after CRE was isolated from a tertiary acute care hospital were selected. Eight samples were collected before and after terminal room disinfection, one each from the bed table, mattress, bed railing, door handle, sink surface, drain, toilet, and toilet railing in each hospital room. For a total of 181 specimens, the relative light units (RUL) and colony forming units (CFU) values were measured using the ATP and culture test, respectively.

Results: Two CRE cases were isolated from the drains of the two hospital rooms before disinfection, one case on the surface of the sink, and the other case from the toilet seat. Both strains were identical as Klebsiella pneumonia and consistent with the patient's strain. The RUL value was highest in the drain before disinfection, and those of all surfaces except the drain decreased after regular terminal disinfection. After disinfection, all except for the drain (50\%) and toilet handrail (90\%) was under the environmental surface cleanliness criterion $(R L U<250)$, and the RLU value for bed table $(p=0.031)$ and the mattress $(p=0.016)$ was significantly different before and after disinfection.

Conclusion: CRE isolation on the surface of CRE patient's room is less than that of previous studies. Regular terminal disinfection is effective to reduce the contamination of most room surfaces except sink drain. Therefore, additional terminal disinfection methods are required for sink drain.

Disclosure of Interest: None declared.

\section{P046}

Nosocomial outbreak caused

by metallo-ßeta-lactamase-producing pseudomonas aeruginosa type IMP linked to contaminated water-related devices in a tertiary hospital, Spain

M. T. Beca", , E. Muñoz', Y. Cogolludo', P. Zamarrón ${ }^{1}$, J. D. Martín ${ }^{1}$, T. Arevalillo', B. Martínez ${ }^{1}$, R. Perea', A. Morano', B. Fernández-Pacheco ${ }^{1}$, H. Burggraaf' ${ }^{1}$, L. Calvo', P. Romero', L. García de la Rosa' ', F. Paulino ${ }^{1}$

${ }^{1}$ Toledo Hospital Complex, Toledo, Spain

Correspondence: M. T. Beca

Antimicrobial Resistance \& Infection Control 10(1): P046 
Introduction: Wastewater has been increasingly implicated in outbreaks with carbapenem-resistant Pseudomonas aeruginosa.

Objectives: The objective of this study is to describe the first nosocomial outbreak of Metallo- $\beta$-lactamase-producing Pseudomonas aeruginosa type IMP in the hemato-oncology ward linked to contaminated water-related devices at our center and to describe the set of measures adopted for its control.

Methods: Between July 26-October 24, 2019, six consecutive isolates of carbapenem-resistant Pseudomonas aeruginosa producing IMP were detected with the same and non-usual antibiogram (sensitive to piperacillin, piperacillin/tazobactam and colistin). The demographic, clinical-epidemiological and evolutionary characteristics of the patients were analyzed. Cases and environmental samples were sent to a National Reference Laboratory for the corresponding molecular characterization by Pulsed Field Gel Electrophoresis (PFGE).

Results: Six patients were affected. Three died. Samples from dry surfaces were negative. Samples from siphons of some patients' rooms sinks were positive; two profiles were found according to molecular characterization by PFGE. The most frequent profile was found in the first four isolated cases in the hemato-oncology ward and traps of sinks of two patients' rooms. The other profile was found in one patient outside hemato-oncology unit and others traps in the ward. There were no more cases after replacement of traps and sinks.

Conclusion: It was an outbreak of infection and colonization by a Metallo- $\beta$-lactamase-producing Pseudomonas aeruginosa type IMP, associated with rapid torpid evolution among hemato-oncology patients. It was also isolated in environmental samples associated with aqueous devices. Its role as a source and facilitator of transmission was determined by the results of the molecular study, and the control measures taken on the environment corroborated the role of these aqueous elements in the transmission.

Disclosure of Interest: None declared.

\section{P047}

Sporadic transmission of VIM-1- and MCR-9-harbouring enterobacter cloacae complex from drains: safe patient care despite persisting biofilms

M. Thieme' ${ }^{1}$, M. Malecki', A. Kirchler'1, R. Hellmuth'1, P. G. Higgins², K. Xanthopoulou ${ }^{2}$, H. Seifert ${ }^{2}$, F. Mattner ${ }^{1}$, A. F. Wendel ${ }^{1, *}$

${ }^{1}$ Institute of Hygiene, Cologne Merheim Medical Centre, University Hospital of Witten/Herdecke, ${ }^{2}$ Institute for Medical Microbiology, Immunology and Hygiene, University of Cologne, Cologne, Germany

Correspondence: A. F. Wendel

Antimicrobial Resistance \& Infection Control 10(1): P047

Introduction: The hospital environment is a source for sporadic transmissions or outbreaks of carbapenemase- or colistin resistance gene-harboring bacteria. The detection of VIM-producing Enterobacter species in four patients hospitalized in two adjacent single rooms of an intermediate care ward over a period of 20 months led to further investigations.

Objectives: This investigation aims to define the molecular epidemiology and transmission dynamics of four cases of hospital-acquired VIM-1-carbapenemase- (some co-harboring mcr-9) E. cloacae complex on a critical care unit and the infection control interventions applied over time.

Methods: Infection prevention and control measures introduced were as follows: contact precautions, environmental sampling of all sinks and shower drains (2019 and 2020), replacement/cleaning and disinfection of the drainage, screening, observations, and trainings. Whole genome sequencing was performed (Illumina MiSeq and MinION, cgMLST analysis by Ridom Seqsphere +, JSpeciesWS, PlasmidFinder and ResFinder).

Results: Several plausible transmission events from the hospital environment were confirmed: one patient-environment-patient transmission with a VIM-1-producing E. hormaechei subsp. hoffmannii (same genotype in patients and drains; patients treated in the same room 16 months apart) and one environment-patient transmission with an E. hormaechei subsp. xiangfangensis coharbouring bla $a_{\mathrm{VIM}-1^{-}}$and $\mathrm{mcr}-9$ (highly related strain found in the sink drain in the adjacent room before the patient was admitted). Observations carried out revealed possible environment-to-patient transmission events as medical equipment and patient care products were stored nearby the washbasin. Despite persisting $b / a_{\mathrm{VIM}-1^{-}}$and $m c r-9-c o n-$ taining biofilms in drains, no more additional patient cases were detected.

Conclusion: Transmission of carbapenemase-producing Enterobacterales arising from the hospital waste-water system are often complex, involving several genotypes and resistance genes. As the biofilm is difficult to eradicate, it is important to emphasize standard precautions and include regular compliance observations to achieve a sustainable infection control.

Disclosure of Interest: None declared.

\section{P048}

Polytherapy as a strategy for the treatment

of carbapenemase-producing klebsiella pneumoniae infections: a two-center, in Bucaramanga-Colombia

K. S. Rodríguez Díaz ${ }^{1, *}$, L. G. Uribe ${ }^{2}$, A. Vega ${ }^{1}$, C. Figueroa ${ }^{1}$, M. P. Torres ${ }^{1}$,

A. Maldonado ${ }^{2}$, J. Lopez Aldana ${ }^{1}$ on behalf of GERMINA (Group

for the Educational Renewal of Internal Medicine)

${ }^{1}$ Industrial University of Santander, ${ }^{2}$ Los Comuneros University Hospital, Bucaramanga, Colombia

Correspondence: K. S. Rodríguez Díaz

Antimicrobial Resistance \& Infection Control 10(1): P048

Introduction: Treatment of infections caused by carbapenemase-producing Enterobacteriaceae leads to high morbidity and mortality due to the limited availability of efficient antibiotic regimens. Monotherapy is associated with higher mortality and the combination of antimicrobials is used as the first choice. This study describes the clinical and microbiological experience of carbapenemase-producing Klebsiella pneumoniae(CPKP)isolates with combination therapy.

Objectives: Describe the characteristics of carbapenemase-producing Klebsiella pneumoniae infections treated with polytherapy in two fourth-level care centers in Bucaramanga. January 2015-January 2020.

Methods: A retrospective cohort study of the Bucaramanga University Hospital. Patients over 18 years of age with carbapenemase-producing Klebsiella pneumoniae infections were included in patients who had completed at least $48 \mathrm{~h}$ with antibiotic combination therapy.

Results: During the study period, 550 patients with CPKP infection were identified. 440 patients were excluded and 110 patients were selected for analysis. The combined antibiotic management regimens were Meropenem plus Polymyxin-B (39\%); Meropenem plus Ertapenem (34\%) and others. The microbiological success was $41 \%$ and the clinical success was $77 \%$ with a failure rate of $23 \%$. Overall mortality was $26 \%$; $85 \%$ of the Kp detected produced class A carbapenemases (KPC), while the rest of the isolates harbored class $B$ Metallo- $\beta$-lactamase genes and class $D$ carbapenemases (OXA). Mortality at the end of hospitalization was significantly higher in patients who presented some severity criterion $(P<0.05)$. On the other hand, the double carbapenems group with Ertapenem presented qualifying mortality compared to the other regimens $(p=0.04)$.

Conclusion: The need for effective management for KPRC persists. This observational analysis shows that the association of ertapenem plus meropenem provides a survival benefit. Larger prospective studies are needed to confirm these findings and to define the role of this unconventional strategy.

Disclosure of Interest: None declared. 
P049

Fecal carriage of extended-spectrum $\beta$-lactamaseand carbapenemase-producing enterobacteriaceae in Moroccan hospitalized patients in a university hospital

S. El Hassouni ${ }^{1, *}$, M. C. Benjelloun ${ }^{1}$, B. Oumokhtar ${ }^{1}$

${ }^{1}$ microbiology, faculty of medicine and pharmacy, fez, Morocco

Correspondence: S. El Hassouni

Antimicrobial Resistance \& Infection Control 10(1): P049

Introduction: The prevalence of extended-spectrum b-lactamaseproducing Enterobacteriaceae (ESBLE) and carbapenemase-producing Enterobacteriaceae (CPE) in hospitalised and community patients is of significant public health concern.

Objectives: This study aimed to investigate the fecal carriage and molecular epidemiology of ESBLE and CPE isolated from patients at admission to a medical ward.

Methods: From January to July 2019, 100 patients admitted to the medical ward via intensive care unit (ICU) and emergency department (ED) were screened for ESBL and CPE carriage at admission. ESBLE were identified by double-disk synergy test. PCR and sequencing were used to characterize the resistance genes.

Results: In the present study, 127 strains of Enterobacteriaceae were collected, the prevalence of faecal carriage of ESBLE was $12.6 \%$ $(16 / 127)$, E. coli was the predominant specie $(9 / 16 ; 56.2 \%)$. A high rate of multiresistance in ESBLE was detected, $87.5 \%$ were resistant to Gentamicin, $81,2 \%$ to SXT, $87.5 \%$ were resistant to Nalidixic Acid and Norfloxacin, and $75 \%$ were resistant to Ciprofloxacin. All isolates were susceptible to Amikacin, Imipenem and Fosfomycin. Among all species, the bla $a_{C T X-M-1}$ group was the most common gene detected (75\%), followed by bla $a_{\text {TEM }}(62.5 \%)$ and $b / a_{S H V}(31.2 \%)$. Carbapenemase production was found in one E. cloacae and was expressed exclusively by the $b / a_{O X A-48}$ gene. Nucleotide sequence analysis of bla CTX-M-1 demonstrated that all strains carried the CTX-M-15 subgroup.

Conclusion: These findings suggest the need for action to better handle the burden of multidrug-resistant bacteria. Education of healthcare workers on basic procedures of infection control is recommended, as well as the implementation of antimicrobial stewardship program in all departments of healthcare facilities.

Disclosure of Interest: None declared.

\section{P050}

KPC rise in an Italian academic hospital during COVID-19 pandemic

G. Giammarini Barsanti ${ }^{1,}{ }^{*}$, M. Driutti ${ }^{1}$, L. Brunelli, ${ }^{1,2}$, R. Cocconi ${ }^{2}$

${ }^{1}$ Dipartimento di Area Medica, Università degli Studi di Udine, ${ }^{2} \mathrm{SOC}$

Accreditamento, Gestione Rischio Clinico e Valutazione delle Perfor-

mance Sanitarie, Azienda Sanitaria Universitaria Friuli Centrale, Udine, Italy

Correspondence: G. Giammarini Barsanti

Antimicrobial Resistance \& Infection Control 10(1): P050

Introduction: Multi Drug Resistant microorganisms (MDR) have been a serious issue for public health for more than fifty years. Carbapenem resistant Klebsiella pneumoniae (KPC) is a highly lethal bacteria, with a mortality rate in order of $40 \%$ worldwide.

Objectives: In this study we examined data collected from the MDR surveillance team to understand how this life-threating infection impacted during COVID-19 pandemic.
Methods: A retrospective observational study was conducted at the about 950 beds Academic Hospital of Udine, Northeast of Italy. Data about new cases of KPC occurred from 2016 to 2021 were collected from internal datasets. Considering bed occupation, we calculated weekly incidence rate of KPC cases. Using Chi-square test, cumulative incidence rates before and after the $10^{\text {th }}$ of October 2020 , considered as the start of the $2^{\text {nd }}$ COVID-19 wave in our hospital, were compared.

Results: During the observation period, 255 new KPC cases were reported on a total of 1,235,177 days of hospitalization. Cumulative and mean incidence rates on the overall observation period resulted 2.064 and 2.075 new KPC cases per 10,000 hospitalization days, respectively. Cumulative incidence rate were calculated in 1.287 before and 7.467 after the second COVID-19 wave, being the two statistically different ( $p<0.001, \mathrm{Cl} 95 \%)$.

\begin{tabular}{|c|c|c|c|}
\hline $\begin{array}{l}\text { Year } \\
\text { s }\end{array}$ & Hospitalization days & $\begin{array}{ll}\text { New } & \text { Eve } \\
\text { nts }\end{array}$ & Mean Weekly Incidence Rate \\
\hline 2016 & 64,831 & 7 & 1.097 \\
\hline 2017 & 278,119 & 65 & 2.304 \\
\hline 2018 & 277,499 & 30 & 1.067 \\
\hline 2019 & 271,174 & 25 & 0.941 \\
\hline 2020 & 249,924 & 16 & 0.638 \\
\hline 2021 & 93,630 & 112 & 11.857 \\
\hline $\begin{array}{l}\text { Tota } \\
\text { | }\end{array}$ & $1,235,177$ & 255 & 2.075 \\
\hline
\end{tabular}

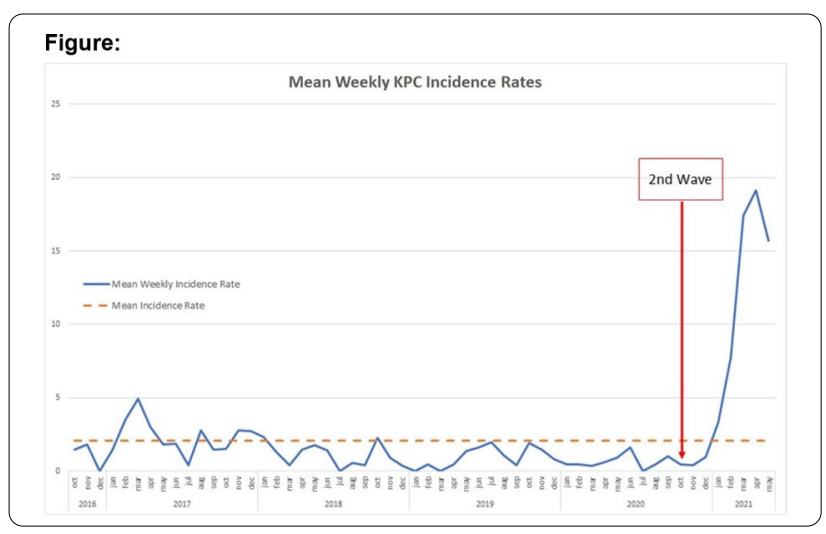

Conclusion: We observed a worrying rise of KPC infection incidence since the $2^{\text {nd }}$ COVID-19 wave began, suggesting an increased KPC outbreaks risk during pandemic. This observation could be explained by a reduced effectiveness of personal protective equipment wore by healthcare workers or its unsuccessfulness in preventing cross infections within COVID units. Even during a pandemic, contact precautions should not be overlooked in healthcare settings and there is an urgent need to reinforce professionals' attention against the spread of MDR to prevent their rise.

Disclosure of Interest: None declared. 
P052

Outcomes of screening for VRE and carbapenemase producing enterobacteriaceae in Tunisian intensive care units: rates and risk factors

W. Dhouib ${ }^{1}$, H. Ghali ${ }^{2,}{ }^{*}$, A. Ben Cheikh², S. Bhiri ${ }^{2}$, R. Bannour ${ }^{1}$, S. Khefacha ${ }^{1}$, H. Said Latiri², M. Ben Rejeb²

${ }^{1}$ Department of prevention and security of care, Sahloul university hospital, ${ }^{2}$ Department of prevention and security of care, Sahloul university hospital, Faculty of Medicine of Sousse, University of Sousse, Sousse,

Tunisia

Correspondence: $\mathrm{H}$. Ghali

Antimicrobial Resistance \& Infection Control 10(1): P052

Introduction: Emerging Highly Resistant Bacteria (eHRB) constitute a major public health problem in Tunisia and in the world. Rates of infections by eHRB are significantly higher in intensive care units (ICUs), justifying the implementation of a screening system for their control.

Objectives: We aimed to describe outcomes of eHBR screening in ICUs of Sahloul university hospital of Sousse (Tunisia) and to determine risk factors of infection by Vancomycin resistant enterococcus (VRE) or Carbapenemase producing Enterobacteriaceae (CPE).

Methods: We conducted a longitudinal observational study during six-month between 1 June and 1 December 2018. Rectal and nasal swab cultures were collected to detect VRE and CPE among patients admitted in six ICUs of Sahloul university hospital of Sousse (Tunisia) and more than three times, at least one week apart.

Results: During the study period 174 patients were screened. Of them, $69.5 \%$ were male and $73.6 \%$ were admitted in surgical ICU. In total, 161 and 152 samples were realized respectively for the detection of CPE and VRE. These samples were positive in $15 \%$ and $8.5 \%$ respectively for CPE and VRE. Klebsiella pneumoniae OXA 48 was the most isolated CPE (80\%).

Independent risk factors for VRE infection were surgery $(\mathrm{aOR}=2.6$, $\left.\mathrm{Cl}_{95 \%}=[1.9-8.2]\right)$, organ failure $\left(\mathrm{aOR}=2.3 \mathrm{Cl}_{95 \%}=[1.5-6.5]\right)$, and exposure to antibiotics during the month before screening $(\mathrm{aOR}=4.2$, $\left.\mathrm{Cl}_{95 \%}=[2.3-7.9]\right)$, and for $\mathrm{CPE}$, exposure to antibiotics during the month before screening $\left(\mathrm{aOR}=3.9, \mathrm{Cl}_{95 \%}=[2.7-10.6]\right)$.

Conclusion: The screening system is a complementary axis to the surveillance of infections with eHRB. Our high rates testify the need for a sensitive, specific and cost-effective screening programm that may help in infection control by early identification of patients.

Disclosure of Interest: None declared.

\section{P053}

CRE carriage: is it for life or can it be cleared?

M. L. Ling ${ }^{1,}$, M. How ${ }^{1}$, M. O. Aung ${ }^{1}$

${ }^{1}$ Infection Prevention \& Epidemiology, Singapore General Hospital,

Singapore, Singapore

Correspondence: M. L. Ling

Antimicrobial Resistance \& Infection Control 10(1): P053

Introduction: Singapore General Hospital has attempted multiple interventions to prevent CRE transmission in inpatient setting. These include active surveillance of high-risk patients, isolation and cohorting, hand hygiene and enhanced environment hygiene programs. However, the number of CRE carriers continued to rise with time because lack of policy to allow untagging the carrier status for returning inpatients to the hospital.

Objectives: The purpose of this study is to determine appropriate period of establishing clearance of CRE in known CRE patients.

Methods: Patients with known history of CRE and discharged more than 3-months ago were recruited into the study, where $1^{\text {st }}$ and $3^{\text {rd }}$ rectal swabs were taken on day 1 and day 7 respectively and $2^{\text {nd }}$ rectal swab was taken between day 2 to day 6 . CRE was detected using the PCR method from the $1^{\text {st }}$ and $2^{\text {nd }}$ samples whilst culture method was used for the $3^{\text {rd }}$ sample.
Results: Out of 76 participants recruited, $50 \%$ of the cases cleared CRE carriage in 304 days ( $95 \% \mathrm{Cl}: 238$ to 369 days) from last date of discharge; and more than $80 \%$ cleared it within 2 years. $56.6 \%$ of study population were male with majority Chinese and median age of 67.0 years. The median length of stay of these 2 groups were 14.0 days for patients who did not clear CRE carriage and 7.0 days for patients who cleared CP-CRE carriage. Sixty-five patients continued antimicrobial treatment after initial discharge, with amoxicillin-clavulanic acid as the frequently used antimicrobial. Median antimicrobial-free days was 118 days for patients who did not clear CRE while for CRE cleared group, it was 281 days.

Conclusion: Our study showed that majority $(80 \%)$ of patients lose CRE carriage if they stayed away from the hospital for at least 2 years. The duration of CRE colonisation is uncertain and is likely to vary between individuals. However, a prospective study with repeated CRE testing at regular interval will be required to determine exact time of CRE clearance before specific guidance can be given as to when one should consider a patient cleared of CRE carriage.

Disclosure of Interest: None declared.

\section{P054}

Cases of bacteremia caused by carbapenem-resistant $E$. coli

O. Orlova ${ }^{1,2,{ }^{*}}$, A. Shelenkov ${ }^{1}$, Y. Mikhaylova ${ }^{1}$, V. Gusarov ${ }^{2}$, N. lumtsunova ${ }^{2}$, L. Petrova $^{2}$, V. Akimkin ${ }^{1}$

${ }^{1}$ «Central Research Institute of Epidemiology» of The Federal Service on Customers' Rights Protection and Human Well-being Surveillance, ${ }^{2}$ Pirogov National Medical and Surgical Center, Russian Federation, Moscow, Russian Federation

Correspondence: $\mathrm{O}$. Orlova

Antimicrobial Resistance \& Infection Control 10(1): P054

Introduction: Genomic analysis of microbial isolates makes it possible to carry out epidemiological decoding of single cases and outbreaks of infectious diseases.

Objectives: To identify an epidemiological link between patients with carbapenem-resistant E. coli.

Methods: During the month of 2020, cases of bacteremia caused by $E$. coli were registered in the Department of Hematology in 10 patients. To determine the identity of the isolates of microorganisms, bacteriological, molecular biological (PCR-RT and MLST) methods were used.

Results: All patients after the TSC were in isolated wards. There were no signs of CAIC in the patients. E. coli sensitive to carbapenems was detected in 8 patients on admission in a rectal smear. E. coli resistant to carbapenems was detected in 6 patients with bacteremia. With the development of bacteremia in the same patients, E. coli resistant to carbapenems was detected in rectal smears. In two patients with the development of bacteremia caused by E. coli sensitive to carbapenems, the genes of carbapenemases NDM and CPC were found in rectal smears. When conducting a microbiological study of objects in the hospital environment and samples of biomaterial (pharynx, nose, hands, rectal smear), E. coli and antibiotic resistance genes were not found in the staff of the Department of Hematology. When conducting a comparative analysis of the complete genomes of Escherichia coli isolates isolated from the blood of 6 patients at all common loci (5045 loci in total), it was found that one of the isolates was slightly different from the others (about 100 substitutions), while in all other pairs there were no more than 10 . These differences may be caused by differences in the plasmid sequences. In this analysis, no plasmids were isolated, but the results already obtained still allow us to attribute all the isolates to one strain.

Conclusion: According to the results of the study, the group colonization of patients with the strain of $E$. coli, due to the translocation of the intestinal flora, was established.

Disclosure of Interest: None declared. 
P055

The importance of monitoring increasing trend of colistin resistance in clinical isolates of Acinetobacter baumannii A. Dedeic-Ljubovic ${ }^{1, *}$, J. Halkovic ${ }^{1}$, Đ. Granov' ${ }^{1}$, Đ. Gačanović ${ }^{1}$, E. Husić ${ }^{1}$, E.-J. Đulić ${ }^{1}$

${ }^{1}$ Clinical Center of the University of Sarajevo, Sarajevo, Bosnia and Herzegovina

Correspondence: A. Dedeic-Ljubovic

Antimicrobial Resistance \& Infection Control 10(1): P055

Introduction: Acinetobacter baumannii attracts a lot of attention, both with its frequency and extremely developed resistance to antimicrobial agents. Once out of use due to numerous side effects, polymyxins such as colistin acts synergistically with other antibiotics and serve as the last therapeutic option for life-threatening infections caused by this extremely resistant pathogen.

For antimicrobial susceptibility testing of colistin, EUCAST recommend the broth microdilution method (BMD). It is a semiquantitative, in vitro method, based on the action of an antimicrobial agent and a bacterial isolate.

Objectives: To investigate an increasing trend of colistin resistance in hospital isolates of Acinetobacter baumannii.

Methods: This retrospective study was conducted at Clinical microbiology Unit, Clinical Center of the University of Sarajevo, the largest tertiary level hospital in Bosnia and Herzegovina. Study included 219 isolates of Acinetobacter baumannii detected from June 2019 to October 2020 by VITEK ${ }^{\circledR}$ COMPACT, BioMerieux.

MIC values were determined by broth microdilution method, according the EUCAST recommendations with "MIC-Strip Colistin", producer Merlin A Bruker Company, Germany.

Results: A.baumannii was isolated most commonly from the ICU 76 (36\%) and pediatric clinic $24(10,96 \%)$. The isolates were recovered mostly from upper respiratory tract $69(31,51 \%)$, wound and drain swabs $62(28,31 \%)$.

Minimum inhibitory concentration (MIC) value for colistin ranged from $0.25-2 \mu \mathrm{g} / \mathrm{ml}$. The highest number of isolates, $125(57 \%)$ had a MIC value of $0.5 \mu \mathrm{g} / \mathrm{ml}$, followed by $1 \mu \mathrm{g} / \mathrm{ml}(64 / 29.22 \%)$ and $0.25 \mu \mathrm{g} /$ $\mathrm{ml}(24 / 10.96 \%)$, respectively. MIC to colistin increased to $2 \mu \mathrm{g} / \mathrm{ml}$ in period April to October 2020 and this value was detected in 6 (2.74\%) of isolates.

Conclusion: Our study showed an increasing trend of colistin MIC value for Acinetobacter baumannii isolates in our hospital. The increase in antimicrobial resistance of this important hospital pathogen has a significant impact on infection control. If this trend continues, simple infections will no longer be curable. Broth microdilution method (BMD) is a good tool for early detection of emerging resistance.

\section{Disclosure of Interest: None declared.}

\section{P056}

Comparison of antibiotic sensitivity of Burkholderia cepacia

and Bukholderia cenocepacia

K. A. Munasinghe ${ }^{1, *}$, O. Ukpu ${ }^{1}$, S. Bakattah ${ }^{1}$

${ }^{1}$ Frostburg State University, Frostburg, United States

Correspondence: K. A. Munasinghe

Antimicrobial Resistance \& Infection Control 10(1): P056

Introduction: Burkholderia cepacia complex (BCC) was tied to various FDA drug recalls such as nasal spray, curl styler, wipes, oral electrolyte, and anesthetic hydro jell. Burkholderia cenocepacia is a clinically important member of the BCC. These are opportunistic pathogens in patients with respiratory tract infections.

Objectives: The main research objective is to compare antibiotic and disinfectant sensitivity of B. cepacia and B. cenocepacia using the Kirby Bauer Method.

Methods: Four replicates of the Muller Hinton culture plates with disks embedded with antibiotics and disinfectants were incubated at $37^{\circ} \mathrm{C}$. After incubation, zones of inhibitions were measured. Bacterial growths were measured at 4, 20, 25, and $37{ }^{\circ} \mathrm{C}$ and $\mathrm{pH} \mathrm{4,6,7}$ and 8 . TSB broth cultures set to $\mathrm{pH} 4,6,7$, and 8 were incubated over night at $4,20,25$, and $37^{\circ} \mathrm{C}$. After incubation, transmission readings were obtained using the Spectrophotometer. Transmission is inversely proportional to bacterial growth. Used the ANOVA with a $\mathrm{P}<0.05$.

Results: The bacteria had resistance to Penicillin (P10), Ampicillin (AMP10), Tetracycline (TE30), Erythromycin (E15), and Streptomycin (S10). Only Chloramphenicol (30 mg) could inhibit the growth of BCC and B. cenocepacia with $22.6 \mathrm{~mm}$ and $22.3 \mathrm{~mm}$ zone of inhibition, respectively. Bacteria were resistant to Roccal, 409, Listerine, Lysol, and Bleach. At $4{ }^{\circ} \mathrm{C}, \mathrm{BCC}$ and $\mathrm{B}$. cenocepacia had $100 \%$ transmission in $\mathrm{pH}$ 4 and $\mathrm{pH}$ 6. At $4{ }^{\circ} \mathrm{C}$, transmission of $\mathrm{BCC}$ in $\mathrm{pH} 7$ and 8 was 100 and $99.4 \%$ and B. cenocepacia was 99.8 and $98.3 \%$, respectively. At $20{ }^{\circ} \mathrm{C}$ transmission of $B$. cepacia in $\mathrm{pH} 4,6,7,8$ was $100,91.9,88.6,93.9 \%$, and $B$ cenocepacia was $99.6,84.8,88.4,93.7 \%$, respectively. At $25^{\circ} \mathrm{C}$, transmission of $\mathrm{BCC}$ in $\mathrm{pH} 4,6,7,8$, was $99,79.6,80.8$, and $79.5 \%$ and B. cenocepacia was $99.6,70.1,77.7$, and $84.0 \%$, respectively. At $37^{\circ} \mathrm{C}$, transmission of $\mathrm{BCC}$ in $\mathrm{pH} 4,6,7,8$, was $99,7.3,59.2$, and $48.8 \%$ and $B$. cenocepacia was $99,10.1,17.0,26.1 \%$, respectively.

Conclusion: Results suggested that BCC and B. cenocepacia have slower growth in any $\mathrm{pH}$ at $4{ }^{\circ} \mathrm{C}$ and in $\mathrm{pH} 4$ at any temperature. Bacterial growth increased with increasing temperature and $\mathrm{pH}$. B. cepacia and $B$. cenocepacia have resistance for many antibiotics, except Chloramphenicol $(30 \mathrm{mg})$, and all disinfectants used.

Disclosure of Interest: None declared.

\section{P057}

fimH and csgA adhesion genes among acinetobacter SPP. isolates and their relation to biofilm formation and antimicrobial resistance pattern

D. M. Ali 1,*, A. Baker ${ }^{2}$, M. eldahshan ${ }^{2}$

${ }^{1}$ microbiology and immunology, faculty of medicine, ${ }^{2}$ microbiology

and immunology, Menoufia university, menofia university, Egypt

Correspondence: D. M. Ali

Antimicrobial Resistance \& Infection Control 10(1): P057

Background: Acinetobacter spp are important opportunistic pathogens responsible for nosocomial infections. Objectives: detection of Es $\beta L$ and carbapenemase, the ability of biofilm formation and their relation to antimicrobial resistance. Methodology: A total of 230 clinical samples from patients admitted to Menoufia University Hospitals were obtained. Acinetobacter spp were identified by standard microbiological methods and Vitek-2 system. The antibiogram of Acinetobacter isolates was tested by the modified Kirby Bauer disk diffusion method, detection of extended-spectrum $\beta$-lactamases and carbapenemase by Es $\beta L$ NDP and CANP tests. Biofilm production was detected by modified Congo red agar and PCR. Results Acinetobacter spp. represented (20.8\%) of all the collected isolates. Vitek-2 system showed that the predominant spp. was Acinetobacter baumannii complex $(80 \%)$. Acinetobacter isolates were highly resistant to cefepime and tobramycin ( $90 \%$ for each), ceftriaxone $(88 \%)$, piperacillin, and ampicillin -sulbactam (86\% for each), piperacillin- tazobactam (84\%) and tetracycline (78\%). About $64 \%$ and $68 \%$ of the Acinetobacter isolates were susceptible to tigecycline and colistin respectively. The sensitivity of Es $\beta$ L NDP for detection of Es $\beta$ L producing Acinetobacter isolates was $93.8 \%$. The Carba NP and carbAcineto NP tests detect carbapenemse production in $6 \%$ and $56 \%$ of Acinetobacter isolates respectively. Biofilm production was found among $56 \%$ isolates by MCRA method, while conventional PCR showed fimH and CsgA genes among $60 \%$ and $18 \%$ isolates respectively. Conclusion: Acinetobacter spp are serious nosocomial pathogens as they can produce ES $\beta$ L and carbapenemase, and produce biofilm that is related to their antimicrobial resistance. Therefore, their adequate prevention and control is imperative.

Disclosure of Interest: D. Ali Employee of: non, Grant/Research support from: non, Speaker bureau of: non, Shareholder of: non, 
Consultant for: non, Paid instructor for: non, Conflict with: non, A. Baker: None declared, M. eldahshan: None declared.

Poster Session: Clostridium difficile

P058

Clostridioides difficile infection in patients not exposed to antibiotics: a 14 years prospective study in a French university hospital

N. Khanafer 1,* , C. U. Edouard Herriot hospital' ${ }^{1}$, L. Oltra' ${ }^{1}$, L. Gerster ${ }^{1}$, O.

Dauwalder ${ }^{1}$, P. Vanhems ${ }^{1}$

${ }^{1}$ Hospices Civils de Lyon, Lyon, France

Correspondence: N. Khanafer

Antimicrobial Resistance \& Infection Control 10(1): P058

Introduction: $C$. difficile infection (CDI) weighs heavily on healthcare system due to increased incidence, morbidity and mortality, as well as costs. CDI is mainly considered as a health-care associated (HCA) after exposure to broad-spectrum antibiotics (ATB). However, CDI has been reported in people previously thought to be at low risk.

Objectives: The objective of this study was to compare characteristics of $\mathrm{CDI}$ cases regarding the previous exposure to ATB in the last 30 days before onset of symptoms.

Methods: Between January 2007 and December 2020, a prospective surveillance study of CDI was conducted in a 900-bed French university hospital. European definitions of CDI case, relapses and origin of acquisition were applied.

Results: A total of 1060 (1182 episodes) patients were included with a mean incidence rate of 2.3 per 1000 hospital-stays. Episodes were HCA (76.7\%), community-acquired (CA) $(17.7 \%)$ or undetermined origin (5.6\%). The mean of age was 66.3 years and $51 \%$ were women Comparison between the two groups $(\mathrm{G})$ of episodes according to previous exposure to ATB (G\#1: 851 (72\%) exposed, G\#2: 331 (28\%) unexposed) showed that the age and sex distribution was similar between the 2 groups. Prior to CDI, exposure to proton pump inhibitors (PPIs), antivirals, immunosuppressants and colonic preparations were significantly more frequent in $\mathrm{G} \# 1$. Patients in $\mathrm{G} \# 1$ had a significantly longer mean length of stay than those in $\mathrm{G} \# 2$ ( 33 vs 25 days; $P=0.007$ ). $C A-C D I$ was significantly more frequent in $\mathrm{G} \# 2$ ( $57.6 \%$ vs $42.4 \%$ for $\mathrm{G} \# 1, \mathrm{P}<0.0001)$. The complication, relapse and death rates were similar between the 2 groups. CDI in low-risk patients (age $<50$ years, no recent exposure to ATB and PPIs) was observed in $4 \%$ of patients (mostly CA, P<0.0001).

Conclusion: Our results show that CDI can affect populations previously thought to be at low risk. These findings suggest that other, no yet known, factors may play a role in the acquisition of CDI. Therefore, it is important for physicians to be aware of the changing epidemiology of CDI in order to remain alert to the possibility of CDI in diarrhoea patients, even in the absence of the common/expected risk factors. Disclosure of Interest: None declared.

\section{P059}

Incidence and risk factors of post-discharge clostridioides difficile infections after hip and knee arthroplasty, a population-based study of 55,842 patients in Poland

E. Jachowicz ${ }^{1}$, M. Gajda ${ }^{2, *}$, M. Brudło ${ }^{3}$, A. Pac ${ }^{4}$, A. Różańska ${ }^{1}$, B. Gryglewska ${ }^{5}$, J. Wójkowska-Mach ${ }^{1}$

${ }^{1}$ Department of Microbiology, ${ }^{2}$ Doctoral School of Medical Sciences and Health Sciences, ${ }^{3}$ Jagiellonian University Medical College, Krakow, Poland, ${ }^{4}$ Department of Epidemiology, Chair of Epidemiology and Preventive Medicine, ${ }^{5} 4$ Department of Internal Medicine and Gerontology, Jagiellonian University Medical College, Krakow, Poland

Correspondence: M. Gajda

Antimicrobial Resistance \& Infection Control 10(1): P059
Introduction: Clostridioides difficile $(\mathrm{Cl})$ is responsible for healthcare associated diarrhea with possible severe progression. Recurrence of the disease induces higher health system costs, as well as exposes patients to additional health risks.

Objectives: The purpose of this study was to determine the incidence and risk factors of post-discharge CD infections (CDI) after hip endoprosthesis.

Methods: The study was conducted using the national database containing the data about 55,842 hip and knee arthroplasties, including 192 cases of CDI from 2017.

Results: The CDI incidence was 34.4 per 10.000 patients, the median stay in hospital for CDI cases was 7 days, this result is in the third quartile of patients without $\mathrm{CDI} ; 48$ patients, $25 \%$ of all $\mathrm{CDIs}$ had a healthcareassociated infections ( $\mathrm{HAl}$ ) other than CDI. At least one antibiotic in postdischarge period before the $\mathrm{CDI}$ symptoms had prescribed 42 patients, $21.9 \%$, (the most common were beta-lactams (J01C and J01D groups), macrolides (308 prescriptions) and quinolone (101 prescriptions). In the multifactorial review, the following turned out to be significant: age $>65$ years of age, taking antibiotics: J01C and/or J01D (beta-lactams and other beta-lactams) and/or J01M (quinolone), HAls other than CDI, trauma as the cause of surgery, stay in the intensive care unit and length of stay in hospital over 7 days.

Conclusion: $\mathrm{C}$. difficile is still one of the most common cause of hospitalacquired infectious. Infection prevention and control, as well as responsible prescribing of antibiotics is an important factor in reducing the incidence of CDI in post-surgery period.

Disclosure of Interest: None declared.

Poster session: control and epidemiologiy of extended spectrum beta-lactamases (ESBL)

P060

Six-year retrospective review of the prevalence

of extended-spectrum B-lactamase-producing Escherichia coli in patients with identified or suspected infection in a tertiary teaching hospital in China

B. Gao ${ }^{1, *}$, W. LV ${ }^{2}$, W. Sun ${ }^{2}$, W. Su${ }^{3}, C . \mathrm{Ma}^{4}, \mathrm{Q} . \mathrm{Fu}^{5}$

${ }^{1}$ Infectious diseases unit, Tianjin 4th Centre Hospital; Graduate Faculty, Tianjin Medical University, ${ }^{2}$ Postgraduate Institute, Tianjin Medical University, ${ }^{3}$ Clinical Laboratory Unit, ${ }^{4}$ Nosocomial Infection Management Department, ${ }^{5}$ ntensive Care Unit, Tianjin 4th Centre Hospital, Tianjin, China

Correspondence: B. Gao

Antimicrobial Resistance \& Infection Control 10(1): P060

Introduction: Escherichia coli is a major pathogen of hospital- and community-acquired infections and causes a tremendous burden on the healthcare system. Investigating the epidemiology of $E$. coli is essential, but rarely performed to manage antimicrobial resistance at the hospital level in China.

Objectives: To manage $E$. coli infections in a local healthcare setting.

Methods: Demographic, epidemiologic and microbiologic data were retrieved from electronic health records in a tertiary teaching hospital in China between 2015 and 2020 to investigate the incidence and resistance profile of $E$. coli. Antibiotic susceptibility testing was performed according to the Clinical Laboratory Standards Institute.

Results: Among 2630 E. coli isolates included after exclusion of stool specimens, extended-spectrum $\beta$-lactamase-producing $E$. coli (ESBL-PE) was identified in 1287 (48.9\%) isolates, and carbapenem-resistant $E$. coli in 17 (1.0\%). Of 413 E. coli isolated from blood, ESBL-PE accounted for $48.7 \%$ and $0.154 / 1000$ patient-days. The 3 hospital units with the highest incidence were the medical/surgical intensive care unit and surgical (gastrointestinal, hepatobiliary, urological) and medical units $(0.611,0.304$ 
and $0.171 / 1000$ patient-days, respectively), which reached statistical significance when compared with obstetrics/gynecology and other surgical units $(0.030$ and $0.007 / 1000$ patient-days, $P=0.00)$. ESBL-PE incidence was higher in hospital-acquired than in community acquired isolates $(P=0.26)$

Conclusion: A comprehensive visualization of routinely-collected laboratory data allows professionals to conduct antimicrobial stewardship interventions and effectively monitor and respond in a timely manner to antimicrobial resistance at the hospital level.

Disclosure of Interest: None declared.

P061

Regional variability in the interpretation of contact precautions for extended-spectrum beta-lactamase-producing enterobacterales (ESBL-E): a cross-sectional survey

A. van Veen ${ }^{1, *}$, M. van Dijk ${ }^{1}$, I. de Goeij ${ }^{1}$, M. Damen ${ }^{2}$, E. Huijskens ${ }^{3}$, S.

Paltansing ${ }^{4}$, M. van Rijn ${ }^{5}$, R. Bentvelsen ${ }^{6}$, M. Vos ${ }^{1}$, J. Severin ${ }^{1}$ on behalf of Infection Prevention \& Antimicrobial Resistance Care Network

South-Western Netherlands

${ }^{1}$ Department of Medical Microbiology and Infectious Diseases, Erasmus MC University Medical Centre, ${ }^{2}$ Department of Medical Microbiology, Maasstad General Hospital, Rotterdam, ${ }^{3}$ Department of Medical Microbiology, Albert Schweitzer Hospital, Dordrecht, ${ }^{4}$ Department of Medical Microbiology and Infection Prevention, Franciscus Gasthuis \& Vlietland, ${ }^{5}$ Department of Medical Microbiology and Infectious Diseases, Ikazia Hospital, Rotterdam, ${ }^{6}$ Department of Infection Prevention, Zorgsaam Hospital, Terneuzen, Netherlands

Correspondence: A. van Veen

Antimicrobial Resistance \& Infection Control 10(1): P061

Introduction: Guidelines recommend the use of contact precautions(CP) when caring for patients colonized or infected with ESBL-E.

Objectives: The aim was to determine regional variability in the application of contact isolation $(\mathrm{CI})$ measures for patients with ESBL-E and to compare these with the ESCMID guideline.

Methods: This study was performed in 8 hospitals in the South-western region of the Netherlands. A cross-sectional survey was developed, and filled out by a researcher during interviews with infection prevention practitioner(s) (November 2020-April 2021). Cl measures were compared to those stated in the ESCMID guideline.

Results: Seven hospitals(87.5\%) applied $\mathrm{Cl}$ measures for patients with ESBL-E. All 7 hospitals agreed in their policy on the personal protective equipment(PPE) used by healthcare workers( $\mathrm{HCWs}$ ) upon room entrance with anticipated patient contact, providing physiotherapy in corridors/stairwells, requiring CP for visitors and in not changing window curtains after $\mathrm{Cl}($ Table 1). Regional variability was observed in the room type used for $\mathrm{Cl}$, PPE use during physiotherapy outside patient rooms, allowing visitors temporary room leave, and cleaning and disinfection products(Table 1). The ESCMID guideline recommends always using PPE upon room entrance. All 7 hospitals followed the ESCMID guideline for HCWs PPE use upon room entrance with anticipated patient contact, however 5 out of 7 hospitals(71.4\%) did not require HCWs to use PPE without anticipated patient contact. Furthermore, the ESCMID guideline recommends isolating patients in single-patient rooms, while 5 out of 7 hospitals(71.4\%) also allow $\mathrm{Cl}$ in multiple-occupancy rooms.

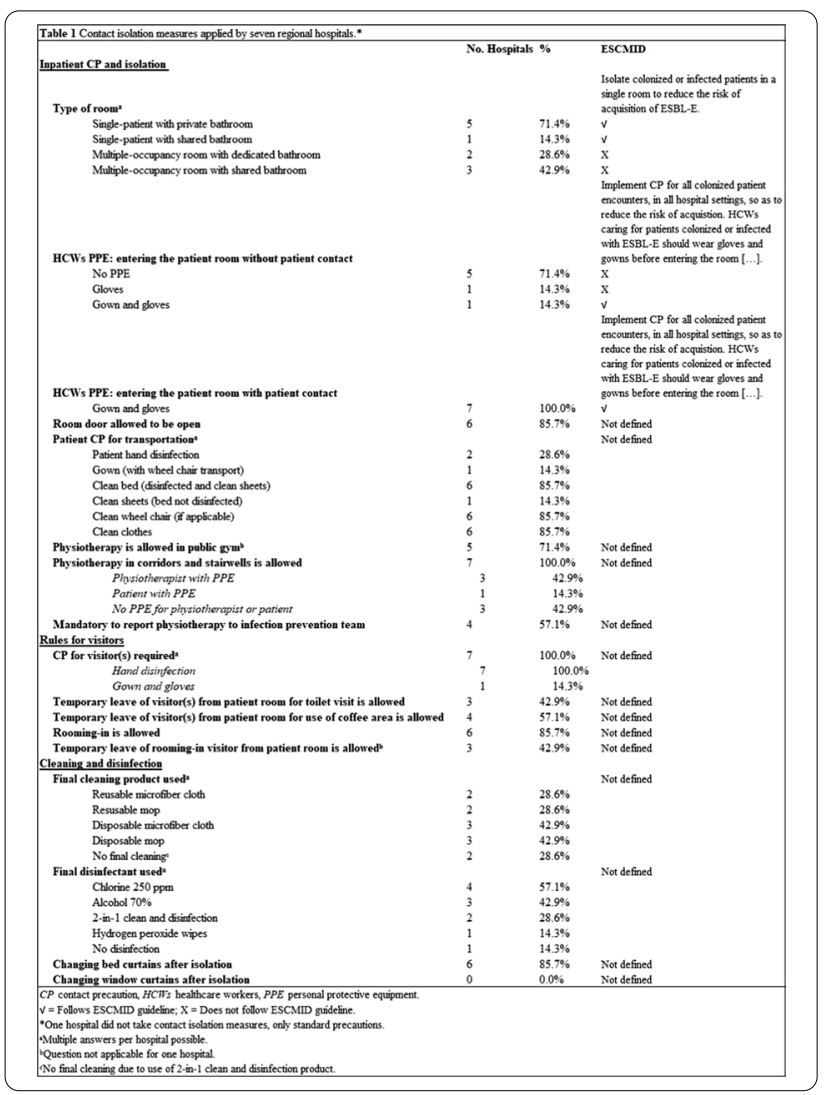

Conclusion: This study showed regional variability in applying $\mathrm{Cl}$ measures for patients with ESBL-E, which can be used to regionally harmonize ESBL-E measures. In the future, the variability in measures can be used to study effects of different elements in a bundle of measures.

Disclosure of Interest: None declared.

P062

Characterization of gram-negative bacteria on ambulance stretchers: a descriptive correlational study

B. C. Santana ${ }^{1, *}$, A. Pereira ${ }^{1}$, P. Oliveira ${ }^{1}$, H. Souza-Junior ${ }^{2}$, E. Watanabe ${ }^{3}$, P. Hermann ${ }^{1}$

${ }^{1}$ University of Brasilia, Ceilândia Sul, ${ }^{2}$ Federal Institute of Goias, Aguas Lindas, ${ }^{3}$ University of Sao Paulo, Ribeirao Preto, Brazil

Correspondence: B. C. Santana

Antimicrobial Resistance \& Infection Control 10(1): P062 
Introduction: Health care-associated infections are a serious problem. The investigation about the role of the ambulance microbiota in healthcare risk is narrow.

Objectives: The objective was to characterize gram-negative bacteria resistant to carbapenem isolated from advanced life support land ambulance stretchers.

Methods: Descriptive correlational study, quantitative, carried out in the Federal District, Brazil, on 6 ambulance stretchers from a public service and 6 from a private service. Performed for three consecutive unannounced days, at the beginning and end of the day shift. The microbiological samples were collected, using Rayon-tipped swabs, moistened in Stuart medium. The bacteria were cultured in Luria Bertani broth (LB) under selection pressure with Vancomycin to exclude gram-positive and subsequent selection of carbapenem-resistant gram-negative by pressure with meropenem in LB broth, according to EUCAST/BrCAST protocol. For the isolation of colonies and the presumptive identification of bacterial species, ESBL chromogenic agar was used, and the final identification of the isolates was done by MALDI-TOF. Antimicrobial susceptibility testing was performed using polisensidisk according to EUCAST/BrCAST. And, by way of PCR, the presence of the $b l a_{K P C}, b l a_{N D M}, b l a_{I M P}$, bla $a_{V I M}$, bla $a_{\text {OXA48, }}$ ESBL CTX-M of groups 1, 2, and 8 genes and of the transposon Tn4401 was searched. Fisher's exact test was used. Statistical significance was defined as $p<0.05$. The study was approved by the Research Ethics Committee.

Results: 25 meropenem-resistant gram-negative bacteria were identified, mainly (36\%) S. maltophlia. Two isolates of A. baumanni stand out, resistant to 5 antibiotics, from 3 different classes. Six bacteria has carried, unusually, bla $a_{C T X-M}$ of group 8 , in S. maltophlia and A. baumanni. Two bla $a_{K P C}$ genotypes in $K$. pneumoniae, associated with the transposon $\mathrm{Tn} 4401$ were found.

Conclusion: The stretchers of ambulances of advanced life support, in this study, were contaminated by meropenem-resistant gram-negative bacteria, most of them S. maltophilia. bla $a_{C T X-M}$ from group 8 and $b l a_{K P C}$ were the only genes identified, with $b l a_{K P C}$ associated with the transposon Tn4401.

Disclosure of Interest: None declared.

\section{P064}

Suppressing klebsiella pneumoniae growth and increasing natural resistance associated macrophage protein 1 MRNA expression after administration of levofloxacin and Miana (coleus scutellarioides (L) benth) leaf extract treatment in BALB/c experimental research

T.D. D. Wahyuni ${ }^{1, *},{ }^{*}$ M. Hatta ${ }^{2}$, A. Bukhari ${ }^{3}$, A. Santoso ${ }^{4}$, M. N. Massi ${ }^{5}$

${ }^{1}$ Internal Medicine, Faculty Medicine Universitas Pelita Harapan, Jakarta,

${ }^{2}$ Molecular Biology and Immunology, ${ }^{3}$ Nutrition, ${ }^{4}$ Pulmonology and Respiration, ${ }^{5}$ Microbiology, Faculty Medicine Universitas Hasanuddin, Makassar, Indonesia

Correspondence: T. D. D. Wahyuni

Antimicrobial Resistance \& Infection Control 10(1): P064

Introduction: The growth of pathogenic bacteria in macrophages is inhibited by Miana (Coleus Scutellariodes [L] Benth).

Objectives: This study aims to determine after giving Miana extract to $B A L B / c$ mice induced by Klebsiella pneumoniae how the expression of mRNA Natural Resistance Associated Macrophage Protein 1 (NRAMP1).

Methods: This experimental research is a post test only control group design on an animal model. Twenty healthy adult male
BALB/c mice were randomly divided into four groups, negative control group (aquades), Levofloxacin $100 \mathrm{mg} / \mathrm{kg}$, intraperitoneal injection, the first treatment group (Miana leaf extract/MLE $510 \mathrm{mg} / \mathrm{kg}$ ) and the second treatment group. (Miana + levofloxacin) for ten consecutive days MLE was administered via gastric gavage. Blood was taken from the first day, the eighth day of the experiment $(2 \mathrm{~h}$ after treatment), and the day 10 . To determine the expression of NRAMP1 mRNA, blood samples were examined by Quantitative Real-Time PCR (qRT-PCR). The growth of Klebsiella pneumonia bacteria in lung tissue was seen using Plate count agar.

Results: After administration of Miana extract for 10 days of treatment, the expression of NRAMP1 mRNA in BALB/c mice increased significantly $(p<0.0001)$. The highest level of mRNA expression was found in the treatment group (Miana + levofloxacin) with an increase before treatment from 8.28 to 13.18 after treatment $p<0.0001$.

Conclusion: In BALB/c mice, NRAMP1 mRNA expression had the highest increase after administration of Miana and Levofloxacin extracts compared to Miana or Levofloxacin alone and was clinically proven to have a comparable effect in suppressing the growth of Klebsiella pneumoniae.

Disclosure of Interest: None declared.

Poster Session: Methicillin-resistant Staphylococcus aureus

P065

Are nursing homes a risk factor of transmission

of methicillin-resistant staphylococcus aureus?

F. F. Battistella (Battistella) $)^{1, *}$

${ }^{1}$ Unité cantonale Hygiène, contrôle et prévention de l'infection, Laus-

anne, Switzerland

Correspondence: F. F. Battistella

Antimicrobial Resistance \& Infection Control 10(1): P065

Introduction: Methicillin-resistant Staphylococcus aureus (MRSA) in Nursing Home $(\mathrm{NHs})$ is directly associated to numerous comorbidities of the residents and to the frequent use of antibiotics. Close contact between residents and non-compliance with Standard Precautions (SP) among healthcare workers might play a crucial role in the spread of multidrug-resistant bacteria.

A study carried out by the Cantonal Unit Hygiene, Prevention and Control of Infection (UHPCi) in 2010-2011 showed that adherence with SP is sufficient to contain the spread of MRSA in NHs and hence new recommendations were issued. Additionally, a surveillance of MRSA was initiated in acute care settings on resident admission to hospital.

In 2019, we observed an increase in the number of new MRSA colonization diagnosis of residents on admission to acute care settings.

Objectives: The objective was to collect and analyze prospectively all news cases of MRSA coming from $\mathrm{NH}$.

Methods: On admission of the resident to hospital, a systematic MRSA screening (nose, throat, inguinal and clinical samples) was performed. In microbiology laboratory, positive MRSA result generated an automatic alert which was sent to the UHPCi. The PCI nurse collected data on risk factors ( $\mathrm{NH}$ of the resident, age, sex, known infections, previous hospitalizations, comorbidities).

Results: A total of 35 residents were screened as newly colonized by MRSA for the time period of $2019-2021$. We collected 23, 7 and 5 cases in 2019, 2020 and early 2021, respectively. Among them, $6 \%$ (2/35) were found to be infected with MRSA on their admission.

The documented risk factors were 1 dialysis, 5 urinary catheters, 5 chronic wounds and 14 with more than two hospitalizations within 
one year. Residents came from different NHs: $11 \mathrm{NHs}$ recorded one resident, $5 \mathrm{NHs} 2$ residents and $3 \mathrm{NHS} 3,4$ and 6 residents, respectively. Conclusion: The limited number of newly diagnosed resident does not confirm if living in a NH is a significant risk factor for MRSA acquisition. Therefore, continuous surveillance of MRSA on admission in acute settings for all resident coming from $\mathrm{NH}$ is needed in order to monitor a potential increase of this pathogen.

Disclosure of Interest: None declared.

\section{P066}

Comparative analysis of methicillin-resistant staphylococcus aureus isolates of clinical and food origin

Y. Mikhaylova 1,* , A. Shelenkov' ${ }^{1}$ A. Egorova ${ }^{1}$, A. Chernyshkov ${ }^{1}$, L. Petrova ${ }^{2}$ M. Zamyatin ${ }^{2}$, V. Akimkin ${ }^{1}$

${ }^{1}$ Central Research Inctitute of Epidemiology, ${ }^{2}$ Pirogov National Medical and Surgical Center, Moscow, Russian Federation

Correspondence: Y. Mikhaylova

Antimicrobial Resistance \& Infection Control 10(1): P066

Introduction: S. aureus is commensal and pathogenic bacteria which can survive in diverse environments and grow in many types of foods. Several surveys have documented the presence of MRSA in foods, but its status as a food-borne pathogen is still unclear.

Objectives: The aim of this work is to perform whole genome sequencing (WGS)-based genomic epidemiology analysis of MRSA isolates of clinical and food origin.

Methods: WGS was performed on Illumina HiSeq platform for 4 MRSA isolates collected from Moscow medical center and 6 MRSA samples isolated from ready-to-eat food. The susceptibility was determined by the boundary concentration method on VITEK2Compact30 analyzer. Genome assemblies and genome comparisons were performed using SPAdes, roary, dnadiff and custom software.

Results: The $S$. aureus isolates studied were characterized by similar profile of antimicrobial susceptibility. They belonged to ST22 and 4 Spa-variants regardless of their origin. All MRSA isolates analyzed had $\mathrm{SCCmec}$ IV type. Another common determinant of antibiotic resistance was penicillin-resistance gene blaZ. All MRSA isolates of food origin carried additional gene of resistance to lincosamide, and two of them also included resistance genes to phenicols and macrolides. The set of virulence factors in S. aureus isolates studied was similar and quite extensive (up to 15 genes), and consisted of both structural components of the cell and secreted products including enterotoxins. Pairwise comparisons of core-genome composition and the number of single nucleotide polymorphisms of the isolates having different origin revealed very close relationship between one food and one clinical isolate.

Conclusion: WGS-based analysis enabled us to reveal unique genome features of MRSA isolates of clinical and food origin. The data obtained will allow us to identify new target molecules for differentiation of MRSA isolates belonging to different genetic variants. We also provide microbiological criteria for MRSA in ready to eat foods, in particular, as an important option for controlling the dissemination of antimicrobial resistance and potential Staphylococcus enterotoxins' production.

Disclosure of Interest: None declared.

\section{P067}

Impact of hand hygiene compliance on meticillin-resistant staphylococcus aureus proportion in healthcare setting over five years period: a retrospective study in Benin

P. F. D. Fonton ${ }^{1, *}$, A. T. Ahoyo ${ }^{1}$, B. Boya ${ }^{2}$

${ }^{1}$ Ecole Polytechnique d'Abomey-calavi, ${ }^{2}$ Laboratoire de Biologie et de Typage Moléculaire en Microbiologie, University of Abomey-calavi,

Cotonou, Benin

Correspondence: P. F. D. Fonton

Antimicrobial Resistance \& Infection Control 10(1): P067
Introduction: Antimicrobial resistance is a global crisis that requires urgent attention and action. We report data on meticillin-resistant Staphylococcus aureus and hand hygiene compliance rate in Benin over five years period.

Objectives: Assess meticillin-resistant Staphylococcus aureus proportion towards hand hygiene compliance in Benin's healthcare settings from 2014 to 2018.

Methods: This was a retrospective study implemented in Benin hospitals between 2014 and 2018. We collected data on meticillin-resistant Staphylococcus aureus proportion and hand hygiene compliance rate. Beta regression was used to conduct statistical analysis. A p-value of $5 \%$ was considered statistically significant.

Results: Hand hygiene compliance rate was 13\%,15\%,26\%,17.5\% and 20\% in 2014, 2015, 2016, 2017 and 2018 respectively, $\mathrm{p}=0.001$. Meticillin-resistant Staphylococcus aureus proportion was $68 \%$, 64\%, 54\% 50\% and 48\% in 2014, 2015, 2016, 2017 and 2018 respectively, $p=0.0001$. From 2014 to 2018 , hand hygiene compliance rate increased while meticillin-resistant Staphylococcus aureus proportion decreased.

Discussion: Hand hygiene compliance increased from a baseline $13 \%$ to $20 \%, p=0.001$. Between pre- and postintervention hand hygiene adherence improved by $29 \%(p<0.001)$ but did not change over the 5 -year assessment (38.3\%; $p=0.47)$ [1]. When hand hygiene compliance increases meticillin-resistant Staphylococcus aureus proportion decreases. Hand hygiene was associated with a decrease in healthcare associated meticillin-resistant Staphylococcus aureus infections in a large health care system [2].

Conclusion: From 2014 to 2018 hand hygiene compliance increased significantly. This suggests the implementation of hand hygiene program in order to control spread of Meticillin-resistant Staphylococcus aureus.

Keywords: Hand Hygiene - Meticillin-resistant Staphylococcus aureus - Five years - Benin.

\section{References}

1. T. Sakihama et al., 2020. Assessing sustainability of hand hygiene adherence 5 years after a contest-based intervention in 3 Japanese hospitals. American Journal of Infection Control.

2. R. Jain et al., 2011. Veterans Affairs Initiative to Prevent Methicillin Resistant Staphylococcus aureus Infections. The new england journal medecine.

Disclosure of Interest: None declared.

\section{P068}

Results of an infection and microbiological surveillance of panton-valentine leucocidin (PVL)-forming S. aureus strains M. M. Neuwirth ${ }^{1, *}$, A. F. Wendel ${ }^{1}$, M. Malecki ${ }^{1}$, F. Mattner ${ }^{1}$

${ }^{1}$ Division of Hygiene and Environmental Medicine, Witten/Herdecke University, Cologne, Germany

Correspondence: M. M. Neuwirth

Antimicrobial Resistance \& Infection Control 10(1): P068

Introduction: The toxin PVL can be produced by both methicillinresistant (MRSA) and methicillin-susceptible (MSSA) Staphylococcus aureus strains. Due to the lysing effect of PVL on leukocytes, severe infections can be caused by the producing bacteria. A microbiological and infection surveillance of PVL-producing S. aureus was performed in three Cologne hospitals between 2014 and 2020 and triggered by the detection of $S$. aureus in combination with recurrent skin infections, severe pneumonia or possible outbreaks.

Objectives: Aim of the study was to investigate if several strains are endemic in the population and if there is an epidemic situation with one strain or a mixture of several strains. The study aimed to investigate if multiple strains are endemic in the population and if there is an epidemic situation with one strain or a mixture of multiple strains. Methods: In case of skin infection or pneumonia and detection of community-acquired S. aureus, bacterial isolates were tested by PCR for the production of PVL by amplifying the encoding gene lukS and included in the surveillance if positive. 46 from 55 bacterial isolates 
were available for further next generation sequencing. Assembled genomes were analysed (core genome MLST by SeqSphere +, Ridom; ResFinder analysis).

Results: A total of 55 patients $\left(300^{*} ; 25 \%\right)$ aged $0-81$ years $(M=15.4 \pm 16.9 ; M D=10)$, with an $S$. aureus strain with lukS gene were recorded (34 patients with PVL-MSSA; 21 with PVL-MRSA). In 10 patients, rehospitalization was necessary due to recurrent infections caused by PVL-positive S. aureus strains. After positive lukS gene detection, decolonization was performed in 9 individuals, no decolonization was performed in 39, and no information was available for 7 individuals. Infections occurred most frequently on the buttocks $(\mathrm{N}=10)$ and thighs $(\mathrm{N}=8)$. A high clonal diversity was detected comprising 16 cgMLST types of PVL-positv S. aureus. 5 small clusters were identified, however transmissions were confirmed in only one cluster (tempo-spatial overlap).

Conclusion: Epidemiologically, an endemic situation with PVL-positv S. aureus and few transmissions were identified in this study. Active surveillance is nevertheless necessary to identify a cluster of PVL-positive cases and possibly an outbreak and to guide therapy and infection control measures.

Disclosure of Interest: None declared.

Poster Session: Surgical site infections

\section{P069}

Experimental study of the pharmacological activity of the combined use of decamethoxin and bupivacaine at local injection into the wound Y. Babina ${ }^{1, *}$, O. Nazarchuk' ${ }^{2}$ D. Dmytriiev ${ }^{1}$

${ }^{1}$ Department of anesthesiology and intensive care, ${ }^{2}$ microbiology, National Pirogov Memorial Medical University, Vinnytsia, Ukraine Correspondence: Y. Babina

Antimicrobial Resistance \& Infection Control 10(1): P069

Introduction: High risk of infections in surgery is associated with microbial contamination by conditionally pathogenic microorganisms of Staphylococcus aureus.

Objectives: Investigation of the efficacy of combined administration of antiseptic and local anesthetic in the area of postoperative infectious wounds.

Methods: Fifty-one male rats weighing $250-300 \mathrm{~g}$ were randomly divided into four groups. Surgical procedures were performed under general anesthesia (intraperitoneal injection of ketamine $75 \mathrm{mg} / \mathrm{kg}^{-1}$ ). The hair on the back of the animal was shaved after loss of corneal reflex and reaction to the retraction of the limbs. The wounds were infected with an additional culture of clinical strain S. aureus 47 (dose $108 \mathrm{CFU} / \mathrm{ml}$ ). The incision was cleaned and two minutes later wiped dry. The incision sites were infiltrated subcutaneously with doses of $3 \mathrm{ml}$ of the study drug: normal saline in the control group (group A, $n=13$ ), antiseptic decamethoxine (gr. $B, n=13), 0.5 \%$ bupivacaine $y$ (gr.C, $n=13$ ) and combinations thereof in a ratio of $1: 1$ (gr.D, $n=12$ ). The effectiveness of antiseptic treatment in the wound area was evaluated using von Frey filaments. Statistical processing was performed using standard biometric methods. Differences at $p<0.05$ were considered significant.

Results: On the third day of the experiment the number of $S$. aureus on the surface of the wounds was found to decrease significantly when decamethoxin and its combination with bupivacaine $0.5 \%$ were used. The use of bupivacaine $0,5 \%$ was accompanied by the moderate microbial invasion in the wound area on the 3rd day. The use of the antiseptic and its combination with bupivacaine on the 10th day revealed almost complete eradication of $S$. aureus on the wound surface, in compared the control group $(p<0,01)$. In the combination antiseptic with bupivacaine the pain sensitivity threshold increased by $48.6 \%$, which was practically the same as in bupivacaine monotherapy (48.9\%).

Conclusion: The combination of antiseptic with bupivacaine $0.5 \%$ in the treatment of wounds is accompanied by an equally high antimicrobial efficacy with a marked anesthetic effect, which opens the prospect of their combined topical application in the treatment of wounds. Disclosure of Interest: None declared.
P070

Reluctance for implementing surgical skin preparation

guidelines: infection control practitioners' opinion

D. Verjat-Trannoy 1,*, C. Daniel ${ }^{1}$, J. Sambourg ${ }^{1}$, K. Lebascle' ${ }^{1}$, P. Astagneau ${ }^{1}$

${ }^{1}$ CPIAS ILE-DE-FRANCE, PARIS, France

Correspondence: D. Verjat-Trannoy

Antimicrobial Resistance \& Infection Control 10(1): P070

Introduction: In the context of a new national surgical site infection (SSI) control program, a study was implemented to evaluate what infection control practitioners (ICP) think about pre-operative skin preparation (PSP) recommendations, updated in 2013 and 2016.

Objectives: The aim of the study was to quantify the integration of the infection control (IC) measures in the local protocols and the potential obstacles for their implementation by surgical teams.

Methods: In 2020, a national on-line questionnaire was addressed to all ICP working in a healthcare facility (HCF) with surgical activity. Data included the ICP opinion on 8 measures of PSP (cf. table). A descriptive statistical analysis of closed responses $(\mathrm{Y} / \mathrm{N})$ was completed by a verbal analysis over the ICP comments.

Results: A total of 485 HCF participated to the study (public: $47 \% /$ private: $53 \%$; representative of the distribution of HCF with surgery). Two first obstacles were identified:1-the unfavorable opinion of ICP about the guidelines 2-the impossible agreement between ICP and surgical teams or in between surgical teams.

\begin{tabular}{|c|c|c|c|}
\hline \multicolumn{4}{|c|}{ Table: Proportion of the protocols integrating the PSP and potential associated obstacles } \\
\hline $\begin{array}{l}\text { Type of IC } \\
\text { measures }\end{array}$ & IC Measures for PSP & $\begin{array}{l}\text { Measures } \\
\text { integrated in the } \\
\text { HCF protocol } \\
\text { (\% of HCF) }\end{array}$ & $\begin{array}{l}\text { Main obstacle } \\
\text { identified for non- } \\
\text { integration of the } \\
\text { PSP measures (\% of } \\
\text { HCF) }\end{array}$ \\
\hline \multirow{3}{*}{$\begin{array}{l}\text { Measures } \\
\text { modified to } \\
\text { improve } \\
\text { infection } \\
\text { control } \\
\text { (priority) }\end{array}$} & $\begin{array}{l}\text { Use of an alcoholic antiseptic } \\
\text { product }\end{array}$ & $94 \%$ & None \\
\hline & No systematic hair removal & $85 \%$ & $\begin{array}{l}\begin{array}{l}\text { Force } \\
(55 \%)\end{array} \text { of habits } \\
\end{array}$ \\
\hline & $\begin{array}{l}\text { No systematic use of } \\
\text { impregnated adhesive drapes }\end{array}$ & $35 \%$ & Force of habits (nc) \\
\hline \multirow{5}{*}{$\begin{array}{l}\text { Minimum } \\
\text { measures to } \\
\text { guaranty } \\
\text { infection } \\
\text { control } \\
\text { without } \\
\text { spending too } \\
\text { much money } \\
\text { or time }\end{array}$} & $\begin{array}{l}\text { Non-antiseptic soap at shower } \\
\text { time }\end{array}$ & $62 \%$ & \begin{tabular}{|ll} 
Lack & of \\
medicalization & of \\
care $(33 \%)$ & \\
\end{tabular} \\
\hline & Single shower & $54 \%$ & 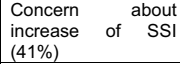 \\
\hline & $\begin{array}{l}\text { Shampooing limited to head } \\
\text { and neck surgery }\end{array}$ & $48 \%$ & \begin{tabular}{|ll} 
Preference for \\
harmonized \\
measure (36\%)
\end{tabular} \\
\hline & $\begin{array}{l}\text { Skin cleaning limited to visible } \\
\text { soiling }\end{array}$ & $35 \%$ & \begin{tabular}{|lrr}
$\begin{array}{l}\text { Concern } \\
\text { increase } \\
(32 \%)\end{array}$ of & SSI \\
\end{tabular} \\
\hline & $\begin{array}{l}\text { Non-antiseptic soap for skin } \\
\text { cleaning }\end{array}$ & $23 \%$ & \begin{tabular}{|lll}
$\begin{array}{l}\text { Force } \\
(36 \%)\end{array}$ & of habits \\
\end{tabular} \\
\hline
\end{tabular}

Conclusion: Our results showed a good integration of priority measures in healthcare protocols, except for impregnated adhesive drapes. As obstacles to implementation varied according to PSP measures, training and communication efforts should focus first on key measures where ICP and surgical teams are the most reluctant.

Disclosure of Interest: None declared.

\section{P071}

The effect of statin medication on surgical site infections or other complications needing revisions after orthopedic surgery

I. Uçkay ${ }^{1, *}$, I. Unterfrauner ${ }^{1}$, M. Olthof ${ }^{1}$, P. Jans ${ }^{1}$

${ }^{1}$ Balgrist University Hospital, Zürich, Switzerland

Correspondence: I. Uçkay

Antimicrobial Resistance \& Infection Control 10(1): P071

Introduction: Statins have anti-inflammatory properties. In several surgical discipülines, statin mediation seems to be preventive of various postoperative complications.

Objectives: The intension was to investigate if a long-term perioperative statin medication, primarily given for cardiovascular disorders, might affect rates of surgical site infections (SSI), or other late noninfectious complications after orthopedic surgeries.

Methods: A single-center cohort of 20,088 patients (median age 53 years, 49\% females, 5\% diabetes) who underwent orthopedic 
surgery during 2014-2019 was investigated to document statin use ( $n=2,486,12 \%, 222$ different brands and doses) and peri- and postoperative complications including SSI. Cox regression analyses, with and without propensity-score matching (nearest neighbor approach) was used to find potential associations.

Results: After a median follow-up of 7 months, 1,414 patients (7\%) of the 20,088 patients needed a surgical revision: $158(0.8 \%)$ due to deep SSI and $1,256(6.3 \%)$ for non-infectious reasons. In multivariate Cox regression analyses, statin use was unrelated to both SSI (hazard ratio (HR) 0.9; 95\% confidence interval (Cl) 0.6-1.4) and non-infectious complications (HR 1.1, 95\% Cl 0.9-1.3). Further, statin use did not increase the risk for $\mathrm{SSI}$ in the subgroup of implant-related surgery $(\mathrm{HR} 0.8,95 \% \mathrm{Cl} 0.4-1.6)$ or arthroplasties (HR 0.8, 95\% $0.3-2.6)$. Propensity-score matched analyses on the variable "statin" equally failed to alter these outcomes.

Conclusion: In our large cohort study with over 20,000 orthopedic interventions, we found no protective effect of a statin medication on deep SSI risks; or on other late non-infectious complications requiring revision surgery.

Disclosure of Interest: None declared.

\section{P072}

Deep surgical site infections following double-dose perioperative antibiotic prophylaxis in adult obese orthopedic patients-a before-after clinical cohort study

I. Uçkay ${ }^{1, *}$, A. Hasler ${ }^{1}$, I. Unterfrauner ${ }^{1}$, M. Olthof ${ }^{1}$, P. Jans ${ }^{1}$

${ }^{1}$ Balgrist University Hospital, Zürich, Switzerland

Correspondence: I. Uçkay

Antimicrobial Resistance \& Infection Control 10(1): P072

Introduction: Obesity is a risk for surgical site infections (SSI). Based on retrospective comparisons and pharmacology, many centers adopted a weight- (or a body mass index (BMI))-related antibiotic prophylaxis to prevent SSIs.

Objectives: This before-and-after study examined whether doubling of the antibiotic prophylaxis can prevent surgical site infections.

Methods: We introduced a hospital-wide double-dose prophylaxis on $3 / 2017$ for patients $>80 \mathrm{~kg}$. In our all-orthopedic cohort, we compared the period 4/2014-3/2017 ("before") to 3/2017-6/2019 ("after") regarding the clinical impact of this dose-doubling on the SSIs.

Results: We compared 9,318 surgeries "before" to 7,455 interventions "after". In both periods, baseline demographics (age, sex, body mass index (BMI), American Society of Anesthesiologists (ASA)-Score, revision surgeries, and duration of surgery) were similar. In the period "after", 3,069 episodes $(3,069 / 16,773 ; 18 \%)$, received a double-dose prophylaxis. Overall, we witnessed 82 deep SSIs $(0.5 \%)$. The pathogens were resistant to the standard cefuroxime prophylaxis during the index surgery in 35 cases $(35 / 82 ; 43 \%)$. By excluding these prophylaxis-resistant cases and all of the five hematogenous SSIs, we remained with 47 SSIs ( $57 \%$ ) that could have been prevented by prophylaxis. The doubledosing of parenteral cefuroxime from $1.5 \mathrm{~g}$ to $3.0 \mathrm{~g}$ in obese patients did not reduce deep SSIs (hazard ratio (HR) $0.7,95 \%$ confidence interval $(\mathrm{Cl})$ 0.3-1.6. In the direct group comparison among obese patients $>80 \mathrm{~kg}$, the administered double-dose prophylaxis equally failed to alter outcome $(3,088 / 16,726$ non-infections vs. $8 / 47$ deep SSI despite double-dose prophylaxis; Pearson-chi ${ }^{2}$-test; $p=0.78$ ).

Conclusion: In our single-center before-and-after-study with almost 17,000 orthopedic surgeries in adult patients, the systemic doubling of the perioperative antibiotic prophylaxis in obese patients clinically failed to reduce the overall deep SSI risks.

Disclosure of Interest: None declared.

\section{P073}

Surgical site infections at donor and recipient sites in patients with iliac crest harvesting for autologous bone grafting-a pilot evaluation

I. Uçkay 1,**

${ }^{1}$ Balgrist University Hospital, Zürich, Switzerland

Correspondence: I. Uçkay

Antimicrobial Resistance \& Infection Control 10(1): P073
Introduction: Surgeons harvest the iliac crest for bone grafting. The epidemiology of surgical site infections (SSI) associated with this procedure at the donor, or recipient site, is unknown.

Objectives: We perform a retrospective pilot evaluation of adult patients undergoing first-time orthopedic surgery at the Balgrist University Hospital between 2014-2019.

Methods: Single-center retrospective cohort study 2014-2019. We excluded patients with infection at the index surgery, diabetic foot surgeries, superficial SSIs, and revision surgeries.

Results: We included 20,088 episodes of primary orthopedic surgery, of which 467 with iliac crest bone sampling (467/20,088; $2 \%)$. Only two iliac sites $(2 / 467 ; 0.4 \%)$ become infected. In contrast, surgeries with iliac crest sampling yielded more SSIs at the recipient site than those without $\left(1.9 \%\right.$ vs. $0.8 \% ; x^{2}$-test; $\left.p<0.01\right)$. These patients equally revealed more co-morbidities such as a longer duration of surgery (median 127 vs. $79 \mathrm{~min}$ ), when compared to the general orthopedic population. In multivariate logistic regression analysis with the outcome "SSI at the recipient site", the iliac harvesting was independently associated with deep SSIs requiring surgical revision (odds ratio 2.1; 95\% confidence interval 1.1-4.2).

Conclusion: In our pilot evaluation with 20,088 primary orthopedic surgeries, the SSI risk of the iliac harvest site was low. In contrast, surgeries with supplementary iliac crest harvesting revealed a higher SSI risk than the general orthopedic population, potentially due to a mix of local independent risks of grafting together with a prolonged surgery time.

Disclosure of Interest: None declared.

\section{P074}

Meningitis after neurosurgical procedures: results of a 10-year surveillance in a Russian neurosurgical ICU

K. Ershova 1,*, O. Ershova², I. Savin², G. Danilov², M. Shifrin², I. Alexandrova², N. Kurdumova ${ }^{2}$

${ }^{1}$ University of Washington, Seattle, United States, ${ }^{2}$ Burdenko National Medical Research Center for Neurosurgery, Moscow, Russian Federation Correspondence: K. Ershova

Antimicrobial Resistance \& Infection Control 10(1): P074

Introduction: Healthcare-associated meningitis after neurosurgical procedures is associated with poor patient outcomes, increased healthcare costs, and is difficult to diagnose due to subtle CSF changes and variable clinical presentation.

Objectives: The goal of this study was to examine the incidence and outcomes of meningitis based on infection surveillance data from a neurosurgical ICU.

Methods: The study was conducted in the neurosurgical ICU at Burdenko Center for Neurosurgery in Moscow from 2011 to 2020. The data was collected prospectively as a part of infection surveillance protocol; all patients with an ICU length of stay $>48 \mathrm{~h}$ were included. Diagnostic criteria for meningitis included status post neurosurgical procedure, CSF glucose $<50 \%$ of blood glucose, increased CSF cell count, positive CSF cultures, and clinical symptoms. The meningitis prevention protocol was introduced in 2011 and included perioperative antibacterial prophylaxis, cross infection control, improved hand hygiene (average 10-year compliance $85 \%$ ), and oxygen-containing antiseptics; closed EVD systems were introduced in 2016.

Results: A total of 4021 patients were included and 359 were diagnosed with meningitis resulting in an incidence of $8.9 \%(95 \% \mathrm{Cl}$ 8.45-9.38). The incidence of meningitis decreased from $15.6 \%$ to $6.5 \%$ during the study period (Figure 1). The mortality rate in patients with meningitis was $23.4 \%(95 \% \mathrm{Cl}: 22.2-243.6)$ that did not change over time. The etiological spectrum was dominated by $\mathrm{CoN}$ Staphilococcus $(30 \%)$ followed by Acinetobacter baumannii $(23 \%)$ and Klebsiella pneumoniae (23\%). The mortality rate was higher in patients infected with Acinetobacter baumannii. The data from other neurosurgical ICUs in Russia is neither available for comparison nor routinely reported to Russia's health surveillance agency. 


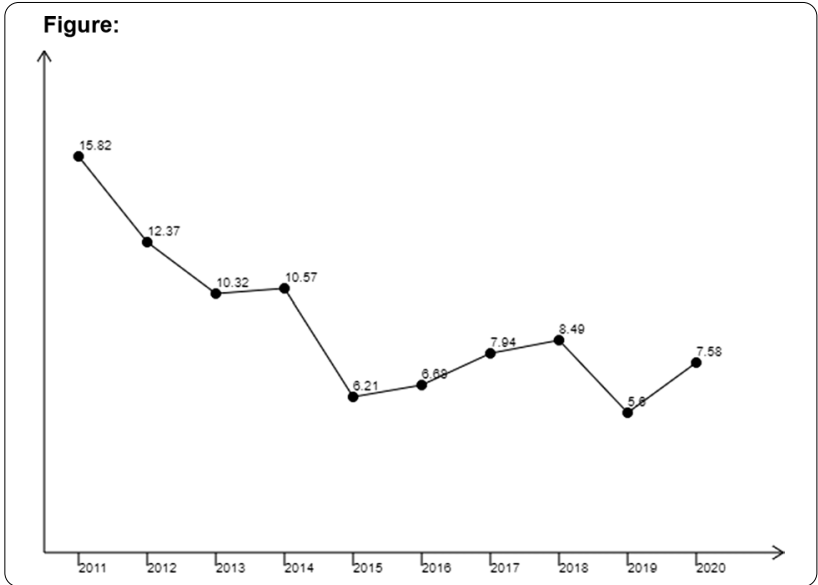

Conclusion: We reported the incidence and mortality rates of healthcare-associated meningitis after neurosurgical procedures in a neurosurgical ICU in Russia. Unique for data gathered from Russia, these results are supported with samples based on a 10-year data collection project, providing high reliability of findings.

Disclosure of Interest: None declared.

\section{P075}

Surveillance of surgical site infections in surgical departments

2017

G. Brahimi ${ }^{1, *}$, S. Ait seddik ${ }^{1}$, A. LARINOUNA ${ }^{1}$, A. Rebouh ${ }^{1}$, N. Cheboub $^{1}$, H. Khellaf', A. Chetitah', A. Boudebouz', W. Djouimaa', A. El kechai , R. Belkaid

${ }^{1}$ CHU BENI MESSOUS, ÉPIDÉMIOLOGIE, ALGER, Algeria

Correspondence: G. Brahimi

Antimicrobial Resistance \& Infection Control 10(1): P075

Introduction: Surveillance of surgical site infections (SSI) is a priority in our hospital. The SSI incidence rate has significantly decreased since the establishment of the active surveillance network in 2006 in certain surgical departments, particularly in ophthalmology.

Objectives: Main objective: Measure the incidence rate of stratified SSIs on the level of risk. Specific objectives: Determine the characteristics of ISOs-Identify the risk factors linked to the occurrence of SSIs. Methods: This is a descriptive longitudinal study for analytical purposes. Data collection was carried out between February 05th and April 10th, 2017 with follow-up until day 30, including all therapeutic interventions in five departments of our CHU: General Surgery, Obstetrics Gynecology, Ophthalmology, ENT and Childhood Surgery. The SSI diagnosis was made according to the criteria of the Atlanta CDC. Data entry and analysis were carried out using the Epi-info 6 software.

Results: A total of 828 patients were included and 778 patients were seen again on day 30 , a postoperative follow-up rate of $93.96 \%$. The average age of operated patients is 53.8 years \pm 31.3 . The average length of hospitalization is 5.3 days \pm 7.0 . The proportion of patients with ASA 1 score was $66.42 \%$ (550/828). The NNIS index could not be calculated in the infantile surgery department due to the lack of traceability of the duration of the intervention, the evaluation of the antibiotic prophylaxis could not be carried out for lack of traceability in almost all of the patient records. Of the 778 patients followed on day 30,39 developed a SSI, an incidence rate of $5.01 \%$. The highest rate was observed in the General surgery department with $16.80 \%$ followed by the Obstetric Gynecology department $6.56 \%$ and the lowest rate of $0.47 \%$ in ophthalmology $(p<0.001)$. The incidence rate of SSI is $8.01 \%$ for the ASA score $\geq 2$ versus $3.48 \%$ for the ASA score $<2$ $(p<0.01)$, as well as for the Altemeier class $\geq 2(10.74 \%$ versus $1.46 \%$, $p<0.001$ ). Stratified on the NNIS index, the incidence rate is $7.62 \%$ for $\mathrm{NNIS} \geq 1$ versus $5.04 \%$ for NNIS $<1$ (DNS) interventions.
Conclusion: Unlike previous years when SSIs occurred in patients at low risk of infection, this year we were able to highlight the usual risk factors. However, efforts must be continued with regard to the traceability of the duration of the intervention and of the antibiotic prophylaxis for better use of the data and risk analysis.

Disclosure of Interest: None declared.

P076

Surveillance of surgical site infections in five surgical

departments 2019

G. Brahimi ${ }^{1}{ }^{1,}$, A. Chetitah' ${ }^{1}$, N. Cheboub ${ }^{1}$, A. El Kechai ${ }^{1}$, A. Boudebouz ${ }^{1}$, S.

Slaouti ${ }^{1}$, K. Chabane ${ }^{1}$, M. Charchari ${ }^{1}$, A. Dahli ${ }^{1}$, S. Ait seddik ${ }^{1}$, A. Larinouna

1 , A. Rebouh ${ }^{1}$, R. Belkaid ${ }^{1}$

${ }^{1} \mathrm{CHU}$ BENI MESSOUS, ÉPIDÉMIOLOGIE, ALGER, Algeria

Correspondence: $\mathrm{G}$. Brahimi

Antimicrobial Resistance \& Infection Control 10(1): P076

Introduction: The incidence rate of surgical site infections (SSI) has significantly decreased in our establishment since the establishment of the active surveillance network in 2006.

Objectives: - Calculate the incidence rate of SSIs.

- Identify the risk factors related to the occurrence of SSI.

Methods: Longitudinal descriptive study for analytical purposes. Data collection was carried out between February 1st and May 30th, 2019 with follow-up until day 30 in five departments: General Surgery, Obstetrics Gynecology, Ophthalmology, ENT and Childhood Surgery. The SSI diagnosis was made according to the criteria of the Atlanta CDC. Data entry and analysis were done using the Epi-info6 software.

Results: A total of 797 interventions were included. 94.10\% (750/797) patients were seen again in consultation on day 30 . The average length of hospitalization is $5.3 \pm 6.5$. $62.90 \%(502 / 797)$ had an ASA score of 1 . The NNIS index could not be calculated for pediatric surgery and ophthalmology due to lack of traceability of the duration of the operation. $26 \%$ of the interventions were carried out urgently. The incidence rate of SSI is $5.70 \%(43 / 750)$, it was significantly higher in gynecology-obstetrics $13.04 \%(21 / 161)$ and in general surgery $12.50 \%(16 / 128)$, no case has been registered in ophthalmology. There are four risk factors, in addition to age which is an endogenous factor $(40.4+-15.9$ years versus $33.8 \pm 18.2 p<0.04)$, it is the average total length of stay $(p<0.01), A S A$ score $\geq 2,(p<0.001)$, Altemeier class $\geq 2$ $(p<0.001)$ and NNIS score $>1(p<0.001)$.

Conclusion: SSI monitoring has highlighted risk factors that must be taken into account in order to improve treatment. We will first aim to reduce the average length of stay, especially in patients with multiple diseases.

Disclosure of Interest: None declared.

P077

The impact of a COVID-19 total lockdown on deep surgical site infections and other non-infectious complications after orthopedic surgery

I. Uçkay ${ }^{1, *}$, I. Unterfrauner ${ }^{2}$, L. Hruby ${ }^{2}$, P. Jans ${ }^{2}$

${ }^{1} \mathrm{Dr}^{2}{ }^{2}$ Balgrist University Hospital, Zürich, Switzerland

Correspondence: I. Uçkay

Antimicrobial Resistance \& Infection Control 10(1): P077

Introduction: The Covid-19 pandemic entailed a lockdown with total restriction of elective orthopedic surgeries. While the access to the hospital and human contacts were limited, hygiene rules were intensified.

Objectives: We investigate the impact of those strict hygiene measures on the risk of deep surgical site infections (SSI), wound healing disorders and other complications after orthopedic surgery, before, during, and after the first Covid-19 lockdown.

Methods: In a single-center study, patients during the first Covid-19 lockdown from March 21, 2020 to April 26, 2020 were compared to a cohort that underwent orthopedic intervention in the pre- and postlockdown phase, respectively from October 1, 2019 to October 31, 2020. Adjusted multivariate analyses were used to investigate the 
occurrence of surgical site infections, wound healing disorders and other complications in three study periods.

Results: We included 5,791 patients in this study. After a mean followup of 7 months, the lockdown cohort showed a significant higher SSI rate compared to the pre- and post-lockdown period ( $2 \%$ versus $1 \%$ and $0.5 \%$ ). The revision rate due to other complications was higher in the pre-lockdown cohort compared to the others (5\% versus $3 \%$ ), whereas there was no difference for wound healing disorders between all cohorts. In multivariate Cox regression analyses, the lockdown phase was unrelated to all SSI (hazard ratio (HR) 1.6; $95 \%$ confidence interval (CI) 0.6-4.8), wound healing disorders (HR 0.7; 95\% Cl 0.1-5.7) and other complications (HR $0.7,95 \% \mathrm{Cl} 0.3-1.5$ ).

Conclusion: The incidences for deep SSI, wound disorders and other complications in orthopedic surgery were not influenced by the promotion of hygiene measures during lockdown.

Disclosure of Interest: None declared.

\section{P078}

Transferability of an infection prevention concept for the reduction of nosocomial infections especially surgical site infections

M. M. Neuwirth ${ }^{1, *}$, S. Maczewski ${ }^{1}$, R. Otchwemah ${ }^{1}$, F. Mattner ${ }^{1}$

${ }^{1}$ Institute for Hygiene, Cologne Merheim Medical Centre, University

Hospital Witten/Herdecke Cologne, Cologne, Germany

Correspondence: M. M. Neuwirth

Antimicrobial Resistance \& Infection Control 10(1): P078

Introduction: The "HygArzt" project investigated the effectiveness, efficiency of infection prevention measures (IPM) implemented by an infection prevention link physician (PLP) in the trauma surgery/ orthopaedics department. In the first phase of the project, the IPM implemented in a pilot hospital led to increases in adherence for all indications of hand hygiene and dressing change as well as to a reduction in the rate of nosocomial infections (NI), especially postoperative wound infections (SSI).

Objectives: In a second phase, based on the process analyses and the results of the pilot hospital, a modular training program for PLP was developed and it was investigated whether the concept could be transferred to other study hospitals.

Methods: In the intervention phase, the PLP was trained in IPM according to the "train-the-trainer approach" (patient decolonization before surgery, standardized wound care, etc.), which he/she subsequently implemented in his/her department. In the post-phase, HHA rates as well as $\mathrm{NI}$ and $\mathrm{SSI}$ rates were collected again.

Results: To examine the impact of implemented IPM on infection rates $(\mathrm{NI}, \mathrm{SSI})$, pre- and post-data were compared. In the pre-phase (237 surgeries), $\mathrm{NI}$ rate $(\mathrm{N}=12)$ was $5.1 \%(\mathrm{Cl} 95 \% 2.4 ; 7.8)$ and $\mathrm{SSI}$ rate $(\mathrm{N}=5)$ was $2.1 \%(\mathrm{Cl} 95 \% 0.3 ; 3.9)$. In the post-phase (177 surgeries), a $\mathrm{NI}$ rate $(\mathrm{N}=7)$ of $4.1 \%(\mathrm{Cl} 95 \% 1.3 ; 7.0)$ was found and no SSI.

The introduction of the dressing change concept significantly increased HHA of all indications (pre $=72.2 \%$; post $=95.6 \%$, $p<0.001$ ). The strongest increase in HHA occurred in the indication "hand disinfection between unclean and clean phase" (pre $=49.3 \%$; post $=86.5 \%, p<0.001$ ). HHA also increased for the other indications "before preparation for dressing change" (pre $=77.0 \%$; post $=98.3 \%$, $p<, 001$ ); "directly before dressing change" (pre $=78.9 \%$; post $=98.3 \%$, $p<0.001$ ); "after dressing change" (pre $=83.5 \%$; post $=100 \%$, $\mathrm{p}<0.001$ ).

Conclusion: By implementing the IPM, infection rates were also slightly reduced and HHA significantly increased for all indications of dressing change in the study hospital. This suggests that the developed bundle of IPM can be transferred to other trauma surgery departments through tailored training. Continuous process analysis could identify individual weaknesses in patient care and adapt intervention concepts.

Disclosure of Interest: None declared.
P079

Assessment of evaluation methodologies of infection preventive efficacy of ventilation systems in operating rooms C. Bulitta ${ }^{1, *}$

${ }^{1}$ Ostbayerische Technische Hochschule (OTH), Weiden, Germany

Correspondence: C. Bulitta

Antimicrobial Resistance \& Infection Control 10(1): P079

Introduction: Microbiological contamination of air in operating room $(\mathrm{OR})$ is a known risk factor for surgical site infections. Evaluation methodology of ventilation systems regarding infection preventive efficacy and cost benefit ratio is still a topic of discussion. To date there are no uniform standardized specifications and validation criteria established. Objectives: We aimed to assess evaluation methodologies of infection preventive efficacy of OR ventilation systems.

Methods: We have systematically reviewed and compared different international standards and the current literature regarding validation of ventilation systems and their infection preventive efficacy. We have analyzed various normative procedures, compared them regarding reliable assessment of efficacy and efficiency and conducted experiments in our research $O R$ and at selected clinical sites.

Results: Key for infection prevention is the reduction of airborne pathogens. The OR must be viewed holistically from a workflow perspective as a complex thermodynamic system with several mutually influencing factors, i.e. room size, convection currents of staff and equipment, flow obstacles and ventilation systems design. Our analysis has shown that protected areas of TAV systems are usually too small. The required size of a protected area can only be determined case by case, unless the operating room as a whole is viewed as protected area like in the concept of temperature controlled airflow (TCAF). Comparative measurements of active and passive air sampling show that only active sampling during live surgery allows reliable assessment of microbiological air burden.

Conclusion: Not only technical execution should be in focus of standards and normative specifications. The "what is to be achieved" should be more important than the "how it is achieved". This enables optimized risk management. It might be useful to follow rather the requirements of EN ISO 14644 and GMP guidelines than countryspecific non-uniform standards for ventilation technology in ORs. As part of the qualification of OR ventilation systems, PQ (Performance Qualification), the examination of the performance under real conditions, plays a central role. The lack of such a "live" qualification as an "acceptance criterion" could explain the controversial study situation regarding the infection preventive efficacy of OR ventilation systems. Disclosure of Interest: C. Bulitta Consultant for: Avidicare AB.

Poster session: long-term care facilities

\section{P080}

Antibiotic consumption in long-term care facilities in Poland and other European countries in the healthcare-associated infections in long-term care facilities HALT-3study

M. Brudło ', E. Jachowicz², A. Różańska², M. Gajda ${ }^{3, *}$, J. Wójkowska-Mach²

${ }^{1}$ Jagiellonian University Medical College, Krakow, Poland, ${ }^{2}$ Department of Microbiology, ${ }^{3}$ Doctoral School of Medical Sciences and Health Sciences, Jagiellonian University Medical College, Krakow, Poland

Correspondence: M. Gajda

Antimicrobial Resistance \& Infection Control 10(1): P080

Introduction: In developed countries, the population of elderly people is steadily increasing, which is associated with increased needs for permanent institutional care in long-term care facilities (LTCF), there about $20 \%$ of infections is due to multi-drug resistant microorganisms. Objectives: The aim of this research was to assess how the situation concerning healthcare associated infections (HAls) and antimicrobial consumption (AMC) in Polish LTCFs relates to that in other European countries.

Methods: The most recent HAls in long-term care facilities Point prevalence survey (HALT PPS) was carried out in European countries (EU/ 
EEA) in 2016-2017, and in Poland it was carried out in April-June 2017 in 24 LTCFs.

Results: The most common HAls was UTI ( $32 \%$ of all), but in Poland skin infection (30.4\% of all). In total 5,035 of the 102,301 eligible residents received at least one antimicrobial agent, with prevalence $4.9 \%$, in Poland, $3.2 \%$. The most frequently used antibacterial were beta-lactams, especially 'amoxicillin and enzyme inhibitor', in total $13.7 \%$, but in Poland in $26 \%$. The statistically significant relationships were found: positive - between the ratios of the elderly persons and physicians and the ratio of physicians and AMC in LTCF, negative - for the availability to nurses' care and the ratio of the elderly persons in the general population; and the share of the elderly population and AMC in LTCF. Conclusion: The presented data indicate major problems regarding antimicrobial consumption in LTCF. The lower AMC in Poland than in the overall was found but this is mainly due to the relatively young population in Poland.

Disclosure of Interest: None declared.

\section{P081}

Impact of mobile infection prevention and control (IPC) teams for nursing homes

K. Trouilloud 1,*, M. Giard ${ }^{2}$, A. Machut ${ }^{2}$, F. Duplatre ${ }^{3}$, O. Baud ${ }^{3}$, P. Fascia ${ }^{4}$, N. Khouider $^{2}$, A. Savey ${ }^{2}$

${ }^{1}$ Equipe Mobile d'Hygiène EHPAD (EMHE), Hospices Civils de Lyon, Saint Genis Laval, ${ }^{2}$ CPias Auvergne-Rhône-Alpes, Regional Coordinating Centre for Infection Prevention and Control, Hospices Civils de Lyon, Lyon, ${ }^{3}$ CPias Auvergne-Rhône-Alpes, Regional Coordinating Centre for Infection Prevention and Control, Centre Hospitalier Universitaire de Clermont-Ferrand, Clermont-Ferrand, ${ }^{4}$ Service d'Hygiène, Epidémiologie, Infectiovigilance et Prévention, Hospices Civils de Lyon, Lyon, France

Correspondence: $\mathrm{K}$. Trouilloud

Antimicrobial Resistance \& Infection Control 10(1): P081

Introduction: Building on the experience of a mobile infection prevention and control team (IPCT) established by Valence hospital since 2007, the Regional Health Agency (ARS) in Auvergne-Rhône-Alpes began financing mobile IPCT for medicalized nursing homes $(\mathrm{NHs})$ in 2013-2014 in the Eastern part, and extended the project to the whole region in 2018-2019.

Objectives: To describe the measured impact of 22 to 27 mobile IPCT in the ARA region from 2017 to 2019 , using benchmarking indicators. Methods: NHs with mobile IPCT or without IPCT available reported annually on main indicator categories that evolved since 2015. They include IPC human resources and organisation, IPC team-led trainings, alcohol handrub consumption (ICSHA), influenza vaccination rates, pneumococcal vaccination policy, and environmental surveillance (mainly for prevention of Legionella risk). Data from hospital-affiliated $\mathrm{NHs}$ (monitored by hospital IPCT) were excluded. Indicators of $\mathrm{NHs}$ with mobile IPCT were compared with those without IPCT.

Results: Before exclusion of hospital-affiliated $\mathrm{NHs}, 59.9 \%$ of requested NHs sent their report about 2019 vs 78.7\% about 2017. A total of 615,610 and $472 \mathrm{NHs}$ were included in the study in 2017, 2018 , and 2019 respectively (Table 1 ).

Overall, means and results indicators were better in $\mathrm{NHs}$ with mobile IPCT compared to $\mathrm{NHs}$ without (Table 2). Human resources allocated were higher (med referent $56.8 \%$ vs $18.5 \%$; paramed referent $77 \%$ vs $48.5 \%$; unit correspondent $78.7 \%$ vs $36.5 \%$ ). More $\mathrm{NHs}$ had IPC training organised (94.2\% vs $61.7 \%)$. ICSHA indicator was significantly higher (35.8\% vs $10.9 \%)$. Influenza vaccination rates for residents were similar in both groups ( $86.3 \%$ vs $85.8 \%$ ), but significantly higher for healthcare workers (HCW) in NHs with IPCT (32.1\% vs $22 \%)$.

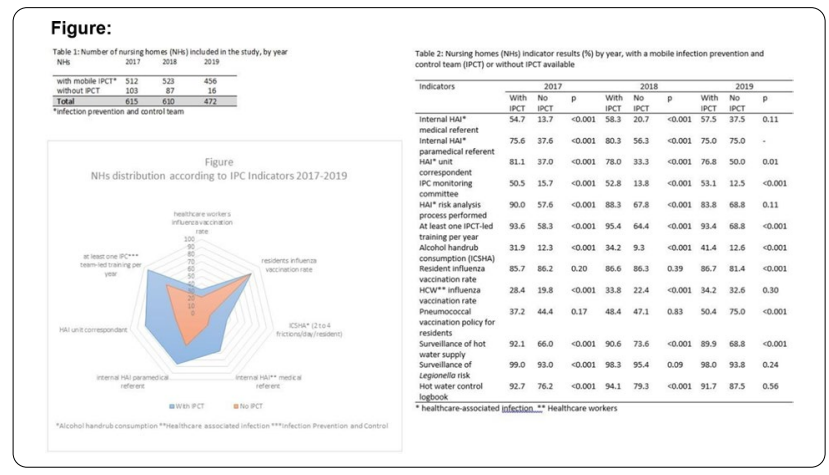

Conclusion: The increasing number of $\mathrm{NHs}$ with mobile IPCT is due to the ongoing extension project to the whole region. Mobile IPC teams had a measurable impact in $\mathrm{NHs}$ over the years in the region, especially in the areas of IPC human resources, IPCT-led training, alcohol handrub use, and HCW influenza vaccination rate. Implementation of such teams should be encouraged all over the country.

Disclosure of Interest: None declared.

P082

Implementation of simulation training in nursing homes

during COVID-19 pandemics

L. Qalla-Widmer ${ }^{1, *}$, D. Héquet ${ }^{1}$, C. Petignat ${ }^{1}$

${ }^{1}$ Unité cantonale HPCI Vaud, CHUV, Lausanne, Switzerland

Correspondence: L. Qalla-Widmer

Antimicrobial Resistance \& Infection Control 10(1): P082

Introduction: From March 2020, the nursing homes (NH) staff has been on the front line of the battlefield linked to the CoVID-19 pandemics. The healthcare workers ( $\mathrm{HCW}$ ) are at the heart of the institutional system as they provide care for residents and fight to prevent the spread of the virus within the institution. CoVID-19 highlighted the lack of knowledge regarding infection control measures in $\mathrm{NH}$. In this context, the Cantonal Unit for Infection Control and Prevention developed a simulation-training program to remain these gaps urgently.

Objectives: The goal was to strengthen the capacity of $\mathrm{NH}$ staff to provide safe and effective care, while protecting the residents and themselves from the spread of infection.

Methods: The infection control nurses defined learning objectives, identified the infection control academic content, developed a teaching strategy fitted to any learning styles and selected interactive teaching resources. After drafting scenarios of simples and complex care situations of targeting learning objectives, they defined the ideal environment and the equipment. Finally, a training was organized and simulations scenarios were recorded on video.

Results: Each session consisted of a simulation conducted by an infection control nurse with a selected scenario. A session averaged 12 participants including nurse, assistant nurse and medico-technical staff. Participants were placed in work situations through role-playing. Before each simulation session, participants were reminded of the mode of transmission of microorganisms, standard precautions and additional precautions. Debriefing was conducted after each session. Pertinent identified issues included the misuse of disposable gown, (droplet precautions), the failure to comply with the standards of hand hygiene and gloves wearing. The lessons learnt from each session were quickly disseminated to other $\mathrm{NH}$ staff.

From June 2020 to March 2021, 67/160 (42\%) NH participated in this simulation-training program. 109 sessions of simulation were conducted over 10 months. Over 1,800 HCW were trained.

Conclusion: Through simulation scenarios, essential elements of infection control can be emphasized. NH staff can be exposed to critical care scenarios and have the opportunity to respond without fear or risk for the resident.

Disclosure of Interest: None declared. 
Poster Session: Occupational exposure

P083

Quality improvement initiative in prevention of occupational exposure to needle-stick/sharp injuries and blood or body fluid exposures in a tertiary care centre in north India

A. Prasad ${ }^{1}$, , V. Singh' ${ }^{1}$, S. Sinha' ${ }^{1}$, P. Kumar Gupta ${ }^{1}$, P. Gupta ${ }^{1}$

${ }^{1}$ All India Institute of Medical Sciences Rishikesh, Rishikesh, India

Correspondence: A. Prasad

Antimicrobial Resistance \& Infection Control 10(1): P083

Introduction: Healthcare workers are exposed to several occupational health and safety hazards,especially to blood-borne disease transmission via sharp injuries or blood/body fluid exposures.

Objectives: To analyse effect of a continuous quality improvement initiative program used for preventing needle stick injuries and blood/ body fluid exposure.To find root cause and take adequate corrective and preventive action.

Methods: The study was conducted at a tertiary care centre in North India and all consecutive needlestick/sharp injuries and blood or body fluid exposures that occurred from January 2020 to April 2021 were included in the study.Based on the analysis of incidence rates of NSI during first 6 months of study period from January 2020 to June 2020(Phase I), a Quality Improvement Initiative plan was formulated using PDSA model.Thorough Root Cause Analysis (RCA) of each case was done using 5 why's method during second phase of study from July,2020 to December 2020.In phase 3,due to COVID19 pandemic,trainings were given to HCWs via virtual sessions to improve practices of HCWs.

Results: The incidence rate decreased from 0.44 incidences per 1000 patient days to 0.11 in December 2020(end of phase 2).Phase 3,rates increased to 0.33 due to new recruitment of untrained students.After training interventions,rate decreased to 0.22. Maximum incidents reported in Nursing officers(46.2\%), sanitation workers(22.6\%) and doctors (21.5\%).No significant difference among males (53.8\%) and females (46.2\%). $65.6 \%$ of the source was non-reactive.Maximum reactive cases were of Hepatitis C.Those expoed to HIV reactive source (3.2\%) were started on ART.Only 2/12 exposed to HBV required HBIG.

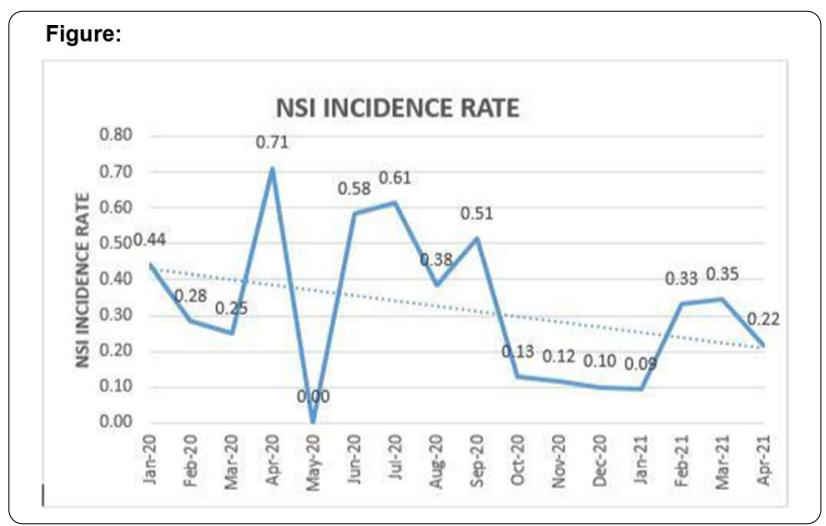

Conclusion: Integrated approach to perform RCA and induction and reinforcement trainings,audits can help in reducing sharp injuries. Disclosure of Interest: None declared.

\section{P084}

Decrease in occupational exposures to communicable diseases other than COVID-19 in a tertiary care center in Lebanon J. Tannous ${ }^{1}$, N. Zahreddine', A. Ibrahim ${ }^{1}$, R. Ahmadieh', S. Kanj 2,* ${ }^{1}$ Infection Control and Prevention Program, ${ }^{2}$ Infectious disease, AMERICAN UNIVERSITY OF BEIRUT MEDICAL CENTER, Beirut, Lebanon Correspondence: S. Kanj

\section{Antimicrobial Resistance \& Infection Control 10(1): P084}

Introduction: Occupational exposures to communicable diseases that are transmitted through droplet or airborne may place healthcare workers (HCW) at risk. Post-exposure interventions such as immunization, prophylactic antibiotics and work restrictions may be indicated.

Objectives: We assessed the unprotected exposures of HCW to communicable diseases before and during the COVID-19 pandemic at the American University of Beirut Medical Center (AUBMC).

Methods: Policies and procedures for standard and isolation precautions as well as post-exposure management are well defined at AUBMC. Unprotected exposures to suspected or confirmed communicable diseases that are transmitted through droplet or airborne routes are immediately reported to the Infection Prevention and Control Program (ICPP). Investigation of the exposures is conducted through staff interviews, direct observations and review of the surveillance cameras in the Emergency Department (ED) and the critical care units. List of the staff identified with unprotected exposures is then forwarded to the Employee Health Unit (EHU) for post-exposure management.

Results: Between 2017 and May 2021, unprotected exposures to communicable diseases such as varicella-zoster virus, pulmonary tuberculosis, pertussis, mumps, measles, Strep group A infections and meningitis caused by Neisseria meningitidis were managed. The highest number of unprotected exposures was identified in 2019 with $400 \mathrm{HCW}$ exposed to 23 communicable diseases. A dramatic decrease in exposures was noted in 2020 and 2021 with 13 and 0 staff, respectively.

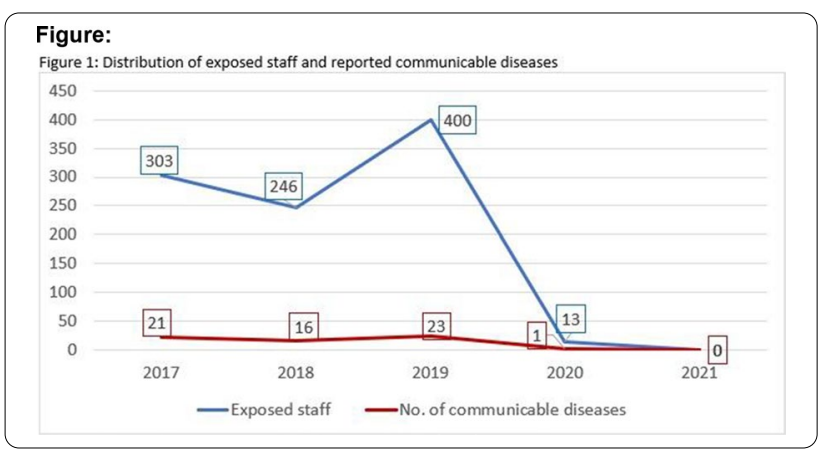

Conclusion: Immediate identification of patients with potential communicable diseases as well as application of standard and transmission-based precautions are essential in halting the transmission of infections among patients and HCW. The mandatory masking policy for patients, visitors and HCW during the COVID-19 pandemic as the well as ongoing educational and training activities including increased awareness about Hand Hygiene practices and environmental decontamination played a vital role in decreasing the occupational exposures at our center.

Disclosure of Interest: None declared.

P085

A journey in reducing percutaneous injuries

M. L. B. Garcia ${ }^{1, *}$, C. Simbulan ${ }^{1}$, F. Mohamed ${ }^{1}$, E. El Magboul ${ }^{2}$, E. Al Omari ${ }^{3}$, F. Ali'

${ }^{1}$ Quality and Patient Safety, Hamad Medical Corporation-Heart Hospital,

${ }^{2}$ Laboratory Department, Hamad Medical Corporation, ${ }^{3}$ Nursing, Hamad Medical Corporation-Heart Hospital, Doha, Qatar

Correspondence: M. L. B. Garcia

Antimicrobial Resistance \& Infection Control 10(1): P085

Introduction: The potential risk of exposure to blood and body fluids particularly needle stick injuries is high particularly among nursing staff during routine nursing care activities and the use of safety engineered devices (SEDs) can reduce the risk of percutaneous exposures. 
In 2018, percutaneous (needles and sharps) injuries in 2018 increased to fifty percent (18 cases) compared to 2017 (9 cases).

Objectives: To reduce percutaneous injuries to $50 \%$ by the end of December 2019 and beyond using safety engineered devices and staff education and training.

Methods: We conducted root cause analysis of all exposures and we developed action plan based on the identified problems. We implemented a multidimensional approach to change the system focused on the physical structures and supplies such as installation of wall-mounted sharp boxes, distribution of available stock items of safety engineered devices and procurement of safety insulin needles. We conducted education and training to all nursing unit preceptors and newly hired nurses about the available safety devices through simulation and with followup of change of practices in the units. Real time reporting of occurrences, counseling of exposed staff for proper management of exposure and feedback to units about preventive measures. Dissemination of blood and body fluid exposure alert and awareness campaigns were organized to increase staff awareness to prevent future occurrences.

Results: After implementation of preventive measures, a significant $56 \%$ reduction of exposed staff from 2018 baseline (18 cases) to 2019 (8 cases) and $38 \%$ reduction in 2020 (5 cases). A 64\% reduction in needle stick injuries in 2019 (4 cases), compared to 11 cases in 2018 and 50\% reduction in 2020 (2 cases). In sharp injuries, 43\% reduction in 2019 (4 cases) compared to 2018 baseline ( 7 cases) and 25\% reduction in 2020 ( 3 cases).

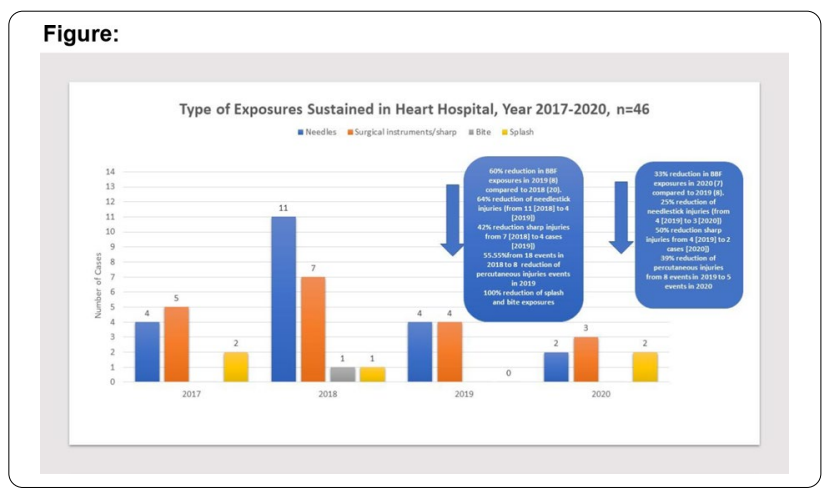

Conclusion: After implementation of preventive measures, a significant $56 \%$ reduction of exposed staff from 2018 baseline (18 cases) to 2019 (8 cases) and 38\% reduction in 2020 (5 cases). A 64\% reduction in needle stick injuries in 2019 (4 cases), compared to 11 cases in 2018 and $50 \%$ reduction in 2020 ( 2 cases). In sharp injuries, $43 \%$ reduction in 2019 ( 4 cases) compared to 2018 baseline (7 cases) and 25\% reduction in 2020 (3 cases).

Disclosure of Interest: None declared.

\section{P086}

Occupational risk of infection with hepatitis C virus: the dentist's perspective

S. S. Kp ${ }^{1, *}$

${ }^{1}$ mes dental college and hospital, malappuram, India

Correspondence: S. S. Kp

Antimicrobial Resistance \& Infection Control 10(1): P086

Introduction: Dental professionals may be exposed to bloodborne viruses (BBV) carried in blood, oral fluids and tissues. Hepatitis $C$ virus $(\mathrm{HCV})$ is one of the principal blood borne pathogens of concern to dentists worldwide. Occupationally acquired hepatitis $C$ viral infection is an important issue in dentistry since there are no known vaccines or effective prophylaxis.

Objectives: The aim of our questionnaire was to determine Dental surgeons' perceptions and attitudes regarding the risks of hepatitis $C$ viral infection as well as the precautions used against infection.

Methods: A Cross-sectional study was done among 200 dental surgeons, at Mangalore, India using pretested structured questionnaire to determine the attitudes and perception of risks of occupational acquired hepatitis $C$ viral infection as well as precautions among dental surgeons.Chi-square and Fisher's exact tests for categorical variables and student $t$-test for continuous variables were performed.

Results: In all, a total of 145 surgeons responded $(72.5 \%)$. Of respondents, $101(70 \%)$ had sustained sharps injuries. Only (29\%) always reported such injuries, although (84\%) expressed concerns of occupationally acquired hepatitis C viral transmission.Most Dentists (58\%) were either extremely or very concerned about the possibility of contracting HCV infection. Dental surgeons were mostly unaware of the true prevalence of hepatitis $C$ in high-risk groups.

Conclusion: The results of this study revealed that post-exposure management was completely inadequate especially the reporting of occupational exposures. However, post-exposure management protocols exist in all dental clinics, but the implementation of the protocol appears to be suboptimal. Needle stick injuries are also of increasing concern to the dentists.Greater awareness of all aspects of hepatitis C infection and its risks to the practice of dentistry is required.

Disclosure of Interest: None declared.

Poster session: influenza + vaccination

P087

Influence of wearing a mask during the COVID-19 pandemic on the influenza vaccination rate among healthcare workers in nursing homes in the canton of Vaud, Switzerland

B. Van Der Kaa ${ }^{1, *}$, D. Hequet ${ }^{1}$

${ }^{1} \cup H P C i$, Canton of Vaud, Switzerland

Correspondence: B. Van Der Kaa

Antimicrobial Resistance \& Infection Control 10(1): P087

Introduction: Vaccination of healthcare workers (HCW) against influenza reduces morbidity and mortality among residents of nursing homes $(\mathrm{NH})$. Therefore, $\mathrm{HCW}$ in $\mathrm{NH}$ are encouraged to be vaccinated against influenza. In the canton of Vaud (Switzerland), the vaccine is provided by Public Health to HCW who can be vaccinated free of charge at their workplace. Following a constant decrease in vaccination coverage among $\mathrm{HCW}$ over several years, wearing a mask became mandatory in $2015 / 16$ for HCW who were not vaccinated, as soon as the epidemic threshold was crossed and throughout the epidemic period.

Objectives: We wish to assess the impact of the obligation to wear a mask for the unvaccinated after 2015/16 as well as the influence of the CoVID19 pandemic with the wearing of a mask required for all professionals on the influenza vaccination coverage in $\mathrm{NH}$ of the canton of Vaud.

Methods: On behalf of Public Health, the Cantonal Unit Hygiene, Prevention and Control of Infection (UHPCi) collects annually the vaccination rates of HCW of every NH in the canton of Vaud. The UHPCi writes a review to the $\mathrm{NH}$ reporting their vaccination rate compared to global vaccination rates of the $\mathrm{NH}$. We analyzed these rates of the 2008/09 to 2020/21 seasons.

Results: The influenza vaccination rate of $\mathrm{HCW}$ in $\mathrm{NH}$ of the canton of Vaud was $42 \%$ at the beginning of the surveillance (2008/09) and then continuously decreased to $35 \%$. Following the directive requiring the wearing of masks for unvaccinated HCW over the epidemic period, this rate gradually increased to $47 \%$ in $2019 / 20$. With the obligation to wear a mask for all staff during the 2020/21 season due to CoVID-19 pandemic, this rate dropped to $37 \%$.

Conclusion: The obligation to wear a mask for the unvaccinated HCW from 2015/16 has made it possible to increase the influenza vaccination coverage for $\mathrm{HCW}$ working in $\mathrm{NH}$. The compulsory wearing of the mask for all in 2020/21 due to the CoVID-19 pandemic had a direct impact on the influenza vaccination rates of HCW with a $10 \%$ drop. The seasonal influenza vaccination rate is directly linked to the protective measures imposed on HCW and therefore on the wearing of masks. Primary prevention in the fight against seasonal influenza should be reviewed with the $\mathrm{HCW}$ of $\mathrm{NH}$, as vaccination remains the gold standard.

Disclosure of Interest: None declared. 


\section{P088}

Longitudinal assessment of team safety culture and leadership regarding influenza and COVID-19 preventive measures: the hospital associated influenza prevention study (HAIP)N D. Nicca ${ }^{1, *}$, I. Kramer ${ }^{2}$, D. Flury ${ }^{3}$, M. Schlegel ${ }^{3}$, M. Schubert ${ }^{2}$

${ }^{1}$ Institut of Epidemiology, Biostatistic and Public Health, University of Zürich, Zürich, ${ }^{2}$ Institute of Nursing, Zurich University of Applied Sciences, Winterthur, ${ }^{3}$ Infectious disease and hospital hygiene, Kantonsspital St. Gallen, St. Gallen, Switzerland

Correspondence: D. Nicca

Antimicrobial Resistance \& Infection Control 10(1): P088

Introduction: Prevention of hospital associated infections requires continuous monitoring and intervention. Team safety culture and leadership are key dimensions associated with infection prevention.

Objectives: The aim of this study was to compare team safety culture and leadership in health care workers (HCW) regarding influenza and COVID-19 preventive measures before and during the COVID-19 pandemic.

Methods: A longitudinal descriptive design was used to analyze data collected in a tertiary hospital in Switzerland across 2 years (2019/20 and 2020/21) reflecting the situation before and during COVID-19. Team safety culture was assessed for hand hygiene, mask wearing and vaccination (influenza/COVID-19 20/21) with adapted items of the safety attitude questionnaire (SAQ, Sexton et al., 2006) and adapted items of the Leadership Practices Inventory (LPI, Kouzes \& Posner, 2003). Data were analyzed descriptively with frequencies and percentages, median and interquartile ranges.

Results: In 2019/2020 $49 \mathrm{HCW}$ participated in the study and 59 in $2020 / 21$. In both years, the majority were nurses, midwives and female (98\%), about two thirds were below 40 years. Overall participants rated team safety culture as high for preventive measures with the exception of influenza vaccination coverage. For all three preventive measures there is an increase from $2019 / 20$ to $2020 / 21$ (table 1a). For $2020 / 21$ only, results show that it is easier to address team safety culture issues regarding COVID-19 vaccination compared to influenza vaccination (figure 1 not attached). Perceived leadership for infection prevention by direct supervisor increased across all items during the COVID-19 pandemic (table2b).

\begin{tabular}{|c|c|c|c|c|c|c|}
\hline \multicolumn{7}{|l|}{ Figure: } \\
\hline \multirow[t]{2}{*}{ 1a Team safety culture n (\% agreement) } & \multicolumn{2}{|c|}{ hand hygiene } & \multicolumn{2}{|c|}{ Mask wearing } & \multirow{2}{*}{\multicolumn{2}{|c|}{$\begin{array}{c}\text { influenza vaccination } \\
\text { coverage }\end{array}$}} \\
\hline & $19 / 20$ & $20 / 21$ & $19 / 20$ & $20 / 21$ & & \\
\hline $\begin{array}{l}\text { In this unit, it is not difficult to speak up if } \mid \text { perceive } \\
\text { a problem regarding influenza and COVID-19 } \\
\text { prevention }\end{array}$ & $40(82)$ & $49(85)$ & $40(82)$ & $52(90)$ & $28(57)$ & $36(62)$ \\
\hline $\begin{array}{l}\text { Health care workers here work together as a well- } \\
\text { coordinated team regarding influenza and COVID- } \\
19 \text { prevention }\end{array}$ & $42(86)$ & 56 (98) & $36(74)$ & 56 (98) & $11(22)$ & $14(25)$ \\
\hline $\begin{array}{l}\text { Mistakes in influenza and COVID-19 prevention are } \\
\text { handled appropriately in this unit, } \mathrm{n} \text { (\% grreement) }\end{array}$ & $43(88)$ & $57(97)$ & $40(82)$ & $56(95)$ & $20(41)$ & 37 (63) \\
\hline $\begin{array}{l}\text { If a patient on this uniti is infected with influenzzo, it } \\
\text { would be eass on this unit to discuss possible } \\
\text { mistakes with influenza prevention, n (\% } \\
\text { argeement) }\end{array}$ & $45(92)$ & 55 (93) & $45(92)$ & 56 (95) & $34(69)$ & $40(70)$ \\
\hline $\begin{array}{l}\text { If patitint on this unit is infected with CoViD-19, it } \\
\text { would be easy on this unit to discuss possible } \\
\text { mistakes with CoviD-19 prevention, } n \text { (\% } \\
\text { agreement) }\end{array}$ & . & $52(90)$ & - & $53(91)$ & - & $46\left(82^{*}\right)$ \\
\hline \multicolumn{2}{|l|}{ 16 Leadership (Score"(median 025, 075)) } & & \multicolumn{2}{|l|}{$19 / 20$} & \multicolumn{2}{|c|}{$20 / 21$} \\
\hline \multicolumn{3}{|c|}{$\begin{array}{l}\text { Sets a personal example of what he / she expects of others } \\
\text { regarding influenza and coviD-19 prevention, Median (Q25, Q75) }\end{array}$} & \multicolumn{2}{|l|}{$6(3,7)$} & \multicolumn{2}{|c|}{$9(7,10)$} \\
\hline \multicolumn{2}{|c|}{$\begin{array}{l}\text { Spends sime and energy making certain that the people he/she } \\
\text { works with adhere to the principles and standards of influenza and } \\
\text { CoviD-19 prevention that we have agreed on. Median }(025,075)\end{array}$} & & \multicolumn{2}{|l|}{$5(4,7)$} & \multicolumn{2}{|c|}{$9(7,10)$} \\
\hline \multicolumn{2}{|c|}{$\begin{array}{l}\text { Follows through on promises and commitments he/she makes } \\
\text { regarding influenza and covio-19 prevention (for example, } \\
\text { information about vaccination or addressing improper w wearing of } \\
\text { masks.) Median (025, 075) }\end{array}$} & & \multicolumn{2}{|l|}{$5(3,7)$} & \multicolumn{2}{|c|}{$8(7,9)$} \\
\hline \multicolumn{2}{|c|}{$\begin{array}{l}\text { Asks for feeddback on how his/her actions regarding influenza and } \\
\text { coviD-19 prevention affect other people's performance. }\end{array}$} & & \multicolumn{2}{|l|}{$2(1,6)$} & \multicolumn{2}{|c|}{$7(3,9)$} \\
\hline \multicolumn{2}{|c|}{ 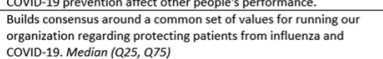 } & & \multicolumn{2}{|l|}{$4(3,7)$} & \multicolumn{2}{|c|}{$8(6,9)$} \\
\hline \multicolumn{2}{|c|}{$\begin{array}{l}\text { Actively listens to and seriously considers diverse points of view } \\
\text { regarding influenza and covp }-19 \text { prevention measures }\end{array}$} & & \multicolumn{2}{|l|}{$4(3,6)$} & \multicolumn{2}{|c|}{$8(6,9)$} \\
\hline \multicolumn{2}{|c|}{$\begin{array}{l}\text { Supports the decisions that people make on their own regarding } \\
\text { influenza and CoviD-19 prevention. Median }(025,075)\end{array}$} & & \multicolumn{2}{|l|}{$6(4.7)$} & \multicolumn{2}{|c|}{$8(7,9)$} \\
\hline \multicolumn{2}{|c|}{$\begin{array}{l}\text { Publicly recognizes people who exemplify committment to shared } \\
\text { values regarding patient protection from influenza and CoviD-19. } \\
\text { Median (025, } 075 \text { ) }\end{array}$} & & \multicolumn{2}{|l|}{$4(2,6)$} & \multicolumn{2}{|c|}{$8(5,9)$} \\
\hline \multicolumn{7}{|c|}{ 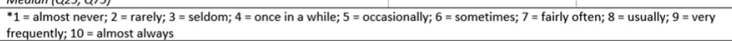 } \\
\hline
\end{tabular}

Conclusion: It will be important to maintain the increase in team safety culture and leadership in 2020/21. The differences in the ease to discuss either influenza or COVID-19 vaccination in respect to safety issues need to be addressed in by prevention initiatives.

Disclosure of Interest: None declared.

\section{P089}

Shift in influenza activity during COVID-19 at a tertiary care center in Lebanon

J. Tannous ${ }^{1}$, N. Kara Zahreddine ${ }^{1}$, A. Ibrahim¹ , R. Ahmadieh ${ }^{1}$, G. Dbaibo², S. Kanj ${ }^{1, *}$

${ }^{1}$ Infection Control and Prevention Program, ${ }^{2}$ Department Pediatrics and Adolescent Medicine, AMERICAN UNIVERSITY OF BEIRUT MEDICAL

CENTER, Beirut, Lebanon

Correspondence: S. Kanj

Antimicrobial Resistance \& Infection Control 10(1): P089

Introduction: Lebanon suffers from a seasonal increase in severe acute respiratory infections caused by Influenza viruses each year. Patients present with mild to severe symptoms which may require hospitalization. The increase in morbidity and mortality is usually noted in elderly and in patients with comorbidities.

Objectives: We studied the Influenza activity at the American University of Beirut Medical Center (AUBMC) before and during the COVID-19 pandemic.

Methods: In Lebanon, the Influenza season extends from September to May with a noted peak during winter. Close monitoring of the Influenza activity is performed at our center by the Infection Control team. Patients with suspected or confirmed Influenza are placed in a single room under droplet precautions. Influenza infections are confirmed by either Rapid Influenza Diagnostic test or Reverse Transcription Polymerase Chain Reaction (RT-PCR) test. During COVID-19 pandemic, public health measures such as lockdown, mandatory masking and physical distancing were implemented in the country with discrepancy in compliance between geographical regions.

Results: Since September 2020, all the respiratory specimens that were tested for Influenza at AUBMC came back negative. While positive results reached $27 \%$ in $2019-2020$ season and $20 \%$ in 2018-2019 Influenza season. The number of specimens tested during season 20-21 was 150 compared to 4592 and 4457 samples tested during the last 2 seasons, respectively.

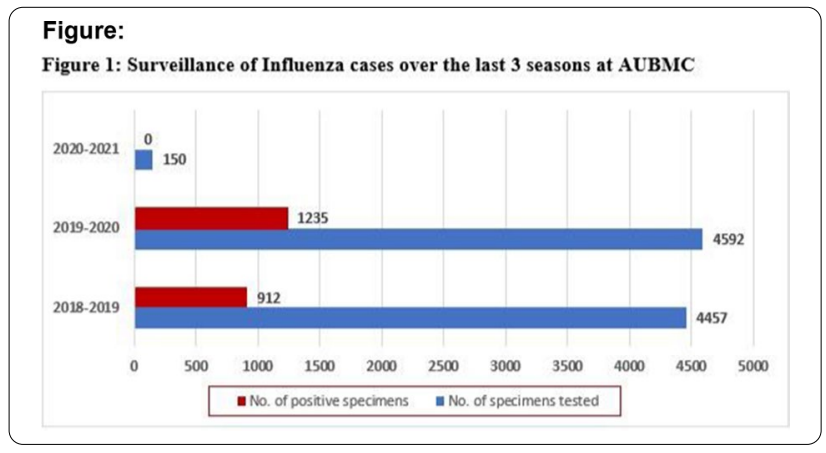

Conclusion: The exceptional zero Influenza cases encountered during the 2020-2021 Influenza season at our center in comparison to the previous 2 seasons is reasonably explained. The dramatic decrease in Influenza cases, hospitalization, morbidities, and mortalities is the result of the public health measures that were imposed by the authorities to control the spread of COVID19 in the country. A similar decline in Influenza cases is similarly observed in the United States where the positivity rate is reported at $0.1 \%$ during the same season.

Disclosure of Interest: None declared. 


\section{P090}

Effect of a multimodal strategy for prevention of nosocomial influenza

M. Gallouche ${ }^{1,2, *}$, H. Terrisse ${ }^{1}$, S. Larrat ${ }^{3}$, P. Morand ${ }^{1,3}$, V. Bonneterre ${ }^{1,4}$, J. L. Bosson ${ }^{1,5}$, C. Landelle ${ }^{1,2}$

${ }^{1}$ Grenoble Alpes University, ${ }^{2}$ Infection Control Unit, ${ }^{3}$ Virology laboratory, ${ }^{4}$ Occupational Health Unit, ${ }^{5}$ Public Health Department, Grenoble Alpes University Hospital, Grenoble, France

Correspondence: M. Gallouche

Antimicrobial Resistance \& Infection Control 10(1): P090

Introduction: A multimodal strategy to prevent nosocomial influenza (NI) was implemented in our institution from the 2015-2016 epidemic season onwards. Three modalities were implemented in all units: promotion of vaccination among healthcare workers (HCW), epidemiologic surveillance and communication campaign. Units receiving a high number of patients with influenza implemented 2 additional modalities: improvement of diagnosis capacities and systematic surgical mask use for HCW and visitors.

Objectives: Our objective was to assess the effectiveness of the 2 strategies for reducing the risk of NI.

Methods: A retrospective study was conducted over 5 epidemic seasons (2014-2015 to 2018-2019) including all adult patients hospitalised with a positive influenza test in our institution. NI was defined as a time interval between admission and symptoms onset superior to $72 \mathrm{~h}$. The weekly number of NI cases was analysed by Poisson regression and incidence rate ratios (IRR) were estimated. Seasons when the strategy was applied were compared to the reference season (2014-2015) and units implementing 3 modalities were compared to those implementing 5 modalities.

Results: A total of 1559 stays were included. The proportion of $\mathrm{NI}$ ranged from $18.10 \%$ to $26.49 \%$ between seasons $(p=0.074)$. There was no significant difference for the occurrence of $\mathrm{NI}$ between the 5 seasons in the units applying only 3 measures. In those implementing 5 measures, there was a rough reduction of $50 \%$ in $\mathrm{NI}$ occurrence over the 4 epidemic seasons when the strategy was applied, compared to the 2014-2015 season. This reduction was statistically significant for 2 seasons $(\mathrm{IRR}=0.56,95 \% \mathrm{Cl}=0.23-1.34$ in 2015-2016; IRR $=0.39,95 \% \mathrm{Cl}=0.19-0.81$ in $2016-2017 ; \mathrm{IRR}=0.50$ $95 \% \mathrm{Cl}=0.24-1.03$ in $2017-2018 ; \mathrm{IRR}=0.48,95 \% \mathrm{Cl}=0.23-0.97$ in 2018-2019).

Conclusion: The application of the strategy with 5 modalities, including systematic surgical mask use and rapid diagnosis, could reduce the risk of NI. Further data, including medico-economic studies, are still necessary.

Disclosure of Interest: None declared.

\section{P091}

Acceptance of influenza vaccination during the COVID-19 pandemic among the Tunisian healthcare workers N. Zammit1, ${ }^{1}$, A. Braham², C. Bennasrallah ${ }^{3}$, E. G. Amani ${ }^{1}$, I. Ayouni ${ }^{1}$, R. Ghammem', S. Ben Fredj ${ }^{1}$, C. Sridi' ${ }^{2}$, A. Chouchane ${ }^{2}$, H. Kalboussi ${ }^{2}$, O. ElMaalel $^{2}$, S. Chatti' ${ }^{2}$, J. Maatoug ${ }^{1}$, H. Ghannem ${ }^{1}$, N. Mrizak ${ }^{2}$ ${ }^{1}$ Department of Epidemiology (LR19SP03), ${ }^{2}$ Department of Occupational Medicine and environmental pathology (LR19SP03), Farhat Hached University Hospital, Faculty of Medicine of Sousse, University of Sousse, Sousse, ${ }^{3}$ Faculty of Medicine of Monastir, Monastir, Tunisia

Correspondence: N. Zammit

Antimicrobial Resistance \& Infection Control 10(1): P091

Introduction: Seasonal influenza vaccination of healthcare workers (HCWs) is recommended in order to protect themselves and patients. During the COVID-19 pandemic, several studies highlighted the benefit of Influenza vaccination in reducing COVID-19 burden especially in developing countries. However, Influenza vaccination coverage among HCWs is unknown in the majority of these countries.
Objectives: To investigate the acceptance of Influenza vaccination during the COVID-19 pandemic among Tunisian HCWs.

Methods: An online cross-sectional study was led between the $7^{\text {th }}$ and the $21^{\text {th }}$ of January 2021 among 493 Tunisian health professionals. A pre-established and pre-tested questionnaire recorded in a free Google form was self-administrated to participants anonymously. The generated online Google Sheet was uploaded and exported to the Statistical Package for the Social Sciences 10.0 software for analysis.

Results: The mean age of participants was $37.4( \pm 9.5)$ years. The males/females ratio was 0.38 .The rates of influenza vaccine acceptance were: $68 \%$ among medical doctors, $65.7 \%$ among pharmacists, $55.8 \%$ among paramedical professionals and 50\% among dental surgeons. Working in the public sector and having a chronic condition predicted more acceptance of influenza vaccine with adjusted odds ratio of 1.9 (95\% Cl: 1.3-3.9) and 2.1 (95\% Cl: 1.3-3.5) respectively. Having been infected by the SARS-COV2 predicted fewer acceptances with and adjusted odds ratio of 0.3 (95\% Cl: $0.1-0.7)$.

Conclusion: More attention should be paid to Tunisian paramedical professionals and dental surgeons in order to increase influenza vaccine uptake among them. Involving HCWs in the national information campaign about COVID-19 and Influenza vaccination would ensure more vaccines acceptance among them.

Disclosure of Interest: None declared.

\section{P092}

Influenza vaccination of HCW and risk of nosocomial influenza in patients hospitalised in short stay units

S. Amour ${ }^{1, *}$, T. Bénet $^{1}$, C. Regis ${ }^{2}$, O. Robert ${ }^{1}$, L. Fontana ${ }^{3}$, B. Lina ${ }^{1}$, B.

Pozzetto ${ }^{3}$, P. Berthelot ${ }^{3}$, P. Vanhems ${ }^{1}$

${ }^{1}$ Hospices Civils de Lyon, ${ }^{2}$ Université de Lyon, LYON, ${ }^{3} \mathrm{CHU}$ Saint-Etienne, Saint-Etienne, France

Correspondence: S. Amour

Antimicrobial Resistance \& Infection Control 10(1): P092

Introduction: Nosocomial influenza (NI) is regularly reported in hospitalised patients. Exposure to healthcare workers (HCW) or patients potentially infected with influenza increases this risk.

Objectives: The objective of this study was to estimate the effect of influenza vaccine coverage (IVC) of HCW on the risk of $\mathrm{NI}$ in hospitalised patients.

Methods: A case-control study was nested in a multicentre prospective cohort study conducted in two university hospitals during two influenza seasons (2013-14 and 2014-15). An influenza-like illness (ILI) was defined as fever $\geq 37.8^{\circ} \mathrm{C}$ (in the absence of antipyretics) and/or cough or sore throat. Each inpatient with ILI included had a nasal swab and a systematic search of influenza by PCR testing. A NI case was a patient with a virological confirmation of influenza with onset of symptoms $\geq 72 \mathrm{~h}$ after admission to the ward. A control was a patient with an ILI during his hospitalisation but with PCR negative for influenza. The IVC rate of HCW in each participating ward was calculated from the data shared by the occupational health departments. A logistic regression was performed with adjustments on patient gender, age, the presence of a potential source of influenza on the ward in the 5 days prior to the start of the ILI, type of ward and influenza season.

Results: A total of 165 patients were included: $24 \mathrm{NI}$ cases and 141 controls. The median age of the patients was 84 years, 94 (57\%) were women and $68(41 \%)$ were vaccinated against influenza. The median IVC rate of HCW on the wards was $18 \%$ (1\% to $71 \%$ ) with a mean of $32 \%$. The percentage of a potential hospital source of influenza was $50 \%$ in $\mathrm{NI}$ cases versus $21 \%$ in controls $\left(\mathrm{P}<10^{-2}\right)$. The crude Odds Ratio (OR) of $\mathrm{NI}$ decreased from 0.52 (95\% confidence interval (Cl95\%) 0.21-1.29) to 0.14 (Cl95\% 0.03-0.63) when the IVC threshold of HCW on the ward increased from 20 to $50 \%$ (figure). Similarly, after adjustment, the Adjusted OR decreased from 0.86 (CI95\% 0.28-2.60) to 0.29 (CI95\% 0.04-1.98). 


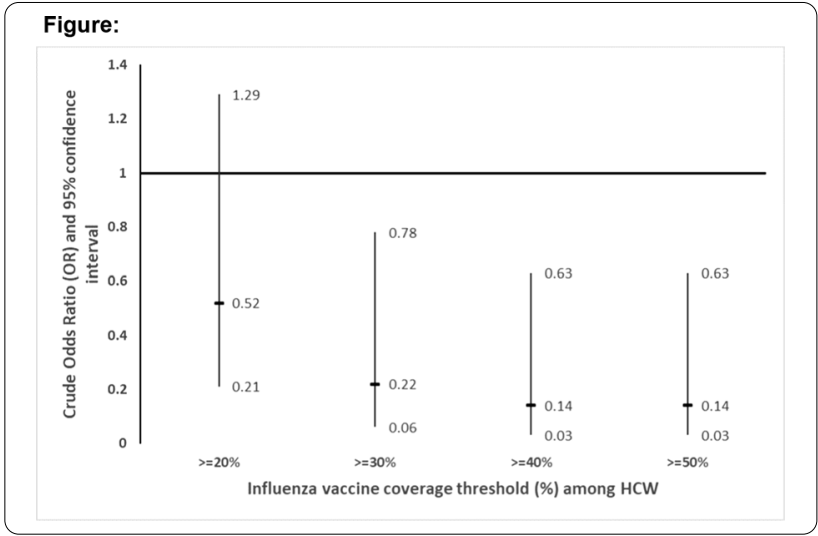

Conclusion: The prevention and control of $\mathrm{NI}$ involves multiple preventive measures including the vaccination of HCW. Based on these observational data, vaccination of HCW is a determinant that contributes strongly to the $\mathrm{NI}$ control.

Disclosure of Interest: None declared.

\section{P093}

Knowledge, attitude and practices (KAP) of physician about adult immunization - a study from developing country I. Khanum, 1,*

${ }^{1}$ Aga Khan University Hospital, Karachi, Pakistan, Pakistan

Correspondence: I. Khanum

Antimicrobial Resistance \& Infection Control 10(1): P093

Introduction: Health care workers (HCWs) are at risk of acquiring vaccine preventable diseases, Increasing awareness of physicians about adult immunization, one of the reason for low adult vaccination rate, will provide success in improving adult vaccination coverage.

Objectives: The aim of current study is to establish knowledge and attitude of physician about adult vaccination.

Methods: A cross sectional survey was performed among physicians via a self-administered questionnaire. Out of 200, 117 complete forms filled by physicians were included in the study.

Results: Majority of the participant were female (62\%).The physicians' own vaccination status was not satisfactory as 108 physicians (92\%) had received HBV vaccine, 54(46\%) influenza vaccine, 41 (35\%) MMR vaccine and 37 (31\%) vaccine against tetanus. Majority ( $n$ 106, 90\%) stated that they are not able to get updated information related to adult vaccine recommendations and main source of knowledge about adult vaccination were books or guidelines. Nearly half of the study participants ( $\mathrm{n} 57,49 \%$ ) had received training about adult immunization at undergraduate level and even less no of participant ( $37 \%$ ) had received formal education related to adult vaccination during postgraduate training period.

Regarding vaccine efficacy, only $82(70 \%)$ of participants believes that vaccine have overall protective effect on community health \& wellness.Physicians believed that high cost of vaccines, lack of vaccination center for adult immunization, lack of structured program or campaign for adult vaccination and lack of awareness regarding adult vaccines among community are important factors for low adult vaccination rate in our population.

Regarding problems related to prescribing vaccines, lack of patient's interest, high patients burden in clinics, non-availability of vaccines and high vaccine cost were considered to be barriers for not advising vaccines to adult patients by study participants.

Conclusion: Healthcare worker's knowledge and attitude play a key role in improving vaccine coverage of community. Education interventions are necessary to improve awareness of HCWs and public in order to protect community against vaccine preventable diseases particularly in countries with low implementation of immunization.

Disclosure of Interest: None declared.

P094

Hindering reasons of children vaccination in one city of Latvia

A. Melbarde-Kelmere, ${ }^{1,}$, V. Sesunova ${ }^{1}$

${ }^{1}$ Nurse and Midwife faculty, Riga Stradins University, Riga, Latvia

Correspondence: A. Melbarde-Kelmere

Antimicrobial Resistance \& Infection Control 10(1): P094

Introduction: In order to maintain high results in the field of vaccineregulated infectious diseases, it is necessary to achieve vaccination coverage of up to $95 \%$ or more, but the level of vaccination tends to decrease. There is a set number of children each year who have not been vaccinated at all against any infectious disease or who have been vaccinated in part, as a result of which vaccination has been delayed (vaccination is not carried out at a certain age).

Objectives: Find out the reasons that hinder children's vaccination in one of Latvian city.

Methods: Quantitative correlation study using questionnaires. Study included 351 parents and 30 medical staff from vaccination institutions. Data were collected and coded in Microsoft Excel, IBM SPSS Statistics 22. descriptive statistics, for the determination of relationships (correlations) Spearman rank correlation coefficient, non-parametric statistical correlation analysis.

Results: Parents are not really clear about vaccines and vaccinations due to very contradictory answers (parents do not know the composition of combined vaccines against which diseases are vaccinated, also not clear and not in a timely manner provided information about vaccination calendar). $55 \%$ of parents consider infectious diseases to be a serious threat to their child 's health, but $45 \%$ do not. Parents most often refuse flu, varicella vaccine, and tick-borne encephalitis vaccine. $65.8 \%$ of parents had not refused vaccination, $32.2 \%$ had refused to be vaccinated and $7 \%$ of respondents indicated that the child had never been offered vaccination.

$86 \%$ of cases vaccination of children is performed in the practice of family doctors.. 1/3 vaccination facilities not working after 17:00, vaccination planned only 2-3 days a week. Information to parents is provided during the visit, less often by telephone, even fewer make reminder notes in the vaccination passport, a very small number of information are provided in letters-letters, invitation.

Conclusion: Timely manner provided information, planned vaccination visits must be organized. Also information regards refused vaccination and individual reminders could contribute vaccination coverage.

Disclosure of Interest: None declared.

\section{P095}

Incidence of vaccine-preventable childhood diseases (VPCD) in the European Union (EU) and in the European free trade association (EFTA) countries

E. Jachowicz ${ }^{1}$, M. Gębicka ${ }^{2}$, M. Gajda ${ }^{3, *}$, D. Plakhtyr ${ }^{4}$, M. Shynkarenko ${ }^{4}$, J. Urbanowicz ${ }^{4}$, J. Czepiel ${ }^{5}$, M. Mach $^{6}$, J. Wójkowska-Mach ${ }^{1}$

${ }^{1}$ Department of Microbiology, ${ }^{2}$ Department of Immunology, ${ }^{3}$ Doctoral School of Medical Sciences and Health Sciences, ${ }^{4}$ Jagiellonian University Medical College, Krakow, Poland, ${ }^{5}$ Department of Infectious and Tropical Diseases, Jagiellonian University Medical College, Krakow, ${ }^{6}$ SGH Warsaw School of Economics, Warsaw, Poland

Correspondence: M. Gajda

Antimicrobial Resistance \& Infection Control 10(1): P095

Introduction: Despite the widespread availability of vaccines, the incidence of vaccine-preventablechildhood diseases (VPCD) started to grow in recent years.

Objectives: The aim of study was comparingthe incidence of selected VPCDs in the EU and EFTA countries in 2014-2019, and thecountryspecific vaccine schedules. 
Methods: VPCD incidence rates in Europe based on "The Surveillance Atlas of Infectious Diseases" by ECDC, vaccination schedules based on ECDC reports.

Results: The obligation to vaccinate is not universal and it generally only applies to 2 preparations: the MMR vaccine and the one against polio. During the study, the situation associated with mumps did not change or improve in individual countries, the median incidence amounted to 18 cases per 100,000 population. The median incidence associated with rubella amounted to 1 case, but in a few countries, it grew very rapidly, i.e. in Germany, Italy and Romania, In Poland, the incidence was clearly decreasing, from 5,923 to 1,532 cases. The most dynamic situation concerns measles. The total median was 2.4 cases per 100,000 population, the only one country with falling incidence was Germany. The diseases associated with Streptococcus pneumoniae and Neisseria meningitidis remained at a stable level in all analysed countries.

Conclusion: Vaccine schedules differ among the countries, so does the epidemiological situation of selected diseases. Morbidity on measles is the most disturbing phenomenon: the incidence rate increased in almost $40 \%$ of all countries, regardless of the obligation to vaccinate. The increasing incidence of VPCD may be due to antivaccine movements, the activity of which is often caused by mistrust and spreading information. In order to better prevent the increase in morbidity, standardisation of vaccine schedules and documentation should be considered in the EU countries.

Disclosure of Interest: None declared.

\section{P096}

\section{The epidemiological patterns of measles in CEE countries}

J. Pekarcikova ${ }^{1, *}$, A. Plskova

${ }^{1}$ Trnava University, Faculty of Health Sciences and Social Work, Trnava, Slovakia

Correspondence: J. Pekarcikova

Antimicrobial Resistance \& Infection Control 10(1): P096

Introduction: Within the WHO European Region, measles occurs mainly in populations with low vaccination coverage, whether children or adults.

Objectives: To describe the current occurrence of measles in selected EU/EAA countries, focusing on selected countries of Central and Eastern Europe and the level of measles vaccination levels in the study population.

Methods: In a descriptive analysis focused on the occurrence of measles in selected EU countries with a focus on the countries of Central and Eastern Europe (CEE) for the period 2014-2019, 6 countries were selected-Bulgaria, the Czech Republic, Hungary, Poland, Romania and Slovakia. Epidemiological data on measles were obtained from the ECDC Atlas for the Surveillance of Infectious Diseases and from the evaluation of administrative vaccination controls in Slovakia. Age-specific morbidity is reported per 1,000,000 people. Vaccination data were available in selected parameters for the last year 2018.

Results: Among the monitored countries of CEE, the prevalence of measles in Romania dominated in 2016-2018. In Romania, measles affected up to $96 \%$ of people without vaccination. No cases of measles have been reported in Hungary. It is interesting to note that the last year-2019 there has been a significant increase in all monitored countries of Central and Eastern Europe, with the exception of Romania, where a slight decrease is observed in new cases. In Slova$\mathrm{kia}$, the measles epidemic affected children under the age of 15 , who could not be vaccinated earlier due to age, and people living in poor hygiene standards. The most significant coverage of MCV1 (95.7\%) and MCV2 (93.5\%) was recorded in the countries of CEE in 2014. The situation in Hungary can be assessed positively, where the vaccination coverage of MCV1 and MCV2 reached $99 \%$. The level of MCV1 coverage in Slovakia ranged from 95 to $99 \%$ and MCV2 between 97 and $98 \%$ in the period under review. The optimal value of the measles vaccination is set at $95 \%$.

Conclusion: Within the EU, measures need to be taken to increase the effectiveness of immunization programs, including through vaccination, to at least $95 \%$ in each EU country, to promote and build effective communication between health professionals in order to increase public confidence in vaccination and motivate each country with best practice. the introduction of compulsory vaccination against preventable diseases.

Disclosure of Interest: None declared.

Poster session: COVID-19: environmental control

\section{P097}

Impact of COVID-19 pandemic on healthcare waste management: a pre experimental study from a Tunisian hospital

R. Bannour 1, ${ }^{*}$, A. Ben Cheikh', S. Bhiri ${ }^{1,2}$, H. Ghali ${ }^{1,2}$, W. Dhouib ${ }^{1}$, S

Khefacha' ${ }^{1}, H^{\prime}$. Said Latiri, ${ }^{1,2}$, M. Ben Rejeb ${ }^{1,2}$

${ }^{1}$ Department of Prevention and Security of Care," Sahloul University Hospital, ${ }^{2}$ Faculty of Medicine of Sousse, University of Sousse, Sousse,

Tunisia, Sousse, Tunisia

Correspondence: R. Bannour

Antimicrobial Resistance \& Infection Control 10(1): P097

Introduction: Worldwide, healthcare waste management (HCWM) is a high priority public health and environmental concern issue. During the Corona Virus Disease 2019 (COVID-19) pandemic, hygiene measures were more strictly applied in hospital setting.

Objectives: The aim of this study was to assess HCWM before and during the COVID 19 pandemic in Sahloul University Hospital (2019-2021).

Methods: A pre experimental study, based on two audits of HCWM, was carried out at Sahloul University Hospital (2019-2021).. The intervention consisted on repeated training sessions on standard hygiene precautions among all healthcare workers. The data was collected by trained auditors using a pre established observation grid based on the manual of good HCWM techniques and practices (ANGED).

Results: Between 2019 and 2021 the overall compliance with the sorting of HCWs has increased from $44.7 \%$ to $68.9 \%$ : it passed from $75 \%$ to $87.2 \%$ for needle containers, from $23.9 \%$ to $48.8 \%$ for yellow bags reserved for infectious risk waste and from X to $70.9 \%$ for black bags reserved for ordinary waste.

For the intra-service collection step the overall score passed from $28.51 \%$ to $58.2 \%$;

The most improved dimensions were "The filling level of the yellow bags is respected" (passed from $28.6 \%$ to $76.6 \%$ ) and "Absence of HCWs outside containers or waste bags" $19.9 \%$ to $90.9 \%$ ).

Nevertheless, a lack of improvement in labeling practice was noted, such as the labeling of bags containing infectious risk wastes, which went from 0 to $0.9 \%$; labeling of needle containersfrom $6 \%$ to $3.2 \%$ and the traceability of the waste disposal time from 0 to $7.3 \%$.

Conclusion: The pandemic may present a unique chance to improve compliance with recommendations regarding HCWsM on the long term, therefore, we should focus on how to maintain high adherence to recommendations and improve deficiencies during and after the COVID-19 pandemic.

Disclosure of Interest: None declared.

\section{P099}

Inactivation of SARS-COV-2 by various surfactants

M. Nishida 1, ${ }^{1}$, Y. Harada ${ }^{1}$, H. Amo ${ }^{1}$, H. Murai ${ }^{1}$, T. Ono ${ }^{1}$, M. Yoshikawa' ${ }^{1}$, Y. Hirata' ${ }^{1}$,T. Sasaki ${ }^{2}$, T. Shioda ${ }^{2}$, M. Ikawa²

${ }^{1}$ Biochemical Laboratory, Saraya Co., Ltd., ${ }^{2}$ Research Institute for Microbial

Diseases, Osaka University, Osaka, Japan

Correspondence: M. Nishida

Antimicrobial Resistance \& Infection Control 10(1): P099

Introduction: The novel severe acute respiratory syndrome coronavirus 2 (SARS-CoV-2) causing the global pandemic is an enveloped virus and expected to be susceptible to some surfactants. The efficacy of the commercially available disinfectants and cleaners against this virus 
has been well examined so far, but only few reports mentioned about the efficacy of each surfactant alone.

Objectives: This study aimed to evaluate the virucidal efficacy of various surfactants against SARS-CoV-2 (JPN/TY/WK-521). In addition, it also aimed to examine the presence of correlation between the virucidal efficacy and cytotoxicity of each surfactant.

Methods: For this study, the experiments dealing with the novel coronavirus were carried out at the Research Institute for Microbial Diseases (Osaka University), while the cytotoxicity tests were performed at the Saraya Microbiologicial Research Center (Saraya Co., Ltd). The virucidal efficacy of each surfactant (cationic, anionic, amphoteric and non-ionic surfactant; $0.01 \% \sim 5.00 \%$ ) was evaluated according to EN 14476 (dirty conditions). Their cytotoxicity was then examined by MTT assay.

Results: The performance of EN 14476 with SARS-CoV-2 demonstrated that the cationic surfactant showed high virucidal efficacy. Although not as high as that of the cationic surfactant, some nonionic and amphoteric surfactants also showed relatively good virucidal efficacy, while the rest did not. After evaluation of cytotoxicity, a good correlation between the virucidal efficacy and the cytotoxicity of each surfactant was observed; the higher the cytotoxicity, the higher the virucidal efficacy.

Conclusion: All of the tested cationic surfactants showed high virucidal efficacy and some nonionic and amphoteric surfactants had relatively highly virucidal efficacy. The evaluation of this strain has been finished. Further studies on the comparison of surfactant efficacy to other enveloped viruses with the original strain would bring some new insights to this research.

Disclosure of Interest: None declared.

Poster session: COVID-19: long-term care facilities

\section{P100}

Investigation of clustered cases of SARS-COV-2 infection (COVID-19) in nursing home settings, Etampes, France, Decembre 2020-Januray 2021

E. Elghouati ${ }^{1, *}$, C. Farrugia ${ }^{2}$, A. Emirian ${ }^{3}$, S. Siami ${ }^{4}$, H. Benhabib ${ }^{5}$

${ }^{1}$ Infection control unit, ${ }^{2}$ Department of Laboratory Medicine, ${ }^{3}$ Depart-

ment of Laboratory Medicine, Intensive care unit, ${ }^{5}$ Department of geriatrics, Centre Hospitalier Sud Essonne, Etampes, France

Correspondence: E. Elghouati

Antimicrobial Resistance \& Infection Control 10(1): P100

Introduction: A cluster of cases of Covid-19 was reported among residents and staff at a nursing home in December 2020.

Objectives: An epidemiological investigation was undertaken in order to describe the outbreak, identify factors associated with SARS-CoV-2 infection and adjust control measures.

Methods: A retrospective cohort study was conducted. SARS-CoV-2 was identified by reverse-transcriptase polymerase chain reaction assay (RT-PCR) or antigen tests.

Questionnaires were documented for demographic and clinical characteristics. Poisson regression was used to estimate the risk factors of infection for Covid-19.

Results: Sixty-two (62) cases of Covid-19 were identified, divided into 46 residents and 16 staff. The mean age of the residents was 86.7 years. The overall Attack Rate (AR) was 31\% (62/200): residents $A R=40.7 \%(46 / 113)$, staff $A R=18.4 \%(16 / 87)$. The fatality rate of residents with confirmed Covid-19 was $19.5 \%$ (9/46).

The distribution of cases over time spanned a period of about two months (from December 2nd 2020 to January 30th 2021). The epidemic curve revealed two successive waves of contamination. Two of the three floors of the facility were affected. No "British variant" of SARS-CoV-2 was identified.

The multi-variate analysis identified history of laboratory confirmed Covid-19 as an independent protective factor for both residents and staff [RRs were respectively $0.03(0.0004-0.19), P<0.001$ and 0.11 $(0.016-0.82), P<0.001]$.
Compared with surgical mask, the use of FFP2 respirator did not prevent the risk of SARS-CoV-2 in healthcare workers $[R R=0.99(0.32$ $-2.99), P=0.91]$.

Conclusion: Fifty-two (52) residents and 31 members of staff had a history of laboratory confirmed Covid-19. Most of them were contaminated during a first Covid-19 outbreak occurred in the facility by the end of March 2020. No case of reinfection was reported. Infection with SARS-CoV-2 appears to provide strong protection from reinfection for up to nine months including for the elderly. Further studies are needed to confirm this finding.

Disclosure of Interest: None declared.

\section{P101}

Local epidemiology of the SARS-COV 2 pandemic in nursing

homes: a Swiss experience

C. Riccio ${ }^{1, *}$, I. Nahimana ${ }^{1}$, L. Qalla Widmer ${ }^{1}$, C. Petignat ${ }^{1}$

${ }^{1}$ Unité cantonale HPCI Vaud, Lausanne, Switzerland

Correspondence: C. Riccio

Antimicrobial Resistance \& Infection Control 10(1): P101

Introduction: The SARS-CoV-2 pandemic has affected many nursing homes $(\mathrm{NH})$ s. Residents are more susceptible to coronavirus disease 2019 (COVID-19) complications due to their age, dependency and sometimes their psychiatric illness. Early identification and care positive residents are a powerful approach to limit the spread of the virus.

Objectives: This study aims to: i) assess the spread of SARS-CoV-2 by collecting data on the $\mathrm{NH}$ of the resident and their microbiologic data in order to control the transmission of the virus. ii) improve the nursing practices of infection control and to adapt the preventive strategies.

Methods: Data were collected from the register of the cantonal office of public health Vaud, from the $\mathrm{NH}$ staff, or from an on-line reporting platform. Immediately upon identification of a case, a cantonal nurse specialized in infection prevention and control (IPC) speaks with the $\mathrm{NH}$ staff or visits the NH. The IPC nurse recommended IPC measures and the screening strategy to control an outbreak. A daily or a biweekly follow-up was required until the resolution of the outbreak. When the NH has no more isolated COVID-19 positive resident, we wrote a final report.

Results: The first wave of the COVID-19 pandemic in NHs lasted from 09.03.2020 to $23.06 .2020 .62 \mathrm{NHs} / 163$ have been affected, $38 \%$ of $\mathrm{NHs}$ in the canton. The mean attack rate was $16 \%(1-62 \%)$. About $700 / 5178$ residents (14\%) have been infected by COVID-19: 400 residents with a laboratory confirmation of infection while 300 were clinical suspicions of COVID-19. There were $5 \%$ of deaths (255/5178). The second wave lasted from 10.08 .2020 to 31.12 .2020 . $104 \mathrm{NH} / 163(64 \%)$ have been affected. The mean attack rate was 38\% (6-91\%). 1914/5178 $(37 \%)$ residents have been infected by the virus; more than twice as much as the first wave. The rate of death of the second wave was $8 \%$ $(426 / 5178)$. The mean duration of outbreak cases was 5 weeks (2-10 Weeks).

Conclusion: $\mathrm{NH}$ s have been highly impacted by the second wave. The systematic screening for SARS-Cov-2 was crucial in detecting asymptomatic residents. The implementation of adequate IPC measures, the $\mathrm{NH}$ collaboration and the interventions of IPC nurses were a successful approach to limit the spread of the virus. Knowledge of NH staff in IPC was improved.

Disclosure of Interest: None declared.

P102

An unprecedented and large-scale departmental support mission to assist living facilities for elderly people facing the COVID-19 pandemic

A. Colas ${ }^{1,2, *}$, A. Baudet ${ }^{1}$, M. Regad ${ }^{1}$, A. Florentin ${ }^{1,2}$

${ }^{1}$ CHRU NANCY, ${ }^{2}$ DHREAS - Université de Lorraine, Vandoeurre les Nancy,

France

Correspondence: A. Colas

Antimicrobial Resistance \& Infection Control 10(1): P102

Introduction: In March 2020, a notable proportion of COVID-19 cases have occurred among Health and Social Institutions. Mid-April, early 
outbreaks among assisted living facilities for elderly people have been recorded in the Meurthe et Moselle French department.

Objectives: The authorities ordered on April, the 10th, an unprecedented and large-scale departmental support mission to assist living facilities at this very critical time.

Methods: Mobile units were formed under the coordination of the infection prevention and control (IPC) department of a teaching hospital in collaboration with the departmental fire station and the departmental council. These IPC teams (IPCT) consisted of three members: a nurse, a fireman and a medical administrator. In total, eight teams were formed and a field visit had been scheduled for each establishment. IPCT went to the facility to meet the management and the ward staff in order to assess the situation and identify any support needs. Report describing strengths, limitations and corrective measures to implement was written and electronically sent to the facilities and to the authorities. Results: Over a five-day period, all the facilities ( $n=66,6029$ places) of the French department were visited. If urgent needs were identified, the IPC department was directly informed by the IPCT to initiate an extensive assistance operation. Although most of the establishments had implemented good management to face the pandemic, the IPC department conducted emergency field support operations in establishments with uncontrolled outbreaks to implement corrective actions and provide staff/equipment.

Conclusion: To our knowledge, this mission is the first support action for Health and Social institutions facing the pandemic in France. As no major outbreaks were later noticed, this mission was a success and met the establishment's need for support in this critical time. Through this mission, many facilities have expressed the need to cooperate with IPC specialists during crises, but also in daily practice. The prospects are obvious: maintain support and implement a permanent collaboration between Health establishments and Health and social Institutions.

Disclosure of Interest: None declared.

\section{P103}

Applying workflow analysis techniques in care homes in the context of IPC and COVID-19

J. Storr ${ }^{1, *}$, C. Kilpatrick ${ }^{1}$, S. Hall ${ }^{2}$, M.-N. Chraiti ${ }^{3}$

${ }^{1}$ KS Healthcare Consulting, ${ }^{2}$ Carer/Relative, Glasgow, United Kingdom,

${ }^{3}$ Geneva University Hospitals, Geneva, Switzerland

Correspondence: J. Storr

Antimicrobial Resistance \& Infection Control 10(1): P103

Introduction: The unprecedented COVID-19 pandemic saw a plethora of infection prevention and control (IPC) recommendations. However, implementing these within a compassionate milieu appeared to present a universal challenge. We highlighted the need for action in an open letter (https://www.nursingtimes.net/opinion/open-letter-infec tion-prevention-and-control-should-never-be-at-the-expense-ofcompassionate-care-16-10-2020/) and identified real life scenarios as one solution to address the perceived IPC-induced compassion catastrophe reported to be taking place in many long term care (LTC) facilities.

Objectives: To apply workflow analysis techniques in LTC in order to present IPC measures in the context of COVID-19.

Methods: First hand narrative descriptions of carer/relative journeys in a LTC facility were obtained. Individuals were asked to recall a recent scenario, breaking it down into its smallest unit of activity, as outlined by WHO in a 2012 document on hand hygiene and long term care. The findings were reviewed and modified by a sample of people working in LTC, relatives/carers and IPC experts, with the experts applying IPC recommendations within the flow of activities.

Results: Simulation of the flow of activity for two commonly encountered LTC scenarios, uniquely from the perspective of carers/relatives, was created. A table and a visual outlining the two scenarios were developed (figure 1 illustrates an example scenario: Spending time with a loved one outside/on a car journey) and launched on a unique website with site traffic monitored. At each step, the relevant IPC measures and their rationale were described with the aim of empowering those in LTCs to act with both compassion and safety.

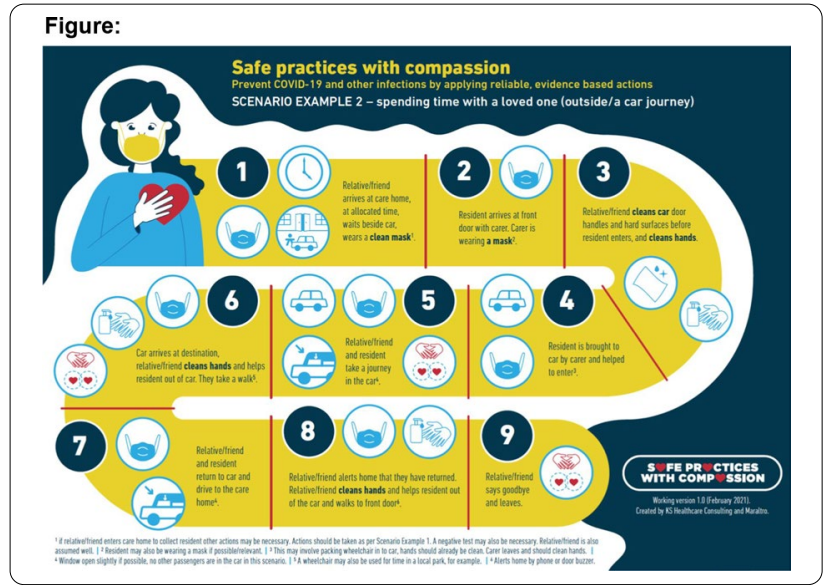

Conclusion: We used a robust, evidence-based approach to develop a data bank of workflow information related to activities within LTC, as well as visual representations. These constitute a de novo implementation resource designed to promote safe access to LTC in the context of a global pandemic. The end products and website (www. enablesafecare.org) go some way to redress the imbalance between IPC and compassion and have been made freely available for universal adaptation and use.

Disclosure of Interest: None declared.

\section{Poster Session: COVID-19: Treatment}

\section{P104}

Virucidal potential of oral rinses and nasal sprays against SARS-COV-2 and their mode of action

T. L. Meister ${ }^{1, *}$, N. Heinen ${ }^{1}$, D. Todt ${ }^{1,2}$, Y. Brüggemann ${ }^{1}$, S. Pfaender ${ }^{1}$, F. H. H. Brill $^{3}$, E. Steinmann ${ }^{1}$

${ }^{1}$ Molecular and Medical Virology, Ruhr University Bochum, Bochum,

${ }^{2}$ European Virus Bioinformatics Center, Jena, ${ }^{3}$ Dr. Brill + Partner GmbH Institute for Hygiene and Microbiology, Hamburg, Germany

Correspondence: T. L. Meister

Antimicrobial Resistance \& Infection Control 10(1): P104

Introduction: The ongoing SARS-CoV-2 pandemic creates a significant threat to global health. Recent studies suggested the significance of throat and salivary glands as major sites of virus replication and transmission during early COVID-19 thus advocating application of oral antiseptics.

Objectives: Here, we evaluated the virucidal activity of different available oral rinses, nasal sprays as well as individual compounds found in oral rinses against SARS-CoV-2. These experiments were performed under conditions mimicking nasopharyngeal secretions and investigated their respective virucidal modes of action.

Methods: According to European guidelines, virucidal activity was determined with a quantitative suspension test with $30 \mathrm{~s}$ exposure time on VeroE6 cells. Mechanistic analysis to reveal the mode of action of antiseptic agents included density gradient centrifugation and a capsid protection assay.

Results: Three of the eight oral rinses as well as two nasal sprays significantly reduced viral infectivity to up to three orders of magnitude to background levels. Mechanistic analysis revealed that treatment with benzalconiumchloride and other antiseptic agents used in mouth rinses primarily disrupted the viral envelope, without affecting viral RNA integrity.

Conclusion: In summary, we provide evidence that SARS-CoV-2 can be efficiently inactivated by commercially available oral rinses and nasal sprays with respect to their compound composition, within 
short exposure times, thus possibly lowering the transmission of SARS-CoV-2.

Disclosure of Interest: None declared.

\section{P105}

Survival analysis of COVID-19 patients treated with different modes of respiratory support in the ICU

Y. Wang ${ }^{1, *}$, B. Deng ${ }^{2}$, F. Qiao ${ }^{3}$, K. Liang ${ }^{4}$, W. Wu ${ }^{5}$, K. Li $i^{6}$, L. Lin ${ }^{7}$, C. Li ${ }^{8}$, Z. Peng ${ }^{2}$, Y. Yuan ${ }^{6}$

${ }^{1}$ Department of Nosocomial Infection Management, Zhongnan Hospital of Wuhan University, ${ }^{2}$ Department of Critical Care Medicine, Zhongnan Hospital of Wuhan University, Wuhan, ${ }^{3}$ Infection Prevention and Control Department, West China Hospital, Sichuan University, Chengdu,

${ }^{4}$ Department of Nosocomial Infection Management, Zhongnan Hospital of Wuhan University, Wuhan, ${ }^{5}$ Hubei University of Medicine, Shiyan,

${ }^{6}$ Hepatobiliary and Pancreatic Surgery, Zhongnan Hospital of Wuhan University, ${ }^{7}$ Hospital Management Institute, Wuhan University, ${ }^{8}$ Information Center, Zhongnan Hospital of Wuhan University, Wuhan, China

Correspondence: $Y$. Wang

Antimicrobial Resistance \& Infection Control 10(1): P105

Introduction: This study is the first to focus on the different respiratory support modes and outcomes of critically ill patients with COVID19 in Wuhan.

The cohort study design is persuasive.

The number of studies retrieved is limited in patients with MDRO cOinfected with COVID-19.

This study only selected critically ill patients with COVID-19 in Leishenshan Hospital.

Objectives: We aimed to describe the clinical characteristics and outcomes of five different modes of respiratory support among critically ill patients with coronavirus disease 2019 (COVID-19).

Methods: This was a hospital-based, retrospective cohort study which setting on Leishenshan hospital in Wuhan, central China. Patients with COVID-19 admitted to the ICU of Leishenshan Hospital from February 8 , 2020 to April 18, 2020 were recruited. The outcome was living status and survival time.

Results: Thirty-five patients died among 114 hospitalization patients (mortality rate, $30.7 \%)$, and 56 patients $(49.12 \%)$ used mechanical ventilation. The mean survival time (days) of patients without respiratory support, noninvasive positive-pressure ventilation (NIPPV), endotracheal intubation, tracheotomy, or endotracheal intubation before and after tracheotomy $(E I+T)$ was $15,34,32,12.5$, and 6 , respectively $(p<0.000)$. Eighteen (15.79\%) patients were co-infected with MDROs, primarily in the $\mathrm{EI}+\mathrm{T}$ group $(83.33 \%)$. The mortality risk of patients treated with NIPPV and $\mathrm{EI}+\mathrm{T}$ was 0.20 and 0.21 times higher than that of patients without any respiratory support (95\% confidence interval $[\mathrm{Cl}]=0.002-0.203 ; 95 \%$ $\mathrm{Cl}=0.002-0.218$ ). The mode of respiratory support was an independent factor affecting the survival of ICU patients with COVID-19.

Conclusion: Mortality risk in patients with NIPPV and EI+T was lower than in those without any respiratory support. Timely and correct respiratory support mode is the key to reducing the death of critically ill patients with COVID-19.

Disclosure of Interest: None declared.

\section{P106}

Inhalation therapy in the context of the COVID-19 pandemic

H.-H. Chang ${ }^{1, *}$, S.-C. Pan ${ }^{1,2}$, Y.-C. Chen ${ }^{1,2}$

${ }^{1}$ Center for Infection Control, ${ }^{2}$ Department of Internal medicine, National Taiwan University Hospital, Taipei, Taiwan, Province of China

Correspondence: $\mathrm{H}$.-H. Chang

Antimicrobial Resistance \& Infection Control 10(1): P106

Introduction: Inhalation therapy allows conveying drugs directly into the airways while minimizing the systemic exposure. Nebulizers can be used by patients unable to use metered-dose inhalers or other inhalers. However, this procedure generates aerosol and causes environmental contamination or disease transmission. Thus, the usage of inhalation therapy should be cautious, especially in the current COVID19 pandemic. Besides, the device can also be a reservoir for pathogens and lead to further infection. The use of nebulizers also requires disassembly and cleaning of after each dose.

Objectives: This survey aimed to evaluate the compliance of hospital policy regarding the use of inhalation therapy.

Methods: This survey was conducted as a part of infection prevention and control program in a 2600-bed teaching hospital. Infection control personnel designed a structured questionnaire and visited the in-patient services during Dec. 1, 2020 to Dec. 31, 2020. The survey included the environment control (single room, close door, or close curtain if more than one bed), cleaning methods after use, and who handle the procedure.

Results: A total of 84 wards were audited. $38 \%$ of the wards did not close the door and $33 \%$ did not close the curtain while performing nebulization. To clean the devise after use, the use of tap water flowed by boiled water was most common (32\%), followed by sterile water $(21 \%)$ and $6 \%$ used tap water only. The cleaning procedure was performed by nurses (30\%) or family/care givers (19\%).

After the survey, Center for Infection Control had made a consensus with Department of Integrated Diagnostics \& Therapeutics and Department of Nursing. The main guidance include: 1 ) suggest use nebulization in single room only and close the door, if not feasible, suggest close the curtain around the patient area; 2) sterile water or boiled water should be used as the last step in cleaning; 3 ) the cleansing procedure should be performed by nurse and trained family/ caregiver.

Conclusion: In the era of COVID-19 pandemic, nebulization therapy may carry the risk of inducing aerosol and increase the risk of disease transmission. Through the survey, we notice the infection control practice of nebulization in the hospital should be strengthen. Isolation methods (door/curtain) and cleaning procedure are the two key points need improvement.

Disclosure of Interest: None declared.

\section{P107}

Efficacy of ixekizumab vs. IL-2 vs. colchicine vs. standard of care for the treatment of hospitalized patients with COVID-19: preliminary results of a randomized clinical trial (struck: survival trial using cytokine inhibitors)

L. Bonifácio ${ }^{1, *}$, E. Ramacciotti ${ }^{2}$, L. Agatii ${ }^{2}$, F. Vilar ${ }^{1}$, A. Tojal ${ }^{1}$, H. Souza ${ }^{1}$, P.

Louzada-Júnior ${ }^{1}$, B. Fonseca ${ }^{1}$, R. Lopes ${ }^{3}$, E. Kallas ${ }^{4}$, F. Bellissimo-Rodrigues ${ }^{1}$

${ }^{1}$ Ribeirão Preto Medical School, University of São Paulo, Ribeirão Preto,

${ }^{2}$ Science Valley Research Institute, São Paulo, Brazil, ${ }^{3}$ Duke University,

Durham, United States, ${ }^{4}$ School of Medicine, University of São Paulo, São Paulo, Brazil

Correspondence: L. Bonifácio

Antimicrobial Resistance \& Infection Control 10(1): P107

Introduction: There are currently scarce approved treatments for Covid-19 and new options are needed.

Objectives: To evaluate the efficacy of an IL-17 inhibitor vs. low-dose IL-2 vs. colchicine vs. standard of care for the treatment of hospitalized patients with Covid- 19.

Methods: This is an on-going multicenter, open-label, prospective, randomized, active-controlled, adaptive 4-arm study. Patients hospitalized with moderate to severe presentation of Covid-19 are being recruited according to predetermined eligibility criteria. Patients are randomized to one of the trial arms: ixekizumab (IL-17 inhibitor), low dose IL-2, colchicine (indirect IL-6 inhibitor), or standard treatment, each according to the pre-established periods and doses. The primary study outcome is the proportion of patients with clinical improvement, defined by an increase of two points on the WHO's ordinal scale at day 28 . Secondary study outcome is in-hospital mortality.

Results: Until May 27, 30 patients with similar baseline clinical and demographic features have been enrolled in the study. Table 1 describes the main efficacy outcomes for each of the study arms. Ixekizumab arm presented the greatest improvement in the WHO scale (71.4\%), while colchicine arm presented the lowest mortality rate $(0 \%)$, but no statistically significant differences have been observed, so far. 


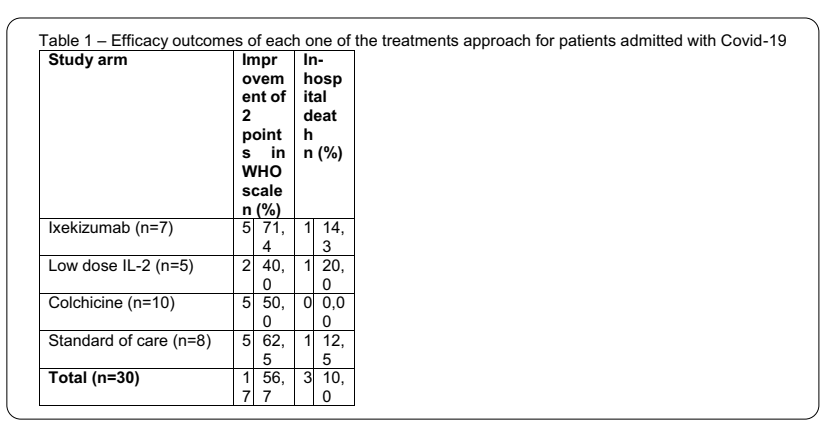

Conclusion: These preliminary results suggest that Ixekizumab and colchicine should be further studied as potential therapeutic drugs for treating patients with moderate to severe Covid-19.

Trial registration: NCT402422/2020-1.

Funding: Rede Vírus (MCTI) and CNPq (402,422/2020-1).

Disclosure of Interest: None declared.

\section{Poster Session: COVID-19: Vaccination}

\section{P108}

Intention to accept COVID-19 vaccine and its associated factors among Tunisian people

A. Maatouk ${ }^{1}$, A. Ammar ${ }^{1,},{ }^{*}$ W. Bannour ${ }^{1}$, O. Ezzi ${ }^{1}$, R. Helali' ${ }^{1}$ B. Trabelsi ${ }^{1}$, M. Njah' ${ }^{1}$, M. Mahjoub 1

${ }^{1}$ Infection Prevention and Control, University Hospital of Farhat Hached, Sousse, Tunisia

Correspondence: A. Ammar

Antimicrobial Resistance \& Infection Control 10(1): P108

Introduction: Coronavirus Disease 2019 (COVID-19) represents an emergence putting public health systems on high alert worldwide. In order to prevent the spread of the coronavirus and its related morbidity and mortality, appropriate strategies are needed such as vaccination. However, this vaccination is the subject of controversy.

Objectives: Our study aimed to determine the intention to accept COVID-19 vaccine and its associated factors among Tunisian people during the COVID-19 pandemic.

Methods: We conducted a cross-sectional study among Tunisian people from December 2021 to January 2021 (before the availability of COVID-19 vaccine in Tunisia) using an online questionnaire developed with Google Forms and submitted through social media. Healthcare professionals were not included in the study. The participation was completely voluntary. Data analysis was performed using the Statistical Package for Social Sciences (SPSS) version 21.0. Factors associated with intention to accept coronavirus vaccine were analyzed using multiple multinomial logistic regression.

Results: A total of 169 Tunisians participated in the study. Most of them were female (85.2\%) with mean age of $48.3 \pm 11.8$ years. In our study, $11.8 \%$ of participants were infected by SARS-CoV-2. Only $33.1 \%$ intended to accept to be vaccinated when COVID-19 vaccine will be available in Tunisia and $22.5 \%$ have not decided yet. Reasons for avoidance or hesitancy concerning the vaccine were suspicion on safety (84\%), on efficacy or quality (53\%), and on utility (26.5\%). Multiple multinomial regression showed that those having a high or very high perceived personal risk of being infected by the SARS-CoV-2 and those who underwent seasonal influenza vaccination were less likely to refuse the vaccine rather than those who have not yet decided about it with respective $\mathrm{OR}_{, 95 \%}[\mathrm{Cl}]: 0.420[0.178 .-0.992]$ and 0.076 [0.015-0.382].

Conclusion: The acceptance rate of COVID-19 vaccine in our study population was moderate. Therefore, a good communication and health education at the community level are needed to increase the COVID-19 vaccine coverage.
Disclosure of Interest: None declared.

\section{P109}

Vaccine hesitancy among healthcare providers during COVID-19

pandemic

F. Algabbani ${ }^{1{ }^{*}}$, A. Algabbani $^{1}$

${ }^{1}$ PSMMC, Riyadh, Saudi Arabia

Correspondence: F. Algabbani

Antimicrobial Resistance \& Infection Control 10(1): P109

Introduction: Public trust in vaccines is a major global health issue.

Objectives: This study aims to assess the vaccine acceptance among healthcare workers and their confidence and hesitancy of the COVID19 vaccine.

Methods: This was a multicenter cross-sectional survey conducted among healthcare providers in Riyadh, the capital of Saudi Arabia. Data collection was carried out between October and November of 2020 through a web-based survey. COVID19 vaccine hesitancy was assessed using eight structured items adapted from the $5 \mathrm{Cs}$.

Results: About $34.6 \%$ (95\% Cl: $27.6 \%>42.4 \%$ ) of participants were willing to vaccinate against COVID-19 and $44 \%$ (95\% Cl: $36.5 \%>51.9 \%)$ will recommend the vaccine to their patients. About $45 \%$ of participants were neutral regarding vaccine safety and $40 \%$ were neutral regarding vaccine effectiveness. Almost $70 \%$ believe that the duration of clinical studies of the COVID-19 vaccines affects their confidence in the efficacy and safety of the vaccine. Those who never hesitated or delayed taking any of the recommended vaccination were more likely to be willing to vaccinate COVID-19 (OR 5.46, 95\% Cl: 2.49-11.98).

Conclusion: Assessing the level of vaccine confidence in the population and associated factors will help implement an effective national vaccine program to enhance vaccination uptake and control COVID19 spread during this pandemic.

Disclosure of Interest: None declared.

P110

Social media and hesitancy towards SARS-COV2 vaccination among the Tunisian health professionals

C. Ben Nasrallah 1,** on behalf of 1, N. Zammit ${ }^{2}$ on behalf of 2, A. Brahem ${ }^{3}$ on behalf of 3, A. El Guedr ${ }^{2}$ on behalf of 2, I. Ayouni ${ }^{2}$ on behalf of 2, R. Ghammam $^{2}$ on behalf of 2, S. Ben Fredj ${ }^{2}$ on behalf of 2, C. Sridi ${ }^{3}$ on behalf of 3, A. Chouchene ${ }^{3}$ on behalf of $3, \mathrm{H}$. Kalboussi ${ }^{3}$ on behalf of 3, O. EIMaale $^{3}$ on behalf of 3, S. Chatti $i^{3}$ on behalf of 3, J. Maatoug ${ }^{2}$ on behalf of 2, N. Mrizak ${ }^{3}$ on behalf of $3, \mathrm{H}$. Ghannem ${ }^{2}$ on behalf of 2.

${ }^{1}$ Faculty of Medicine of Monastir, Monastir, ${ }^{2}$ Department of Epidemiology (LR19SP03), Farhat Hached University Hospital, ${ }^{3}$ Department of Occupational Medicine and environmental pathology (LR19SP03), Farhat Hached University Hospital, Faculty of Medicine of Sousse, University of Sousse, Sousse, Tunisia

Correspondence: C. Ben Nasrallah

Antimicrobial Resistance \& Infection Control 10(1): P110

Introduction: Health professionals are the leaders of the war against the SARS-COV2 pandemic. Their adherence to the vaccination against this new virus is crucial to ensure a sufficient coverage in the community.

Objectives: To evaluate hesitancy towards SARS-COV2 vaccines among the Tunisian health professionals and their use of information sources about these vaccines.

Methods: A cross-sectional study was led online between the $7^{\text {th }}$ and the $21^{\text {th }}$ of January 2021 among Tunisian health professionals. A number of at least 460 participants were required. Data were collected anonymously using a pre-established and pre-tested questionnaire recorded in a free Google form.

Results: A total of 493 responses were obtained. The mean age of participants was $37.4( \pm 9.5)$ years. Females represented $70.2 \%$ of participants. The rate of hesitancy towards COVID-19 vaccines was $51.9 \%$. Lack of information about the COVID19 vaccines was reported by $81.7 \%$ of participants. Social media were the most used source of information by them (66.9\%) while $7.9 \%$ of them used the national information site for health professionals. Use of social media more than two hours per day 
was positively associated with hesitancy towards vaccination against SARS-COV2 with an OR of 2.5 (95\% Cl: 1.5-4.2) contrary to the use of the national website for information for health professionals which was negatively associated with hesitancy with an OR of 0.5 (95\% Cl:0.2-0.9).

Conclusion: The current information strategy should be reinforced in Tunisia. Social media may represent a good channel for disseminating valid messages and tackling misinformation. Engaging health care professionals in social media to counter the vaccine related misinformation would boost the national information strategy.

Disclosure of Interest: None declared.

\section{P111}

COVID-19 vaccine perception and attitude among Tunisian healthcare workers

A. Maatouk ${ }^{1}$, A. Ammar ${ }^{1, *}$, O. Ezzi ${ }^{1}$, W. Bannour ${ }^{1}$, S. Chelly ${ }^{1}$, R. Helali', B.

Trabelsi ${ }^{1}$, M. Njah ${ }^{1}$, M. Mahjoub ${ }^{1}$

${ }^{1}$ Infection Prevention and Control department, University Hospital

of Farhat Hached, Sousse, Tunisia

Correspondence: A. Ammar

Antimicrobial Resistance \& Infection Control 10(1): P111

Introduction: During COVID-19 pandemic, vaccination is a required strategy to overcome this disease especially for healthcare workers (HCWs) given their increased risk of exposure to COVID-19. However, vaccination hesitancy is one of the significant obstacles to make this strategy successful.

Objectives: We aimed in this study to determine perception and attitude toward COVID-19 vaccine among HCWs in Tunisia, and factors associated with their acceptability of this vaccine.

Methods: We conducted a cross-sectional study among Tunisian HCWs from December 2021 to January 2021 using an online questionnaire submitted electronically using the most popular online groups of HCWs on Facebook. Data analysis was performed using the Statistical Package for Social Sciences (SPSS) version 21.0.

Results: A total of $257 \mathrm{HCWs}$ participated in the study with median age of $40[34 ; 50]$ years. Among them, $21 \%$ were tested positive to SARS-CoV-2. Almost $44 \%$ of the respondents were caring for COVID-19 patients. Almost a third of the respondents $(27.6 \%)$ had no intention to be vaccinated, and $21.8 \%$ were hesitant. COVID-19 vaccine avoidance or hesitancy was justified by the suspicion on safety $(92.1 \%)$, on efficacy or quality (66.1\%), and on utility (15.7\%). Most of the HCWs who had no intention to accept COVID-19 vaccine, did not receive seasonal influenza vaccination $(84.5 \%$ ) compared to $59.2 \%$ of those accepting the vaccine and $73.2 \%$ of hesitant HCWs $(p=0.001)$. Most of the HCWs who had the intention to accept COVID-19 vaccine were female (80\%) $(p=0.16)$ and had a high or very high perceived risk of being infected by the SARS-CoV- 2 in $60 \%$ of cases. Most HCWs who had the intention to refuse COVID-19 vaccine did not have serious cases of COVID-19 among their patients $(73.2 \%)(p=0.89)$.

Conclusion: Almost half of HCWs were either hesitant or not accepting COVID-19 vaccine in our study. This high percentage may be explained by the lack of communication and information about the vaccine and can represent an obstacle to the achievement of COVID-19.

Disclosure of Interest: None declared.

\section{P112}

\section{Predictors of COVID-19 vaccination refusal among Tunisian}

\section{healthcare personnel}

C. Ben Nasrallah ${ }^{1, *}$ on behalf of 1, A. Brahem ${ }^{2}$ on behalf of $2, \mathrm{~N}^{2}$ Zammit $^{3}$ on behalf of 3, I. Ayouni ${ }^{3}$ on behalf of 3, A. El Guedr ${ }^{3}$ on behalf of 3, S. Ben Fredj ${ }^{3}$ on behalf of 3, R. Ghammam ${ }^{3}$ on behalf of 3, A. Chouchene ${ }^{2}$ on behalf of 2, C. Sridi ${ }^{2}$ on behalf of 2, H. Kalboussi ${ }^{2}$ on behalf of 2, S. Chatti $^{2}$ on behalf of 2, O. ElMaalel ${ }^{2}$ on behalf of 2, J. Maatoug ${ }^{3}$ on behalf of 3, N. Mrizak ${ }^{2}$ on behalf of 2, H. Ghannem ${ }^{3}$ on behalf of 3

${ }^{1}$ Faculty of Medicine of Monastir, Monastir, ${ }^{2}$ Department of Occupational Medicine and environmental pathology (LR19SP03), Farhat Hached University Hospital, ${ }^{3}$ Department of Epidemiology (LR19SP03), Farhat Hached University Hospital, Faculty of Medicine of Sousse, University of Sousse, Sousse, Tunisia.

Correspondence: $C$. Ben Nasrallah

\section{Antimicrobial Resistance \& Infection Control 10(1): P112}

Introduction: Healthcare personnel (HCP) are at the frontline of the COVID-19 pandemic and are recognized as a priority target group for COVID-19 vaccines. However, the acceptance or refusal of vaccination among HCP is debated.

Objectives: To investigate the prevalence and the predictors of COVID-19 vaccination refusal among Tunisian HCP.

Methods: A cross sectional study was conducted using an anonymous online survey among Tunisian HCP from 7 to $21^{\text {th }}$ of January 2021. Primary endpoints were the intention to decline vaccination against COVID-19 if a vaccine was available. Logistic regression analysis was used to assess the factors associated with the intention to decline the COVID-19 vaccination.

Results: Of the 546 responses, 493 were retrieved. Among included HCP, 292 (59.2\%) were physicians, 53 (10.8\%) were paramedical personnel, $70(14.2 \%)$ were pharmacists and $78(15.8 \%)$ were dentists. Overall, 62 of 493 HCP (12.6\%) stated their intention to refuse vaccination. Logistic regression analysis revealed that being aged more than 40 years-old was a predictor of COVID-19 vaccination refusal among $\mathrm{HCP}$ with an adjusted odds ratio (AOR) of 2 (95\%Cl: 1.2-3.6). A history of vaccination against influenza during the current season and the use of television as a source of information about COVID-19 vaccination predicted a lower risk of refusing COVID-19 vaccination with AORs of $0.2(95 \% \mathrm{Cl}: 0.1-0.4)$ and 0.5 (95\%Cl: $0.3-0.8)$ respectively.

Conclusion: The current information campaign about COVID-19 vaccination should be reinforced among Tunisian HCP. Older ones may be involved in this campaign as leaders. The use of the official media seems to be a good communication channel for this campaign.

Disclosure of Interest: None declared.

\section{P113}

The impact of SARS-COV-2 and SARS-COV-2 vaccination on workforce absenteeism in an academic, tertiary care, four-hospital medical center

T. Lee ${ }^{1, *}$, S. McClanahan ${ }^{2}$

${ }^{1}$ Infection Prevention/ Employee Health, ${ }^{2}$ Infection Prevention/Employee Health, Charleston Area Medical Center, Charleston West Virginia, United States

Correspondence: $\mathrm{T}$. Lee

Antimicrobial Resistance \& Infection Control 10(1): P113

Introduction: We conducted prospective surveillance of workforce (WF) illness related to SARS-Cov-2 as routine Employee Health Department (EHD) activities. We then evaluated associated absenteeism and the introduction of SARS-Cov-2 vaccination on absenteeism patterns to determine the impact of those vaccinations.

Objectives: 1. Examine trends in absenteeism among medical center WF during the SARS-Cov-2 pandemic. 2. Demonstrate impact of SARSCov-2 vaccination on WF absences.

Methods: This was an observational descriptive study. Data related to WF illnesses, exposures, and positive SARS-Cov-2 test results were obtained using a questionnaire, telephone interviews, and review of laboratory records. Case investigation was started when the healthcare personnel (HCP), a laboratory, or public health worker reported a hospital WF exposure, illness, or positive test result to EHD or to Infection Prevention Department. Reason for WF absence was documented daily for each occurrence. Vaccination data were obtained via vaccination databases within the hospital or the State immunization database.

Results: Formal data collection began on 01/05/20. Daily absences continued to rise, with the highest peak of 193 in December 2020 On December 15, prior to community vaccination programs, prime doses of a two-dose SARS-Cov-2 vaccine (Pfizer-BioNTech) were administered to members of high-risk departments of the WF, with expanded WF vaccinations administered over the next 5 months. In the weeks following the first prime vaccinations, WF daily absences dropped to as low as 89 but began to increase until just after the booster doses of vaccine were begun on $5 / 01 / 21$. By $1 / 03 / 21$, daily absences had decreased to between 9 and 35. A total of 103 SARS-Cov- 2 cases occurred among WF between 19/02/21 and 25/05/21, the majority of which were among WF 
not vaccinated. Of those cases, 17 were breakthrough cases (among fully vaccinated WF) but were clinically mild events.

Conclusion: Absenteeism due to SARS-Cov-2 was reduced and sustained when WF SARS-Cov- 2 vaccinations were administered.

Disclosure of Interest: None declared.

\section{P114}

\section{Seroprevalence in Argentinian health care workers}

after vaccination with Sputnik V

D. Santonato ${ }^{1, *}$, A. Malvicini ${ }^{1}$, A. Novau' ${ }^{1}$, S. Torres ${ }^{2}$, A. Siaba ${ }^{2}$, L. Pereyra ${ }^{3}$, V. Romano ${ }^{4}$, P. Brenzoni ${ }^{4}$, W. Cornistein ${ }^{4}$

${ }_{1}^{1}$ Infection Control Department, ${ }^{2}$ Paediatric Intensive Care Unit, ${ }^{3}$ Infectious Diseases, ${ }^{4}$ Central Laboratory, Hospital Universitario Austral, Pilar, Argentina

Correspondence: D. Santonato

Antimicrobial Resistance \& Infection Control 10(1): P114

Introduction: Health care workers (HCW) are at increased risk of Coronavirus Disease 2019 (COVID-19). It is essential to guarantee protection in this population. In Argentina, the vaccination campaign began on December 29, 2020 and HCW were among first beneficiaries. The first available vaccine was Sputnik V. Previous studies showed efficacy over $91 \%$. We aimed to corroborate this.

Objectives: To determine presence and quantity of Anti-RBD IgG antibodies in HCW at Hospital Universitario Austral.

Methods: Seroprevalence study in HCW 3 or more weeks after the second dose of Sputnik V. We excluded those with history of COVID19 (positive RT-PCR, positive IgG before vaccination, symptoms not evaluated). Participants had a blood sample taken and processed using Abbott ${ }^{\circledR}$ SARS-CoV-2 IgG II Quant. A chemiluminescent microparticle immunoassay (CMIA) used for qualitative and quantitative determination of IgG antibodies to SARS-CoV-2 on the Alinity and ARCHITECT i Systems. Quantitative results were used in analyses. As per manufacturer's instructions, a value $\geq 50 \mathrm{AU} / \mathrm{ml}$ was considered positive. Descriptive analysis; Stata 10.0 Software.

Results: Characteristics of the cohort (Table 1). All of the participants developed Anti-RBD IgG. Antibody concentrations (Picture 1). No difference was found in antibody concentrations between 20 to 40 days versus 40 to 100 days from second vaccine dose.

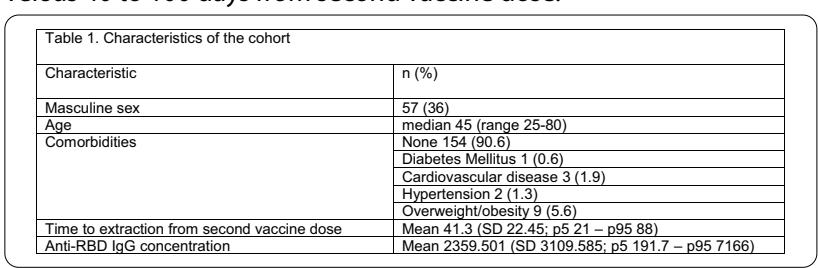

Figure:

Table 1. Time to extraction from second vaccine dose

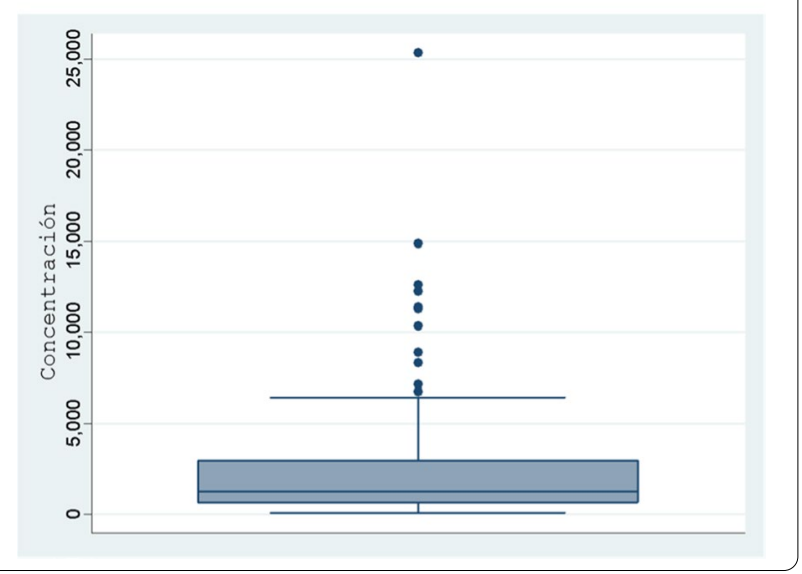

Conclusion:: The entire cohort developed antibodies after immunization with Sputnik $V$ with no difference in concentration between extraction 20-40 days from second dose and more than 40 days. Further studies are needed to determine duration of immunity, influence of variants of concern, and to establish concentrations of IgG correlated with neutralizing antibodies.

Disclosure of Interest: None declared.

P115

Effect of COVID-19 vaccination on prevention of COVID-19 in a tertiary care center in Lebanon

J. Tannous ${ }^{1}$, N. Kara Zahreddine ${ }^{1}$, C. Sakr ${ }^{2}$, A. Ibrahim¹', R. Ahmadieh ${ }^{1}$, R. Mahfouz ${ }^{3}$, U. Musharrafieh ${ }^{4}$, S. Kanj $j^{5, *}$

${ }^{1}$ Infection Control and Prevention Program, ${ }^{2}$ Employee Health Unit, ${ }^{3}$ Pathology and Laboratory Medicine, ${ }^{4}$ University Health Services, ${ }^{5}$ Infectious Diseases, AMERICAN UNIVERSITY OF BEIRUT MEDICAL CENTER, Beirut, Lebanon

Correspondence: S. Kanj

Antimicrobial Resistance \& Infection Control 10(1): P115

Introduction: Lebanon received its first batch of Pfizer-BioNTech vaccines on February 13, 2021 to launch a free nationwide vaccination campaign led by the Ministry of Public Health. The country has suffered an increase in cases and fatalities after the Beirut explosion in August 4, 2021.

Objectives: We analyzed the efficacy of vaccination on preventing COVID-19 among staff at the American University of Beirut Medical Center (AUBMC).

Methods: The COVID-19 vaccination campaign started in different centers including AUBMC on February 14,2021. Two doses of vaccine were administered at 3 weeks interval. Infection Control (IC) Program and Employee Health Unit (EHU) continued to raise awareness and investigate COVID-19 infections among staff. Positivity of cases is confirmed by antigen and/or PCR testing.

Results: Till May 26, 2021, Lebanon reported 538,991 COVID cases ( $8 \%$ of population) and 7,690 deaths. While at AUBMC, a total of 1,133 (29\%) staff developed COVID-19. To date, $8.6 \%$ of the population received the first dose of the vaccine, and $4.5 \%$ received the second dose. Eighty five percent of the hospital staff received the first dose and $80 \%$ received the second dose. The trend depicting COVID-19 cases at AUBMC followed the one observed in Lebanon. Following the end of year's celebrations an increase of cases was observed at AUBMC and in Lebanon during January. Inversely, we observed a decrease in the number of positive cases in May 2021 nationwide and at AUBMC.

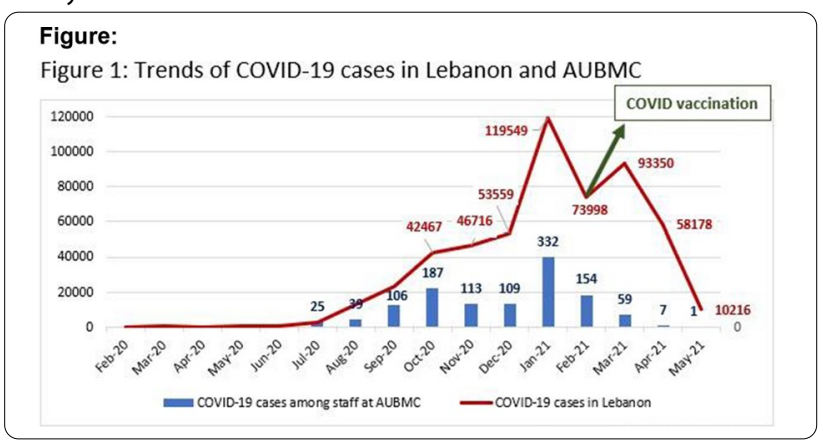

Conclusion: The trend in COVID-19 cases declined at the national level starting April 2021 similar to our trend. The decline at AUBMC was more pronounced most likely because of the rapidity of the immunization as most of the workforce was vaccinated within 4 weeks. The spread of the virus in Lebanon decreased due to vaccination and the natural immunization secondary to the infection. IC practices, mandatory masking, hand hygiene, and physical distancing, remain instrumental in halting COVID19 transmissions in the community in view of the slow vaccination rollout in Lebanon.

Disclosure of Interest: None declared. 
P116

Decrease in demand for hospital beds in a tertiary care center in Lebanon following COVID-19 vaccination and governmental lockdown measures

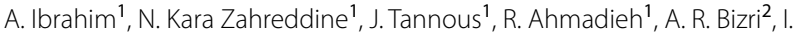
Bou $\mathrm{Akl}^{3}$, S. Kanj ${ }^{1,2, *}$

${ }^{1}$ Infection Control and Prevention Program, ${ }^{2}$ Infectious Diseases, ${ }^{3}$ Department of Internal Medicine, AMERICAN UNIVERSITY OF BEIRUT MEDICAL

CENTER, Beirut, Lebanon

Correspondence: S. Kanj

Antimicrobial Resistance \& Infection Control 10(1): P116

Introduction: The outbreak of COVID-19 in Lebanon created substantial demand for hospital beds. Hospitals were bound to equip units to admit such patients. A national vaccine rollout was planned by the Ministry of Public Health (MOPH).

Objectives: We evaluated the impact of a COVID-19 vaccination campaign and governmental measures on reducing hospitalization and mortality among COVID-19 patients at a tertiary care center in Lebanon.

Methods: Nationwide lockdown measures were imposed following a rise in COVID-19 cases. Our facility, a tertiary care center, designated a building in March 2020 to accommodate COVID-19 patients (regular and critical). During the second week of February 2021, the COVID-19 vaccination rollout started. Two doses of Pfizer-BioNTech vaccine were administered at 3 weeks interval. Other vaccines such as Oxford-AstraZeneca and Sputnik V were administered by the private sector.

Results: In Lebanon, the daily number of COVID-19 cases showed a rapid increase in January 2021. The highest percentages were recorded during the first quarter of 2021 with $7 \%$ infections and $6 \%$ mortality. At AUBMC, $100 \%$ bed occupancy with increased mortality were noted in the COVID-19 dedicated units during the same period. The drop in cases started to appear when the country reached $4 \%$ to $7 \%$ vaccinated persons with first and second dose, respectively. In May 2021, bed occupancy continued to drop and the pattern was comparable to the decrease in new COVID-19 cases and deaths at the national level.
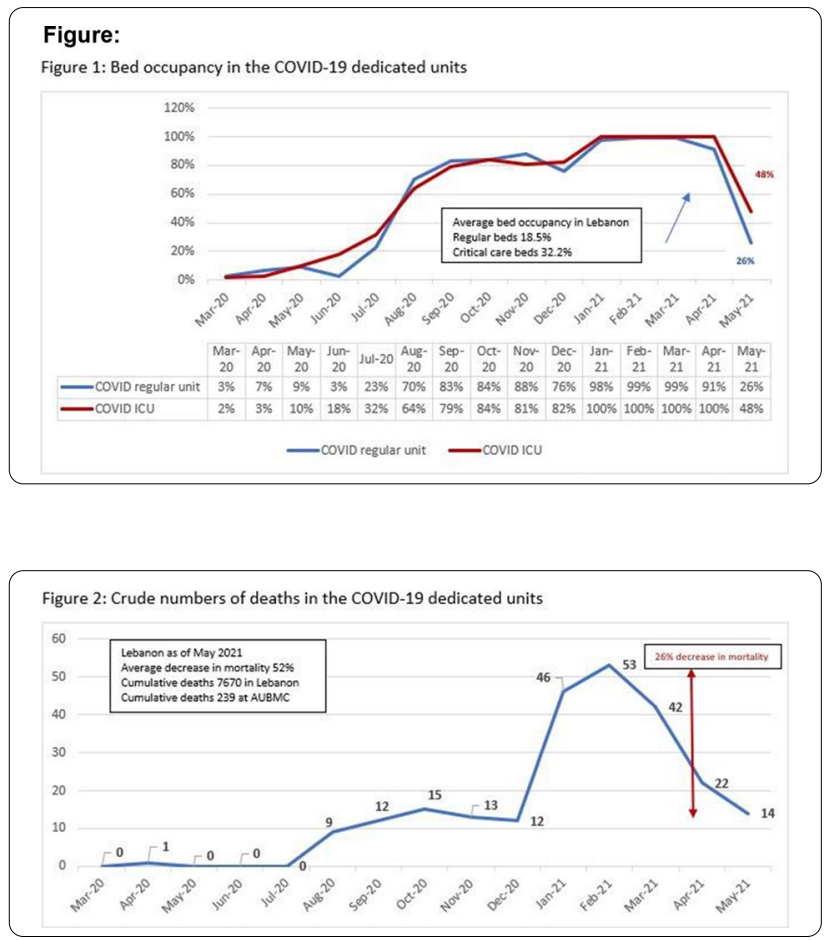

Conclusion: The trends illustrating occupied beds and mortality rates in Lebanon were similar to our trends at AUBMC. The decline in cases and in deaths is primarily related to offering the vaccines based on occupation, age and medical condition. Improving the preparedness at our center is a lesson learnt in the event of another threat. Despite the decline in cases, hospital staff are at risk. The continued importance of masking is mandatory and indispensable in poorly ventilated areas or crowded places.

Disclosure of Interest: None declared.

Poster session: COVID-19 among healthcare workers

P117

Is the daily use of public transport facilities an enhanced epidemiological risk for diagnosing COVID-19 among healthcare workers with mild respiratory symptoms?

L. Steinwender ${ }^{1}$, D. Holy ${ }^{1}$, J. Burkhard ${ }^{1}$, I. Uçkay ${ }^{1, *}$

${ }^{1}$ Balgrist University Hospital, Zürich, Switzerland

Correspondence: I. Uçkay

Antimicrobial Resistance \& Infection Control 10(1): P117

Introduction: Many people in Switzerland think that the daily use of the public transport facilities, during a pandemic peak wave, could be associated with an enhanced risk for Covid-19.

Objectives: To investigate the association between the daily use of the public transport and acquisition of Covid-19 in Zurch during a peak wave of the pandemics.

Methods: Retrospective cohort analysis of hospital-own databases at the Balgrist University Hospital in Zurich, between 1 October and 31 December 2020. We specifically interviewed our healthcare workers (HCW) about the use of public transport (with mandatory masking). Results: During the three most intensive months of the $2^{\text {nd }}$ wave, we investigated 376 episodes of Covid-19-compatible respiratory symptoms and exposures among our HCW (median age 37 years), of which 94 (25\%) revealed a positive PCR result for Covid-19. Overall, $225 \mathrm{HCW}$ (225/376; 60\%) reportedly used the public transport (train, bus, tramway). In group comparison, the HCWs using the public transport system acquired no more Covid-19 than using a private transport (58/225 vs. 36/151; Pearson-chi ${ }^{2}$-test; $p=0.67$ ).

We added a logistic regression model with the outcome "Covid-19 infection" to adjust for the case-mix of different localizations or opportunities of potential contamination such as a documented exposure in the hospital, among the team members, in the family, or after a close contact to a PCR-confirmed case. In the multivariate results, using the public transport was irrelevant concerning the acquisition of Covid19 (odds ratio (OR) $0.98,95 \% \mathrm{Cl} 0.59-1.62$ ), in contrast, for example, to being exposed to a sick team member (OR 2.28, 95\%Cl 1.20-4.34).

Conclusion: In Zurich, the daily use of public transport was not associated with an additional risk of being diagnosed with Covid-19 among the young population of HCWs, not even during the peak of a pandemic wave. Other factors are more relevant.

Disclosure of Interest: None declared.

P118

Risk of incident SARS-COV-2 infection among healthcare workers residing in Egyptian quarantine hospitals

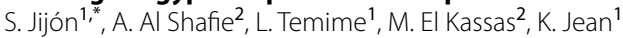

${ }^{1}$ Laboratoire MESuRS, Conservatoire National des Arts et Metiers, Paris,

France, ${ }^{2}$ Faculty of Medicine, Helwan University, Cairo, Egypt

Correspondence: S. Jijón

Antimicrobial Resistance \& Infection Control 10(1): P118

Introduction: In response to the COVID-19 epidemic, Egypt established a unique care model based on quarantine hospitals where only externally-referred confirmed COVID-19 patients were admitted, and healthcare workers (HCWs) resided continuously over 1- to 2-week working shifts. 
Objectives: To estimate the risk of COVID-19 infection among HCWs in quarantine-hospital settings and assess the relative contribution of HCW-to-HCW (HtoH) and patient-to-HCW (PtoH) transmissions.

Methods: Detailed longitudinal data was collected in two Egyptian healthcare facilities (hereafter denoted by Hosp1 and Hosp2), during the 2020 first wave of the COVID-19 epidemic (Hosp1: March $14^{\text {th }}$-August 1st; Hosp2: June $6^{\text {th }}$-July 11 th). In both hospitals, only HCWs with no SARS-CoV-2 antibodies were allowed to start working shifts. During shifts, HCWs were tested using RT-PCR on nasopharyngeal swabs: i) routinely at the end of the shift, ii) upon symptoms, and iii) in case of outbreak suspicion ( $>2$ positive tests among HCWs).

Using a stochastic compartmental model for the spread of SARS-CoV-2 in each hospital, we assessed the risk of SARS-CoV-2 acquisition overall and by transmission route ( $\mathrm{HtoH}$ vs $\mathrm{PtoH})$. We estimated the model parameters using Markov Chain Monte Carlo approaches.

Results: Over a total follow-up of 6,601 person-days (PD), we estimated an incidence rate of 0.97 (95\% Crl: 0.56-1.53) per 100 PD at Hosp1 and 8.98 (95\% Crl: 3.81-17.75) per 100 PD at Hosp2. The probability for a HCW to be infected at the end of a shift was $12.8 \%$ (95\% Crl: 7.6\%-19.5\%) for a 2-week shift at Hosp1, which lies within the range of risk levels previously documented in standard healthcare settings, whereas it was >threefold higher for a 7-day shift at Hosp2 $(48.2 \%$, $95 \% \mathrm{Crl}: 23.8 \%-74.5 \%)$. Infection risk was mostly driven by HtoH transmission in both hospitals, although a substantial contribution from PtoH transmission was also found in Hosp2.

Conclusion: The large variation in the infection risk found between the two quarantine hospitals we studied suggests that HCWs may face a high risk of infection, but that, with sufficient anticipation and infection control measures, especially those preventing patient-to-HCW transmission, this risk can be brought down to levels similar to those observed in standard healthcare settings.

Disclosure of Interest: None declared.

\section{P119}

Risk of COVID-19 in healthcare workers working in intensive care setting

M. Alshamrani1 ${ }^{1,}$, A. El-Saed ${ }^{1}$, Y. Arabi ${ }^{2}$, M. Al Zunitan ${ }^{3}$, F. Farahat ${ }^{1}$, M.

Matalqa ${ }^{1}$, S. Almohrij ${ }^{4}$

${ }^{1}$ Infection control, ${ }^{2}$ Critical Care Medicine, King Abdulaziz Medical City,

${ }^{3}$ Infection control, King Abdullah Specialist Children Hospital, ${ }^{4}$ Chief

medical office, King Abdulaziz Medical City, Riyadh, Saudi Arabia

Correspondence: M. Alshamrani

Antimicrobial Resistance \& Infection Control 10(1): P119

Introduction: The higher risk of COVID-19 in healthcare workers (HCWs) is well-known. However, the risk within HCWs is not fully understood.

Objectives: The objective was to compare the COVID-19 risk in intensive care unit (ICU) versus non-ICU locations. Additionally, to examine the interaction between hospital locations and professional category.

Methods: A prospective surveillance study was conducted among HCWs at a large tertiary care facility in Riyadh between March 1st to November 30th, 2020. HCWs included both clinical (provide direct patient care) and non-clinical positions (do not provide direct patient care).

Results: A total 1,594 HCWs with COVID-19 were included; 103 (6.5\%) working in ICU and 1491 (93.5\%) working in non-ICU locations. Compared with non-ICU locations, ICU had more nurses $(54.4 \%$ versus $22.1 \%, \mathrm{p}<0.001)$ and less support staff $(2.9 \%$ versus $53.1 \%, \mathrm{p}<0.001)$. COVID-19 infection was similar in ICU and non-ICU locations $(9.0 \%$ versus $9.8 \%, p=0.374)$. However, it was significantly higher in ICU nurses $(12.3 \%$ versus $6.5 \%, p<0.001)$. Support staff had higher risk than other HCWs, irrespective of ICU working status $(15.1 \%$ versus $7.2 \%, p<0.001$ ). The crude relative risk (RR) of COVID-19 in ICU versus non-ICU locations was $0.92,95 \%$ confidence interval [Cl] was $0.76-1.11$ $(p=0.374)$. However, RR adjusted for professional category was significantly increased to $1.23,95 \% \mathrm{Cl} 1.01-1.50(p=0.036)$.

Conclusion: ICU had a significantly higher risk of COVID-19 infection only after adjusting for the distribution and risk of different professional categories. The finding underscores the importance of strict implementation of preventive measures among all HCWs, including those performing non-clinical services.

Disclosure of Interest: None declared.

\section{P120}

Assessment the risk factors for SARS-COV-2 infection in a tertiary hospital healthcare worker

W. A. Mazi ${ }^{1, *}$, S. Abdelhafeez ${ }^{1,2}$, W. Althubaiti ${ }^{3}$, A. Felimban ${ }^{1}$

${ }^{1}$ Infection Prevention and Control, King Abdulaziz Specialist Hospi-

tal-Taif, ${ }^{2}$ Infectious Disease Control Directorate, Directorate of Health

Affairs, ${ }^{3}$ Training and Education Department, King Abdulaziz Specialist

Hospital-Taif, Taif, Saudi Arabia

Correspondence: W. A. Mazi

Antimicrobial Resistance \& Infection Control 10(1): P1 20

Introduction: The spread of severe acute respiratory syndrome coronavirus 2 (SARS-CoV-2) is accompanied by uncertainty its ability to spread in the human population and its virulence. In May 2020, World Health Organization (WHO) released a protocol assessment of risk factors for coronavirus disease 2019 (COVID-19) in health workers.

Objectives: To characterize and assess the risk factors for COVID-19 infection in a tertiary hospital health worker.

Methods: A descriptive cross sectional study was conducted in a tertiary hospital among 62 nasopharyngeal swabs confirmed infected with COVID-19 healthcare workers from July and August 2020, Saudi Arabia. Demographic, adhesion to prevention measures, pre-exciting condition and symptoms data were collected and analyzed as demonstrated by the WHO protocol. Hospital COVID-19 acquired infection was defined as the onset of clinical features of COVID-19 7 days or more after admission or between days 3 and 6 after admission if epidemiologically linked to hospital exposure.

Results: About $61 \%$ Nurses with mean age 34 years old were the major exposed to COVID-19 infection. $37 \%$ were considered as hospital acquired COVID-19 infection. About $66-72 \%$ of victims had headache, muscle ache, fatigue and sore throat, $51-58 \%$ had loss of small, fever, diarrhea, cough and lose of appetite. Obesity was the most frequent $(\mathrm{N}=6,9.7 \%)$ followed by diabetes mellitus and asthma $(\mathrm{N}=3,4.8 \%$ of each). All healthcare workers were fully protected when dealing with the patient, however $20 \%$ of healthcare workers were not sure about wearing surgical mask when contacted with colleagues. Fortunately, $98 \%$ had mild infection with home or quarantine isolation and recovered.

Conclusion: COVID-19 is a community-based infection affects all ages. Although all healthcare workers were fully protected during patient care, acquiring COVID-19 infection could be expected during either from the community or within the hospital environment from asymptomatic carriers of healthcare workers and consequences spread between healthcare workers in a short time period. Minor symptomatic healthcare workers should be considered for suspected of SARSCoV-2 carrier and early laboratory detection is highly recommended to avoid the spread on COVID-19 infection between healthcare workers.

Disclosure of Interest: None declared.

P121

Risk factors of COVID-19 in frontline healthcare workers in Mashhad university hospitals

A. Saeidinia ${ }^{1}$, A. Khorsand Vakilzadeh ${ }^{2}$, L. Golnari ${ }^{3}$, G. Ghanbari ${ }^{1}$, B. Bonyadi ${ }^{3}$, M. H. Aelami, ${ }^{3, *}$

${ }^{1}$ Pediatrics, ${ }^{2}$ Acupuncture, ${ }^{3}$ Infection Control and Hand Hygiene Research Center, Imam Reza Hospital, MASHHAD UNIVERSITY OF MEDICAL SCI-

ENCES, Mashhad, Iran, Islamic Republic Of

Correspondence: M. H. Aelami

Antimicrobial Resistance \& Infection Control 10(1): P121

Introduction: After emerging COVID-19, it became a worldwide concern. Healthcare workers (HCWs) are at high risk of getting infection and they have the capability to transmit virus to patients and also to coworkers. There are few studies about risk factors of frontline HCWs. 
Objectives: In this study, we evaluated the risks of infection in frontline HCWs in Mashhad university hospitals.

Methods: This was a cross sectional study in HCWs of Mashhad University Hospitals between March till July 2020. Participants were asked by telephone about the risk factors of getting COVID-19 infection. The checklist was researcher-made and was on the basis of WHO risk factors regarded to COVID-19, MERS and SARS.

Results: In this study, 534 HCWs with positive PCR test for COVID19 were enrolled. The response rate in our study was $64.04 \%$. The mean age of participants was $36.02 \pm 8.5$ years. Among them 176 and 239 were participated in infection control workshop and hand hygiene workshop before COVID-19 pandemic respectively. Most of the frontline HCWs were nurse. Malaise $(269,78.7 \%)$, fever $(233,68.1)$ and headache $(189,55.3)$ were the most common symptoms. Having underlying disease was related to hospitalization $(P=0.012)$. Being smoker and participating in intubation procedure were independent predictors of hospitalization in HCWs.

Conclusion: Frontline HCWs had a significantly increased risk of COVID-19 infection. Although adequate supplies of PPE were necessary, it did not completely mitigate high-risk exposures. Being smoker and participating in intubation procedure were independent predictors of hospitalization in HCWs.

Disclosure of Interest: None declared.

\section{P122}

\section{SARS-COV-2 Risk factors in health care workers: a systematic} review

C. Ben Nasrallah ${ }^{1, *}$ on behalf of 1, I. Zemni ${ }^{1}$ on behalf of 1, M. Kacem on behalf of 1, A. Ben Cheikh ${ }^{2}$ on behalf of 2, W. Dhouib' ${ }^{1}$ on behalf of 1 , D. Ben Hassine ${ }^{1}$ on behalf of $1, H$. Abroug ${ }^{1}$ on behalf of 1, M. Ben Fredj ${ }^{1}$ on behalf of 1,1 . Bouanène ${ }^{1}$ on behalf of $1, A$. Belguith Sriha ${ }^{1}$ on behalf of 1

${ }^{1}$ Department of Preventive Medicine and Epidemiology, Fattouma Bourguiba University Hospital, Monastir, ${ }^{2}$ Department of Prevention and Care Safety, Sahloul University Hospital, Sousse, Tunisia

Correspondence: C. Ben Nasrallah

Antimicrobial Resistance \& Infection Control 10(1): P122

Introduction: Health-care workers (HCWs) are at the frontline of response to coronavirus disease 2019 (COVID-19) and subsequently at higher risk of acquiring the disease.

Objectives: We aimed to investigate SARS-CoV-2 risk factors among HCWs using a systematic review.

Methods: A systematic review was carried out from January 2020 to $20^{\text {th }}$ March 2021 on COVID-19 risk factors among HCWs in PubMed and Google Scholar. Medical subject headings (MeSH) were searched using Boolean operators "OR/AND". The search terms were: ("coronavirus infection" OR "COVID-19" OR "SARS-CoV-2")" AND ("health personnel") AND ("risk factors" OR "risk assessment"). We included in our review only papers published in peer-reviewed journals.

Results: Twenty-one articles were included in this review. The main associated factors of COVID-19 infection among HCWs were personal protective equipment (PPE) shortage, exposure to infected patients mainly through working in high-risk departments, aerosol generating procedures, working overload, lack of knowledge on SARS-CoV-2 infection control and suboptimal hand hygiene. Our review showed a higher risk of infection among physicians compared with nurses and general services employees. Pre-existing medical conditions, age and male gender were also associated with COVID-19 infection among HCWs.

Conclusion: Lack of PPE, exposure to infected patients, work overload, poor infection control, and preexisting medical conditions put HCWs at risk of COVID-19 infection. Identifying these factors is of paramount importance to develop sustainable measures that protect HCWs especially those with higher risk levels.

Disclosure of Interest: None declared.
P123

Risk factors and transmission dynamics of COVID-19

among resident doctors-a retrospective analysis in a non-COVID tertiary care centre

V. K. HB ${ }^{1}$, E. Manuel $\left.\right|^{2, *},{ }^{\prime}$. $S^{1}$

${ }^{1}$ Neuromicrobiology, National Institute of Mental Health and Neurosciences, ${ }^{2}$ Neuromicrobiology, NIMHANS, Bengaluru, India

Correspondence: E. Manuel

Antimicrobial Resistance \& Infection Control 10(1): P123

Introduction: The national registry of Indian Medical Association (IMA) shows that 747 doctors have died of COVID 19 as on 1.4.2021 \& there is paucity of data on the risk factors for COVID 19 among healthcare workers from India.

Objectives: To identify the risk factors and challenges in breaking the chain of infection among COVID infected resident doctors.

Methods: A cross sectional study using a structured questionnaire to interview 67 COVID affected resident doctors, was done at National Institute of Mental Health and Neurosciences, a non-COVID tertiary healthcare centre, Bengaluru, India, during March 2020 to April 2021. The data was analysed statistically using the SPSS software version 17. Results: 67 resident doctors participated, with a median age of 29 years Male to female ratio was 3:2. Patient-facing resident doctors $(91 \%)$ were affected more than the nonpatient-facing( $9 \%) 0.55 \%$ were fully vaccinated against COVID, $9 \%$ infected after first dose, $3 \%$ infected within 14 days of the second dose. $33 \%$ were unvaccinated All were symptomatic and $97 \%$ resumed duty after 14 days of isolation. No mortality reported.

Table 1: Summary of risk factors \& challenges in breaking the chain of infection
\begin{tabular}{|l|l|}
\hline Risk factors & Percentage \\
\hline Prioritised practices in the emergency room & 24 \\
\hline Long(>6) working hours & 39 \\
\hline Overcrowding & 3 \\
\hline Inadequate ventilation & 3 \\
\hline Care of psychiatry patients & 3 \\
\hline Interviewing patient bystanders (Psychiatry OPD \& wards) & 3 \\
\hline${ }^{*} 25 \%$ did not identify any risk factor. & \\
\hline Challenges & $\%$ \\
\hline Compromised social distancing in the hospital & 45 \\
\hline Inadequate work distribution & 21 \\
\hline Delay in testing & 15 \\
\hline
\end{tabular}

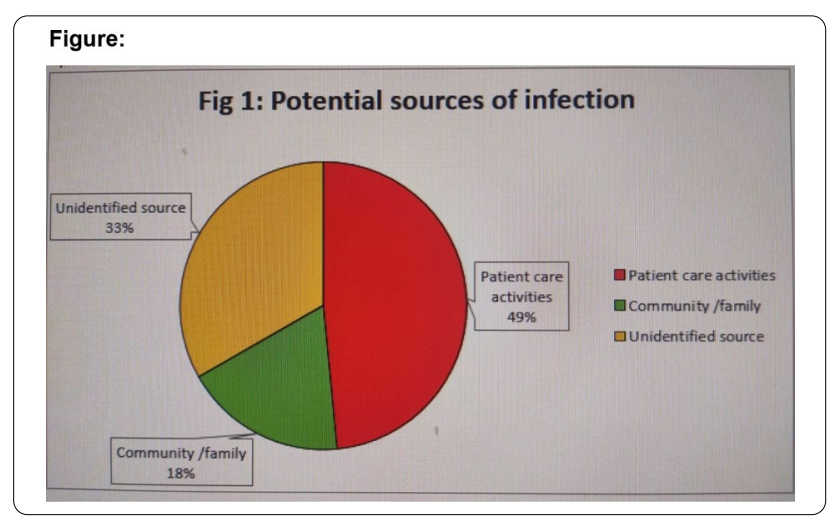

Conclusion: The above observations expose the vulnerability of resident doctors in contracting the infection during patient care due to longer exposure, unavoidable suboptimal compliance to infection prevention \& control practices, inadequate work redistribution strategies etc. 
The analysis of these risk factors is pointing to the need for more effective interventions and occupational health policies. Limitations of the study is that, being a non-covid speciality, the outcome cannot be extrapolated onto a covid care facility.

Disclosure of Interest: None declared.

\section{P124}

Clustering of COVID-19 infections among healthcare workers: experience from a tertiary care center in Saudi Arabia B. S. Molaeb ${ }^{1, *}$, M. Saad ${ }^{1}$, Z. Almoosa ${ }^{2}$ on behalf of Infection Prevention and Control Department: Dr. Ahmed Sanad, Mrs. Shaymaa El Gamal, Mr. Fadi Mahmoud, Mrs. Bindu Sureendran, Mrs. Carmela Maranon, Mrs. Basma Mowafy

${ }^{1}$ Infection Prevention and Control Department, ${ }^{2}$ Academic Affairs Department, Almoosa Specialist Hospital, Al-Ahsa, Saudi Arabia

Correspondence: B. S. Molaeb

Antimicrobial Resistance \& Infection Control 10(1): P124

Introduction: Healthcare workers (HCWs) face an increased risk of infection with the novel coronavirus disease (COVID-19) when effective infection prevention and control (IPC) measures are not carefully implemented. Little is known about the COVID-19 acquired infections in HCWs and how clusters of infections occur inside and outside hospitals.

Objectives: This study aims to provide an overview of the sources of COVID-19 infections in HCWs with emphasis on the identified clusters and highlight the measures taken to mitigate further transmissions at our institution during the COVID-19 pandemic.

Methods: We conducted a retrospective non-interventional study of all HCWs who were diagnosed with COVID-19 infection based on real-time reverse transcription polymerase chain reaction $(P C R)$ test. Clusters were defined as 2 or more COVID-19 infected HCWs with a unique source exposure. Cases were included from April 1, 2020 until February 28, 2021. Contact tracing, active screening and IPC surveillance were performed to investigate all cases and guide effective IPC measures to contain COVID-19 spread among exposed HCWs.

Results: A total of $327 \mathrm{HCWs}$ were diagnosed with COVID-19 infection; the majority of cases (58\%) were community-acquired. Ten clusters involving $55 \mathrm{HCWs}$ were identified. Nine clusters were related to HCWs who shared hospital accommodations (34 HCWs) and had social/personal interactions such as sharing meals and gathering in same place (19 HCWs). One cluster involved 2 HCWs who were exposed to a COVID-19 patient and was a consequence of failing to abide with IPC measures. None of the clusters occurred in the COVID-19 designated units in which HCWs were more adherent to the IPC measures. Active screening and effective IPC plans (including universal masking, optimized cleaning, and raising awareness) resulted in preserving continuity of service during the ongoing pandemic. No transmissions from HCWs to patients occurred.

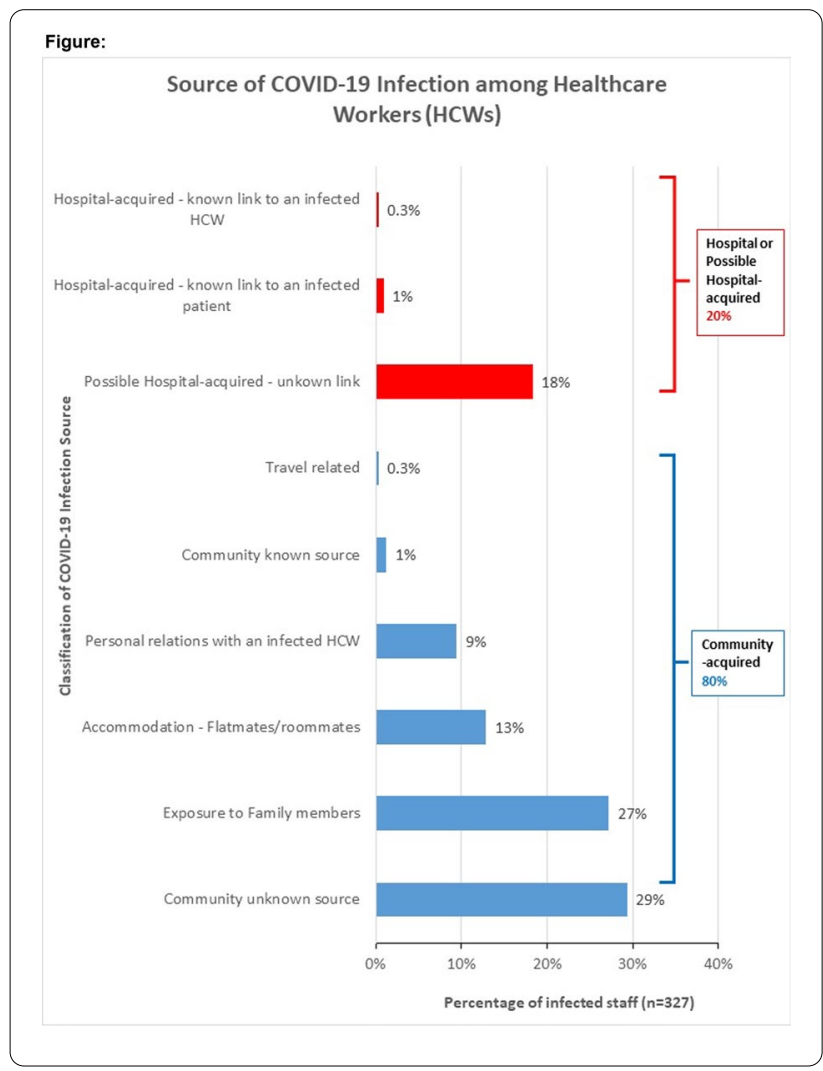

Conclusion: Clustering of COVID-19 infection was common among HCWs in our setting. Working in COVID-19 designated wards was not associated with an increased risk of acquiring COVID-19 infection. Individual-level compliance among HCWs in addition to an integrated surveillance strategy and outbreak management are crucial to support the ongoing COVID-19 response.

Disclosure of Interest: None declared.

P125

COVID-19 In healthcare workers: an estimate of the rate of infection in a population sample from Saudi Arabia R. Alazmi ${ }^{1, *}$, M. AlAhmari ${ }^{2}$, H. Alyousif ${ }^{1}$, S. Alsuwaie ${ }^{1}$, Z. Alabdrabalnabi $^{1}$

${ }^{1}$ Infection Preventionist, ${ }^{2}$ Respiratory Care, Eastern Health Cluster, Dammam, Saudi Arabia

Correspondence: R. Alazmi

Antimicrobial Resistance \& Infection Control 10(1): P125 
Abstract video clip description: Introduction: Coronavirus disease 2019 (COVID-19) is a devastating ongoing pandemic that has led to millions of deaths in the world. Healthcare workers (HCW) on the frontline have also lost their lives and have been at the highest risk of infection compared to the general population [1]. This is the first study to estimate the rate of infection among $\mathrm{HCW}$ in the Eastern Province of Saudi Arabia.

Methods: This is a retrospective cross-sectional study of HCWs working at the Eastern Health Cluster in the Eastern Province of Saudi Arabia. Subjects diagnosed with COVID-19 were identified from the health cluster database and from phone interviews of HCWs with laboratory-confirmed SARS-CoV-2 infection. Data related to the possible source of infection, socio-demographics, the containment zone, the type of exposure, and symptoms were collected. The data were analyzed using GraphPad Prism 9 software. The descriptive statistics for the categorical variables were given as frequencies and percentages (n [\%]).

Results: A total of $4104 \mathrm{HCWs}$ were included in this study. Most of the HCWs (87.1\%) with COVID-19 were working in hospitals, and $13.4 \%$ were working at primary health care facilities (Table 1). The majority (82.1\%) of the HCWs with COVID-19 were symptomatic, and $17.9 \%$ were asymptomatic, with only about $2.41 \%$ having a travel history. Table 2 shows that $79 \%$ of the HCWs acquired the virus in the community, while only $15 \%$ were infected at the hospital. Of those who were infected at the hospital, $62 \%$ were infected by co-workers, while $38 \%$ were infected by patients. Due to the country's lockdown, only $2.4 \%$ carried COVID-19 from other countries in the world.

Conclusions: Health care workers are considered to have the highest risk of getting SARS-CoV-2. In this study, the majority of infected HCWs acquired the infection in the community and suffered from mild symptoms.

\section{References}

1. Transmission of SARS-CoV-2: implications for infection prevention precautions: scientific brief, 09 July 2020 [Internet]. Geneva: World Health

Organization; 2020. (https://apps.who.int/iris/handle/10665/333114,acces sed 22 May 2021).

Disclosure of Interest: None declared.

\section{P127}

Impact of the hospital triage system on SARS-COV2 infection among frontline health personnel in Kinshasa, Democratic Republic of Congo (DRC).

B. Houndjo ${ }^{1, *}$, N. T. Guinko ${ }^{2}$, R. OKUM ${ }^{3}$, P. Mirindi ${ }^{4}$

${ }^{1} \mathrm{IPC}, \mathrm{WHO}$, Conakry, Guinea, ${ }^{2} \mathrm{WHO}$, kinshasa, Congo, The Democratic Republic of the, ${ }^{3} \mathrm{IPC}, \mathrm{WHO}$, kinshasa, Congo, The Democratic Republic of the, ${ }^{4} \mathrm{CIP}(\mathrm{IPC})$, World Health Organization, Conakry, Guinea

Correspondence: B. Houndjo

Antimicrobial Resistance \& Infection Control 10(1): P127

Introduction: In the fight against the pandemic, no country knows the exact number of SARS-CoV-2 infected people, especially among health workers: infections in healthcare account for $12-29 \%$ of cases $(1,2)$, and the SARS-Cov-2 severity and mortality in healthcare settings are higher than in the community (3). Thus, health Personnel constitutes a priority of infection prevention and control activities when implementing a triage system.

Objectives: Describe the impact of implementing a triage system at health facility level on health workers SARS-CoV2 infection.

Methods: We proceed to an in-depth investigation of the SARS-Cov2 reported cases among healthcare workers and the isolation of suspected cases from triages at a SARS-Cov2 treatment center between Mar 10 and Dec 31, 2020
Results: By end 2020, DRC notified 17,850 cases of SARS-Cov2, with $78.8 \%$ in Kinshasa. 679 frontline staff (FLS) were confirmed positive for SARS-Cov2, 38.0\% of them in Kinshasa. Table I below shows the infected FLS by occupational category.
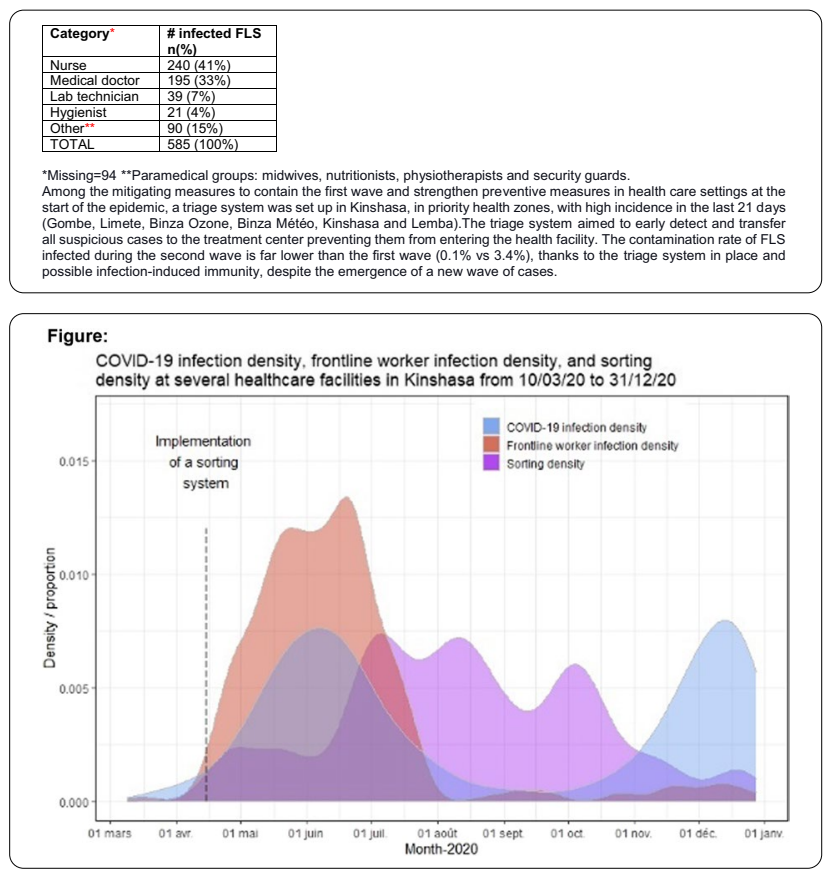

Conclusion: With this SARS-CoV-2 pandemic still ongoing, it remains imperative to continue work on infection control in healthcare settings to protect FLS and already vulnerable patients.

Disclosure of Interest: None declared.

\section{P128}

A qualitative study on safety perception among healthcare workers of a tertiary academic care center during the SARS-COV-2 pandemic

S. Ehrenzeller ${ }^{1, *}$, A. Durovic ${ }^{1}$, R. Kühl ${ }^{1}$, A. Martinez ${ }^{1}$, M. Battegay ${ }^{1}$, A. Fringer ${ }^{2}$, M. von Rotz ${ }^{1}$, S. Tschudin Sutter ${ }^{1}$

${ }^{1}$ Division of Infectious Diseases and Hospital Epidemiology, University Hospital Basel, Basel, ${ }^{2}$ School of Health Professions, Institute of Nursing, Winterthur, Switzerland

Correspondence: S. Ehrenzeller

Antimicrobial Resistance \& Infection Control 10(1): P128

Introduction: Protecting healthcare workers ( $\mathrm{HCWs)} \mathrm{from} \mathrm{exposure}$ to SARS-CoV-2 during patient care is central to managing the current pandemic. Higher levels of trust in personal protective equipment (PPE) and infection prevention and control (IPC) strategies have been previously related to lower levels of emotional exhaustion, yet little is known on how to achieve such a perception of safety.

Objectives: We sought to identify institutional actions, strategies and policies related to HCW's safety perception during the early phase of the pandemic at a tertiary care center in Switzerland by interviewing HCWs from different clinics, professions and positions.

Methods: For this qualitative study, 36 face-to-face semi-structured interviews were performed. Interviews were based on a guide 
addressing the perception of institutional strategies and policies during the first phase of the pandemic in March 2020. The participants included doctors $(n=19)$ and nurses $(n=17)$ in senior and non-senior positions from eight clinics in the University Hospital Basel, Switzerland, all involved in patient care. All interviews were audio-recorded and transcribed verbatim. Data were analyzed using qualitative content analysis and organized using MAXQDA (VERBI Software GmbH, Berlin).

Results: Five recurring themes were identified to affect perceived safety of HCWs during the SARS-CoV-2 pandemic: 1) transparency and clarity of information, 2) communication on the availability of PPE (with provision of information alone increasing the feeling of safety even if supplies of PPE were reported to be low), 3) uniformity and consistency of guidelines, 4) digital resources to support face-to-face teaching (with personal information transfer still being considered superior in terms of strengthening safety perception) and 5) support and appreciation for the work performed.

Conclusion: This study identifies institutional policies and actions, the most important of which being transparent communication, influencing HCW's safety perception during the first wave of the COVID-19 pandemic. Such knowledge reveals potential areas of action critical to improving preparedness and management in hospitals faced with an infectious disease threat.

Disclosure of Interest: None declared.

\section{P129}

Healthcare workers' knowledge and perception of COVID-19 pandemic in Faranah, Guinea

L. Landsmann 1, ${ }^{1,}$, A. O. Diallo ${ }^{2}$, S. K. Abrokwa ${ }^{1}$, A. Borodova', C. Rocha ${ }^{1}$, R. Wood $^{1}$, M. Arvand ${ }^{1}$, M. Diallo ${ }^{2}$, M. Borchert ${ }^{1}$

${ }^{1}$ Robert Koch Institut, berlin, Germany, ${ }^{2}$ Regional Hospital Faranah, Faranah, Guinea

Correspondence: L. Landsmann

Antimicrobial Resistance \& Infection Control 10(1): P129

Introduction: In response to the COVID-19 pandemic, the WHO launched a strategic preparedness and response plan, outlining public health measures to support countries worldwide.

Objectives: Our study aimed to assess the level of preparedness of health care workers (HCWs) towards COVID-19 and strenghten HCWs knowledge and perception of the SARS-COV-2 pandemic and its related Infection prevention and control (IPC) measures in Faranah, Guinea.

Methods: We conducted a cross-sectional pre- and post-intervention study between april 2020 and march 2021. This consisted of a baseline assessment, in-person training, an immediate post-training follow-up and a three months follow-up. Participants were HCWs from the Faranah Regional Hospital (HRF) and two health care centres (HCC). The assessment was carried-out using a questionnaire developed based on WHO recommendations. Data was analysed using STATA Version 17.

Results: There was no significant difference in the overall mean knowledge score during baseline $(32.67 \pm 5.81)$ and second follow-up $(33.96 \pm 4.04 p<0.467)$. Subgroup analysis found significant improvement in knowledge for the HCC, but not for the hospital, comparing baseline $(29.35 \pm 6.89)$ and second follow-up $(34.38 \pm 3.89, p<0.007)$. Overall, there was significant improvement in knowledge among Auxiliary Nurses in the HCC (baseline $28.21 \pm 6.68$, follow-up $33.87 \pm 4.26$ $\mathrm{p}<0.010$ ), while no significant improvement was observed in other profession groups in both HRF and HCC. Assessment of perception towards Covid-19 pandemic revealed that $48.6 \%$ of participants responded feeling helpless regarding the pandemic at baseline, while only $12.9 \%$ responded this way after second follow-up.

Conclusion: The study highlighted optimal level of preparedness towards COVID-19 and its IPC measures among HCWs in both HRF and HCC as reflected by the good knowledge score noted in both baseline and follow-ups. Knowledge was further strengthened in the HCC - relatively low resourced facilities as compared to HRF, as shown by improvement in knowledge after training. Furthermore, IPC training was a key component in improving certain perceptions HCW's had towards COVID-19.

Disclosure of Interest: None declared.

\section{P130}

Perception and attitude of Tunisian healthcare workers

towards COVID19 infection prevention

S. Ben Fredj ${ }^{1}$, R. Ghammem ${ }^{2}$, N. Zammit ${ }^{2}$, C. Ben Nasrallah 2, ${ }^{2}$, J. Maatoug ${ }^{2}$, H. Ghannem²

'Service d'Epidémiologie, "LR 19SP033", Hôpital Farhat Hached, '2Service

d'Epidémiologie, "LR 19SP033", Université de Sousse, Faculté de Médecine de Sousse, Hôpital Farhat Hached, Sousse, Tunisia

Correspondence: C. Ben Nasrallah

Antimicrobial Resistance \& Infection Control 10(1): P130

Introduction: The covid19 pandemic has been associated with significant challenges for Healthcare workers (HCWs) due to the high risk to be infected. Inadequate attitudes among HCWs can directly influence practices and lead to spread of disease in the healthcare-settings.

Objectives: To assess the attitudes of the Tunisian HCWs towards the COVID-19 disease and the perception of preventive measures in the worksite.

Methods: This cross-sectional healthcare-setting study of HCWs in private sector, Tunisia, was conducted during the last quarter of 2020, from September to December 2020. Two-stage cluster sample was performed for needed sample size of 399 participants. For the data collection we used a pretested self-administered questionnaire. sociodemographic characters, psychosocial data, perception of preventive measure implementation in the worksite and attitudes towards COVID19 were recorded. The data analysis was performed using SPSS software 20.

Results: A total of $506 \mathrm{HCWs}$ were enrolled in the study with a mean age of $33.8 \pm 8.4$ years. The sex-ratio was 0.33 . About $63 \%(n=314)$ of the participants were afraid to contract the COVID19 infection and $93 \%(n=461)$ were frightened of transmetting the virus to their families. More than half of the HCWs declared that they washed hands regularly and weared masks more frequently than the previous infection wave. Nearly $91 \%$ of HCWs respected the social distanciation. Three in four respondents (74\%) totally agreed that preventive measures were implemented and $62 \%$ claimed that the responsibles at the worksite take care of the employees' health. the majority of HCWs (99\%) respected the preventive measures and declared that the clinics' responsibles will support them in infection case.

Conclusion: HCWs reported in this study a high rate of positive perceptions and attitudes towards the preventive measures to slow down the COVID19 spreading. Adequate attitudes represent a key component for HCWs' well-being as part of public health measures during this pandemic.

Disclosure of Interest: None declared. 
P131

Control measures of COVID-19 outbreak among non-clinical healthcare workers

H. Ibrahim ${ }^{1, *}$, S. Alghamdy', S. Abdulhafeez², W. Mazi ${ }^{3}$

${ }^{1}$ Public Health, ${ }^{2}$ Academic Affairs and Training, ${ }^{3}$ Infection Prevention

and control, King Abdulaziz Specialist Hospital, Taif, Saudi Arabia

Correspondence: $H$. Ibrahim

Antimicrobial Resistance \& Infection Control 10(1): P131

Introduction: Coronavirus disease (COVID-19) is a disease caused by the severe acute respiratory syndrome coronavirus 2 (SARS-CoV-2) and transmitted by both respiratory droplets and contact with respiratory secretions. Community, hospital, and residency outbreaks have been reported worldwide.

Objectives: To describe the pattern and control measures of COVID19 outbreak among non-clinical healthcare workers.

Methods: A retrospective study to control COVID-19 outbreak among non-clinical healthcare workers was applied from June to August 2020 in a tertiary hospital, Saudi Arabia. Active surveillance and traced their contacts were conducted using the $\mathrm{MOH}$ standards tracing form. Nasopharyngeal swabs were taken for SARS-CoV-2 screening. Infected staff was quarantined immediately. Intervention measures were implemented including, hand hygiene, social distance restrictions, universal masks, and daily monitoring of symptomatic workers. Isolation of infected staff and restriction from the work for 14 days was applied and followed by clinical assessment for back to the work. Room size was standardized to 4 square meters for two persons as recommended by the CDC guidelines. Male residency is completely physically separated from the female hostel.

Results: Ninety-eight non-clinical healthcare workers were confirmed positive for SARS-CoV-2. There were sixty-five cases reported in June and gradually declined to thirty, three, and zero in July, August, and September, respectively. The bulk of the cases occurred among the male gender of biomedical, general engineering staff, and administration personnel (36\%) followed by other categories. The most affected age group was (30-39 years old) with five hospitalized and two deaths. All male of biomedical staff was got the infection within two weeks which is correlated to the congested basement work location. However, none of the biomedical female staff were reported positive due to fully physical separation workplace area; compared to the male section.

Conclusion: Congestion with closed and poor ventilated workplace areas increase the risk of SARS-CoV-2 transmission. Physical separation limits the spread transmission of the infection. Infection prevention measures should be implemented for all healthcare workers including non-clinical healthcare workers.

Disclosure of Interest: None declared.

P133

Safely re-opening a healthcare facility in Nairobi, Kenya

after closure due to SARS-COV-2 infection in healthcare personnel

L. K. Ndegwa ${ }^{1, *}$, D. Kimani ${ }^{2}$, M. Njeru ${ }^{2}$

${ }^{1} \mathrm{DGHP},{ }^{2} \mathrm{DGHT}, \mathrm{CDC}$, Nairobi, Kenya

Correspondence: L. K. Ndegwa

Antimicrobial Resistance \& Infection Control 10(1): P133
Introduction: The first case of COVID-19 in healthcare personnel (HCP) in Kenya was reported on March 30, 2020 in Nairobi, leading to the closure of a health facility for 41 days from April 1, 2020. This resulted in the cessation of healthcare services in this 16-bed primary health facility with 61 staff, attending to 300 outpatients and 600 methadone access treatment clients daily.

Objectives: To describe steps taken to re-open a facility after closure due to SARS-CoV2.

Methods: The Kenya Ministry of Health and the United States Centers for Disease Control and Prevention (CDC) jointly conducted a risk assessment of the facility, using a modified World Health Organization six-element infection prevention and control (IPC) facility risk assessment tool.

Results: Of the six domains assessed, the IPC program scored $40 \%$ across all elements, personal protective equipment (PPE) $60 \%$, and cleaning and disinfection $70 \%$. Key findings from the assessment included breaches in IPC measures including poor ventilation in most service delivery areas; observed lack of physical distancing; inadequate information, education, and communication (IEC) materials related to COVID-19; lack of standard operating procedures (SOPs) on cleaning and disinfection of the high touch areas, insufficient IPC training, inadequate hand hygiene facilities and PPE supplies, and an inactive IPC committee.

We recommended improving the ventilation and changing patient flow patterns in the facility and serving pharmacy clients outside, enhanced cleaning and disinfection of high touch surfaces, providing PPE to HCP and clients, installing PPE donning and doffing stations, displaying IEC materials in strategic locations, disseminating SOPs for $\mathrm{HCP}$ risk assessments, activating the IPC committee, and establishing an OSH program. The health facility re-opened on May 11, 2020.

Conclusion: The IPC facility risk assessment tool was useful in identifying problem areas leading to remediation measures.

Disclosure of Interest: None declared.

P134

Assessment of protective measures for healthcare workers in the context of the COVID-19 pandemic at university hospital center of Angre, (Abidjan - Cote D'ivoire)

I. Kouakou, ${ }^{1, *}$, N. E. fatto ${ }^{2}$

${ }^{1}$ medical biology chu angre, université felix houphouet boigny, abidjan,

${ }^{2}$ medical radiology, université felix houphouet boigny, abidan, Côte

d'Ivoire

Correspondence: I. Kouakou

Antimicrobial Resistance \& Infection Control 10(1): P134

Introduction: Healthcare personnel are at an increased risk of acquiring COVID-19 infection especially in resource-restricted healthcare settings. Health workers are crucial to the successful implementation of infection prevention and control strategies to limit the transmission of SARS-CoV-2 at healthcare facilities.

Objectives: The aim of our study was to determine the personal protective equipment (PPE) knowledge and attitudes of health workers in tertiary hospital. 
Methods: A cross-sectional study design was used. The knowledge, attitudes and practices (KAP) survey was part of a rapid appraisal of the COVID-19 occupational health and safety response in the intensive care and emergency departments of the hospital of Angre. A structured self-administered questionnaire was used. Only doctors, nurses and nursing assistants were included in may 2021.

Results: A total of 50 health workers at different levels of care participated in the survey. Nurses were the first to know the usefulness of PPE in $51 \%$ of cases followed by doctors $37 \%$. Regarding the surgical masks, $90 \%$ of the participants knew their usefulness. only $23.8 \% \mathrm{knew}$ the characteristics of the mask type N95 and 21\% did not know which of the masks protected most against covid-19. A few participants did not know the need to wear gloves. Half of participants indicated they receive training on the correct use of PPE (58\%).

Conclusion: Our results showed that personal protective equipment are used inappropriately by healthcare workers (HCWs). We recommended that public health education be targeted towards the categories of HCWs with high-risk practices and attitudes to achieve the necessary control measure been instituted by the hospital.

Disclosure of Interest: None declared.

P135

From isolation to safe opening; a proposal for safe healthcare delivery in Al-Azhar university hospitals

R. Alsharnoby ${ }^{1}$, S. Fawaz ${ }^{2}$, A. Hamada ${ }^{3}$, S. Hatmalla ${ }^{4}$, E. Gamal ${ }^{1}$, M Abdelnasser,"

${ }^{1}$ Infection Control, ${ }^{2}$ Quality, ${ }^{3}$ Director, Deputy, ${ }^{4}$ Maintenace, Al-Azhar Specialized Hospital, ${ }^{5}$ Microbiology \& Immunology, Faculty of Medicine,

Al-Azhar University, Cairo, Egypt.

Correspondence: $M$. Abdelnasser

Antimicrobial Resistance \& Infection Control 10(1): P135

Introduction: In March 2020, when the WHO announced COVID-19 virus as a pandemic, Al-Azhar University Specialized Hospital has been allocated as an isolation hospital. In June 2020, the number of admitted Covid-19 patients was decreased gradually. So, an integrated plan has been designed for safe opening.

Objectives: The aim of this plan was to control and limit the spread of Covid-19 virus, in order to ensure the safety of the hospital's employees and for the hospital's keenness to work proactively.

Methods: A general framework for proactive measures to restart and return to work was laid by a group of infection control, quality improvement, safety, maintenance and administration in May 2020. The implementation steps were based on three axes: environmental insurance, safe work environment and employee safe return.

Results: The implementation of this plan has been started in July 2020. In the $1^{\text {st }}$ axis, the hospital was closed for one week with a complete clearance of the work site and maintenance of all floors and walls was done using antibacterial paints. In the $2^{\text {nd }}$ axis, offices were redistributed (at least $1.5 \mathrm{~m}$ between desks) and cleaned on a daily basis. Thermal examination was done at external hospital gates. Staff health clinic was ready just in case for those with symptoms of high temperature, fatigue, etc. Awareness panels on hand hygiene were distributed. A timetable was made for daily collection of waste. Elevators were cleaned and disinfected daily and as required. In the $3^{\text {rd }}$ axis, employees were asked to conduct Covid-19 markers and CT Scan (Chest). Approval to return was taken by chest consultant, directors of infection control and human resources.

Conclusion: Safe opening was a challenge in in our hospital. A plan with proactive measures are still in place. The plan was designed to ensure safe hospital and work environment. It also stressed on safe return for employees and visitors. A triage clinic was also launched for both employee and patients,

Disclosure of Interest: None declared.
P136

Outcomes of asymptomatic hospital employees

in COVID-19-post-exposition quarantine during the 2 nd pandemic wave in Zurich

L. Steinwender ${ }^{1}$, J. Burkhard ${ }^{1}$, D. Holy ${ }^{1}$, I. Uçkay ${ }^{1, *}$

${ }^{1}$ Balgrist University Hospital, Zürich, Switzerland

Correspondence: I. Uçkay

Antimicrobial Resistance \& Infection Control 10(1): P136

Introduction: The benefit of routinely putting asymptomatic hospital employees, after close contact to Covid-19 cases, into a ten-days' postexposition quarantine must be evaluated.

Objectives: Evaluate the clinical accuracy of the auarantine measures among young asymptomatic healthcare workers. To define substrata fpr exposition, fow which the quarantine is rasonable.

Methods: Retrospective cohort analysis of hospital-own databases of the Services of Human Resources, Occupational Medicine, and of Infection Control at the Balgrist University Hospital in Zurich, Switzerland, between October $1^{\text {st }}$ and 31 December 31, 2020.

Results: During the most intensive months of the pandemic $2^{\text {nd }}$ wave, we assessed 376 episodes of Covid-19-compatible symptoms; of whom $94(25 \%)$ were positive. Among all positive episodes, the proportion of those with prior quarantine was $11 \%$ (10/94 cases). The reason for the quarantine order was transient exposure in 278 cases, and an iterative (permanent) exposure (e.g. within the family) in 98 episodes. Among the 81 employees in quarantine, ten $(12 \%)$ became symptomatic after a median interval of five days following the last exposure (range, 3-11 d). All ten secondary Covid-19 cases during quarantine were due to a permanent exposure. No employee with transient exposure developed symptoms. The quarantine led to an estimated loss of 810 full workdays within three months.

Conclusion: Among our hospital employees and during the peak months of the $2^{\text {nd }}$ pandemic wave, the risk for developing a symptomatic, PCR-confirmed, Covid-19 disease during a post-expositional quarantine was $12 \%$, and essentially concerned persons with a history of iterative exposure. In hospitals, the decision for quarantine among employees should equally base on nature of the individual exposure.

Disclosure of Interest: None declared.

Poster session: SARS-CoV2: infection control measures and transmission

P137

Infection control in the COVID hospital

O. Orlova $a^{1, *}$, O. Karpov ${ }^{1}$, V. Gusarov ${ }^{1}$, A. Pivkina ${ }^{1}$

${ }^{1}$ Pirogov National Medical and Surgical Center, Moscow, Russian Federation Correspondence: O. Orlova

Antimicrobial Resistance \& Infection Control 10(1): P137

Introduction: The increase in the incidence of new coronavirus infection (COVID-19) has led to the re-profiling of hospitals for the treatment of this group of patients.

Objectives: To convert the surgical center into a COVID Hospital in compliance with infection safety measures.

Methods: Carrying out a complex of organizational, engineering and epidemiological measures.

Results: Within three days, the building was completely cleared of patients, the territories for finding patients (infectious zone) and medical personnel (clean zone) were determined, which were separated by erected sanitary checkpoints for medical personnel. In order to minimize the risk of inhalation of the viral aerosol, the infectious zone of the sanitary checkpoint was divided into two rooms, the first - to remove the protective suit, the second-to remove the second pair of gloves, a respirator, a cap and further treatment of exposed areas of the body with $70 \%$ alcohol. The separation of the traffic flow of cars, employees and patients was carried out. The traffic flows in the Hospital were determined, and elevators for traffic flows of different epidemiological significance were identified and marked. In the clean area of the Hospital, rooms were organized for food, rest and work of the staff, with the installation of dispensers to ensure regular hand treatment. The 
Hospital deployed autonomous clinical diagnostic and microbiological laboratories to exclude the possibility of delivering potentially dangerous material to other buildings of the Center, which was especially important given the ongoing planned work in other buildings of the Center. A disinfection chamber and a site for decontamination of medical waste were allocated. Prior to the admission of patients, practical training was conducted for all Hospital staff. In order to prevent the spread of COVID-19, a smear test for SARS-CoV-2 was organized for all Hospital staff once a week. In the premises of the clean zone in accessible places were installed skin antiseptics for hand treatment.

Conclusion: The result of the conversion was the deployment of a hospital with 280 beds, including 33 intensive care units. The hospital was open for three months. During the operation of the hospital, 1149 patients were treated, of which $154(13.4 \%)$ were treated in intensive care units.

Disclosure of Interest: None declared.

\section{P138}

Standard precautions and nursing professionals: a pilot study on adherence in times of COVID-19 pandemic

J. F. F. C. Cordeiro ${ }^{1, *}$, D. C. Cordeiro ${ }^{1}$, T. A. D. Cruz ${ }^{1}$, I. F. L. Fracarolli ${ }^{1}$, T. G. Santos $^{1}$, E. B. G. Gomide', D. D. Andrade', A. P. D. Santos ${ }^{1}$

${ }^{1}$ Escola de Enfermagem de Ribeirão Preto, Ribeirão Preto, Brazil

Correspondence: J. F. F. C. Cordeiro

Antimicrobial Resistance \& Infection Control 10(1): P138

Introduction: Standard precautions (SP) are measures recommended by the World Health Organization for infection control. There are widespread recommendations of security measures during the COVID-19 pandemic.

Objectives: to investigate adherence of a nursing professionals regarding SP during the COVID-19 pandemic and to examine the association among length of experience, professionals with graduate degrees and workplace setting with the percentage of adherence.

Methods: A pilot cross-sectional study was conducted using a validated questionnaire. Nursing professionals working in the front line of the COVID-19 pandemic in the State of São Paulo, Brazil, participated in the study. Data were analyzed using descriptive statistics and presented using absolute and relative frequency. Pearson's Chi-square test $\left(X^{2}\right)$ was used to verify the association between length of experience (years working in nursing), completion of postgraduate studies (certification, master's and doctorate), workplace (public or private) of nursing professionals, and the percentage of total adherence to SP.

Results: 169 nursing professionals were evaluated, being 49 (29\%) aged between 18 and 24 years old, $44(26 \%)$ with $\leq 1$ year of experience in nursing, 81 (48\%) with postgraduate studies, and 91 (53.8\%) were employed in private health institutions. In terms of adherence to the SP, 157 (92.9\%) performed hand hygiene in the intervals of care, with greater adherence after being in contact with biological material 165 (97.6\%). The majority of the participants reported always using gloves. There was no adequate adherence to the use of safety glasses, hats, handling with sharps or the notification of accidents at work. Chi-square test did not reveal significant association among the conditions: length of experience, completion of graduate studies and workplace of nursing professionals, with the percentage of total adherence to $S P\left(X^{2}{ }_{(1)}=0.033 ; p=0.855\right),\left(X^{2}{ }_{(1)}=0.006 ; p=0.940\right),\left(X^{2}{ }_{(2)}=1.031\right.$; $p=0.597)$, respectively.

Conclusion: Nursing professionals' adherence to SP was found to be below the ideal recommendation. Length of experience, having an advanced degree or workplace setting did not influence adherence to SP.

Disclosure of Interest: None declared.

\section{P139}

Improving wash and IPC readiness and behaviors in five low-income countries in response to COVID-19

J. Lopez ${ }^{1, *}$, T. Ashengo ${ }^{1}$, S. Sara ${ }^{1}$

${ }^{1}$ Momentum Country and Global Leadership, Washington, United States Correspondence: J. Lopez

Antimicrobial Resistance \& Infection Control 10(1): P139
Introduction: In July of 2020, USAID's MOMENTUM and Country Global Leadership began implementation of a water, sanitation, and hygiene (WASH) and Infection Prevention and Control (IPC) intervention aimed at improving outbreak readiness and behaviors in five countries in 154 primary and secondary facilities: Sierra Leone (26), Ghana (25), Uganda (25), India (61), and Bangladesh (17).

Objectives: The project conducted initial readiness and behavioral observations followed by training using quality improvement methodologies, provision of IPC supplies and equipment, and infrastructure rehabilitation and will be followed by an endline assessment. This presentation will explore the findings of these assessments.

Methods: The project developed a standard weighted IPC and WASH readiness assessment tools based on the WHO Infection Prevention and Control Assessment Framework and the WHO/UNCIEF Joint Monitoring Program indicators for WASH in healthcare facilities, then incorporated additional questions based on national tools and guidelines. Data was collected using digital tools and dashboards were developed to facilitate data sharing. The data collected guided a quality improvement strategy using a hub and spoke approach using both in-person and virtual trainings along with training visits by staff specializing in IPC and moderated WhatsApp groups to facilitate dialogue between Quality Improvement facilitators and facility staff. Monthly behavioral audits were also conducted to gauge improvements in hygiene behaviors and use of personal protective equipment.

Results: Except for Ghana, the initial readiness assessment scores for the facilities were low: Sierra Leone (39.35\%), Ghana (83.52\%), Uganda (50.89\%), India (45.71\%) and Bangladesh (41.07\%). Final assessments are to be completed by July 2021, except for Sierra Leone, which completed its endline assessments in April 2021. Sierra Leone saw its facility IPC readiness scores improve from 39.35\% in October 2020 to $67.77 \%$ in February 2021. Behavioral audits also saw improvement in outpatient handwashing behaviors increaseing from 49 to $84 \%$. PPE use increase by $25 \%$ (64\% to $89 \%$ ).

Conclusion: Preliminary findings demonstrate the importance of adequate supplies and infrastructure coupled with training on best practices and monitoring of behaviors, to improve IPC readiness.

Disclosure of Interest: None declared.

\section{P140}

Does COVID-19 trigger changes in attitudes and behaviour of respiratory infection prevention measures? First results of health-care-associated influenza prevention project (HAIP) D. Flury, ${ }^{1,}$, M. Schlegel ${ }^{1}$, I. Kramer ${ }^{2}$, M. Schubert ${ }^{2}$, D. Nicca ${ }^{3}$

${ }^{1}$ Infectious disease and hospital hygiene, Kantonsspital St.Gallen, St.Gallen, ${ }^{2} Z$ Zurich university of applied Science, University of applied Science, Winterthur, ${ }^{3}$ Institute for Epidemiology, Biostatistics and Prevention, University of Zürich, Zürich, Switzerland

Correspondence: D. Flury

Antimicrobial Resistance \& Infection Control 10(1): P140

Introduction: Results of the HalP showed that during the last influenza seasons attitudes and adherence with infection preventive measures against the transmission of influenza remained stable.

Objectives: To describe changes in attitudes and behaviour of health care worker (HCW) in infection prevention measures (wearing mask, hand hygiene, Influenza vaccination) during winter season before and after COVID-19.

Methods: We collected data on 3 pilot wards during Influenzaseason 19/20 and 20/21 in the cantonal hospital of St.Gallen, a tertiary 700-bed hospital in eastern Switzerland. Adherence with hand hygiene was recorded by clean care monitor from Swissnoso according to the 5 moments (WHO). Data on Influenza and COVID19 vaccination rates were obtained by personal medical services. HCW's perceived importance of- and self-reported behaviour of influenza preventive measures were assessed with a questionnaire. In 20/21 the questions were adapted to cover for COVID-19. Results: Observed adherence with hand hygiene increased significantly from 68 to $78 \%(p<0.001)$ as did self-reported adherence to wearing mask from 10 to $98 \%(p<0.001)$. The other observed behaviours where not significant (table1a). Influenza vaccination 
rate reminded low over both seasons. Self-reported and observed Covid-19 vaccination rate was significantly higher than influenzavaccination rate $(32 \%$ versus $17 \%$ and $27 \%$ versus $10 \%)$. The perceived importance of hand hygiene remained high, the attitudes toward own influenza vaccination did not change over the seasons(Table1b). A relevant change can be observed in mask wearing which was perceived as with low importance $(2$ out of 10 ) before the SARS-CoV2 pandemic and most important(10out of 10) thereafter.

\begin{tabular}{|c|c|c|c|c|}
\hline \multicolumn{5}{|l|}{ Figure: } \\
\hline & $19 / 20$ & \multicolumn{2}{|c|}{$20 / 21$} & p value \\
\hline Hand Hygiene & & \multirow{3}{*}{\multicolumn{2}{|c|}{$\begin{array}{c}100(58 / 58) \\
78(623 / 789)\end{array}$}} & \\
\hline self-reported behaviour correct, $\%$ & $96(47 / 49)$ & & & \\
\hline observed behaviour, \% & $68(608 / 894)$ & & & $p<0.001$ \\
\hline Mask & & \multirow{2}{*}{\multicolumn{2}{|c|}{$98(58 / 59)$}} & \\
\hline self-reported behaviour, \% 1 & $10(5 / 49)$ & & & $p<0.001$ \\
\hline Vaccination & & & & \\
\hline self-reported behaviour, $\%$ & $9(4 / 49)$ & $17(10 / 59)$ & $32(19 / 59)$ & $\begin{array}{c}n . s^{2}{ }^{2} \\
p=0.06^{3}\end{array}$ \\
\hline observed behaviour, $\%$ & $14(21 / 145)$ & $10(11 / 107)$ & $27(29 / 107)$ & $\begin{array}{c}\text { n.s. } \\
0 . .^{2} \\
0=001^{3}\end{array}$ \\
\hline
\end{tabular}

\begin{tabular}{|c|c|c|c|c|}
\hline & \multirow{2}{*}{$19 / 20$} & \multicolumn{2}{|c|}{$20 / 21$} & \multirow{2}{*}{ p value, } \\
\hline & & Influenza & COVID-19 & \\
\hline $\begin{array}{l}\text { Hand Hygiene } \\
\text { attitude (importance) } \\
\text { Mask }\end{array}$ & $10(10,10)$ & $10(10,10)$ & $10(10,10)$ & n.s. ${ }^{2}$ \\
\hline attitude (importance) & $2(0.5,7)$ & $10(8,10)$ & $10(9,10)$ & $\begin{array}{l}p<0.001^{1} \\
p=0.005^{2}\end{array}$ \\
\hline Vaccination & & & & \\
\hline attitude (importance for themselves) & $4(2,6)$ & $5(2.6)$ & $7(5 / 10)$ & $\begin{array}{c}n . s^{1} \\
p>0.001^{2}\end{array}$ \\
\hline attitude (importance for patient) & $8(5,9)$ & $8(5,10)$ & $8(7,10)$ & n.s. ${ }^{1}$ \\
\hline 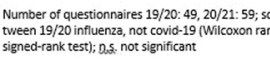 & $\begin{array}{l}\text { tuntil } 10 \mathrm{im} \\
\text { ue between }\end{array}$ & $\begin{array}{l}\text { (median (Q) } \\
\text { za and covid }\end{array}$ & $\begin{array}{l}\text { ) }{ }^{1} \text { p-value be } \\
21 \text { (Wilcoxox }\end{array}$ & \\
\hline
\end{tabular}

Conclusion: The emergence of the COVID-19 pandemic had an important impact on attitudes and behaviour of infectious disease prevention measures. As expected the change could be perceived in attitude and the behaviour toward wearing a mask but also increased the adherence with hand hygiene. Vaccination coverage has been and remains low for both COVID-19 and Influenza but the significantly lower readiness for influenza underscores the specific vaccination scepticism about influenza.

Disclosure of Interest: None declared.

\section{P141}

Compliance with preventive measures before and during home quarantine among a Tunisian cohort of COVID-19 patients

R. Bannour 1,*, N. Zammit ${ }^{1,2}$, R. Ghammam ${ }^{1,2}$, S. Ben Fredj ${ }^{1,2}$, A. Eleuchi ${ }^{1}$, I. Mlouki', J. Maatoug ${ }^{1,2}$, H. Ghannem ${ }^{1,2}$

${ }^{1}$ Department of Epidemiology (LR19SP03), university hospital Farhat Hached Sousse, 2Faculty of Medicine of Sousse, University of Sousse, Sousse, Tunisia, Sousse, Tunisia

Correspondence: R. Bannour

Antimicrobial Resistance \& Infection Control 10(1): P141

Introduction: Until now, there is no specific treatment to beat the COVID-19 disease. Regarding the scarcity of vaccines, compliance with collective and individual preventive measures remain the most important weapon against this new disease in developed countries.

Objectives: To assess compliance with collective and individual preventive measures among COVID-19 patients before and during home quarantine in the governorate of Sousse.

Methods: A prospective longitudinal study of three months was led among a cohort of 375 patients with COVID-19 isolated at home.
Participants were randomly selected from the new declared cases in the governorate of Sousse during November 2020.

Data were collected using a pre-established and pre-tested questionnaire administered during phone calls interviews with trained medical doctors. The frequency of the compliance with hygiene measures was evaluated using a five item scale with the following possible responses: ("do not remember"; "never";"sometimes"; "often" and "all the time").

Results: A total of 375 participants were included. The median age of participants was 40.0 (IQR: 29.75-54.25) years. Females represented $60 \%$ of them. Among participants, 359 (95.7\%) consider that social distancing is an efficient collective preventive action. Before the COVID-19 infection episode, this measure was not easy to respect for $121(32.3 \%)$ participants. Besides, compliance all the time with mask wearing, coughing into the elbow and hand hygiene were reported by $49.1 \%, 41.9 \%$ and $58.9 \%$ of participants respectively. Otherwise, during the confinement, 95 (25.4\%) participants declared not respecting the quarantine and $111(29.6 \%)$ participants transmitted the infection to their family members.

Conclusion: The current national awareness compaign should be reinforced in Tunisia in order to enhance compliance with collective and individual preventive measures. Use of social media, involving leaders and enforcing the law may increase people's adherence to hygiene rules.

Disclosure of Interest: None declared.

\section{P142}

SARS-COV-2 infection control measures in a Spanish tertiary

hospital: a multimodal strategy

P. Puche ${ }^{1, *}$, M. Herranz ${ }^{1}$, C. Hidalgo ${ }^{1}$, S. Suclupe ${ }^{1}$, A. Paez ${ }^{1}$, S. Ruiz ${ }^{2}$, M. M.

Montero $^{3}$, C. Gonzalez ${ }^{1}$

${ }^{1}$ Infection Control Program, Department of Epidemiology and Evaluation,

${ }^{2}$ Occupational Health Department, ${ }^{3}$ Infectious Disease Department, Parc

Salut Mar, Barcelona, Spain

Correspondence: P. Puche

Antimicrobial Resistance \& Infection Control 10(1): P142

Introduction: There are different elements of a multimodal strategy to implement preventive measures and control the nosocomial transmission by SARS-CoV-2.

Objectives: To describe multimodal strategies used with the frontline healthcare staff (HCS) to implement SARS-Cov-2 preventive measures in the hospital.

Methods: Design: Descriptive study of preventive measures in Hospital del Mar, from August 2020 to February 2021.

Inclusion Criteria: Medical and surgical departments; Exclusion Criteria: Outpatient visits department.

Strategies implemented: Strategies were led by the nurses of Infection Control Program (ICP) with interested allies across the hospital.

Education and Training: HCS were taught when and what type of Personal Protective Equipment (PPE) to use and how both donning and doffing them.

Monitoring and feedback: Observations, one to one conversations, and supportive supervision helped the staff to improve performance. Reminders and communications: To promote protocols and standardize the desire actions at the right time, illustrated friendly reminders were posted on the door of rooms.

Results: Education and training: The ICP and the occupational health department carried out training sessions for HCS leaders from each department and shift. There were 78 participants among nurses, auxiliary nurses, medical doctors, porters, and general service staff. More than half were nurses $(53.8 \%)$, and the medical area staff had more participation than surgical, emergency, ICU, and midwifery area.

Monitoring and feedback: From 696 observations across wards, in the beginning, the compliance of the appropriate use of PPE was $58.6 \%$ however in February increased to $81.3 \%$.

Reminders and communications: Each door of the rooms had a reminder about the use of preventive measures: hand hygiene when entering and leaving the room, use of gloves, gown, and facemask 
inside the room, use shields/goggles if necessary, and keep the door closed unless the patient has no respiratory symptoms.

Conclusion: Multimodal strategies may be applicable to promote preventive measures in the pandemic context. Improvement in PPE compliance was observed after training, monitoring, and feedback.

Disclosure of Interest: None declared.

\section{P143}

Practice of standard precautions by Tunisian teaching hospital health care workers (HCW) within the COVID-19 epidemic in March 2020

A. Gara ${ }^{1, *}$, B. Sana ${ }^{1,2}$, A. Ben Cheikh ${ }^{1,2}$, H. Ghali, ${ }^{1,2}$, W. Dhouib ${ }^{1}$, R. Bannour ${ }^{1}$, S. Khfecha' ${ }^{1}$, H. Said Latiri ${ }^{1,2}$, M. Ben Rejeb ${ }^{1,2}$

${ }^{1}$ Department of Prevention and Security of Care, Sahloul university hospital, Sousse, Tunisia, Sahloul university hospital, Sousse, Tunisia, ${ }^{2}$ Faculty of Medicine of Sousse, University of Sousse, Sousse, Tunisia, sousse, Tunisia

Correspondence: A. Gara

Antimicrobial Resistance \& Infection Control 10(1): P143

Introduction: The current outbreak of the SARS-CoV-2 all over the world requires strengthening the practice of preventing measures particularly in healthcare settings. Standard Precautions (SP) are the minimum infection prevention practices designed to both protect and prevent from spreading infection among patients. Within this framework we aimed to assess the practice of standard precautions among healthcare workers (HCWs) in a tunisian teaching hospital over this outbreak in March 2020.

Objectives: degree of compliance with standard precautions by health care workers and readness to face the Covid-19 epidemic in a tunisian teaching hospital over this outbreak in March 2020.

Methods: This is an audit of the practice of SP by direct observation over a period of one month during the first wave of the covid- 19 outbreak (March 2020).We implemented an observation grid gathering recommandations relative to the six axes of SP. This grid was built considering the new recommandations supported by French Society of Hospital Hygiene in 2017.

Results: The highest compliance was for excreta management $87.2 \%$, followed by prevention of accidents with exposure to blood $79.4 \%$, then Respiratory Hygiene $69.6 \%$, environmental management $68.1 \%$, Personal Protective Equipment $60.9 \%$ and Hand Hygiene was the lowest $57.1 \%$.

Conclusion: These results demonstrate an acceptable level of engagement in the prevention and control of the infectious risk in the hospital besides of an institutional mobilization to face the Covid-19 epidemic. therefore, it is recommended to multiply the evaluations to verify these conclusions. Likewise, the training axis is essential to perpetuate the optimal practice of standard precautions.

Disclosure of Interest: None declared.

\section{P144}

Isolation precautions and hand hygiene compliance facing COVID-19 pandemic: analysis of a group of Brazilian hospitals L. M. Abraão ${ }^{1, *}$, D. Cais ${ }^{1}$, S. Lessa Pires ${ }^{1}$, F. Colombari ${ }^{2}$

${ }^{1}$ Infection Control Service, ${ }^{2}$ Medical Practices, Americas Medical Services_-UHG Brazil, Sao Paulo, Brazil

Correspondence: L. M. Abraão

Antimicrobial Resistance \& Infection Control 10(1): P144

Introduction: To COVID-19's facing strategies is important to ensure the rational and appropiate use of aff PPE, not only masks, wich requires correct and rigorous behavior from heath care workers$\mathrm{HCWs}$, but particularly in doffing procedures off personal protective equipment- PPE and hand hygiene-HH practices (WHO,2020).

Objectives: The objective of this study was to evaluate the appropriate use of PPE in the isolation precautions-IP, the $\mathrm{HH}$ compliance among HCWs and the hospital's structure to support these practices. Methods: A prevalence study was conducted in a group of 16 hospitals from four different states in Brazil, during the period of three months
(April to June 2020). Our approach consisted of assessing the HCWs regarding the IP (contact+droplets) and $\mathrm{HH}$ compliance, associated with a evaluation of hospital structure to supporting HCWs in these practices. For this assessment, a check list was built based on the WHO recommendations for prevention of COVID-19. We considered: the professional category; appropriate PPE use according to the activity performed; PPE proper placement; PPE proper withdrawal; $\mathrm{HH}$ at the right moments (5 moments), and proper hospital structure (including: availability of PPE and alcoolic solution in the point of care).

Results: 1867 observations were performed among 225 health care professionals. The HCWs were: nurses, nursing technicians, doctors and physiotherapists. The data were stratified into IP conformity, hand higyene compliance and appropriate struture of hospitals to supporting the IP and $\mathrm{HH}$ (table 1). The overall rate conformity of HCWs related the IP were $84 \%$. General HH compliance of HCWs evaluated were $56 \%$. Considering the structure of the hospitals to supporting IP and $\mathrm{HH}$ practices, we identified that $81 \%$ of the hospitals had an appropriate structure.

\begin{tabular}{|c|c|c|c|c|c|c|c|c|}
\hline \multicolumn{9}{|l|}{ Figure: } \\
\hline \multirow[t]{2}{*}{ HCWs } & \multicolumn{2}{|c|}{$\begin{array}{l}\text { Appropriate PPE use } \\
\text { according to the activity } \\
\text { performed }\end{array}$} & \multicolumn{2}{|c|}{ PPE proper placement } & \multicolumn{2}{|c|}{ PPE proper withdrawal } & \multicolumn{2}{|c|}{$\begin{array}{l}\text { HH at the right moments (5 } \\
\text { moments) }\end{array}$} \\
\hline & $\begin{array}{c}\text { Observations } \\
(\mathrm{N})\end{array}$ & \begin{tabular}{|l|} 
Conformity \\
rate $(\%)$ \\
\end{tabular} & $\begin{array}{c}\text { Observations } \\
\text { (N) }\end{array}$ & \begin{tabular}{|c|} 
Conformity \\
rate $(\%)$ \\
\end{tabular} & $\begin{array}{c}\text { Observations } \\
\text { (N) }\end{array}$ & \begin{tabular}{|c|} 
Conformity \\
rate $(\%)$ \\
\end{tabular} & \begin{tabular}{|c|}
$\begin{array}{c}\text { Obsenvetions } \\
\text { (N) }\end{array}$ \\
\end{tabular} & $\begin{array}{c}\text { Conformity } \\
\text { rate }(\%) \\
\end{array}$ \\
\hline Nurses & 30 & $25(83 \%)$ & 30 & $17(56 \%)$ & 21 & $15(71 \%)$ & 29 & $15(52 \%)$ \\
\hline \begin{tabular}{|l|}
$\begin{array}{l}\text { Nuring } \\
\text { technicians }\end{array}$ \\
\end{tabular} & 25 & $19(76 \%)$ & 25 & $13(52 \%)$ & 25 & $16(64 \%)$ & 25 & $14(56 \%)$ \\
\hline Doctors & 115 & $107(93 \%)$ & 110 & $84(76 \%)$ & 99 & $66(67 \%)$ & 115 & $65(56 \%)$ \\
\hline Physiotherapists & 23 & $23(100 \%)$ & 21 & $13(61 \%)$ & 18 & $10(56 \%)$ & 23 & $13(56 \%)$ \\
\hline TOTAL & 193 & $174(50 \%)$ & 186 & $127(68 \%)$ & 163 & $107(66 \%)$ & 192 & $107(56 \%)$ \\
\hline
\end{tabular}

Conclusion: We identified an important weakness in adherence to hand hygiene among health professionals, despite the most part of hospitals presented an appropriated structure to supporting this practice and the IP. These findings can be explained by the fear that professionals would become infected at the beginning of the COVID-19 pandemic. This led us to work specifically and strongly to align this practice, ensuring the safety of patients and HCWs.

Disclosure of Interest: None declared.

\section{P145}

Development of three aide-memoires to improve standard, droplet/contact and airborne transmission practices and behaviours

C. Kilpatrick ${ }^{1, *}$, A. P. Coutinho-Rehse ${ }^{1}$

'WORLD HEALTH ORGANISATION, Copenhagan, Denmark

Correspondence: C. Kilpatrick

Antimicrobial Resistance \& Infection Control 10(1): P145

Introduction: The World Health Organization (WHO) recommends the use of multimodal improvement strategies (MMIS) to implement IPC interventions. These include each item of standard and transmission-based precautions according to national guidelines or standard operating procedures and under the coordination of the national IPC focal point (or team, if existing). In 2020, a rapid review of WHO IPC guidance, including for COVID-19, was undertaken to address WHO European region country needs with regards to implementation and improvement tools.

Objectives: To develop targeted improvement tools to support IPC guidance into action (GTA) in WHO European region countries.

Methods: The topics for new improvement tools were decided upon and text drafted. A first online consultation (29 June-13 July 2020) included WHO and country experts. The questions took account of theory of change concepts, resource use, values and preferences (figure 1). Feedback informed significant changes to the tools. The second consultation, November 2020, involved WHO headquarters and regional office experts. It comprised an interactive 90-min virtual discussion, with materials shared in advance and by asking three key 
questions, iteratively seeking final validation. A final follow up exercise in 2021 verified accuracies in the final products.

Results: A new WHO Europe document consisting of three focused, improvement tools called aide-memoires was launched in May 2021, targeted at IPC focal points. The three aide-memoires are; 1 ) respiratory and hand hygiene, 2) personal protective equipment and 3) environmental cleaning, waste and linen management. Each consists of action checks structured around the five elements of the MMIS https:// www.euro.who.int/en/health-topics/disease-prevention/antimicrob ial-resistance/publications/2021/infection-prevention-and-controlguidance-to-action-tools-2021.

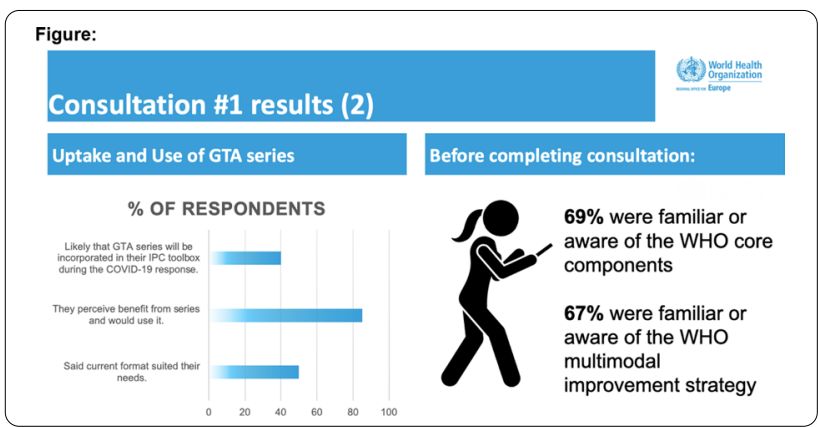

Conclusion: These are the first GTA tools to focus on the detail of 'how' to improve standard and transmission-based precaution practices and behaviours by describing actions that comprise the five elements of the WHO MMIS. The action checks included in each are informed by WHO IPC guidance, as well as input from experts, and are importantly rooted in a proven improvement approach acting as reminders of things to do in the real world.

Disclosure of Interest: None declared.

P146

Improving compliance to workplace prevention and control (IPC) measures during the COVID-19 pandemic in a non-healthcare setting

A. Wailagala ${ }^{1, *}$, R. Ayebare ${ }^{1}$, F. Mayanja ${ }^{1}$, M. Kesande ${ }^{1}$, D. Omagor ${ }^{1}$, M.

Laker', S. Lamunu', M. Lamorde', A. Kambugu'

${ }^{1}$ Infectious Diseases Institute, Kampala, Uganda

Correspondence: A. Wailagala

Antimicrobial Resistance \& Infection Control 10(1): P146

Introduction: Information on non-healthcare setting Infection control measures, especially in the Context of the COVID-19 pandemic is scarce. The Ministry of Health Uganda instituted national lockdown to minimize the spread of the SARS CoV2 virus, which has progressively eased since July 2020. The Infectious Diseases Institute (IDI) established measures to ensure workplace safety for staff, visitors, and service providers. However, monitoring compliance is crucial for the standards to have an impact.

Objectives: This survey aimed to monitor and improve staff compliance with the prescribed criteria for workplace safety for over two months.

Methods: A baseline survey using a pretested tool was done in August 2020 to check staff compliance to the different IPC mitigation measures at IDI. This survey's feedback informed an enhancement of workplace safety measures, including encouraging working from home, reducing office occupancy to about $30 \%$ of the pre-pandemic capacity, and mandatory use of face coverings. The evaluation was followed by a second survey in October 2020 to assess changes in compliance following the enhancement of IPC mitigation measures.

The metrics assessed include; Compliance with screening procedures at the entrance (Hand hygiene, face mask possession, Temperature measurement, and Registration). Assessment of proper face-covering use and number of staff carrying personal ABHR was done by directly observing and asking a convenient sample of staff on each of the building complex's six levels, respectively.
Results: Over the two months, the overall staff compliance with screening efforts increased from 59 to $89 \%$, with the most considerable improvements seen in mask possession and recording of contact details at the entrance. Correct mask use within the premises improved from 23 to $47 \%$ and ownership of personal ABHR from $39 \%-61 \%$.

Conclusion: Strengthened administrative controls lead to measurable improvement in compliance with IPC. Workplace settings should actively monitor for compliance, with constant reminders on best practices for occupational exposure to COVID-19 since changing behaviour takes time. Disclosure of Interest: None declared.

P147

Strengthening infection prevention and control practises at grassroot level

V. Singh ${ }^{1, *}$, A. Prasad ${ }^{1}$, P. Gupta ${ }^{2}$, S. Rao ${ }^{3}$, P. Gupta ${ }^{1}$

${ }^{1}$ Microbiology, All India Institute of Medical Sciences Rishikesh, Rishikesh,

${ }^{2}$ Microbiology, AllMS Bilaspur, Bilaspur, ${ }^{3}$ Pathology, All India Institute

of Medical Sciences Rishikesh, Rishikesh, India

Correspondence: $V$. Singh

Antimicrobial Resistance \& Infection Control 10(1): P147

Introduction: The emergence of COVID-19 pandemic across the globe has underlined the need to enable the medical graduates to be prepared for the unknown. The health sector has been severely affected by the COVID-19 pandemic and there is acute need to maximize trained health care task force and improve the existing healthcare facilities. Due to manpower crisis, the government of India announced to utilize services of final year MBBS students. This study was aimed to train the medical students and prepare them to work as frontline workers.

Objectives: The objective of the study was to assess the effectiveness of flipped classroom teaching of MBBS students for infection prevention and control practices.

Methods: A quasiexperimental cross-sectional study with pre-test and post-test design, using Google Questionnaire forms for tests, was conducted at a tertiary care center in North India. Pre-test was conducted 2 days prior to training class and then the students were provided with study material including powerpoint and video lectures pertinent to standard and additional precautions. Data was analysed using descriptive statistics, Chi-square test, Fisher's t-test and ANOVA.

Results: The results of study suggested that the training format was efficient with significant increase in level of knowledge post training from $16.3 \%$ of students with scores more than $70 \%$ in pre-test to $57 \%$ of students with $>70 \%$ scores post-test. The mean scores of students in pre and post tests are shown in Table 1. There was significant association found in between gain of knowledge and gender and status of previous training received during last 6 months.Comparison of Mean knowledge scores.

\begin{tabular}{|l|l|ll|l|}
\hline TOTAL TEST SCORE $=36$ & PRE-TEST & \multicolumn{2}{|l|}{ POST-TEST } & Sig (2-tail) \\
\hline MEAN (S.D.) & $22.76(2.74)$ & $26.39 \quad(4.06)$ & 0.001 \\
\hline
\end{tabular}

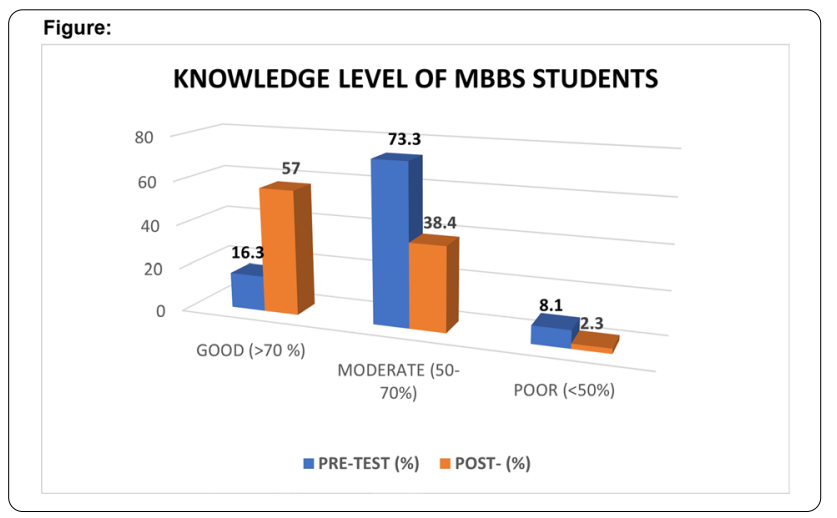


Conclusion: In the present scenario, there is constant need to train the medical students regarding infection control especially regarding standard and additional precautions. Infection prevtion \& control should be included in curriculum of medical students. The Flipped Classroom model may not be perfect answer for training of MBBS students amidst COVID-19, nonetheless this model was found to be immensely relevant in this study.

Disclosure of Interest: None declared.

\section{P148}

Impact of the COVID-19 pandemic on infection control practices in a tertiary-care Spanish hospital: preliminary results from a survey

G. Abelenda-Alonso 1,", M. Puig-Asensio ${ }^{1}$, A. Hornero ${ }^{1}$, A. Rombauts ${ }^{1}$, C. Gutiérrez ${ }^{1}$, E. García-Lerma ${ }^{1}$, E. Jiménez-Martínez' ${ }^{1}$ C. Tebé ${ }^{1}$, M. Pujol ${ }^{1}$, J. Carratalà

${ }^{1}$ Bellvitge University Hospital, Barcelona, Spain

Correspondence: $\mathrm{G}$. Abelenda-Alonso

Antimicrobial Resistance \& Infection Control 10(1): P148

Introduction: Hospitals rapidly prepared to identify, isolate, and take care of SARS-COV-2 cases while ensuring health-care workers (HCWs) safety. Initial shortages in personal protective equipment (PPE) and lack of hospital preparedness posed at risk infection control practices.

Objectives: To assess the impact of COVID-19 pandemic on infection control practices in a tertiary-care hospital in Spain.

Methods: An electronic survey was sent to 753 frontline HCWs on March, 2021. The survey included questions about hand hygiene, catheter maintenance, and PPE. More questions focused on the impact of COVID-19 on infection preventionists daily routine.

Results: A total of 163 HCWs (21.6\%) completed the survey [Intensive care unit: 32.5\%; Infectious diseases unit: 22.7\%; Pulmonology unit: 20.4\%]. Sixty-seven were physicians, sixty-four nurses, twenty nursing assistants, four infection preventionists and eight other groups. Low compliance with hand hygiene prior to an aseptic procedure and after touching patient's environment was reported in $70.6 \%(115 / 163)$ and $82.2 \%(134 / 163)$ respondents. Barriers to infection control practices were: 1) Glove use was an interfering factor for compliance with the 5 -moments of hand hygiene in $44.8 \%(53 / 163) ; 2)$ regarding catheter care, $23.1 \%(15 / 65)$ of nurses reported problems with catheter dressings and, among them, $53.3 \%$ stated that dressing change was difficult with glove use. Further, $65.6 \%$ of nurses $(42 / 65)$ recognized drawing blood cultures from the central venous catheter, instead of venipuncture 3 ) the incorporation of untrained personnel during the pandemic was perceived as a limiting factor for appropriate infection control practices in $47.2 \%(77 / 163)$. Finally, infection preventionists reported that during the first COVID-19 wave (March-June 2020), 70-90\% of their activities shifted to COVID-19 issues, and this interfered in other duties.

Conclusion: COVID-19 pandemic negatively affected infection control practices, namely hand hygiene and catheter use. The use of gloves and the need to rapidly incorporate untrained personnel were perceived as barriers for optimal infection control practices.

Disclosure of Interest: None declared.

\section{P149}

How to COVID-19 pandemic infection control and prevention In

Cho Ray hospital, Vietnam

T. M. Phung Manh ${ }^{1, *}$, T. Nguyen Tri ${ }^{2}$

${ }^{1}$ Infection Control, ${ }^{2}$ Arrhythmia, Cho Ray hospital, Ho Chi Minh City, Viet Nam

Correspondence: T. M. Phung Manh

Antimicrobial Resistance \& Infection Control 10(1): P149
Introduction: Cho Ray is the largest general hospital in the south of Vietnam with daily 3,000 inpatients and 6,000 outpatients. Daily, Cho Ray hospital (CRH) receives 20,000 people including; patients, patient's relatives, and healthcare workers. Annually, CRH deals with several outbreaks such as season influenza $\mathrm{A} / \mathrm{H} 1 \mathrm{~N} 1$ and a potential invasion of other severe infection from foreign countries such as bird flu A/ H5N1, A/H7N9, Ebola, MER-CoV. COVID-19 pandemic appeared as the first case in CRH and a second case outside of China in January, 2020. To successful handle of COVID-19 pandemic, we make cross-sectional descriptive study to find factors to contribute the success in COVID-19 pandemic control and prevention in Cho Ray hospital.

Objectives: Finding factors to contribute for well COVID-19 control and prevention in Cho Ray hospital.

Methods: Using infection surveillance system and cross-sectional descriptive study to review all factors that can be effect on COVID-19 control in Cho Ray hospital.

Results: Our research demonstrates that these factors are keywords to succeed COVID-19 control and prevention in Cho Ray hospital including; 1 ) administrative control; 2) establishment of protocols and treatment zones for COVID-19 in preparation for all scenarios of increasing outbreak; one department knockdown, more than two departments knockdown, and hospital knockdown; 3) infection control training for healthcare workers in pandemic: 2000 healthcare workers annual and pandemic training. 4) proactive preparation of PPE, environment and surface disinfectant for all hospitals: 300 special and 1000 common PPE suit available; 5) enhancement of hospital environment, and usage of medical mask, hand hygiene: over $90 \%$ wearing mask and $81 \%$ hand hygiene compliance; 6 ) establishment of COVID-19 urgent response team: supported more 10 provinces; 7) screening SARSCoV-2 test: $100 \%$ people in hospital.

Conclusion: Our study reveals that leadership, proactive infection control and prevention, flexible strategy based on COVID-19 dynamic are the most important factors to well handle COVID-19 pandemic in Cho Ray hospital, but still remains developing economy.

Disclosure of Interest: None declared.

P150

Examining the discourse regarding the delivery of occupational health and safety training in infection prevention and control guidelines

M. O. Qureshi ${ }^{1,},{ }^{*}$, A. Chughtai ${ }^{1}$, H. Seale ${ }^{1}$

${ }^{1}$ University of New South Wales, Sydney, Australia

Correspondence: M. O. Qureshi

Antimicrobial Resistance \& Infection Control 10(1): P150

Introduction: Events such as the COVID-19 pandemic, continue to highlight that healthcare providers are at heightened risk of acquiring healthcare associated infections. Reducing the risk requires a multimodal approach and a culture of safety. Occupational health and safety (OHS) training, focused on infection prevention and control (IPC) is one element of this approach. Currently, there is limited understanding of the framing and dialogue focused on OHS (IPC) training globally.

Objectives: This study aimed to examine IPC guidelines from selected high, low, and middle-income countries (LMIC) and other transnational health agencies, to examine the inclusion of OHS (IPC) training and to report on areas of inconsistencies and gaps.

Methods: For this review the IPC guidelines from the World Health Organization, US Centers for Disease Control and Prevention, Gulf Cooperation Council, as well as three high-income countries (Australia, Canada, and United Kingdom) and four LMIC (India, Indonesia, Pakistan and Philippines) were reviewed. 
Results: All the guidelines recommended job specific IPC training but only two discussed the need for delivery of OHS training on IPC at undergraduate and/or post graduate level, as well as part of a workplace induction. The recommendations around mode of delivery and curriculum elements differed across the guidelines and only two acknowledged the need to adopt adult learning principles. Lastly, none of the LMIC guidelines included recommendations about evaluating training programs.

Conclusion: Although all the guidelines suggest OHS (IPC) training be provided to HCPs, there is a lack of focus on the impact that HCPs prior experience should have on the content development, style of approach and evaluation of training programs. This may lead to undesirable outcomes like poor delivery of quality OHS (IPC) training programs. Developing a culture of learning in healthcare organizations, along with integration of appropriate adult learning principles would help to improve the development of training programs.

Disclosure of Interest: None declared.

P152

\section{Largely varying yield of universal SARS-COV-2 admission} screening by population incidence

T. Scheier ${ }^{1}$, A. Wolfensberger ${ }^{1}$, D. Saleschus ${ }^{1}$, R. Kouyos ${ }^{1}$, M. Vazquez $^{1}$, W. Zingg ${ }^{1}$, P. W. Schreiber ${ }^{1, *}$

${ }^{1}$ Division of Infectious Diseases and Hospital Epidemiology, University Hospital Zurich, Zürich, Switzerland

Correspondence: P.W. Schreiber

Antimicrobial Resistance \& Infection Control 10(1): P152

Introduction: A- or presymptomatic SARS-CoV-2 infected individuals pose a potential threat for health care facilities due to the risk of secondary cases. To prevent unnoticed introduction of SARS-CoV-2, several institutions implemented universal admission screening. However, little is known on the yield of this approach in different epidemiological settings.

Objectives: We aimed to describe the rate of asymptomatic SARSCoV-2 infected patients identified in universal admission screening, and its correlation with the population incidence.

Methods: Publicly available cantonal data were used to describe the population incidence. All patients admitted to the University Hospital Zurich, Switzerland, were tested for SARS-CoV-2 by polymerase chain reaction (PCR). SARS-CoV-2 positive patients were retrospectively categorized as symptomatic or asymptomatic at admission and history for prior COVID-19 assessed by medical chart review. For correlation analyses, we calculated the positivity rates per calendar week for the admission screening and incidence rates per calendar week and $100^{\prime} 000$ inhabitants for the cantonal data.

Results: In the canton of Zurich, median SARS-CoV-2 incidence was 34.3 infections per calendar week and $100^{\prime} 000$ inhabitants (interquartile range: 9.9 to 250.2 ). A total of $21^{\prime} 512$ admissions screenings were performed in a 44-week period between calendar week 182020 and calendar week 8 2021. SARS-CoV-2 PCR was positive in 647 (3.0\%) individuals. In 89 (13.8\%) individuals, the positive test result was considered as residual viral replication after recent COVID-19 and not reflecting contagiousness. 457 (70.6\%) SARS-CoV-2 positive individuals presented with COVID-19 symptoms at admission, whereas 101 (15.6\%) SARS-CoV-2 individuals did not show any COVID-19 symptoms. Both, the rate of SARS-CoV-2 positive individuals $(r=0.88$, $\mathrm{P}<0.001)$ and the rate of asymptomatic SARS-CoV-2 positive individuals $(r=0.84, P<0.001)$ correlated with the population incidence (Figure).

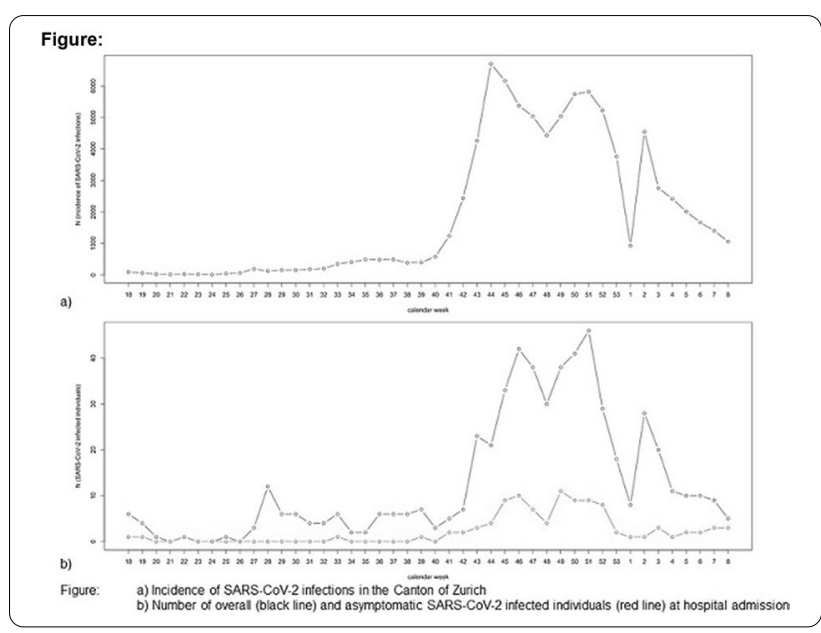

Conclusion: In line with prior studies, approximately $0.5 \%$ of all admission screenings were positive in asymptomatic individuals. A strong correlation of the rate of asymptomatic SARS-CoV-2 infected individuals with the population incidence was detected.

Disclosure of Interest: T. Scheier: None declared, A. Wolfensberger: None declared, D. Saleschus: None declared, R. Kouyos: None declared M. Vazquez: None declared, W. Zingg: None declared, P. W. Schreiber Grant/Research support from: "Filling the Gap" of the Medical Faculty of the University of Zurich, Conflict with: speaker honoraries from Pfizer, travel grant support from Pfizer and Gilead outside of the submitted work.

P153

Prevalence of COVID-19 in individuals without respiratory symptoms

S. Yatsyshina' ${ }^{1}$, M. Mamoshina ${ }^{1, *},{ }^{*}$ M. Elkina ${ }^{1}$, V. Akimkin $^{1}$

${ }^{1}$ Central Research Institute of Epidemiology, Moscow, Russian Federation

Correspondence: M. Mamoshina

Antimicrobial Resistance \& Infection Control 10(1): P153

Introduction: Asymptomatic COVID-19 persons may be involved in the spreading of SARS-CoV-2.

Objectives: The aim of our study was to determine the prevalence of SARS-CoV-2 in people without respiratory symptoms and evaluate the effectiveness of surgical masks for the prevention of COVID-19.

Methods: The study, conducted in August and October 2020 in 26 regions of Russia, involved 14,119 persons ( 4582 children) without respiratory symptoms in the last 2 weeks, who have not traveled outside the country. Naso-oropharyngeal swabs were sampled and tested for detection of SARS-CoV-2 RNA with the "AmpliSens Cov-Bat-FL" reagent kits (CRIE, Moscow). Participants were questioned about occupation, if they were wearing surgical masks and if they had had close contact with someone with a respiratory infection within last 2 weeks. Results: The SARS-CoV-2 RNA frequency increased from $0.49 \%$ in August to $4.02 \%$ in October $(p<0.001)$. Concentration of SARS-CoV-2 RNA varied from approximately $10^{10}$ in $1 \mathrm{ml}$ of respiratory swab to the limit of detection (1000 copies/ml). Close contact with respiratory infection person reported $3.14 \%$ of participants. SARS-CoV-2 RNA was found in $3.8 \%$ of such persons in comparison to $1.66 \%$ in whole group. Persons whose profession is associated with a high level of social contacts in October were infected less often than representatives of the same age group: SARS-CoV-2 was detected in $3.4 \%$ and $6.8 \%$, respectively $(p=0.001)$.

Conclusion: Individuals with asymptomatic COVID-19 may have high concentrations of SARS-CoV-2 and serve as a dangerous source of infection, especially when not wearing medical masks. 
The use of surgical masks reduced the risk of infection with SARSCoV-2 by $34 \%$. In order to provide more effective protection during prolonged contact with a COVID-19 patient, healthy individuals should use a respirator. Wearing of medical masks in community is a necessary anti-epidemic measure, since it reduces the spread of the virus and the likelihood of infection. Higher compliance with anti-epidemic measures of persons whose profession is associated with frequent social contacts contributed to a decrease in the level of their infection.

Disclosure of Interest: None declared.

\section{P154}

SARS-COV-2 infection in children in close contact with households with COVID-19

A. Hosseininasab", ${ }^{1, *}$, A. Khalooei ${ }^{2}$

${ }^{1}$ Pediatrics, ${ }^{2}$ Social medicin, KMU, Kerman, Iran, Islamic Republic Of

Correspondence: A. Hosseininasab

Antimicrobial Resistance \& Infection Control 10(1): P154

Introduction: Many vital issues, characteristics, quarantine measures and the treatment of children live in infected families with SARS-CoV-2 remain unknown.

Objectives: This study aimed to assess clinical features and frequency of PCR positive test among children in close contact with household COVID-19 cases.

Methods: In this study, on 111 children in family clusters with confirmed COVID-19 infection we defined the characteristics of children with COVID-19 infection from infected family clusters and addresses the need for medical attention to children infected with COVID-19 under the current viral crisis.In this study, on 111 children in family clusters with confirmed COVID-19 infection wedefined the characteristics of children with COVID-19 infection from infected familyclusters and addresses the need for medical attention to children infected with COVID-19 under the current viral crisis.

Results: The mean (SD) and median age of the children were 8.18 (3.96) and 8 years,respectively. Almost half $(n=55)$ of the children had at least one sign or symptomrelated to COVID-19. More than $42 \%(n=47)$ of all the children had at least onegeneral sign or symptom related to COVID-19, followed by respiratory $(27.9 \%, n=31)$ and gastrointestinal $(26.1, \mathrm{n}=29)$ signs and symptoms. Of the children exposed tocases with COVID-19 in their families, $42.3 \%(n=47)$ were PCR positive for COVID-19and only four cases were hospitalized. The frequencies of SARS-CoV-2 infection insymptomatic children and asymptomatic ones were $43.6 \%$ and $41.1 \%$, respectively $(P>0.5)$. Also, the frequency of SARS-CoV-2 infection showed no significant difference interms of sex, age, child birth order, and number of family members, underlyingdisease, and a history of drug use $(P>0.05)$.

Conclusion: Almost all SARS-CoV-2 infection is mainly asymptomatic or mild during childhood in this study. The importance of asymptomatic pediatric infection in the transmission of the disease to others needs further study.

Disclosure of Interest: None declared.

\section{Poster session: SARS-CoV2: PPE}

\section{P155}

Development of wearing practice and common use errors of mouth-nose coverings as well as preference in the choice of masks in the population during the COVID-19 pandemic in two cities of North Rhine-Westphalia, Germany

M. M. Neuwirth ${ }^{1, *}$, M. Neuwirth ${ }^{2}$, V. Montilva Ludewig ${ }^{1}$, F. Mattner ${ }^{1}$, R Otchwemah ${ }^{1}$

${ }^{1}$ Institute for Hygiene, Cologne Merheim Medical Centre, University Hospital Witten/Herdecke Cologne, Cologne, ${ }^{2}$ Department of Technology and Operations Management, University Duisburg-Essen, Duisburg, Germany

Correspondence: M. M. Neuwirth

Antimicrobial Resistance \& Infection Control 10(1): P155
Introduction: The mandatory wearing of mouth-nose covers is, in addition to maintaining distance, a central component of the transmission prevention of COVID-19 and is intended to protect potential contact persons from ingesting pathogen-containing material.

Objectives: The study examined whether the population's motivation to comply with the oral-nasal coverage requirement was higher immediately after its implementation than in the weeks that followed, as declining coronavirus disease-19 (COVID-19) case rates and the end of the initial lockdown on May 15,2020, may have led to a decrease in risk awareness and thus noncompliance with the established behavioral rules.

Methods: In a covert observational study in mask-required settings (public transportation and retail stores), mask types used and observed mask use errors were recorded over two time periods in a total of 9131 individuals. In addition, the importance of individual mask features, wearing behavior, and reasons for choosing current mouth-to-nose coverage, as well as attitudes toward COVID-19 protective measures, were collected in an online survey. Individuals observed in public and individuals in the online surveys represent two separate cohorts.

Results: Over the time (07.05.-13.05.2020/12.06.-08.07.2020), the mask wear adherence increased in from $97.2 \%$ to $98.6 \%(p<0.001)$. In addition, a percentage decrease in the number of people without mouth-nose cover $(p<0.001)$ and an increase in the correct mask application was observed $(p=0.024)$. In the online survey, $84 \%$ of respondents identified comfort/convenience and $81 \%$ of respondents identified fit as the most important mask attributes. The reasons for the choice of the mouth-nose cover used were heterogeneous and varied. The respondents stated that they found the wearing of a mouth-nose-covering annoying (57\%) but useful (60\%), but would not wear a mask if there was no mask obligation or recommendation to wear the masks (67\%).

Conclusion: Despite improvement, a high frequency of errors (31\%) in wearing face masks were still observed, indicating the need for better clarification of the population on the correct wearing of a mask.

Disclosure of Interest: None declared.

\section{P156}

Knowledge, attitude, and practice regarding the use of personal protective equipment among healthcare workers during COVID-19 pandemic in Tunisian health care facilities A. Ammar ${ }^{1, *}$, A. Maatouk ${ }^{1}$, O. Ezzi ${ }^{1}$, N. Omri ${ }^{1}$, W. Bannour ${ }^{1}$, R. Helali ${ }^{1}$, M. Njah' ${ }^{1}$, M. Mahjoub ${ }^{1}$

${ }^{1}$ Infection Prevention and Control, University Hospital of Farhat Hached,

Sousse, Tunisia

Correspondence: A. Ammar

Antimicrobial Resistance \& Infection Control 10(1): P156

Introduction: Working at the frontlines against the COVID-19 pandemic, healthcare workers (HCWs) should have adequate knowledge, positive attitude, and proper practice of personal protective equipment (PPE) to get protection from COVID-19 infection.

Objectives: we aimed in this study to assess the knowledge, attitude, and practice (KAP) regarding personal protective equipment (PPE) among HCWs in the University hospital of Farhat Hached (Sousse, Tunisia).

Methods: A cross-sectional study during COVID-19 pandemic using an online self-administered French language questionnaire. The questionnaire was submitted electronically during November and December 2020 using the most popular online groups of HCWs on Facebook in Tunisia. Data analysis was performed using the Statistical Package for Social Sciences (SPSS) version 21.0.

Results: In total, 140 health workers from 6 different Tunisian university hospitals participated in this study with median age of $31[28$, $38]$ years. Most of them were doctors (64\%). In total, participants responded correctly in $74 \%$ of questions related to knowledge about COVID-19 pandemic and proper use of PPE. According to $41.1 \%$ of the HCWs, PPE were not sufficiently available in their departments. Only $56 \%$ of them considered that their departments were adequately prepared to manage COVID-19 patients, and $68 \%$ of them perceived that 
they have received a good training for COVID-19 prevention. Most of the participants $(66.2 \%)$ managed a suspected or confirmed COVID-19 patient. Whatever the type of care provided, they always wear a protective mask, an overblouse or combination, gloves, protective glasses or a visor and headdress in respectively $71.4 \%, 57.1 \%, 62.6 \%, 47.3 \%$ and $68.1 \%$ of cases. Undressing PPE was considered safe in only $65.9 \%$ of cases.

Conclusion: The findings demonstrated that the HCWs had an overall good knowledge but a poor practice regarding PPE. This finding relieves the need for further training and practice evaluation concerning the use of PPE among HCWs.

Disclosure of Interest: None declared.

\section{P157}

Personal protective equipment and hand hygiene status in healthcare workers during COVID-19 pandemic in Mashhad university hospitals

N. Pourbadakhshan ${ }^{1}$, M. Parhizkar ${ }^{2}$, M. Mohammadi Keshouk2 ${ }^{2}$, F. Pelian², M. H. Aelami ${ }^{3, *}$

${ }^{1}$ Hand Hygiene and Infection Control Research Center, Imam Reza Hospital, ${ }^{2}$ Hand Hygiene and Infection Control Research Center Imam Reza Hospital, ${ }^{3}$ Hand Hygiene and Infection Control Research Center Imam Reza Hospital, MASHHAD UNIVERSITY OF MEDICAL SCIENCES, Mashhad, Iran, Islamic Republic Of

Correspondence: M. H. Aelami

Antimicrobial Resistance \& Infection Control 10(1): P157

Introduction: Health- care workers (HCWs) are in great danger of infection with COVID-19. They can transmit virus to coworkers and also their patients. Proper hand hygiene $(\mathrm{HH})$ and correct usage of personal protective equipment (PPE) could prevent healthcare associated infections including COVID-19.

Objectives: The aim of the study was to evaluate correct usage of PPE and compliance of hand hygiene among HCWs before and after touching patients.

Methods: During a 6 months period from June till November2020,we observed HCWs in two university hospitals in Mashhad, Iran. Standard questionnaire using WHO compliance check list for $\mathrm{HH}$ and WHO check list for PPE were filled by infection control nurses. Data were analyzed using SPSS software 2016.

Results: Seventy-one HCWs in medical and ICU wards of two university hospitals entered our study. There were 21 men and 50 women, most of them were nurses $62(87.3 \%)$. Thirty HCWs (42.3\%) from ICU wards and $37(52.1 \%)$ from medical wards and $4(5.6 \%)$ from surgical ward entered our research. Thirty-two (45.1\%) of them washed their hands with alcohol -based hand rub correctly. The $\mathrm{HH}$ compliance rate was $45.1 \%$ before touching patients and $67.6 \%$ after touching patients.Most HCWs in our study $69(97.2 \%)$ wore masks the right way.Wearing personal protective kit in a proper method was done in $42(59.2 \%)$ of them and taking off only in $34(47.9 \%)$ of HCWs. Hand hygiene compliance rate before touching patients was higher in medical wards compared to ICU and surgical wards. $p$ value $=0.05$.

Conclusion: Frequent education and training of HCWs is necessary for the standard of care.

Disclosure of Interest: None declared.

Poster Session: Catheter-associated urinary tract infections (CAUTI)

\section{P158}

Comparison of bacterial species and antimicrobial resistance between catheter and non-catheter associated urinary tract infections

S. D'incau ${ }^{1, *}$, A. Atkinson ${ }^{1}$, L. Leitner ${ }^{2}$, A. Kronenberg ${ }^{3}$, T. M. Kessler ${ }^{2}$, J. Marschall ${ }^{1}$

${ }^{1}$ Infectious Diseases, Inselspital, Bern University Hospital, Bern,

${ }^{2}$ Neuro-Urology, Balgrist University Hospital, Zürich, ${ }^{3}$ Institute for Infectious Diseases, University of Bern, Bern, Switzerland

Correspondence: S. D'incau

\section{Antimicrobial Resistance \& Infection Control 10(1): P158}

Introduction: Urinary tract infections (UTI) represent at least $40 \%$ of all hospital-acquired infections. The majority of cases are catheter-associated.

Objectives: Our goal was to clarify whether uropathogens and antimicrobial resistance (AMR) characteristics vary depending on catheter association.

Methods: We analyzed a dataset containing 27,158 urine cultures from the Swiss Centre for Antibiotic Resistance (ANRESIS) database from calendar year 2019. Group differences in the proportions of bacterial species and antibiotic-resistant isolates from catheter-associated UTI (CAUTI) and non-CAUTI samples were investigated using the chi-squared test (or variants). A two-sided $p$-value of $<0.05$ was considered statistically significant. We analyzed the 4 most common pathogens and clinically relevant antibiotics.

Results: The most often identified pathogens were E.coli, K.pneumoniae, P.aeruginosa, P.mirabilis (cf Table). E.coli from CAUTI samples were more often resistant compared to E.coli from non-CAUTI samples. Third generation cephalosporin (surrogate for ESBL), ciprofloxacin (CIP), norfloxacin (NOR) exhibited large differences $(p<0.001)$ (cf Figure). For K.pneumoniae, difference in susceptibility was highly significant for CIP $(p=0.001)$ and NOR $(p=0.03)$. For P.mirabilis, difference in susceptibility was significant only for NOR $(p=0.01)$. P.aeruginosa from non-CAUTI samples were significantly less often resistant to cefepim $(p=0.02)$ and piperacillin-tazobactam $(p=0.04)$.

\begin{tabular}{|c|c|c|c|}
\hline & CAUTI & Non-CAUTI & $p$ \\
\hline$n$ & 5317 & 21841 & \\
\hline \multicolumn{2}{|l|}{ Pathogen (\%) } & & $\begin{array}{l}<0.00 \\
1\end{array}$ \\
\hline E. coli & 1694 (31.9) & $11960(54.8)$ & $\begin{array}{l}<0.00 \\
1\end{array}$ \\
\hline K.pneumoniae & $591(11.1)$ & $2162(9.9)$ & 0.008 \\
\hline P.aeruginosa & $535(10.1)$ & $711(3.3)$ & $\begin{array}{l}<0.00 \\
1\end{array}$ \\
\hline P.mirabilis & $283(5.3)$ & $870(4.0)$ & $\begin{array}{l}<0.00 \\
1\end{array}$ \\
\hline
\end{tabular}

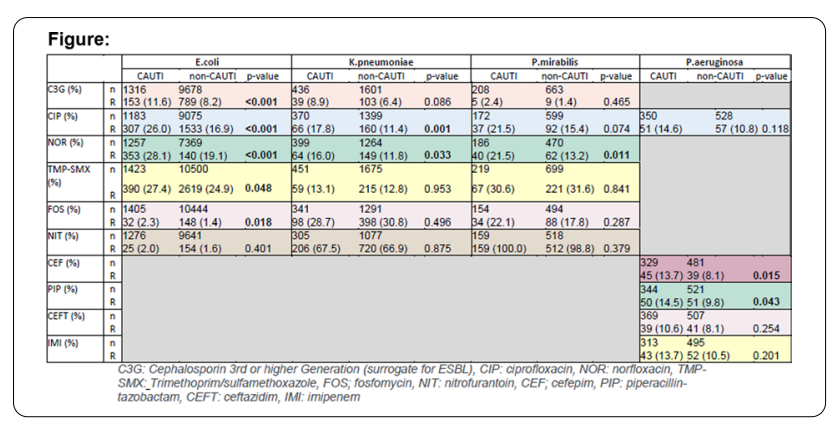

Conclusion: The pathogen distribution found in CAUTI and nonCAUTI samples was similar, with the exception of Pseudomonas being more often detected in CAUTI samples. CAUTI pathogens exhibited antibiotic resistance more frequently than non-CAUTI pathogens. Of note, some often-prescribed antibiotics for empirical treatment such as CIP and TMP-SMX exhibited resistance rates of up to $30 \%$ in some of the most common pathogens. This emphasizes the need for urine sampling for culturing before initiating a therapy in case of CAUTI and the need for therapeutic alternatives.

Disclosure of Interest: None declared. 
P159

Reducing urinary catheter use and urinary tract infections in geriatric patients - results of a pilot interventional study L. Mrziglod 1", , S. Saydan², P. Gastmeier ${ }^{2}$, S. Hansen ${ }^{2}$

${ }^{1}$ Department of Infectious Diseases, ${ }^{2}$ Institute of Hygiene and Environmental Medicine, Charité - Universitätsmedizin Berlin, Berlin, Germany Correspondence: L. Mrziglod

Antimicrobial Resistance \& Infection Control 10(1): P159

Introduction: Indwelling urinary tract (UT) catheters (UTC) are a wellknown risk factor for UT infection (UTI).

Objectives: Since geriatric patients are at high risk for infections an intervention was initialized in 4 geriatric wards of a secondary care hospital.

Methods: Between 11/2018 and 1/2020 unit-based data on UTC use and nosocomial UTI were collected according to methods of the German national surveillance system KISS. Wards received data feedback in $5 / 2019$. From $7 / 2019$ to $1 / 2020$ the intervention focusing on i.) education and training on UTC insertion and care, ii.) daily assessment of UTC need with so called "catheter indication sheets (CS)" in patients with UTC $\geq 2$ days and iii.) timely removal of unnecessary UTCS was realized. A team-based approach was initiated by introducing the intervention to ward physicians and nurses.

UTC use, incidences and incidence densities for UTC-associated (ass.) UTI were calculated before and during the intervention and differences were tested. Additionally, the use of CS in patients with UTC $\geq 2$ days was analyzed during the intervention.

Results: 3,564 patients with 53,954 patient days (pd) and 9,208 UTC days were included. Overall the pooled UTC use rate decreased from $19.1 / 100 \mathrm{pd}$ to $15.2 / 100 \mathrm{pd}(\mathrm{p}<0.001)$. The pooled incidence of UTCass. UTI decreased from 2.07 to 1.40 UTC-ass. UTI per 100 patients $(p=0.128)$ and the pooled incidence density of UTC-ass. UTI decreased from 1.34 to 0.95 UTC-ass. UTI per $1000 \mathrm{pd}(\mathrm{p}=0.18)$. During the intervention 186 from 351 patients with a UTC $\geq 2$ days received a CS for daily UTC assessment (53\%). Patients with a CS had in median 9 UTC days (IQR 5,15) whereby patients without CS had 10 UTC days (IQR $7,16)(p=0.07)$.

Conclusion: Our data show that a surveillance-based prevention intervention reduces UTC use and UTC-ass. UTI in geriatric patients. Further studies are needed to show an effect in larger populations. To what extent the usage of checklists for daily UTC assessment affects UTI prevention has to be further investigated.

Disclosure of Interest: None declared.

Poster Session: Catheter-related bloodstream infection

\section{P161}

Bloodstream infection among the patients infected with COVID-19 in a tertiary hospital in Iran

G. Pouladfarr,* on behalf of Zahra Jafarpour

${ }^{1}$ Professor Alborzi Clinical Microbiology Research Center, Shiraz University of Medical Sciences, Shiraz, Iran, Islamic Republic Of

Correspondence: G. Pouladfar

Antimicrobial Resistance \& Infection Control 10(1): P161

Introduction: Bacterial co-infection, especially bloodstream infection (BSI) could be a leading cause of morbidity and mortality in hospitalized patients with coronavirus disease 2019 (COVID19) infection, demanding a proper empirical antibacterial therapy.

Objectives: This study aimed to investigate the bacterial etiology of BSI in two groups of patients (i.e., COVID-19 and non-COVID-19) in a tertiary hospital in Iran.

Methods: We investigated all bacterial etiologies of BSIs among the patients hospitalized in Nemazi Teaching Hospital in Shiraz, during March 2020-March 2021. Using an automated blood culture system for BSI diagnosis, we detected 919 pathogenic bacteria by standard biochemical methods: 132 in COVID-19 group and 787 in non-COVID-19 one.
Results: Totally, $62 \%$ and $54 \%$ of the patients were male in COVID-9 and non-COVID-19 groups, respectively. In COVID-19 group, 98 (74.2\%) were adult and 34 (25.8\%) children (under 18 years of age). The age of 147 patients in non-COVID-19 group was unknown (19.2\%) and $381(48.5 \%)$ were adult and 259 (32.3\%) children. As revealed, in COVID-19 group, adults with BSI outnumbered the children ( $74.2 \%$ vs. $25.8 \%$, respectively); however, the difference in non-COVID-19 groupwas less $(48.5 \%$ vs. $32.3 \%$, respectively), even if all the patients with unknown age in the latter were considered to be adult $(528,67 \%)$. The two most common bacterial isolates in COVID-19 group were Achromobacter species (spp.) $(24,18 \%)$ and Klebsiella app. In non-COVID-19 group, the most common pathogens were Escherchia coli $(89,11 \%)$ and Staphylococcus aureus $(103,13 \%)$.

Conclusion: BSI among hospitalized adult patients with COVID-19 infection was more frequent than that among children. It could be explained by the fact that the total number of adult patients with COVID-19 who needed hospitalization is much higher than children. Also, the two most frequently isolated pathogens associated with BSI were different in two groups of patients hospitalized in a tertiary hospital of a developing country, that is, Iran. Nevertheless, the etiology of BSI in hospitalized patients with COVID-19 needs further investigations which could help physicians to choose the most effective empiric therapy in respective patients.

Disclosure of Interest: None declared.

\section{P162}

Successful implementation of chlorhexidine-impregnated dressings in adults with central venous catheter

Q. Liu ${ }^{1,}$, , H. X. Toh ${ }^{1}$, L. C. Lee ${ }^{1}$, K. B. How ${ }^{1}$, M. L. Ling ${ }^{1}$

${ }^{1}$ Infection Prevention and Epidemiology, Singapore General Hospital,

Singapore, Singapore

Correspondence: Q. LiU

Antimicrobial Resistance \& Infection Control 10(1): P162

Introduction: Singapore General Hospital (SGH) has been using Central line-associated bloodstream infections (CLABSIs) insertion and maintenance bundles as part of our efforts to prevent CLABSIs in patients. CDC (2017) updated the recommendations on the use of chlorhexidine-impregnated (CHG) dressings for prevention of intravascular catheter-related infections. Thereafter, SGH embarked on CDC's recommended implementation.

Objectives: Implementation of CHG dressings in adults with CVC during COVID-19 pandemic.

Methods: Approval was obtained in 2019 to implement its use but we were only able to launch implementation from 16 July 2020. During the COVID-19 pandemic, training was conducted via e-learning platforms. Infection Prevention Liaison Officers (IPLOs) of the wards were trained and tasked with cascading information on CHG dressing to their respective wards. Infection Prevention Nurses (IPNs) conducted audits via interviews with the ward nurses on their knowledge and compliance to usage of CHG dressing in August and November 2020. In addition, audits on proper application and documentation on the usage of CHG dressing was done from January 2021 to March 2021.

Results: Interview results.

In the August 2020 interviews, 7 out of 37 wards (19\%) were unaware of the implementation, where the products were kept and unable to describe the indications of the CHG dressing. Immediate action was taken to remind ward nurses through the IPLO newsletter as well as through daily huddles done by IPNs with ward IPLOs. By November 2020 , only 2 out 40 wards (5\%) interviewed were unaware of the above - a substantial improvement.

Practices results.

In January 2021, audits on proper application and documentation on the usage of CHG dressing showed a low compliance of $71 \%$, which subsequently improved to $100 \%$ compliance in February and March 2021.

Conclusion: We were able to successfully implement the use of CHG dressing during the pandemic through the education of stakeholders. Regular audits were conducted to validate successful implementation. 
In conclusion, engaging IP champions, training, and audits greatly aided in the successful implementation of a new practice in the clinical setting despite challenges posed by the pandemic.

Disclosure of Interest: None declared.

P163

Opinions of nurses in ICU on the importance and utilization of the CLABSI prevention bundle in an academic hospital in Gauteng

D. $\mathrm{Ng}^{\prime} \mathrm{ambi}^{1,}, \mathrm{~S}$. Armstrong ${ }^{2}$

${ }^{1}$ Kamuzu Central Hospital, Lilongwe, Malawi, ${ }^{2}$ University of the Witwatersrand, Johannesburg, South Africa

Correspondence: D. Ng'ambi

Antimicrobial Resistance \& Infection Control 10(1): P163

Introduction: The Central Line Associated Blood Stream Infections (CLABSI) prevention bundle has proven to prevent CLABSI when all elements are utilized. While the bundle is evidence based, if nurses do not believe it is important, they are unlikely to use it. The purpose of the study was to describe the nurses' opinions on the importance and utilization of the CLABSI prevention bundle with a view, at a later stage, to inform the infection control unit on what to consider when teaching and implementing the CLABSI prevention bundle.

Objectives: To describe the opinions of the nurses in ICU of the importance and the usefulness of the CLABSI prevention bundle. To describe the relationship between the importance and utilization of the CLABSI prevention bundle based on the nurses' opinions.

Methods: This study used a descriptive research design using $Q$ methodology approach. Q methodology is one of the methods used to study opinions. The study was done at one of the public academic hospitals in Gauteng. Data was collected using a $\mathrm{Q}$ sort technique from thirty nurses in Intensive Care Unit (ICU). The nurses had to sort a set of thirty statements about the CLABSI prevention bundle. The sorting ( $Q$ sort) was done on a $Q$ diagram under specific conditions of instruction. Pictures of completed $Q$ sorts were taken and transcribed on smaller $Q$ diagram and that was used for data analysis. Factor analysis was used to interpret data by means of a software PQ Method version 2.35 package.

Results: The overall finding is that opinions are related to human behaviour. The results of the study revealed mixed opinions among the nurses in ICU. Some value aseptic technique during insertion of the central line. Some value maintenance of the central line, while some value some elements on both the insertion and maintenance of the central line. The study revealed that those elements that the nurses believe to be important, are the ones that they also utilize.

Conclusion: Nurses opinions differ from one another and judging by their opinions, it is evident that not all CLABSI prevention bundle elements are believed to be important, nor are all bundle elements utilized. Recommendations have been proposed for nursing education, practice, management and research.

Disclosure of Interest: None declared.

\section{P164}

Risk of catheter associated bloodstream infection by catheter type in neonatal intensive care unit

G. Catho ${ }^{1, *}$, V. Sauvann ${ }^{1}$, F. Rosa Mangeret ${ }^{2}$, M.-N. Chraiti ${ }^{1}$, O. Baud ${ }^{1}$, S. Harbarth ${ }^{1}$, N. Buetti ${ }^{1}$

${ }^{1}$ Infection Control Division, ${ }^{2}$ Division of Neonatology and Pediatric Intensive Care "Geneva University Hospitals and Faculty of Medicine, Geneva, Switzerland

Correspondence: G. Catho

Antimicrobial Resistance \& Infection Control 10(1): P164

Introduction: Little is known about the bloodstream infection (BSI) risk associated with umbilical intravascular catheter in neonates.

Objectives: The objective of this study was to assess the risk of intravascular catheter-associated BSI (CABSI) among the different catheter types using a large prospective database at the neonatal intensive care unit (NICU) in the Geneva University Hospitals.
Methods: We included all neonates hospitalised in NICU with at least one central intravascular catheter insertion from January 2017 to December 2020. Dwell time was considered as the number of full days a catheter was in place. Firstly, we described CABSI according to the catheter type (arterial $[A U C]$, venous umbilical catheters [VUC]) during the catheter maintenance. Secondly, we used univariable and multivariable marginal Cox regression models for clustered data to evaluate the CABSI-risk according to the catheter type among umbilical catheters.

Results: We observed 1103 intravascular catheters in 574 neonates. Among them, $55(9.6 \%)$ were very low birth weight $(<1000 \mathrm{~g})$ and 323 (56.27\%) were low birth weight $(<2500 \mathrm{~g})$. Overall, $388(67.6 \%)$ were preterm and among them $101(17.6 \%)$ were extremely preterm, 170 (29.6\%) were very preterm and $117(20.4 \%)$ were moderate to late preterm. 264 were female (46\%). We identified 581 VUC, 198 AUC and 324 PICC. The median dwell-time was 2 (IQR: 0; 4), 3 (IQR: 2; 5) and 6 (IQR: $4 ; 13)$ days for UVC, AUC and PICC, respectively. We identified 4 and 17 CABSIs in AUC and VUC, respectively. The CABSI risk were increased after two days of catheter maintenance for umbilical catheters (Figure). Among umbilical catheters and using univariable Cox models, we observed a similar CABSI-risk between VUC and AUC (HR 1.09, 95\% $\mathrm{Cl}$ : 0.38-3.08., $\mathrm{p}=0.87)$. After adjustment for sex, prematurity and gestational age, we observed a similar CABSI-risk between VUC and AUC (HR 0.57, 95\% Cl 0.156-2.081 p=0.394).

Conclusion: Implementing a strategy to reduce duration of umbilical catheters in NICU may be an effective measure to reduce CABSI in this population and requires further exploration.

Disclosure of Interest: None declared.

P165

Survey of central venous catheter care bundle compliance in medical wards, hospital Pulau Pinang, Malaysia

L. Kok Soon 1,*, L. Wei Chyuan², W. Peng Shyan², L. Kar Nim², C. Ting Soo²

${ }_{1}^{1}$ Infectious Disease, Hospital Sultanah Aminah, Johor Bahru, ${ }^{2}$ Infectious

Disease, Hospital Pulau Pinang, Pulau Pinang, Malaysia

Correspondence: L. Kok Soon

Antimicrobial Resistance \& Infection Control 10(1): P165

Introduction: Central venous catheter (CVC) care is important in preventing line-related infection. Periodic surveillance in Hospital Pulau Pinang showed a compliance rate of $63.2 \%$ for CVC care bundle and 9 out of 24 patients diagnosed to have bloodstream infection were associated with a central line in April 2018.

Objectives: This project aims to improve the compliance of CVC care bundle, identify barriers in compliance and establish CLABSI incidence in the Medical wards of Hospital Pulau Pinang, Malaysia.

Methods: Subjects audited ( $>18$ years old) were in-patients with central venous catheters inserted during working hours.

This audit is divided into 3 phases:

1st phase: Baseline audit on subjects with CVC inserted in medical wards, CVC insertion subjects identified, and follow-ups conducted.

During the CVC insertion, the process was audited with a checklist with the information such as site selection, correct antiseptic use and maximum barrier protection.

A checklist form produced by the Ministry of Health was given to nurses for daily review.

The 2nd phase consisted of interventions were as follows:

- Continuous medical education using lectures and training videos

on proper CVC bundle care during line insertion.

- Short quiz on central catheter care maintenance.

- Regular hand hygiene awareness and promotion.

- Dedicated CVC carts/procedure room for CVC insertion.

3rd phase: a post-intervention audit on CLABSI rate and data analysis. 
Results:

\begin{tabular}{|l|l|l|}
\hline \multicolumn{2}{|c|}{} \\
\hline Age (mean) & Pre intervention Survey ( $\mathrm{n}=36)$ & Post Intervention Survey ( $\mathrm{n}=34)$ \\
\hline Gender & 59 years & 52 years \\
Male & $41.7 \%$ & $55.8 \%$ \\
Female & $58.3 \%$ & $44.2 \%$ \\
\hline Types of Catheters & $8.3 \%$ & $5.8 \%$ \\
Double lumens & $38.8 \%$ & $32.4 \%$ \\
Triple lumens & $61.8 \%$ \\
\hline Triple lumens (dialysis) & $52.7 \%$ & \\
\hline Indication & & $8.8 \%$ \\
Inotropic support & $8.3 \%$ & $6.5 \%$ \\
Intravenous & $30.5 \%$ & $61.7 \%$ \\
Haemodialysis & $58.3 \%$ & $0 \%$ \\
\hline CVP monitioring & $2.7 \%$ & 6 days \\
\hline Mean Duration & 8 days \\
\hline
\end{tabular}

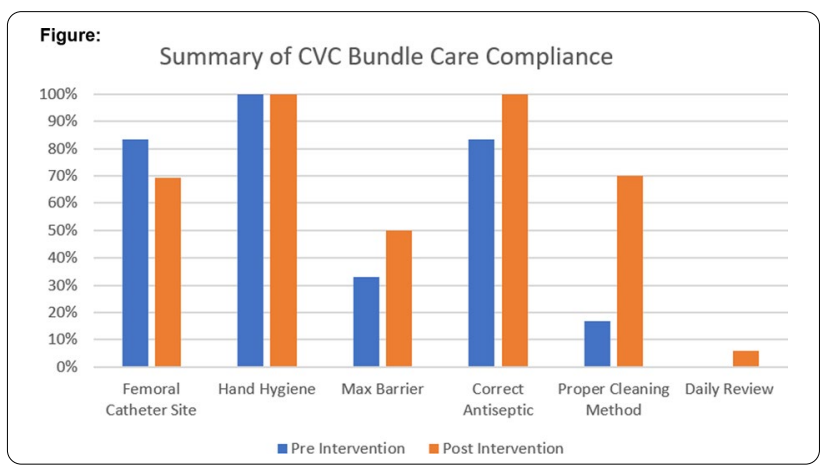

Conclusion: This project indicated components of the CVC bundle care with improved compliance, notably maximal barrier usage, correct antiseptic, and proper cleaning method. Areas for improvement are choice of site for CVC insertion and daily CVC review. Recommendations from this audit are for employment of continuous medical education and regular audits to increase knowledge and awareness of CVC care bundles. Due to a low sample size, the CLABSI rate was unable to be established.

Disclosure of Interest: None declared.

\section{P166}

Short-course versus long-course systemic antibiotic treatment for uncomplicated intravascular catheter-related bloodstream infections due to gram-negative bacteria, enterococci or coagulase-negative staphylococci: a systematic review S. Muff ${ }^{1, *}$, A. Tabah ${ }^{2}$, Y.-A. Que ${ }^{3}$, J.-F. Timsit ${ }^{4}$, L. Mermel ${ }^{5}$, S. Harbarth ${ }^{1}$, N. Buetti $^{1}$

${ }^{1}$ University of Geneva Hospitals and Faculty of Medicine, Geneva, Switzerland, ${ }^{2}$ Redcliffe Hospital, Brisbane, Australia, ${ }^{3}$ University Hospital Bern, Bern, Switzerland, ${ }^{4}$ University of Paris, Paris, France, ${ }^{5}$ Rhode Island Hospital and Department of Medicine, Providence, United States

Correspondence: S. Muff

Antimicrobial Resistance \& Infection Control 10(1): P166

Introduction: The optimal duration of systemic antimicrobial treatment for catheter-related bloodstream infections (CRBSI) is unknown.

Objectives: In this systematic review, we aimed to assess the efficacy of short-course treatment for CRBSI due to Gram-negative bacteria, coagulase-negative staphylococci and enterococci.

Methods: We systematically searched the electronic bibliographic databases MEDLINE, EMBASE and Cochrane Library for studies published before February 2021. All studies that investigated the duration of adequate systemic antibiotic treatment in adult patients with uncomplicated intravascular catheter infections due to Gram-negative bacteria, coagulase-negative staphylococci or enterococci were eligible for inclusion. Studies including concomitant treatment with antibiotic lock therapy were excluded. The primary outcomes were clinical failure/cure, mortality and microbiologic-confirmed relapse.

Results: Seven retrospective cohort studies and one case-cohort study met the inclusion criteria. No randomized controlled studies met inclusion criteria. The quality of the included studies was low $(n=7)$ to moderate $(n=1)$. No significant differences were observed regarding mortality and microbiological relapse between short-course and long-course systemic antibiotic treatment in patients with CRBSI due to coagulase-negative staphylococci or Gram-negative bacteria. No association was found between mortality and treatment duration in the two studies assessing enterococcal CRBSI.

Conclusion: The limited data available suggests that shorter systemic antibiotic treatment duration may be sufficient for uncomplicated CRBSI. Further well-designed prospective studies are needed to confirm these findings.

Disclosure of Interest: S. Muff: None declared, A. Tabah: None declared, Y.-A. Que: None declared, J.-F. Timsit Grant/Research support from: Astellas, $3 \mathrm{M}, \mathrm{MSD}$, and Pfizer, Consultant for: $3 \mathrm{M}, \mathrm{MSD}$, Bayer Pharma, Nabriva, and Pfizer, Conflict with: 3 M, MSD, Pfizer, and Biomerieux, L. Mermel Consultant for: Citius Pharmaceuticals, Marvao Medical, Leonard-Meron Biosciences, Destiny Pharma, and Nobio unrelated to the submitted work, S. Harbarth: None declared, N. Buetti: None declared.

\section{P167}

Prevalence and death-associated factors in healthcare-associated bacteremia in the department of infectious and tropical diseases of Fann hospital, Dakar

D. KA ${ }^{1, *}$, A. Massaly ${ }^{1}$, N. M. Fall ${ }^{1}$, V. M. P. Cisse Diallo ${ }^{1}$, C. T. Ndour ${ }^{1}$, M. Seydi ${ }^{1}$ ${ }^{1}$ Dakar, Centre Hosptalier de Fann, Université Cheikh Anta Diop de Dakar, Dakar, Senegal

Correspondence: D. KA

Antimicrobial Resistance \& Infection Control 10(1): P167

Introduction: Healthcare-associated bacteremia is a real public health problem because of its high morbidity and mortality.

Objectives: The objectives of this study were to describe the characteristics of bacteremia and to identify death - associated factors.

Methods: This was a retrospective, descriptive and analytical study based on the records of patients hospitalized in the department of infectious and tropical diseases, whose diagnosis of healthcare-associated bacteremia was retained during the study period from January 1 , 2016 to December 31, 2017.

Results: Fifty-two cases of healthcare-associated bacteremia were collected. The hospital prevalence was $2.6 \%$. Male sex was predominant with a sex ratio $=1.2$. The average age was $42 \pm 16$ years. Twenty-two patients were HIV-infected. The majority of patients (32 cases) had been on antibiotics before their current hospitalization. Regarding the reasons for hospitalization, pulmonary signs dominated the series, followed by neurological and gastrointestinal signs with respectively 27,26 and 18 cases. Invasive devices were dominated by peripheral venous catheters $(100 \%)$ followed by urinary catheterization $(87 \%)$. The main germs found were Staphylococci (26.6\%), Enterobacter spp (23.5\%), Klebsiella pneumonia (18.7\%) and Escherichia coli (14.1\%). Staphylococci were highly resistant to cefoxitin (88.2\%) and methicillin (70\%). There was a high level of resistance of gram-negative bacilli to 3 rd line cephalosporin. Case fatality was $35 \%$. Acute renal failure $(p=0.01)$ and male gender $(p=0.05)$ were associated with the occurrence of death.

Conclusion: Healthcare-associated bacteremia is a real public health problem. Standard hygiene measures play an important role in the control of these infections.

Disclosure of Interest: None declared. 
Poster Session: Diagnostic Stewardship

P168

Performance evaluation of genexpert SARS-COV-2 in early

diagnosis of COVID-19

S. M. Aljohani ${ }^{1,2,3,4, *}$, A. Alshehri ${ }^{5}$, A. ALSHEHRI ${ }^{5}$, T. Alsalmi $^{5}$

${ }^{1}$ Consultant Microbiologist, KAMC-Riyadh, ${ }^{2}$ Associate professor, King Saud Bin AbdulAziz University for Health Sciences, ${ }^{3}$ Adjunct Associate Professor, King Abdullah International Medical Research Centre, ${ }^{4}$ Editor-in-Chief, Journal Of Infection and Public Health, ${ }^{5}$ Molecular Microbiology, KAMC-R, RIYADH, Saudi Arabia

Correspondence: S. M. Aljohani

Antimicrobial Resistance \& Infection Control 10(1): P168

Introduction: Rapid diagnostics of SARS-CoV-2, are a very critical in critically ill patients. The routinely used real-time RT-PCR is sensitive, specific and able to process large batches of samples. However, turnaround time is long and in cases where fast obtained results are critical, molecular point of care tests (POCT) can be an alternative.

Objectives: In our study, we evaluate Cepheid Xpert Xpress SARS-CoV-2 point-of-care test for rapid and accurate diagnosis of SARS-CoV-2.

Methods: The Xpert Xpress SARS-CoV-2 assay was evaluated against the routine real-time RT-PCR assays using RealStar ${ }^{\circledR}$ SARS-CoV-2 RTPCR Kit 1.0 (Altona). A sensitivity and specificity was calculated using different samples with different concentration and different viral load. A total of 300 samples were enrolled into this evaluation. 45 positive samples and 255 negative samples.

Results: Xpert Xpress SARS-CoV-2 point of care test showed great performance compared to routine in-house testing with a limit of detection. Other seasonal respiratory viruses were not detected. In clinical samples Xpert Xpress SARS-CoV-2 reaches an agreement of $100 \%$ compared to all in-house RT-PCRs. The PPA was $97.8 \%$ (95\% Cl: $88.4 \%-99.6 \%)$ and the NPA was $95.6 \%$ (95\% Cl: 85.2\%—98.8\%).

Conclusion: Cepheids GeneXpert Xpert Xpress SARS-CoV-2 is a valuable addition for laboratories in situations where rapid and accurate diagnostics are of the essence. It's great tool to diagnose/ role out patients in Emergency room, Labor \& delivery wards and pre-emergency surgeries or interventions.

Disclosure of Interest: None declared.

\section{P169}

Predicting PCR test necessity using inteligent methods

A. Taherzadeh ${ }^{1, *}$, E. M. Farkhani ${ }^{2}$, I. Mostafavi ${ }^{2}$

${ }^{1}$ Isfahan University of technology, Tehran, ${ }^{2}$ Mashhad University of Medical Sciences, Mashhad, Iran, Islamic Republic Of

Correspondence: A. Taherzadeh

Antimicrobial Resistance \& Infection Control 10(1): P169

Introduction: With the outbreak of the Covid-19 pandemic in late 2019 , Polymerase chain reaction (PCR) assay has become the accepted standard for diagnosing this disease. As the prevalence rate increases, some countries face shortages of test kits. A growing need for a method that can determine the necessity of laboratory testing using clinical symptoms is an inevitable outcome of this pandemic.

Objectives: The objective of this study was to design and develop an algorithm using intelligent methods, to predict the necessity of PCR using the patient's clinical symptoms.

Methods: The dataset used in this study consists of 226,777 suspected cases of Covid-19 with known PCR results (145,752 positive and 81,025 negative), with one or more clinical symptoms including fever, shortness of breath, chest pain, cough, sore throat, and chills. Furthermore, information such as age, gender, BMl, background disease, being in a high-risk group, and any close contact with a positive covid-19 patient was taken from the cases. Using three supervised methods (support vector machine (SVM), K-Nearest Neighbor (KNN), and Decision Tree (DT)), the dataset was classified into two categories: probable positive PCR and probable negative PCR. KNN and DT had higher accuracy (> 80\%) and less processing time. The risk of classifying a positive PCR as negative is greater than classifying a negative PCR as positive. Therefore, weighted matrices were involved in the algorithm so that the false positive classification error was much greater than the false negative classification error.

Results: Eventually, the KNN algorithm with $82 \%$ precision, $91 \%$ recall, and $63 \%$ specificity was selected as the optimal method and was used for predicting PCR necessity. This expresses that the designed algorithm is able to suggest $91 \%$ of patients with future positive PCR to take a PCR, and $82 \%$ of patients that the designed algorithm introduced to undergo a PCR, will have positive PCR result. In addition, using the designed algorithm will reduce taking PCR from patients with future negative PCR result, down to $37 \%$.

Conclusion: Applying the designed algorithm in countries with a shortage of PCR kits will optimize resources with minimum inaccuracy. The outcome of this study and the designed algorithm can be used at health centers as a criterion to determine whether a patient should undergo a PCR.

Disclosure of Interest: None declared.

P170

Diagnostic stewardship in emergency medicine: results

of a retrospective cohort study

C. Berninghausen 1,* F. Schwab², L. Hottenbacher ${ }^{3}$, R. Somasundaram ${ }^{4}$, P.

Gastmeier ${ }^{2}$, S. Hansen ${ }^{2}$

${ }^{1}$ Department of Cardiology and Intensive care, Vivantes Wenckebach

Klinikum, ${ }^{2}$ Institute of Hygiene and Environmental Medicine, Charité

- Universitätsmedizin Berlin, ${ }^{3}$ Department of Emergency Medicine,

Vivantes Auguste-Viktoria Klinikum and Vivantes Wenckebach Klinikum,

${ }^{4}$ Department of Emergency Medicine, Charité - Universitätsmedizin

Berlin, Berlin, Germany

Correspondence: $C$. Berninghausen

Antimicrobial Resistance \& Infection Control 10(1): P170

Introduction: Once sepsis is suspected, a minimum of 2 sets of blood cultures (BCs) should be obtained prior to administration of antimicrobial therapy (AT) to support identification of pathogens and to allow de-escalating AT.

Objectives: Appropriate BC (2 sets of BCs prior to administration of AT) sampling was investigated in three emergency departments (EDs).

Methods: We performed a retrospective cohort study of patients admitted via one of 3 EDs with the hospital discharge diagnosis "sepsis" according to ICD-10 System A40- A41 in 2018. Exclusion criteria were nosocomial sepsis, elective admissions and transfers from external hospitals and direct transfers to intensive care units.

Administrative and clinical data were retrospectively collected from patients' charts. Independent factors associated with appropriate $\mathrm{BC}$ were analyzed by multivariable logistic regression analysis. Ethical approval was obtained from Charité - Universitätsmedizin Berlin. Results: From 1,761 admitted patients 1,143 (676 male, 466 female) patients were analyzed. 542 (31\%) patients were immunocompromised, $522(46 \%)$ presented with a temperature $>38^{\circ} \mathrm{C}$. 993 patients $(87 \%)$ showed organ dysfunction. 946 patients $(83 \%)$ received BCs whereby $520(45 \%)$ patients received 1 set and 426 (37\%) patients at least 2 sets.

349 (31\%) patients received appropriate sampling of $\geq 2$ BC sets before AT. Factors independently associated with appropriate BC collection are shown in table 1. 


\begin{tabular}{l} 
Figure: \\
Table 1: Results of the multivariable analysis: Factors associated with appropriate blood culture \\
sampling \\
\begin{tabular}{|l|l|c|c|c|}
\hline Parameter & Category & Odds ratio & $95 \% \mathrm{Cl}$ & p-value \\
\hline Gender & Male & 1.53 & $(1.15-2.03)$ & 0.003 \\
\hline Mental status & Notaltered & $1=$ Reference & & \\
\hline & Altered & 1.00 & $(0.71-1.42)$ & 0.989 \\
\hline & NK & 0.56 & $(0.38-0.82)$ & 0.003 \\
\hline Nemperature $\left({ }^{\circ} \mathrm{C}\right)$ & Normal (36-<38) & $1=$ Reference & & \\
\hline & $<36$ & 1.50 & $(0.97-2.34)$ & 0.069 \\
\hline & $\geq 38$ & 1.86 & $(1.36-2.54)$ & $<.0001$ \\
\hline $\begin{array}{l}\text { Systolic blood pressure } \\
\text { (mmHg) }\end{array}$ & Normal (>100) & $1=$ Reference & & \\
\hline & $\leq 100$ & 1.41 & $(1-1.98)$ & 0.052 \\
\hline & NK & 0.29 & $(0.17-0.51)$ & $<.0001$ \\
\hline Immunodeficiency & Yes & 1.50 & $(1.13-1.98)$ & 0.004 \\
\hline Emergency Department & $\mathrm{A}$ & $1=$ Reference & & \\
\hline & $\mathrm{B}$ & 0.33 & $(0.21-0.53)$ & $<.0001$ \\
\hline & $\mathrm{C}$ & 0.18 & $(0.12-0.28)$ & $<.0001$ \\
\hline
\end{tabular} \\
\hline
\end{tabular}

Conclusion: Data show that BC sampling varies among EDs and among individual patients. Awareness for appropriate $B C$ sampling should be increased in order to provide adequate AT to all patients with the suspicion of sepsis.

Disclosure of Interest: None declared.

\section{P171}

The effect of multifaceted interventions on storage and transport of samples for microbial culture

M. J. Cho', J. S. Jeong, ${ }^{2,}$, Y. H. Kim¹', I. S. Jeong ${ }^{3}$, S. H. Kim ${ }^{1}$

${ }^{1}$ Department of Nursing, Asan Medical Center, Seoul, ${ }^{2}$ Clinical Nursing

University of Ulsan, Ulsan, ${ }^{3}$ College of Nursing, Pusan National University, Busan, Korea, Republic Of

Correspondence: $\mathrm{S}$. Jeong

Antimicrobial Resistance \& Infection Control 10(1): P171

Introduction: Storage and transportation of samples for microbial culture is critical for correct diagnosis and treatment of patient diseases. When samples are collected from the ward, in most cases, nurses are responsible for storing and transporting samples.

Objectives: The purpose of this study is to provide multifaceted interventions for nurses and to confirm the changes on knowledge, sample management practice and blood culture transport time.

Methods: A one-group pretest-posttest experimental design was conducted with 41 nurses in two general wards of tertiary acute care hospital in Seoul, South Korea. Multifaceted interventions including education, feedback, posting guidelines and reminders, and system improvement were provided to ensure that proper storage and timely transport of samples from May 2019 to January 2020. The outcomes were measured in three ways before and after intervention; the knowledge on sample management using self-reported questionnaire, sample management practice at wards by observation, and time from blood sampling to arrival at the laboratory.

Results: Among the participants, $31.7 \%$ have trained in storage and transportation of samples. After intervention, the average knowledge was significantly increased from 1.20 points $( \pm 1.12)$ to 5.10 points $( \pm 3.20)$ out of 9 for transportation $(p<0.001)$. The average knowledge on sample storage was also increased but insignificant. The proportion of appropriate sample storage room, condition and transportation time were significantly increased from $43 \%(46 / 107)$ to $77.1 \%$ $(84 / 109)(p<0.001)$. Average transportation time to the laboratory of blood sample significantly decreased from $3 \mathrm{~h} 36 \mathrm{~min}( \pm 1 \mathrm{~h} 52 \mathrm{~min})$ to $3 \mathrm{~h} 01 \mathrm{~min}( \pm 1 \mathrm{~h} 41 \mathrm{~min})$, and the arrival within $2 \mathrm{~h}$ increased significantly from $22.9 \%$ to $39.2 \%$ ( $p=0.021$ ).

Conclusion: Multifaceted interventions for this study are effective for nurse to improve the knowledge and practice to store and transport samples properly. However, some samples remain in the ward longer than expected after intervention and require further intervention.

Disclosure of Interest: None declared.
P172

Place of blood cultures in sepsis in a resource-limited setting, Abidjan, Côte D'ivoire

G. A.-J. Bahan ${ }^{1, *}$, A. D. Yapi ${ }^{1}$, Y. M. Coulibaly ${ }^{1}$, N. A. Kacou ${ }^{1}$

${ }^{1}$ Bacteriology Unit, Medical Biology Department, Abidjan, Côte d'Ivoire

Correspondence: G. A.-J. Bahan

Antimicrobial Resistance \& Infection Control 10(1): P172

Introduction: Sepsis have a substantial impact on morbidity and mortality. Its early management requires appropriate antibiotic therapy which involves detection of the pathogens agents by blood culture. Blood cultures are the reference method for diagnosis of sepsis.

Objectives: The purpose of this study was to determine the blood culture completion rate in sepsis at the Treichville University Hospital Center.

Methods: It's a cross-sectional study included hospitalized patients in the intensive care unit and tropical infectious diseases unit at the Treichville University Hospital Center, from 2016 to 2018. Data collection was based on socio-demographic, clinical, microbiological (blood culture) characteristics and biological infectious markers such as blood count, C-reactive protein and procalcitonin.

Results: The overall prevalence of sepsis was $15.52 \%$ ( 232 cases out of 2191 hospitalized patients). The mean age was 40 years ( \pm 12 years). The sex ratio was 0.96 . The frequent sources of infection were pulmonary $(17.67 \%)$, urogenital $(9.48 \%)$ and digestive $(8.62 \%)$. Blood cultures had been performed to 49 patients $(21.12 \%)$. The positivity rate was $46.93 \%$ (23/49). Gram negative bacilli was dominated with 11 isolates of Enterobacteriaceae (11/23). The most common bacteria species were Escherichia coli in 5, Enterobacter cloacaein 3, Klebsiella pneumoniaein 2 and Enterobacter aerogenes in 1 patients. Biological infectious markers were performed in $42(22.41 \%)$ for the C-Reactive Protein, $31(13.36 \%)$ for procalcitonin and $230(99.13 \%)$ for the whole blood count. Antibiotics associations have been used empirically in $96.98 \%$ (225/232) including bi-antibiotic therapy (141) and tri-antibiotic therapy (84). The most used antibiotics were ceftriaxone $(27.47 \%)$, gentamycin $(24.67 \%)$ and metronidazole (14.95\%). Deaths occurred in 150 cases $(64.65 \%)$.

Conclusion: Blood culture may help to improve sepsis management in low-income countries.Antibiotic therapy should be guided by the patterns of bacteria and their susceptibility to antibiotics.

Keywords: Blood culture, Sepsis, Biomarkers, Abidjan.

Disclosure of Interest: None declared.

Poster Session: Environment: Surface contamination and decontamination

\section{P173}

Development of a training resource focused on those who clean health care facilities

C. Kilpatrick ${ }^{1, *}$, J. Storr ${ }^{1}$, K. Lee ${ }^{2}$, S. Nurse Findlay ${ }^{3}$, W. Graham ${ }^{4}$

${ }^{1}$ KS Healthcare Consulting, Glasgow, ${ }^{2}$ Independent researcher, Dundee, United Kingdom, ${ }^{3}$ World Health Organization, Geneva, Switzerland, ${ }^{4}$ London School of Hygiene and Tropical Medicine, London, United Kingdom Correspondence: C. Kilpatrick

Antimicrobial Resistance \& Infection Control 10(1): P173

Introduction: The workforce who clean health care facilities are overlooked and undervalued. Strengthening the training of this important cadre of staff in all countries will support infection prevention and control (IPC), water, sanitation and hygiene and antimicrobial resistance (AMR).

Objectives: To explore existing materials and develop a new World Health Organization (WHO) training resource aimed at those who clean, initially in maternity wards.

Methods: Electronic databases were searched using mesh terms for cleaners/cleaning, IPC and AMR, low and middle income countries, maternal and newborn health, training and development. Relevant web portals were also searched. After an initial assessment of all 
sourced publications, four scientific publications and 44 resources were further reviewed against a list of criteria.

Results: An existing product entitled TEACH CLEAN was identified as suitable for modification. Permission was granted and adaptations and updates were made. A consultation was performed and a twopart WHO prototype was created. Other publications featuring the latest standards on IPC and environmental cleaning were also identified and informed the content. The components of the package stayed true to the original educational concept, which has been tested in a range of countries. An outline of the contents of the WHO resource is in Figure 1.

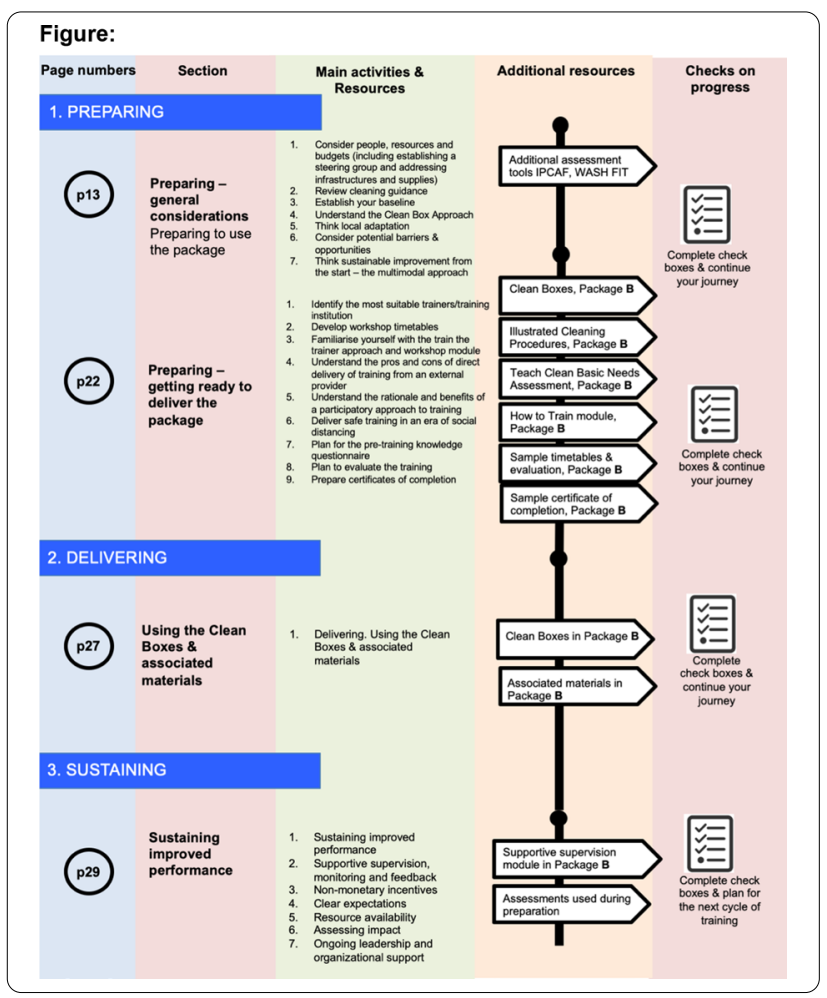

Conclusion: This resource is the first WHO-developed training curriculum aimed at those who clean, a priority that has assumed greater significance in recent years including due to COVID-19. Further validation of the prototype is planned in a sample of WHO regions.

Disclosure of Interest: None declared.

\section{P174}

Clean frontline: a stepped wedge cluster trial of an environmental hygiene educational intervention across thirteen Cambodian hospitals

G. Gon ${ }^{1, *}$ on behalf of Clean Frontline working group

${ }^{1}$ Infectious Disease Epidemiology, London School of Hygiene and Tropical Medicine, London, United Kingdom

Correspondence: G. Gon

Antimicrobial Resistance \& Infection Control 10(1): P174

Introduction: Environmental hygiene is a key component of infection prevention in healthcare, and a driver of healthcare associated infections. Staff who clean in many low resource countries receive no formal training on cleaning, waste disposal and linen handling. This issue has been execrated by the COVID-19 pandemic. The only recommended training on environmental hygiene for low resourced facilities, TEACH CLEAN, uses a training of trainers model. A selected cadre "champions" which in turn train their peers with responsibilities on environmental hygiene at the facility level. Early pilot data to test its effecteveness of this training package are very promising.

Objectives: The main objective is to evaluate the effectiveness of an environmental cleaning bundle to improve microbiological cleanliness in Cambodian hospitals.

Methods: TEACH CLEAN will be implemented across all hospitals (13) of three provinces in Cambodia. A stepped wedge randomised trial will be used to evaluate the effectiveness of TEACH CLEAN to improve microbiological cleanliness in Cambodian hospitals. All facilities will receive the intervention. Hospitals are arranged in groups of three or four based on the randomisation with staggered commencement dates of the intervention at four distinct time points. The design will include nine months of data collection. Intervention months will vary between two and seven. We expect one month gap between the training of champions and the training of staff at the facility level. The main outcome is microbiological cleanliness $(<2.5 \mathrm{cfu} /$ $\mathrm{cm} 2=$ clean; $\geq 2.5 \mathrm{cfu} / \mathrm{cm} 2=$ not clean) measured using a non-specific agar on one side for measuring total Aerobic Colony Counts (ACC/ $\mathrm{cm} 2$ ). A secondary outcome will be presence/absence of S.Aureus. With 30 sampling sites in each hospital and with a pre-training cleanliness proportion ranging from 30 to $50 \%$ will give us over $85 \%$ power to detect a $10 \%$ absolute post-intervention increase in cleanliness.

Results: NOTE: This is a trial protocol presentaiton as the trial will commence in June 2021.

Conclusion: Evidence from the this trial will contribute to future policy and practice guidelines about hospital environmental hygiene and ultimately reduce healthcare associated infections. This would be the first randomised trial on environmental hygiene in low resource settings.

Disclosure of Interest: None declared.

\section{P175}

Determining staffing levels for environmental cleaning: time measurements in non-critical care units in an acute care hospital in Brussels

A. Caluwaerts ${ }^{1, *}$, L. Pintelon ${ }^{2}$, J. Hopman ${ }^{3}$

${ }^{1} \mathrm{IPC}$, Kliniek Sint Jan, Brussels, ${ }^{2} \mathrm{CIB}, \mathrm{KUL}$, Leuven, Belgium, ${ }^{3}$ Quality

and Safety, Radboudumc, Nijmegen, Netherlands

Correspondence: A. Caluwaerts

Antimicrobial Resistance \& Infection Control 10(1): P175

Introduction: Environmental cleaning plays an important role in the prevention of healthcare associated infections. A multimodal improvement strategy includes the creation and uptake of standard operational procedures through appropriate staffing, training, monitoring and feedback.

Objectives: Given the lack of (inter)national standards, the objective of this study was to determine staffing levels for environmental cleaning in non-critical adult care units in an acute care hospital in Brussels. Methods: We performed time measurements to define the time needed to accomplish the daily cleaning of single patient rooms. Only rooms that were considered thoroughly cleaned, based on fluorescent marking prior to cleaning, were included in the analysis. Build-upon the time measurements and findings from a systematic literature review, we estimated the time needed to perform different types of cleaning for different types of rooms, cleaning of non-patient areas and non-cleaning tasks performed by a cleaner in a unit. Bed management data of 2019 were used to calculate the average number of times the different types of cleaning occurred per day in a unit. Finally, we calculated the full-time equivalents (FTE) in accordance with data from the human resources department.

Results: The mean time to perform a daily cleaning of a single room was 18,87 (Cl 15,09 - 22,65) minutes. With bed occupancy rates between 59 and $86 \%$ in units with on average 30 beds in single and double rooms, we need on average $6 \mathrm{~h} 37 \mathrm{~min}$ per unit to clean the patient rooms. Once we add the $107 \mathrm{~min}$ to perform the cleaning of non-patient areas and other tasks of the cleaning staff, we end up with an average need of $8 \mathrm{~h} 24$ min per day, which results in 1,96 FTE per unit (given the 10 public holidays, 25 leave and 20 absenteeism days per year). 
Conclusion: We were able to determine staffing levels for environmental cleaning in non-critical adult care units in an acute care hospital in Brussels. The presented benchmarks need to be validated to determine the degree of applicability in other settings. We underline the need of a transparent, easy to use and effective tool to calculate staffing levels for environmental cleaning in the healthcare setting. Disclosure of Interest: None declared.

\section{P176}

Establishing a new standard method to assess the effectiveness and improve the environmental cleaning and disinfection in an acute district general hospital

M. M. Dalauidao ${ }^{1, *}$

'King's College Hospital NHS Foundation Trust, London, United Kingdom Correspondence: M. M. Dalauidao

Antimicrobial Resistance \& Infection Control 10(1): P176

Introduction: Environmental cleaning and disinfection is paramount in minimising and preventing hospital acquired infections. One of the most important responsibilities of healthcare facilities is to ensure high standards of the environmental cleaning and disinfection. However, there is a lack of standard process to check and assess cleaning effectiveness.

Objectives: To be able to establish a new standard method to assess the effectiveness and improve the environmental cleaning and disinfection in an acute district general hospital.

Methods: A quality improvement model based on Plan Do Study Act (PDSA) Cycle approach was utilised for this project. Process mapping, stakeholder analysis and a driver diagram were undertaken to understand the current practice. A questionnaire was developed for cleaning staff in the hospital in order to understand and establish their current knowledge and practice. This survey helped to identify gaps and establish the focus of the intervention. An invisible fluorescent marker gel was used as the method of assessment in conjunction with the traditional visual inspection, feedback and education. Ten high touch areas around the patient's bed space were identified where the fluorescent gel was applied and these were checked using a portable ultraviolet light by the author and later on the project he was joined by the zonal managers from the cleaning team at a weekly to twice weekly intervals.

Results: The survey showed that there is a need for further training among the cleaning staff. At the initiation of the new assessment method in February 2021, compliance with the cleaning and disinfection standard was $27 \%$. Weekly to twice weekly assessment, education and feedback was commenced. At the end of the implementation phase, the compliance had increased to $91 \%$. In addition, the cleaning staff morale had increased and they now feel more valued and appreciated. This is demonstrated by the improvement in quality of cleaning they have consistently delivered throughout the duration of the project.

Conclusion: The introduction of the fluorescent marker gel and the portable UV light, in conjunction with visual inspection, training and feedback has improved and sustained the quality of environmental cleaning and disinfection.

Disclosure of Interest: None declared.

\section{P177}

Environmental surface disinfection monitoring by adenosine triphosphate (ATP) bioluminescence based assay in a tertiary health care setting

H. Gautam ${ }^{1, *}$, A. H. Choudhary², S. Khullar', B. K. Das ${ }^{1}$, A. Kapil $^{1}$

${ }^{1}$ Microbiology, ${ }^{2}$ Hospital Administration, AllMS, New Delhi, New Delhi, India

Correspondence: $\mathrm{H}$. Gautam

Antimicrobial Resistance \& Infection Control 10(1): P177

Introduction: Environmental surfaces in hospitals can be a possible reservoir for microorganisms. Proper cleaning and disinfection of hospital environmental surfaces has become even more crucial during this ongoing COVID-19 pandemic. Appropriate disinfection practices play a crucial role in their prevention.

Objectives: This study aimed to observe the effect of monitoring of cleaning and disinfection practices using an ATP bioluminescence assay during the ongoing COVID-19 pandemic.

Methods: The study was performed over a period of two and a half months in a tertiary health care centre in India. High touch surfaces were identified where cleaning with freshly prepared $1 \%$ Sodium hypochlorite was performed as a part of routine cleaning. Post cleaning of these surfaces, samples were collected and tested using Adenosine Triphosphate bioluminescence (ATP) based assay.

Results: A total of 416 testing at various sites were performed during the study duration. Results were non satisfactory (>250 RLU) at 123 sites and the testing was repeated after repeat cleaning and disinfection. All the tests except two performed following repeat disinfection were satisfactory $(<250 \mathrm{RLU})$. Decrease in ATP content (RLUs) following repeat cleaning at these 123 sites was significant $(p<0.0001)$. A decreasing trend in the ATP content as measured in the first reading was noted over a period of two and a half months.

Conclusion: The role of monitoring cleaning and disinfection practices in healthcare settings cannot be underscored. ATP bioluminescence based assay is a rapid, easy to perform test providing early feedback which can result in an early corrective action. Though ATP bioluminescence based assay cannot specifically detect SARS-CoV-2, however, give a measure of general cleanliness. However, use of sodium hypochlorite is an EPA (Environmental Protection Agency) approved disinfectant for SARS-CoV-2 along with satisfactory hospital cleaning and disinfection practices may indirectly indicate satisfactory disinfection against SARS-CoV-2.

Disclosure of Interest: None declared.

\section{P178}

Wastes and surfaces as reservoir of antibiotic resistant bacteria in Nigeria

I. Yusuff, ", Z. Muhammad Damji ${ }^{1}$, M. Danladi Shuaibu' ${ }^{1}$, N. Hamza' ${ }^{1}$, N. Abdullahi Bako ${ }^{1}$

${ }^{1}$ Microbiology, Bayero University, Kano, Kano, Nigeria

Correspondence: I. Yusuf

Antimicrobial Resistance \& Infection Control 10(1): P178

Introduction: Acquisition of community-associated methicillin resistant Staphylococcus aureus (MRSA) and extended spectrum beta-lactamase (ESBL) producing Gram negative bacteria from environmental sources and surfaces is widely reported in Nigeria.

Objectives: A study was designed to assess the role of discharged environmental wastes, hands and frequently shared and touched surfaces as potential reservoirs of antibiotic resistant pathogens.

Methods: A total of 818 samples of wastes which include sewage, sullage, diapers, food remnants, sands, plastics, water sachets, and swabs of restroom floors, corridors, door handle, bowls and hands of vulnerable and non vulnerable children (VC and NVC) were analyzed and susceptibility of isolates recovered from them to different antibiotics were determined. The moderately resistant $S$. aureus and Gram negative bacilli were further screened for methicilin resistance and ESBL production.

Results: Result showed that sewage, sullage, diapers, food remnants and restroom floors were frequently contaminated with PPB that are highly and moderately resistant to penicillins and flouroquinolones. Nearly all the isolates were susceptible to a carbapenem and cephalosporins. Hands and high-touch surfaces of VC such as bowl were contaminated with E. coli, S. aureus and K. pneumoniae. No ESBL-E. coli was isolated from all the samples. Two ESBL-K.pneumoniae, 2 MRSA and 1 ESBL- $P$. aeruginosa were recovered from VC bowls, sewage, sullage, and diapers.

Conclusion: Sewage, sullage, some solid wastes, restroom floors, VC's hand, and their bowl serve as potential reservoir for some resistant bacteria.

Disclosure of Interest: None declared. 
P179

Results of an audit on bio-cleaning in the operating room in 2019 authors

G. Brahimi ${ }^{1, *}$, K. Chabane ${ }^{1}$, A. Dahli ${ }^{1}$, M. Charchari ${ }^{1}$, A. Boudebouz ${ }^{1}$, S. Ait seddik ${ }^{1}$, S. Slaouti ${ }^{1}$, M. Charchari ${ }^{1}$, A. Larinouna ${ }^{1}$, A. El kechai ${ }^{1}$, A. Chetitah ${ }^{1}$, A. Rebouh ${ }^{1}$, R. Belkaid ${ }^{1}$

${ }^{1} \mathrm{CHU}$ BENI MESSOUS, ÉPIDÉMIOLOGIE, ALGER, Algeria

Correspondence: $G$. Brahimi

Antimicrobial Resistance \& Infection Control 10(1): P179

Introduction: The bio-cleaning of the premises may seem like a very trivial act. However, it takes on all its importance in a hospital environment and more particularly in the operating theater (OR), a place with a high risk of infection.

Objectives: Evaluate the compliance of bio-cleaning practices for floors and surfaces in relation to the standards in the BOs of our $\mathrm{CHU}$.

Methods: The audit took place in the eight medico-surgical departments with operating rooms (General surgery, Urology, ENT, Ophthalmology, Pneumo-phthisiology, Pneumo-allergology, $\mathrm{CCl}$ and Gynecology) from December 8 to 19, 2019. Data was collected by observation of staff during the performance of their duties. Data entry and processing were carried out using EPI info 6 software.

Results: A total of 17 agents were audited, of which $58.8 \%(10 / 17)$ are affiliated with the hospital and $41.17 \%$ with two private companies (H.N. and E.N.). 41.2\% of those audited have an average level of education and $35 \%$ participated in the training on bio-cleaning. Each employee has his own equipment, but it isn't appropriate for the requirements of the hospital. Disinfection of floors is done by bleach in $62.5 \%$ and by disinfectant detergent in $37.5 \%$. The disinfection products used are not labeled in $62.5 \%$ of the departments. $100 \%$ of staff do not respect the storage, dilution and contact time of bleach. Agents affiliated with the hospital do not maintain the BO when the room opens in $40 \%$ and between two operations in $60 \%$. Cleaning of horizontal surfaces is not performed by $60 \%$ of agents affiliated with the hospital. Between two interventions, $77 \%$ of those audited do not wet sweep and $69.2 \%$ of those audited do not clean the furniture. At the end of the program, $64.7 \%$ of those audited do not clean the walls at mid-height. All of the HN Company staff, $60 \%$ of the E. N. Company's bio-cleaning agents and $60 \%$ of the hospital's bio-cleaning agents perform weekly maintenance. The logical order in the of operations is not respected and the notion of clean to dirty and from top to bottom within the same room is unknown by all staff.

Conclusion: Despite the training carried out on the application of the protocols, discrepancies are observed, this is due to the turnover of bio-cleaning agents, hence the need to repeat the training and display the development of the protocols.

Disclosure of Interest: None declared.

\section{P180}

Bacterial cross-transmission between inanimate surfaces

\section{and patients in ICU rooms}

E. Kuczewski ${ }^{1, *}$, L. Henaff $^{2}$, N. Khanafer ${ }^{1}$, S. Loeffert ${ }^{3}$, P. Cassier ${ }^{1}$, T.

Rimmelé ${ }^{1}$, L. Argaud ${ }^{1}$, P. Vanhems ${ }^{1,2}$

${ }^{1}$ Hospices Civils de Lyon, ${ }^{2}$ University Lyon 1, Lyon, ${ }^{3}$ Laboratoires

ANIOS-ECOLAB, Lezennes, France

Correspondence: E. Kuczewski

Antimicrobial Resistance \& Infection Control 10(1): P180

Introduction: Contaminated environment plays an important role in the acquisition of nosocomial pathogens by patients hospitalized in Intensive Care Units (ICUs). Immediate zones around patient's bed can be a reservoir of potential pathogens, where they can survive for many months.

Objectives: In this study conducted in a French University Hospital, we aimed to describe the microbial ecology of two rooms in two ICUs, and to explore the potential link between environmental contamination and patient's colonization and/or infection.

Methods: In 2020 during 5 months, environmental samples from room surfaces (i.e. foot side bed barriers; worktop of nurse cart; computer keyboard and mouse; adaptable; washbasin and lever hydroalcoholic solution/soap; dedicated stethoscope) have been carried out with sterile wipes once monthly. Fifty samples were performed, just before daily cleaning. Wipes were incubated $48 \mathrm{~h}$ at $30^{\circ} \mathrm{C}$ in tryptone soy broth, and then samples were plated on four culture media. Identified bacteria were compared to those isolated from patients hospitalized in the same rooms.

Results: The main bacteria found in the environment were: E.faecalis (15,5\%); P.agglomerans (11,7\%); E.faecium (10,7\%); B.cereus $(5,8 \%)$; A.baumannii $(5,8 \%)$. In six cases, the same bacteria were found in both environmental and clinical samples with a time interval $<10$ days. For five of them, a transmission environment-patient was suspected (table 1).

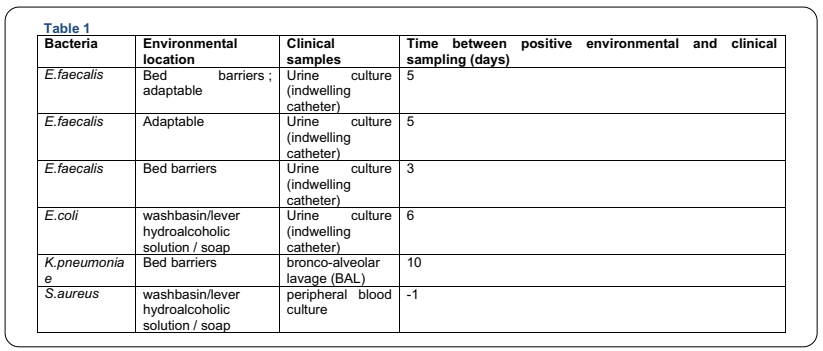

Conclusion: This study confirms that the close environment of the patient is a reservoir of opportunistic or pathogenic microorganisms. This source of infection should not be underestimated in the occurrence of infections in critically ill patients.

Disclosure of Interest: E. Kuczewski Grant/Research support from: Anios, L. Henaff: None declared, N. Khanafer: None declared, S. Loeffert Employee of: Anios, P. Cassier: None declared, T. Rimmelé: None declared, L. Argaud: None declared, P. Vanhems: None declared.

P181

Evaluation of the environmental monitoring after an outbreak of bacillus cereus infections in a neonatal intensive care unit

(NICU)

A. Davias ${ }^{1}$, C. Terreaux-Masson ${ }^{1}$, I. Pelloux ${ }^{2}$, C. Giner ${ }^{1}$, M.-R. Mallaret ${ }^{1}$, C. Landelle ${ }^{1, *}$

${ }^{1}$ Infection Control Unit, ${ }^{2}$ Bacteriology Laboratory, Grenoble Alpes University Hospital, Grenoble, France

Correspondence: C. Landelle

Antimicrobial Resistance \& Infection Control 10(1): P181

Introduction: In 2009, our French University Hospital has known an outbreak of Bacillus cereus infections in newborns of NICU. Nine patients have got a positive biologic sample to $B$. cereus, 5 of them had a real infection. An important cleaning has been realized in the unit and an environmental monitoring was implemented since 2009.

Objectives: Our objective was to evaluate the efficacy of the environmental monitoring on the onset of infections in new-born hospitalized in neonatology, considering the absence of any guidelines developing acceptable $B$. cereus thresholds in hospital environment.

Methods: We conducted a retrospective observational study in our 3 units of a neonatology ( 1 NICU, 1 neonatal intermediate care unit and 1 neonatal medicine unit) of 36-beds between 2009 and 2019. We defined a case of $B$. cereus infection as a newborn with clinical symptoms of infection and with at least one biological sample positive to $B$. cereus. Every room of NICU was controlled twice by year and every room of neonatology was controlled once by year, with a campaign of 10 environmental samples. Samples were made with Count-Tact ${ }^{\circledR}$ agar and swabs. The average proportion of B. cereus and Bacillus spp by campaign were calculated.

Results: Since 2009, 18 patients were identified with a positive biological sample to $B$. cereus and 11 patients had a real infection. Number of cases decreased until 2011 and fluctuated between 2011 and 2019. All units combined, environmental B. cereus and total Bacillus proportion 
averages have both decreased since the establishment of the environmental monitoring and never exceeded $12 \%$ and $7 \%$ respectively since 2011.

Conclusion: A strengthened environmental monitoring during 11 years in 3 units receiving patients susceptible to $B$. cereus infections is showing a decrease of Bacillus spp proportions in the environment, and a decrease of $B$. cereus contaminations and infections in hospitalized new-born. It didn't allow to completely stop the occurrence of cases but to greatly decrease them.

Disclosure of Interest: None declared.

\section{P182}

Whole genome sequencing excluded the environment as the source of infection of pseudomonas aeruginosa in a neonatal intensive care unit

D. S. Blanc 1,*, L. Senn ${ }^{1}$, I. Federli ${ }^{1}$, I. Koening ${ }^{1}$, E. Giannoni ${ }^{1}$, F. Legault ${ }^{1}$, M. Roth-Kleiner ${ }^{1}$, B. Grandbastien ${ }^{1}$

${ }^{1}$ Centre Hospitalier Universitaire Vaudois, Lausanne, Switzerland

Correspondence: D. S. Blanc

Antimicrobial Resistance \& Infection Control 10(1): P182

Introduction: Pseudomonas aeruginosa is a rare pathogen in neonatal intensive care unit (NICU). Following P.aeruginosa bacteremia in two NICU patients hospitalized in two different rooms with dedicated nurses, we performed an epidemiological investigation.

Objectives: To find environmental sources and routes of transmission of $P$. aeruginosa infections in NICU patients using standard and genomic typing methods.

Methods: All humid environments were screened for P.aeruginosa. This included tap water, faucets, sink traps, water reservoirs of incubators, cosmetics, and disinfectants. Sink traps were dismantled and 10 samples were done at different sites of each sink trap. Isolates were first typed by double locus sequence typing (www.dlst.org/ Paeruginosa) and all isolates identical to the three patient's isolates (DLST 28-77), were further analysed by whole genome Multi Locus Sequence Typing (wgMLST, BioNumerics v.7.6.3). As control, we analysed 15 isolates of DLST 28-77 not related to the outbreak.

Results: Among the 287 environmental samples analysed for the presence of P.aeruginosa, 99 were positive, mostly from sink traps. Typing revealed 35 isolates belonging to DLST 28-77. Whole genome sequencing revealed that all DLST28-77 isolates $(N=53)$ belonged to the MLST ST395. wgMLST analysis showed differences of 0 to 180 loci between all isolates. Only 0 to 2 loci differences were observed between the three isolates from the two NICU patients. The closest environmental isolates from the two NICU patients had $>70$ loci differences and the closest patient isolate had 27-30 loci difference. The later was recovered from a patient in the adult ICU with no link with the NICU.

Conclusion: The thorough investigation of the environment of our NICU showed that sink traps were the main reservoir of P.aeruginosa. However, none of the environmental isolates was genetically close enough to be considered as the source of infection. The sole use of standard molecular typing (DLST) would have lead us to a false conclusion that sink traps were the source of infection.

Disclosure of Interest: None declared.

\section{P183}

Environmental cleaning practices and microbiome of high-touch surfaces in covid and non-COVID ICUs-a pilot study

E. Y. Kumoto ${ }^{1, *}$, A. Guimarães ${ }^{1}$, C. Silva ${ }^{1}$, R. Chaves ${ }^{2}$, A. Pereira ${ }^{1,3}$ on behalf of Impacto MR group

${ }^{1}$ Intensive Care Unit, ${ }^{2}$ Department of Anesthesiology, ${ }^{3}$ Big Data, Hospital Israelita Albert Einstein, São Paulo, Brazil

Correspondence: E. Y. Kumoto

Antimicrobial Resistance \& Infection Control 10(1): P183

Introduction: In the context of the pandemic caused by SARS-CoV-2, reports of increased incidence of Health Care-Related Infections have been published. Variability in environmental cleaning practices and differences in the microbiome composition of high-touch surfaces may be related to this trend.

Objectives: To assess hospital cleaning practices and microbiome compositions of COVID and non-COVID ICUs.

Methods: Prospective observational study in COVID and non-COVID intensive care units (ICU) of 3 Brazilian' hospitals. After informed consent, environmental cleaning practices were studied by mean of a qualitative instrument through Plan-Do-Study-ACT cycles that was composed by a leadership, an operation/cleaning professionals assessment, and the core component 8 of the Infection Prevention and Control Assessment Framework. 38 swabs of high-touch surfaces were collected to evaluate bacterial profile and resistance genes, using large-scale DNA sequencing and real-time PCR.

Results: Environmental hygiene practices observed were different among the 3 hospitals regarding. IPCAF scores were similar. Surface microbiomes in COVID/non-COVID ICUs were similar within hospitals, but each one presented an unique diversity profile. Low impact was found of terminal cleaning processes in gram-positive bacteria (specially, Corynebacterium, Strepococcus e Staphylococcus). In 2 out 12 sanitizers samples were positive for Burkholderia Cenocepacia, Pseudomonas, Enterobacteriaceae, Stenotrophomonas. Resistance genes mec-A were found in more than $50 \%$ of the samples in 2 out 3 hospitals, reaching higher still higher rates in COVID units (71.1\% and $86.7 \%)$. KPC were also found in all ICUs, indistinctly, followed by OXA23 and VanA (in much lower rate, not in all ICUs).

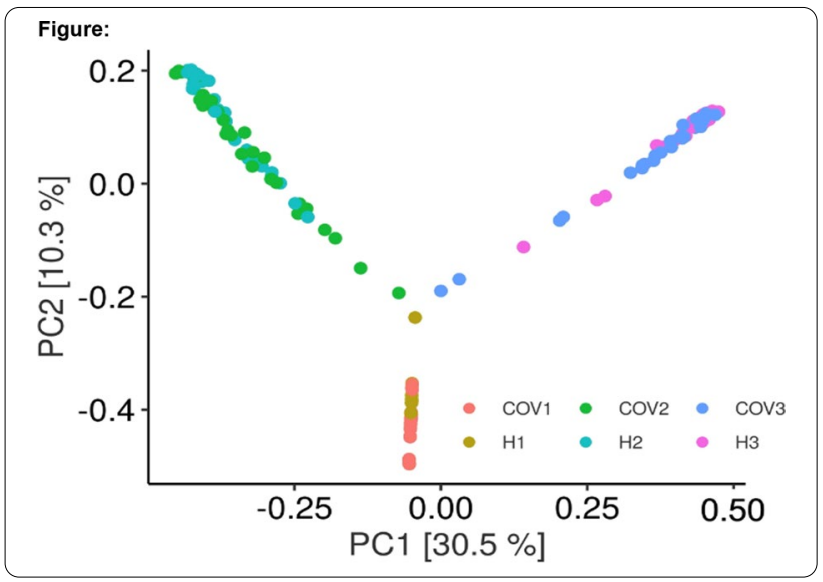

Conclusion: Surface microbiome within hospitals in COVID and nonCOVID ICUs of did not present significant differences. Conversely, each hospitals presented very distinct diversity profiles. Although presumed, clinical impact of environment-related findings, as well as differences between environmental hygiene practices and contamination of sanitizing agents deserves further investigation future studies.

Disclosure of Interest: None declared.

P184

Evaluation of environmental sampling kit to monitoring

of microbial contamination in immunohematology and transfusion unit of general hospital in North Italy

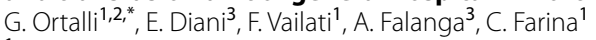

${ }^{1}$ Microbiology Institute, ASST "Papa Giovanni XXIII, Bergamo, ${ }^{2}$ Laboratorio di Microbiologia e Virologia, Hosptal Santa Chiara, Trento, ${ }^{3}$ Division

of Immunohematology and Transfusion Medicine, ASST "Papa Giovanni XXIII, Bergamo, Italy

Correspondence: G. Ortalli

Antimicrobial Resistance \& Infection Control 10(1): P184

Introduction: Healthcare associated infections (HAl) in hospital are caused by environment agents transferred between surfaces.

Objectives: Aim of this study was to monitor environmental contamination in Division of Immunohematology and Transfusion 
Medicine of Papa Giovanni XXIII General Hospital in Bergamo (Italy), comparing Copan SRK ${ }^{\circledR}$ with FLOQSwabs ${ }^{\circledR}$ to traditional cotton swabs and contact plates.

Methods: On a seasonal basis for 1 year, collection points (workbenches, trolleys, glasses, handles and keyboards) were identified and sampled. Flat surfaces were tested comparing contact plates (55 mm TSA) and SRK samples. Articulated surfaces were crosssampled, to avoid analysis bias, comparing cotton swabs and SRK samples. After sampling, SRK medium and cotton swabs were inoculated and incubated on $90 \mathrm{~mm}$ TSA plates $\left(35 \pm 2{ }^{\circ} \mathrm{C}\right.$, aerobic atmosphere, 7 days). Identification of isolates was performed with MALDI-TOF.

Results: Data from environmental samples, are resumed in table 1. Probably due to a wider sampling area, contact plates from flat surfaces showed more species detected compared to SRK, but a lower value of total CFU in summer and autumn, probably related to a better sampling procedure with flocked swabs. From articulated surfaces: SRK detected more species and isolates compared to cotton swabs, allowing to detect Gram negative bacteria (such Escherichia coli), yeast and filamentous fungi on the joint surfaces.

\begin{tabular}{|c|c|c|c|c|c|}
\hline \multicolumn{2}{|l|}{ Figure: } & \multirow{3}{*}{$\begin{array}{c}\begin{array}{c}\text { Spring* } \\
\left(\mathrm{N}^{* *}=112\right)\end{array} \\
16(50)\end{array}$} & \multirow{3}{*}{$\begin{array}{c}\begin{array}{c}\text { Summer* } \\
\left(\mathrm{N}^{* *}=123\right)\end{array} \\
13(96)\end{array}$} & \multirow{3}{*}{$\begin{array}{c}\begin{array}{c}\text { Autumn* } \\
\left(\mathrm{N}^{* *}=106\right)\end{array} \\
15(106)\end{array}$} & \multirow{3}{*}{$\begin{array}{c}\begin{array}{c}\text { Winter* } \\
\left(\mathrm{N}^{* *}=106\right)\end{array} \\
9(115)\end{array}$} \\
\hline & & & & & \\
\hline Fl & Contact plate & & & & \\
\hline Flat & SRK & $13(23)$ & $8(176)$ & $12(185)$ & $8(100)$ \\
\hline \multirow{2}{*}{ Articulated } & Cotton swab & $11(23)$ & $11(378)$ & $7(74)$ & $3(28)$ \\
\hline & SRK & $13(114)$ & $11(233)$ & $12(241)$ & $4(60)$ \\
\hline
\end{tabular}

Conclusion: Copan SRK, associated with FLOQSwabs, showed to be effective for sampling procedure where compared with standard methods. While results for flat surfaces were slightly different, on a seasonal basis, between reference and test methods, for articulated surfaces Copan SRK showed to be more effective in monitoring microbial environmental contamination.

\section{Disclosure of Interest: None declared.}

\section{P185}

\section{An investigation down the drain}

T. Inkster ${ }^{1, *}$, M. Weinbren ${ }^{2}$

${ }^{1}$ Microbiology, Queen Elizabeth University Hospital, Glasgow, ${ }^{2} \mathrm{NHS}$

Assure, NSS, Edinburgh, United Kingdom

Correspondence: T. Inkster

Antimicrobial Resistance \& Infection Control 10(1): P185

Introduction: Hospital drainage systems are becoming increasingly implicated as a source of outbreaks with organisms such as CPEs. It is therefore important to give thought to the design of drainage systems when building new hospitals, to mitigate the risk.

Objectives: We aim to describe the abnormalities in sink drains found in a new build hospital.We discuss how improvements could be made in future design and the importance of addressing human behaviour in mitigating risk from drains.

Methods: Drains were investigated following reports of relux of material back up into sinks by staff and patient infections. Drain components were removed for inspection and analysis by the laboratory. Assessment of biofilm reaction, bacterial burden and chemical analysis of drain debris was undertaken.

Results: Drains were found not to be flush with the back of the sink with a lip present where water was stagnating. Excess sealant was present in the drains and there was corrosion of an aluminium component. Several plastic objects were retrieved and some scrub sinks were completely occluded by surgeons nail picks. Mature biofilm and high bacterial counts were detected along with evidence of nutrients in the drains.

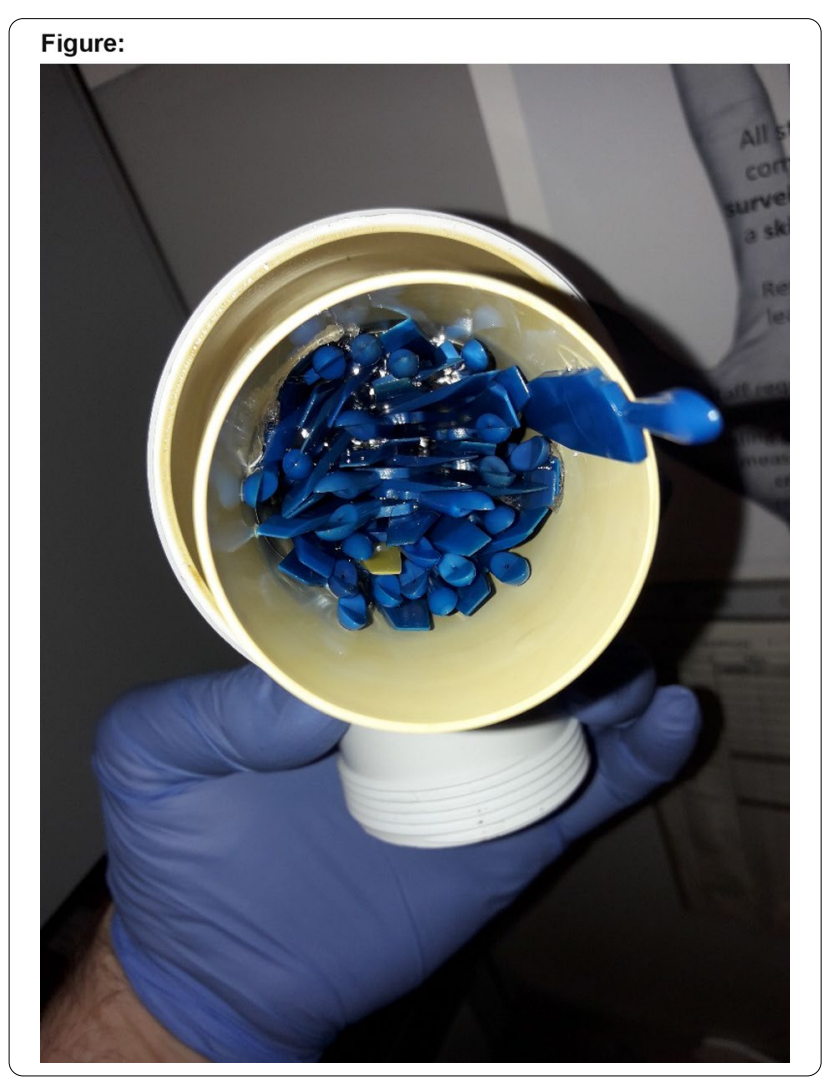

Conclusion: Several problems with drain design and installation were identified. Firstly the drain was not contiguous with the back of the sink which promoted stagnation and pooling of water. Excess sealant present in the drain led to occlusion and further excacerbated stagnation as the drain was not free flowing. Corrosion and splitting of an aluminimum spigot enhanced the occlusion and provided an uneven damaged surface for bacteria to adhere to. Together these conditions promoted biofilm formation,excacerabted by the presence of foreign material and nutirients.

There are several lessions from these findings that should be applied to future design of drains. 1) The drain should be completlely flush with the back of the sink 2) Overzealous use of sealant should be avoided as this can lead to obstruction and poor flow 3) Components used in drains should be with a material not liable to corrosion 4) Workmanship should be of a high standard.

It is also important to address behavioural aspects and infection control teams should ensure staff/ patients are aware of the importance of drain hygiene. Objects and fluids should not be decanted in drains and alternative disposal methods should be provided.

Disclosure of Interest: None declared.

Poster Session: Environmental control: Medical devices

P186

Bacterial contamination of healthcare workers' mobile phones; case study at two referral hospitals in Uganda

F. Tusabe ${ }^{1, *}$, M. Kesande ${ }^{1}$, J. Nanyondo ${ }^{1}$, H. A. Afreenish ${ }^{2}$, I. Olivia ${ }^{3}$, R. R. Ayebare $^{1}$

${ }^{1}$ Global health security, Infectious Diseases Institute, Kampala, Uganda, ${ }^{2}$ Microbiology, NIH, Pakistan, Islamabad, Pakistan, ${ }^{3}$ Policy, 3Barbaricum

LLC Washington,"Washington, United States

Correspondence: F. Tusabe

Antimicrobial Resistance \& Infection Control 10(1): P186 
Introduction: Hospital and Community-acquired infections are escalating and pose significant public health unhealthiness worldwide. The advancements of telemedicine and automation of healthcare records are supported by cell phones, laptops and wearable devices. Objectives: To establish incidence of healthcare workers' mobile phones becoming contaminated with pathogenic bacteria and their possible roles as vehicles of transmission of antimicrobial-resistant bacteria.

Methods: This was a cross-sectional study at two referral hospitals in Uganda between May and October 2020. Self-administered questionnaires were provided to the participants after informed consent. Mobile phones of the healthcare workers in different departments of the hospitals were randomly swabbed. Samples were collected, transported to the microbiology laboratory for bacterial culture and antimicrobial susceptibility tests. Results were entered into Microsoft excel for analysis.

Results: Our study revealed that $93 \%$ of the screened mobile phones were contaminated with one or more species of bacteria, interestingly $46 \%$ were multi-drug resistant; the majority of them being resistant to penicillin, cotrimoxazole, ciprofloxacin and Gentamycin respectively, this is a time bomb of MDR transmission. and only $8 \%$ of the participants cleaned their hands after using a mobile phone. Organisms isolated were E.coli, Micrococcus spp, CoNS and Bacillus spp. 15\% of the isolated organisms are potential pathogens $(n=13)$.

Conclusion: Realization of the role the Healthcare workers' mobile phones play in the spread of hospital-acquired infections is paramount. Advancements in telemedicine and automated healthcare have not been matched by advancements in sanitizing and decontaminating protocols specifically for phones and other mobile devices, this study provides strong evidence for developing and strengthening disinfection protocols of mobile phones.

In future, there is a need to correlate the bacterial isolates and resistance patterns with nosocomial infections identified in similar settings. The need to study the efficacy of different mobile phone disinfection protocols is also paramount.

Disclosure of Interest: None declared.

\section{P187}

The impact of a multidisciplinary infection control intervention on contamination of portable computerized devices in healthcare settings

M. Alshamrani 1, ${ }^{1}$, K. Yassine ${ }^{2}$, A. El-Saed ${ }^{1}$, K. Ameer ${ }^{2}$, M. Hawthan ${ }^{1}$, S. Ramou ${ }^{3}$, B. Al Alwan ${ }^{3}$, F. Othman ${ }^{1}$, M. Al Zunitan ${ }^{2}$

${ }^{1}$ Infection control, King Abdulaziz Medical City, ${ }^{2}$ Infection control, ${ }^{3}$ Microbiology laboratory, King Abdullah Specialist Children Hospital, Riyadh,

Saudi Arabia

Correspondence: M. Alshamrani

Antimicrobial Resistance \& Infection Control 10(1): P187

Introduction: Use of portable computerized devices on multiple patients carry the risk of spreading healthcare-associated infections (HAls), if appropriate infection control practices are not strictly followed.

Objectives: To quantify the bacterial burden of these devices and to assess the impact of a multidisciplinary intervention on reducing the risk of cross-contamination.

Methods: A pre and post-interventional study was carried out in different units of a tertiary care hospital. The study target was Close Loop Medication Administration (CLMA) device. In April 2019, environmental swap samples were collected from the surfaces of CLMA devices and tested for bacterial contamination. This was followed by the formulation and implementation of a multidisciplinary intervention that included training of 200 healthcare workers (HCWs). In March 2020, environmental swap samples were re-collected from CLMA devices and tested in the same way.

Results: Out of 111 CLMA devices tested in the pre-interventional assessment, $57(51.4 \%)$ were positive. Out of 128 microorganisms retrieved, $74(57.8 \%)$ were Gram-positive and $54(42.2 \%)$ were Gramnegative. They included Bacillus species (19.5\%), Micrococcus species (17.2\%), Pseudomonas aeruginosa (16.4\%), Coagulase-negative staphylococci (10.9\%), Klebsiella pneumonia (9.4\%), Escherichia coli (7.0\%), Dephtroids (6.3\%), Acinetobacter spp. (5.5\%), Enterobacter spp. (3.9\%), Staphylococcus aureus (2.3\%), and Streptococcus (1.6\%). Out of 113 CLMA devices tested in the post-interventional assessment, 19 (16.8\%) were positive with one Gram positive microorganism. They included Coagulase-negative staphylococci (84.2\%), Micrococcus species (10.5\%), and Bacillus species (5.3\%).

Conclusion: The findings confirm the success of a multidisciplinary intervention focused on improving disinfection and infection control practices in reducing the bio-burden on portable devices used in tertiary care setting. In our hospital where the majority of HAls are caused by Gram-negative pathogens, this intervention many positively impact the fight against HAls.

Disclosure of Interest: None declared.

\section{P188}

Pilot study on medical tape contamination in a hospital setting

H. Reuter ${ }^{1, *}$, D. Emberger ${ }^{2}$, N. Parohl ${ }^{3}$, W. Popp ${ }^{3}$, M. Roßburg ${ }^{4}$

${ }^{1} 3$ M Deutschland GmbH, Neuss, ${ }^{2}$ Marien Hospital Herne St. Elisabeth

Gruppe $\mathrm{GmbH}$, Herne, ${ }^{3}$ HyKoMed GmbH, ${ }^{4}$ MVZ Dr. Eberhard \& Partner, Dortmund, Germany

Correspondence: $\mathrm{H}$. Reuter

Antimicrobial Resistance \& Infection Control 10(1): P188

Introduction: Medical tapes are used routinely and stored in various patient areas, supply carts, and healthcare personnel pockets once removed from packaging. Many publications discuss how common practices around medical tapes can lead to infection transmission. To minimize risk, some manufacturers already offer tape rolls with antimicrobial spools or for single-patient use.

Objectives: Our purpose was to quantify the contamination of tape rolls found in different locations within a hospital in Germany.

Methods: A test method was developed using RODAC plates and swabs to optimize conditions. Each roll was sampled in 4 sites: top layer of the tape, deeper layer of the tape, side of the roll, and inside ring of the spool. Results were normalized to colony forming units (CFU) per plate $\left(24 \mathrm{~cm}^{2}\right)$. Since no microbial standard limits exist for the acceptability of medical tape other than "low bacterial contamination with absence of pathogens", we categorized our results based on limits offered by GMP (European Pharmacopoeia) and the hygienic monitoring of air conditioning systems (VDI 6022). Categories were: no bacterial detection ("very good"), 1-24 cfu/plate ("good"), 25-49 cfu/ plate ("conspicuous"), 50-100 cfu/plate ("borderline"), or $>100 \mathrm{cfu} /$ plate ("insufficient").

Results: 32 tape rolls ( 19 with an antimicrobial spool and 13 without) were examined. Of 128 total samples, 71 had evidence of skin and environmental bacteria, 1 had Enterococcus sp, and 56 had no bacteria detected. 53 samples had 1 species, 16 had 2 species, and 3 had 3 species. The large majority of CFUs came from the top layer of the tape and the side of the roll, regardless of the presence of an antimicrobial spool. Two rolls ranked "very good", 16 ranked "good", 4 ranked "conspicuous", 2 ranked "borderline", and 8 ranked "insufficient". None of the rolls were contaminated with pathogenic bacteria, but only 2 rolls had no bacterial detection at all.

Conclusion: Tape rolls used repetitively in a hospital very often carry microbial flora and therefore may contribute to bacterial transmission in hospitals. An antimicrobial spool does not offer complete protection since bacteria can be found even on most of those tape rolls.

Disclosure of Interest: None declared.

\section{P189}

Main risk factors for patient's infection associated with reprocessing endoscopes in Russia

T. Grenkova ${ }^{1, *}$, E. Selkova ${ }^{1}$

${ }^{1}$ Moscow Gabrichevsky Research Institute of Epidemiology and Microbi-

ology, Moscow, Russian Federation

Correspondence: T. Grenkova

Antimicrobial Resistance \& Infection Control 10(1): P189 
Introduction: About 14 million endoscopic interventions are carried out in Russia every year and a few score of HAl cases, which are related to them are officially registered. The epidemiological safety system (ESS) of endoscopic interventions and the method of safety level detection in a certain medical facility have been worked out in the country.

Objectives: Reveal the risk factors for patient's infection at endoscopy departments to improve the ESS.

Methods: 460 of doctors and nurses of endoscopy hospital departments from 80 regions of Russia were interviewed. The form contained 47 questions. Some questions have not been answered by all respondents.

Results: There are no reprocessing rooms arranged in 75 (16.3\%) of 460 endoscopic departments. $369(91,1 \%)$ of 405 respondents do not violate the precleaning procedure; $29.9 \%$-carry out the leak test irregularly. $325(73,5 \%)$ of 442 respondents never violate the guidelines when carrying out the manual cleaning, $7.2 \%$ skip the step of soaking in washing solution, $5.7 \%$ reduce a number of brushing passes through channels, $6,8 \%$ of respondents miss out the rinsing before an endoscope is connected to an AER/EWD. High-level disinfection (HLD) in AER is performed at $65.2 \%$ of endoscopy departments. Among HLD applied agents the aldehyde share is $47,9 \%$, oxygen active compounds-38,7\%, the rest $13,4 \%$ of agents are not included into the list of recommended for such a purpose. More than a third of endoscopy departments do not check the minimum effective concentration in a high-level disinfection solution. Final manual rinsing of gastrointestinal $(\mathrm{Gl})$ endoscopes are made by tap water at all endoscopy departments which were questioned. Microbiological studies of the effectiveness of the endoscopes reprocessing cycles are not carried at $56(12.7 \%)$ of 460 endoscopy departments, at 82 departments (17.9\%) it is performed less than once per a quarter. Unsatisfactory results were received for $4.4 \%$ of samples.

Conclusion: Obtained results analysis revealed the main risk factors for patient's infection associated with ineffectively reprocessed endoscopes and has allowed to improve the requirements to separate components of ESS: technical fit-out and material support of the endoscopes processing, microbiological water quality for final rinsing of $\mathrm{Gl}$ endoscopes, carrying out of cleaning quality control and HLD of endoscopes.

Disclosure of Interest: None declared.

\section{P190}

Epidemiolodgycal safety of ultrasound diagnostics

E. Selkova ${ }^{1}$, V. Mitkov' ${ }^{2}$, T. Grenkova ${ }^{1, *}$, M. Mitkova ${ }^{2}$

${ }^{1}$ Moscow Gabrichevsky Research Institute of Epidemiology and Microbiology, ${ }^{2}$ Russian Medical Academy of Continuous Professional Education, Moscow, Russian Federation

Correspondence: T. Grenkova

Antimicrobial Resistance \& Infection Control 10(1): P190

Introduction: Infection prevention and control (IPC) in ultrasound (US) examination remains a subject of serious disagreement among specialists. Several countries developed and implemented guidelines to ensure IPC in this area. In Russia, there are no such national guidelines, but the need for them is recognized.

Objectives: To analyze the type of ultrasound transducers (probes) reprocessing and the use of single-use covers for them (for external, intracavitary and intraoperative procedures) in medical organizations of the Russian Federation.

Methods: 869 ultrasound doctors from 77 regions of Russia were interviewed. The questionnaire contained 21 questions about the reprocessing of US probes and the use of single-use probe covers for them.
Results: Percutaneous probes clean 280 (38.7\%), clean and disinfect $443(61.3 \%)$ of the 723 respondents; $132(18.2 \%)$ of the respondents say that they never use disposable transducer covers.

Intracavity probes were only cleaned by $105(16.8 \%)$ out of the 627 respondents, cleaned and low-level disinfected by 294 (46.9\%), cleaned and high-levels disinfected (HLD) by 228 (36.3\%). Only 31.6\% of the respondents use effective disinfectants for HLD standards achieving. Single-use transducers covers were used in $97.0 \%$ of intracavity examinations, but $34.3 \%$ of them were not sterile.

All 54 respondents, performing intraoperative ultrasound procedures, clean and disinfect probes after use, but only half of them sterilize or perform HLD additionally. $90.7 \%$ of respondents use sterile single-use transducer cover on a permanent basis.

Ultrasound practitioners named three main reasons that impede the safety of ultrasound diagnostics: the lack of conditions for the introduction of HLD (57.7\%), the lack of national guidelines (48.2\%), and insufficient knowledge in this area (32.2\%).

Conclusion: The study revealed the potential risks of infection for patients during ultrasound examinations associated with the lack of standardized approaches for ensuring this medical technology epidemiological safety. The development and implementation of IPC national guidelines in ultrasound diagnosis into clinical practice is a priority.

Disclosure of Interest: None declared.

Poster session: environmental control: others

P191

Importance of the hospital environment in the transmission of infections by multidrug-resistant microorganisms: a systematic review of literature

A. Guimarães ${ }^{1, *}$, A. Pereira, 1,2, R. Chaves ${ }^{2,3}$, C. Vallone $^{1}$, E. Kumoto $^{1}$

on behalf of IMPACTO MR Group

${ }^{1}$ Big Data, ${ }^{2}$ Critical Care Unit, ${ }^{3}$ Anesthesiology, Hospital Israelita Albert

Einstein, São Paulo/SP, Brazil

Correspondence: A. Guimarães

Antimicrobial Resistance \& Infection Control 10(1): P191

Introduction: Despite of healthcare environment is an important reservoir for multidrug-resistant microorganisms (MDROs), the impact of environmental interventions in clinically relevant outcomes is unclear.

Objectives: To review the literature about the impact of environmental interventions (non-related to sanitizers) in the clinical impact of MDROs.

Methods: Studies were identified through electronic literature search of PubMed, CINAHL, and SCOPUS, by two independent authors. Any type of environmental interventions in surgical centers, ICUs and wards were evaluated. No restrictions regarding patients and microorganisms were applied. Bench studies, MDROs inocula on surfaces, studies based on sanitizers (disinfectants or detergents) or animal studies were excluded. Quality of randomized controlled trials and non-randomized ones were evaluated by the Cochrane Collaboration's tool, and Newcastle-Ottawa scale, respectively.

Results: The search strategy identified 1173 studies. Of those, 20 studies were included in systematic review. Only 4 studies were randomized, and those, only 1 (a bundle-based intervention), assessed the impact on clinical outcomes. Bundle of interventions (including educational procedures) were the most frequently non-sanitiser method found (7 studies). Ultraviolet light (UV, 2 studies) and hydrogen peroxide vapor (HPV, 4 studies) seemed to be promising methods to improve cleaning processes. Wipe-based methods were assessed in 2 prospective studies, and one of them highlights the potential of spread MDROs in case of not properly used. Other cleaning methods (for instance, testing more cloths, electrolysed water, extra cleaning etc.), were evaluated in 5 studies. 


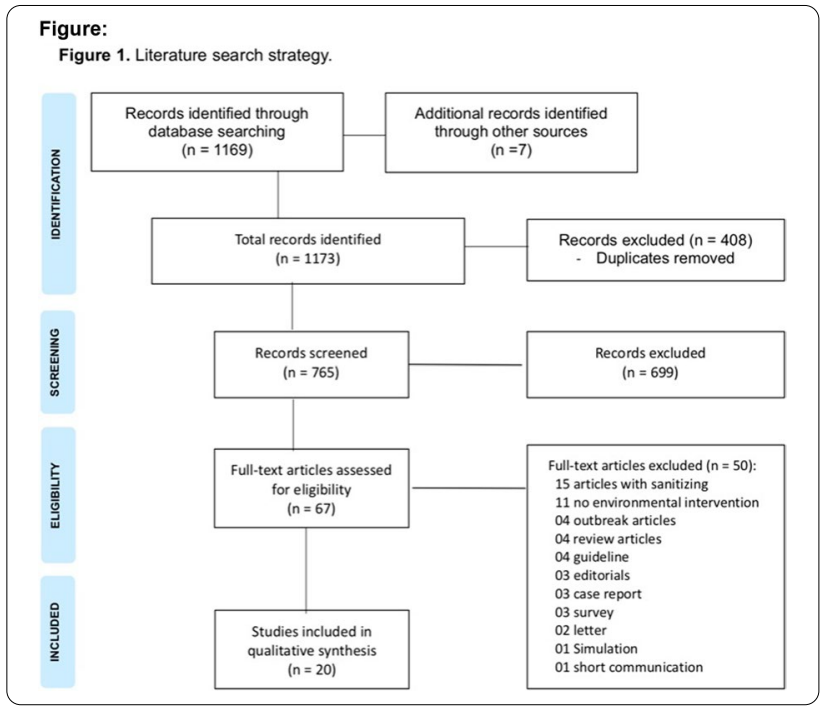

Conclusion: UV, HPV, and bundle-based approaches seem to be potential complementary environmental interventions to sanitizers in hospitals. However, only few, low-quality interventional studies are available, most of them focused in microbiological, and not clinical outcomes. Future research in this area should focus in randomized clinical trials (including cluster) evaluating the potential intervention in clinically relevant outcomes.

Disclosure of Interest: None declared.

\section{P192}

Optimizing air monitoring procedures in critical areas of a third level hospital

K. Bliek Bueno ${ }^{1, *}$, C. Lapresta Moros ${ }^{1}$, A. Del Cura Bilbao ${ }^{1}$, J. M. Celorrio Pascual ${ }^{1}$, T. Giménez Júlvez ${ }^{1}$, A. M. Cortés Ramas ${ }^{1}$, M. J. Hernández Navarrete ${ }^{1}$

${ }^{1}$ Preventive Medicine and Public Health, Miguel Servet University Hospital, Zaragoza, Spain

Correspondence: K. Bliek Bueno

Antimicrobial Resistance \& Infection Control 10(1): P192

Introduction: Critical areas in hospitals in Spain (operating rooms, intensive care units, burns units, transplant units, etc.) follow the particle counting and microbiological sampling limits in the air established in the ISO 171340:2012 and 14,644-1 standard requirements. Existing literature is contradictory when determining the association between particle counts and microbiological colony-forming units (CFU), and a particle count cut-off point has never been determined to safely guarantee the absence of microbiological contamination.

Objectives: The objective of this study was to establish a cut-off point in the number of air particles in critical areas in hospitals that could potentially avoid microbiological sampling.

Methods: Diagnostic validity and reliability study conducted using particle counting (number of $0.3,0.5$ and $5.0 \mu \mathrm{m} / \mathrm{mm} 3$ particles) and microbiological sampling (bacterial and fungal CFUs) in the air of critical areas of the Miguel Servet University Hospital collected between January 2010 and August 2019. The particle cut-off point with greater diagnostic validity was established using the area under the ROC curve (AUC), the Youden index, and the positive (PPV) and negative (NPV) predictive values.

Results: We obtained 442 particle counts with their corresponding microbiological samples. Out of the three particle diameters, we selected $0.3 \mu \mathrm{m}$ particles for having the highest diagnostic performance. Our selected cut-off points were 770,000 particles (AUC $=0.80$, $\mathrm{NPV}=94 \%$ ) for fungi and 9,900,000 particles (AUC $=0.76$, $\mathrm{NPV}=96.5 \%)$ for bacteria, which could have avoided $58.6 \%$ and $85 \%$ of microbiological sampling procedures for fungi and bacteria respectively.

Conclusion: Microbiological contamination varies significantly with the number of particles in the air. A reasonable cut-off point can be established to guarantee environmental biosecurity in hospitals while reducing monitoring time and economic costs.

Disclosure of Interest: None declared.

P193

Healthcare environmental hygiene: preliminary results of an international sentinel survey of hospital practices in healthcare environmental hygiene

A. Peters ${ }^{1, *}$, M. N. Schmid ${ }^{2}$, M. DeKraker ${ }^{1}$, D. Pittet ${ }^{1}$

${ }^{1}$ Infection Control Programme \& WHO collaborating Centre on Patient Safety, University of Geneva Hospitals and Faculty of Medicine of Geneva, ${ }^{2}$ University of Geneva, Geneva, Switzerland

Correspondence: A. Peters

Antimicrobial Resistance \& Infection Control 10(1): P193

Introduction: Good practices in healthcare environmental hygiene (HEH) are key to infection prevention and control, and decrease the risk of healthcare associated infections. $\mathrm{HEH}$ includes anything in the healthcare environment not directly on the patient, including surfaces, sterilization, device reprocessing, air, water management, laundry and waste management. HEH is often a neglected field, and little is known about the topography of practices and challenges globally.

Objectives: Our survey aims to show how HEH programs work around the world and what specific challenges they face. The larger objective of this work is to use the data collected to develop a Healthcare Environmental Hygiene Self-Assessment Framework (HEHSAF), in order to help hospitals improve their HEH in line with the Clean Hospitals ${ }^{\circledR}$ project.

Methods: The online survey is built according to the 5 components of the WHO multimodal improvement strategy. 729 healthcare facilities (HCF) from 175 countries received the HEH survey: $30 \mathrm{HCF}$ answered from 12 high, 9 high-middle, 5 low-middle and 4 low incomes countries. 4 facilities from each income level were selected for semi-structured interviews to obtain qualitative data.

Results: Preliminary results indicate that most HCF believe HEH is important (71\%). Often, budget allocated for cleaning and disinfection is not sufficient (58\%) and the responders do not make decisions for the budget (63\%). Detergent $(77 \%)$, bleach or chlorine-based disinfectant $(80 \%)$ and mop and bucket systems $(87 \%)$ are the most frequent products and supplies used. UV disinfection machines are used in 33\% HCF surveyed. Environmental service (EVS) staff do not have the possibility to complete certification programmes (83\%) nor to advance in management roles (75\%). No events about HEH are hosted in over half of the HCF surveyed. Communication between EVS staff and nurse staff is frequent on the workfloor (47\%) but in $27 \%$ of cases, there is no formal meeting between the EVS staff and nursing staff.

Conclusion: There are major differences among HEH programs and the importance they are given by HCF. New approaches in products and supplies are often not used, and there are problems with workplace culture. There is definitely room for improvement on a global level.

Disclosure of Interest: None declared. 
P194

Essential supply list for infection prevention and control in healthcare facilities

J. Lopez ${ }^{1, *}$, T. Ashengo ${ }^{1}$, S. Sara ${ }^{1}$

${ }^{1}$ Momentum Country and Global Leadership, Washington, United States

Correspondence: J. Lopez

Antimicrobial Resistance \& Infection Control 10(1): P194

Introduction: Maintaining infection prevention and control (IPC) standards is vital to ensuring that healthcare staff provide services that are safe for patients and staff. To meet basic levels of IPC readiness, healthcare facilities need access to essential supplies and equipment like soap and water, proper medical waste receptacles, and sufficient stocks of personal protective equipment (PPE) for staff, patients, and visitors.

Objectives: In response, USAID's Momentum Country and Global Leadership (MCGL) project developed an Essential Supply List for healthcare facilities (HCFs) to maintain basic standard IPC practices for all healthcare service levels and contexts. The list complements existing guidance and standard documents by providing centralized global guidance on essential IPC supplies and equipment, along with transmission-based precautions, minimum supply specifications and recommended stocking quantities. While certain clinical contexts may require additional standards and supplies, these basic standards remain universal.

Methods: The list is non-exhaustive and focuses on the essential supplies required for IPC based on a review of the literature, interviews with IPC experts and review of available guidance. While this document was not written to respond directly to the COVID-19 pandemic it does include essential IPC supplies required for delivery of safe healthcare services in the context of COVID-19.

Results: The final list of essential supplies was compiled into the following sections: Healthcare workers, Envrionmental Cleaning, Instrument Processing, Wate Managment, and Infrastructure and Maintenance. The sections are further divided by IPC materials and PPE.

Conclusion: The presentation will provide an overview of the docu ment and how it can support national and sub-national health authorities by incorporating it into their Logistic Management information Systems (LMIS) and inventory control systems, along with development of forecasting plans for PPE and IPC consumables that prioritize needs, stockpiling and deployment plans and continued communication with facilities to ensure supply.

Disclosure of Interest: None declared.

P195

Epidemiology of exophiala dermatitidis in a Glasgow hospital, potential hospital sources and control measures

T. Inkster ${ }^{1, *}$, C. Peters ${ }^{1}$, G. Macgregor ${ }^{2}$

${ }^{1}$ Microbiology, ${ }^{2}$ Respiratory Medicine, Queen Elizabeth University Hospi-

tal, Glasgow, United Kingdom

Correspondence: T. Inkster

Antimicrobial Resistance \& Infection Control 10(1): P195

Introduction: Exophiala dermatitidis is a saprophytic black yeast associated with respiratory, soft tissue and blood stream infections. Its prevalence in Cystic Fibrosis (CF) patients ranges from 1-19\% worldwide.Whilst previous studies have found evidence of $E$. dermatitidis in domestic sources there is a paucity of literature on hospital acquisition, sources and relevant control measures.

Objectives: To discuss the epidemiology of E. dermatitidis in our hospital, the investigations undertaken to investigate potential hospital sources and recommendations for future control measures.

Methods: Data between January 2016 and June 2019 was extracted for all patient isolates of $E$. dermatitidis from all sample types. Deduplication of positive isolates was undertaken with the first isolate in each patient included in the analysis.

Environmental sampling of chilled beams, dishwashers, washing machines, linen and drains was undertaken using cotton swabs. These were plated on to a Sabaroud Agar ( SAB) plate and incubated at $37 C$ for 5 days. E dermatitidis was identified using MALDI.

Results: $\mathrm{E}$ dermatitidis was isolated predominantly from patients with Cystic fibrosis and more frequently from adults with the condition compared with children. Other patient groups with positive isolates included those with chronic lung conditions and haematological disorders. The majority of sample types were respiratory although five patients had positive blood cultures during the study period The number of affected patients reached a peak in quarters 3 and 4 of 2017 and fell following the identification of environmental sources and implementation of control measures.

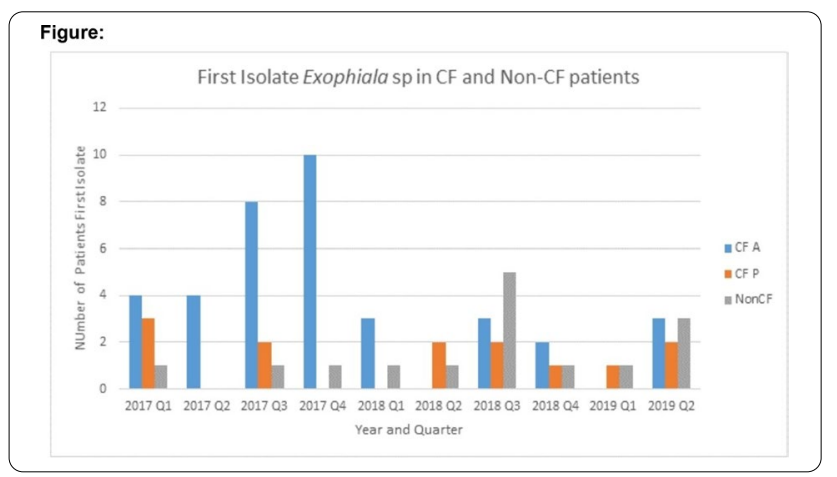

Conclusion: In our study we found evidence of hospital sources of $E$ dermatitidis. The yeast was found in dishwashers, chilled beams and from air sampling. Another potential source was damage to shower flooring and the presence of visible black mould. We implemented the following control measures; 1) Dishwashers were removed from wards housing immunocompromised patients. 2)An increase in cleaning frequency was introduced for chilled beams 3) Damaged showers were repaired. Infection control teams should be aware of the environmental conditions which might promote growth of E dermatitidis and how to mitigate the risks.

Disclosure of Interest: None declared.

\section{P196}

Recognition on the high-touch surfaces among nurses and inpatients

Y. J. Heo ${ }^{1,2}$, J. S. Jeong ${ }^{1, *}$, H. R. Choi ${ }^{1}$, I. S. Jeong ${ }^{3}$, Y. H. Kim ${ }^{1}$

${ }^{1}$ Department of Clinical Nursing, University of Ulsan, Seoul, ${ }^{2}$ Department of Infection Control, H Plus Yangji Hospital, Seoul, ${ }^{3}$ College of Nursing,

Pusan National University, Yangsan, Korea, Republic Of

Correspondence: J. S. Jeong

Antimicrobial Resistance \& Infection Control 10(1): P196

Introduction: As hospital environment can be a cause of healthcareassociated infection, environment control plays an important role in preventing the spread of pathogens. Particularly, high-touch surfaces frequently contacted by patients or healthcare workers are more easily contaminated than other surfaces, so it is recommended to clean them regularly or more often. Though, the examples of the high-touch surfaces are provided in the guidelines, it has never been studied how frequently the surrounding are contacted, or how consistent is the recognition of the contract frequency between patients or healthcare workers.

Objectives: This study was aimed to compare the recognition on the high touch surfaces between nurses and inpatients, and to prioritize cleaning and disinfection.

Methods: The participants were 122 nurses working at general wards and 56 patients hospitalized at the 299-bed general hospital in Seoul. Data were collected from 6 to 10 April 2020 using a self-reported questionnaire including 38 items in four sections; 5 items on the medical devices, 13 items on toilets, 10 items on the equipment around sink and door, and 10 items on the bedside area and furniture. 
Results: Average recognition on high touch surfaces was significantly higher in nurses than patients. $(5.96 \pm 1.47$ vs. $2.35 \pm 1.98, p<0.001)$. Top seven high touch surfaces included IV poles, bed side rail, bed height remote control, bed table, restroom door handle, bedside personal locks, and restroom seat, the ranking of which was the different between nurses and patients. The recognition on high touch surfaces was related to the position $(p=0.020)$, work department $(p=0.004)$, and work experience at current department $(p=0.013)$ for nurses and gender $(p<0.001)$ and ability to move alone $(p=0.003)$ for patients.

Conclusion: Though nurses and patients show consensus on major high touch surfaces, there is a big difference in touch frequency. Further researches are required to confirm high touch surfaces through observation.

Keywords: Patient's rooms; Environment; Disinfection; Recognition; Nurses; Patient.

Disclosure of Interest: None declared.

\section{P197}

A first report of qac $\delta e 1$ detection in pseudomonas species from hospital environment in Slovakia

M. Havrisko ${ }^{1}$, J. Brnova ${ }^{1, *}$, L. Reizigova ${ }^{1}$, L. Pazderka ${ }^{1}$, J. Prnova ${ }^{1}$, A. Liskova ${ }^{2}$, Z. Sirotna ${ }^{3}$, A. Streharova ${ }^{4}$, V. Krcmery ${ }^{5}$

${ }^{1}$ Centre for Microbiology and Infection Prevention, School of Health Sciences and Social Work, Trnava University, Trnava, ${ }^{2}$ Department of Clinical Microbiology, University Hospital Nitra, Nitra, ${ }^{3}$ National Reference Centre of Environmental Microbiology, Public Health Authority, Bratislava, ${ }^{4}$ Department of Infectious Diseases, Trnava Univeristy Hospital, Trnava, ${ }^{5}$ Tropicteam, St. Elisabeth University, Bratislava, Slovakia

Correspondence: J. Brnova

Antimicrobial Resistance \& Infection Control 10(1): P197

Introduction: Quaternary ammonium compounds (QAC) are often employed as disinfectants in hospitals. The resistance mechanism to disinfectants is usually determine by genes qac $\Delta \mathrm{E} 1$ that are found in Gram-negative bacteria, mainly in the Enterobacteriaceae family and with Pseudomonas species.

Objectives: The aim of this study was to analysed the frequency of biocide resistant gene qac $\triangle \mathrm{E} 1$ in Pseudomonas species isolated from hospital environment during HOSPITAL-ENVIRO-REZ study in 20152019 in Slovakia.

Methods: In this study 274 environmental isolates of Pseudomonas species from 93 Slovakia hospitals were screened for the presence of biocide resistant genes qac $\triangle E 1$ (by the PCR and visualised by ELFO) and resistance to antibiotics genes bla $_{\mathrm{KPC}}, \mathrm{bla}_{\mathrm{NDM}}$, $\mathrm{bla}_{\mathrm{VIM}}$,

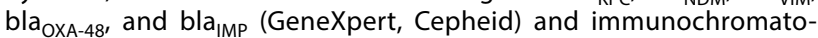
graphic rapid tests (NG-Test CARBA 5, NG Biotech).

Results: Out of the 274 isolates of Pseudomonas species from hospital environment 78 isolates $(28,5 \%)$ were positive for qac $\Delta \mathrm{E} 1$ gene. Overall $62(79,5 \%)$ were from hospital sanitary facilities (sinks, sink drains, faucets, showers, washing basins and soaps). Within all qac $\Delta E 1$ Pseudomonas spp. isolates were $57(73 \%)$ multidrug resistant. Carbapenem resistance were detected in $32(41 \%)$ isolates, of which two were bla IMP $_{1}$ and six bla $\mathrm{VIM}_{\mathrm{VIM}}$ positive.

Conclusion: This study shows that the multidrug resistant Pseudomonas species with qac $\Delta \mathrm{E} 1$ gene which harboured resistance to quaternary ammonium compound biocides are widespread in hospital sanitary facilities in Slovakia.

This study was supported by a research grant from the MŠVVaŠ SR and grant fromTrnava University.

Disclosure of Interest: None declared.

\section{P198}

Peculiarities of formation of biofilms by pathogens and microorganisms of damage of food production

A. V. Tutelyan ${ }^{1, *}$, Y. Yushina ${ }^{2}$ on behalf of A.A. Semenova, E.V.Zayko, D.S.

Bataeva, M. Grudistova², V. Akimkin ${ }^{3}$

${ }^{1}$ Central Research Institute of Epidemiology, ${ }^{2}$ V. M. Gorbatov Federal

Research Center for Food Systems, ${ }^{3}$ Central Researche Institute of epide- miology, Moscow, Russian Federation

Correspondence: A. V. Tutelyan

Antimicrobial Resistance \& Infection Control 10(1): P198

Introduction: Microorganisms form biofilms on any surface. The danger for the food in-dustry is the development of biofilms on abiotic surfaces. The abiotic surface of food production is rough, porous, with joints, seams and a huge number of hard-to-reach places, i.e. there are all conditions that contribute to the localization of biofilms. The structure of the matrix serves as a barrier to prevent the penetration of disinfectants and detergents.

Objectives: A study was carried out on the ability of microorganisms Listeria and Pseudomonas species to form biofilms.

Methods: As objects of research, 26 strains of various types of microorganisms were selected, isolated from the objects of the production environment of a meat processing plant and a poultry plant, as well as from meat products. Biofilm formation was studied in vitro on microtiter plates. The ability to form biofilms was evaluated $+4^{\circ} \mathrm{C}$, the growth rate of the culture was assessed in a photometer at a wavelength of $540 \mathrm{~nm}$. Wells filled with sterile broth served as a control. The excess of the optical density of the crystal violet over the control indicated the formation of biofilms by bacteria.

Results: As a result of the studies carried out, it was found that at a low positive temperature, all strains of pathogens formed stable biofilms during the first three days of cultivation. This ability was possessed not only by pathogenic species of Listeria (L. monocytogenes), but also by non-pathogenic ones- $L$. welshimeri and $L$. innocua. The results showed that all Salmonella spp. possessed the ability to form biofilms at low positive temperatures, especially intensively during $96 \mathrm{~h}$ of incubation. None of the strains formed a large amount of biofilm until $24 \mathrm{~h}$. Of the two studied bacterial strains Pseudomonas gessardi and Pseudomonas azotoformans, both strains showed a high ability to form biofilms at low positive tem-peratures.

Conclusion: The ability to form biofilms by pathogens and spoilage microorganisms poses a serious threat to the production of safe products and is one of the reasons for the stable circulation of microorganisms in the conditions of meat processing plants.

Disclosure of Interest: None declared.

\section{P199}

Impact of health education on knowledge of hospital staff regarding healthcare wastes management in Tunisian university hospital

R. Bannour ${ }^{1, *}$, H. Ghali, ${ }^{1,2}$, A. Ben Cheikh ${ }^{1,2}$, S. Bhiri, ${ }^{1,2}$, W. Dhouib 1 , S.

Khefacha ${ }^{1}$, H. Said Latiri $^{1,2}$, M. Ben Rejeb ${ }^{1,2}$

${ }^{1}$ Department of Prevention and Security of Care, ${ }_{1}$ Sahloul University

Hospital, ${ }^{2}$ Faculty of Medicine of Sousse, University of Sousse, Sousse,

Tunisia, Sousse, Tunisia

Correspondence: R. Bannour

Antimicrobial Resistance \& Infection Control 10(1): P199

Introduction: Inappropriate healthcare wastes (HCWs) management may lead to many psychological, environmental and health hazards. Therefore, there has been growing interest among medical organizations in strategies to reduce the quantity of HCWs and developing the recycling programs..

Objectives: The aim of this study was to assess healthcare professional knowledge regarding HCWs management before and after an educational intervention session.

Methods: A pre-experimental study design was applied to assess the impact of an intervention program on knowledge and practice regarding HCWs management during March 2021 in Sahloul university hospital. The same questionnaire used in the pre-test was used immediately after the end of the intervention program.

Results: A total of 63 healthcare professionals participated in our study. Overall, a significant increase in knowledge after the intervention was detected among all knowledge items. Regarding the knowledge of possible risks caused by healthcare waste, identification of toxicity as a risk increased from $5.7 \%$ to $22.5 \%$; of psychological impact 
from 0 to $32.5 \%$; injuries from 31.4 to $52.5 \%$; infections from $62.9 \%$ to $80 \%$ and environmental contamination from $28.6 \%$ to $57.5 \%$.

Concerning the different types of healthcare wastes and their types of packaging; the rate of correct answers passed from $0 \%$ for "Flammable or explosive waste"; "Radioactive Hazardous Health Activity Waste" and "Anatomical parts and placentas" to 10\%;10\% and 5\% respectively.

Conclusion: The health professional recorded an improvement in their knowledge with regard HCWs management in post-test, but the majority of answers still not exceed $50 \%$.

Therefore, we suggest implementing practical training workshops targeting all health personnel.

Disclosure of Interest: None declared.

Poster session: environmental control: products

\section{P200}

Ewash-a multi-period cross-over trial comparing hypochlorous acid and bleach on hospital surfaces

G. Gon ${ }^{1, *}$ on behalf of EWASH working group

${ }^{1}$ Infectious Disease Epidemiology, London School of Hygiene and Tropical Medicine, London, United Kingdom

Correspondence: G. Gon

Antimicrobial Resistance \& Infection Control 10(1): P200

Introduction: Environmental hygiene is crucia for preventing hospital associated infections. Cleaning practices in low-income hospitals continue to rely on sodium hypochlorite, which is corrosive, requires accurate dilution on a daily basis and is toxic if in contact with skin or mucous membranes. Neutral Electrolyzed Water (EW) is an innovative surface disinfectant produced by passing an electrical current through saline water, whose active ingredient is hypochlorous acid. EW is neither toxic nor corrosive and it presents no environmental hazard, as it slowly reverts to saline during the period of chemical relaxation. The proven broad-spectrum antimicrobial activity of EW enables high-level surface disinfection. Surprisingly, the use of EW is currently largely unknown in the health sector.

Objectives: The main objective is to evaluate the efficacy of Electrolyzed Water for disinfection of near-patient hospital surfaces as compared to standard disinfection.

Methods: A multi-period cluster randomised trial (EWASH trial) will be used to evaluate the efficacy of EW for surface disinfection compared to standard disinfectant (chlorine-based bleach) in three hospitals in Abuja, Nigeria, over six weeks. Four wards will be selected in each hospital and will be randomly allocated to one of the two interventioncontrol sequences. Each week either EW or the standard disinfectant is provided to these wards for surface cleaning.

Microbiological cleanliness will be measured using double sided dipslides with non-selective agar for Aerobic Colony Count $(<2.5 \mathrm{cfu} /$ $\mathrm{cm} 2=$ clean; $\geq 2.5 \mathrm{cfu} / \mathrm{cm} 2=$ not clean). The presence of S.aureus will be a secondary outcome. The study design is expected to have $87 \%$ power to demonstrate non-inferiority at a $5 \%$ margin with $15 \mathrm{sam}$ pling sites in each ward (cohort structure) and a baseline of cleanliness ranging from 30 to $50 \%$.

Focus group discussions (FGDs) will be conducted with the cleaners to understand their perception of the product, barriers and enablers.

Results: The trial is under way and data completion is planned for July 2021.

Conclusion: EW has never been tested in low-resource settings, the EWASH study will contribute to provide evidence on its efficacy for environmental hygiene. The study could provide evidence for further applications of EW in hospital settings such as cleaning medical equipment, use for burns wounds and diabetic foot infections.

Disclosure of Interest: None declared.
P201

The effect of various surface disinfectants and their method of use on bactericidal efficacy

J. Ochi ${ }^{1, *}$, R. Matsumura ${ }^{1}$, Y. Hirata ${ }^{1}$

${ }^{1}$ Biochemical Laboratory, Saraya Co., Ltd., Osaka, Japan

Correspondence: J. Ochi

Antimicrobial Resistance \& Infection Control 10(1): P201

Introduction: Quaternary ammonium compounds (QAC) are common disinfecting agents used for environmental disinfection of health care facilities in Japan. Products are usually available in spray and wet wipe types to disinfect environmental surfaces. However, there are still a lot to understand about environmental disinfectants containing QAC in terms of their types and different methods of use.

Objectives: This study aimed to the bactericidal efficacy of QAC-containing surface disinfectants with different method of use.

Methods: QAC-containing liquid formulation was evaluated with EN13727 and EN13697.

Different types of QAC-containing surface disinfectants were evaluated according to EN16615 in their actual use. Spray and wet wipe types were used in test. For spraying, two types of methods were used: 1) Spray directly on the environmental surface and wipe off with a dry wipe, 2) Spray on a dry wipe and wipe the environmental surface. The wet wipe type was used as is.

Results: The high efficacy of QAC-containing liquid formulation was confirmed with EN13727 and EN13697. Regarding bactericidal efficacy of QAC-containing surface disinfectants in their actual use, the results of EN16615 varied depending on their type and method of use.

Conclusion: Although bactericidal efficacy of liquid formulation is important for surface disinfectants, it is also important to understand the performance characteristics of each type in before use.

Disclosure of Interest: None declared.

P202

Liquid hydrogen peroxide efficacy compared with standard cleaning care on surfaces disinfection

C. Hidalgo 1,* , M. Posso ${ }^{1}$, A. Ramírez ${ }^{2}$, L. Martínez ${ }^{3}$, R. Bover ${ }^{4}$, M. Herranz $^{1}$, E. Padilla ${ }^{2}$, C. González ${ }^{1}$

${ }^{1}$ Epidemiology \& Evaluation Department, Parc de Salut Mar, ${ }^{2}$ Microbiology Department, LRC, ${ }^{3}$ Traumatology \& Orthopedics Department, ${ }^{4}$ General Services Department, Parc de Salut Mar, Barcelona, Spain

Correspondence: $C$. Hidalgo

Antimicrobial Resistance \& Infection Control 10(1): P202

Introduction: In our hospital, sodium hypochlorite and quaternary ammonium + amines are the used products for desinfecting the room surfaces (our standard clinic care: SCC), but they have disadvantages.

Objectives: To compare the effectiveness of liquid hydrogen peroxide with SCC on reducing surface contamination.

Methods: Pre-post quasi-experimental study with 2 phases comparing the effectiveness of liquid hydrogen peroxide (phase 1) versus SCC (phase2). Quantitative and qualitative evaluation was assessed from February 2019-February 2020, on 5 high-touch hospital room surfaces (upper and lower surfaces of food table, call botton, toilet flap and mattress) in 2 hospitalization units (one surgical and one medical)at Hospital del Mar. For each surface: adenosine triphosphate (ATP), visual assessment (fluorescent marker) and aerobic colony counts (ACC) measurements were collected before and after cleaning. Mann-Whitney $\mathrm{U}$ test was used for assessing medians differences in the ATP and ACC. Stratified analysis by hospitalization unit, isolated status and contact surface was performed. P values lower than 0.05 were considered statistically significant.

Results: We evaluated 310 surfaces:217 from phase 1 and 93 from phase 2. Compared to post-cleaning values, pre-cleaning surfaces showed a statistically higher median of ATP relative light units (RLU) and ACC for both products: a) hydrogen peroxide: 316 vs. 52 ATP RLU, and 30 vs. 7 ACC; and b) standard cleaning 136 vs. 23 ATP RLU, and 34 vs. 5 ACC. Medical unit surfaces presented higher pre-cleaning RLU rates than in surgical unit, in both cases a significant reduction was 
achieved after cleaning with both products. Statistically significant reduction was observed for isolated and not isolated patients. Interestingly, not isolated patient's room's surfaces revealed higher median pre-cleaning RLU and ACC values than in isolated patient's.

Conclusion: Both cleaning procedures have a similar effectiveness in cleaning and disinfection. It's important to emphasize the relevance of performing properly these processes by the housekeepers.

Disclosure of Interest: C. Hidalgo Grant/Research support from: This study was partly funded by Diversey, The authors did not receive direct funding from the sponsor, M. Posso Grant/Research support from: This study was partly funded by Diversey, The authors did not receive direct funding from the sponsor, A. Ramírez Grant/Research support from: This study was partly funded by Diversey, The authors did not receive direct funding from the sponsor, L. Martínez Grant/Research support from: This study was partly funded by Diversey, The authors did not receive direct funding from the sponsor, R. Bover Grant/Research support from: This study was partly funded by Diversey, The authors did not receive direct funding from the sponsor, $M$. Herranz Grant/Research support from: This study was partly funded by Diversey, The authors did not receive direct funding from the sponsor, E. Padilla Grant/ Research support from: This study was partly funded by Diversey, The authors did not receive direct funding from the sponsor, C. González Grant/Research support from: This study was partly funded by Diversey, The authors did not receive direct funding from the sponsor.

\section{P203}

\section{Antimicrobial activity in the gasphase with hypochloric acid}

D. Boecker ${ }^{1, *}$, R. Breves ${ }^{2}$, C. Bulitta ${ }^{3}$, Z. Z. Zhang ${ }^{4}$

${ }^{1}$ TOTO Consulting, San Jose, United States, ${ }^{2}$ Corporate Microbiology, Henkel AG \& Co KGaA, Duesseldorf, ${ }^{3}$ Institut für Medizintechnik (IfMZ), Ostbayerische Technische Hochschule Amberg-Weiden, Amberg Weiden, ${ }^{4}$ TOTO Consulting, Berlin, Germany

Correspondence: D. Boecker

Antimicrobial Resistance \& Infection Control 10(1): P203

Introduction: Virus-carrying aerosol particles are recognized as infection carriers in the current Corona pandemic, but their high-risk potential is often underestimated and represents the infection route that has been least systematically countered to date. As a result, aerosols currently represent an insufficiently contained mode of disease transmission at public indoor spaces (e.g.: offices, schools, gastronomy).

Objectives: The study investigated if the disinfecting potential of $\mathrm{HOCl}$ in suspensions are transferrable to in-air cleaning applications and to what extent aerosolized $\mathrm{HOCl}$ solutions can deactivate indoor microbial contaminations in-air at or below legal limits.

Methods: For the liquid disinfection we used a standard suspension disinfection test protocol. For the in-air tests we conducted several experiments where aerosolized bacterial suspensions were injected into lab chambers preloaded with different $\mathrm{HOCl}$ gas concentrations.

Results: In suspension experiments we found sufficient efficacies for all studied organisms (Staphylococcus aureus, Pseudomonas aeruginosa, Escherichia coli K12 and Staphylococcus warnerii according to EN 1276, C. albicans according to EN 13,624 as well as vaccinia virus according to EN 14,476) at minimum concentrations of $200 \mathrm{ppm} \mathrm{HOCL}$. The in-air measurement set-up allows to follow microbe deactivation by $\mathrm{HOCl}$ interaction. The deactivation rate increases with the $\mathrm{HOCl}$ concentration, and the values are greatest for Gram-negative germs.

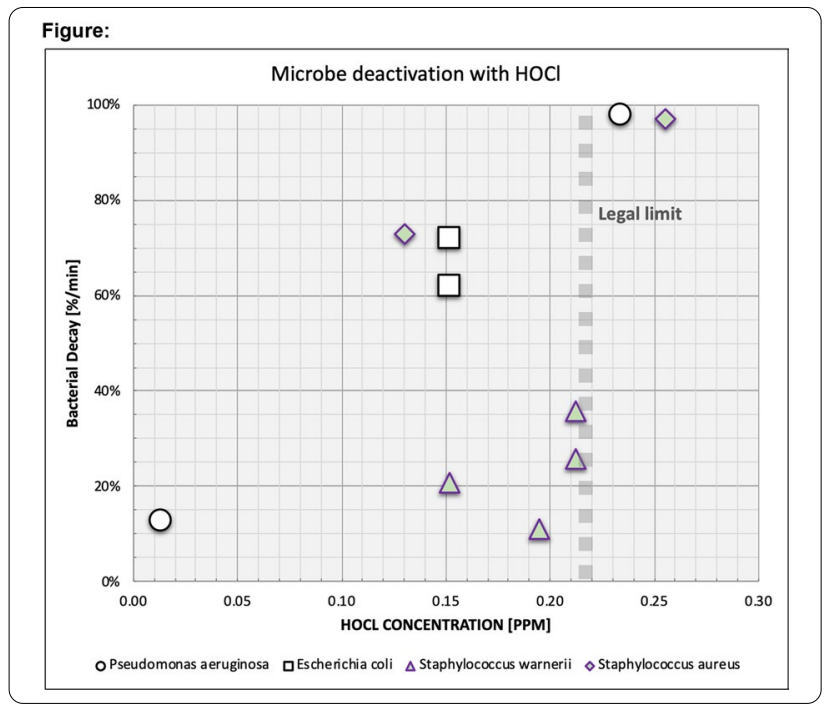

Conclusion: We validated the disinfecting effects of $\mathrm{HOCl}$ in suspensions. We confirmed our hypothesis of the high disinfecting power of $\mathrm{HOCl}$ in-air at safe levels for populated indoor places. The investigated Gram-positive and -negative bacteria can be understood as a general model system for infectious particles, including enveloped viruses (to which Coronavirus belongs). These early results suggest that $\mathrm{HOCl}$ should be further evaluated as an air-cleaning method which may complement established concepts.

Disclosure of Interest: D. Boecker Consultant for: Consultant to the device company, R. Breves Conflict with: Member of oji scientific advisory panel, C. Bulitta Conflict with: Member of oji scientific advisory panel, Z. Zhang Consultant for: Consultant to the device company.

P204

Factors influencing the durability of antimicrobial coatings on different surfaces

S. Buhl $l^{1, *}$, A. Stich ${ }^{1}$, J. Vogt ${ }^{1}$, R. Brückner ${ }^{2}$, C. Bulitta ${ }^{1}$

${ }^{1}$ medical engineering, Ostbayerische Technische Hochschule Amberg-Weiden, Weiden in der Oberpfalz, ${ }^{2} \mathrm{HECOSOL} \mathrm{GmbH}$, Bamberg, Germany

Correspondence: S. Buhl

Antimicrobial Resistance \& Infection Control 10(1): P204

Introduction: Infection through contaminated surfaces is not an abstract risk, but has already been shown by several publications. It has been shown that antimicrobial surfaces can significantly reduce microbiological contamination on surfaces in the long term. However, there have been few studies on how surface properties and reprocessing affect the durability of these coatings.

Objectives: This work will therefore examine the influence of different materials and reprocessing methods on the durability of antimicrobial surface technologies.

Methods: Test samples made of different materials (glass, plastic) are subsequently provided with an $\mathrm{TiO} 2 \mathrm{Ag}$ based antimicrobial coating (HECOSOL $\mathrm{GmbH}$, Bamberg) by electrospray technique and tested for their durability and remaining activity by standard methods (ISO 22196). In a second step, abrasion tests and microbiological activity tests were done on coated wallpaper bonded to plasterboard using various cleaning and disinfecting agents and cloth systems (microfiber cloth, cotton cloth, foam cloth).

Results: While strong antimicrobial efficacy can still be demonstrated on glass surfaces even after several hundred abrasion cycles, this is no longer present on plastic surfaces after just a few cycles. The abrasion tests with various cleaning agents, disinfectants and wipe systems also showed an influence of the material and the preparation on the antimicrobial efficacy. All test samples in our experimental setup showed 
at least slight efficacy. However, only 5 of the 47 tested samples showed a remaining strong efficacy.

Conclusion: Our results suggest that the underlying surface material and also the selection of cleaning and disinfection procedures and wipe systems appears to influence the durability of the coatings on the surfaces. In order to be able to make a statement about the longterm activity of these surface technologies, the effectiveness should be tested in the finished product and after several reprocessing cycles. Disclosure of Interest: None declared.

\section{P205}

Antibacterial activities of metal oxides on copper alloy surfaces H. Komura ${ }^{1, *}$, H. Kawakami ${ }^{1}$

${ }^{1}$ Osaka city university, Sugimoto, Sumiyoshi-ku, Osaka-shi, Japan Correspondence: $\mathrm{H}$. Komura

Antimicrobial Resistance \& Infection Control 10(1): P205

Introduction: Copper alloys are frequently used for hand touched places. As copper alloys have higher antibacterial properties, it is expected to reduce contact infection through environmental surfaces. Oxides are formed on the copper alloy surface in an atmosphere. If a treatment method of producing a highly antibacterial oxides can be developed, the antibacterial activities of the copper alloy surface can be maintained. As a result, a reduction of bacterial transmission through the environmental surface is expected.

Objectives: We investigated the antibacterial activities of metal oxides on the copper alloy surface, using brass as a sample.

Methods: Oxygen-free copper (OFC) (99.96\%) and brass ((mass\%) $\mathrm{Cu}$ : 70.0, Zn: 30.0) were used as samples. Coupon specimens ( $25 \mathrm{~mm} \times 25 \mathrm{~mm} \times 2 \mathrm{~mm}$ ) were cut out from commercially available plates. Test surface of specimens were polished with emery paper (\# 320). The polished specimens were degreased with acetone, and then ultrasonically cleaned in ethanol. Such specimens were called specimen A. Specimen $B$ and $C$ were prepared by applying following treatments to the specimen $A$. For specimen $B$, the specimen $A$ was immersed in a $1 \mathrm{M}$ $\mathrm{LiCl}$ aqueous solution for $1 \mathrm{~h}$. Then, the specimen was kept at $300^{\circ} \mathrm{C}$ for $3 \mathrm{~h}$ in atmosphere. For specimen $\mathrm{C}$, the specimen $\mathrm{A}$ was kept at $350^{\circ} \mathrm{C}$ for $100 \mathrm{~min}$ in atmosphere. The surface products of the specimens were analyzed using X-ray photoelectron spectroscopy (XPS). The antibacterial activities were evaluated by film contact method designated in ISO 22196 (contact time $30 \mathrm{~min}$ ). Escherichia coli (NBRC3972 E. coli) was used as the test bacterium.

Results: Both $\mathrm{Cu}$ and $\mathrm{Cu}_{2} \mathrm{O}$ were detected on the OFC specimen $\mathrm{A}$ (OFC-A) surface. Only $\mathrm{Cu}_{2} \mathrm{O}$ was detected on the OFC-B surface, while only $\mathrm{CuO}$ on the OFC-C surface. Antibacterial activities were confirmed in all specimens. The viable cell counts of the OFC-A and B were about 3 order lower than the value for the negative control $\left(3.5 \times 10^{4} \mathrm{CFU} /\right.$ $\mathrm{cm}^{2}$ ). OFC-C had a higher viable cell count in comparison with A and $B$, indicating lower in antibacterial activity.

For Brass- $\mathrm{A} \mathrm{Cu}, \mathrm{Cu}_{2} \mathrm{O}, \mathrm{Zn}, \mathrm{ZnO}$ were detected, while only $\mathrm{ZnO}$ was on the Brass- $B$ and $C$ surfaces. The viable cell counts of Brass- $B$ and $C$ were about 2 order higher than that of the Brass-A.

Conclusion: The antibacterial activities of metal oxides on a brass are inferior to them on an untreated brass.

Disclosure of Interest: None declared.

\section{P206}

\section{Antibacterial activity of brass post-cleaning/disinfection} treatments

E. Dauvergne ${ }^{1, *}$, D. Declerck ${ }^{2}$, C. Lacquemant ${ }^{2}$, C. Mulllié ${ }^{1}$

${ }^{1}$ Laboratoire AGIR-UR UPJV 4294, Université de Picardie, Amiens, ${ }^{2}$ FAVI

S.A., Hallencourt, France

Correspondence: E. Dauvergne

Antimicrobial Resistance \& Infection Control 10(1): P206

Introduction: Brass can be used as an antimicrobial surface in healthcare facilities to help reducing Hospital Acquired Infections (HAI) mediated by an environmental transmission. However, few studies have so far evaluated the impact of hospital cleaning and disinfection practices on brass antimicrobial properties.

Objectives: This work therefore aimed to assess the impact of commonly used disinfectants on the antibacterial activity of a commercially available brass $\left(A B+{ }^{\circledR}\right)$.

Methods: Stainless steel (control) and brass samples were therefore treated 365 times with a commonly used hospital disinfectant containing $0.3 \%(\mathrm{~W} / \mathrm{W})$ quaternary ammonium (QA) according to the manufacturer's recommendations to simulate one year of cleaning/disinfection. Treatment with an aerosolized product used for terminal decontamination (peracetic acid (PA) 1200 ppm and hydrogen peroxide) was also carried out 30 times on some of these samples.The antibacterial efficacy of variously treated samples was evaluated on six multidrug-resistant clinical strains representing major species involved in $\mathrm{HAl}$, as previously described (Dauvergne et al., Antibiotics 2020, 9, 245).

Results: Results compiled in Table 1 show that brass samples retain a fairly good antibacterial activity after $Q A \pm P A$ treatment, whatever the strain.

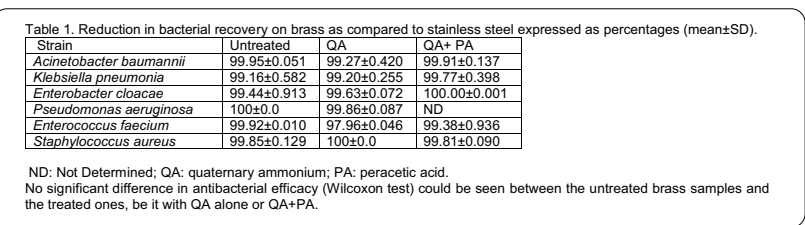

Conclusion: The simulated cleaning/disinfection process therefore did not impair significantly the antibacterial activity of $A B+{ }^{\circledR}$ brass on the panel of strains tested. These preliminary results support the use of this type of surface in healthcare settings to reduce environmental transmission of pathogens.

Disclosure of Interest: E. Dauvergne Employee of: FAVI S.A., Grant/ Research support from: PhD grant funded by ANRT and FAVI S.A., D. Declerck Employee of: FAVI S.A., C. Lacquemant Employee of: FAVI S.A., C. Mulllié: None declared.

Poster session: hand hygiene: alcohol handrub efficacy, tolerability and system change

\section{P207}

Publication trends in hand hygiene: pandemics serve as wake-up calls N. Lotfinejad ${ }^{1, *}$, D. Pires ${ }^{1}$, D. Pittet ${ }^{1}$

${ }^{1}$ Infection Control Programme, University of Geneva Hospitals and Faculty of Medicine, Geneva, Switzerland

Correspondence: N. Lotfinejad

Antimicrobial Resistance \& Infection Control 10(1): P207

Introduction: Hand hygiene with alcohol-based handrub (ABHR) is the global standard of care in preventing healthcare-associated infections that are known among the most common adverse events in healthcare settings. There has been a growing body of knowledge and evidence in this area in the past few decades in order to improve this lifesaving action.

Objectives: To investigate the annual number of publications using hand hygiene-related keywords in the medical literature and analyze publication trends in this field.

Methods: This review is based on Medline search until December 2020, using MEdical Subject Headings (MESH) search terms and keywords. We searched the most commonly used words to describe hand hygiene and analyzed the number of publications retrieved yearly. Results: From 1921 to December 2020, we found 12,737 publications using hand hygiene-related keywords. The most prevalent keywords were hand hygiene, hand disinfection, hand washing, handrub, hand sanitizer and ABHR. Searching all keywords revealed that the number of publications in the field of hand hygiene has doubled in $2020 \mathrm{com}$ pared to 2019. We observed a significant growth in using the hand hygiene keyword, over the past 20 years, with a major peak in 2020 . 


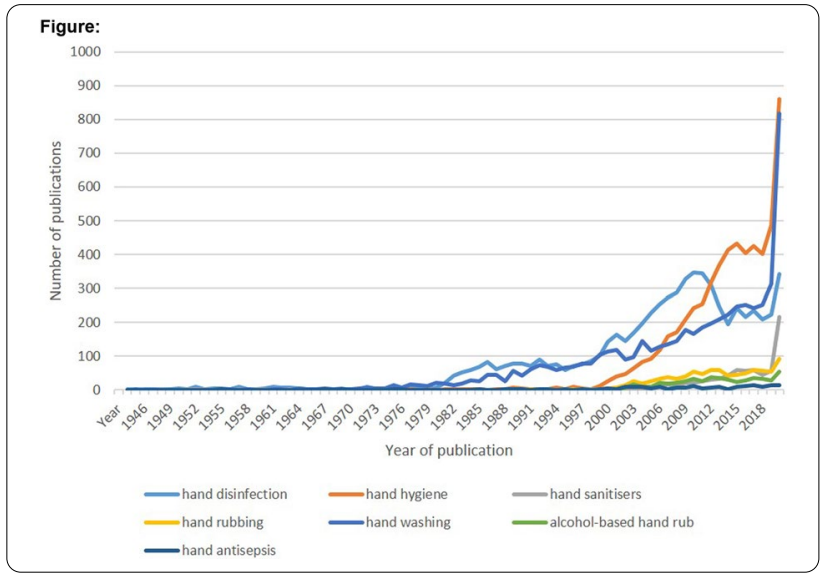

Conclusion: Advances in the hand hygiene literature have been remarkable over the past few years and particularly significant during the recent COVID-19 pandemic. We identified significant changes over time, with a major shift from using soap and water to the preference of ABHR. Higher peaks of research studies during the recent major pandemics, in particular the COVID-19, highlight the capacity for continuous improvements in hand hygiene science and practices.

Disclosure of Interest: None declared.

\section{P208}

Standard monitoring of handrub dispensers' time-dependent behavior

S. Bansaghi ${ }^{1, *}$, T. Haidegger ${ }^{2}$

${ }^{1}$ Doctoral School of Health Sciences, Semmelweis University, ${ }^{2}$ University

Research and Innovation Center (EKIK), Óbuda University, Budapest, Hungary

Correspondence: S. Bansaghi

Antimicrobial Resistance \& Infection Control 10(1): P208

Introduction: Hand hygiene with alcohol-based handrub is essential in every hospital to avoid the spread of infections, thus, ABHR dispensers belong to the critical infrastructure. The decrease in dispensed volume over time was reported to be a generic phenomenon among commonly used dispensers. To prevent under-dosage, dispensers should be audited even before purchasing, and periodically thereafter. Objectives: The aim of this work was to create an effortless yet reliable protocol for that regular audit.

Methods: Properly assessing time-dependency of the dispensing is time-consuming, and therefore not feasible in clinical settings, while the quality-assurance driven goal is to audit all dispensers. To comply with all such requirements, a short protocol was developed, where the whole procedure takes $15 \mathrm{~min} /$ dispenser. This is considered to be a quick test, built on the following: push the dispenser at least 3-times, to set it back to its baseline. Push the dispenser 5 times, collect the dispensed handrub. Let the dispenser rest for $5 \mathrm{~min}$, then collect the first 2 doses separately. Repeat this last, 5-min cycle. The result of this "quick" test was compared with a 3-day extensive, ground-truth test by outcome.

Results: From 11 various dispensers, 234 quick test results were collected. If the volume-loss in 5 min exceeded a threshold value (varied between 5-15\%), the dispenser was categorized as 'Problematic'. In each case, the result of the quick test was compared with the dispenser's baseline test, and the measurements was categorized as false negative, true negative, false positive or true negative. Sensitivity, specificity, positive and negative predictive value was determined in case of different threshold values (Figure 1).

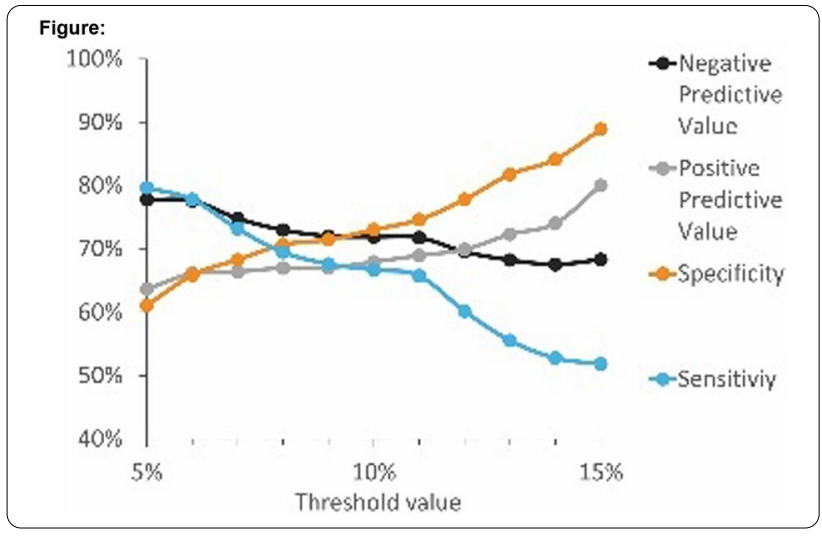

Conclusion: Periodically auditing handrub dispensers is necessary, feasible, yet cumbersome. Evaluation criteria should be wisely chosen to maximize the predictive potential of this test. Optimizing the time requirement of any new quality test is a critical factor towards clinical applicability.

Disclosure of Interest: S. Bansaghi Grant/Research support from: S.B. is supported by the ÚNKP-20-3-II-SE-24 New National Excellence Program of the Ministry for Innovation and Technology from the source of the National Research, Development and Innovation Fund., T. Haidegger: None declared.

P209

A randomized crossover study to test the tolerability of three alcohol-based handrubs: preliminary results

A. Peters ${ }^{1},{ }^{*}$, C. Cave ${ }^{2}$, J. Carry ${ }^{3}$, J. Sauser ${ }^{1}$, D. Pittet ${ }^{1}$

${ }^{1}$ Infection prevention and control, HUG, Geneva, Switzerland, ${ }^{2}$ Faculty of Pharmacy, University of Bordeaux, Bordeaux, France, ${ }^{3}$ Faculty of Medicine, University of Geneva, Geneva, Switzerland

Correspondence: A. Peters

Antimicrobial Resistance \& Infection Control 10(1): P209

Introduction: Hand hygiene with alcohol-based handrubs (ABHR) is the gold standard of care in healthcare facilities (HCF), and prevents healthcare-associated infections. The COVID-19 pandemic caused a global shortage of $A B H R$, which resulted healthcare facilities often using different products than normally. Consumption of ABHR more than doubled at HUG, and compliance stayed the same. Other HCF showed a decrease in compliance among healthcare workers (HCWs). Some of this effect could be due to the differences in tolerability of various formulations. Low tolerability is a known barrier to hand hygiene compliance, but there is little literature about which types of handrub have better dermal tolerance.

Objectives: This crossover study aimed to compare the tolerability and acceptability of 3 different ABHR formulations in 39 volunteers. The ABHRs tested are already used in HCF and have passed the EN1500 norms. The main objective of the study is to determine which types of ABHRs and emollients are most likely to have high dermal tolerability in heavy use settings.

Methods: The methods for this study were adapted from the WHO Protocol for evaluating handrub tolerability (Method 2). The participant population consisted of people that did not already use ABHR heavily, thus excluding most categories of HCW. 39 participants were randomized to 3 different ABHR formulations: 3 are ethanol-based (one with superfatting agents), and one is isopropanol-based. Participants tested each of the 3 solutions over a series of 5-day intervention weeks, each followed by a 9-day washout week. They kept a daily 
log of handrubbing sessions, and returned to investigators for a skin assessment after each intervention period.

Results: Product acceptability and skin condition and were evaluated both by the participants themselves and a trained observer. A total of 24 components related to tolerability were analyzed for each participant weekly. Preliminary results show a marked difference in the acceptability of the formulations. Full results will be analyzed in July, 2021.

Conclusion: Compliance of caregivers is crucial for patient safety, and determining which ABHRs have the best tolerability is likely to increase $\mathrm{HCW}$ satisfaction and compliance.

Disclosure of Interest: None declared.

\section{P210}

Efficacy of hand hygiene products against SARS COV-2

K. Ormandy ${ }^{1, *}$, G. Oxley ${ }^{1}$, D. Falbo ${ }^{2}$, S. Zhou ${ }^{3}$, C. Wilde ${ }^{3}$

${ }^{1} \mathrm{SC}$ J Professional, Denby, United Kingdom, ${ }^{2} \mathrm{SC}$ Johnson, Racine, ${ }^{3}$ Microbac Laboratories, Sterling, United States

Correspondence: K. Ormandy

Antimicrobial Resistance \& Infection Control 10(1): P210

Introduction: Hand hygiene is the primary measure in preventing transmission of pathogens to patients and healthcare workers. With more than 150 million reported COVID-19 cases worldwide of which many require hospital care, it is vital that essential hand hygiene products are sufficiently efficacious against SARS-CoV-2.

Objectives: Microbicidal actives (e.g. ethanol) have been shown to be efficacious against other coronaviruses. The objective of this study is to demonstrate viricidal activity of a range of formulated skin care products against SARS-CoV-2 specifically.

Methods: Testing was conducted using ASTM E1052-20 and EN14476:2013 + A2:2019 test methods. The test product was challenged with an aliquot of viral inoculum as well as an interference substance (for EN14476) then held for an exposure (contact) time. Immediately after the contact time, the reaction mixture was neutralized. The neutralized sample was assayed for infectious virus via a cell culture-based viral infectivity assay (TCID50 assay). The results were compared to a mock-treated Virus Recovery Control to determine the virucidal activity ( $\log _{10}$ reduction) by the test product.

Results: Table I shows activity against SARS-CoV-2 following exposure to 4 products according to EN14476 and 1 product according to E1052.

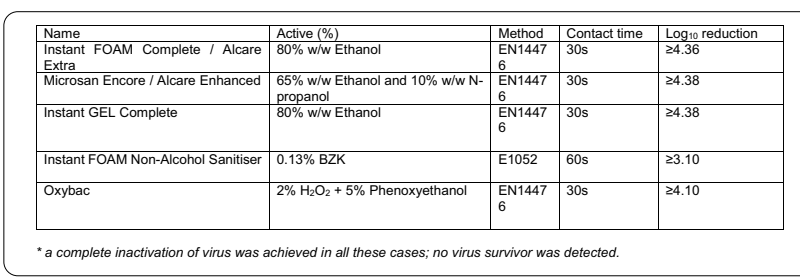

Conclusion: Alcohol-based sanitizers are effective against SARS$\mathrm{CoV}-2$. This effectiveness is not strongly affected either by product format or by alcohol composition if a sufficient concentration is applied.

SCJ Professional BZK based hand sanitizer and Hydrogen Peroxide/ Phenoxyethanol based antimicrobial foam soap are also both effective against SARS-CoV-2.

As a result, we would recommend the use of product formulations meeting those specifications in health care, community, and home environments as part of hand hygiene practices to control the transmission of COVID-19.

Disclosure of Interest: None declared.
P211

Alcohol-based hand rub in vitro activity against SARS-COV-2 and candida auris

A. Capezzone De Joannon ${ }^{1, *},{ }^{*}$ A. Testa ${ }^{1}$, N. Falsetto ${ }^{1}$, M. Procaccini ${ }^{1}$, L. Ragni $^{1}$

${ }^{1}$ Angelini ACRAF s.p.a., ancona, Italy

Correspondence: A. Capezzone De Joannon

Antimicrobial Resistance \& Infection Control 10(1): P211

Introduction: Alcohol-based hand sanitizers (ABHS) are highly effective at inactivating virus and other microbials. For this reason, their use is strongly recommended as an infection prevention measure, in particular, for healthcare personnel when caring for infected patients. Of note, $\mathrm{ABHS}$ sales have grown exponentially in the first months of the COVID-19 pandemic, extending their use to the whole population and generating a constant need for ABHS on the market.

Objectives: The aim of this study is to evaluate the activity of a $74 \%$ ethanol ABHS (Amuchina gel X-GERM) against SARS-CoV-2, according to the European Standard EN14476:2013 + A2:2019 guidelines and against Candida auris, according to the European Standard EN 13,624:2013 guidelines.

Methods: Virucidal activity of the study product was evaluated against the SARS-CoV-2 strain USAWA1/2020 (BEI Resources NR-52281) in the presence of $0.3 \mathrm{~g} / \mathrm{l}$ of bovine serum albumin, for 30 and $60 \mathrm{~s}$. Dilutions of the neutralized reaction mixture were inoculated onto Vero E6 cells to evaluate cytopathic effect.

To assess the activity against $C$. auris DSM $21,092,8 \mathrm{~mL}$ of the product were added to $1 \mathrm{~mL}$ of yeast in presence of $0.3 \mathrm{~g} / \mathrm{l}$ of bovine serum albumin, for 30 and $60 \mathrm{~s}$. The resulting suspension was neutralized, plated onto $\mathrm{SAB}$ agar and incubated at $37^{\circ} \mathrm{C}$ for $48 \mathrm{~h}$.

Results: The study product, in the presence of an interfering substance, reduced the SARS-CoV-2 below detectable limits, with a reduction of the TCID50 greater than $4 \log _{10}$ already after $30 \mathrm{~s}$ of exposure (Table $1 \mathrm{~A}$ ). In addition, the study product showed a reduction of more than $4 \log _{10}$ of C. auris DSM 21,092, already after 30 s (Table 1B).

Figure:
\begin{tabular}{|c|c|c|c|}
\hline Table 1a. 74\% ethanol ABHS activity on SARS-CoV-2 \\
\hline European Standard Guidelines & Strain & Contact Time & $\begin{array}{c}\log _{10} \text { TCID50 reduction } \\
\text { mean } \pm \text { SD) }\end{array}$ \\
\hline \multirow{2}{*}{ EN14476:2013+A2:2019 } & $\begin{array}{c}\text { SARS-CoV-2 } \\
\text { USAWA1/2020 }\end{array}$ & 30 seconds & $\geq 4.11 \pm 0.12$ \\
\cline { 2 - 4 } & 60 seconds & $\geq 4.11 \pm 0.12$ \\
\hline Table 1b. 74\% ethanol ABHS activity on C. auris & \multicolumn{1}{|l|}{} \\
\hline European Standard Guidelines & Strain & Contact Time & $\log _{10}$ reduction \\
\hline \multirow{2}{*}{ EN 136242013} & Candida auris & 30 seconds & $>4.53$ \\
\cline { 3 - 4 } & DSM 21092 & 60 seconds & $>4.53$ \\
\hline
\end{tabular}

Conclusion: Obtained results on the activity of a $74 \%$ ethanol ABHS (Amuchina gel X-GERM) against SARS-CoV-2 and C. auris support the effectiveness of this alcohol-based formulation as a prevention measure for COVID-19 illness and in healthcare C. auris-associated infections.

Disclosure of Interest: A. Capezzone De Joannon Employee of: Angelini Pharma S.p.A., A. Testa Employee of: Angelini Pharma S.p.A., N. Falsetto Employee of: Angelini Pharma S.p.A., M. Procaccini Employee of: Angelini Pharma S.p.A., L. Ragni Employee of: Angelini Pharma S.p.A.

P212

The impact of H1N1 and COVID-19 on the consumption of alcohol-based handrub at the university of Geneva hospitals, Switzerland

N. Lotfinejad ${ }^{1, *}$, A. Iten ${ }^{1}$, D. Pittet ${ }^{1}$

${ }^{1}$ Infection Control Programme, University of Geneva Hospitals and Fac-

ulty of Medicine, Geneva, Switzerland

Correspondence: N. Lotfinejad

Antimicrobial Resistance \& Infection Control 10(1): P212 
Introduction: Hand hygiene with alcohol-based handrub (ABHR) is the standard of care against healthcare-associated infections, which represents a major adverse event when providing care at hospitals. Continuous monitoring of hand hygiene compliance is one of the main components of the WHO multimodal hand hygiene improvement strategy. Measuring the consumption of ABHRs is considered as a surrogate marker of hand hygiene compliance.

Objectives: To study the impact of the most recent major pandemics, H1N1 and coronavirus disease 2019 (COVID-19), on ABHR consumption at University of Geneva Hospitals (HUG).

Methods: We analyzed the ABHR consumption amounts at HUG from 1994 to 2020. The hospital pharmacy gathers data regarding the volume of $A B H R$ consumption as L/1000 patient-days, first started in 1994. The data provided from the pharmacy refers to the hospital as a whole.

Results: We observed two peaks in the consumption of ABHR at HUG, in 2009 and 2020. The consumption of ABHR increased from $35.38 \mathrm{~L} / 1000$ patient-days in 2008 to $50.33 \mathrm{~L} / 1000$ patient-days in 2009, when H1N1 pandemic emerged. The rise in using ABHRs was even more significant during the COVID-19 pandemic, which increased from $41.75 \mathrm{~L} / 1000$ patient-days in 2019 to $99.33 \mathrm{~L} / 1000$ patient-days in 2020 with the emergence of the current pandemic.

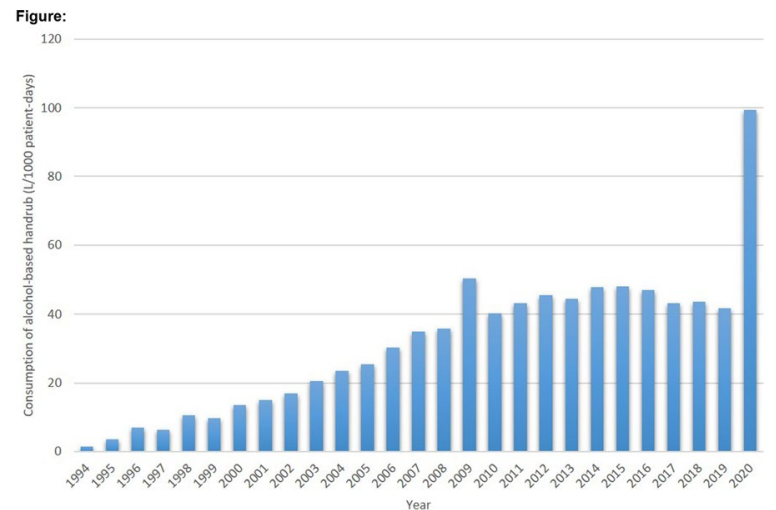

Conclusion: ABHRs are increasingly used at HUG and more than $99 \%$ of hand hygiene actions are currently performed with ABHRs. During major infectious disease outbreaks, the consumption of $A B H R$ increases dramatically, considering its efficacy against cross-transmission of various microorganisms. Therefore, ensuring the global and equitable access to high quality ABHRs and considering local production in times of crisis are necessary in order to prevent lack of supplies, especially during pandemics.

Disclosure of Interest: None declared.

\section{P213}

Assessment of ethanol exposure from alcohol-based hand rub use among healthcare workers: potential for developmental toxicity in nursing offspring

A. Quiñones-Rivera ${ }^{1, *}$, A. Han $^{2}$, A. Buerger ${ }^{2}$, M. Vincent ${ }^{2}$, H. Allen², A. Maier ${ }^{2}$ ${ }^{1} \mathrm{GOJO}$ Industries, Inc., Akron, ${ }^{2}$ Cardno ChemRisk, Cincinnati, United States Correspondence: A. Quiñones-Rivera

Antimicrobial Resistance \& Infection Control 10(1): P213

Introduction: Alcoholic beverage ingestion during pregnancy is associated with adverse effects on fetal development. There is a gap in understanding the developmental toxicity potential in nursing children exposed to ethanol via breastmilk from mothers using ethanolcontaining products, such as alcohol-based hand rubs (ABHRs). A previous study showed that $A B H R$ use by healthcare workers (HCWs) resulted in low internal ethanol doses and thus low developmental toxicity potential.
Objectives: We assessed blood ethanol concentrations (BECs) from $A B H R$ use compared to health related benchmarks for lactational exposures in response to regulatory initiatives in Europe.

Methods: Dose-response relationships between ethanol exposures and developmental effects in nursing offspring were examined. Maternal BECs in animals were determined at the estimated onset dose for offspring neurotoxicity. BECs were predicted for breastfeeding women who consume alcohol per public health guidelines. These BECs were compared to predicted BECs of HCWs following various ABHR uses. Exposures were compared to health benchmarks to identify potential risks for adverse developmental outcomes. Margins were calculated by dividing the BEC from the relevant comparator health benchmark (toxicology or health guideline based) by the BEC from each ABHR use. A margin greater than 1 for health guideline benchmarks and a margin greater than 10 for the toxicology based benchmark are expected to represent low concern.

Results: Epidemiology results showed no clear indication of doseresponse relationships between lactation exposure and developmental effects; but the studies have several limitations. Animal studies reported neurodevelopmental effects in nursing pups at ethanol doses that also caused maternal toxicity. Margins of 2.2-1,000 exist between BECs associated with ABHR use and BECs associated with neurotoxicity or oral intake within public health guidelines.

Conclusion: Estimated BECs from even intensive use of ABHR by HCWs are below those likely to create a health concern for a breastfeeding child.

Disclosure of Interest: A. Quiñones-Rivera Grant/Research support from: GOJO Industries, A. Han Grant/Research support from: GOJO Industries, A. Buerger Grant/Research support from: GOJO Industries, M. Vincent Grant/Research support from: GOJO Industries, H. Allen Grant/Research support from: GOJO Industries, A. Maier Grant/ Research support from: GOJO Industries.

\section{P214}

Assessing the quality of hand hygiene in the community

H. Soule ${ }^{1, *}$, N. Akoum ${ }^{1}$, D. Pires ${ }^{1}$, C. Fankhauser ${ }^{1}$, J. Sauser ${ }^{1}$, D. Pittet ${ }^{1}$

${ }^{1}$ Infection Control Programme and WHO Collaborating Centre on Patient

Safety, Geneva University Hospitals, Geneva 4, Switzerland

Correspondence: $\mathrm{H}$. Soule

Antimicrobial Resistance \& Infection Control 10(1): P214

Introduction: To protect against coronavirus, hand hygiene $(\mathrm{HH})$ of good quality, i.e. covering all the surfaces of the hands with alcohol based handrub (ABHR), is paramount. It was shown that the WHO method is rarely performed correctly by healthcare workers. It is thus likely that $\mathrm{HH}$ performance in the community is even worse.

Objectives: In this descriptive study, we measured the quality of $\mathrm{HH}$ performed by visitors at the entry of an hospital according to different $\mathrm{HH}$ methods.

Methods: Due to the Covid-19 pandemic, visitors passed on a checkpoint at the entry of Geneva Hospitals where they had to sanitize their hands with an ABHR containing a fluorescent component. We performed 2 interventions that consisted in placing posters at the checkpoint explaining 2 simplified HH methods: "Fingertips first" with focus given to the fingertips and "Spray based" with a controlled volume of ABHR sprayed on the hands. We also formed a control group without any instructions. Visitors ignored that their $\mathrm{HH}$ would be assessed. We used the Semmelweis Hand Hygiene Scanner ${ }^{\circledR}$ (SHHS) to evaluate the quality of $\mathrm{HH}$. The investigator approached the visitors after they passed the checkpoint to evaluate their hands. During each period of observations, 30 visitors were enrolled in each of the 3 arms of the study. The primary outcome was the $\%$ of hand surface covered by ABHR as measured by the SHHS. It was good when the result was superior to $95 \%$.

Results: The study lasted from February to April 2021. In the control group, $\mathrm{HH}$ of 405 visitors were assessed; the $\%$ of hand coverage was $80.4 \%$ and $29.6 \%$ of the visitors performed good $\mathrm{HH}$. In the "Fingertips first" group, out of 425 participants, the result was good for $27.5 \%$ and the coverage was $81.2 \%$. And, in the "Spray based" group, $26.9 \%$ 
out of 498 visitors obtained a good result and the coverage was $83.3 \%$. The results were not significantly different between the 3 groups.

Conclusion: The quality of $\mathrm{HH}$ was better than expected. The quality was not different with or without instructions and between the methods with a regular dispenser and a spray dispenser. We actually observed that visitors didn't paid attention to the instructions. Those results may overestimated $\mathrm{HH}$ in the community because our study took place in an hospital and visitors rubbed their hands with $A B H R$ more carefully not to contaminate the patients.

Disclosure of Interest: None declared.

Poster session: hand hygiene: behaviour \& compliance

P215

A systematic review on hand hygiene compliance in hospitals as the most effective measure for patient safety

S. Damianova Radeva ${ }^{1, *}$, A. B. Arredondo Provecho ${ }^{1}$

${ }^{1}$ Hospital Universitario Fundación Alcorcón, Alcorcón, Spain

Correspondence: S. Damianova Radeva

Antimicrobial Resistance \& Infection Control 10(1): P215

Introduction: Hand hygiene $(\mathrm{HH})$ is the most effective, inexpensive and easy-to-implement measure that effectively prevents cross-transmission of pathogens and microorganisms that cause healthcare-associated infections (HAls).

Objectives: A systematic review is written with the purpose to recall that HCWs' hands are the main source by which nosocomial infections can be transmitted to the patients. Moreover, with this review is demonstrated that sanitary hand hygiene $(\mathrm{HH})$ is the main way to protect patients from HAls.

Methods: A literature search and analysis of the literature on sanitary hand hygiene $(\mathrm{HH})$, compliance with the "five-moments for hand hygiene", the implementation of multimodal strategies in hospitals, the implementation of new strategies for monitoring $\mathrm{HH}$ and HCWs compliance with hand hygiene $(\mathrm{HH})$, was carried out.

Results: HAls can increase morbimortality and public health social costs. It is true that there is sufficient evidence which demonstrates that $\mathrm{HH}$ reduces $75 \%$ of HAls cases, but protocols established by the World Health Organization (WHO) are still not correctly followed by healthcare workers (HCWs). Also, another reason why HCWs do not follow WHO's protocols is because they claim that there is lack of scientific information of the definitive impact of improved $\mathrm{HH}$ on nosocomial infections rates.

Conclusion: So, what this systematic review demonstrates is that the correct compliance with hand hygiene can considerably reduce the number of healthcare-associated infections (HAls) and other emerging viruses as SARS-CoV-2, in patients and healthcare workers.

Disclosure of Interest: None declared.

\section{P216}

Overconfidence regarding one's own hand hygiene compliance among physicians and nurses in surgery: results of the WACH-trial's baseline survey in six non-university hospitals in Germany J. Lamping ${ }^{1}$, I. Tomsic ${ }^{1}$, I. Hartlep², P. Schipper ${ }^{2}$, M. Stolz ${ }^{3}$, C. Krauth ${ }^{3}$, I. F. Chaberny $^{2}$, T. von Lengerke ${ }^{1, *}$

${ }^{1}$ Department of Medical Psychology, Hannover Medical School, Centre of Public Health and Healthcare, Hannover, ${ }^{2}$ Institute of Hygiene, Hospital Epidemiology and Environmental Medicine, Leipzig University Hospital, Centre for Infection Medicine, Leipzig, ${ }^{3}$ Institute of Epidemiology, Social Medicine and Health Systems Research, Hannover Medical School, Centre of Public Health and Healthcare, Hannover, Germany

Correspondence: T. von Lengerke

Antimicrobial Resistance \& Infection Control 10(1): P216
Introduction: One barrier to participate in interventions to increase one's own hand hygiene $(\mathrm{HH})$ compliance can be overconfidence in terms of overestimating one's performance [1, 2]. In this context, so far no study has compared self-reports of overall compliance vs. compliance specific to the 5 moments of $\mathrm{HH}$.

Objectives: Given findings from cognitive psychology that items with a higher difficulty (here: overall compliance) tend to be more strongly associated with overconfidence effects [3], we tested empirically whether $\mathrm{HH}$ overestimation is stronger for self-reported overall compliance than for an index calculated based on self-reported momentspecific compliance rates weighted by the 5 moments' occurrence frequencies.

Methods: In the WACH-trial ("SSI and Antibiotics Use in Surgery"; German Clinical Trials Register-ID: DRKS00015502), a self-administered questionnaire survey on SSI-preventive compliance was conducted in 2019 among $\mathrm{N}=93$ physicians (response rate: $28.4 \%$ ) and $\mathrm{N}=225$ nurses (30.4\%) in 9 surgical departments in 6 non-university hospitals in Germany. Self-reported HH compliance was assessed using percentage scales. The 5 moments' occurrence frequencies were estimated based on a systematized review by the 1st author. For t-tests for paired

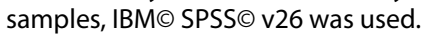

Results: Among physicians, there was a tendency towards greater overestimation in the self-reported than in the calculated overall compliance rate (means: 87.2 vs. $84.6 \%, p=0.072$; nurses: 84.4 vs. $85.9 \%$, $\mathrm{p}=0.153$ ). Compared with $\mathrm{HH}$ compliance rates during ward rounds observed in the study hospitals before the survey (physicians: $59.1 \%$, nurses: $65.9 \%$ ), overestimation was higher among physicians as well $(+9.6 \%$ based on self-reported overall compliance and $+5.5 \%$ based on the calculated rates).

Conclusion: The tendency for $\mathrm{HH}$ overestimation among physicians (not nurses) is consistent with the general finding of lower HH compliance in this professional group, and suggests that overconfidence effects may be relevant causal mechanisms.

Ref.:

1. https://doi.org/10.1016/j.ajic.2018.10.022

2. https://doi.org/10.1017/ice.2019.156

3. https://doi.org/10.1007/s11409-019-09198-4

Disclosure of Interest: None declared.

P217

The hawthorne effect (HE) in hand hygiene (HH)

in a subpopulation of volunteer healthcare professionals (HCPS) followed by direct observation (DO) during 6 months

C. Fankhauser ${ }^{1, *}$, J. Sauser ${ }^{1}$, F. Timurkaynak ${ }^{1}$, A. Peters ${ }^{1}$, D. Pires ${ }^{1}$, D. Pittet ${ }^{1}$

${ }^{1}$ Infection Control Program, Geneva university hospital, Geneva, Switzerland

Correspondence: $C$. Fankhauser

Antimicrobial Resistance \& Infection Control 10(1): P217

Introduction: The HE is when individuals change behaviour due to their awareness of being observed. It is considered the main bias of DO, increasing $\mathrm{HH}$ compliance by $30 \%-50 \%$. In a clinical trial, volunteer HCPs wore an electronic device delivering feedback on duration of handrubbing and customized AHBR volume for their hands. The device provoked no improvement on $\mathrm{HH}$ compliance. A gradual decline of $\mathrm{HH}$ compliance was observed, evoking habituation of the HCPs to the observer, or a regression of the HE.

Objectives: To assess the impact of habituation to HH-DO on HCPs and to compare $\mathrm{HH}$ compliance differences between consecutive observations.

Methods: From June 1, 2017 to January 6, 2018, in a 300 bed-Geriatrics hospital in Geneva, Switzerland, 89 voluntary HCPs participated in a cluster randomized, stepped-wedge clinical trial described 
elsewhere*. $\mathrm{HH}$ observers were trained and validated according to the WHO method. Each HCP was observed at least once a month for a minimum of 6 opportunities. To model $\mathrm{HH}$ compliance, a linear mixedeffects model was used with the intervention, observation number and observation time as fixed effects. A subject-specific random intercept was considered. Observation numbers 10 to 12 were excluded from the analysis due to small sample size. Pairwise comparisons were performed between observation numbers to estimate their effect on HH compliance.

Results: Table 1 summarizes $\mathrm{HH}$ compliance according to the observation order and the number of HCPs included. Table 2 presents a Model-based estimated effects between observation numbers. Estimate shows the difference in compliance between observation numbers. There is a significant decrease in $\mathrm{HH}$ compliance as from the $4^{\text {th }}$ observation, $4.1 \%$ up to the $9^{\text {th }}, 19.4 \%$.

Figure:
Table1. Summary statistics for compliance by observation number
\begin{tabular}{|c|c|c|c|c|}
\hline $\begin{array}{c}\text { Observation } \\
\text { number }\end{array}$ & $\mathrm{n}$ & HH compliance (\%) & Lower Cl$^{*}$ & Upper Cl \\
\hline 1 & 89 & 74.4 & 70.4 & 78.4 \\
\hline 2 & 88 & 71.1 & 66.5 & 75.7 \\
\hline 3 & 86 & 71.1 & 66.3 & 76.0 \\
\hline 4 & 83 & 70.8 & 66.1 & 75.5 \\
\hline 5 & 83 & 65.1 & 60.5 & 69.7 \\
\hline 6 & 81 & 61.3 & 56.5 & 66.2 \\
\hline 7 & 70 & 63 & 57.9 & 68.1 \\
\hline 8 & 52 & 64.6 & 59.9 & 69.3 \\
\hline 9 & 31 & 59 & 50.9 & 67.0 \\
\hline 10 & 11 & 56 & 42.1 & 69.8 \\
\hline 11 & 5 & 48.3 & 27.3 & 69.4 \\
\hline 12 & 3 & 55.2 & 42.4 & 68.1 \\
\hline
\end{tabular}

Table 2. Normal model-based estimated effects between observation numbers

\begin{tabular}{|c|c|c|c|c|}
\hline Effect & Estimate (\%) & Lower Cl $^{*}$ & Upper Cl $^{\boldsymbol{*}}$ & p-value \\
\hline Obs1 & & & & \\
\hline Obs2-Obs1 & -4.7 & -10.2 & 0.9 & 0.09776 \\
\hline Obs3-Obs1 & -5.3 & -11.2 & 0.5 & 0.07399 \\
\hline Obs4-Obs1 & -6.0 & -12.1 & 0.2 & 0.05688 \\
\hline Obs5-Obs1 & -12.2 & -19 & -5.5 & 0.00035 \\
\hline Obs6-Obs1 & -17.1 & -24.5 & -9.6 & 0.00001 \\
\hline Obs7-Obs1 & -15.6 & -23.5 & -7.6 & 0.00012 \\
\hline Obs8-Obs1 & -14.5 & -23.1 & -6.0 & 0.00088 \\
\hline Obs9-Obs1 & -19.4 & -29.1 & -9.8 & 0.00008 \\
\hline
\end{tabular}

Conclusion: The HE seems to decrease with habituation of HCP of being observed. The reduction on $\mathrm{HH}$ compliance after repetitive observations ranges from $4.1-19.4 \%$.

Disclosure of Interest: None declared.
P218

A cluster randomised trial in residential homes testing a nudging intervention to improve hand hygiene compliance among health workers in Northern Italy

B. Braut ${ }^{1, *}$, T. Medda ${ }^{1}$, D. Pennesi ${ }^{1}$, G. Gon ${ }^{2}$

${ }^{1}$ University of Turin, Torino, Italy, ${ }^{2}$ London School of Hygiene and Tropical Medicine, London, United Kingdom

Correspondence: B. Braut

Antimicrobial Resistance \& Infection Control 10(1): P218

Introduction: SARS-CoV-2 pandemic has aroused attention to infectious diseases' transmission in long term facilities. While other measures adopted to fight transmission in these structures are temporary, hand hygiene is here to stay.

Objectives: We develop a nudge strategy aimed to increase hand hygiene, especially through the use of hydro-alcoholic gel. We focus on health workers hand hygiene compliance during the morning washing routine. The intervention is based on three main drivers of poor hand hygiene identified during formative research: accessibility of hand hygiene material, knowledge and instrumental beliefs. The intervention is cost-effective and scalable. We provided health workers with a handrub bottle placed in a portable basket. Health workers are trained to bring the bottle near the patient zone. We introduce a delimitation mark indicating the patient zone. The interventions were preceded by a briefing and accompanied by iconographic representations which explained the interventions. The intervention is conducted in three residential homes in Northern Italy.

Methods: The study is a cluster randomised control trial where each health worker pair (they work in pairs) is randomised to receiving the handrub bottle or not. In addition, each guest room is randomised to receive the demarcation line or not. The primary outcome is hand hygiene compliance across the four moments prescribed by the WHO for residential homes. The outcome will be measured over three weeks after the intervention. The study design is expected to have $80 \%$ power to demonstrate superiority of the intervention (compared to the control: pairs without handrub and rooms without demarcation) at a $5 \%$ margin with 30 observations per health worker pair/room, and 20 pairs/rooms, an expected effect size of 1.3 and a baseline of hand hygiene compliance ranging from 30 to $50 \%$.

Results: The trial is under way and data completion is planned for July 2021.

Conclusion: Effective hand hygiene intervention studies in residential homes are lacking, the present epidemic has emphasized this void. This study could provide evidence of successful strategies to be implemented in this context to improve hand hygiene and hence prevent healthcare associated infections.

Disclosure of Interest: None declared. 
P221

Assessing sustainability of hand hygiene compliance 33 months after change agents-based intervention

Y. F. Lee ${ }^{1, *}$, W. Zingg ${ }^{2}$, H. U. Ngian ${ }^{1}$, Z. H. Chin ${ }^{3}$, M.-L. McLaws ${ }^{4}$, S. Amir Husin $^{5}$, H. H. Chua ${ }^{1}$, W. H. Lai ${ }^{6}$, J. Wang ${ }^{7}$, D. Pittet ${ }^{8}$

${ }^{1}$ Sarawak General Hospital, Kuching, ${ }^{2}$ Department of Infectious Diseases and Hospital Epidemiology, University Hospital Zurich, Zurich, ${ }^{3}$ Sarawak State Health Department, Kuching, Malaysia, ${ }^{4}$ University of New South Wales, Sydney, Australia, ${ }^{5}$ Infection Prevention \& Control Unit, $\mathrm{MoH}$, Putrajaya, ${ }^{6}$ Clinical Research Centre, Kuching, Malaysia, ${ }^{7}$ Otto von Guericke University, Magdeburg, Germany, ${ }^{8}$ Infection Control Programme and WHO Collaborating Centre on Patient Safety, University of Geneva, Geneva, Switzerland

Correspondence: Y. F. Lee

Antimicrobial Resistance \& Infection Control 10(1): P221

Introduction: Hand hygiene is an effective, low-cost practice to reduce health care-associated infections. Given that hand hygiene is a behavior-driven action, innovative improvement strategies should be promoted using suitable change agents for sustained compliance.

Objectives: Our study aimed to evaluate the 33 months sustainability of hand hygiene compliance between wards exposed to change agents with different leadership style.

Methods: We conducted a randomized-controlled study using the World Health Organization hand hygiene gold standard auditing method in two acute wards of a public tertiary care hospital in Malaysia. The intervention was hand hygiene promotion delivered by peer-identified change agents (PICAs) in study arm 1 or by Management-Selected change agents (MSCAs) in study arm 2. Primary outcome was hand hygiene compliance rate in four periods; preintervention, intervention, 8-weeks post-intervention, and 33-months post-intervention.

Results: Mean hand hygiene compliance rate improved significantly by 10 percentage points (PPs) from 48 to 58\%, and 14 PPs from 50 to $64 \%$, between the pre- to the 8 -weeks post- intervention period $(P<0.001)$ for study arms 1 and 2, respectively. Mean Hand hygiene compliance rate improved significantly by 15 PPs from 48 to $64 \%$, and $21 \mathrm{PPs}$ from 50 to $70 \%$ between the pre- and 33-months post-intervention period $(P<0.001)$ for study arms 1 and 2 , respectively. There was no statistically significant difference in hand hygiene improvement 33 months post-intervention between the two study arms $(P=0.25)$.

Conclusion: Both PICAs and MSCAs-led interventions achieved significant sustained improvement in hand hygiene compliance 33-months after the original intervention.

Disclosure of Interest: None declared.

\section{P222}

\section{Compliance with hand hygiene $(\mathrm{HH})$ practices under standard} precautions (SP) in non COVID-19 wards in a tertiary care hospital C. Fankhauser ${ }^{1,}$, F. Timurkaynak ${ }^{1}$, E. Sallansonnet ${ }^{1}$, I. Grangier ${ }^{1}$, V. Belpaire ${ }^{1}$, L. Baltazar ${ }^{1}$, A. Dumps ${ }^{1}$, A. Peters ${ }^{1}$, V. Camus ${ }^{1}$, A. Iten ${ }^{1}$, D. Pittet ${ }^{1,2}$

${ }^{1}$ Infection Control Program, ${ }^{2}$ Faculty of Medicine, Geneva University

Hospitals, Geneva, Switzerland

Correspondence: C. Fankhauser

Antimicrobial Resistance \& Infection Control 10(1): P222

Introduction: Since the beginning of the COVID-19 pandemic, HUG took care of a large number of patients suspected of having or affected by COVID; the majority of HUG wards became temporarily COVID wards. When the situation returned to normal, the practice of SP needed to be strengthened. Among SP, $\mathrm{HH}$ is the most important. Monitoring $\mathrm{HH}$ by direct observation (DO) and performance feedback are essential to improve and sustain compliance. Glove use remains a challenge for $\mathrm{HH}$ compliance, particularly before donning or after doffing. Immediate individual feedback (IIF) is very useful for the understanding of $\mathrm{HH}$ by HCWs during patient care.

Objectives: To evaluate $\mathrm{HH}$ compliance improvement when immediate or delayed feedback is provided and to assess the impact of glove use on compliance.

Methods: The study was conducted at HUG from 1 January to 30 June 2021. Trained, validated and interrater reliability-tested observers monitored $\mathrm{HH}$ using the WHO DO method, for a minimum of 30 opportunities/ward/month. Feedback were applied using a series of approaches: immediate oral individual feedback (IIF) to HCW, a monthly delayed feedback by the IPC nurse through a poster figuring the average ward compliance observed and goal setting for the next month. A written report of $\mathrm{HH}$ compliance including the rate of missed actions while the $\mathrm{HCW}$ was wearing gloves was also provided. Final results for the total study period with statistical evaluation will be provided at ICPIC.

Results: Between 1 Jan and 30 April 2021, a total of 10,332 opportunities were observed. Table 1 shows preliminary results of overall monthly $\mathrm{HH}$ compliance, levels of IIF and frequencies of missed $\mathrm{HH}$ actions in the presence of gloves.

\begin{tabular}{|c|c|c|c|c|c|c|c|}
\hline \multicolumn{8}{|c|}{ Table1. Non COVID-19 wards } \\
\hline Months & Opportunities & \begin{tabular}{|c|}
$\mathrm{HH}$ \\
actions
\end{tabular} & Compliance (\%) & $111 F^{*}(\%)$ & $\begin{array}{c}\text { Missed HH } \\
\text { actions }\end{array}$ & $\begin{array}{l}\text { Missed HH actions } \\
\text { with Gloves }\end{array}$ & $\begin{array}{l}\text { Missed HH actions with } \\
\text { Gloves }(\%)\end{array}$ \\
\hline January & 2513 & 1741 & $69.28(\mathrm{Cl} \pm 14.1)$ & 41.03 & 772 & 176 & $22.80(\mathrm{Cl} \pm 5.47)$ \\
\hline February & 2636 & 1729 & $65.59(\mathrm{C} \pm 14.8)$ & 31.26 & 907 & 161 & $17.75(\mathrm{Cl} \pm 4.19)$ \\
\hline March & 2748 & 2020 & 74.31 (C+19.9) & 52.50 & 698 & 98 & $14.04(\mathrm{Cl} \pm 3.81)$ \\
\hline April & 2435 & 1832 & $75.24(\mathrm{Cl} \pm 13.5)$ & 60.16 & 603 & 59 & $9.78(\mathrm{Cl} \pm 3.76)$ \\
\hline
\end{tabular}

Conclusion: The intervention was associated with improvement in $\mathrm{HH}$ compliance. Glove use was associated with a high number of missed $\mathrm{HH}$ actions. Reduction of glove use together with individual feedback were associated with a decreased frequency of missed actions.

Disclosure of Interest: None declared.

P223

Hand hygiene behaviors in the intensive care unit

J. Huang ${ }^{1, *}$, Q. Xu ${ }^{1}$, M. Kong ${ }^{1}$, A. Jerde ${ }^{1}$, D. Cepulis ${ }^{2}$, Y. Liu ${ }^{2}$, P. Liu ${ }^{2}$

${ }^{1}$ University of Louisville, Louisville, ${ }^{2}$ Microsensor Labs, Chicago, United

States

Correspondence: J. Huang

Antimicrobial Resistance \& Infection Control 10(1): P223

Introduction: Hand hygiene $(\mathrm{HH})$ compliance is hard to improve and underlying behaviors are not clearly understood among healthcare workers.

Objectives: We aim to use an electronic $\mathrm{HH}$ system to study the $\mathrm{HH}$ compliance and patient contact time among ICU providers by day of the way, hour of the day, and dispenser location.

Methods: An automated electronic hand hygiene system was installed in a 10-bed surgical intensive care unit. $\mathrm{HH}$ opportunities and compliance rates were captured automatically by the system with time and location stamps at individual levels.

Results: For different day of the week, $\mathrm{HH}$ compliance rate decreased from $31.4 \%$ on Mondays to $27.2 \%$ on Sundays with Wednesdays being the highest (35.2\%), and there was no significant time effect $(P=0.077)$. For different hour of the day, the $\mathrm{HH}$ compliance rate decreased from $36.8 \%$ in $7 \mathrm{AM}-8 \mathrm{PM}$ to $20.4 \%$ in 7PM-8PM with 7AM-8PM being the highest, and there was no significant time effect $(P=0.224)$. For alcohol solution dispenser locations, the $\mathrm{HH}$ compliance rates of going out of the room events was significantly higher than those of coming into the room events $(37.3 \%$ vs $26.1 \%$, P-value $<0.001)$. For patient contact time, the hourly average patient contact time per provider per day decreased from 872 s on Mondays to 709 s on Sundays, with Mondays being the longest, and no significant time effect $(P=0.399)$. The hourly average patient contact time per provider per hour had 8AM-9AM being the highest (1284 s) and 5PM-6PM being another peak (901 s), and there was no significant time effect $(P=0.125)$. 


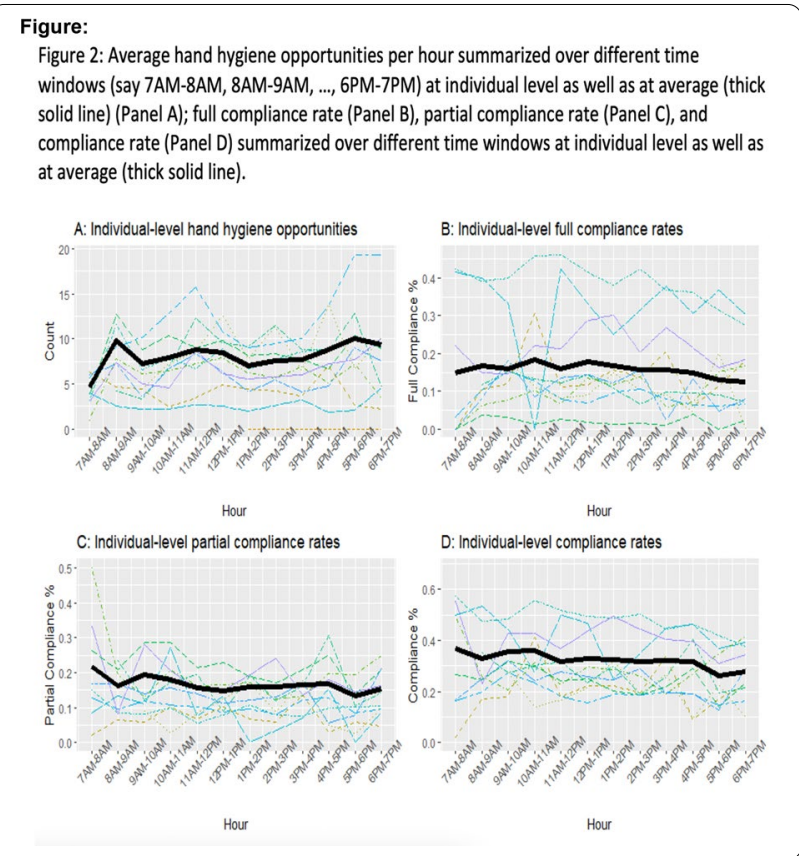

Conclusion: Hand hygiene behaviors are complex and provider dependent. $\mathrm{HH}$ compliance and patient contact time vary by day of the week, hour of the day, and dispenser locations.

Disclosure of Interest: J. Huang Shareholder of: Microsensor Labs, Q. Xu Grant/Research support from: Microsensor Labs, M. Kong Grant/ Research support from: Microsensor Labs, A. Jerde Grant/Research support from: Microsensor Labs, D. Cepulis Employee of: Microsensor Labs, Y. Liu Shareholder of: Microsensor Labs, P. Liu Shareholder of: Microsensor Labs.

\section{P224}

Hand hygiene practices assessment among health care workers at an university hospital in Tunisia during the COVID-19 pandemic: an observation audit

N. Omri ${ }^{1,2,3, *}$, I. Mlouki ${ }^{1,2,3}$, F. Ben youssef ${ }^{1,2,3}$, H. Sfar ${ }^{1}$, S. El mhamdi ${ }^{1,2,3}$

${ }^{1}$ Preventive and Community Medicine, University Hospital Tahar Sfar

Mahdia, Mahdia, ${ }^{2}$ Community Medicine, Faculty of Medicine of Monastir, ${ }^{3}$ Research laboratory, Epidemiology Applied to Maternal and Child Health, Monastir, Tunisia

Correspondence: N. Omri

Antimicrobial Resistance \& Infection Control 10(1): P224

Introduction: The ongoing Covid-19 pandemic provides the opportunity to demonstrate the potential benefit of the good hand hygiene practices (HHP) in controlling health care-associated transmission of Covid-19.

Objectives: The present study aims at evaluating hand hygiene practices of health care workers (HCWs) during the first wave of Covid-19 pandemic at the university hospital Tahar Sfar Mahdia (Tunisia).

Methods: We carried out an observation audit among HCWs staff at the university hospital Tahar Sfar Mahdia in Tunisia during the first wave of Covid-19 pandemic (April-May 2020) using four pretested and reliable audit sheets. The first one concerned the simple washing hand, the second and third sheet were used to evaluate respectively the hygienic washing and the hydro alcoholic friction and the fourth sheet to assess the compliance and conformity rates. We excluded the outpatients departments.
Results: A total of 214 observations were enrolled of which $143 \mathrm{HCWs}$ were observed. Approximately a quarter $24.6 \%$ were medical professionals and $53.2 \%$ were nursing personnel with a female predominance (sex-ratio $=0.33$ ). Nearly two thirds of the observations $61.5 \%$ concerned high-risk departments and $56.6 \%$ were collected during the morning shift. The Half of HCWs observed $50.3 \%$ met the requirements of efficacy for hand hygiene. The majority $91.3 \%$ did not respect the technique of simple washing hand against $75 \%$ the technique of hydro alcoholic friction. The hand hygiene compliance rate before observation was $19 \%$ compared to $20.3 \%$ after. The overall conformity rate was $6.5 \%$.

Being male $(p<0.001)$, working at a high risk department $(p<0.01)$ and working during the morning shift $(p=0.003)$ were found to have significant relationship with good HHP.

Conclusion: HCWs must modify their behaviors to face the risk propensity of the Covid-19 pandemic. However, to improve the poor compliance a culture of rigorous HHP should be established.

Disclosure of Interest: None declared.

\section{P225}

Hand hygiene adherence at a university hospital: a comparative study between the first and the second COVID-19 waves in Tunisia I. Mlouki ${ }^{1,2,3, *}$, N. Omri ${ }^{1,3}$, F. Ben youssef $1^{1,2,3}$, H. Sfar $^{4}$, F. Chebbi ${ }^{1}$, N. Rezg ${ }^{1}$, A. Khouildi', A. Hadj Sassi ${ }^{1}$, S. El Mhamdi ${ }^{1,2,3}$

${ }^{1}$ Departement of Preventive and Community Medicine, University Hospital Taher Sfar, Mahdia, ${ }^{2}$ Department of Community Medicine, ${ }^{3}$ Research laboratory "Epidemiology Applied to Maternal and Child Health" 12SP17, Faculty of Medicine of Monastir, Monastir, ${ }^{4}$ Departement of Preventive and Community Medicine, Hopital Taher Sfar Mahdia, Mahdia, Tunisia Correspondence: I. Mlouki

Antimicrobial Resistance \& Infection Control 10(1): P225

Introduction: Hand hygiene is the most effective measure in reducing healthcare-associated infections. Accordingly, it has been recommended as an important strategy to prevent the spread of COVID-19 in health care settings.

Objectives: We aimed to measure the adhesion and the relevance of hand washing during the first and the second COVID-19 waves at the University Hospital Taher Sfar Mahdia (Tunisia).

Methods: We performed a prospective study recruiting a cohort of randomly selected health care professionals at the University Hospital Taher Sfar Mahdia. Direct hand hygiene observations were conducted on July 2020 (during the first wave of COVID-19 in Tunisia) and January 2021 (during the second wave), then evaluated using the hand hygiene audit developed by WHO.

Results: We performed 378 observations of hand hygiene (214 on July 2020 and 166 on January 2021) among 286 health workers with a female predominance ( $74.8 \%$ and $66.4 \%$, respectively). About $26.6 \%$ and $37.1 \%$ of the sample were medical professionals during the first and the second waves respectively. The rate of health workers in Intensive Care Units (ICU) ranged from $38.5 \%$ to $36.4 \%$ between the two periods.

From July 2020 to January 2021, the global relevance rate significantly increased from $6.5 \%$ to $21.3 \%(p<0.001)$. Hand hygiene adherence 'before patient contact' improved from $19 \%$ to $31.4 \%$, and 'after patient contact' from $20.3 \%$ to $31.9 \%$. The correct execution of hand hygiene technique increased from $8.7 \%$ to $50 \%(p<0.001)$.

The rates of hand hygiene relevance were higher in males $(p=0.003)$, in ICU $(p<0.001)$ and the morning shift period $(p=0.01)$.

Conclusion: Although the COVID-19 improved hand hygiene adhesion rates, relevance remains challenging. It is important to continue the promotion of hand washing with the same enthusiasm and commitment even after this outbreak.

Disclosure of Interest: None declared. 


\section{P226}

The impact of the COVID-19 prevention and control program on hand hygiene compliance at Sahloul university hospital Tunisia S. Bhiri ${ }^{1,2}$, W. Dhouib ${ }^{1}$, A. Ben Cheikh ${ }^{1,2}$, H. Ghali ${ }^{1,2,}{ }^{*}$, R. Bannour ${ }^{1}$, A. Gara ${ }^{1}$, S. Khefacha ${ }^{1}$, H. Said Latiri, ${ }^{1,2}$ M. Ben Rejeb ${ }^{1,2}$

${ }^{1}$ Department of Prevention and Security of Care, sahloul university hospital, ${ }^{2}$ Faculty of Medicine of Sousse „, University of Sousse, Sousse, Tunisia Correspondence: $H$. Ghali

Antimicrobial Resistance \& Infection Control 10(1): P226

Introduction:

Background

Hand Hygiene $(\mathrm{HH})$ has been recommended as an important strategy to help prevent the spread of COVID-19 in hospitals. Monitoring $\mathrm{HH}$ compliance is considered as a critical aspect of an effective $\mathrm{HH}$ program.

The aim of our study was to assess the impact of the COVID-19 pandemic through the direct observation on $\mathrm{HH}$ compliance of health care workers(HCW) in hospitals.

Objectives: The aim of this study was to assess the impact of the COVID-19 prevention and control program on $\mathrm{HH}$ compliance witin health care workers(HCW) at Sahloul University Hospital 2019-2020.

Methods: We conducted a pre-experimental study based on a onegroup pretest-posttest among HCWs at Sahloul University Hospital. The intervention consisted in COVID-19 prevention and control program conducted by the prevention and security of care department As part of this programme, we organised theoretical and practical workshops on standard precautions (mainly $\mathrm{HH}$ ).

All categories of HCW (medical and paramedical staff) working in the various departments were included. The evaluation tool was a WHO observation form provided among the tools of the multimodal strategy.

Results: Prior to pandemic (2019),1515 opportunities for $\mathrm{HH}$ were observed and 996 ones in 2020. Overall compliance among all HCW was better during the pandemic than before the pandemic (38.3\% vs $49.3 \%$.; $<<0.001$ ). Hand washing remained more frequently practiced than hand rubbing. However, the latter has doubled in percentage after the intervention in 2020 (from7.3 to 14.2\%; $p<0.001$ ). The paramedical stuff were the most observant to the $\mathrm{HH}$ indications during the 2 years. In 2019, the intensive care units were the most compliant $(50.5 \%$ vs $54.1 \% ; P<0.001)$ while,in 2020 , the medical services were the most compliant (38.4 vs $56.5 \% ; \mathrm{P}<0.001$ ).During the 2 -year period, the most respected indication to $\mathrm{HH}$ was "after touching the patient"(43.6 in 2019 vs $57.0 \%$ in $2020 ; P<0.001$ ).

Conclusion: COVID-19 prevention and control program seems to have a positive impact on the $\mathrm{HH}$ compliance. Obtained Data are useful to provide $\mathrm{HCW}$ with feedback, to guide further intervention aiming at sustainably $\mathrm{HH}$ compliance improvement.

Disclosure of Interest: None declared.

\section{P228}

"Investigating the determinants of hand hygiene compliance among birth attendants in Kenya and Malawi using the service provision assessments (SPA)"

L. Dansero ${ }^{1, *}$, G. Gon ${ }^{1}$

${ }^{1}$ Infectious Disease Epidemiology, London School of Hygiene and Tropical Medicine, London, United Kingdom

Correspondence: L. Dansero

Antimicrobial Resistance \& Infection Control 10(1): P228

Introduction: Hand hygiene $(\mathrm{HH})$ is an essential element of Infection Prevention and Control (IPC). Birth attendants' $\mathrm{HH}$ is pivotal to preventing new-born and maternal infection. Compliance with $\mathrm{HH}$ and its determinants are underinvestigated in Low and Middle-Income Countries. We analysed the Kenya and Malawi Service Provision Assessments (SPA, 2010 - 2013), which are nationwide surveys that provides an extensive overview of a country's health service.

Objectives: The main objective was to analyse $\mathrm{HH}$ compliance and its determinants during delivery in Kenya and Malawi using the SPA dataset.
Methods: We analysed 785 observed deliveries (407 in Kenya and 378 in Malawi) across 392 facilities. The outcomes were hand washing/ rubbing 1)before any initial examination, 2)before labour, and 3)after birth. We used descriptive statistics to assess outcome levels and multivariate multilevel logistic regressions to investigate several determinants. All analyses accounted for survey characteristics.

Results: $\mathrm{HH}$ compliance in Kenya and Malawi was respectively $37.2 \%$ (Cl:32.2-42.5) and 60.8\% (Cl:65.0-74.3) before any initial examination; 34.7\% (Cl:29.8-39.9) and 63.7\% (Cl:58.8-68.4) before labour, 80.7\% $(\mathrm{Cl}: 75.6-84.9)$ and $83.1 \%(\mathrm{Cl}: 78.9-86.5)$ after birth. After accounting for potential confounders, greeting the woman $\left(\mathrm{OR}_{1}=10.65, \mathrm{Cl}: 2.71-\right.$ 41.8, p-value: 0.001; $\mathrm{OR}_{2}=14.12, \mathrm{Cl}: 3.08-64.73$; $\mathrm{p}$-value: 0.001$)$, birth attendants' years of delivery experience $\left(\mathrm{OR}_{1}=1.14, \mathrm{Cl}: 1.04-1.24, \mathrm{p}\right.$ value: $0.004 ; \mathrm{OR}_{2}=1.16, \mathrm{Cl}: 1.07-1.27, \mathrm{p}$-value:0.001) and managing authority of the facilities $\left(\mathrm{OR}_{1}=4.21, \mathrm{Cl}: 1.02-17.27, \mathrm{p}\right.$-value: 0.046$)$ were found to be associated with $\mathrm{HH}$ before initial examination and labour in Kenya. In Malawi greeting the woman $\left(\mathrm{OR}_{1}=4.05, \mathrm{Cl}: 0.82-\right.$ 19.93, p-value: $0.085 ; \mathrm{OR}_{2}=12.5, \mathrm{Cl}: 1.4-112.2$, $\mathrm{p}$-value:0.024) and the presence of any danger signs for the woman $\left(\mathrm{OR}_{2}=2.26, \mathrm{Cl}: 1.15-4.47\right.$, $\mathrm{p}$-value:0.018; $\mathrm{OR}_{3}=3.04 \mathrm{Cl}: 0.96-9.5, \mathrm{p}$-value:0.057) were associated with $\mathrm{HH}$ before labour and after birth. Other variables (e.g. availability of hand-disifectant) were not associated with $\mathrm{HH}$ in this dataset.

Conclusion: Hand hygiene compliance was low in both countries, but substantially lower in Kenya. Greeting the woman, years of delivery experience, presence of danger signs were important determinants of $\mathrm{HH}$ compliance in Kenya and Malawi.

Disclosure of Interest: None declared.

\section{P229}

Understanding hand hygiene behavior in a public hospital in Benin using the theoretical domain frameworks C. Yehouenou ${ }^{1,2, *}, A$. ABEDINZADEH ${ }^{3}$, R. HOUNGNIHIN ${ }^{4}$, F. DOSSOU ${ }^{5}, A$ Simon ${ }^{6}$, O. DALLEUR ${ }^{7}$

${ }^{1}$ Université catholique de Louvain/Louvain drug research institute, Brussels, Belgium, ${ }^{2}$ Reference laboratory of Mycobacteria, Cotonou, Benin,

${ }^{3}$ Pharmacy, Clinique universitaire Saint Luc;Université catholique de Louvain, Brussels, Belgium, ${ }^{4}$ Laboratoire d'Anthropologie Médicale Appliquée (LAMA), Université d'Abomey-Calavi, ${ }^{5}$ Department of surgery and surgical specialties, Faculty of Health sciences,Campus universitaire Champs de Foire, PO Box; 01BP118, Cotonou, Benin, ${ }^{6}$ centres hospitaliers joliment, Prevention et contrôle des infections, Groupe Jolimont sable, Rue Ferrer, 159-7100 Haine-Saint-Paul., ${ }^{7}$ Pharmacy, clinique universitaire Saint-Luc, Clinical Pharmacy Research group (CLIP), Louvain Drug Research Institute (LDRI), Université catholique de Louvain, Brussels, Belgium

Correspondence: $C$. Yehouenou

Antimicrobial Resistance \& Infection Control 10(1): P229

Introduction: Hand hygiene $(\mathrm{HH})$ is widely recognized to be one of the most successful and cost-effective measures for reducing the incidence of healthcare-associated infections (HAls). The compliance of healthcare workers (HCWs)with best practice has nonetheless been sub-optimal.

Objectives: To use the Theoretical Domain Frameworks (TDF) to identify the behavioral determinants that may impact HCW'shand-hygiene compliance in a public hospital in Benin.

Methods: A qualitative design comprising face-to-face semi-structured interviews with eight sampled HCWs. the interviews included questions on transmission of infections, hand-hygiene practices, problems with their implementation, and way to improve adherence with $\mathrm{HH}$ recommendations. Interviews were coded into 14 behavioral domains using the TDF and were subdivided into themes. Interviews transcrips were analyzed using thematic content analysis involving a systematic 3-step approach:coding, generationof specific beliefs, and identification of relevant domains.

Results: One of the barriers to good $\mathrm{HH}$ adherence most cited in our study was environmental context and resources (such as empty hand-gel dispensers). the interviewees believed that role models had a significant impact on the good practices of others HCWs. Participants were confident of their capabilities to perform appropriate 
$\mathrm{HH}$ behaviors. almost all of them (7/8) reported having the necessary knowledge and skills and believed they could carry out appropriate $\mathrm{HH}$ behavior.

Conclusion: The theoretical Domains Framework provides a useful tool for understanding $\mathrm{HH}$ behavior. But while knowledge is necessary for behavior change, it is not sufficient. This study identified several key behavioral constructs aligned with the TDF that can be targeted when developing new $\mathrm{HH}$ interventions. These may increase the $\mathrm{HH}$ compliance and patient safety.

Disclosure of Interest: None declared.

Poster session: hand hygiene: communication and promotion across the globe

\section{P230}

The effectiveness of hand hygiene interventions for preventing transmission or acquisition of viral infections in the community: findings from a systematic review

L. Gozdzielewska ${ }^{1, *}$, C. Kilpatrick ${ }^{2}$, J. Reilly', S. Stewart ${ }^{1}$, J. Butcher ${ }^{1}$, A. Kalule $^{1}$, O. Cumming ${ }^{3}$, J. Watson ${ }^{3}$, L. Price ${ }^{1}$

${ }^{1}$ Research Centre for Health, Glasgow Caledonian University, ${ }^{2}$ KSHealthcare Consulting, Glasgow, ${ }^{3}$ Department of Disease Control, London School of Hygiene and Tropical Medicine, London, United Kingdom Correspondence: L. Gozdzielewska

Antimicrobial Resistance \& Infection Control 10(1): P230

Introduction: The World Health Organization recommends improving hand hygiene $(\mathrm{HH})$ practices of the general public as one aspect of controlling the transmission of novel coronaviruses and influenza virus epidemics or pandemics.

Objectives: To systematically review the evidence on the effectiveness of $\mathrm{HH}$ interventions for preventing transmission or acquisition of viral infections in the community.

Methods: PubMed, MEDLINE, CINAHL and Web of Science databases were searched for empirical studies published between 2002-May 2020, on $\mathrm{HH}$ in the general public and acquisition or transmission of novel coronavirus infections or influenza. Study selection, data extraction and quality assessment were conducted by one reviewer, with all decisions checked by another. We conducted a sub-set analysis of intervention studies included in this review, by calculating the effect estimates.

Results: The review identified four intervention studies, all of which used cluster randomised designs evaluating the effectiveness of $\mathrm{HH}$ education paired with provision of $\mathrm{HH}$ products or hand washing with soap and water (HW) against influenza transmission or acquisition amongst the populations of schoolchildren $(n=2)$ or the general public $(n=2)$. Three indicated a protective effect of $\mathrm{HH}$ interventions (Figure); yet, this effect was significant for only one school-based intervention, which consisted of the provision of $\mathrm{HH}$ education and performing $\mathrm{HW}$ twice a day (OR: $0.64 ; 95 \% \mathrm{Cl} 0.51,0.80)$. However, the risk of bias of this study was assessed as unclear; whereas the remaining three studies were assessed as high risk.

\begin{tabular}{|c|c|c|c|c|c|c|c|}
\hline \multicolumn{8}{|c|}{$\begin{array}{l}\text { Figure: } \\
\text { Forest plot showing the individual effects of hand hygiene interventions on influenza } \\
\text { transmission or acquisition }\end{array}$} \\
\hline Study & & & & & & $\begin{array}{l}\text { Odds Ratio } \\
\text { with } 95 \% \mathrm{Cl}\end{array}$ & $\begin{array}{l}\text { Weight } \\
(\%)\end{array}$ \\
\hline Stobbins 2011 & 51 & 1,644 & 53 & 1,612 & $\longrightarrow$ & $0.94[0.64,1.39]$ & 27.25 \\
\hline Talaat 2011 & 125 & 1,582 & 281 & 2,271 & - & $0.64[0.51,0.80]$ & 38.55 \\
\hline Cowling 2009 & 14 & 243 & 28 & 251 & & $0.52[0.27,1.00]$ & 14.99 \\
\hline \multirow[t]{2}{*}{ Larson 2010} & 29 & 917 & 24 & 880 & $\square$ & $-1.16[0.67,2.01]$ & 19.21 \\
\hline & & & & & $1 / 2$ & 2 & \\
\hline
\end{tabular}

Conclusion: There is some limited evidence demonstrating that hand hygiene interventions were effective in preventing influenza in school children. Thus, whilst provision of $\mathrm{HH}$ education to school children will be beneficial from a public health perspective, it's impact on influenza transmission is unclear. Research is needed to evaluate the effectiveness of $\mathrm{HH}$ interventions for prevention of respiratory infections, including SARS-CoV-2, amongst more diverse groups of the general public populations.

Disclosure of Interest: None declared.

\section{P231}

What should have worked but didn't really: addressing hand

hygiene misinformation in the press

A. Peters ${ }^{1, *}$, D. Pittet ${ }^{1}$, S. Ghasemi ${ }^{1}$

${ }^{1} \mathrm{HUG}$, Geneva, Switzerland

Correspondence: A. Peters

Antimicrobial Resistance \& Infection Control 10(1): P231

Introduction: Misinformation about infection prevention is everywhere, and especially widespread and harmful during a destabilizing situation such as a pandemic. Published sources of misinformation in online newspapers are among the most widely read by laypeople. It is important to respond to fake news, though it is difficult to know the impact of such a response.

Objectives: We wanted to measure the impact of our ability to reduce the damage done by an article published in the The Express during the COVID-19 pandemic. This article contained a large amount of false and misleading information about hand hygiene. Our objective was to make the publisher correct the misleading article and to analyze how feasible this was, if we had ample time and resources to devote to one piece of fake news.

Methods: After discovering the misleading article, we sent an email reporting and denouncing the false information to the publisher. We contacted the Independent Press Standards Organisation (IPSO) to file a complaint and provided a detailed analysis of which points of their Editors' Code of Practice were violated in the concerned article. We contacted the British Institute of Cleaning Science where the "expert" cited in the newspaper article was reported to be a consultant, in order to find out more from them. All inaccurate or misleading elements of the article were analyzed, with full documentation of the scientific literature.

Results: Though each inaccuracy was addressed specifically with plenty of evidence, the newspaper refused to take any action to correct or remove the article, claiming to be confident about the content. IPSO rejected the claim we filed, as they considered that the article contained the point of view of the interviewed person. The British Institute of Clean Science specified that the "expert" was an unpaid consultant and released a statement (also sent to IPSO) that specified that the views of their unpaid consultant did not reflect their own.

Conclusion: Even with ample resources, it proved to be very difficult, if not impossible, to have a measurable impact on infection prevention misinformation in the lay press. More work needs to be done to address this divide between academics and the lay press, as it is crucial for infection prevention and public health.

Disclosure of Interest: None declared.

\section{P232}

Perception of healthcare workers towards healthcare-associated infections and hand hygiene exposed to different change agents Y. F. Lee ${ }^{1, *}$, W. Zingg ${ }^{2}$, H. U. Ngian ${ }^{1}$, Z. H. Chin ${ }^{3}$, M.-L. McLaws ${ }^{4}$, S. Amir

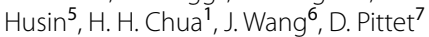

${ }^{1}$ Sarawak General Hospital, Kuching, ${ }^{2}$ Department of Infectious Diseases and Hospital Epidemiology, University Hospital Zurich, Zurich, ${ }^{3}$ Sarawak State Health Department, Kuching, Malaysia, ${ }^{4}$ University of New South Wales, Sydney, Australia, ${ }^{5}$ Infection Prevention \& Control Unit, $\mathrm{MoH}$, Putrajaya, Malaysia, ${ }^{6}$ Otto von Guericke University, Magdeburg, Germany, ${ }^{7}$ Infection Control Programme and WHO Collaborating Centre on Patient Safety, University of Geneva, Geneva, Switzerland

Correspondence: Y. F. Lee

Antimicrobial Resistance \& Infection Control 10(1): P232 
Introduction: Healthcare-associated infections (HAls) have affected hundreds of millions of individuals worldwide. Taking that hand hygiene is behaviour driven, improvement strategies can be improved by fostering collaborative multimodal strategies via change agents.

Objectives: To understand how the different attitude of change agents shaped the perception of healthcare (HCWs) workers towards HAls and hand hygiene.

Methods: Two acute wards of a tertiary hospital in Malaysia were randomly exposed to hand hygiene improvement interventions delivered by peer-identified change agents (PICAs) in study arm 1 , and management selected change agents (MSCAs) in study arm 2. Primary outcome was perception of HCWs towards (i) impact of HAls and the importance of hand hygiene, (ii) strategies and tools for hand hygiene compliance and; (iii) facilitators' importance for optimum hand hygiene, measured using the WHO Perception Survey for HCWs (revised 2009), applied in the pre- and post- intervention periods. Secondary outcome was perceived leadership styles of PICAs and MSCAs by HCWs, vocalised during question and answer sessions.

Results: The number of respondents in pre- and post-intervention was 72 and 70 , and 75 and 71 in study arms 1 and 2, respectively. From the pre- to post intervention periods, the perception of HCWs on the impact of HAls, and the importance of hand hygiene were similar and remain unchanged in both study arms. In the post-intervention period, the proportion of HCWs that perceived improvement in safety climate for hand hygiene was significantly higher in PICA led-ward compared to MSCA led-ward ( $91 \%$ vs $65 \%, P<0.001)$. Proportion of HCWs on the perception of the facilitators' effectiveness on hand hygiene was significantly higher with PICAs compared to MSCAs. Healthcare workers perceived that PICAs lead by example, while MSCAs were authoritative and more desirable for hand hygiene improvement.

Conclusion: Although HCWs preferred the authoritative leadership style of MSCAs, they still perceived PICAs to be more effective in improving hand hygiene compliance.

Disclosure of Interest: None declared.

\section{P233}

Is the WHO multimodal hand hygiene strategy applicable and effective at the primary care level in resource-limited settings?-a quantitative analysis in healthcare centers of Faranah, Guinea

S. Müller, ${ }^{1,}$, L. Landsmann ${ }^{1}$, A. O. K. Diallo², R. Wood', C. Rocha', O. Tounkara ${ }^{2}$, M. Arvand ${ }^{1}$, M. Diallo ${ }^{2}$, M. Borchert ${ }^{1}$

${ }^{1}$ Robert Koch Institute, Berlin, Germany, ${ }^{2}$ Regional Hospital, Faranah, Guinea

Correspondence: S. Müller

Antimicrobial Resistance \& Infection Control 10(1): P233

Introduction: Healthcare-associated infections (HAI) are the most frequent adverse events in healthcare worldwide. The Multimodal Hand Hygiene $(\mathrm{HH})$ Improvement Strategy by the WHO aims at reducing HAl. Hitherto, there is little evidence of the applicability and effectiveness of the WHO HH Strategy at primary care level, especially in healthcare centers $(\mathrm{HCC})$ of resource limited settings such as Guinea.

Objectives: The objectives of the present study were to improve $\mathrm{HH}$ knowledge and compliance of healthcare workers (HCWs) in two HCC in the region of Faranah, Guinea; to increase the availability of alcoholbased handrub (ABHR) by supplying HCC with locally produced ABHR; and to assess the effectiveness of the WHO Multimodal HH Improvement Strategy on primary care level.

Methods: $\mathrm{HH}$ knowledge, perception and compliance of HCWs were assessed in baseline prior to the intervention and compared to two follow-up assessments, one immediately and one six months after the intervention. The intervention consisted of $\mathrm{HH}$ training and the continuous supply of HCW with ABHR, which was produced in the Regional hospital Faranah. The monthly consumption of ABHR after the training was monitored with respect to consultations.

Results: Baseline knowledge score was $11 / 25$, increased significantly to $16 / 25$ upon first and decreased slightly to $15 / 25$ in second followup. Compliance with $\mathrm{HH}$ showed a significant increase from $15.6 \%$ to $84.4 \%(p<0.001)$ in first follow-up. Compliance in second follow-up was lower than in first follow-up $(53.2 \%, p<0.001)$, but was still more than $37 \%$ higher than in baseline $(p<0.001)$. ABHR consumption averaged $0.77 \mathrm{~mL}$ per consultation.

Conclusion: This study shows that the WHO HH Strategy is an appropriate method to improve $\mathrm{HH}$ compliance and knowledge in primary care level, but needs some adjustment of assessment and training procedures. Assessments should include the observation of $\mathrm{HH}$ action correctness and trainings should further emphasize on proper $\mathrm{HH}$ performance and ABHR amount usage. Moreover, our results highlight the need for continued training and education to maintain a high level of $\mathrm{HH}$.

Disclosure of Interest: None declared.

\section{P234}

How to go beyond the WHO strategy and build a hand hygiene culture in the regional hospital of Faranah

A. Diallo 1,*, S. Müller', L. Landsmann², C. Rocha ${ }^{2}$, R. Wood ${ }^{2}$, O. Tounkara', M. Borchert ${ }^{2}$, M. Arvand ${ }^{2}$, M. Diallo ${ }^{1}$

${ }^{1}$ Regional Hospital of Faranah, Faranah, Guinea, ${ }^{2}$ Robert Koch Institute, Berlin, Germany

Correspondence: A. Diallo

Antimicrobial Resistance \& Infection Control 10(1): P234

Introduction: The Multimodal WHO Hand Hygiene (HH) Strategy is a widely used and well recognized approach. However, the evidence on the sustainability in low-resource settings is still scarce. The WHO strategy was implemented at the Regional Hospital of Faranah (HRF), but context specific challenges developed.

Objectives: We aimed to promote $\mathrm{HH}$ culture by addressing these challenges and implementing appropriate solutions.

Methods: Over a period of 3 years, the project team used quantitative and qualitative methods to assess $\mathrm{HH}$ improvement. Local staff was trained and instructed to track the development, identify barriers and find solutions to build a sustained HH culture.

Results: Four main barriers were identified and addressed by targeted measures. 1) The lack of staff for local production was overcome by implementing a train the trainer approach. The initially trained pharmacist trained four other team members. To further strengthen the production team, a national solution of training and sending staff is proposed by the HRF. 2) Difficulties in obtaining production ingredients in high quality were addressed by purchasing large quantities and the establishment of a storage room in the hospital pharmacy. 3) To prepare for sustainable local production, the return, recycling and exchange of empty bottles was planned by opening a register of distribution. 4) To stop the decrease in knowledge and compliance over time, a HH championship every 6 months was initiated. All hospital departments were invited to participate and staff assessed via the WHO knowledge questionnaires and $\mathrm{HH}$ observations. Furthermore, refresher trainings and demonstrations of $\mathrm{HH}$ were given in daily staff meetings.

Conclusion: To build HH culture is feasible in low-resource hospitals such as the HRF. However, context specific challenges require locally designed solutions such as the introduction of recycling systems for ABHR bottles, organizing $\mathrm{HH}$ championships, or promoting daily staff trainings to maintain the improvement on $\mathrm{HH}$ practises and creating a $\mathrm{HH}$ culture. This development of a $\mathrm{HH}$ culture and its sustainable local production helped the HRF to respond to the current COVID-19 pandemic.

Disclosure of Interest: None declared. 


\section{P235}

Development of a cost-effective water dispenser for hand washing at a rural/resource limited setting

S. Parangimalai Madan Kumar ${ }^{1, *}$

${ }^{1}$ Vidya Mandir Senior Secondary School, Chennai, India

Correspondence: S. Parangimalai Madan Kumar

Antimicrobial Resistance \& Infection Control 10(1): P235

Introduction: Being the second most populous country, India houses around 1.4 billion people, with major of its population in rural setting, who have limited access to health and infrastructural facilities. Lack of clean water coupled with access to contaminated water has only resulted in increase in spread of infectious diseases, especially among the rural children. In addition, there still exists the problem of open defecation, especially in rural India which acts as a nidus in the spread of infection. In spite of the continued efforts by the governmental and private agencies to encourage the habit of adequate hand washing, especially before and after eating and defecation, lack of basic facilities including absence or malfunction of water storage tanks and taps hinder this process.

Objectives: The author has proposed a new design of Water Dispenser for hand washing which can be constructed using locally available resources and thus reduces the cost involved in the construction of tanks and the necessary tap facilities.

Methods: The design consists of a panel of a round wooden log, preferably of bamboo or any wood which is native to that particular region. The log panel structure consists of two supporting pillars, along with a horizontal wooden platform to support the water dispenser with the strings, as shown in Figure 1. The users are encouraged to wash their hands, after sensitizing them about the health benefits, along with the handwashing technique and the duration of the same. The user can pull the string to pour the water from the dispenser for washing his/her hands and feet.

Results: The feasibility of this design was field tested at a rural setting and around 30 users were questioned regarding the comfort and the usability factors on a scale of 0 (not useful) to 5 (Highly useful). Most of the participants expressed satisfaction in terms of the ease of installation and usage of this model for hand washing.

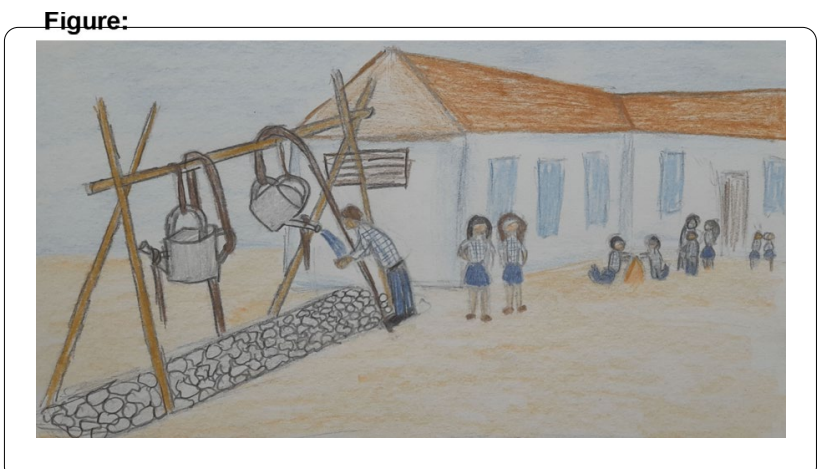

Conclusion: The advantages of this methods included ease of installation, low cost and avoidance of container to draw water from the water source and hence contaminating the source of water. Though this model has some limitations, the proposer feels that this model could be a viable alternative for water dispensing for hand washing purposes in rural or resource limited settings.

Disclosure of Interest: None declared.

\section{P236}

One minute to keep coronavirus away, teaching community good hand hygiene practices

M. Fawwaaz ${ }^{1, *}$

${ }^{1}$ Atlantique, Clinique Centrale de Calavi, Abomey-Calavi, Benin

Correspondence: M. Fawwaaz

Antimicrobial Resistance \& Infection Control 10(1): P236
Abstract video clip description: The coronavirus pandemic has disrupted lifestyles for almost two years across the planet. The fight for its prevention in the absence of its eradication involves the implementation of barrier measures including the treatment of hands. So, as part of the International Hand Hygiene Day this year with the theme "One Minute to Save Lives"; health workers from the Calavi Central Clinic in the Republic of Benin, have decided to increase their hand hygiene compliance rate by organizing an awareness and training day for users of the establishment. So each caregiver came out of his office to show the hand washing technique to a user (patient, attendant, or other non-clinical user). This activity, which lasted three hours, saw the participation of 21 clinic agents who taught 42 users. This operation, which deserves to be repeated, showed the good technique of simple hand washing to more than forty users in one morning.

Keywords: coronavirus, prevention, hand hygiene.

General objective: Participate in the reduction of the transmission of the coronavirus by the practice of hand washing.

Specific objectives: Promote hand hygiene in the minds of users.

Demonstrate the correct and simple hand washing method to the community.

Method and materials: Each health worker revisits their simple handwashing protocol, explains it to a user, and the two do it together.

Materials: Two hand washing stations, soap, disposable hand towels, garbage cans.

Results: The operation was monitored by all the medical practitioners present at the station, ie $100 \%$, of 06 nurses and 12 health and pharmacy auxiliaries. These health workers of all categories showed the technique to 42 users who practiced simple hand washing, more than $80 \%$ of the people who went to the clinic this morning.

Conclusion: The practice of hand hygiene is in the headlines as stated by one user. Everyone saw their caregiver come out and discuss the technique and importance of hand washing with them. This will inevitably improve their knowledge of this very important method in infection prevention and control (IPC) in healthcare settings.

Disclosure of Interest: None declared.

P237

Hand hygiene in the soap's realm

S. M. Filipe Gonçalves ${ }^{1, *}$, D. Moderno ${ }^{1}$, A. Agostinho ${ }^{2}$, F. Paiva-Santos ${ }^{3}$, E. Noriega ${ }^{4}$

${ }^{1}$ Figueira da Foz District Hospital, Figueira da Foz, Portugal, ${ }^{2}$ Geneva University Hospitals, Geneva, Switzerland, ${ }^{3}$ Nursing School of Coimbra, Coimbra, ${ }^{4}$ National Infection Prevention and Control Group, Lisbon, Portugal Correspondence: S. M. Filipe Gonçalves

Antimicrobial Resistance \& Infection Control 10(1): P237

Introduction: Promoting hand hygiene among healthcare workers every year is a constant challenge. The material provided by "Clean Care is Safer Care" from the World Health Organization (WHO) team helps to mobilize all professionals as a whole, however year after year they resent the tiredness leading to a decrease in hand hygiene compliance rates. The use of innovative local strategies can help to overcome this tiredness and input a new dynamic, thus maintaining and even improving hand hygiene compliance.

Objectives: Revitalize the hand hygiene campaign through an innovative strategy in order to improve the compliance rate.

Methods: Figueira da Foz Hospital is a secondary district hospital who joined the WHO's hand hygiene campaign in 2014 and thenceforth, every 5th may, healthcare workers are mobilized to celebrate the World's Hand Hygiene Day. In the first 3 years we used updated posters, banners and screen-savers. In 2018 we decided to bring a new approach: we made a small illustrated book with a story for the healthcare workers children: Once upon a time... in Soap's Realm, an imaginary story in a kingdom where hand hygiene was widely recognised as an important way to keep people healthy. King McClean invested in infrastructures, produced the best world's soap and there even was a troubadour to keep hand washing in people's routines. Until the day the good old king died, his work wasn't continued and everyone got ill after an invasion of microbes. But like all children's stories, this one also has a happy end! 
Results: The book met a huge success. The pooled hand hygiene compliance rate between periods increased from $62,0 \%$ to $72,4 \%$ ( $95 \%$ Cl: $5,34-15,35 ; p<, 001)$; before the intervention it was $63,3 \%$ in $2015,57,1 \%$ in 2016 and $59,9 \%$ in 2017 and after the intervention the compliance improved to $66,4 \%$ in 2018 and $73,5 \%$ in 2019 . The rate of nosocomial infections also improved from $5,2 \%$ in 2015 to $3,5 \%$ in 2019, according to institutional prevalence.

Conclusion: A different way of communication can change the way we receive the same message, create awareness for hand hygiene compliance thus preventing healthcare associated infections.

Disclosure of Interest: None declared.

Poster session: hand hygiene: multimodal implementation including electronic monitoring

\section{P238}

Knowledge of medical/nursing (MED/NURS) leaders about hand hygiene and standard precautions (SP) in non COVID-19 wards in a tertiary care hospital

A. Iten ${ }^{1}$, V. Camus ${ }^{1, *}$, C. Fankhauser ${ }^{1}$, Z. Horcik ${ }^{2}$

${ }^{1}$ Infection control program, ${ }^{2}$ Learning center, HUG, Genève, Switzerland

Correspondence: $\mathrm{V}$. Camus

Antimicrobial Resistance \& Infection Control 10(1): P238

Introduction: Since the beginning of the COVID-19 pandemic, HUG are taking care of patients suspected of having or affected with COVID19. The majority of the HUG wards became COVID wards; CONTACT and DROPLETS measures replaced SP. When the situation returned to normal, the use of personal protective equipment was inappropriate. A corrective program was implemented. One of its objectives was to address the role of the MED/NURS leaders of the non-COVID wards. In the initial phase of the program, the PCl knowledge of these managers was assessed and updated. The majority of the wards became COVID wards; CONTACT and DROPLETS measures replaced SP.

Objectives: To assess the knowledge of MED/NURS leaders of nonCOVID wards regarding hand hygiene and wearing of gloves and to evaluate the choice of personal protective equipment by MED/NURS managers in non-COVID wards for frequent clinical situations.

Methods: The MED/NURS managers of the non-COVID wards were invited to participate in a training session. 3 ICP professionals and 1 adult education specialist: 1 . described the context and presented the project; 2.tested the participants' knowledge about hand hygiene, gloves' wearing and personal protective equipment to be used in frequent clinical situations. The quiz was built with an educational purpose.

The participants' answers were recorded anonymously by an online survey software. Aggregated responses were presented and commented during the session.

Results: 11 training sessions were held from 27.01.2021 to 26.02.2021. 286 MED/NURS leaders were invited: 177 (61.8\%) attended. More than $75 \%$ of the participants appreciated this type of training.

\begin{tabular}{|l|cc|}
\hline Topic of questions & \multicolumn{2}{|c|}{$\begin{array}{c}\text { Correct answers } \\
\%\end{array}$} \\
\hline Hand hygien & 87.15 & $(\mathrm{Cl} \pm 8.53)$ \\
\hline Gloves & 92.85 & $(\mathrm{Cl} \pm 1.73)$ \\
\hline Clinical situations & 59.32 & $(\mathrm{Cl} \pm 5.56)$ \\
\hline
\end{tabular}

Conclusion: Knowledge about hand hygiene and gloves' wearing is satisfactory: it was enough to answer true or false to a statement. The percentage of correct answers given to clinical situations is insufficient: knowing which personal protective equipment to use with regard on transmission risk remains complicated. This is one of the topics on which our program must focus.

Disclosure of Interest: None declared.
P239

Health care workers knowledege about hand hygiene

within the COVID-19 epidemic in Tunisia 2020

A. Gara ${ }^{1, *}$, A. ben cheikh ${ }^{1}$, S. bhiri ${ }^{1,2}$, H. ghali ${ }^{1,2}$, W. dhouib ${ }^{1}$, R. bannour ${ }^{1}$, S. khfecha ${ }^{1}$, H. Said Latiri ${ }^{1}$, M. ben rejeb ${ }^{1,2}$

${ }^{1}$ Department of Prevention and Security of Care, Sahloul university hospital, Sousse, Tunisia, ${ }^{2}$ Faculty of Medicine of Sousse, University of Sousse,

Sousse, Tunisia, sousse, Tunisia

Correspondence: A. Gara

Antimicrobial Resistance \& Infection Control 10(1): P239

Introduction: The coronavirus COVID-19 pandemic is the defining global health crisis of our time.

Current evidence indicates that the COVID-19 virus is transmitted through respiratory droplets or contact. So, to reduce the speed spread of the infection, hand hygiene $(\mathrm{HH})$ is considered as a corner stone measure. We aimed to describe the knowledge about $\mathrm{HH}$ of health care workers (HCWs) in a Tunisian teaching hospital over this outbreak in May 2020.

Objectives: The aim of this study was to determine the knowledge regarding hand hygiene practices amongst health care workers.

Methods: It was a descriptive study of the knowledge of HCWs about $\mathrm{HH}$ performed over a period of one-month (May) 2020. We implemented a self-administered questionnaire written in French focusing on the importance of $\mathrm{HH}$ in prevention and control of infection, then general information about $\mathrm{HH}(5$ moments for $\mathrm{HH}$, handwashing vs hand rub, conditions before $\mathrm{HH}$ ).

Results: A total of 466 participants (67.4\%) were enrolled to the study, among them $50.2 \%$ were doctors $(n=234)$ vs $49.8 \%$ were staff nursing $(n=234)$. The majority of participants belonged to surgical services $(n=234)$, then medical services $(n=153)$ then annexes $(n=79)$ with respective rates of $50 \%, 33 \%$ and $17 \%$. The importance of $\mathrm{HH}$ in the reduction of the risk of Covid- 19 was recognized by $52.6 \%$ of HCWs. Fifty eight percent of them knew that the virus SARS-CoV-2 survived only $10 \mathrm{~min}$ on the hands.

Staff nursing' $\mathrm{HH}$ knowledge in different items was higher than that of the medical staff.

Conclusion: Opting for sustainable programs to control the COVID-19 outbreak, it appears that $\mathrm{HH}$ promotion and training is crucial especially among doctors.

Disclosure of Interest: None declared.

\section{P240}

Train-the-trainers program to improve hand hygiene promotion capacity in Iranian hospitals

N. Lotfinejad ${ }^{1,}$, M. H. Aelami ${ }^{2}$, E. Tartari ${ }^{1}$, C. Fankhauser-Rodriguez ${ }^{1}$, D. Pittet $^{1}$

${ }^{1}$ Infection Control Programme, University of Geneva Hospitals and Faculty of Medicine, Geneva, Switzerland, ${ }^{2}$ Pediatrics \& Infection Control and Hand Hygiene Research Center, Mashhad University of Medical

Sciences, Mashhad, Iran, Islamic Republic Of

Correspondence: N. Lotfinejad

Antimicrobial Resistance \& Infection Control 10(1): P240

Introduction: Healthcare-associated infections (HAls) are considered to be the most prevalent harmful event in healthcare that threaten patients worldwide. Hand hygiene is the cornerstone of preventing HAls and it is promoted by the World Health Organization (WHO) multimodal hand hygiene improvement strategy (MHHIS). The hand hygiene self-assessment framework (HHSAF) and the Train-The-Trainers (TTT) program are among the tools to improve hand hygiene practices according to the MHHIS.

Objectives: To determine the hand hygiene promotion capacity of Iranian public hospitals through the HHSAF application, as well as evaluating the impact of TTT program.

Methods: A two-part program on implementation of the MHHIS was conducted by a group of four infection prevention and control 
practitioners from Geneva. A total of 13 university hospitals from different regions of Iran participated in the HHSAF survey and a 3-day TTT hands-on simulation-based hand hygiene training program took place in Shiraz, Iran.

Results: In total, 53 individuals participated in completing the HHSAF survey during the survey period from September 2018 to December 2019. We found that facility scores varied, with the majority at intermediate or advanced levels. The TTT program was performed from 16 to 19 April in Shiraz, Iran, with 44 participants, among which $37 \mathrm{com}$ pleted both pre and post TTT course tests. The overall mean of correct answers of the tests before and after the TTT course were $62.41 \%$ and $75.30 \%$, respectively.

Conclusion: This report presents the current status of Iranian facilities on the hand hygiene improvement continuum. Further completion of the HHSAF should be undertaken throughout the country in support of hand hygiene action. According to the TTT test results, participants' knowledge improved following this training program. This standard training method should be applied in different settings worldwide to disseminate knowledge to health care workers.

Disclosure of Interest: None declared.

\section{P241}

University training as the key point for student nurses' behaviour on hand hygiene compliance

A.-P. Ana Belén ${ }^{1, *}$, L. G.-M. Sara ${ }^{2}$, D. R. Silvia ${ }^{3}$

${ }^{1}$ Preventive Medicine, Hospital Universitario Fundación Alcorcón. Adjunct faculty at Rey Juan Carlos University, ${ }^{2}$ Alumna enfermería, Universidad Rey Juan carlos, Madrid, ${ }^{3}$ Universidad de Alcalá de Henares., Alcalá de Henares.Madrid, Spain

Correspondence: A.-P. Ana Belén

Antimicrobial Resistance \& Infection Control 10(1): P241

Abstract video clip description: Introduction: Hand hygiene $(\mathrm{HH})$ is the most effective, inexpensive and easy-to-implement measure which prevents healthcare-associated infections (HAls). The SARSCoV-2 pandemic has highlighted its importance not only in patient care, but also in preventing the transmission of microorganisms in both inside and outside the hospital.

Objectives: to describe the knowledge level about $\mathrm{HH}$, to detect the weaknesses and to plan unified training activities for the 4th year student nurses at Madrid's Community public universities.

Methodology: A cross-sectional study was done. It consisted on filling the World Health Organisation survey about $\mathrm{HH}$ knowledge in a voluntary and anonymous way. It was distributed, on the social networking sites of the 4 th year student nurses, through google drive, to the 4 public universities to which this study was intended for.

Results: The survey was answered by $22.4 \%$ of the 4 th year student nurses of Madrid's public universities. Of all the participants, $43.7 \%$ were from Rey Juan Carlos University, 20.4\% were from Autónoma University of Madrid, 19\% were from Complutense University of Madrid and $16.9 \%$ were from University of Alcalá de Henares. $84.5 \%$ of the participants were female, and $15.5 \%$ were male. Among the different items included in the survey, the following are worth mentioning. $96.5 \%$ of the survey respondents affirm that during their degree they have received training about $\mathrm{HH}$. The overall average number of correct answers was 17.18 out of 25 .

Discussion: Nevertheless, certain deficiencies in terms of knowledge about the importance, timing and technique of Hand Hygiene were observed. So, some unified training activities of a theoretical-practical nature are proposed to reinforce knowledge of $\mathrm{HH}$ in the four universities to which the survey was aimed at.

We considered introducing simulation as a method of learning $\mathrm{HH}$ moments in care tasks. This method allows reaching an agreement about some of the systematic activities, since it implements the different moments on $\mathrm{HH}$ during care tasks, in this way it ensures patient safety. In addition, its use and evaluation show positive results.

Disclosure of Interest: None declared.
P242

Integrating infection prevention and control training

into advanced cardiac life support training-a single center study S. Hansen ${ }^{1, *}$, F. Schwab ${ }^{1}$, M. Weisker ${ }^{1}$, T. Jeske ${ }^{1}$, R. Somasundaram² ${ }^{2}$, B. A. Leidel $^{2}$

${ }^{1}$ Institute of Hygiene and Environmental Medicine, ${ }^{2}$ Department of Emergency Medicine, Charité - Universitätsmedizin Berlin, Berlin, Germany

Correspondence: S. Hansen

Antimicrobial Resistance \& Infection Control 10(1): P242

Introduction: Hand hygiene $(\mathrm{HH})$ compliance of healthcare workers (HCW) in emergency departments (ED) has been shown low and described as potentially infeasible especially during resuscitation [Haac 2017].

Objectives: To what extent Infection Prevention and Control (IPC) training is practicable during Advanced Cardiac Life Support (ACLS) training was analyzed in an observational study.

Methods: Prospective analysis of $\mathrm{HH}$ compliance observation (CO) and feedback during an American Heart Association certified ACLS training of ED HCW in 03-04/2021. CO were realized according to WHO's 5 moments by experienced IPC staff during 3 consecutive ACLS cases in each of 8 training sessions. Compliance rates (CR) were calculated as ratio of performed and recommended $\mathrm{HH}$ procedures. Feedback was given after each case. Further IPC aspects were discussed when recognized by EDs' HCW. Pooled CR were calculated, differences were tested between the 1 st and 3 rd ACLS case.

Results: $60 \mathrm{HCWs}$ participated in the training (22 nurses, 7 physicians). 486 recommended $\mathrm{HH}$ opportunities were observed, the majority related to indication $2(204 ; 42 \%)$. As shown in table 1 pooled CR increased for WHO indications $1-4$ and in total from $41.3 \%$ to $77.8 \%$ $(p<0.001)$.

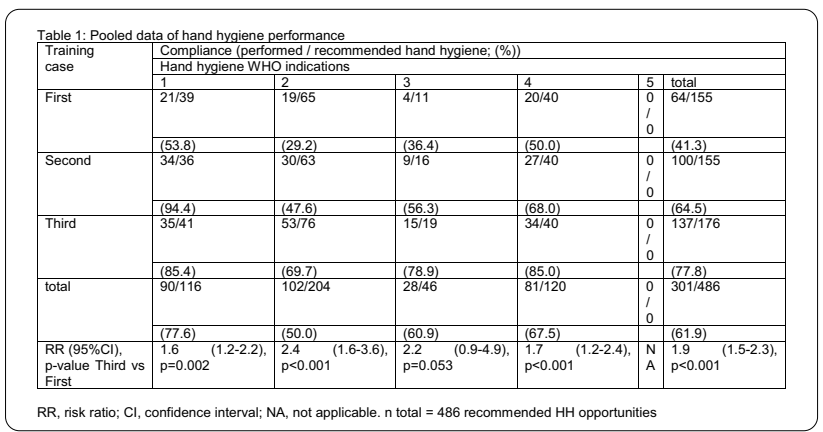

Conclusion: $\mathrm{HH} \mathrm{CO}$ and immediate feedback improves HCWs $\mathrm{HH}$ adherence during ACLS training. Further evaluation is needed to analyze translation of IPC training into real patient care.

Disclosure of Interest: None declared.

P243

Electronic hand hygiene systems can be used to assess clinically relevant interventions

M. Stangerup ${ }^{1}$, R. Hansen², L. Sode ${ }^{2}$, M. B. Hansen 3,*, B. Hesselbo ${ }^{1}$, H. Calum ${ }^{1,4}$, B. Olesen ${ }^{5}$

${ }^{1}$ Infection Control Unit, Bispebjerg and Frederiksberg Hospitals, ${ }^{2}$ Department of Orthopaedic, Bispebjerg University Hospital, ${ }^{3}$ Konduto ApS,

${ }^{4}$ Department of Clinical Microbiology, Amager and Hvidovre Hospitals,

${ }^{5}$ Department of Clinical Microbiology, Herlev, Gentofte and Nordsjæellands Hospitals, Copenhagen, Denmark.

Correspondence: M. B. Hansen

Antimicrobial Resistance \& Infection Control 10(1): P243

Introduction: There is a need to identify the most effective hand hygiene interventions. A previous study showed that hand hygiene compliance $(\mathrm{HHC})$ of hospital visitors increased using colourful tape on the floor as a visual reminder at the main entrance next to alcoholbased hand rub (ABHR) dispensers. 
Objectives: We aimed to assess the effect of this low-cost, low-effort approach on healthcare workers' (HCWs) HHC in patient rooms using an electronic hand hygiene system.

Methods: To avoid the risk of bias associated with direct observations, an electronic hand hygiene system (sani nudge ${ }^{\mathrm{TM}}$ ) was used to obtain HHC data from doctors $(n=6)$ and nurses $(n=18)$ in a surgical ward from Dec 2020-Feb 2021.

The study was divided into a baseline and an intervention period where pink tape was placed on the floor at the entrance of each patient room next to the ABHR dispensers. The pink colour was identical to the colours of stickers and brochures used during the last hygiene campaign.

The hypothesis was that the HCWs would associate the pink-coloured tape (nudge) with hand hygiene, resulting in more frequent $\mathrm{ABHR}$ and increased HHC. We used Student's t-test to assess differences between baseline and intervention.

Results: The HHC of the doctors was not associated with a significant change from baseline to intervention either before patient contact ( $25 \%$ vs. $23 \%, p=0.76)$ or after patient contact $(52 \%$ vs. $57 \%, p=0.73$ ). As with the doctors, the $\mathrm{HHC}$ of the nurses did not change from baseline to intervention either before patient contact ( $31 \%$ vs. $35 \%$, $p=0.10$ ) or after patient contact ( $49 \%$ vs. $46 \%, p=0.46$ ).

Conclusion: This study demonstrates that interventions can be assessed with an electronic hand hygiene system. We found that colourful tape on the floor at patient room entrances did not have an impact on the HHC of HCWs. The lack of effect may be due to banner blindness due to frequent exposure. More dynamic nudges may be needed to change the hygiene behaviour of HCWs.

Disclosure of Interest: None declared.

\section{P244}

Use of an electronic hand hygiene monitoring system

is associated with decreased staff absenteeism

M. B. Hansen ${ }^{1}$, H. J. Kolmos ${ }^{2, *}$

${ }^{1}$ Medical \& Science, Konduto, København K, ${ }^{2}$ Clinical Microbiology, University of Southern Denmark, Odense, Denmark

Correspondence: $\mathrm{H}$. J. Kolmos

Antimicrobial Resistance \& Infection Control 10(1): P244

Introduction: The World Health Organization promotes the use of alcohol-based hand rubs (ABHR) at the point of care to protect patients and staff from healthcare-associated infections.

Objectives: We aimed to assess the effect of implementing an electronic hand hygiene monitoring system (EHHMS) on staff hand hygiene compliance (HHC) and staff absenteeism.

Methods: An EHHMS (sani nudge ${ }^{\mathrm{TM}}$ ) was implemented in an internal medicine department at a university hospital for an 18-months period (Aug 2017-Jan 2019). Anonymous sensors were placed on existing ABHR dispenser solutions, staff name badges and at patient beds to measure hand hygiene opportunities and actions.

The study was designed with a 7-months baseline period followed by an 11-months intervention period. The intervention consisted of datadriven group feedback at biweekly staff meetings in which ward management presented the $\mathrm{HHC}$ levels according to profession and room types. A copy of the results was put on a bulletin board after each meeting to serve as a reminder. Monthly HHC goals were decided and communicated by management to help focus their improvement work.

Staff absenteeism was defined as short term sick leave between 1-28 days and averaged for each month.

We used Student's t-test to assess differences in HHC between baseline and intervention, and Pearson correlation test to assess correlations between HHC and staff absenteeism. Two-sided $p$ values $<0.05$ were considered statically significant.

Results: We included 100 front-line healthcare workers (doctors, $n=31$; nurses, $n=69$ ). The average HHC at baseline was $31 \%$. During the intervention period, the average $\mathrm{HHC}$ significantly increased to $51 \%(p<0.0001)$ which was associated with a significant decrease in staff absenteeism from 7 to $3 \%(p<0.0001)$. There was a strong negative correlation between $\mathrm{HHC}$ and staff absenteeism $(r=-0.8$, $p=0.0001$ ).

Conclusion: The increase in $\mathrm{HHC}$ after introducing a data-driven group feedback intervention using an EHHMS was associated with a significant decrease in staff absenteeism. The demonstrated relationship between staff HHC and absenteeism shows a return of investment opportunity for hospital management while also increasing the staff and patient safety.

Disclosure of Interest: None declared.

\section{P245}

Implementing an electronic hand hygiene system improved compliance in the intensive care unit

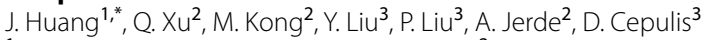

${ }^{1}$ Anesthesiology \& Perioperative Medicine, ${ }^{2}$ University of Louisville, Louisville, ${ }^{3}$ Microsensor Labs, Chicago, United States

Correspondence: J. Huang

Antimicrobial Resistance \& Infection Control 10(1): P245

Introduction: Hand hygiene $(\mathrm{HH})$ compliance is low and difficult to improve among healthcare workers.

Objectives: We aim to validate an electronic $\mathrm{HH}$ system and assess the impact of this system on $\mathrm{HH}$ compliance and quality changes over time at both group and individual levels.

Methods: An automated electronic hand hygiene system was installed in a 10-bed surgical intensive care unit.

Results: The full $\mathrm{HH}$ compliance rate increased significantly from $8.4 \%$ in week 1 to $20.5 \%$ in week 16 with week 10 being the highest (27.4\%). The partial compliance rate maintained relative consistency between $13.2 \%$ to $20.0 \%$. The combined compliance rate (full compliance rate + partial compliance rate) increased from $23.5 \%$ in week 1 to $34.6 \%$ in week 16 with week 10 being the highest (41.4\%). We found significant variations among providers in terms of $\mathrm{HH}$ opportunities per shift, full compliance, partial compliance and combined compliance rates. The average duration of hand rubbing over time in partial compliance occurrences did not change significantly over time.

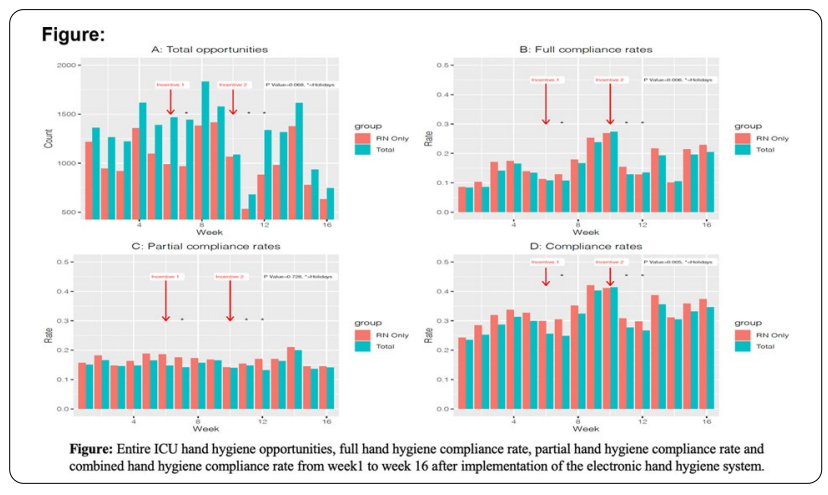

Conclusion: A sensor-based platform with automated $\mathrm{HH}$ compliance and quality monitoring, real time feedback and comprehensive individual level analysis, improved providers' HH compliance in an ICU. There were significant variations among individual providers.

Disclosure of Interest: J. Huang Shareholder of: Microsensor Labs, Q. Xu Grant/Research support from: Microsensor Labs, M. Kong Grant/ Research support from: Microsensor Labs, Y. Liu Shareholder of: Microsensor Labs, P. Liu Shareholder of: Microsensor Labs, A. Jerde Grant/ Research support from: Microsensor Labs, D. Cepulis Employee of: Microsensor Labs. 
Poster Session: Healthcare-associated infections-General issues

\section{P246}

Impact of infection prevention and control precautions taken during COVID-19 pandemic on healthcare associated infections (HAIS)

M. L. Ling ${ }^{1, *}$, M. How ${ }^{1}$, M. O. Aung ${ }^{1}$

${ }^{1}$ Infection Prevention \& Epidemiology, Singapore General Hospital,

Singapore, Singapore

Correspondence: M. L. Ling

Antimicrobial Resistance \& Infection Control 10(1): P246

Introduction: From the start of the COVID-19 pandemic, heightened awareness and diligent efforts are made to ensure zero healthcareassociated COVID-19 amongst healthcare workers. Hand hygiene, environment hygiene and use of appropriate personal protective equipment (PPE) are emphasized and daily audits are done to ensure compliance.

Objectives: We are interested to know if these enhanced measures help towards reducing healthcare associated infections (HAls) amongst inpatients at the Singapore General Hospital, a 1750-bedded acute tertiary care hospital.

Methods: We compared standardized infection ratios (SIRs) of healthcare-onset (HO)-MRSA bacteremia, HO-Clostridium difficile, CLABSI and CAUTI rates pre-COVID (Nov 2017-Dec 2019) and during COVID19 pandemic (Mar-Sep 2020). SIRs were computed following NHSN methodology.

Results: SIR values were less than 1 for HO-MRSA bacteremia, HOClostridium difficile, CLABSI during the peak of COVID-19 pandemic, Mar-Sep 2020 (0.38, 0.44 and 0.31 respectively). Control charts plotted from Nov 2017 confirmed that there was a shift in data points for CLABSI from Sep 2019 - Oct 2020. However, there was no significant impact on the other 3 indicators. Our institution has zero healthcare associated COVID-19 amongst staff to date.

Conclusion: The enhanced IPC precautions instituted during COVID19 pandemic succeeded in sustaining decreased CLABSI rates likely because of increased compliance to CLABSI bundle. We have demonstrated that enhanced infection prevention and control horizontal measures viz. hand hygiene, environment hygiene help towards keeping HAls low in our hospital. These should continue post-pandemic as they are part of Standard Precautions.

Disclosure of Interest: None declared.

\section{P247}

Mortality and its etiological factors in a COVID-19 intensive care unit in Tunisia

I. Mlouki ${ }^{1, *}$, H. Khelij ${ }^{1}$, N. Sebai ${ }^{1}$, M. Khedhr ${ }^{1}$

${ }^{1}$ Department of Microbiology, University Hopital Taher Sfar, Mahdia, Tunisia

Correspondence: I. Mlouki

Antimicrobial Resistance \& Infection Control 10(1): P247

Introduction: High death rates were recorded during the COVID-19 pandemic with a major negative impact worldwide.

Objectives: We aimed at determining etiological factors of mortality among COVID-19 patients admitted in intensive care unit in the region of Mahdia (Tunisia).

Methods: We performed a cross sectional study between October 2020 and February 2021 among all patients admitted in the COVID-19 intensive care unit of the University Hospital Taher Sfar Mahdia. We collected data about nosocomial infection, characteristics of etiological bacterial and fungal species and death.

Results: A total of 95 patients admitted in the COVID intensive care unit were enrolled with a mean age of $60.6 \pm 13.5$ years. Regarding the stay duration, we recorded a median of 16 days (IQR 9-25).

About $67.4 \%$ of participants had nosocomial infection with 49 patients had at least 2 different episodes of infection. The most common infection types were urinary tract infection (50/152), ventilator-associated pneumonia (44/152) and bacteremia (44/152). About $55.7 \%$ of isolated bacteria were multi-resistant to antibiotics.

The overall mortality was $53.7 \%$. It was associated significantly with a higher mean age $(54.2 \pm 11.5$ vs $40.8 \pm 12.9$, OR $2, \mathrm{Cl} 95 \% 1.5-4.8$; $\mathrm{p}=0.02$ ), nosocomial infection (OR $5, \mathrm{Cl} 95 \% 2.2-15 ; \mathrm{p}<0.001)$ and a higher number of co-infection ( $\geq 2$ episodes) (OR 7, Cl 95\% 1.3-10.8; $\mathrm{p}=0.01$ ).

Conclusion: These alarming findings emphasize the urgent need of a proper management of bacterial nosocomial infection in COVID-19 intensive care units to prevent mortality and critical situations.

Disclosure of Interest: None declared.

\section{P248}

Hospital-acquired infections associated to COVID-19 intensive care unit at a Tunisian hospital: determinants factors

I. Mlouki ${ }^{1,2,3,},{ }^{*}$, H. Khelij ${ }^{4}$, O. Jaoued ${ }^{5}$, F. Ben youssef ${ }^{1,6},{ }^{1}$ N. Omri ${ }^{1}, H$.

Bayoudh ${ }^{7}$, H. Sfar ${ }^{1}$, N. Sebai ${ }^{8}$, H. Nouira ${ }^{5}$, M. F. Hassen ${ }^{5}$, S. Atrouss ${ }^{5}$, S. El Mhamdi $1,2,3$

${ }^{1}$ Preventive and Community Medicine, University Hospital Taher Sfar Mahdia, Mahdia, ${ }^{2}$ Community Medicine, ${ }^{3}$ Research laboratory "Epidemiology Applied to Maternal and Child Health"12SP17, Faculty of Medicine of Monastir, Monastir, ${ }^{4}$ Department of Microbiology, University Hospital

Taher Sfar, Intensive Care Unit, University Hospital Taher Sfar Mahdia, Mahdia, ${ }^{6}$ Preventive and Community Medicine, Faculty of Medicine of Monastir, Monastir, ${ }^{7}$ Family Medicine, ${ }^{8}$ Department of Microbiology, University Hospital Taher Sfar Mahdia, Mahdia, Tunisia

Correspondence: I. Mlouki

Antimicrobial Resistance \& Infection Control 10(1): P248

Introduction: Hospital-acquired infections pose a serious challenge to healthcare workers during the COVID-19 pandemic.

Objectives: We aimed at assessing associated factors to hospitalacquired infection in the COVID-19 Intensive Care Unit (ICU) in the region of Mahdia (Tunisia).

Methods: We conducted a prospective study from September 2020 to February 2021 among COVID-19 patients in the ICU of the University Hospital Taher Sfar Mahdia from hospital admission to discharge or death. We used the "Rapid core case report form" developed by World Health Organization (WHO) for the COVID-19 data plateform.

Results: A total of 114 patients were enrolled with a mean age of $61.28 \pm 12.19$ years and median length stay of 14 days (IQR 9-24). Diabetes (43.4\%) and hypertension (38.6\%) were the most frequent underlying diseases. Fifty-four patients (54.2\%) developed nosocomial infection after a median of 7 days (IQR 4-11) of admission. Pneumonia (29.8\%) and bacteremia (28\%) were the most common infection sites. In $34.2 \%$ of cases, infection was related to resistant bacteria ( $100 \%$ of multi-resistance for Acinetobacter baumanii and $90.5 \%$ for Klebsiella pneumonia).

Infection was significantly associated with severe acute respiratory distress syndrome (OR 4.4, 95\% $\mathrm{Cl} 1.3-10.9, \mathrm{p}=0.018$ ) and longer invasive ventilation (more than 7 days) (OR 10.4, 95\% Cl 9.7-20.5, $\mathrm{p}<0.001$ ).

Conclusion: Measures to reduce Hospital-acquired infections such as implementing antimicrobial stewardship programmes are needed in order to achieve better therapeutic outcomes.

Disclosure of Interest: None declared.

P249

The burden of healthcare-associated infections in COVID-19 patients in Trnava university hospital during first year of pandemic

J. Prnová ${ }^{1,2,{ }^{*},}$, Z. Škvarková ${ }^{1}$, H. Straková1, A. Strehárová ${ }^{2}$, J. Brňová ${ }^{1,2}$

1 Department of Hospital Hygiene and Epidemiology, University Hospital

Trnava, ${ }^{2}$ Centre for Microbiology and Infection Prevention, School

of Health Sciences and Social Work, Trnava University, Trnava, Trnava

University, ${ }^{3}$ University Hospital Trnava, Trnava, Slovakia.

Correspondence: J. Prnová

Antimicrobial Resistance \& Infection Control 10(1): P249 
Introduction: The COVID-19 pandemic has brought new challenges in the field of the healthcare-associated infection (HCAl) caused by multidrug-resistant microorganisms.

Objectives: The objective of the study was to describe the burden, epidemiology and outcome of healthcare-associated infection in hospitalized patients with COVID-19.

Methods: We conducted a retrospective cohort study which included COVID-19 positive adult ( $\geq 18$ years) admitted between 16 March 2020 and 31 March 2021 to the 638-bed University Hospital Trnava in Slovakia. We analyzed epidemiological and microbiological features as well as outcome data. The European Centers for Disease Control and Prevention definition of HCAl and statistical package R-project were used for data analysis.

Results: Overall 1517 patients were hospitalized with COVID-19 during the study period (median age 69 years; IQR $57-78 ; 54.3 \%$ male). A total of $330(22.0 \%) \mathrm{HCAl}$ were diagnosed in 220 patients (14.6\%) with the median 9 days (IQR 4-14) upon hospital admission. Respiratory infection $(38.8 \%)$, urogenital infection $(27.9 \%)$, bloodstream infection (14.5\%), and infection caused by Clostridioideus difficile (14.2\%) were the most frequent. Overall 550 different microorganisms were isolated: $64,5 \%$ gram-negative, $15.3 \%$ fungi. The most common cause of HCAI was Klebsiella spp. (24.2\%; $40.6 \%$ carbapenem producing Klebsiella spp., KPC) and Pseudomonas aeruginosa present in $21.5 \%$ of infections (56.2\% carbapenem resistant Pseudomonas aeruginosa). The mortality of patients developing HCAI was $19.2 \%$. In-hospital mortality was significantly higher in comparison with patients without HCAI $(41.1 \%$ vs. $28.6 \% \mathrm{p}<0.001)$. Patients who developed HCAl did have higher risk of the dead (OR 1.7; 95\% Cl 1.2-2.1; $p<0.001)$.

Conclusion: Healthcare-associated infections in COVID-19 patients are an important cause of mortality and morbidity. We identified a higher rate of HCAl in comparison with a reported study which can be caused by a large influx of COVID-19 patients in the second wave of the pandemic.

Disclosure of Interest: None declared.

\section{P250}

Impact of the COVID-19 outbreak on the trend

of healthcare-associated infection rate in a teaching hospital of Tunisia

S. Bhiri ${ }^{1}$, H. Ghali ${ }^{1}{ }^{*}$, A. Ben Cheikh ${ }^{1}$, W. Dhouib ${ }^{2}$, R. Bannour ${ }^{2}$, S. Khefacha ${ }^{2}$, H. Said Latiri ${ }^{1}$, M. Ben Rejeb ${ }^{1}$

${ }^{1}$ Department of prevention and security of care, Sahloul university hospital, Faculty of Medicine of Sousse, University of Sousse, ${ }^{2}$ Department of prevention and security of care, Sahloul university hospital, Sousse, Tunisia

Correspondence: $\mathrm{H}$. Ghali

Antimicrobial Resistance \& Infection Control 10(1): P250

Introduction: The coronavirus disease 2019 (COVID-19) pandemic has had an enormous impact on healthcare systems globally. Although these efforts have understandably taken immediate priority, the impacts on traditional healthcare-associated infection (HAl) surveillance and prevention efforts remain concerning.

Objectives: This study aimed to describe the trends of healthcareassociated infection rate at Sahloul university hospital of Sousse, Tunisia within the current COVID-19 outbreak.

Methods: Since 1991, the infection control team of the prevention and security of care department has conducted annual point prevalence surveys in March and early April, as part of a quality improvement program promoted by our department in Sahloul university hospital for the prevention and control of HAls. A cross-sectional design was used to assess the HAI rate during and before COVID-19 outbreak in our hospital.

Results: The total rate of HAls was $10.1 \%$ in 2021 during the COVID-19 outbreak, which was $15.5 \%$ in the past year $(p=0.06)$. This decrease was observed in all of the three types of wards (surgical, intensive care and medical). However, it was only significant for intensive care units $(p=0.01)$ and medical wards $\left(p<10^{-3}\right)$. The prevalence of infected patients decreased from $14.9 \%$ in 2020 to $9.6 \%$ in 2021 . However, this decrease was not statistically significant. Except for surgical site infection, the rates of pneumonia/lower respiratory tract infection and other infections, decreased significantly compared the past year $\left(p<10^{-3}\right)$. A 6.5-point increase was observed in the rate of urinary tract infection and a 19.6-point increase in blood stream infection (BSI) during the COVID-19 outbreak compared with the past year; however, this was statistically significant only for BSI.

Conclusion: The results of the present study showed a reduction in the rate of HAls during the COVID-19 outbreak. Therefore, HAI can be minimized by increasing awareness, creating a positive attitude, improving staff hygiene behaviors, and providing facilities to comply with the standards of infection control protocols.

Disclosure of Interest: None declared.

\section{P251}

Prevalence of health care-associated infections during the COVID-19 pandemic at a university hospital in Sousse. Tunisia 2021

W. Dhouib ${ }^{1}$, S. Bhiri ${ }^{1,2}$, A. Ben Cheikh ${ }^{1,2}$, H. Ghali, ${ }^{1,2},{ }^{*}$, R. Bannour ${ }^{1}$, S.

Khefacha ${ }^{1}$, H. Said Latiri ${ }^{1,2}$, M. Ben Rejeb ${ }^{1,2}$

${ }^{1}$ Department of Prevention and Security of Care, sahloul university hospital, ${ }^{2}$ Faculty of Medicine of Sousse, University of Sousse, Sousse "T Tunisia

Correspondence: $\mathrm{H}$. Ghali

Antimicrobial Resistance \& Infection Control 10(1): P251

Introduction: Worldwide, Health Care-Associated infections (HCAls) have a significant impact on morbidity, mortality and quality of life. At the local level, the prevalence of HCAls at Sahloul Hospital was $6.1 \%$ in $2018,9.5 \%$ in 2019 and $15.5 \%$ in 2020 (following the first wave of COVID-19 in Tunisia).

In this particular context, we conducted our annual prevalence study.

Objectives: The objective was to determine the prevalence of HCAls at Sahloul hospital in 2021; the typology of infections as well as the antibiotic resistance profile.

Methods: We conducted a cross-sectional study of HCAls prevalence with a single passage in March. The agreed definition of an HCAls was any infection that occurs during a hospital stay of at least $48 \mathrm{~h}$ and if it was not present at admission. All hospital departments were included in the survey.

Results: A total of 228 patients were included in the study with a mean age of 58 years and a sex ratio of 1.15. A total of 23 HCAls were identified, representing a prevalence of HCAls of $10.0 \%$. The prevalence of infected patients was particularly higher (20.0\%) in intensive care units (ICU) than in other departments of the hospital. The most common infections were urinary tract infections (31\%) and bacteremia (26\%). Of the 23 HCAls identified, 20 were microbiologically documented, 23 different germs were isolated (the sample contained two germs in three patients,). Of these 23 isolated germs, $47 \%(n=11)$ were Gram Negative Bacilli and $30 \%$ had a high bacterial resistance.

Conclusion: The 2021 prevalence does not differ from the rates usually observed in recent years (around 10\%) with the exception of the previous year 2020 (15.5\%). This return to the "normal" HCAls prevalence rate in 2021 could be explained by the increasingly rigorous application and adherence to standard hygiene precautions during the COVID-19 pandemic.

Disclosure of Interest: None declared.

\section{P252}

Nosocomial infection: a comparative study between COVID19 patients and other hospitalized patients in a Tunisian university hospital

R. Bannour ${ }^{1, *}$, S. Bhiri ${ }^{1,2}$, A. Ben Cheikh ${ }^{1}$, H. Ghali ${ }^{1,2}$, W. Dhouib ${ }^{1}$, S.

Khefacha ${ }^{1}$, H. Said Latiri $^{1,2}$, M. Ben Rejeb ${ }^{1,2}$

${ }^{1}$ Department of Prevention and Security of Care, Sahloul University

Hospital, ${ }^{2}$ Faculty of Medicine of Sousse, University of Sousse, Sousse,

Tunisia, Sousse, Tunisia

Correspondence: R. Bannour

Antimicrobial Resistance \& Infection Control 10(1): P252 
Introduction: Nosocomial infections (NI) are major patient safety problems in hospitals. As patients infected by SARS-CoV- 2 need prolonged hospital stays and heavy treatments, they may be present higher incidence of NI.

Objectives: The aim of this study is to compare $\mathrm{NI}$ rates and its risk factors between patients presenting COVID 19 infection and other hospitalized patients.

Methods: A point-prevalence study was conducted in Sahloul university hospital on March 2021 among patients hospitalized for more than $48 \mathrm{~h}$.

Data collection was carried out through anonymous standardized survey record inspired from the NosoTun plug. Data extracted from medical records included diagnoses, laboratory results, microbiological data, and antibiotic use. Microbiologically-confirmed bacterial and fungal pathogens from clinical cultures were evaluated to characterize community- and $\mathrm{NI}$.

Results: A total of 228 patients were included with a total prevalence of $\mathrm{NI}$ of $10.08 \%$ ( 23 cases). Schematically patients presenting $\mathrm{NI}$ were departed into two groups according to their COVID 19 status; the prevalence of $\mathrm{NI}$ among patients with COVID19 was $15.4 \%$ vs $8.9 \%$ among other patients $(p=0.2)$.

Concerning risk factors; there was a significant difference between the two groups in term of mean age (66 vs 47 years respectively; $\mathrm{p}=0.014)$; medical history of diabetes $(100 \%$ vs $22.2 \%$; $p=0.01)$; presence of urinary catheter ( $100 \%$ vs $27.8 \% ; p=0.015)$; peripheral venous catheter $(100 \%$ vs $27.8 \% ; p=0.01)$ and mechanical ventilation $(100 \%$ VS $16.7 \% ; p=0.005)$.

Respiratory infection and Bacteremia were the most common sites of infection in COVID-19 patients and the isolated microorganisms were acinetobacter; while it was sataphylococcus auerus for other patients. Conclusion: Minimizing NI transmission remains a challenge, especially in the COVID 19 pandemic context that has placed a large burden on hospitals and healthcare providers. it is crucial to deepen our understanding transmission pathways of $\mathrm{NI}$ in order to implement infection prevention guidelines and enhance protection of patients. Disclosure of Interest: None declared.

\section{P253}

Trend of healthcare associated infection's prevalence over 10 years (2010-2020) in a teaching hospital in Tunisia H. Hannachi $i^{1,2}$, D. Chebil ${ }^{1,2}$, D. ben hassine ${ }^{1}$, S. chelly ${ }^{1,2}$, E. arfaoui ${ }^{1}$, L. merzougui ${ }^{1,2, *}$ on behalf of all the staff of prevention an infection control ${ }^{1}$ prevention and infection control department, kairouan, ${ }^{2}$ Faculty of medicine of Sousse, University of Sousse, SOUSSE, Tunisia Correspondence: $L$. merzougui

\section{Antimicrobial Resistance \& Infection Control 10(1): P253}

Introduction: Healthcare associated infections (HAls) have been identified as a problem of public health in all over the world. In Tunisia, national surveys on the prevalence of HAls have been routinely taken place in health facilities. In our hospital, we conduct a prevalence survey yearly as part of the prevention and control program of HAls.

Objectives: We aimed to describe the trend of HAls'S prevalence in Ibn ElJazzar Hospital over 10 yearsas well as the most frequent identified site infections.

Methods: It is a cross-sectional study of one-day prevalence with a single pathway, including all patients who had been hospitalized for at least $48 \mathrm{~h}$. Data collection was carried out using Noso-Tun form (national HAl prevalence survey).

Results: over 10 years, the prevalence of HAls ranged from $11.9 \%$ in 2010 to $3.19 \%$ in 2015 and then to $10.32 \%$ in 2020 . The most frequent site infectionthat was found in 2010, 2012, 2013 was urinary tract infection with respectively the proportions of $36.7 \%(11 / 30)$, $31.3 \%(5 / 16)$ and $37.5 \%(3 / 8)$. Blood stream infection was the most important infection localization in 2011 (38.5\%), in 2014(57.14\%) and in 2020 (22.3\%). Pneumonia had the higher prevalence over 2 years (2016 and 2017). The bacteriological analysis was performed in $60.3 \%$ of HAls cases. In $46.3 \%$ of cases $(n=19)$, isolated bacteria
wasEscherichia Coli. In second position, Klebsiellapneumoniae was identified in $26.8 \%$ of cases $(n=11)$ followed by enterobacteriaccaein $19.5 \%(n=8)$.

Conclusion: This prevalence survey showed obviously the evolution of healthcare associated infections over 10 years. It could help us adjust the infection control program in our hospital and implement the different strategies to prevent the increasing rate of HAls.

Disclosure of Interest: None declared.

P254

Prevalence and determinants of healthcare associated infection in a teaching hospital in a country of North Africa D. chebil ${ }^{1,2,}{ }^{*}, H$. hannachi ${ }^{1,2}$, D. ben hassine ${ }^{1}$, S. chelly ${ }^{1,2}$, E. arfaoui ${ }^{1}$, L. merzougui ${ }^{1}, 2$

${ }^{1}$ prevention and infection control department, kairouan, ${ }^{2}$ Faculty of medicine of Sousse, University of Sousse, SOUSSE, Tunisia

Correspondence: D. chebil

Antimicrobial Resistance \& Infection Control 10(1): P254

Introduction: Healthcare Associated Infection (HAls) are a major safety concern for both health care providers and patients. They continue to increase at an alarming rate.

Objectives: Aim of the study was to determine prevalence and associated factors of healthcare care associated infections in the University Hospital IbnElJazzar in Kairouan, Tunisia.

Methods: This was a cross sectional study conducted in 13 wards in the Teaching HospitallbnElJazzar in Kairouan, Tunisia in March 2020 All patients hospitalized over $48 \mathrm{~h}$ were included in the study. Data were provided from patients' files. Data entry and analysis was done using SPSS version 22.

Results: A total of 184 patients were enrolled in this study. The prevalence of healthcare acquired infections was $10.32 \%(19 / 184)$. The ratio of infection/infected was $1.35(19 / 14)$. Urinary tract infection was the most frequent site infection (26.3\%), followed by:blood stream infection, respiratory tract infection $(15.7 \%)$, and surgical site infection(10.5\%). Risk factors that were associated to healthcare associated infections were: Obesity $\left(p=\leq 10-^{3}\right)$, immunodeficiency $(p=0.008)$, urethral catheterization $(p=0.002)$, central venous catheter $(p=0.016)$, peripheral venous catheter $(p=0.005)$, artificial ventilation ( $p=0.001)$, parenteral nutrition $(p=0.005)$, surgery $(p=0.041)$. Conclusion: The prevalence of healthcare associated infection is relatively high. Thus, many recommended measures should be soon taken set up a robust infection control program, describe procedures related to medical device handling, encourage healthcare professionals to respect standard and complementary hygiene measures.

Disclosure of Interest: None declared.

P255

Healthcare-associated infections in a hospital in central eastern Tunisia: particularities and prevention strategies

M. Mohamed ${ }^{1}$, S. Chelly ${ }^{1,}$, O. Ezzi ${ }^{1}$, A. Asma ${ }^{1}$, B. Trabelsi ${ }^{1}$, M. Sahnoun ${ }^{1}$, M. Njah ${ }^{1}$

${ }^{1}$ Hospital hygiene department Farhat Hached teaching hospital, Sousse,

Tunisia

Correspondence: S. Chelly

Antimicrobial Resistance \& Infection Control 10(1): P255

Introduction: Healthcare-associated infections constitute a real public health problem. To limit the damage in terms of morbidity and mortality, and to develop effective prevention strategies, knowledge of its extent and predisposing factors is essential.

Objectives: Determine the prevalence of healthcare-associated infections, identify the risk factors associated and guide prevention strategies. Methods: It was a cross-sectional prevalence study carried out over 7 days, from December 28, 2017 to January 3, 2018 in 19 clinical departments of the Farhat Hached CHU in Sousse. The definition of healthcare-associated infections was that of the Centers for Disease Control and Prevention (CDC). Trained investigators collected data from the medical file and follow-up sheets using standardized sheets. Results: A total of 371 patients were included with an $M / F$ sex ratio of 0.75 and a median age of 38 years. The prevalence of infected patients 
was $15.9 \%$ and the number of healthcare-associated infections was $18 \%$. Lung infections were the most common (22.3\%). Multivariate analysis showed that age $\leq 16\left(\mathrm{OR}_{\mathrm{A}}=3.69\right.$ [1.88-7.26]), immunosuppression $\left(\mathrm{OR}_{\mathrm{A}}=2.07\right.$ [1.04-4.12]), antibiotic therapy in the six months before admission $\left(\mathrm{OR}_{\mathrm{A}}=2.11\right.$ [1.13-3.92]), admission by transfer $\left(\mathrm{OR}_{\mathrm{A}}=2.33[1.08-5]\right)$, gastric catheterization $\left(\mathrm{OR}_{\mathrm{A}}=3.72[]\right)$, peripheral vascular catheterization $\left(\mathrm{OR}_{\mathrm{A}}=2.42[1.13-5.19]\right)$ and central vascular catheterization $\left(\mathrm{OR}_{\mathrm{A}}=4.41[1.65-11.77]\right)$ were independent risk factors for healthcare-associated infections.

Conclusion: The epidemiological surveillance of healthcare-associated infections makes it possible, on the one hand, to orient and better target prevention programs and, on the other hand, makes it easier to evaluate control actions. Prevention can only be conceived in the form of comprehensive and multidisciplinary action.

Disclosure of Interest: None declared.

P256

Assessment of trends in healthcare-associated infections in a Tunisian university hospital through repeated point-prevalence surveys (2012 - 2020)

H. Ghali ${ }^{1, *}$, A. Ben Cheikh ${ }^{1}$, S. Bhiri ${ }^{1}$, W. Dhouib ${ }^{2}$, R. Bannour ${ }^{2}$, S. Khefacha ${ }^{2}$ H. Said Latiri ${ }^{1}$, M. Ben Rejeb ${ }^{1}$

${ }^{1}$ Department of Prevention and Security of Care, Sahloul university hospital, Faculty of Medicine of Sousse, University of Sousse, ${ }^{2}$ Department of Prevention and Security of Care, Sahloul university hospital, Sousse, Tunisia

Correspondence: $\mathrm{H}$. Ghali

Antimicrobial Resistance \& Infection Control 10(1): P256

Introduction: Healthcare-associated infections (HAls) represent substantial burden on health care systems in developing countries. Surveillance is one of the most effective prevention methods of HAls in hospitals worldwide.

Objectives: The aim of our study is to describe trends in HAls in a Tunisian university hospital through repeated point-prevalence surveys over nine years $(2012-2020)$, and to identify associated factors of HAI.

Methods: The current study focused on data collected from 2012 to 2020 from all departments except emergency and hemodialysis services. All types of HAls as defined by the Centers for Disease Control and Prevention (CDC) were included. Data collection was carried out using NosoTun plug. Patient data form was structured according to the following sections: demographic data, admission data, clinical data, antimicrobials (AM) use and HAI data. Univariate and multivariate logistic analysis were used to identify HAI risk factors.

Results: Overall, 2729 patients were observed in the nine surveys; the mean age was $48.3 \pm 23.3$ years and $57.5 \%$ were male. We identified 267 infected patients (9.8\%) and 296 HAls (10.8\%). Pneumonia/lower respiratory tract infections were the most frequent $\mathrm{HAI}(24 \%)$, followed by urinary tract infection $(20.9 \%)$. The prevalence of infected patients increased from $10.6 \%$ in 2012 to $14.9 \%$ in 2020 . However this increase was not statistically significant. The prevalence of HAls increased significantly from $12.3 \%$ to $15.5 \%(p=0.003)$. The only decrease involved bloodstream infections (from 2 to $1 \%$ ).

Independent risk factors significantly associated with HAI were undergoing surgical intervention $(\mathrm{aOR}=1.7)$, the use of antibiotic treatment in 6 months $(a O R=1.8)$, of peripheral line $(a O R=2)$, parenteral nutrition $(\mathrm{aOR}=2.4)$, urinary tract within 7 days $(\mathrm{aOR}=2.4)$, central line $(\mathrm{aOR}=6.3)$, and prosthesis $(\mathrm{aOR}=12.8)$, length of stay $(\mathrm{aOR}=3)$, and the year of the survey. Young age was found as protective factor $(\mathrm{aOR}=0.98)$.

Conclusion: Sahloul university hospital has a long history of activities aimed at risk management and infection control, based on a multimodal and multidimensional approach which were reinforced after the coronavirus pandemic.

Disclosure of Interest: None declared.
P257

Prevalence of healthcare-associated infections at a Tunisian university hospital

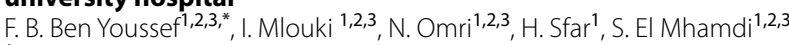

${ }^{1}$ Preventive and Community Medicine, University Hospital Taher Sfar,

${ }^{2}$ Community Medicine, Faculty of Medicine of Monastir, ${ }^{3}$ Research

laboratory, "Epidemiology Applied to Maternal and Child Health", Mahdia,

Tunisia

Correspondence: F. B. Ben Youssef

Antimicrobial Resistance \& Infection Control 10(1): P257

Introduction: The fight against Healthcare Associated Infections ( $\mathrm{HAI})$ is a challenge to health care providers. The epidemiological surveillance strategy for HAl is based on several actions such as carrying out regular local prevalence surveys in hospitals.

Objectives: The aim of the study was to estimate the prevalence of HAl at the University Hospital Tahar Sfar Mahdia (Tunisia).

Methods: A cross sectional study was performed from the $1^{\text {st }}$ to the $6^{\text {th }}$ of February 2021 among patients admitted for $\geq 48 \mathrm{~h}$ in all departments of the Hospital of Tahar Sfar Mahdia.. We used a standardised measurement tool developed by experts in the Department of Preventive Medicine.

Results: We included 128 patients ( $52.3 \%$ male) with a mean age of $49.15 \pm 26.01$ years and extremes ranging from 2 days to 96 years. Among them, 41 (32\%) were infected and 61 (47.7\%) were taking antibiotics the day of the study. About $16.4 \%$ of patients were hospitalized in intensive care units.

Twenty three participants had at least one HAI, for a prevalence rate of infected patients of $18 \%$.

A total of 33 co-infections were identified among these patients ( 7 patients with two infections each and 1 patient with 3 infections), for a prevalence of nosocomial infections of $25.7 \%$. The most common infection sites were: urinary tract infection (33\%), septicemia (21.2\%) and Respiratory infection (15.1\%).

Pseudomonas Aeruginosa, Escherichia coli and Acinetobacter baumannii were the most frequently responsible for nosocomial infections (respectively $21 \%, 18.7 \%$ and $18.7 \%$ ) with $100 \%$ of bacterial resistance for Acinetobacter (6/6).

Conclusion: The surveillance of $\mathrm{HAl}$ is crucial in order to measure the level of infectious risks, define the prevention policy to be carried out, and evaluate the effectiveness of this prevention policy.

Disclosure of Interest: None declared.

P258

Point prevalence survey (PPS) of healthcare associated infections (HCAI) in selected hospital in Malaysia

N. F. Bakhtiar ${ }^{1}$ on behalf of Infection Control Unit, S. Amir Husin 1,* on behalf of Infection Control Unit

${ }^{1}$ Infection Control Unit, Ministry of Health Malaysia, Putrajaya, Malaysia Correspondence: S. Amir Husin

Antimicrobial Resistance \& Infection Control 10(1): P258

Introduction: Surveillance on HCAI helps to determine the epidemiological of HCAl and one of the tools to prevent HCAI. In Ministry of Health (MOH), National HCAl surveillance was conducted since 2012.

Objectives: The aim of this study is to determine the prevalence of HCAI, the types of HCAl, the organisms causing HCAl and the risk factors associated with $\mathrm{HCAl}$.

Methods: A one-day hospital wide PPS conducted over a period of 2 weeks in November 2019 involving 20 Ministry of Health (MOH) and 3 University Hospitals. The survey is based on the existing $\mathrm{MOH}$ guidelines and Center for Disease Prevention and Control (CDC)/National Healthcare Safety Network (NHSN) Surveillance Definition of HCAl and Criteria for Specific Types of Infections in the Acute Care Setting and European Centre for Disease Prevention and Control (ECDC), PPS of HCAl and antibiotics use in European acute care hospitals- protocol version 4.3. Stockholm: ECDC; 2012.

Results: A total of $962 \mathrm{HCAl}$ cases were reported from 23 participating hospitals in November 2019. The HCAl prevalence was 5.2 per 100 
admissions. The prevalence of each type of HCAl as shown in Table 1. Meanwhile the prevalence of devices related HCAls for VAP and CLABSI was $12.6 \%$ and $6.7 \%$ respectively. The most commonly found risk factors in patient with HCAI were underlying diseases, prolonged hospitalisation and history of surgery. The top 3 antimicrobial resistance (AMR) markers isolated were Methicillin-resistant Staphylococcus aureus (MRSA), 3rd Generation Cephalosporin Resistant Extended-spectrum beta-lactamases (ESBL) producing Klebsiella pneumoniae, and 3rd Generation Cephalosporin Resistant Extended-spectrum beta-lactamases (ESBL) producing Escherichia coli. The top 3 department with highest HCAl prevalence were Critical Care Unit (20.0\%) followed by Hematology (7.6\%) and Nephrology department (7.3\%).

Table 1: Prevalence of each type of $\mathrm{HCAl}$
\begin{tabular}{|l|l|}
\hline Types of HCAl & Incidence per 1000 admission \\
\hline Pneumonia & 17.9 \\
\hline Surgical site infection (SSI) & 11.3 \\
\hline Blood stream infection (BSI) & 11.0 \\
\hline Urinary tract infection (UTI) & 2.0 \\
\hline Clinical sepsis (CS) & 4.5 \\
\hline Others & 4.7 \\
\hline
\end{tabular}

Conclusion: National surveillance of $\mathrm{HCAl}$ is an important component in infection prevention and control (IPC) program as part of the quality improvement tool in reducing HCAl in healthcare facility.

Disclosure of Interest: N. F. Bakhtiar Employee of: Ministry of Health Malaysia, S. Amir Husin Employee of: Ministry of Health Malaysia.

\section{P259}

Incidence and microbiological profile of healthcare-associated infections in a Tunisian surgical intensive care unit

O. Ezzi ${ }^{1, *}$, A. Ammar ${ }^{1}$, Y. Ayedi ${ }^{1}$, F. Ferhi ${ }^{1}$, K. Ben Jazia' ${ }^{1}$, M. Njah ${ }^{1}$, M.

Mahjoub ${ }^{1}$

${ }^{1} \mathrm{CHU}$ Farhat Hached_Sousse_Tunisie, Sousse, Tunisia

Correspondence: O. Ezzi

Antimicrobial Resistance \& Infection Control 10(1): P259

Introduction: Healthcare-associated infections (HAls) occurring in patients treated in an intensive care unit (ICU) are serious complications in the treatment process. Their impact implies prolonged hospital stay, long-term disability, increased resistance of microorganisms to antimicrobials, a massive additional financial burden for health systems, high costs for patients and their families, and excess deaths.

Objectives: The aim of the study was to determine the incidence and microbiological profile of healthcare-associated infections in a Tunisian surgical intensive care unit.

Methods: A prospective cohort study was carried out in surgical ICU in a Tunisian university hospital.

An active surveillance method was used to detect HAls in adult patients who spent over $48 \mathrm{~h}$ in a surgical ICU ward located in Central Eastern Tunisia from January to December 2019.

Results: During the study period 84 patients were treated in the ICU, for more than $48 \mathrm{~h}$ each. Sex-Ratio was 2.43. Mean age was 50.7 years $(\mathrm{SD}=19.7)$. Median length of stay was 4 days $(\mathrm{IQR}=2-9)$.

A HAls incidence rate of 50 per 100 admissions and incidence of 44.6 per 1,000 patient days was observed. Among 42 HAls reported, pathogens could be identified for $33 \mathrm{HAl}$ cases (78\%).

The main types of $\mathrm{HAl}$ detected in ICU patients included the following: Pneumonia (60\%), Central Line Associated Infections (CLAl) (10\%) and Urinary infection (10\%).

Acinetobacter baumanii was the most common organism isolated (45\%) followed by Pseudomonas aeruginosa (21\%) and Klebsielle pneumonia (15\%).

Conclusion: Our results highlight the importance of implementation of active surveillance program.

In Tunisia, infection control programmes are a challenge for the future, and their implementation requires increasing the awareness of both medical staff and hospital management.

Disclosure of Interest: None declared.
P260

Risk factors of healthcare associated infections (HAIS)

in a Tunisian hospital in 2021

R. Bannour ${ }^{1, *}$, S. Bhiri ${ }^{1,2}$, A. Ben Cheikh ${ }^{1}$, H. Ghali, ${ }^{1}$, , W. Dhouib ${ }^{1}$, S.

Khefacha ${ }^{1}$, H. Said Latiri ${ }^{1,2}$, M. Ben Rejeb ${ }^{1,2}$

${ }^{1}$ Department of Prevention and Security of Care, Sahloul University

Hospital, ${ }^{2}$ Faculty of Medicine of Sousse, University of Sousse, Sousse,

Tunisia, Sousse, Tunisia

Correspondence: R. Bannour

Antimicrobial Resistance \& Infection Control 10(1): P260

Introduction: Healthcare-associated infection (HAI) is a major public health concern. Controlling this fatal scourge should begin with continuous monitoring of HAl frequency and its associated factors.

Objectives: The aim of this study was to determine independent factors of HAl in a Tunisian university hospital in 2021.

Methods: A cross-sectional survey was conducted within the epidemiological monitoring program at Sahloul University Hospital. The onset of infection within $48 \mathrm{~h}$ of hospitalization was used to define HAl. Multivariate analysis by a binary logistic regression step by step descendent was performed to assess the Independent factors of HAI.

Results: Overall, 228 patients were enrolled with a median age of 53.5 years old. Among them, 22 had at least one $\mathrm{HAl}$ and one patient had two, giving a prevalence of infected patients of $9.6 \%$ and a prevalence of infections of $10.08 \%$. The prevalence of infected patients was particularly higher (25\%) in intensive care units (ICUs) than in other departments of the hospital. Multivariate analysis of HAl gave these independent risk factors: Diabetes (OR $=3.97$ [1.23 - 12.81], Central venous catheter $(\mathrm{OR}=17.69$ [2.74- 113.94], and history of prosthesis during the previous year (OR=31.84 [5.17-196.07]).

Conclusion: Although aware of its limits, cross sectional study remains a simple research design suitable for HAI monitoring, feasible in our context of reduced resources. The prevalence HAl was $9.6 \%$. which drew a fairly stable trend in recent years, at around $10 \%$. Device-associated infections, particularly in critically ill patients should be targeted when scheduling Incidence studies and when tailoring HAI prevention actions.

Disclosure of Interest: None declared.

\section{P261}

What's the problem? How infection prevention and control teams find valuable problems with healthcare-associated infections

L. F. Irgang ${ }^{1, *}$, F. Gama ${ }^{1}$, M. Holmén ${ }^{1}$ on behalf of CIEL-Center

for Innovation, Entrepreneurship and Learning research

${ }^{1}$ School of Business, Innovation and Sustainability, Halmstad University, Halmstad, Sweden

Correspondence: $L$. F. Irgang

Antimicrobial Resistance \& Infection Control 10(1): P261

Introduction: Reducing healthcare-associated infections (HAls) is heavily contingent on proficient infection prevention and control (IPC) teams, with the ability of finding valuable problems that need to be solved. From a Problem-Findind and Problem-Solving Perspective, a valuable problem means a gap between a current and a desired situation, which in this study includes failures, deviations and opportunities for improvements in IPC practices. Once solved, valuable problems generate superior knowledge.

Objectives: This study aims to understand how IPC teams find valuable problems relating to HAls.

Methods: We performed a multiple case study of 3 hospitals located in Brazil and Sweden that recently faced outbreaks caused by Acinetobacter baumannii. We collected data from 3 exploratory and 13 semi-structured interviews with nurses and physicians enrolled in IPC teams. Supplementary documents were used for data triangulation. Data was analyzed using the thematic analysis technique.

Results: Our findings suggest an approach based on two different sets of activities for finding valuable problems: practices for HAI prevention and for HAI control. Practices for HAI prevention comprises routinely and elective actions whereas practices for $\mathrm{HAl}$ control involve ad hoc and emergent actions. The practices are organized 
into problem-detection, problem-framing, and problem-formulation activities.

Our study provides a framework (see Figure) to guide IPC teams' attention on how to find valuable problems relating to HAl prevention and control, as well as the criteria on how to prioritize the most important problems that need to be prioritized and solved. This ultimately culminates in valuable knowledge and in high-value solutions to be implemented to reduce HAl occurrence. Besides, our approach provides a range of practices that support IPC teams on how to timely shift from prevention to control decision-making modes.

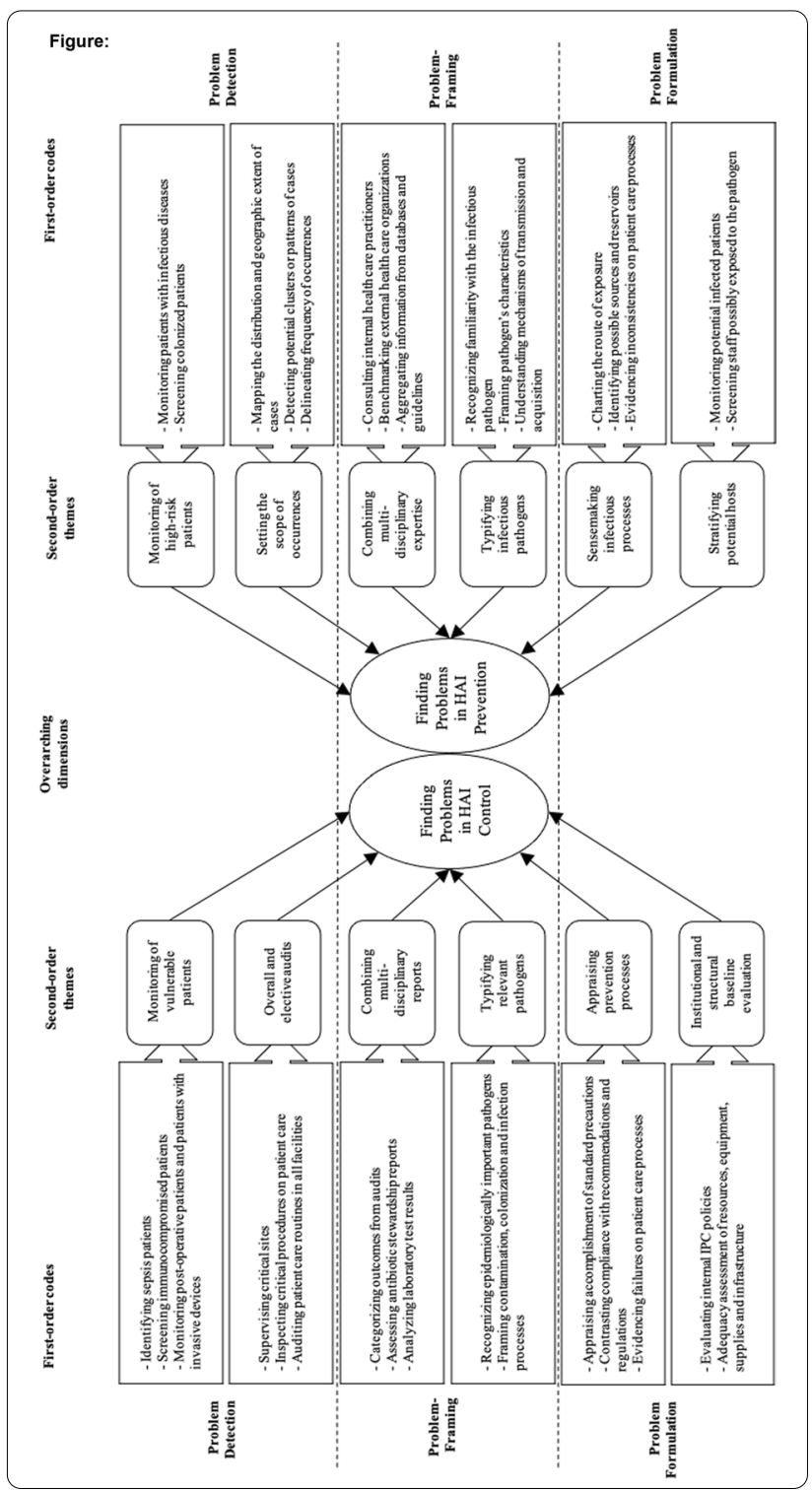

Conclusion: The study concludes that IPC teams that find valuable problems more quickly and efficiently reduce the occurrence of HAls and prevent outbreaks. Additionally, IPC teams that differentiate prevention practices from control practices and know when and how to shift from prevention to control decision-making modes obtain valuable problems and high-value solutions in terms while consuming less resources. Disclosure of Interest: None declared.
P262

Improving the peritoneal dialysis program in a pediatric hospital using six sigma methodology

S. E. Rivera Molina1, L. A. Madrid Rivera ${ }^{2,}$, , C. M. Romero Quiroz ${ }^{3}$, N. Rubio Zerón $^{4}, Y$. V. Donaire ${ }^{4}$

${ }^{1}$ Infectious Diseases, ${ }^{2}$ Medical Student, ${ }^{3}$ General Doctor, ${ }^{4}$ IPC Nurse, Hospital Maria Especialidades Pediátricas, Tegucigalpa, Honduras

Correspondence: L. A. Madrid Rivera

Antimicrobial Resistance \& Infection Control 10(1): P262

Introduction: Peritoneal dialysis (PD) improves survival in chronic kidney disease. Unfortunately there are complications associated with the use of PD catheters, $85 \%$ are of infectious origin carrying high burden of morbidity and mortality.

Objectives: Describe the PD program improvement recolecting data from an active 3-year surveillance.

Methods: We conducted a PD infection surveillance from 3 years to improve quality of care. An event of peritonitis was diagnosed with at least $2 / 3$ criteria:1.Clinical signs or symptoms(cloudy effluent or abdominal pain with fever or vomiting);2.Altered peritoneal fluid cell count(after a dwell time of $2 \mathrm{~h}: W B C$ above 100 cells/mm3 in an uncentrifuged sample, with at least 50\% neutrophils;or any WBC count with at least $50 \%$ neutrophils if the dwell time was less than $2 \mathrm{~h}$ );3.Positive peritoneal fluid culture. Patient and event data were recorded and analyzed using descriptive statistics.(StataCorp. 2019. Release 16.)

Results: We present results from December 1st, 2017 through December 31 st, 2020 in 112 pediatric patients who required $\mathrm{PD}(13,327$ catheter-days). 45 peritonitis episodes occurred in 42 individuals(42/112, 37.5\%). Peritonitis rate: 1.23 infections per patient-years(ideally<0.67). Thirty eight $(84.4 \%)$ of events were healthcare-associated. The median time from catheter placement to the event was 28 days(1-500 days). All patients had clinical signs or symptoms of peritonitis. Peritoneal fluid cultures were positive in only 18 events (40\%):61\% Gram-negative(Escherichia coli and Klebsiella pneumoniae the most common),33\% Gram-positive(Staphylococcus epidermidis the most common) and 1 Aspergillus spp. Thirteen events(29\%)required removal and permanent transfer to hemodialysis, six(13\%)died due to infection.

Conclusion: Recolecting data of PD infections during this period allowed us to identify risk factors and quantify the magnitude of the problem to recognize the major issues. This supported the infection prevention and control team with to implement and improve preventive measures for the PD Program.

Disclosure of Interest: None declared.

P263

Mortality in intensive care units: risk factors and attributable risk of healthcare associated infections, Tunisia (2018)

A. Ben Cheikh¹, N. Haddad ${ }^{2}$, S. Bhiri ${ }^{1}$, H. Ghali1,*, S. Khefacha ${ }^{2}$, H. Said Latiri $^{1}$, M. Ben Rejeb ${ }^{1}$

${ }^{1}$ Department of Prevention and Security of Care, Sahloul university hospital, Faculty of Medicine of Sousse, University of Sousse, ${ }^{2}$ Department of Prevention and Security of Care, Sahloul university hospital, Sousse, Tunisia

Correspondence: $\mathrm{H}$. Ghali

Antimicrobial Resistance \& Infection Control 10(1): P263

Introduction: The mortality in Intensive Care Units (ICUs) is estimated to represent one of the higher hospital mortality rates. Moreover, patients in ICUs are at a higher risk to develop Health care associated infections (HAls) than other patients.

Objectives: To determine the risk factors of mortality ICUs in Tunisia and to identify the attributable mortality of HAls.

Methods: This was a longitudinal descriptive study performed during 3 months in all ICUs of our University Hospital in Sousse, Tunisia in 2018. All patients admitted at least $48 \mathrm{~h}$ during the study period were investigated. HAls were defined as infections that occurred at least $48 \mathrm{~h}$ after admission to the ICU. Risk factors with a P-value less than 0.20 were initially included in the binary logistic regression model to determine the independent risk factors for hospital mortality. Then, $P$ values $\leq 0.05$ were considered statistically significant. The adjusted Population-Risk Attributable to HAls was calculated by the following formula: $(\mathrm{aPAR})=[\mathrm{p}(\mathrm{aOR}-$ $1) / p(a O R-1)+1] \times 100$. P: was the incidence rate of HAls. aOR: was the adjusted Odds Ratio. 
Results: A total, 202 patients were enrolled in the study period. The Median age was 53 years old (IQR: $24-66$ ). The Sex ratio was 1.62 . The total mortality incidence was $19.3 \%, \mathrm{IC}_{95 \%}$ [13.9-24.7]. The incidence rate of HAls was $32.6 \%$. The independent risk factors of ICU mortality were: Intubation [OR 7.54;IC $\mathrm{C}_{95 \%}(1.85$ - 30.74)], Tracheotomy [OR 3.01; IC $\left.C_{95 \%}(1.11-8.78)\right]$, Dialysis [OR 31.71; IC $C_{95 \%}$ (5.37- 187.27)] and HAls [OR 3.30;IC $\left.C_{95 \%}(1.11-9.80)\right]$.The attributable mortality of ICU acquired HAls was about $45 \%$ with an attributable mortality of pneumonia at $7 \%$ and bloodstream infection at $26 \%$.

Conclusion: This study suggested that Intubation, tracheotomy, dialysis and HAls are predictors of mortality in ICUs in Tunisia and that $45 \%$ of deaths in ICUs were attributable to HAls. Paying attention to reduce this major preventable complication would only translate better outcomes. Disclosure of Interest: None declared.

\section{P264}

Incredible cost of non-acting in infection prevention and control in Turkey

E. Alp ${ }^{1, *}$, H. O. Ari ${ }^{1}$, Y. Ozatkan ${ }^{1}$, E. Islek ${ }^{1}$, C. H. Hekimoglu ${ }^{1}$, M.-L. McLaws ${ }^{2}$

${ }^{1}$ Ministry of Health, Ankara, Turkey, ${ }^{2}$ NSW Health Department, Sydney, Australia Correspondence: E. Alp

Antimicrobial Resistance \& Infection Control 10(1): P264

Introduction: The implementation of national infection prevention and control programme (IPC) including national surveillance system and multi-modal hand hygiene strategy is essential for decreasing healthcare associated infections (HAls) and cost.

Objectives: A cost modeling study to show how cost-effective the reduction in hospital infection rates.

Methods: The structured IPC (infection control committees, training, surveillance, multimodal hand hygiene activities) has been put into practice since 2006 by $\mathrm{MoH}$. All these activities since 2006 significantly reduced HAls in Turkey and published in the literature (Gozel et al.). We conducted an analysis to reveal the gain in health expenditures from the reduction seen in Device associated (DA)-HAls types examined in the article. The simulation study was carried out under the assumption that if the infection control program mentioned in the study had not been applied, the rates of hospital infections in 2008 would remain the same in the following years. As a first step, we considered the patient day's figures between 2008 and 2017 years in the intensive care units in Turkey. Next, we made a simulation analyze in order to obtain the number of infections expected to occur for each year taking account the number of patient days for each year and the infection rates in 2008. In this way, we estimated the number of infections that would have occurred if the infection control program was not implemented. Cost data was obtained from cost studies conducted by Ağırbaş et al. regarding Device associated (DA)-HAls for hospitals in Turkey in 2011. The costs obtained in the relevant study were updated for each year by considering the inflation rates published by Turkish Statistical Institute for the following years and used in the calculations.

Results: It has been found that, due to interventions for the prevention of hospital infections, especially in intensive care units in Turkey, between 2009-2017 it is estimated that a total of 3,107,192,116 TRL cost savings were obtained. This corresponds to approximately 1,159,174,148 USD. Conclusion: Interventions implemented to reduce hospital infections have significantly reduced the costs of hospital infections and have had a decreasing effect on health expenditures in Turkey.

Disclosure of Interest: None declared.

Poster session: hospital-acquired and ventilator-associated pneumonia (HAP and VAP)

\section{P265}

Incidence, risk factors and clinical implications of post-discharge pneumonia after HIP arthroplasty, a population-based study of $\mathbf{5 5 , 8 4 2}$ patients in Poland

M. Gajda 1,* A. Pac² B. Gryglewska ${ }^{3}$ A. Różańska ${ }^{4}$, P. Gajda², J. Wójkowska-Mach ${ }^{4}$

${ }^{1}$ Doctoral School of Medical Sciences and Health Sciences, ${ }^{2}$ Department of Epidemiology, Chair of Epidemiology and Preventive Medicine,

${ }^{3} 4$ Department of Internal Medicine and Gerontology, ${ }^{4}$ Department of Microbiology, Jagiellonian University Medical College, Krakow, Poland Correspondence: M. Gajda

Antimicrobial Resistance \& Infection Control 10(1): P265

Introduction: Hip endoprostheses are one of the most frequently performed procedures, due to their impact on quality of life.

Objectives: The purpose of this study was to determine the incidence, risk factors, and clinical implications of post-discharge pneumonia (PNU) after hip endoprosthesis in Polish adults.

Methods: This retrospective study was conducted using the database of the National Health Fund, about 55,842 hip arthroplasties performed in 2017. The comparison between groups was based on the Chi-squared test or Fisher's exact test for variables with a very small number of observations. The risk of PNU development was assessed in the multivariable logistic regression model.

Results: ost-discharge PNU was identified in 371 patients and accounted for $26.6 \%$ of all post-discharge infections, (incidence rate of $0.7 \%$ ).

Multivariable analysis showed a significantly higher risk of PNU in patients aged 65-and-older (OR 3.47, 95\% Cl 2.40-5.03), urgently admitted (OR 3.97, 95\% Cl 3.16-4.98), operated in the winter (OR 1.7, $95 \% \mathrm{Cl} 1.37-2.11$ ) and hospitalized in the intensive care unit (OR 5.88, $95 \% \mathrm{Cl} 3.65-9.46)$. Preventative factors were diseases of the musculoskeletal system (OR $0.73,95 \% \mathrm{Cl} 0.59-0.91)$ and post-operative rehabilitation (both outpatient and inpatient OR $0.32,95 \% \mathrm{Cl} 0.10-0.99$, and $\mathrm{OR} 0.65,95 \% \mathrm{Cl} 0.42-0.99$, respectively). The influenza vaccine in 208 patients $(0.5 \%)$ had no significant impact on the risk of PNU $(p=0.708)$. The in-hospital case fatality rate in observed post-discharge PNU was $21.4 \%$.

Conclusion: PNU is one of the most common postoperative infections after hip endoprosthesis, especially in the winter. Postoperative rehabilitation reduces the risk of PNU. The low rate of influenza vaccination is resulting in no impact on the risk of PNU.

Disclosure of Interest: None declared.

P267

Implementing an oral care protocol in the hospital setting

to reduce non-ventilator hospital acquired pneumonia

J. Simmons ${ }^{1, *}$

${ }^{1}$ Purdue University Global, Kirkland, United States

Correspondence: J. Simmons

Antimicrobial Resistance \& Infection Control 10(1): P267

Introduction: The interaction between main risk factors of oral microbiota, aspiration, and patient condition has potential to create the opportune atmosphere for hospital acquired pneumonia (HAP) to develop. Oral care is a modifiable risk factor for Non-Ventilator Hospital Acquired Pneumonia (NV-HAP). Yet oral care provision at the selected facility was inconsistent due to inaccessible or unavailable oral care supplies, possible low priority with nursing staff, and lack of a standard oral care protocol.

Objectives: An oral care protocol was implemented within the hospital setting to reduce NV-HAP. The protocol included all inpatient adult units, excluding labor and delivery along with the centers for mental health units.

Methods: Evaluation of the project was conduction through pre and post intervention comparison of ICD-10 codes, nursing documentation of oral care, oral care prevalence survey, and nursing knowledge survey.

Results: Post implementation evaluation data showed an increase in oral care practice based on nursing documentation, availability and use of oral care supplies. Improved understanding of providing oral care to prevent HAP, made oral care a higher priority for nursing staff. While data was limited, a decrease in NV-HAP was noted the month following the oral care protocol implementation.

Conclusion: Implications for practice was a change in culture. While nursing knowledge post implementation survey showed no change in nursing's understanding of the impact of oral care on the patient's overall health, previous perceptions of oral care being a low priority task for patient comfort has changed. This is reflected by increased nursing documentation of oral cares provided, increased patient 
access and use of oral care supplies, more reported patient assistance and education, as well as knowledge of independent patient's oral care habits during hospitalization. Oral care has become a higher priority for nursing staff.

Disclosure of Interest: None declared.

Poster session: infection prevention and control: Implementation and patient safety

\section{P268}

What will infection prevention look like in 2030—results of the first round of a global crystal ball exercise

H. Sax 1,*, L. Clack ${ }^{2}$, S. Kuster ${ }^{1}$, J. Marschall ${ }^{3}$, M. Schlegel ${ }^{4}$, P. W. Schreiber ${ }^{1}$, A. Wolfensberger ${ }^{1}$ on behalf of Future IPC Collective \& Swissnoso

${ }^{1}$ Infectious Diseases and Hospital Epidemiology, University Hospital Zurich, University of Zurich, ${ }^{2}$ Implementation Science In Healthcare, University Zurich, Zurich, Infectious Diseases, Bern University Hospital and University of Bern, Bern, ${ }^{4}$ Cantonal Hospital St Gallen, St Gallen, Switzerland

Correspondence: H. Sax

Antimicrobial Resistance \& Infection Control 10(1): P268

Introduction: Healthcare delivery is currently undergoing radical changes and so will the demands for effective Infection prevention in healthcare (IPC).

Objectives: We initiated a global collaboration among IPC professionals to imagine the status of IPC in 2030 to inform the present.

Methods: In JAN 2019, a purposive sample of 44 IPC professionals around the globe were invited to answer a 10 -item online questionnaire, including 2 demographics, 4 housekeeping and 4 open-ended core questions: (Q1) "status of IPC in 2030", (Q2) "position \& people in charge of IPC", (Q3) "necessary skills", and (Q4) "open questions". The latter were each submitted to inductive content analysis and displayed by semi-quantitative network mapping, the remainder reported descriptively.

Results: Overall, 18 of 44 (41\%) invited responded JAN-MAR 2019 (6 US, 2 CA, 2 CH, 1 FR, DE, MX, NL, SG, UK, each; 15 with physician, 3 with nurse, and 1 with management background; all in senior positions). The main emerging themes for Q1 were "multidrug-resistant organisms"; "automatisation of data collection, processing \& feedback" with the sub-themes 'robotics', 'monitoring', 'surveillance', 'short-circuit feedback'; "system integration \& broadening of IPC" with 'patient participation'; "global perspective" with 'low/middle income country challenges', 'outpatient'; "behaviour"; and "implementation" (Figure). The views were predominantly positively (66\%) oriented. Q2 and Q3 yielded a broad range of professional profiles, ranging from data, behaviour, implementation, communication know-how and skills, positioning IPC highly in healthcare institutions and beyond. Similarly, Q4 covered a large area including medicine, life-science, data science, social science, organisational and political questions.

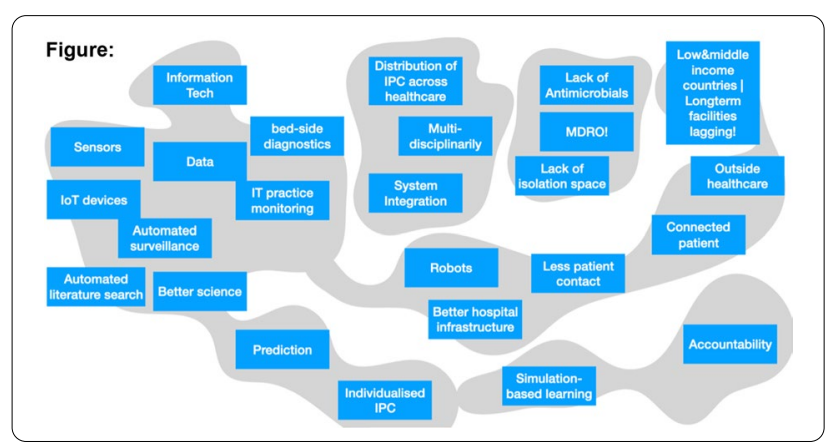

Conclusion: The first round of the Future IPC project produced a mainly positive picture of ICP in 2030. The project will continue with further rounds of multi-method inquiry with evolving participation (including an assessment of changes attributable to insights gained during the SARS-CoV-2 pandemic) to serve as a roadmap for developing this critical field of medicine.

Disclosure of Interest: None declared.

\section{P269}

Strategies to improve infection control link nurse programs

M. Dekker ${ }^{1}{ }^{*}$, I. Jongerden ${ }^{2}$, M. de Bruijne ${ }^{2}$, C. Vandenbroucke-Grauls ${ }^{1}$, R. van Mansfeld ${ }^{1}$

${ }^{1}$ Department of Medical Microbiology and Infection Prevention, ${ }^{2}$ Department of Public and Occupational Health, Amsterdam UMC, Amsterdam,

Netherlands

Correspondence: M. Dekker

Antimicrobial Resistance \& Infection Control 10(1): P269

Introduction: Infection control link nurses (ICLN) experience various barriers in daily practice. Identification of strategies to address these barriers can improve current ICLN programs and guide their future implementation.

Objectives: To identify strategies for effective implementation of ICLN programs using the Consolidated Framework for Implementation Research (CFIR)-Expert Recommendations for Implementing Change (ERIC) Implementation Strategy Matching tool.

Methods: We conducted a Delphi study. A panel of eight experts mapped 19 barriers, found in our previous studies, to the most fitting CFIR constructs. Subsequently, (dis)agreements were discussed and barriers were further clarified. The CFIR- ERIC Matching Tool generated a list of strategies to address these barriers. Outcomes were discussed with the panel and with end-users of these programs (e.g. ICLN and infection control practitioners).

Results: Seven main barriers for the implementation of a link nurse program were identified (table 1). These barriers corresponded with CFIR constructs, predominantly from the domains 'inner setting' (characteristics of the implementing organization) and 'process (stages of implementation). With the ERIC tool strategies were identified to overcome these barriers; they are listed in order of priority in Table 2.

\section{Table 1. Barriers}

Infection control has no priority at the hospital level

The role of link nurses is not defined

ICLN are not accepted by medical staff

ICLN programs are initiated, developed and implemented solely by infection control practitioners

Responsibility to educate link nurses lies with infection control practitioners

Interconnecting link nurses from various departments to exchange experiences and best practices is challenging Only half of link nurse programs are evaluated 
Table 2. ERIC Strategies

Identify and prepare champions

Conduct local consensus discussions

Assess for readiness and identify barriers and facilitators

Inform local opinion leaders

Facilitate interactive problem solving

Conclusion: Application of the CFIR-ERIC tool points to the identification and preparation of champions for the successful implementation of ICLN programs. Further strategies can be tailored to each context with the help of the identified barriers and the use of the tool. Disclosure of Interest: None declared.

\section{P270}

Let's get in infection control modus! ALL the medical students in Nijmegen IPC trained and examined before starting their clinical rotations

O. Coenen ${ }^{1, *}$, M. Tingen ${ }^{1}$, E. Bowles ${ }^{1}$, A. Tostmann ${ }^{1}$

${ }^{1}$ Medical Microbiology, dept Infection Control, Radboudumc, Nijmegen, Netherlands

Correspondence: $\mathrm{O}$. Coenen

Antimicrobial Resistance \& Infection Control 10(1): P270

Introduction: Education in infection prevention and control (IPC) is an indispensable part of an adequate IPC program. For medical students, IPC education should be theoretical and practical, so the student can provide safe patient care.

Objectives: At our medical faculty, until 2019 IPC education for medical students consisted of three hours of teaching. Evaluations with students and teachers showed that the program was not considered challenging and the importance of IPC did not affect the student. The teachers indicated there was too little time to cover the important aspects of IPC. Therefore, we developed a new and more inspirational educational IPC program for medical students at the Radboudumc medical faculty.

Methods: Since 2019, medical students are educated in IPC according to our newly developed educational program. The program was developed by the IPC team, the medical education director and several peers within the medical and educational domain. The IPC education is planned in the preparatory clinical rotations module. Students must pass an exam before they can start their clinical rotations.

The education program consists of:

- Self-study: scientific literature, theoretical assignments and an inhouse developed IPC E-learning module.

- Q\&A session with IPC expert.

- Practical training: in small groups IPC measures are practiced in an simulated patient environment.

- Individual examination: a practical exam in a realistic isolation setting. Students are tested on donning, doffing, hand hygiene, cleaning and disinfection and communication.

Results: The new educational program for medical students has been in place for two years now. There is a $100 \%$ attendance during the teaching activities and the students are actively involved. Feedback shows the program reflects the daily practice and students feel confident to perform IPC measures in a clinical setting. Also they realize what it means for a patient to be isolated, this emphasizes the importance of clear communication on IPC measures.

Conclusion: We are the only medical faculty in the Netherlands where $100 \%$ of the graduated medical students are fully IPC trained and examined. We believe that the mandatory exam at the end of the program shows the importance of IPC in patient care.

At this moment we are evaluating the program.

Disclosure of Interest: None declared.

\section{P271}

Effect of training on knowledge regarding infection control measures among healthcare workers in 10 selected states in Sudan, 2021

H. Azrag 1,*

${ }^{1}$ Infection Control Directorate, Federal Ministry of Health, Khartoum, Sudan

Correspondence: $\mathrm{H}$. Azrag

Antimicrobial Resistance \& Infection Control 10(1): P271

Introduction: Healthcare workers are at a high risk of infection. Infection prevention and control (IPC) is a practical, evidencebased approach preventing patients and health workers from being harmed by avoidable infections.

Objectives: This study was conducted to assess the effect of the training on the knowledge of the healthcare workers on how to protect themselves and their patients from being infected during COVID 19 pandemic in Sudan.

Methods: During March 2021, 471 healthcare workers were selected from the most COVID-19 Pandemic affected States in Sudan. The Knowledge of the participants was assessed before and after conduction of training using a questionnaire consisted of two sections. Section A elicited responses on demographic variables of participants. Section B elicited information on the participants' knowledge about standard precautions. A significant difference was defined as $P$ value $<0.05$ (2-tailed). Continuous data were compared with paired t-test. Statistical analysis was performed with SPSS (Version 21).

Results: The knowledge of health care workers differed significantly before and after the training $(P=0.01)$, emphasizing that the impact of the training was important to improve the knowledge of participants. The average value of improvement of healthcare workers' knowledge was about $26.5 \%$.

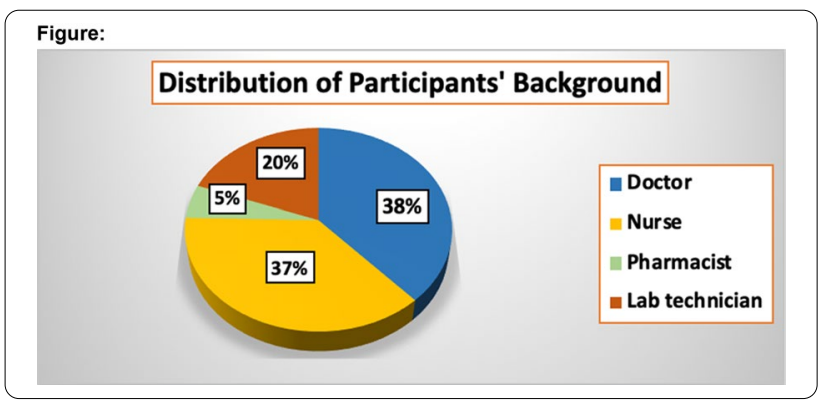

Conclusion: The findings emphasized the role of the training to improve the knowledge and education of health care workers about standard precautions as well as prevention of infection during COVID 19 pandemic. Education and training which is the third core component of the IPC should be reinforced for the better health of patients and medical staff, and be considered as one of the key factors in any pandemic response.

Disclosure of Interest: None declared. 


\section{P272}

Identification of resources to inform a new country level infection prevention and wash training package

C. Kilpatrick ${ }^{1, *}$, J. Storr ${ }^{1}$, K. Smith ${ }^{2}$, H. Hamilton ${ }^{2}$, N. Mwenda ${ }^{3}$, M

Narracott $^{2}$, A. Woodland ${ }^{2}$

${ }^{1} \mathrm{KS}$ Healthcare Consulting, Glasgow, ${ }^{2}$ WaterAid, London, United Kingdom, ${ }^{3}$ WaterAid, Lilongwe, Malawi

Correspondence: $C$. Kilpatrick

Antimicrobial Resistance \& Infection Control 10(1): P272

Introduction: The World Health Organization (WHO) Core components of infection prevention and control (IPC) programmes state that patient care activities should be undertaken in a clean, hygienic environment that facilitates safe practices. This includes all elements of water, sanitation and hygiene (WASH) infrastructure and services and includes training as a component. In many low and middle income countries (LMICS), activities are both aimed at and led by WASH and IPC focal points. Feedback from previous trainings and anecdotal evidence suggest that IPC resources are mainly clinically focused and do not fully embrace all aspects of WASH in health care to fulfill country needs.

Objectives: To identify relevant resources to inform a new country level WASH and IPC training package as part of a Wimbledon Foundation funded WaterAid project in Malawi.

Methods: A desk research exercise was undertaken through a search of Google and key web portals using key words aimed at capturing freely available international, regional and country level resources. Criteria were established to categorise the resources which were finally listed as guidance $(G)$, training $(T)$, assessment $(A)$ or implementation (I) and noted for their focus on LMICs and if they had undergone testing/evalution.

Results: In total, 66 resources were found, 33 categorised as training, primarily focused on IPC. A WHO WASH in health care facilities training package was reviewed, however, the cross over with IPC was noted as being limited. A number of relevant international and national guidelines were also identifed, that present the evidence base for informing training materials. Similarly, assessment and implementation materials exist, though most often not combined as IPC/WASH but presented as separate documents. Still they provide content to inform training materials on the aspect of monitoring.

Conclusion: A range of training materials are freely available. However the majority of these are focused on IPC and aimed at frontline, clinical improvements and do not fully embarace associated aspects of WASH. The need for a new practical resource to engage WASH focal points appropriately in IPC is deemed necessary to address country needs.

Disclosure of Interest: None declared.

\section{P273}

Infection prevention and control staffing and programs in Middle

\section{Eastern countries}

E. Tannous ${ }^{1, *}$, A. El-Saed ${ }^{2}$, K. Ameer $^{2}$, A. Khalaf ${ }^{3}$, S. Mohammad ${ }^{1}$, B.

Molaeb ${ }^{4}$, F. Othman ${ }^{2}$, M. Alshamrani ${ }^{2}$

1 Infection control, Cleveland Clinic Abu Dhabi, Abu Dhabi, United Arab

Emirates, ${ }^{2}$ Infection control, King Abdulaziz Medical City, Riyadh, Saudi

Arabia, ${ }^{3}$ Infection control, Sheikh Khalifa Medical City, Abu Dhabi, United

Arab Emirates, ${ }^{4}$ Infection control, Al-Moosa Specialist Hospital, Al-Ahsa,

Saudi Arabia

Correspondence: E. Tannous

Antimicrobial Resistance \& Infection Control 10(1): P273

Introduction: Infection prevention and control (IPC) programs are well-established in North American and the European countries. On the other hand, these programs are still evolving in the Middle Eastern and North African (MENA) countries. Gulf Cooperation Council (GCC) states have much better financial resources than the rest of MENA countries.

Objectives: To characterize IPC personnel and programs in MENA countries, with special emphasis on the differences between GCC and non-GCC hospitals.
Methods: A cross-sectional online survey was conducted in 2019 among IPC staff joining the Arab Countries Infection Control Network The survey focused on three domains; demographic and professional characteristics of IPC personnel, organizational structure, and IPC program characteristics.

Results: A total 269 participants aged $39.9 \pm 8.4$ years were included in the study. The majority of participants were female $(67.7 \%)$, nurses (63.7\%), and of Middle Eastern origin (57.3\%). Only $32.2 \%$ of participants had certification board of infection control (CBIC) while $80.1 \%$ of those who did not have it planned to get it. Only $22.7 \%$ of participants were extremely or very satisfied with their current compensation. Surveillance was the most time consuming task $(26.6 \%)$, followed by isolation $(12.4 \%)$, investigation of outbreaks (12.1\%), and identification of infection (11.4\%). The most frequent ratio of IPC personnel per bed was one per 100 beds (39.5\%). The majority of facilities had enoughsupported IPC program (63.9\%), formal IPC committee (93.7\%), IPC plan (91.4\%). Compared with non-GCC countries, GCC countries had significantly more frequent Asian professionals $(<0.001)$, nursing staff $(p=0.006), C B I C$ certification $(p=0.003)$, training in educational services $(p=0.038)$ and cleaning/sterilization $(p=0.010)$, higher ratio of IPC personnel per bed $(p=0.047)$, enough-supported IPC program $(p=0.010)$, formal IPC committee $(p=0.001)$, and IPC plan $(p=0.001)$. Conclusion: The findings showed generally satisfactory IPC staffing and programs in the MENA region, with considerable variability between countries of different resources. The findings may help policy makers in improving IPC staffing and programs.

Disclosure of Interest: None declared.

P274

Prevention and infection control for short-term volunteers of a non-governmental organization in a developing country

J. Barrio Cortes ${ }^{1, *}$, C. Rojas Muñoz ${ }^{1}$, M. Á. Acosta Benito ${ }^{1}$, Á. Hidalgo Baz ${ }^{1}$, Á. Vicario Merino ${ }^{1}$, M. T. Beca Martínez ${ }^{1}$, M. Ruiz López ${ }^{1}$

${ }^{1}$ Camilo José Cela University, Madrid, Spain

Correspondence: J. Barrio Cortes

Antimicrobial Resistance \& Infection Control 10(1): P274

Introduction: Short-term volunteers are susceptible to a wide spectrum of morbidities, mostly infectious diseases preventable with general hygiene and preventive measures.

Objectives: This study aims to describe the pre-travel consultation and pre-travel health education received by European short-term volunteers for preventing infectious diseases and to identify the infections that they encountered during their one month stay with a nongovernmental organization (NGO) in Cambodia.

Methods: A cross-sectional descriptive observational study was conducted on short-term volunteers who collaborated with an NGO in Cambodia during August 2018. The informed consent and the sociodemographic, clinical and health prevention related questionnaire data were filled in by 198 volunteers before the travel. The health problems they encountered were registered in a local health clinic by healthcare professionals (doctors and nurses). Univariate and bivariate analyses were performed.

Results: The volunteers average age was 24.2 years (SD $=7.6), 64 \%$ were women, $60.1 \%$ were Spanish, 35.4\% French and the remaining $4.5 \%$ of other nationalities such as British, German, Belgian, Dutch or Italian. Some $18.2 \%$ had allergies, $8.6 \%$ had pre-existing health conditions and $10.6 \%$ were under regular treatment. $77.3 \%$ visited the pre-travel consultation clinic, $21.7 \%$ took malaria prophylaxis, $92.4 \%$ received vaccination against hepatitis $A, 82.3 \%$ against typhoid fever vaccination, $82.3 \%$ against typhoid, $57.1 \%$ against tetravalent meningitis; $38.4 \%$ against rabies; $36.4 \%$ against Japanese encephalitis and $6.6 \%$ against cholera. A total of $39.9 \%$ completed a specific pre-travel health course. Medical assistance was sought by 112 (57.3\%) volunteers. The most prevalent infections were upper respiratory infections (19\%), diarrhoea (12.5\%) and skin infections (10.4\%).

Conclusion: Short-term volunteers experienced a high prevalence of infections during their stay in Cambodia but most of them were mild, preventable and were quickly resolved. Pre-travel consultation 
and specific pre-travel health training seemed to increase prevention measures and disease awareness.

Disclosure of Interest: None declared.

\section{P276}

Identification and implementation of infection prevention and control (IPC) priorities in health care (HC) facilities in post-disaster contexts in low- and middle-income countries (LMIC)

J. K. R. E. Letsch ${ }^{1, *}$, F. Mattner ${ }^{2}$, D. Peter ${ }^{2}$

${ }^{1}$ Orthopaedic surgery, Vivantes Klinikum Spandau, Berlin, ${ }^{2}$ Institute of Hospital Hygiene, Kliniken Köln, Lehrstuhl für Hygiene und Umweltmedizin, Witten/Herdecke University, Germany

Correspondence: J. K. R. E. Letsch

Antimicrobial Resistance \& Infection Control 10(1): P276

Introduction: Natural disasters are often associated with an increased incidence of infectious diseases (ID), especially if population displacement occurs (1).

Objectives: To identify HC-based IPC priorities implemented in postdisaster contexts in LMIC.

Methods: Scoping review (2): Database search using defined search terms, followed by a review of citation indices of the included articles. Only peer-reviewed full text articles published in English between 2000-2020 were included.

Results: 12 studies were included in the final analysis. The following IPC priorities were identified:

1. ID Surveillance (outbreak detection) at HC facility level and timely reporting of data to guide IPC interventions.

2. Monitoring of compliance with IPC measures at HC facility level.

3. Implement screening of outpatients for signs of ID.

4. Avoid crowding of $\mathrm{HC}$ facilities by referring inpatients to other facilities.

5. Use of hand- and surface disinfection and appropriate personal protective equipment.

6. Safe waste- and water management.

7. Dead body management.

In none of the studies it was described how IPC measures were implemented nor was their effect on the incidence of ID evaluated.

Conclusion: The identified post-disaster IPC priorities can be found in the WHO core components of IPC in HC facilities, except 8. (3). While the benefit of IPC in post-disaster contexts has been clearly stated, future research is needed to examine the effect of IPC priorities on the disaster related incidence of ID and how IPC can be successfully implemented in a post-disaster context.

\section{References:}

1. Kouadio IK et al. Infectious diseases following natural disasters: prevention and control measures. Expert Rev Anti Infect Ther. 2012 Jan;10(1):95-104. https://doi.org/10.1586/eri.11.155. PMID: 22149618

2. Tricco, AC et al. PRISMA extension for scoping reviews (PRISMA-SCR): checklist and explanation. Ann Intern Med. 2018,169(7):467-473. https://doi. org/10.7326/M18-0850.

3. Guidelines on core components of infection prevention and control programmes at the national and acute health care facility level. Geneva: World Health Organization; 2016. Licence: CC BY-NC-SA 3.0 IGO.

Disclosure of Interest: None declared

\section{P277}

Learning organization and continuous improvement

M. Mahjoub ${ }^{1}$, B. Trabelsi ${ }^{1}$, C. Souhir ${ }^{1, *}$, O. Ezzi ${ }^{1}$, A. Asma $^{1}$, A. Abbadi ${ }^{1}$, M. Njah'

${ }^{1}$ Hospital hygiene department, Farhat Hached teaching hospital, Sousse, Tunisia

Correspondence: C. Souhir

Antimicrobial Resistance \& Infection Control 10(1): P277
Introduction: Improvement requires commitment from healthcare professionals and continuous evaluation, but at the beginning it is necessary to is necessary to carry out an inventory of their practices in order to determine the priority points to be improved.

Objectives: is to compare the level of the learning organization and continuous improvement as a part of the patient safety culture among doctors and paramedical staff at the maternity services of public health structures in the region of Sousse (Tunisia).

Methods: It was an analytical cross-sectional study carried out in in the region of Sousse in 2017 from 1/15/2017 to 03/15/2017, among health professionals, all categories combined (medical and paramedical), working in structures of public health specializing in gynecoobstetrics (central and peripheral maternity hospitals). The study was conducted using a questionnaire specially designed for the purposes of this work.

Results: The overall score for this concept was $73.11 \%$. The distribution of responses showed that $83.4 \%$ of respondents "judged that the implementation of a process to assess the degree of satisfaction of patients on discharge from maternity could improve safety of care". On the other hand, $85.7 \%$ of respondents said that "practice of simulation exercises contributed to improve the quality of care". All the items were perceived more by doctors compared to paramedics. However, this difference was only significant for the item "In our department, errors led to positive changes: implementation of procedures" $(82.6 \%$ versus $59.3 \% ; p=0.029$ ).

Conclusion: It is essential to operationalize an integrated approach to improve the patient safety culture. The prospects are to develop this type of study in other health establishments in order to better understand the level of development of this concept with the aim of a better adjustment and standardization of our policies and action strategies.

Disclosure of Interest: None declared

\section{P278}

Impact of education intervention on knowledge of infection control practices among healthcare providers during COVID-19 pandemic

I. Khanum ${ }^{1}{ }^{1}$, K. Habib $^{1}$, B. Jamil ${ }^{1}$

${ }^{1}$ Aga Khan University Hospital, Karachi, Pakistan, Pakistan

Correspondence: I. khanum

Antimicrobial Resistance \& Infection Control 10(1): P278

Introduction: Healthcare workers (HCWs) are at an increased risk of exposure to SARS-CoV-2 infection. There is a need for urgent intervention to incease knowledge and practices related to infection prevention and control (IPC) during the current pandemic.

Objectives: The objectives of the current study are to assess knowledge, attitude and practices among HCWs regarding IPC practices related to COVID-19 and whether training session can be used as an effective educational tool to improve knowledge.

Methods: This cross sectional study was conducted among HCWs from Sindh. After assessment of baseline knowledge, attitude and practices via pre test, a virtual session on COVID-19 IPC practices was conducted, which was followed by post test.

Results: Among 240 participant, 141 (59\%) were frontline workers dealing with patients with COVID-19. Only $76(31 \%)$ had previous training on IPC before pandemic and even during pandemic, few $(n=95,40 \%)$ had attended a training workshop. Majority $(70.4 \%)$ of participants were working in a facility with an established IPC department. There was an overall statistically significant improvement in knowledge before and after the education workshop ( $p$ value $<0.01$ ). The majority of HCWs believed that poor compliance with personal protective equipment (PPE) was due to hot climate, interference with daily work, increased workload and long working hours. The knowledge and reported compliance of hand hygiene were good among majority of participants ( $>90 \%$ ). A large number of HCWs (88\%) carry hand sanitizers all the time and frequently clean their belongings during current pandemic. Although $75 \%$ of HCWs believed that PPE can 
protect them from contracting infections, a poor compliance of wearing PPE was reported while dealing with patients with COVID-19.

Conclusion: Frequent awareness sessions can help in improving knowledge related to infection control and practices among HCWs.

Disclosure of Interest: None declared

\section{P279}

Applying the who twinning partnerships for improvement (TPI) 6-step model to drive infection prevention and control (IPC) during the COVID-19 pandemic: setting objectives and delivering activities to achieve outcomes during crisis

cM. Bingham ${ }^{1, *}$, J. Storr ${ }^{2}$, K. Aparicio Reyes ${ }^{3}$, S. Syed ${ }^{3}$

${ }^{1}$ Independent Consultant, Denver, United States, ${ }^{2}$ Independent Consultant, London, United Kingdom, ${ }^{3}$ Integrated Health Services, WHO, Geneva, Switzerland

Correspondence: I. khanum

Antimicrobial Resistance \& Infection Control 10(1): P279

Introduction: Implementation of a validated twinning partnership approach with clear objectives while maintaining flexibility of inputs and activities during a pandemic, can yield improvements in IPC as a cornerstone of quality of care.

Objectives: To apply the World Health Organisation (WHO) TPI 6-step model to guide twinning partners through a systematic process to drive quality of care in health settings.

Methods: A partnership between Macau Health Bureau and Timor-Leste Cabinet of Quality Assurance in Health was initiated, guided by WHO's TPI objectives and 6-step model. The 2-year TPI included partnership formation, a situational analysis, co-developing a quality improvement (QI) action plan, joint implementation of action and bi-directional learning. IPC at the national, subnational and facility level was as prioritized by the partnership. Three Timor-Leste facilities were chosen for IPC improvements. The WHO IPC assessment framework at the facility level (IPCAF) was used to gather data at baseline and at intervals over the 2-year TPI.

Results: Based on the IPCAF results, the action plan focused on 4 areas: 1) establishing IPC team in each of the facilities; 2) training and capacity building in standard precautions; 3 ) facility infrastructure improvements (including water sanitation and hygiene); and 4) hand hygiene advocacy. The structured TPI methodology and direct partner support resulted in clean water being consistently available at the 3 facilities and capacity building in standard precautions, transmission-based precautions and PPE. COVID-19 realities required adaptation and re-scheduled infrastructure and training activities.

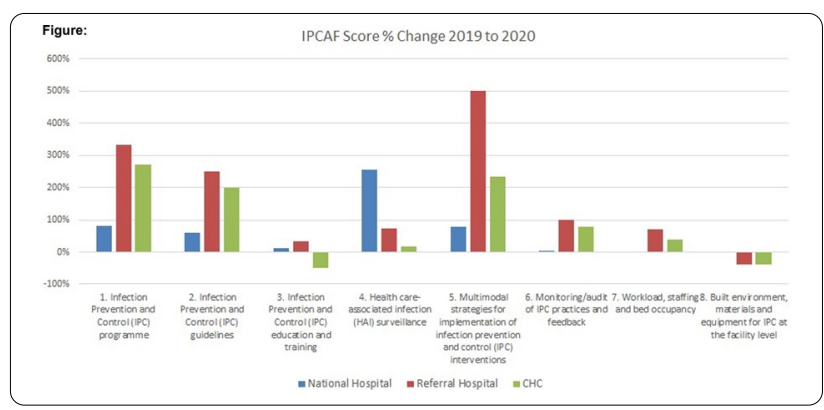

Conclusion: Implementation of the partnership plan using the structured TPI approach catalyzed IPC improvements at the three facilities and beyond. Although COVID-19 required adjustment of activities, a robust QI plan supported innovation, flexibility and commitment. Lessons from the experience can be applied to other twinning partnerships. Disclosure of Interest: None declared
P280

Safe PPE: user needs for an interactive ppe training tool

G. Lacey ${ }^{1, *}$, J. Cahill'2, A. Kay², V. Howard², R. Harte ${ }^{3}$, F. O'Reilly ${ }^{3}$, E.

Ziampra $^{4,5}$, F. Fitzpatrick ${ }^{4,5}$, B. Mulcahy ${ }^{6}$, S. George ${ }^{6}$, R. Nolan $^{7}$

${ }^{1}$ Computer Science and Statistics, ${ }^{2}$ Psychology, ${ }^{3}$ Learnovate, Trinity Col-

lege Dublin, ${ }^{4}$ Royal College of Surgeons, ${ }^{5}$ Beaumont Hospital, Dublin,

${ }^{6}$ Bon Secours Hospital, Cork, ${ }^{7}$ Tallaght University Hospital, Dublin, Ireland

Correspondence: G. Lacey

Antimicrobial Resistance \& Infection Control 10(1): P280

Introduction: Staff competency and access to PPE training became critical early in the COVID 19 pandemic. This placed demands on infection prevention \& control staff when already busy with COVID-19-related tasks.

Objectives: To be prepared for the next pandemic, we investigated the role of mindfullness and technology in PPE training.

Methods: Human factors research involving three clinical sites $(\mathrm{N}=174)$ consiting of interviews, workshops and participatory codesign sessions. This was extended by interviews with 14 IPC experts from 7 sites in UK and USA.

Results: Existing PPE \& HH Training:

- Addressed the lived experience of using PPE

- Variety of training formats e.g. classroom (15\%) and in-unit training (85\%)

- One $(1 / 10)$ site assessed PPE competency

- Four $(4 / 10)$ sites used a Virtual Learning Environment to track the learner journey

- One $(1 / 10)$ site used a mobile phone based training

Performance Shaping Factors:

- Training, Fatigue, Distraction, Rushing, Stress, etc.

- Changing types of PPE with different quality and fit

- Changing PPE guidelines as IPC knowledge evolved

Emerging Mobile Learning App Concept \& Requirements:

- All trainers expressed concerns of low engagement with phonebased training

- Online and in-person training must be consistent

- Assessment should be carried out on-site, with special-purpose equipment

- PPE and Hand Hygiene training should incorporate self-care \& mindfulness

- Scenario-based training needed to support different clinical roles and needs

- Customise training to local guidelines

Conclusion: Mindfulness is a protective factor for effective and safe performance. Organisations should consider integrating wellness into IPC training. Technology can support in-person training but it is not a replacement and must be available $24-7$. A variety of training access pathways are good but the Learner Journey should be tracked to ensure everyone is trained and skills are maintained over time

Disclosure of Interest: G. Lacey Consultant for: surewash, J. Cahill: None declared, A. Kay: None declared, V. Howard: None declared, R. Harte: None declared, F. O'Reilly: None declared, E. Ziampra: None declared, F. Fitzpatrick: None declared, B. Mulcahy: None declared, S. George: None declared, R. Nolan: None declared

P281

Frequency and reporting of adverse events: dimension of patient safety culture

M. Mahjoub ${ }^{1}$, S. Chelly ${ }^{1, *}$, O. Ezzi ${ }^{1}$, A. Asma ${ }^{1}$, B. Trabelsi ${ }^{1}$, A. Abbadi ${ }^{1}$, M.

Njah ${ }^{1}$

${ }^{1}$ Hospital hygiene department Farhat Hached teaching hospital, Sousse,

Tunisia

Correspondence: S. Chelly

Antimicrobial Resistance \& Infection Control 10(1): P281

Introduction: Adverse events represent not only a problem of safety and quality of care for patients, but also an economic problem which their financial consequences were considerable. 
Objectives: to measure and analyze the degree of development of the concept "Frequency and reporting of adverse events" relating to the of patient safety culture of healthcare professionals.

Methods: A cross-sectional study was carried out in 2017 using a validated questionnaire among all health professionals in the five public health structures specializing in gyneco-obstetrics in the governorate of Sousse (Tunisia) ( $\mathrm{N}=251$ and 217 respondents).

Results: The overall score for frequency and reporting of adverse events was $69.7 \%$. The distribution of the different items in this dimension showed that $83.4 \%$ of respondents felt obliged to report errors that have consequences on patients to their hierarchical superiors, on the other hand only $65.9 \%$ felt required to report an error that occurs and is corrected before affecting the patient. All the items in this dimension were perceived without significant difference between physicians and paramedics.

Conclusion: The establishment of a surveillance system for adverse events is a central link in any policy to improve the quality and safety of care in health establishments, which must involve all staff (workers, technicians, nurses, doctors...).

Disclosure of Interest: None declared

\section{P282}

Healthcare worker-patient relationship and patient safety culture M. Mahjoub ${ }^{1}$, B. Trabelsi ${ }^{1}$, S. Chelly 1, ${ }^{1}$, O. Ezzi ${ }^{1}$, A. Asma ${ }^{1}$, A. Abbadi ${ }^{1}$, M. Njah ${ }^{1}$ ${ }^{1}$ Hospital hygiene department, Farhat Hached teaching hospital, Sousse, Tunisia

Correspondence: S. Chelly

Antimicrobial Resistance \& Infection Control 10(1): P282

Introduction: A good relationship between healthcare worker and patient is a main link of the care safety.

Objectives: Measure the level of healthcare provider relationship within the framework of healthcare safety culture with health professionals practicing in our establishment.

Methods: It was a cross-sectional descriptive study, carried out in 2017 for two months, of interest to all health professionals (medical and paramedical (nurse, midwife and senior technician)) working in public health structures specializing in gyneco-obstetrics in the region of Sousse (Tunisia).

Results: The overall score for this concept was $66.8 \%$. The distribution of responses for the different items of this dimension showed that $82.9 \%$ of the respondents found that "adverse events could affect the trust relationship between doctor and patient". However, only $37.3 \%$ of all healthcare professionals believed that "patient should be informed of the fault as soon as it is committed and of the consequences that may". On the other hand, $66.8 \%$ of respondents said that "traditional medicine practices are an obstacle to improving the safety of care". The doctors reported significantly more than the paramedics, the fact that "patient must be informed of the fault as soon as it is committed and of the consequences that may", "the patient has a role to play in improving safety", and finally, traditional medicine practices can constitute an obstacle to improving the safety of care" respectively $(60.9 \%$ versus $34.5 \% ; p=0.014),(100 \%$ versus $82.5 \% ; p=0.029)$ and $(87 \%$ versus $65.4 \% ; p=0.03)$.

Conclusion: Our study allowed us to obtain an inventory and a global vision of the perception of healthcare professionals relating to patient safety and to identify gaps. This concept is a central link in the patient safety culture and an inseparable pillar of the strategy for improving the quality and safety of care within a healthcare establishment.

Disclosure of Interest: None declared

\section{P283}

Overall perception of patients' safety among health professionals working in the maternity sector in public health structures

in Sousse (Tunisia)

M. Mahjoub ${ }^{1}$, S. Chelly, ${ }^{1,}$, O. Ezzi , A. Asma ${ }^{1}$, B. Trabelsi ${ }^{1}$, A. Abbadi ${ }^{1}$, M. Njah'

${ }^{1}$ Hospital hygiene department Farhat Hached teaching hospital, Sousse, Tunisia Correspondence: $\mathrm{S}$. Chelly

Antimicrobial Resistance \& Infection Control 10(1): P283
Introduction: Patient's safety culture reflects the perceptions of norms, processes, and attitudes relating to a culture of preventable errors.

Objectives: To measure and assess the level of development of the concept of "global perception of patient safety" relating to the patient safety culture among health professionals working in the maternity sector in public health structures in Sousse.

Methods: A cross-sectional study was carried out in 2017 using a validated questionnaire among all health professionals in five public health structures specializing in gyneco-obstetrics in the governorate of Sousse (Tunisia) ( $\mathrm{N}=251$ of which 217 responded).

Results: The overall score for the overall perception of patient safety was $44.1 \%$. Analysis of the responses to the different items showed that only $37.3 \%$ of respondents admitted that they had no problems related to the safety of care in their department. But when there is an antenatal death or a complication in the delivery, $79.3 \%$ of respondents said they felt this event could have been avoided. Among all the items, there was not a significant difference between physicians and paramedics except the item "the competent physician does not make errors that could harm the patient" which was significantly more perceived among paramedics than physicians. ( $35.6 \%$ versus $8.7 \% ; p=0.009$ ).

Conclusion: The risk of error in therapeutic choices and in care exists despite the fact that the doctors are highly qualified and competent, which confirms that the error is human and that "zero risk" does not exist. Disclosure of Interest: None declared

\section{P284}

Patient safety culture based on teamwork and human resources of healthcare professionals in the maternity services in Sousse (Tunisia)

M. Mahjoub ${ }^{1}$, S. Chelly ${ }^{1,}$, O. Ezzi ${ }^{1}$, A. Asma ${ }^{1}$, B. Trabelsi ${ }^{1}$, A. Abbadi ${ }^{1}$, M. Njah ${ }^{1}$

${ }^{1}$ Hospital hygiene department Farhat Hached teaching hospital, Sousse, Tunisia

Correspondence: S. Chelly

Antimicrobial Resistance \& Infection Control 10(1): P284

Introduction: An effective teamwork is essential for patient safety in the context of a complex healthcare system, it minimizes adverse events caused by miscommunication.

Objectives: is to measure and analyze the level of development of teamwork and human resources concept of patient safety culture among healthcare professional.

Methods: An analytical cross-sectional study was carried out in 2017 using a validated questionnaire among all health professionals in five public health structures specializing in gyneco-obstetrics in the governorate of Sousse (Tunisia) ( $\mathrm{N}=251$ of which 217 responded).

Results: The overall score for "teamwork in the department" was $64.4 \%$. Analysis of the responses to the different items showed that a difference in favour of physicians was raised; $65 \%$ said they were ready to combine their efforts as a team when the workload is heavy $(62.4 \%$ versus $37 \%$; $p=0.019), 66.8 \%$ thought that everyone considers others with respect $(64.4 \%$ versus $37 \% ; p=0.03)$ and $6.8 \%$ thought that more experienced staff will be available on the first call if the situation requires their presence $(95.7 \%$ versus $63.4 \% ; p=0.002)$. The overall score for "Teamwork between services in the facility" was $59.9 \%$. Doctors have affirmed significantly more than paramedics that when transferring a patient to another service we provide all the results of imaging or biology exams that have been performed, in addition to the fact that the services of the institution did not coordinate well with each other (respectively $95.7 \%$ versus $61.3 \% ; p=0.001$ and $87 \%$ versus $64.4 \% ; p=0.03$ ). The overall "Human Resources" score was $65.65 \%$. Among its items; $45.6 \%$ believed that they had enough staff to cope with the workload and $85.7 \%$ said that too long working hours could have consequences for patient safety $(100 \%$ of doctors against $84 \%$ of paramedics $(p=0.0038)$ ).

Conclusion: Teamwork as well as having sufficient staff is one of the factors influencing the quality of care, the evaluation of health care providers and the satisfaction of patients. Thus, professionals who shared information have a marked improvement in the efficiency of the work unit.

Disclosure of Interest: None declared 
P285

Culture of patient safety based on non-punitive response to error and freedom of expression of healthcare professionals

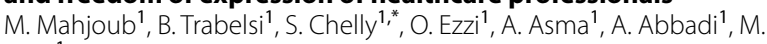
Njah $^{1}$

${ }^{1}$ Hospital hygiene department, Farhat Hached teaching hospital, Sousse, Tunisia

Correspondence: S. Chelly

Antimicrobial Resistance \& Infection Control 10(1): P285

Introduction: Freedom of expression and the non-punitive response to error is a central concept of culture of patient safety among healthcare professionals since it reflects the climate and general atmosphere of the professional environment.

Objectives: Measure and analyze the degree of development of the concept Freedom of expression and non-punitive response to error in the culture of patient safety.

Methods: This is an analytical cross-sectional study carried out in 2017 over two months (from 1/15/2017 to 03/15/2017) among health professionals, all categories combined (medical and paramedical), working in structures of public health specializing in gyneco-obstetrics (central and peripheral maternity hospitals) in the region of Sousse (Tunisia). The study was conducted using a questionnaire specially designed for the purposes of this work.

Results: The overall score was $43.2 \%$. The distribution of responses showed that $40.1 \%$ of respondents said that, in the services, they discussed the means to be put in place in order to avoid repeating the mistakes made. Only $30.4 \%$ of respondents said they perceive staff to feel free to argue the decisions or actions of their superiors. A significant difference was noted in favor of physicians for item "the staff express themselves freely if they see something in the care that can have negative consequences on the patients" (78.3\% versus $50.5 \%$; $p=0.012$ ). On the other hand, there were significant differences in favor of paramedics for items "in the departments, we discuss the means to put in place so that errors no longer occur", "Staff feel free to discuss the decisions or actions of their superiors" and "staff are afraid of asking questions when something does not seem to be correct" respectively ( $42.8 \%$ versus $17.4 \% ; p=0.019)$, (33\% versus $3.7 \%$; $p=0.017)$ and (48.5\% versus $21.7 \% ; p=0.015)$.

Conclusion: This concept has generally been shown to be underdeveloped in our establishment. We must learn to analyze our mistakes, to improve our procedures and our protocols, to better manage and govern our systems, to improve our communication, information and awareness policies.

Disclosure of Interest: None declared

\section{P286}

Risk of HCV infection associated with different hospital-based invasive procedures: a systematic review and meta-analysis P. Henriot ${ }^{1, *}$, M. Castry ${ }^{2}$, Y. Shimakawa ${ }^{3,4}$, K. Jean ${ }^{1,4}$, L. Temime ${ }^{1,4}$

${ }^{1}$ MESURS Laboratory, Conservatoire national des arts \& métiers, ${ }^{2}$ INSERM, IAME, ${ }^{3}$ Emerging Disease Epidemilogy Unit, Institut Pasteur, ${ }^{4}$ PACRI Unit, Institut Pasteur-CNAM, Paris, France

Correspondence: P. Henriot

Antimicrobial Resistance \& Infection Control 10(1): P286

Introduction: Healthcare settings may play an important role in the transmission dynamics of blood-borne pathogens when invasive procedures are performed while compliance with infection control precautions remains imperfect.

Objectives: This study aims at summarizing the evidence on the risks of hepatitis $C$ virus (HCV) infection across hospital-based invasive procedures and understanding the role of these procedures in $\mathrm{HCV}$ transmission.

Methods: We conducted a systematic review and meta-analysis to identify recent studies reporting association measures of HCV infection risk that are linked to iatrogenic procedures performed in hospital settings. Invasive procedures were categorized into 10 groups based on an expert opinion for which pooled measures were calculated. Finally, the relationship between pooled measures and the country-level HCV prevalence or the Healthcare Access and Quality (HAQ) index was assessed in meta-regressions.

Results: A total of 73 studies were included in the analysis. The most evaluated procedures were blood transfusion and surgery (49 and 34 studies). The pooled OR associated with HCV infection was highest for haemodialysis $(6.48,95 \% \mathrm{Cl}$ : $0.43-97.7)$ and lowest for endoscopy (1.44, 0.98-2.11). The OR for blood transfusion was higher for transfusions performed before $1996(3.84,2.46-6.00)$ than for those without a specified date $(2.38,1.88-3.01)$. Finally, there was no significant association between the HAQ index and the country-level overall risk for all procedures, but a significant one was found for HCV prevalence. In procedure-specific analyses, the HCV infection risk was significantly negatively associated with the HAQ for endoscopy and positively associated with HCV prevalence for endoscopy and surgery.

Conclusion: Many iatrogenic procedures were found to be related to the risk of HCV infection. However, care must be taken to interpret the results because of: i) limited number of studies for some procedures, leading to uncertainty in the estimates; ii) lack of adjustment on confounding factors in some studies; and iii) heterogeneity in the way the exposure has been assessed across studies.

Disclosure of Interest: None declared

\section{P287}

Knowledge, attitude and practice regarding hepatitis prevention among medical professionals of Banfora (Burkina Faso)

K. C. C. Sawadogo ${ }^{1, *}$, I. F. BAKO ${ }^{1}$, B. BAGUE ${ }^{1}$, M. S. HIEN ${ }^{2}$

${ }^{1}$ Health science department, Joseph KI-ZERBO University, Ouagadougou, ${ }^{2}$ Day hospital, Regional hospital of Banfora, Banfora, Burkina Faso

Correspondence: K. C. C. Sawadogo

Antimicrobial Resistance \& Infection Control 10(1): P287

Introduction: Knowledge of hepatitis seem to be insuffisant in the hospital of Banfora. It can negatively impact the quality of care in this hospital. This is why we conducted the study to evaluate the knowledge, attitude and practice regarding hepatitis prevention among medical professionals of Banfora.

Objectives: The main objective is to evaluate the kwoledge, attitude and practice regarding hepatitis prevention among medical professionals of Banfora.

The secondary objectives are:

1. To determine socio-demographic caracteristics of the study population

2. To determine the proportion of population which did training on hepatitis prevention

3. To determine the proportion of population who got hepatitis $B$ immunization

Methods: This was a descriptive cross-sectional study that took place from November 19 to December 18, 2018, at the Banfora Regional Hospital Center. The study covered all categories of health professionals likely to be victims of hepatitis exposure accident. A self-questionnaire on the subject was given to health professionals.

Results: The questionnaire was completed by 202 health professionals, representing a $68 \%$ completion rate. The average age of the health workers was 38.67 years. Nurses predominated with $62.81 \%$ of the sample, followed by hospital hygiene technicians (11.06\%). Health care workers who had ever had an hepatitis exposure accident represented $39.80 \%$ of our sample. Midwives were the most affected by hepatitis exposure accident with $64.29 \%$. Only $35.86 \%$ of patients had received training on hepatitis exposure accident during their professional career. Non-reporting of hepatitis exposure accident was mainly due to personal negligence. The majority of caregivers stated that the hepatitis B virus had the highest infectious potential. In our study, $98 \%$ of our sample had been vaccinated against hepatitis B and $61 \%$ had received at least 3 doses. A total of $86.94 \%$ of the health care workers were aware of the precautions for hepatitis exposure accident.

Conclusion: Knowledge of hepatitis exposure accident needs to be improved given the high number of healthcare professionals who have already been victims. Continuing education is therefore needed to increase hepatitis prevention.

Disclosure of Interest: None declared 


\section{P288}

Comparison of perceptions of well-treatment between medical and paramedical staff in a teaching hospital in Sousse-Tunisia M. Mahjoub ${ }^{1}$, S. Chelly ${ }^{1, *}$, O. Ezzi ${ }^{1}$, A. Asma ${ }^{1}$, B. Trabelsi ${ }^{1}$, M. Besbes ${ }^{1}$, M. Njah $^{1}$

${ }^{1}$ Hospital hygiene department, Farhat Hached teaching hospital, Sousse, Tunisia

Correspondence: S. Chelly

Antimicrobial Resistance \& Infection Control 10(1): P288

Introduction: The promotion of well-treatment and its corollary the prevention of mistreatment are currently major issues for health systems.

Objectives: To compare the perceptions of well-treatment among the different categories of health professionals.

Methods: This is a cross-sectional descriptive study carried out over a period of four months (from May 1, 2018 to August 31, 2018) among health professionals of all categories working in the 19 care services of the Farhat Hached hospital in Sousse (Tunisia). Our study was carried out using a questionnaire inspired by the Health authority of health (HAS).

Results: During treatment, significant differences in favor of medical staff are reported for the adaptation of the words and actions to the patient's condition, communication and medical data information respectively $88.7 \%$ versus $71 \% ; p<0.001,68.7 \%$ versus $54 \% ; p=0.006$ and $94 \%$ versus $81 \% ; p<0.001$. On the other hand, significant difference in favor of paramedical staff for the items "be careful not to make noise" (94\% versus $86 \% ; p=0.011)$, knock on the door and wait before entering the patient's room $(66.5 \%$ versus $54.7 \% ; p=0.024)$ and pay attention to non-verbal communications ( $93.5 \%$ versus $86.7 \%$; $p=0.03$ ). In the service, significant differences in favor of the paramedical manager are raised with regard to screening for nutritional disorders (70\% versus $56.7 \% ; \mathrm{p}=0.01)$ and screening for pressure ulcer risk ( $77 \%$ versus $66.7 \% ; p=0.032$ ). During the course of care, the medical manager reported significantly more perceptions in favor of well-treatment for the information provided to the patient as well on his state of health $(77.3 \%$ versus $66.5 \% ; p=0.027)$ than on the adverse events that occurred ( $76 \%$ versus $66 \% ; p=0.043$ ).

Conclusion: The realization of our study among health professionnal at the Farhat Hached hospital of Sousse should argue the need to establish a whole "culture of well contracting" with the implementation of a strategy promoting the development of this state of mind among healthcare professionals.

Disclosure of Interest: None declared

\section{P289}

Ell-treatment in hospitals: perceptions of health professionals in a central tunisian hospital

M. Mahjoub ${ }^{1}$ S. Chelly ${ }^{1,}{ }^{*}$, O. Ezzi ${ }^{1}$, A. Asma ${ }^{1}$, B. Trabelsi ${ }^{1}$, M. Besbes ${ }^{1}$, M. Njah ${ }^{1}$ ${ }^{1}$ Hospital hygiene department, Farhat Hached teaching hospital, Sousse, Tunisia

Correspondence: S. Chelly

Antimicrobial Resistance \& Infection Control 10(1): P289

Introduction: Promoting well-treatment means strengthening a model of comprehensive patient care centered on their needs, expectations, preferences and rights.

Objectives: To specify the degree of application of certain concepts of well-treatment according to the perceptions of healthcare professionals.

Methods: It was a cross-sectional descriptive study carried out during four months (from May 1, 2018 to August 31, 2018) among health professionals of all categories working in the 19 care services of the Farhat Hached hospital in Sousse. The collect was carried out using a questionnaire developed in 2012 by the high authority of health (HAS).

Results: In total, response rate was $66.4 \%$ (350/527). More than three quarter of the participants $(78.6 \%)$ said that they adapt their words and actions to the patient's condition. Of the respondents, $99.4 \%$ said that they provided care with respect for privacy and $94.6 \%$ said that they respected the functional autonomy of the patient. Respondents declared, in more than two third of cases, that patient information is organized in the same way as the collection of the trusted person. Only one percent of respondents said that regular information is provided to the patient on his condition throughout his care.

Conclusion: Therefore, it is necessary to integrate into the Farhat Hached hospital project a prioritization of well-treatment among the primary concerns of the institution requiring immediate support, which implies the mobilization of all institutional actors and that of professionals. Disclosure of Interest: None declared

Poster Session: Nosocomial COVID-19 outbreaks

P290

Risk factors of nosocomial COVID-19 at Grenoble Alpes university hospital

C. Dinh ${ }^{1, *}$, M. Gallouche ${ }^{1,2}$, H. Terrisse ${ }^{1}$, K. Gam ${ }^{1}$, C. Giner ${ }^{2}$, J. Giai1 ${ }^{1,3}$, J.-L. Bosson $^{1,3}$, S. Lambert-Lacroix ${ }^{1}$, C. Landelle ${ }^{1,2}$

${ }^{1}$ Grenoble Alpes/CNRS University, ${ }^{2}$ Hospital Hygiene Service, ${ }^{3}$ Public health department, CHU Grenoble Alpes, Grenoble, France

Correspondence: $C$. Dinh

Antimicrobial Resistance \& Infection Control 10(1): P290

Introduction: During the COVID-19 pandemic, hospitals implemented infection prevention strategies to reduce nosocomial transmission. Nevertheless, these strategies sometimes failed and determination of risk factors of transmission is crucial.

Objectives: Our main objective was to determine the risk factors of nosocomial Covid-19 at Grenoble Alpes University hospital (CHUGA).

Methods: A case-control study was conducted at CHUGA. A retrospective data collection was performed between 01/09/2020 and $31 / 01 / 2021$. Adults patients hospitalized in medicine or surgery units were included. Infants or patients hospitalized in ICU were excluded. Case patients were patients with a nosocomial Covid-19 (clinical symptoms and positive PCR for SARS-CoV-2 or positive PCR for SARSCoV- $\leq 28 C T$ ); control patients were patients without infection (negative PCR for SARS-CoV-2). They were matched by their length of stay and their period of admission. Bivariate and multivariate analysis were performed with a conditional logistic regression by Stata 12.0.

Results: A total of 1393 patients with Covid-19 were hospitalized; 722 patients were included in the case-control analysis $\left(n_{\text {case }}=281\right.$; $\left.\mathrm{n}_{\text {control }}=441\right)$. In bivariate analysis, case patients were significantly older (OR:1.25; Cl95\% [1.12; 1.40]), had more often a roommate (OR:1.74; Cl95\% $[1.23 ; 2.43])$, more often a co-infection (OR:1.73; Cl95\% [1.26; 2.36]), more often a severity risk factor of Covid-19 (OR:2.06; CI95\% [1.14; 3.71]) and a higher Charlson comorbidity index (OR:1,09; CI95\% [1,01; 1,20] than control patients. In an adjusted model that included the admission in the emergency room and the existence of a severity risk factor, the risk factors of nosocomial Covid-19 were: older age (aOR:1.24 per 10 years; CI95\% [1.08; 4.41]), having a roommate (aOR:1.63; CI95\% [1.14; 2.33]), and having a co-infection (aOR:1.62; C195\% [1.17; 2.26]).

Conclusion: Older patients with co-infection hospitalized in a multiple room were more susceptible to nosocomial Covid-19. These preliminary results need to be consolidated taking into account exposition to contagious healthcare workers or contagious patients.

Disclosure of Interest: None declared

\section{P291}

Does the COVID-19 patients have a higher cross infection prevalence? A cross sectional study on healthcare aquired infection at the Tunisian university hospital in 2021

W. Dhouib 1 , S. Bhiri ${ }^{1,2}$, A. Ben Cheikh ${ }^{1,2}$, H. Ghali ${ }^{1,2, *}$, R. Bannour ${ }^{1}$, S. Khefacha ${ }^{1}$, H. Said Latiri ${ }^{1,2}$, M. Ben Rejeb ${ }^{1,2}$

${ }^{1}$ Department of Prevention and Security of Care, sahloul university hospital, 2Faculty of Medicine of Sousse, University of Sousse, Sousse „Tunisia Correspondence: $\mathrm{H}$. Ghali

Antimicrobial Resistance \& Infection Control 10(1): P291 
Introduction: Since the second of March 2020 Tunisia has faced three COVID-19 waves. Until May 16, 2021, the total number of COVID-19 cases reached 327,473 , with a fatality rate of $3.63 \%$. The rate of hospitalized patients in public and private hospitals was $4.7 \%$. Health CareAssociated infections (HCAls) could worsen hospital management and increase the burden of COVID-19.

Objectives: The objective was to determine the prevalence of HCAls among patients with Covid-19 diagnosis at a Tunisian university hospital in 2021.

Methods: The Prevention and Security of Care Department, carried out the prevalence survey for the year 2021. It was a cross-sectional study of HCAls prevalence with a single passage in March. The agreed definition of an HCAls was any infection that occurs during a hospital stay of at least $48 \mathrm{~h}$ and if it was not present at admission. All hospital departments were included in the survey. Patients with Covid-19 diagnosis were hosted in three departments rearranged for this purpose: an Intensive Care Unit (ICU) for the most severe patients, a semi critical unit and a non critical unit. Results: At our passage in the COVID-19 Departments, 25 patients were included in the study, representing $10.9 \%$ of the total sample that included 228 patients. The median age of these Covid-19 patients was 69 years IQR[61-73] with a sex ratio of 0.47 . The prevalence of HCAls in COVID-19 patients was $20 \%(5 / 25)$ with a $95 \% \mathrm{Cl} 16,86-23,13]$. All the five HCAls were notified in the ICU, corresponding to a prevalence of 71.4 with a $95 \% \mathrm{Cl}[67.8$ - 74.9]. Two of the 5 infections were highly resistant bacterimia with acinetobacter baumanii complex, 1 multiresistant germ urinary infection and 2 nonresistant germ respiratory infection.

Conclusion: The prevalence of HAls in COVID-19 patients was particularly high in patients with severe conditions requiring hospitalisation in ICU. This could highlight the need to strengthen training on standard precautions. In addition, an incidence study is required to determine risk factors for HAIS acquisition in COVID-19 patients.

Disclosure of Interest: None declared
P292

Determinants of nosocomial COVID-19: a prospective, multicenter hospital-based study

K. Chen', S. Ghantous ${ }^{1}$, L. Henaff', M. Saadatian-Elahi ${ }^{1}$, P. Vanhems ${ }^{1}$, C. Elias ${ }^{1, *}$

${ }^{1}$ Lyon University Hospital, Lyon, France

Correspondence: C. Elias

Antimicrobial Resistance \& Infection Control 10(1): P292

Introduction: Accurate attribution of nosocomial COVID-19 is challenging, particularly given wide variation in incubation period.

Objectives: Within the multicenter hospital-based prospective NOSOCOR project, we aimed at characterizing the determinants of nosocomial COVID-19.

Methods: An observational, prospective and multicenter study was conducted in a university hospital of 5,300 beds. Any patient diagnosed positive for COVID-19, presenting with a cough and/or fever above $37.8{ }^{\circ} \mathrm{C}$, admitted to the hospital and having stayed there for more than $24 \mathrm{~h}$ was included. The European Center for Disease Prevention and Control (ECDC) defined nosocomial COVID-19 according to three thresholds: $48 \mathrm{~h}$, seven days and 14 days. Comparison of clinical characteristics of COVID-19 hospitalized patients were performed according to the three ECDC thresholds using a multivariate analysis. Results: A total of 1078 patients were included in the study. The median age was 77 [64-86] years old and a sex ratio equal to 1.06 . $996(92.1 \%)$ patients presented at least one comorbidity, with 665 $(61,7 \%)$ patients presenting a cardiac condition. In addition, 341 (45.5\%) patients had a contact in a health care facility with a COVID19 suspected or confirmed case in the 14 days before symptom onset. Patients aged above $75(p<0.01)$ and having a contact in a healthcare facility with a COVID-19 case 14 days before symptom onset $(p<0.001)$ were found as determinants of nosocomial COVID-19 regardless of the threshold (Table 1a, 1b, 1c). Diabetes was significantly associated with nosocomial COVID-19 $(p<0.05)$ when the delay between admission and symptom onset was greater than 7 days.

\begin{tabular}{|c|c|c|c|c|c|c|}
\hline & \multicolumn{4}{|c|}{ Univoriate analyyis } & \multicolumn{2}{|c|}{ Multivariate anolysis } \\
\hline & $\begin{array}{l}\left.\mathrm{J}-{ }^{-}, 2\right] \\
\mathrm{N}=968(89.8 \%)\end{array}$ & $\begin{array}{l}12,+\infty-1] \\
N=110(10.2 \%)\end{array}$ & OR (C1 95\%) & p-value & OR (C 95\%) & p-value \\
\hline Age [median, IQR] & $75[62-86]$ & $85[80-90]$ & & & & \\
\hline$[18-75]$ & $491(50.7 \%)$ & $18(16.4 \%)$ & Ref & & & \\
\hline [76-102] & 477 (49.3\%) & $92(83.6 \%)$ & $5.2[3.2-9.1]$ & 50.001 & $3.7[1.97-7.69]$ & 0.001 \\
\hline BMI & $25.8[22.6-29.3]$ & $23.4[20.4-26.9]$ & & & & \\
\hline $230 \mathrm{~kg} / \mathrm{m}^{2}$ & $31(4.6 \%)$ & $3(3 \%)$ & $0.6[0.14-1.71]$ & 0.61 & & \\
\hline \multicolumn{7}{|l|}{ Sex } \\
\hline Male & $515(53.2 \%)$ & $39(35.5 \%)$ & $2.1[1.4-3.1]$ & $\$ 0.001$ & & \\
\hline $\begin{array}{l}\text { Female } \\
\text { Ward }\end{array}$ & 453 (46.8\%) & $71(64.5 \%)$ & Ref & Ward & & \\
\hline Medical, Surgery, Obstetric & 761 (78.6\%] & $110(100 \%)$ & $1.6 \mathrm{et} 7[5.7-5 \mathrm{e}+82]$ & $\$ 0.001$ & & \\
\hline \multirow{2}{*}{\multicolumn{7}{|c|}{ Comorbidities }} \\
\hline & & & & & & \\
\hline Cardiovascular disease & 576 (59.5\%) & $89(80.9 \%)$ & $2.88[1.8-4.8]$ & $\$ 0.001$ & & \\
\hline Diabetes & $229(23.7 \%)$ & $30(27.3 \%)$ & $1.21[0.77-1.87]$ & 0.4 & & \\
\hline Immunodeficiency & $69(7.1 \%)$ & $9(8.2 \%)$ & $1.16[0.53-2.28]$ & 0.83 & & \\
\hline Liver disease & $66(6.8 \%)$ & $8(7,3 \%)$ & $1.07[0.46-2.17]$ & 1 & & \\
\hline Renal disease & $154(16 \%)$ & $24(21.8 \%)$ & $1.48[0.9-2.4]$ & 0.11 & & \\
\hline Hypothyroidism & $84(8.7 \%)^{\circ}$ & $15(13.6 \%)$ & $1.66[0.89-2.92]$ & 0.09 & & \\
\hline Rheumatic disease & $145(15 \%)$ & $26(23.6 \%)$ & $1.76[1.08-2.78]$ & 0.02 & & \\
\hline Chronic lung diseare & $126(13 \% 6)$ & $17(15.5 \%)$ & $1.2[0.68-2.1]$ & 0.48 & & \\
\hline Malignancy & $217(22.4 \%)$ & $34(30.9 \%)$ & $1.55[1-2.4]$ & 0.05 & & \\
\hline \multicolumn{7}{|c|}{$\begin{array}{l}\text { Contoct with suspected or confirmed } \\
\text { case }\end{array}$} \\
\hline case & & & & & & \\
\hline Healthcare setting & 262 (39.9\%) & $79(84.9 \%)$ & $8.5[4.9-16]$ & $<0.001$ & 5.9 [3.3-11.2] & $\$ 0.001$ \\
\hline Work place & $19(2.9 \%)$ & $0(0)$ & $4 e-7[0-1.4]$ & 0.15 & & \\
\hline Family setting & $147(22.4 \%)$ & $0\langle 0\rangle$ & $\operatorname{Se}-8[0-0.13]$ & $<0.001$ & & \\
\hline
\end{tabular}

BMI : Body Mass Index; IQR : Interquartile Range : OR : Odds-Ratio : IC : Confidence Interval 


\begin{tabular}{|c|c|c|c|c|c|c|}
\hline & \multicolumn{4}{|c|}{ Univariate anolygis } & \multicolumn{2}{|c|}{ Multivariate analygis } \\
\hline & $\begin{array}{l}1-, 7] \\
N=995 \text { (92.3\%) }\end{array}$ & $\begin{array}{l}{[7,+\infty[1} \\
N=83(7.7 \%)\end{array}$ & OR (C1 95\%) & p-value & OR (C 95\%) & p-value \\
\hline Age [median, IQR] & $75[62-86]$ & $86[90-90]$ & & & & \\
\hline$[18-75]$ & 499 (49.9\%) & $10(12.8 \%)$ & Ref & & & \\
\hline$[76-102]$ & 501 (50.1\%) & $68(87.2 \%)$ & $6.8[3.6-14]$ & $\$ 0.001$ & $4.5[2.1-11.3]$ & $\infty 0.001$ \\
\hline BMI & $25.8[22.6-29.3]$ & $23.1(20-26]$ & & & & \\
\hline $230 \mathrm{~kg} / \mathrm{m}^{2}$ & $33(4.8 \%)$ & $1(1.3 \%)$ & $0.26[0.01-1.25]$ & 0.24 & & \\
\hline \multicolumn{7}{|l|}{ Sex } \\
\hline Male & $524(52.7 \%)$ & $30(36.1 \%)$ & $1.97[1.2-3]$ & 0.004 & & \\
\hline Female & 471 (47.3\%) & $53(63.9 \%)$ & Ref & & & \\
\hline \multicolumn{7}{|l|}{ Word } \\
\hline Medical, Surgery, Obstetrics & 788 (79.2\%] & $83(100 \%)$ & $3.3 e+7[6.7 e-5-2.6 e+126]$ & $\infty .001$ & & \\
\hline Intensive Care Unit & $207(20.8 \%)$ & $0(08 \%)$ & Ref & & & \\
\hline \multicolumn{7}{|l|}{ Comorbidities } \\
\hline Cardiovascular disease & 596 (59.9\%) & $69(83.1 \%)$ & $3.3[1.92-6.2]$ & $\$ 0.001$ & & \\
\hline Diabetes & 230 (23.1\%) & $29(34.9 \%)$ & $1.79[1.1-2.9]$ & 0.02 & $2[1-3.5]$ & 0.01 \\
\hline Immunodeficiency & $71(7.1 \%)$ & $7(8.4 \%)$ & $1.2[0.49-2.5]$ & 0.83 & & \\
\hline Liver disease & $67(6.7 \%)$ & $7(8.4 \%)$ & $1.28[0.52-2.7]$ & 0.72 & & \\
\hline Renal disease & $157(15.8 \%)$ & $21(25.3 \%)$ & $1.8[1.05-3]$ & 0.02 & & \\
\hline Hypothyroidism & $87(8.7 \%)$ & $12(14.5 \%)$ & $1.76[0.88-3.3]$ & 0.08 & & \\
\hline Rheumatic disease & $153(15.4 \%)$ & $18(21.7 \%)$ & $1.5[0.86-2.6]$ & 0.13 & & \\
\hline Chronic lung diseare & $133(13,4 \%)$ & $10(12 \%)$ & $0.89[0.48-1.7]$ & 0.73 & & \\
\hline & 230 [23.1\% & $21(25.3 \%)$ & $1.13[0.66-1.86]$ & 0.65 & & \\
\hline \multicolumn{7}{|c|}{ Contact with suspected or confirmed } \\
\hline & & & & & & \\
\hline Healthcore setting & 279 (41.3\%) & $62(84 \%)$ & $7.4[4-14.6]$ & $\infty 0.001$ & $5.1[2.7-10.7]$ & $\$ 0.001$ \\
\hline Work place & $19(2.8 \%)$ & $0(0)$ & $6 e-07[0-8]$ & 0.24 & & \\
\hline Family setting & $147(21.7 \%)$ & $0(0)$ & $6 e-08[0-29]$ & $\$ 0.001$ & & \\
\hline
\end{tabular}

Table 1c. Determinants of nosocomial COVID-19 defined with a 14 days threshold

\begin{tabular}{|c|c|c|c|c|c|c|}
\hline & \multicolumn{4}{|c|}{ Uniwariate analysis } & \multicolumn{2}{|c|}{ Multivoriate anolysis } \\
\hline & $\begin{array}{l}1-, 14] \\
N=1024(95 \%)\end{array}$ & $\begin{array}{l}114,+\infty-1 \\
N=54(5 \%)\end{array}$ & OR (C1 95\%) & p-value & OR (Cl 95\%) & p-value \\
\hline Age [median, IQR] & $76[63-86]$ & $85[85-86]$ & & & & \\
\hline [18-75] & $502(49 \%)$ & $7(13 \%)$ & Ref & & & \\
\hline$[76-102]$ & $522(51 \%)$ & $47(87 \%)$ & $6.5[3.1-15.8]$ & $\infty .001$ & $4.3[1.7-12.7]$ & 0.003 \\
\hline BMI & $25.7[22.5-29]$ & $23.4[20-27]$ & & & & \\
\hline $230 \mathrm{~kg} / \mathrm{m}^{2}$ & $33(4.6 \%)$ & $1(2 \%)$ & $0.42[0.02-2.04]$ & 0.72 & & \\
\hline \multicolumn{7}{|l|}{ Sex } \\
\hline Male & $535(52.2 \%)$ & $19(35.2 \%)$ & $2[1.2-3.6]$ & 0.01 & & \\
\hline Female & $489(47.8 \%)$ & $35(64.8 \% 6)$ & Ref & & & \\
\hline \multicolumn{7}{|l|}{ Ward } \\
\hline Medical, Surgery, Obstetric & $795(77.6 \%)$ & $54(100 \%)$ & $2.1 e+7(5.3 e-7-6.5 e+115)$ & $\$ 0.001$ & & \\
\hline Intensive Care Unit & $207(20.2 \%)$ & $0(0 \%)$ & Ref & & & \\
\hline \multicolumn{7}{|l|}{ Comorbidities } \\
\hline Cardiovazcular disezese & $620(60.5 \%)$ & $45(83.3 \%)$ & $3.3[1.7-7.2]$ & $\infty 0.001$ & & \\
\hline Diabetes & 236 (23\%) & $24(42.9 \%)$ & $2.5[1.4-4.3]$ & 0.001 & $2.8[1.5-5.3]$ & 0.001 \\
\hline Immunodeficiency & $75(7.3 \%)$ & $3(5.6 \%)$ & $0.74[0.18-2.1]$ & 0.79 & & \\
\hline Liver disease & $71(6.9 \%)$ & $3(5.6 \%)$ & $0.79[0.19-2.2]$ & 1 & & \\
\hline Renal disease & $169(16.5 \%)$ & $9(16.7 \%)$ & $1.01[0.46-2.01]$ & 0.98 & & \\
\hline Hypothyroidism & $92(9 \%)$ & $7(13 \%)$ & $1.5[0.6-3.2]$ & 0.32 & & \\
\hline Rheumatic disease & $161(15.7 \%)$ & $10(18.5 \%)$ & $1.22[0.57-2.37]$ & 0.58 & & \\
\hline Chronic lung diseare & $138(13,5 \%)$ & $5(9.3 \%)$ & $0.66[0.22-1.52]$ & 0.37 & & \\
\hline Malignancy & $239(23.3 \%)$ & $12(22.2 \%)$ & $0.94[0.47-1.78]$ & 0.85 & & \\
\hline \multirow{2}{*}{\multicolumn{7}{|c|}{$\begin{array}{l}\text { Contoct with suspected or confirmed } \\
\text { cose }\end{array}$}} \\
\hline cose & & & & & & \\
\hline Healthcore setting & $300(42.8 \%)$ & $41(82.7 \%)$ & $6.9[3.3-16]$ & $\infty .001$ & $4.6[2.2-10.9]$ & $<0.001$ \\
\hline Work place & $19(2.7 \%)$ & $0(0 \%)$ & $3 e-7[0-32]$ & $\infty 0.001$ & & \\
\hline Family setting & $147(21 \%)$ & $0(0 \%)$ & $3 e-8[0-35]$ & $\$ 0.001$ & & \\
\hline
\end{tabular}

Conclusion: Age above 75 and being identified as a contact case from a healthcare facility were found to be three to five times more at risk of developing a nosocomial COVID-19. Little heterogeneity was found in the application of nosocomial COVID-19 definition. However, in practice a balance between a sensible and specific definition taking into account population at risk would be helpful in this timely framework. Disclosure of Interest: None declared
P293

Comparison between healthcare-associated and community-acquired hospitalised COVID-19 cases from the Swiss prospective COVID-19 cohort

G. Catho ${ }^{1, *}$, A. Atkinson ${ }^{2}$, S. D'incau ${ }^{2}$, N. Buetti ${ }^{1}$, J. Marschall2, S. Harbarth'1, A. Iten ${ }^{1}$

${ }^{1}$ Division of Infection Control, Geneva University Hospitals and Faculty of Medicine, Geneva, ${ }^{2}$ Department of Infectious Diseases, Bern University Hospital and University of Bern, Bern, Switzerland

Correspondence: G. Catho 


\section{Antimicrobial Resistance \& Infection Control 10(1): P293}

Introduction: Numerous reports of healthcare-associated COVID19 (HA-COVID-19) outbreaks have highlighted that hospitals can be a platform for SARS-CoV-2 transmission. Uncertainty remains with regards to clinical outcomes of patients who contracted SARS-CoV-2 in healthcare facilities compared to those hospitalized after community acquisition (CA-COVID-19).

Objectives: The objective of this study was to describe and compare characteristics and clinical outcomes of patients with HA-COVID-19 versus CA-COVID-19.

Methods: We used data from 16 hospitals included in the prospective national surveillance on COVID-19 in Switzerland. We included all hospitalized COVID-19 adult cases with a laboratory confirmed infection. HA-COVID-19 cases were defined as those detected $>5$ days after hospital admission. Only the first hospital stay after diagnosis for CACOVID-19 cases, and during diagnosis for HA-COVID-19 cases, were considered. Cases with no information on place of acquisition were excluded.

Results: Between February and December 2020, 1'389 HA-COVID-19 cases and 9'139 CA-COVID-19 were included. HA-COVID-19 patients were older than CA-COVID-19 (median [IQR] age: 79 [70-86] versus 70 [57-80], predominantly female (48.2\% versus $39.6 \%)$, and were more likely to have a Charlson comorbidity index $>4$ (78.2\% versus $54.9 \%)$. At the time of diagnosis, HA-COVID cases were most frequently hospitalized in general medical $(570,41 \%)$ and Geriatric/Rehabilitation wards $(409,29.4 \%)$. Length of stay was shorter for CA-COVID-19 cases (median 15, IQR [10-23] days from admission) than for HA-COVID-19 (17 [9-30] days from COVID-19 diagnosis). Fewer HA-COVID-19 patients stayed in intermediate or intensive care units (ICU) (223 [16.1\%] versus $2^{\prime} 031$ [22\%] of CA-COVID-19 cases) ( $\left.p<0.001\right)$, and fewer HA-COVID-19 cases experienced any COVID-19 complications (770 (65.7\%) versus 6665 (83.5\%), $\mathrm{p}<0.001)$. Overally, 350 (26.6\%) HA-COVID-19 and 1225 (13.9\%) CA-COVID-19 died.

Conclusion: Patients who acquired COVID-19 within the hospital were older and more comorbid. They were less frequently transferred to the intermediate or ICU and experienced fewer COVID-19 complications, but suffered from higher rates of hospital mortality.

Disclosure of Interest: None declared

P294

Nosocomial COVID-19 in a tertiary care center - incidence

and secondary attack rates after exposure

A. Wolfensberger ${ }^{1, *}$, P. W. Schreiber ${ }^{1}$, A. Frey ${ }^{1}$, V. Schärer ${ }^{1}$, C. O. Hundal ${ }^{1}$, D. Saleschus ${ }^{1}$, M. Vazquez ${ }^{1}$, W. Zingg ${ }^{1}$

${ }^{1}$ Department of Infectious Diseases and Hospital Epidemiology, UNIVERSITY HOSPITAL ZÜRICH, Zürich, Switzerland

Correspondence: A. Wolfensberger

Antimicrobial Resistance \& Infection Control 10(1): P294

Introduction: Patients not suspected to be infected with SARS-CoV2 but suffering from COVID-19 or patients hospitalized during the incubation period of COVID-19 pose a risk for transmitting SARS-CoV2 to fellow patients.

Objectives: We aimed to describe the incidence of patients hospitalized in our tertiary care center with community- and healthcareassociated COVID-19, calculate the number of patients who exposed other patients to SARS-CoV2 and the secondary attack rate of exposed patients, and investigate risk factors for SARS-CoV2 transmission.

Methods: In this retrospective study, all patients admitted to the University Hospital Zurich, Switzerland, with a positive SARS-CoV2 PCR were included. Community- or healthcare-associated COVID-19 were defined according to the European Centre for Disease Prevention and Control (ECDC) criteria. Patients receiving standard care during their period of contagiousness were defined as potential index patients. Exposed patients were patients sharing a room with a potential index patient 1) on the general ward for any time, 2) on the intermediate or intensive care unit (IMC/ICU) for $\geq 6 \mathrm{~h}$, or 3 ) on the IMC/ICU for any time with an index undergoing aerosol-generating procedures.
Results: Of 1131 patients with a positive SARS-CoV2 PCR or labeled to be a patient with COVID-19 between October 2020 and April 2021 $90.6 \%(n=1025)$ had community-associated illness, 3.5\% $(n=40)$ had probable and $3.8 \%(n=43)$ had definite healthcare-associated COVID19 , and $2.0 \%(n=23)$ had indeterminate illness. Of all patients, $19.0 \%$ $(n=215)$ were potential index patients, and $56.3 \%(n=121)$ of these exposed at least one other patient. Of 292 exposed patients, 47 were later diagnosed with COVID-19. The mean secondary attack rate per index patient was $14.4 \%$. Transmission of SARS-CoV2 was higher if contact time was longer (adjusted Odds Ratio (aOR): $1.03,95 \% \mathrm{Cl}$ : 1.02 1.04 ) and if CT-value of index was lower (aOR: $0.92,95 \% \mathrm{Cl}: 0.87-0.97$ ).

Conclusion: The secondary attack rate after exposure to SARS-CoV2 of patients in the hospital setting is comparable to rates reported from the household setting. The risk of an incident SARS-CoV2 infection increases with longer duration of exposure and higher viral load of index patient

Disclosure of Interest: None declared

P295

A SARS-COV-2 (B.1.1.7) outbreak at a non-COVID inpatient ward at a hospital: lessons learned

M. Dekker ${ }^{1, *}$, J. Koopsen ${ }^{1}$, J. Schinkel ${ }^{1}$, I. Spijkerman ${ }^{1}$

${ }^{1}$ Department of Medical Microbiology \& Infection Prevention, Amsterdam UMC, Amsterdam, Netherlands

Correspondence: $M$. Dekker

Antimicrobial Resistance \& Infection Control 10(1): P295

Introduction: The severe acute respiratory syndrome coronavirus 2 (SARS-CoV-2) lineage B.1.1.7 has an increased transmissibility compared to predecessor lineages. Healthcare workers (HCWs) are at an increased risk of being exposed to SARS-CoV-2 but also of being a source of transmission.

Objectives: To describe the lessons learned from a B.1.1.7 outbreak in a tertiary hospital.

Methods: An Outbreak Management Team developed a mitigation strategy.

1) The importance of the prevailing infection control measures, including social distancing, capacity limits for rooms, universal masking in case of $<1.5 \mathrm{~m}$ distance and the early testing and domestic quarantine of $\mathrm{HCW}$ with symptoms was stressed.

2) An infection control practitioner visited the ward each working day during the outbreak period, to advise and observe practice.

3) Contacts were traced and divided into high-risk and low risk contacts in the workplace and in household/social contacts.

4) Voluntary nasopharyngeal swabs were taken twice a week, to detect asymptomatic cases.

5) Potential weak links of infection control measures were discussed with nurses.

Results: Nine nurses and two informal caregivers tested RT-PCR positive for SARS-CoV-2 in December 2020. The index nurse tested positive following the earlier infection of a household contact. The outbreak was recognized a day later when the second nurse tested positive and was confirmed by Whole Genome Sequencing. Of the remaining nine cases which tested positive in the following 11 days, one case tested positive after a documented SARS-CoV-2 infection 83 days prior.

We found a primary attack rate within the department of $18 \%$ and a secondary attack rate of $54 \%$ among contacts of positive cases.

Short conversations in changing rooms without masks, short periods of lack of social distancing during breaks and the incorrect wearing of masks were mentioned as potential causes for transmission.

Conclusion: Two keys point were taken from this outbreak with lineage B.1.1.7. First, it was controlled by adherence to guidelines in place, despite increased transmissibility of the variant. Second, reinfections with lineage B.1.1.7 can occur rapidly after primary infection.

These lessons, reiterate the importance of adherence to prevailing infection prevention methods to prevent transmission among HCW.

Disclosure of Interest: None declared 


\section{P296}

Would the reinforcement of standard precautions help to reduce the occurrence of nosocomial COVID cases in non-COVID wards? A. Iten ${ }^{1, *}$, V. Camus ${ }^{1}$, C. Fankhauser ${ }^{1}$, M. Ares ${ }^{1}$, A. Graindorge ${ }^{1}$, F. Favre ${ }^{1}$, M. Rousseau-Chadegg ${ }^{1}$, M. Steiner ${ }^{1}$, G. Golaz ${ }^{1}$, J. Carry ${ }^{1}$, J. Castro ${ }^{1}$, L. Thivolet ${ }^{1}$, D. Pittet $^{1}$

${ }^{1}$ Prevention Control Program, HUG, Genève, Switzerland

Correspondence: A. Iten

Antimicrobial Resistance \& Infection Control 10(1): P296

Introduction: Since the beginning of the COVID-19 pandemic, HUG hospital is mandated to care for patients suspected of having or affected by COVID-19. The majority of the wards (W) at HUG became COVID W; CONTACT and DOPLETS measures replaced standard precautions (SP). Additional measures were implemented to prevent nosocomial (NOSO) cases: control of visitors and staff at the entrance of the hospital, surgical mask wearing, screening of patients and staff, epidemiological surveillance. Vaccination started in January 2021. On 26.05.2021, $17.3 \%$ of the general population and $57.5 \%$ of the hospital staff received at least one dose.

Objectives: To reduce the occurrence of NOSO cases in non-COVID W

Methods: The program covers the period from 01.01 to 30.06 .2021 and concerns non-COVID units. It is based on several axes of intervention, including: 1.Training: medical-nursing managers of the non-COVID W were invited to participate in a training session on the topic "SP". Trainers were 3 IPC professionals and 1 adult education specialist. 2. Monitoring of compliance with hand hygiene, mask wearing and provision of alcohol based handrub at the $\mathrm{W}$ entrance. Observers are trained, validated and interrater reliability tested. Feedback was applied: oral immediate feedback; monthly delayed feedback using a poster for each $W$ with SP' compliance, explaining the results with the hierarchy, and set the goal for next month. A written report was sent to the hospital management.

Patients' data are from the prospective national surveillance on COVID-19 identified hospitalized COVID-19 adult cases with a laboratory confirmed infection. NOSO cases were defined as those detected $>5$ days after hospital admission.

Results: From 24.08.2020 to 23.05.2021, 1863 community acquired COVID cases and 497 NOSO cases were collected. The percentage of NOSO cases decreased after the introduction of the program (before $426 / 1589$ (26.8\%) after $71 / 771(9.2 \%), p<0.001)$.

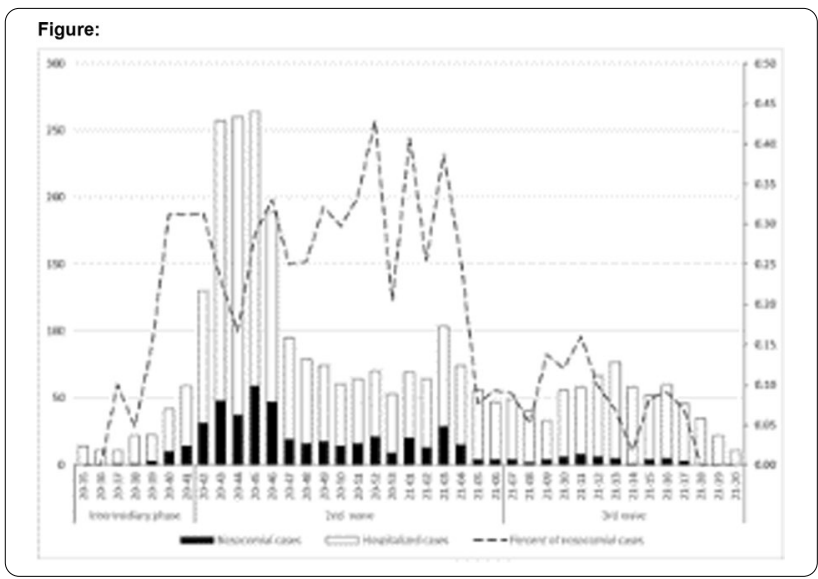

Conclusion: Following the intervention, the incidence of NOSO cases has markedly decreased

Disclosure of Interest: None declared
P297

Control of nosocomial transmission of SARS-COV-2 in the service of internal medicine in Lausanne university hospital

E. Kampouri ${ }^{1,2, *}$, E. Glampedakis ${ }^{1}$, E. Moulin ${ }^{1}$, G. Chaillou ${ }^{1}$, I. Federli ${ }^{1}$, M. Gouffon $^{3}$, J. Vaucher ${ }^{3}$, C. Sartori ${ }^{3}$, P. Vollenweider ${ }^{3}$, B. Grandbastien ${ }^{1}$, L.

Senn ${ }^{1}$

${ }^{1}$ Service of Hospital Preventive Medicine, ${ }^{2}$ Infectious Diseases Service,

${ }^{3}$ Service of Internal Medicine, Lausanne University Hospital and University of Lausanne, Lausanne, Switzerland

Correspondence: E. Kampouri

Antimicrobial Resistance \& Infection Control 10(1): P297

Introduction: Transmission of SARS-CoV-2 inside the hospital has significant implications for patients and healthcare workers.

Objectives: We describe a nosocomial outbreak of SARS-CoV-2 infections in the Service of Internal Medicine, the implemented infection prevention and control (IPC) measures and their impact.

Methods: We report all consecutive nosocomial cases in a 206-bed service from February 8 until April 8, 2021 (weeks 6-14). Nosocomial COVID-19 cases were defined as microbiologically documented cases by $P C R$, diagnosed $\geq 5$ days after admission. A cluster was defined based on the detection of $\geq 2$ nosocomial COVID-19 cases within a given period $(<72 \mathrm{~h}$ ) or geographically linked. IPC measures implemented on week 10 to control SARS-CoV-2 transmissions in all nonCOVID units were: systematic screening at day 3 after admission; weekly screening of all patients by PCR on saliva; and decreasing the occupancy of all patient rooms with 5 patients to 3 .

Results: 69 cases were identified, with 57 attributed to 21 clusters and 12 cases considered isolated. Median age was 74 years (IQR 65, 80) and $40.6 \%$ were female. All cases had a previous negative test and median time from admission to positive test was 12 days (IQR 8,17$) .45 .1 \%$ of nosocomial cases were completely asymptomatic. $60.9 \%$ of patients stayed in rooms with 5-patients. Death occurred in $23.2 \%$ of cases. The incidence of nosocomial cases was the highest between weeks 8 and 10 with 18 and 17 new cases per week, respectively. After the introduction of IPC measures on week 10, there was a rapid decrease in the number of cases until complete absence of new cases on week 14.

Conclusion: Nosocomial infections were frequently asymptomatic potentially hampering fast diagnosis which is crucial for control of transmission, and were associated with high mortality rate. The implementation of additional IPC measures led to a gradual decrease in nosocomial transmissions and allowed controlling the outbreak.

Disclosure of Interest: None declared

P298

Nosocomial transmission of SARS-COV-2: experience from the contact tracing activity of the infection control unit for the university hospital of lausanne

E. Glampedakis ${ }^{1}{ }^{*}$, E. Kampouri ${ }^{1}$, E. Moulin ${ }^{1}$, F. Boiron ${ }^{1}$, P. Bressin ${ }^{1}$, I. Federli Hanachi ${ }^{1}$, G. Chaillou ${ }^{1}$, D. Gustin ${ }^{1}$, M. Gyger Wanzenried ${ }^{1}$, L. Kalbermatter ${ }^{1}$, I. Koenig ${ }^{1}$, A. Lacot ${ }^{1}$, M. Limet-Dutoit ${ }^{1}$, G. H. Salehi ${ }^{1}$, M.-J. Thevenin ${ }^{1}$, E. Viaccoz ${ }^{1}$, A. Vital-Heilbronn¹, B. Grandbastien', L. Senn ${ }^{1}$

${ }^{1}$ Service de médecine préventive et Hospitalière, Département de Médicine, Centre Hospitalier Universitaire Vaudois (CHUV), Lausanne, Switzerland

Correspondence: E. Glampedakis

Antimicrobial Resistance \& Infection Control 10(1): P298

Introduction: The high transmissibility of SARS-CoV-2 is of particular concern for hospitals as hospitalized patients are at risk of severe COVID-19 and related death.

Objectives: Describe the contact tracing activity related to nosocomial SARS-CoV-2 cases in our hospital.

Methods: The Infection Control Unit received alerts for positive PCR results performed by the Microbiology laboratory in hospitalized patients. Nosocomial infections, occurring after at least 5 days of hospitalization, were further investigated. Contact tracing was performed via an institutional software allowing tracing index patients' movements and their roommates. Patients were considered "contacts" at risk and were placed in quarantine if they shared the same room 
with the index-case up to $72 \mathrm{~h}$ before index's first positive PCR or first symptoms. Contacts systematically had nasopharyngeal SARS-CoV-2 PCR testing at days 0,10 counting from last contact with the index or at symptom onset.

Results: Between November $1^{\text {st }} 2020$ until March $31^{\text {st }} 2021,322$ nosocomial SARS-CoV-2 cases were identified, of whom 195 (61\%) had previously been in contact with another known case while for 127 (39\%) source of infection was unknown. Median time from admission to positive PCR was 13 days (IQR 8 - 25). Symptoms where present in $67 \%$ of cases. Median age was 76 years (IQR $64-84$ ). Of all episodes, $75 \%$ were diagnosed in medical units (including 50 cases, $16 \%$ in geriatric sections), $24 \%$ in surgical sectors and $1 \%$ in the ICU. Contact tracing activity identified 605 contacts with a median of 2 contacts per index case (range $0-19$ ). Of these, $32 \%$ had a positive PCR result during follow-up.

Conclusion: A thorough contact tracing with systematic PCR screening is necessary after detection of a nosocomial SARS-CoV-2 case as transmissibility is high and more than $1 / 3$ of cases are asymptomatic. The non-identification of a source for more than a third of cases raises concerns of potential implication of healthcare workers in transmission.

Disclosure of Interest: None declared

\section{P299}

Clusters of patients who had initial negative RT-PCR then converted to positive COVID-19

N. Dagunton 1,*, H. Rahman ${ }^{1}$, J. Rimmer², N. Rosso ${ }^{3}$, M. Brizuela', K. Erbeck ${ }^{3}$, H. Vyas ${ }^{3}, G$. Espiritu ${ }^{3}$ on behalf of no

${ }^{1}$ Infection Control, ${ }^{2}$ medical staff, ${ }^{3}$ nursing, Hoboken University Medical

Center, Hoboken, United States

Correspondence: N. Dagunton

Antimicrobial Resistance \& Infection Control 10(1): P299

Introduction: Diagnosing SARS-CoV-2 infection is essential in stopping the spread of COVID19, yet we have to take into account that onset of symptoms often vary among our exposed patients.

Objectives: Investigate the probable causes of conversions among the clusters of patients with negative RT-PCR COVID19 results upon admission who converted to positive PCR after 2-18 days in one of the acute care hospitals in New Jersey, USA.

Methods: A descriptive study was conducted to identify cases, trace exposed contacts, and identify the probable causes of conversion. A confirmed case was defined as a positive reverse-transcription polymerase reaction test for SARS-COV-2. COVID19 cases were categorized as community-acquired, and or hospital-acquired. Hospital-acquired is defined as an occurrence of a positive PCR result or onset of symptoms on or after 7 days post admission.

Results: There were 18 patients admitted, all tested negative, and were not place on isolation. Within 7 days post admission 11 (61\%) tested positive. Review of records showed that 14 (78\%) were community-acquired infection and 4 (22\%) were HAls. Symptomatic upon admission $11(61 \%)$ and asymptomatic $7(39 \%)$. Twelve (67\%) with 2 or more comorbidities and the rest none.

During their stay 5 patients $(28 \%)$ required high flow oxygenation, 1 required intubation. Thirteen (72\%) on nasal cannula/room air. Five (28\%) expired, 12 recovered/discharged, and 1 still in the hospital.

Conclusion: The conversions among these patients could be due to full reliance on the diagnostic RT-PCR and not considering clinical conditions, breaches in infection control, and gaps in identifying exposed patients from the community prior to admission.

Thus, strict infection control measures were implemented which included enhanced surveillance screening through RT-PCR on day 1 , day 3 and day 5; more rigid environmental terminal cleaning even in non-Covid units where most of the conversion occurred; increased awareness among physicians and staff for any subtle changes in clinical condition to place in isolation and repeat testing, dedication of staff and equipment, and refresher courses on the use of personal protective equipment.

Disclosure of Interest: None declared
P300

Outbreaks of seasonal OC43 coronavirus with nosocomial transmission during COVID-19 pandemic setting: when a coronavirus hides another

E. Moulin ${ }^{1, *}$, E. Kampouri ${ }^{1}$, E. Glampedakis ${ }^{1}$, M. Gyger Wanzenried ${ }^{1}$, E. Gilles de Pélichy², O. Opota ${ }^{3}$, L. Senn ${ }^{1}$

${ }^{1}$ Infection Control, ${ }^{2}$ Psychiatry Department, ${ }^{3}$ Departement of Pathology and Laboratory Medicine, Centre Hospitalier Universitaire Vaudois, Lausanne, Switzerland

Correspondence: E. Moulin

Antimicrobial Resistance \& Infection Control 10(1): P300

Introduction: Management and control of COVID-19 outbreaks in hospital settings represent a major challenge. Any suspicion of nosocomial transmissions require prompt investigations and control measures.

Objectives: To describe the management of 2 consecutive clusters of patients presenting suspect symptoms of Covid-19 in a 19-bed psychogeriatric unit, hosting concomitantly 2 patients with SARS-CoV-2 infection confirmed by RT-PCR on nasopharyngeal swabs (lineage B.1.1.7, viral loads of $6.9 \mathrm{E}+8$ and $8.0 \mathrm{E}+7$ copies $/ \mathrm{ml}$, respectively) among whom one was a nosocomial case with persistence of a high viral load at day $14(8.9 \mathrm{E}+6$ copies $/ \mathrm{ml})$.

Methods: After identification of the SARS-CoV-2 cases, control measures were promptly applied. During the following weeks, the clinicians of the ward additionally reported 2 clusters of 3 patients with suspect symptoms. All of these suspect cases were isolated with Droplet Precautions and were investigated by a nasopharyngeal swab for SARSCoV-2 testing. If the first SARS-CoV-2 RT-PCR was negative, a second test was performed within $24 \mathrm{~h}$. Additionally, we proposed to complete investigations by an extended respiratory multiplex RT-PCR.

Results: Among the 6 symptomatic patients, all had 2 consecutively negative SARS-CoV-2 RT-PCR. The respiratory virus panel test revealed a positive $\mathrm{PCR}$ for $\mathrm{OC} 43$ coronavirus in $5 / 6$ patients, with viral load ranging from $3 \mathrm{E}+5$ to $3 \mathrm{E}+9$ copies $/ \mathrm{ml}$, confirming a nosocomial outbreak of a seasonal coronavirus. For the remaining patient, infection by OC43 coronavirus was considered possible, regarding the close contacts with positive cases during the hospital stay.

Conclusion: Even if the actual Covid-19 epidemic setting should conduct to promptly research a SARS-CoV-2 infection in symptomatic hospitalized patients, this report highlights the possibility of cocirculation of different respiratory virus within the same ward. More extended microbiological investigations with specific RT-PCR analysis in symptomatic patients repeatedly tested negative for Covid-19, can conduct to a better understanding of nosocomial outbreaks. Sometimes a coronavirus can hide another!

Disclosure of Interest: None declared

Poster Session: COVID-19 outcomes and prediction

P301

Factors associated with prolonged intubation among COVID-19 patients in the region of Mahdia (Tunisia)

H. B. Bayoudh ${ }^{1, *}$, F. Ben Youssef $f^{2,3,4}$, O. Jawed $^{5}$, I. Mlouki ${ }^{2,3,4}$, N. Omri $^{2,3,4}$, H. $\mathrm{Sfar}^{3}$, H. Nouira ${ }^{5}$, M. F. Hassen ${ }^{5}$, S. el atrous ${ }^{5}$, S. El Mhamdi ${ }^{2,3,4}$

${ }^{1}$ Department of Family Medicine, University Hospital Tahar Sfar, Mahdia,

${ }^{2}$ Department of Community Medicine, Faculty of Medicine, Monastir,

${ }^{3}$ Department of Preventive and Community Medicine, University Hospital Tahar Sfar, ${ }^{4}$ Research laboratory, "Epidemiology Applied to Maternal and Child Health" 12SP17, ${ }^{5}$ Department of Intensive Care Medecine,

University Hospital Tahar Sfar, Mahdia, Tunisia

Correspondence: H. B. Bayoudh

Antimicrobial Resistance \& Infection Control 10(1): P301

Introduction: The high mortality among intubated COVID-19 patients in Intensive Care Unit (ICU) has given rise to a debate over risk factors related to a prolonged intubation period.

Objectives: In this study, we aimed to identify individual risk factors associated with prolonged intubation ( $\geq 7$ days) among patients with COVID-19. 
Methods: A prospective study was carried out between September 2020 and february 2021 among patients admitted in the COVID-19 ICU at the University Hospital Tahar Sfar Mahdia. The measurement tool was the "RAPID CORE CASE REPORT FORM" developed by WHO.

Results: A total of 114 participants were enrolled with an average age of $61.4 \pm 12.2$ years.

About $51.4 \%$ of patients needed intubation. During the hospitalisation, $12(21.1 \%)$ were eventually extubated and 45 (78.9\%) died. Prolonged intubation was recorded in $43.8 \%$ of cases. Among them, $35.4 \%$ were diabetic, $30.6 \%$ have hypertension and $6.1 \%$ had chronic heart disease.

The main factors significantly associated with longer intubation were: Nosocomial infection $(80.8 \%$ vs $7.7 \% ; \mathrm{p}<0.01)$, acute respiratory distress syndrome $(60.6 \%$ vs $17.8 \% ; p<0.01)$, and ionotropic use during the first $24 \mathrm{~h}(82.4 \%$ vs $36 \% ; p<0.01)$.

Multivariable logistic regression analysis identified nosocomial infection (OR, 20.54 [95\% Cl, 5.44-77.57] and early invasive intubation (during the first $24 \mathrm{~h})(\mathrm{OR}, 4.65[95 \% \mathrm{Cl}, 1.1-19.56])$ as predictive factors of prolonged intubation.

Conclusion: As COVID-19 infection continue to rapidly consume the health care system's limited resources, technicians consulted for airway management should consider these factors in their decision making

Disclosure of Interest: None declared

\section{P302}

Associated factors with extended hospital stay among COVID-19 patients in intensive care unit at an university hospital in Tunisia

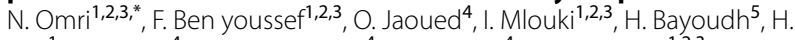
$\mathrm{Sfar}^{1}$, H. Nouira ${ }^{4}$, M. Fekih hassen ${ }^{4}$, S. El atrous ${ }^{4}, \mathrm{~S}$. El mhamdi ${ }^{1,2,3}$

${ }^{1}$ Preventive and Community Medicine, University Hospital Tahar Sfar Mahdia, Mahdia, ${ }^{2}$ Community Medicine, Faculty of Medicine of Monastir, ${ }^{3}$ Research laboratory, Epidemiology Applied to Maternal and Child Health, Monastir, ${ }^{4}$ Intensive care Medicine, ${ }^{5}$ Family Medicine, University Hospital Tahar Sfar Mahdia, Mahdia, Tunisia

Correspondence: N. Omri

Antimicrobial Resistance \& Infection Control 10(1): P302

Introduction: In Tunisia, the Covid-19 pandemic threatens to overwhelm scarce clinical resources especially in Intensive Care Units (ICUs)

Objectives: This study aims at analyzing clinical presentation on admission and the determinants associated with extended hospital stay in (ICUs).

Methods: We carried out a prospective study on 117 hospitalized patients in ICUs at the university hospital Tahar Sfar Mahdia which is located in the Central-East part of Tunisia from September 2020 to February 2021 using a valid and reliable tool inspired from the WHO2019-nCOV-Clinical platform observation.

Results: A total of 117 patients were recruited with a mean age of $61.4 \pm 12.2$ years. Males outnumbered $(60 \%$, sex ratio $=0.67)$. The majority $85.5 \%$ had pre-existing chronic diseases mainly hypertension $38.5 \%$ and Diabetes $42.2 \%$. The median length of stay was 14 (IQR: $24-9$ ) days. Approximately the half $47 \%$ had an extended stay (>14 days). Of the 117 patients included, $46.2 \%$ died in hospital and almost the half $50.1 \%$ required mechanical ventilation.

Our survey shows a significantly longer hospital stay among older patients $(52.9 \%)$, patients having oxygen saturation $<90 \%(51 \%)$ and those who have developed a health care associated infection $63.5 \%$. The main factor associated with extended hospital stay was invasive intubation (77.6\%).

Acquiring a nosocomial infection $(p=0.001)$, developing a complication $(p<0.001)$, resorting to invasive intubation $(p<0.001)$ and administering vasopressor drugs $(p=0.001)$ were found to have significant relationship with extended hospital stay.

Conclusion: This close look at the length of Covid-19 IUCs stay could provide an overview and would improve the prognosis of Covid-19 patients.

Disclosure of Interest: None declared
P303

Evaluation of the long-term impact of COVID-19 on the quality of life among disease survivors: preliminary results of a prospective cohort study (Recovida)

L. Bonifácio ${ }^{1, *}$, F. Barbosa-Júnior ${ }^{1}$, V. Csizmar ${ }^{1}$, A. P. Pereira ${ }^{1}$, F. Carvalho ${ }^{1}$, F. Guioti ${ }^{1}$, A. Dal Fabro' ${ }^{1}$, J. P. Souza ${ }^{1}$, F. Bellissimo-Rodrigues ${ }^{1}$

${ }^{1}$ Ribeirão Preto Medical School, University of São Paulo, Ribeirão Preto,

Brazil

Correspondence: L. Bonifácio

Antimicrobial Resistance \& Infection Control 10(1): P303

Introduction: There is scarce information on how long-term clinical, psychological and social consequences of Covid-19 affects the quality of life of people surviving it.

Objectives: We aimed to follow up patients surviving Covid-19 to evaluate and to compare their quality of life status before and after the acute phase of disease.

Methods: RECOVIDA is a prospective cohort study conducted in a public tertiary hospital in Brazil. From May 1 to December 31, 2020, we recruited participants on the Hospital Post-Covid-19 outpatient clinic, which offers clinical follow-up to adult patients, most of them discharged after being admitted due to Covid-19. Quality of life was evaluated through an interview based on The World Health Organization Quality of Life (WHOQOL-BREF) tool.

Results: 175 patients were included in the study and interviewed up to 120 days after symptoms onset. $51.4 \%$ of participants were female with average age of $53 \pm 14$ years. Table 1 describes scores obtained on the WHOQOL-BREF before and after Covid-19. Table 2 focuses on the current situation, where it is shown that most of patients were satisfied with their life after Covid-19. Pairing the individual data of 168 respondents, we observed that for 49 (29.6\%) of them quality of life worsened after Covid-19, for 98 (58.3\%) it remained on the same level and for $21(12.5 \%)$ it actually improved after Covid-19.
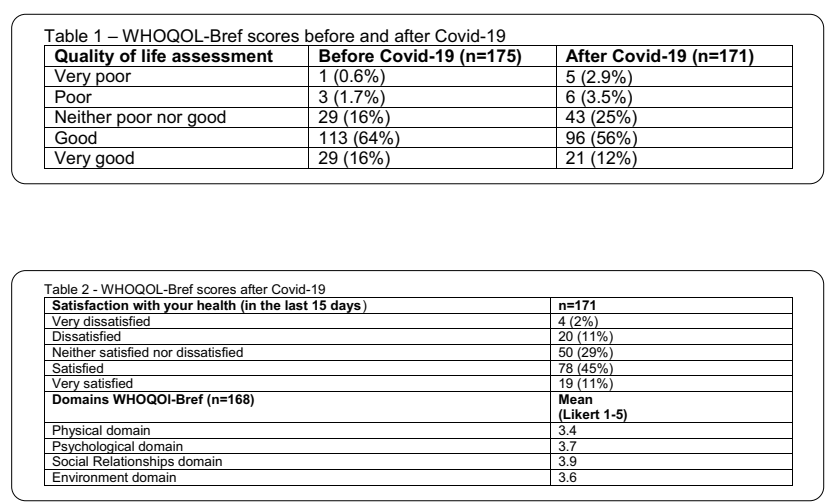

Conclusion: Covid-19 disease may last longer than its acute phase. Around $30 \%$ of patients have their quality of life significantly compromised up to 120 days after the disease onset.

Disclosure of Interest: None declared

\section{P304}

Evaluation of long-term clinical findings among COVID-19 survivors: preliminary results of a prospective cohort study (Recovida)

L. Bonifácio ${ }^{1}$, V. Csizmar ${ }^{1}$, A. P. Pereira ${ }^{1}$, F. Barbosa-Júnior ${ }^{1}, V^{\prime}$. Bollela', R. Santana', J. P. Souza', F. Bellissimo-Rodrigues ${ }^{1, *}$

${ }^{1}$ Ribeirão Preto Medical School, University of São Paulo, Ribeirão Preto, Brazil

Correspondence: F. Bellissimo-Rodrigues

Antimicrobial Resistance \& Infection Control 10(1): P304

Introduction: Little is known about long-term clinical consequences of Covid-19. 
Objectives: We aimed to follow-up patients surviving the acute phase of disease to identify and eventually describe persistent symptoms related to it.

Methods: RECOVIDA is a prospective cohort study conducted in a public-affiliated tertiary-care hospital, in Brazil. From May 1 to December 31, 2020, we recruited participants on the Post-Covid-19 ambulatory of the study facility, which offers clinical follow-up to adult patients, most of them discharged after being admitted due to Covid19. We classified their acute disease following WHO severity of disease criteria.

Results: 175 patients were included in the study and evaluated up to 120 days after the onset of symptoms (mean $=57$ days). Regarding demographic features, $51.4 \%$ of participants were female, and average age was $53 \pm 14$ years. Past medical history included hypertension $(37 \%)$, diabetes mellitus (28\%) and obesity (mean body mass index of $31.7 \pm 7.3$ ). By the time of evaluation, $80 \%$ of participants still experienced at least one long-term symptom, as described in Table 1.

\begin{tabular}{|c|c|c|c|c|}
\hline 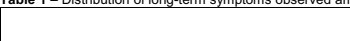 & Mild/Moderate $(n=35)$ & $\begin{array}{l}\text { Severe } \\
(n=80)\end{array}$ & $\begin{array}{l}\text { Criticial } \\
(n=60)\end{array}$ & \begin{tabular}{|l} 
Total \\
$(n=175$
\end{tabular} \\
\hline $\begin{array}{l}\text { Average time from disease onset to observation (days) } \\
\text { Prevalence of long-term symptoms }\end{array}$ & $\begin{array}{l}51 \pm 27 \\
25(71.4 \%)\end{array}$ & \begin{tabular}{|l|l}
$48 \pm 23$ \\
59 \\
$173.8 \%$
\end{tabular} & \begin{tabular}{|l|}
$72 \pm 26$ \\
56 \\
$(93.3 \%)$
\end{tabular} & $\begin{array}{l}57 \pm 27 \\
140 \\
(80 \%)\end{array}$ \\
\hline $\begin{array}{l}\text { Respiratory symptoms } \\
\text { Dyspnea } \\
\text { Cough }\end{array}$ & $25.7 \%$ & $27.5 \%$ & $25 \%$ & $\begin{array}{l}26.3 \% \\
228 \%\end{array}$ \\
\hline $\begin{array}{l}\text { Cardiovascular symptoms } \\
\text { Swelling } \\
\text { Hypertension } \\
\text { Hypotension }\end{array}$ & $\begin{array}{l}5.7 \% \\
2.8 \% \\
0 \% \\
0 \%\end{array}$ & \begin{tabular}{|l}
$7.5 \%$ \\
$0 \%$ \\
$1.2 \%$ \\
\end{tabular} & \begin{tabular}{|l}
$8.3 \%$ \\
$1.6 \%$ \\
$1.6 \%$ \\
\end{tabular} & \begin{tabular}{|l}
$7.4 \%$ \\
$1.1 \%$ \\
$1.1 \%$ \\
\end{tabular} \\
\hline $\begin{array}{l}\text { Neurological symptoms } \\
\text { Headache } \\
\text { Altered skin sensitivity } \\
\text { Hypogeusia/Dysgeusia } \\
\text { Hyposmia } \\
\text { Brain fog }\end{array}$ & \begin{tabular}{|l|}
$28.6 \%$ \\
$2.8 \%$ \\
$25.7 \%$ \\
$20 \%$ \\
$5.7 \%$ \\
\end{tabular} & \begin{tabular}{|l}
$20 \%$ \\
$5 \%$ \\
$16.2 \%$ \\
$8.7 \%$ \\
$3.7 \%$ \\
\end{tabular} & \begin{tabular}{|l|}
$16.6 \%$ \\
$35 \%$ \\
$16.6 \%$ \\
$66.6 \%$ \\
$5 \%$
\end{tabular} & $\begin{array}{l}20.5 \% \\
14.8 \% \\
18.3 \% \\
10.3 \% \\
4.5 \%\end{array}$ \\
\hline $\begin{array}{l}\text { Gastrointestinal symptoms } \\
\text { Diarrhea } \\
\text { Dyspepsia }\end{array}$ & \begin{tabular}{|l|l}
$\%$ & \\
$5.7 \%$
\end{tabular} & $\begin{array}{l}2.5 \% \\
0 \%\end{array}$ & $\begin{array}{l}6.6 \% \\
0 \%\end{array}$ & $\begin{array}{l}3.4 \% \\
1.1 \%\end{array}$ \\
\hline $\begin{array}{l}\text { Other symptoms } \\
\text { Fatigue } \\
\text { Weakness } \\
\text { Vision problem }\end{array}$ & $\begin{array}{l}28.5 \% \\
8.5 \% \\
2.8 \%\end{array}$ & $\begin{array}{l}28.7 \% \\
13.7 \% \\
1.2 \% \\
3.7 \%\end{array}$ & $\begin{array}{l}35 \% \\
35 \% \\
13.3 \%\end{array}$ & $\begin{array}{l}30.8 \% \\
20 \% \\
5.7 \%\end{array}$ \\
\hline
\end{tabular}

Conclusion: Covid-19 disease does not necessarily end with the end of its acute phase. In fact, a large proportion of patients, even after a mild disease, persist with clinically meaningful symptoms up to 120 days after the disease onset.

Disclosure of Interest: None declared

\section{P306}

Predictors of mortality in hospitalized COVID-19 patients in a Tunisian university hospital

D. chebil ${ }^{1,2, *}$ S. rouis ${ }^{2,3}$, E. arfaoui ${ }^{1}$, H. hannachi $i^{1,2}$, L. merzougui ${ }^{1,2}$

${ }^{1}$ prevention and infection control department, kairouan, ${ }^{2}$ Faculty of medicine of Sousse, University of Sousse, SOUSSE, ${ }^{3}$ infectious diseases department, kairouan, Tunisia

Correspondence: D. chebil

Antimicrobial Resistance \& Infection Control 10(1): P306

Introduction: Mortality rates of coronavirus disease 2019 (COVID-19) continue to rise across the world. The predictors of mortality in COVID19 patients are under study.

Objectives: we aim to evaluate the risk factors associated with the death of patients with COVID-19 pneumonia.

Methods: We analysed data of laboratory confirmed hospitalized patients at the COVID-19 unit of a university hospital in the Tunisian center, between September 2020 and January 2021.Patients' characteristics, comorbidities, and laboratory abnormalities were recorded. Potential predictors of in-hospital mortality were identified by multivariable Cox regression model.

Results: A total of 256 patients were included.Mean age was 66 years (SD, 14 years) and $62 \%(n=158)$ patients were males. Forty-eight percent $(n=123)$ of patients had at least two co-morbidity with hypertension and diabetesas the most common comorbidities.Severe cases accounted for $52 \%$ of the study population.The overall in-hospital mortality rate was $29 \%(n=72)$. multivariate analysisrevealed that Age $>=60$ years (HR 5.87, $\mathrm{p}<0.001)$; The presence of coronary heart disease (HR 2.47, $p=0.027$ ) and leukocytes $\geq 10,000$ (HR 2.25, $p=0.012$ ), were independently associated with increased in-hospital mortality.

Conclusion: Predictors could assist clinicians to identify patients with a poor prognosis at an early stage to reduce the Covid-19-related mortality and rationalize the use of limited medical resources.

Disclosure of Interest: None declared

P307

Mortality risk factors for COVID-19 patients in the region

of Mahdia (Tunisia)

F. B. Youssef ${ }^{1,2,3, *}$, O. Jawed ${ }^{4}$, I. Mlouki 1,2,3, N. Omri 1,2,3, H. Bayoudh ${ }^{5}, H$. Sfar $^{1}$, H. Nouira ${ }^{4}$, M. F. Hassen ${ }^{4}$, S. El Atrous' ${ }^{4}$, S. El Mhamdi ${ }^{1,2,3}$

${ }^{1}$ Preventive and Community Medicine, University Hospital Taher Sfar,

${ }^{2}$ Community Medicine, Faculty of Medicine of Monastir, ${ }^{3}$ Research laboratory, "Epidemiology Applied to Maternal and Child Health", ${ }^{4}$ Intensive Care Medicine, ${ }^{5}$ Family Medicine, University Hospital Taher Sfar, Mahdia, Tunisia Correspondence: F. B. Youssef

Antimicrobial Resistance \& Infection Control 10(1): P307

Introduction: Timely identification of COVID-19 patients at high risk of mortality is crucial to improve patient management and resource allocation in hospitals.

Objectives: The aim of this study was to identify mortality risk factors at the COVID-19 Intensive Care Unit (ICU) in the region of Mahdia Methods: We conducted a prospective study including patients with severe COVID-19 infection admitted to the ICU of The University Hospital Tahar Sfar Mahdia. The survey was carried out between September 2020 and February 2021. We used "The RAPID CORE CASE REPORT FORM" developed by the World Health Organization.

Results: We analyzed the records of 117 patients (59.8\% were males). Mean age was $61.4 \pm 12.2$ years. Common symptoms were shortness of breath $(82.6 \%)$, cough $(71.1 \%)$ and fever $(69.3 \%)$. The median length of stay in the unit was 18.3 days (IQR: 2-66). About $48.7 \%$ of patients had invasive ventilation, $30.3 \%$ required vasopressors intake and $51.4 \%$ required prone position.

The overall mortality rate was $46.2 \%$. Longer stay in the hospital ( $>14$ days) and longer intubation duration ( $\geq 7$ days) were associated with a higher risk of mortality $(61.8 \%$ vs $32.3 \% ; p<0.001$ and $77.6 \%$ vs $20.6 \% ; p<0.001$ respectively). Mortality was also related to acute respiratory distress syndrome $(66.7 \%$ vs $13 \% ; p<0.001)$ and nosocomial infection $(80.8 \%$ vs $17 \% ; \mathrm{p}<0.01)$.

Multivariable logistic regression analysis showed increasing odds of mortality with nosocomial infection (OR, 12.41 [95\% Cl, 3.3-46.57] respiratory distress syndrome (OR $7.52[95 \% \mathrm{Cl} .1 .71-32,95])$ and inotropes or vasopressors intake (OR 7.39 [95\% Cl, 1.57-34.69]). Higher oxygen saturation on admission was found to be a protective factor against mortality (OR $0.91[95 \% \mathrm{Cl}, 0.85-0.97])$.

Conclusion: Interventions that prevent these risk factors are needed to improve the prognosis of Covid-19 patients.

Disclosure of Interest: None declared

P308

Clinical characteristics, outcomes, and risk factors for mortality

in hospitalized patients with COVID-19 from the start

of the pandemic in Sahloul university hospital

A. Ben Cheikh ${ }^{1}$, H. Ghali ${ }^{1, *}$, S. Bhiri ${ }^{1}$, R. Bannour ${ }^{2}$, W. Dhouib ${ }^{2}$, S. Khefacha ${ }^{2}$, H. Said Latiri', M. Ben Rejeb ${ }^{1}$

${ }^{1}$ Department of Prevention and Security of Care, Sahloul university hospital, Faculty of Medicine of Sousse, University of Sousse, ${ }^{2}$ Department of Prevention and Security of Care, Sahloul university hospital, Sousse,

Tunisia

Correspondence: $\mathrm{H}$. Ghali

Antimicrobial Resistance \& Infection Control 10(1): P308

Introduction: Tunisia was one of the countries with the highest COVID-19 mortality worldwide, especially during this last stage of the pandemic. Although risk factors for severe disease in COVID19 patients have been described in Chinese and United States (US) 
cohorts, studies reporting on clinical characteristics, risk factors and outcome of Tunisian COVID-19 patients are still scarce.

Objectives: The aim of our study was to describe the clinical characteristics of COVID-19 patients in a tertiary care center since the start of the pandemic (from first hospitalization until May 5), and identify independent risk factors for hospital mortality.

Methods: Since the start of the pandemic, the prevention and security of care department has been recording all new cases of COVID_19 hospitalized in the various units taking care of these patients. Daily monitoring of the evolution of the patient's condition is provided by the hygienist technicians of our department.

Results: From when the first patient with COVID-19 was admitted to May 5, 752 laboratory-confirmed COVID-19 patients had been admitted to our hospital. Most patients were male $(55.5 \%)$, the mean age was $62.3 \pm 14.5$ years, with 279 patients $(37.1 \%)$ being $<60$ years of age. The median length of stay was 11 days (IQR 5-14). The most common comorbidities were hypertension (47.7\%), diabetes (46.2\%), and coronary artery disease (19.9\%). Most patients had an important degree of hypoxemia, with $53.7 \%$ of patients not requiring supplemental oxygen, and $22.4 \%$ needed invasive mechanical ventilation. The overall case fatality rate was $23 \%$. More patients died in the intensive care units (65.8\%). Multivariate analysis model showed that history of comorbidity $(p=0.015)$, Non-invasive ventilation $(p=0.041)$, and mechanical ventilation $(p=0.028)$ were associated with an increased risk of death.

Conclusion: The risk factors found allow the identification of vulnerable groups in whom monitoring and early identification of symptoms should be prioritized in order to reduce mortality.

Disclosure of Interest: None declared

\section{P309}

Evolution of mortality over time: results from the COVID-19 Swiss hospital surveillance system (CH-SUR)

M. Roelens ${ }^{1, *}$, A. Martin ${ }^{1}$, B. Vidondo ${ }^{2}$ on behalf of $\mathrm{CH}$ Sur Consortium,

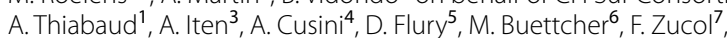
C. Balmelli ${ }^{8}$, P. Zimmermann ${ }^{9}$, N. Troillet ${ }^{10}$, D. Vuichard-Gysin ${ }^{11}$, P. W. Schreiber ${ }^{12}$, S. Bernhard-Stirnemann ${ }^{13}$, S. Tschudin-Sutter ${ }^{14}, Y$. Nussbaumer-Ochsner ${ }^{15}$, R. Sommerstein ${ }^{16}$, R. Gaudenz ${ }^{17}$, J. Marschall ${ }^{18}$, F. Maximiano Sousa', B. Friker', G. Schuepbach' ${ }^{2}$, M. Maeusezahl ${ }^{19}$, M. Wymann ${ }^{19}$, S. Harbarth ${ }^{20}$, C. Gardiol ${ }^{19}$, O. Keiser ${ }^{1}$ on behalf of CH Sur Consortium

${ }^{1}$ University of Geneva, Geneva, ${ }^{2}$ University of Bern, Bern, ${ }^{3}$ Cantonal Hospital Geneva, Geneva, ${ }^{4}$ Cantonal Hospital Graubünden, Chur, ${ }^{5}$ Canton Hospital St Gall, St Gall, ${ }^{6}$ Cantonal Hospital Lucerne, Lucerne, ${ }^{7}$ Cantonal Hospital Winterthur, Winterthur, ${ }^{8}$ Cantonal Hospital Ticino, Ticino, ${ }^{9}$ University of Fribourg, Fribourg, ${ }^{10}$ Valais Hospitals, Sion, ${ }^{11}$ Thurgau Hospital Group, Münsterlingen, ${ }^{12}$ Zurich University Hospital, Zurich, ${ }^{13}$ Children Hospital Aarau, Aarau, ${ }^{14}$ University Hospital Basel, Basel, ${ }^{15}$ Cantonal Hospital Schaffhausen, Schaffhausen, ${ }^{16} \mathrm{Hospital}$ Hirslanden Central Switzerland, Lucerne, ${ }^{17}$ Cantonal Hospital Nidwalden, Stans, ${ }^{18}$ Cantonal Hospital Bern (Insel), ${ }^{19}$ Swiss Federal Office Public Health, Bern, ${ }^{20}$ University Hospital

Geneva, Geneva, Switzerland

Correspondence: M. Roelens

Antimicrobial Resistance \& Infection Control 10(1): P309

Introduction: The assessment of COVID-19 associated mortality is crucial to evaluate the impact of the pandemic and to assess the effectiveness of measures.

Objectives: We aimed to investigate trends in COVID-19 related mortality over time in Switzerland, using data from the COVID-19 Hospitalbased Surveillance (CH-SUR) database.

Methods: Considering four different time periods of COVID-19 epidemic, we calculated crude and adjusted mortality rates and performed competing risks survival analyses for all patients and for patients admitted to intensive care (ICU).

Results: Overall, 16,967 COVID-19 related hospitalizations and 2,307 deaths of adult patients were recorded. Crude hospital mortality rates were $15.6 \%$ in the 1 st and $14.4 \%$ in the 2 nd wave; for ICU patients it was $24 \%$ and $31.3 \%$, respectively. The overall adjusted risk of death was lower for hospitalised patients during the 2nd compared to the 1st wave ( $\mathrm{HR} 0.75,95 \% \mathrm{Cl} 0.73-0.77)$. In contrast, patients admitted to ICU as well as patients with invasive ventilation presented a higher risk of death during the 2 nd wave (HR 1.62, 95\% Cl 1.54-1.70 and HR 2.10, $95 \% \mathrm{Cl} 1.99-2.20$, respectively).

Conclusion: Our findings may be explained by various changes in the COVID-19 patient management in Swiss hospitals, e.g. with the use of effective drugs against complications or with different guidelines for ICU admission and invasive ventilation use.

Disclosure of Interest: None declared

\section{P310}

Changes of lipoxina4 levels following early hospital management of patients with COVID-19

F. Jamali', B. Shahrami ${ }^{1}$, F. Najmeddin ${ }^{1}$, A. Haddadi ${ }^{2}$, M. Sharifzadeh ${ }^{3}$, A. A. A. Arabzadeh ${ }^{4, *}$, M. Mojtahedzadeh ${ }^{1}$

${ }^{1}$ Department of Clinical Pharmacy, ${ }^{2}$ Department of Infectious Disease,

${ }^{3}$ Department of Pharmacology, Tehran University of Medical Sciences,

Tehran, ${ }^{4}$ Department of Surgery, Ardabil University of Medical Sciences, Ardabil, Iran, Islamic Republic Of

Correspondence: A. A. A. Arabzadeh

Antimicrobial Resistance \& Infection Control 10(1): P310

Introduction: LipoxinA4 (LXA4) is an anti-inflammatory biomarker that participates in the active process of inflammation resolution which is suggested to be beneficial in infectious and inflammatory diseases like the coronavirus disease 2019 (COVID-19).

Objectives: This study aimed to determine early changes of LXA4 levels in the hospitalized patients with confirmed COVID-19 following the clinical management as well as its correlation with common used inflammatory markers, including Erythrocyte Sedimentation Rate (ESR), C-reactive protein (CRP), and ferritin.

Methods: Thirty-one adult hospitalized patients with the non-severe COVID-19 were included. LXA4, ESR, and CRP serum levels were collected on the first day of hospitalization, and LXA4 levels were measured $48-72 \mathrm{~h}$ later as well. Moreover, the maximum serum ferritin level during the five days of following patients was collected.

Results: The mean age of patients was $61.9 \pm 17$ years, and the male to female ratio was $18: 13$. LXA4 levels were significantly increased at $48-72 \mathrm{~h}$ compared to the baseline concentrations $(9.9 \pm 0.7 \mathrm{vs}$. $21.7 \pm 15.1 \mathrm{ng} / \mathrm{L} ; \mathrm{P}<0.05)$. The mean baseline concentrations of CRP and ESR and the mean maximum concentration of ferritin were $74.7 \pm 57.3 \mathrm{mg} / \mathrm{L}, 55.7 \pm 34.4 \mathrm{~mm} / \mathrm{h}$, and $568.7 \pm 530 \mathrm{ng} / \mathrm{mL}$, respectively. Besides, CRP and ESR levels at the time of admission and maximum ferritin levels during the hospitalization were positively correlated to an increase of LXA4 levels $(R=0.499,0.535,0.398 ; P=0.007$, $0.005,0.043$, respectively).

Conclusion: LXA4 may be a valuable marker to assess the treatment response compared to ESR, CRP, and ferritin in hospitalized patients with COVID-19. Furthermore, LXA4 could be considered a potential treatment option in inflammatory conditions. Further studies are necessary to clarify LXA4 role in COVID-19 pathogenesis, as well as the balance between such pro-resolving mediators and inflammatory parameters.

Disclosure of Interest: None declared

Poster Session: Public Health issues of COVID-19

\section{P311}

Examining the discourse regarding the delivery of occupational

health and safety training to healthcare workers: a review

of pandemic plans of 23 countries

M. O. Qureshi ${ }^{1}{ }^{*}$, A. Chughtai ${ }^{1}$, H. Seale ${ }^{1}$

${ }^{1}$ University of New South Wales, Sydney, Australia

Correspondence: M. O. Qureshi

Antimicrobial Resistance \& Infection Control 10(1): P311

Introduction: COVID-19 pandemic has highlighted gaps in health service responses and has exposed ongoing risks for healthcare providers 
(HCPs). Mitigating these risks requires multiple measures, including the delivery of pandemic specific occupational health and safety (PSOHS) training focused on infection prevention and control (IPC).

Objectives: This study aimed to compare national and transnational pandemic plans and COVID-19 guidelines for the inclusion of PSOHS (IPC) training, as well as strategies for managing the surge in PPE needs and staffing.

Methods: For this review pandemic plans and COVID-19 (IPC) guidelines of the World Health Organization (WHO), the European Centre for Disease Prevention and Control (ECDC) and 23 countries from six WHO defined world regions were reviewed including United States of America, Peru, Chile, Canada from Region of the Americas (PAHO); Spain, United Kingdom, Italy, Germany from European Region (EURO); Nigeria, Algeria from African Region (AFRO); Saudi Arabia, Oman, Bahrain, Jordan from Eastern Mediterranean region (EMRO); Bangladesh, Thailand, Sri Lanka, Myanmar from South-East Asia region (SEARO); China, Singapore, Philippines, Japan and Australia from Western Pacific Region (WPRO).

Results: The WHO, ECDC and nine countries reported PSOHS (IPC) training; however only four discussed training HCPs on correct PPE use; two countries listed strategies to manage the surge in demand, but only one country discussed managing the shortage of PPE. None of the COVID-19 guidelines recommended training HCPs for correct reuse or extended use of PPE and only one country's guideline outlined mandatory HCP attendance and delivery of training in a regional language.

Conclusion: Pandemic plans should be revised to include guiding principles regarding the delivery of pandemic specific OHS (IPC) training, as well as on managing the surge in demand of PPE and HCPs. There is also a need to provide guidance on when countries should consider reuse and extend use of PPE. This discourse should also be reflected in disease specific pandemic guidelines, like COVID-19 (IPC) guidelines. This review may assist international health agencies in subsequent guideline updates.

Disclosure of Interest: None declared

\section{P312}

Rationale for different quarantine durations during the COVID-19 pandemic

B. K. Burmen ${ }^{1, *}$, D. bii ${ }^{2}$

${ }^{1}$ Center for Respiratory Disease Research, KENYA MEDICAL RESEARCH INSTITUTE, Nairobi, ${ }^{2}$ KENYA MEDICAL RESEARCH INSTITUTE, Kisumu, Kenya

Correspondence: B. K. Burmen

Antimicrobial Resistance \& Infection Control 10(1): P312

Introduction: All contacts of individuals with a confirmed or probable COVID-19 should be quarantined in a designated facility or at home for 14 days from their last exposure. Although shorter quarantine periods may lessen health system's burdens especially when new infections are rapidly rising, prolonging the quarantine duration could be prudent for high-risk scenarios and in regions with insufficient test resources.

Objectives: To describe the rationale for different COVID-19 quarantine durations during the on-going pandemic in select countries.

Methods: An online search was conducted to document countries' rationale for different quarantine durations during the ongoing COVID-19 pandemic. Only countries that stated a quarantine duration and its rationale were eligible for inclusion. A country was stated to have no quarantine, reduced, or prolonged its COVID-19 quarantine duration if it recommended $0,1-<14$, or $>14$ days. Country's names were anonymized based on the focus of this study.

Results: Countries that did not impose a quarantine period recommended self-isolation or quarantine on a case-by-case basis. E.g. persons who had been at sea for more than 14 days did not have to undergo quarantine. Countries that increased their quarantine period found persons testing positive after the recommended 14-day period, identified mutated viral strains, and did so in response to increased COVID-19 activity. Countries that reduced their quarantine period based it on the virus' incubation period, a day 7 COVID-19 test result, a traveler's vaccination status, the reduced risk of contagion over time and the country's prevailing COVID-19 cases. Quarantine duration was reduced based on a traveler's COVID-19 risk profile, his or her country of origin, to encourage compliance and lessen cost of governmentfunded quarantine.

Conclusion: Quarantine durations was adjusted to in country disease burden, disease dynamics, the natural history of the disease, COVID19 vaccination status, existence of mutant strains and for efficient resources' use. Countries also wanted to encourage vaccination, testing and boost quarantine guideline's compliance. A comprehensive country-specific longitudinal analysis ought to incorporate testing guidelines and vaccination status for each quarantine duration and each country's epidemic curve would provide useful information on best practices.

Disclosure of Interest: None declared

P313

Web-queries as an evaluation marker for epidemiological situation of SARS-COV-2 infection in Russia

K. Momynaliev 1,*, D. Khoroshun ${ }^{1}$, V. Akimkin ${ }^{1}$

${ }^{1}$ Federal Budget Institution of Science "Central Research Institute

of Epidemiology" of The Federal Service on Customers Rights Protection and Human Well-being Surveillance, Moscow, Russian Federation

Correspondence: K. Momynaliev

Antimicrobial Resistance \& Infection Control 10(1): P313

Introduction: In recent years, the study of large amounts of Internet data has become a valuable way of monitoring and analyzing public health behavior. Changes in smell and taste are characteristic symptoms of COVID-19, so monitoring dynamics of these queries on the Internet can provide early information on the spread of COVID-19 in order to assess the effectiveness of anti-epidemic measures against COVID-19.

Objectives: Evaluation of the usefulness of Internet queries related to the smell to assess the effectiveness of anti-epidemic measures of prevent the spread of COVID-19 in some regions of Russia.

Methods: Data on new (confirmed) cases of the disease by weeks in the indicated regions were obtained from the Internet resources https://стопкоронавирус.рф. For the analysis of Internet queries, we used the Yandex.Wordstat database of search queries. Searches were analyzed that included the words "smell". Data on were received weekly from 16-22.03.2020 to 15-21.02.2021.

Results: A strong temporal relationship has been shown between selfreported odor changes (for example, "loss of smell") and new cases of COVID-19 infection in the studied areas of the Central region of Russia. A correlation was found between the number of queries related to smell and new confirmed cases of COVID-19 infection: very high for Moscow city $(r=0.96)$, high for Bryansk and Ryazan regions $(r>0.70)$, average for Vladimir, Ivanovo, Kaluga, Moscow, Smolensk and Tula regions $(0.5<r<0.7)$. During the "second wave" of the pandemic in Russia, the increase in the number of queries related to the smell outpaced the increase in the number of new confirmed cases in Moscow (city), Moscow (region), Ryazan, Smolensk, Vladimir and Ivanovo regions by 2 weeks, for the Tula region-by 3 . For Bryansk and In Kaluga regions, no such dependence was observed.

Conclusion: The rise in sudden interest in smell among Internet users can be seen as a valuable minimally invasive indicator of the spread of the coronavirus in the population, as well as to assess the effectiveness of anti-epidemic measures against COVID-19.

Disclosure of Interest: None declared

P314

Alerte infection, a tool for an innovative public management of health care professionals to prevent epidemics in the vaud canton

M. K. M. Ku Moroni ${ }^{1, *},{ }^{*}$ C. Petignat ${ }^{1}$

${ }^{1}$ Office du Médecin Cantonal, HPCI Vaud, Lausanne, Switzerland

Correspondence: M. K. M. Ku Moroni

Antimicrobial Resistance \& Infection Control 10(1): P314 
Introduction: With a first observation of information problem in epidemic cases, it was noted that health care professionals don't have time to get informed by themselves about hygiene hospital news or protocols last updates. The cantonal unit of Hygiene Prevention and Control of Infection in Vaud (HPCi Vaud) in charge of epidemics monitoring in health facilities, worked on a strategy to push in the fastest possible way important information to the health care professionals to prevent diseases' spread as soon as a situation has been detected.

Objectives: The objective of this public management is to control epidemics by reducing the communication time in early detection between Public Health (OMC/HPCi Vaud) and the health care professionals who are main actors on the field, in order to improve their responsiveness in decision or action. The global goal of this Public Health administration is to protect patients in acute care and nursing home residents from infections and communicable diseases in the Vaud canton.

Methods: As an information problem. This solution has been managed according to communications methods and strategies. On that occasion, the long process of management and communication from public health passing by several intermediaries to reach the healthcare workers as been optimised to be shorter with no intermediary.

Results: A free mobile application has been released at the end of 2019 for care workers in Vaud health facilities. This app sends HPCI alerts classified by category that users can enable/disable according to their needs.

Results of a sample of users showed that over $95,2 \%$ say they are more responsive to adapt their decision and action according to the information notification, and $83.1 \%$ say that the alerts helps to protect patients and residents.

Conclusion: In the current context of the CoVID-19 pandemic, this tool has been very useful to communicate as fast as possible with the health care professionals about the details of the patient care and the multiple updates of pandemic recommendations. The effective impact of responsiveness thanks to the tool confirmed by users' feedback is very positive.The mobile app has not been yet installed by all the care workers in the Vaud canton and this implicates launching complementary communications and thinking about new improvements.

\section{Disclosure of Interest: None declared}

\section{P315}

The use of drones for the delivery of supplies, medical equipment, diagnostic testing, and medical treatments to remote first nations communities in Alberta during COVID-19

W. Hawkins ${ }^{1}$, B. Baylis ${ }^{2,3}$, A. Khan ${ }^{4}$, A. Kirkpatrick ${ }^{2,3}$, K. Whitehead ${ }^{1}$, S.

Moeini', S. Lapointe ${ }^{1}$, B. Berenger ${ }^{3}$, K. Flemons ${ }^{2}$, J. Conly ${ }^{2,3, *}$

${ }^{1}$ Southern Alberta Institute of Technology, ${ }^{2}$ University of Calgary, ${ }^{3}$ Alberta

Health Services, ${ }^{4}$ Stoney Health Services, Calgary, Canada

Correspondence: J. Conly

Antimicrobial Resistance \& Infection Control 10(1): P315

Introduction: For members of remote Indigenous communities, accessing healthcare often requires travel to off-reserve locations, which is both burdensome to the individual and increases the risk of contracting COVID-19. Remotely Piloted Aircraft Systems (RPAS), or drones, offer a potentially cost-effective method for reducing that burden and risk by removing geographic barriers and increasing timeliness and accessibility of supplies, equipment, and remote care.

Objectives: To demonstrate the potential of RPAS-delivered technology and supplies in diagnosis, evaluation, and treatment, including RPAS-delivered ultrasound (US) machines; to co-develop governance and operating manuals with local Indigenous communities; and to use this work as a scalable model for fully operational RPAS-based medical delivery systems.

Methods: Our team is developing and pilot-testing innovative RPASbased medical delivery solutions at the systems, regulatory, technological (software and hardware), and procedural levels. This includes working with Transport Canada for Beyond Visual Line of Sight approval; RPAS fleet development and testing; custom payload system design and fabrication (i.e. refrigerated unit for vaccine delivery) procedural development (i.e. risk and safety assessments); Detect and Avoid System testing and refinement; and pilot-testing COVID-19 test kit fidelity and remote US functionality.

Results: We have successfully delivered PPE (gloves, gowns, masks) and COVID-19 test kits. We confirmed that the delivered RNA COVID19 test samples contained no decay in signal or testing capabilities. We also delivered a sanitized Tele-mentored Self-Diagnostic US and successfully Tele-mentored a patient in performing a proficient selfadministered US at a remote location.

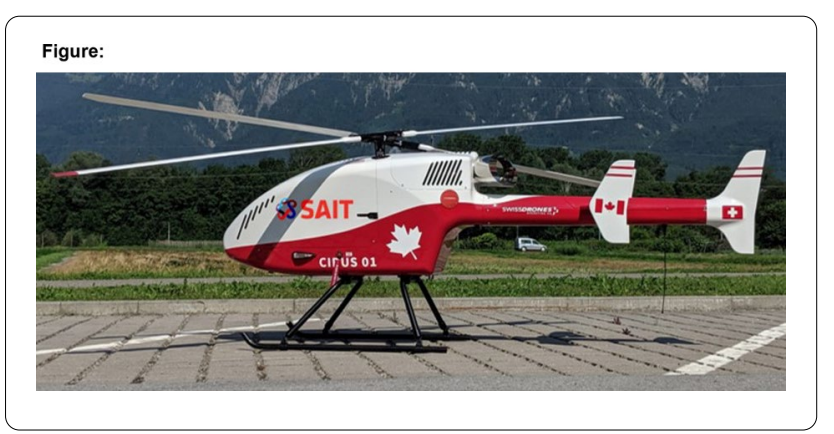

Conclusion: RPAS-based medical delivery models offer an innovative approach to addressing longstanding issues of healthcare access and equity, and are particularly relevant in the context of COVID-19 and preventing pandemic spread.

Disclosure of Interest: None declared

\section{P316}

A public health response to the global COVID-19 pandemic: the Nigerian story, January-May,2020

S. C. Mba ${ }^{1, *}$, C. Dan-Nwafor ${ }^{1}$, C. Ochu' ${ }^{1}$, E. Ilori' ${ }^{1}$, J. Oladejo ${ }^{1}$, C.

Umeokonkwo ${ }^{2}$, N. Mba $^{1}$, P. Nguku ${ }^{2}$, C. Ihekweazu ${ }^{1}$

${ }^{1}$ Nigeria Centre for Disease Control, ${ }^{2}$ African Field Epidemiology Network, Abuja, Nigeria

Correspondence: S. C. Mba

Antimicrobial Resistance \& Infection Control 10(1): P316

Background: The novel coronavirus disease (COVID-19) was reported in Wuhan China in December 2019. SARS-CoV-2 is of a zoonotic origin and is transmitted between humans via respiratory droplets and fomites. As COVID-19 spread globally, Nigeria instituted its pandemic preparedness measures in January 2020 preceding detection of its index case on February 28, 2020. We report the emergence of COVID19 in Nigeria, describing the public health response up till May 2020.

Method: We reviewed the Nigeria's country-level response to COVID19 at the National level from January - May 2020.

Results: The national public health response to the COVID-19 pandemic rapidly progressed from initial containment measures, with early closure of the international airspace and intensive contact tracing, to lockdown in high-burden states, closure of schools, nonessential businesses, and non-pharmaceutical interventions. A level three EOC was activated, the highest level of response in the country intended for public health measures. Following the ease of lockdown, mitigation strategies to address ongoing community transmission include increased testing, isolating all confirmed cases and tracing all contacts. By May 31, 2020, Nigeria had recorded 10,162 confirmed COVID-19 cases from all but one state, with three states Lagos, Kano and Federal Capital Territory, accounting for $64.5 \%$ of the cases.

Conclusion: Nigeria launched a swift and aggressive response to COVID-19, leveraging on its existing epidemic preparedness and learning from other parts of the globe where transmission began earlier. Future response efforts are focused on targeted non-pharmaceutical interventions at sub-national level. Mobilizing local governments, civil 
society, and citizens to take ownership of these strategies in addition to a cohesive whole-of-government approach is critical to combating the pandemic in Nigeria. By rapidly implementing this set of interventions, Nigeria likely slowed down the rate of virus transmission and bought extra time to implement a robust case detection, testing, and treatment centre capacity.

Key words: COVID-19, Nigeria, Pandemic, Response

Disclosure of Interest: None declared

\section{P317}

The COVID-19 pandemic through the eyes of youth: exploratory study in Geneva, Switzerland

C. Tuor ${ }^{1, *}$, R. Rey ${ }^{1}$, P. Bigarella ${ }^{1}$, A. Peters ${ }^{1}$, P. Hudelson ${ }^{1}$, C. Guitart ${ }^{1}$, D.

Pittet $^{1}$

${ }^{1}$ Faculty of Medicine, Infection Control Programme, and Primary Care

Medicine, University of Geneva Hospitals and Faculty of Medicine,

Geneva, Switzerland

Correspondence: C. Tuor

Antimicrobial Resistance \& Infection Control 10(1): P317

Introduction: During the SARS-CoV-2 pandemic, youth have accounted for a large share of confirmed cases compared to other age groups. This has led to portrayals of youth during the pandemic as reckless and irresponsible.

Objectives: The objective of our study was to explore the perceptions and experiences of youth during the first wave of the SARS-CoV-2 health crisis, with the aim of providing information useful for adapting COVID-19 prevention information and activities aimed at youth.

Methods: We conducted street interviews with a convenience sample of youth in several selected locations in Geneva, Switzerland, and key informant interviews with adults who had frequent contact with youth in Geneva.

Results: We approached 107 youth; 101 (94.4\%) participated. We interviewed 101 youth between 15 and 29 years old (51/101 were female) and 8 key informants. Eight respondents reported having been previously tested for COVID-19, two because of family contact tested postitive for COVID-19. Only one respondent reported a family member being hospitalized for COVID-19. The average duration of the interview was $10 \mathrm{~min}$. Most youth in our study did not fear for their own health but did worry about their family and loved ones. They described the emotional and behavioral impact of the pandemic and of misinformation about COVID-19.

Conclusion: Our study results challenge some of the preconceived ideas about youth during COVID-19, and illustrate their difficulties and concerns during the pandemic.

Disclosure of Interest: None declared

Video Clip Award

\section{C01}

DR. Radtke's routine (karusell (DR. Radtkes Metode))

E. F. Snøfugl ${ }^{1}$, A. Radtke ${ }^{2, *}$, K. H. McDonagh ${ }^{1}$, C. O. Kalland ${ }^{1}$, N. Lian' ${ }^{1}$

${ }^{1}$ Department of communications, ${ }^{2}$ Unit for Infection Control, St. Olavs

hospital HF, Trondheim University Hospital, Trondheim, Norway

Correspondence: A. Radtke

Antimicrobial Resistance \& Infection Control 10(1): C01

\section{Abstract video clip description:}

Introduction: In order to promote hand hygiene at St. Olavs Hospital a song and video were composed, recorded and filmed by the Department of communications in cooperation with the Unit for Infection Control.

Methods: The song and video focused mainly on the technique of applying and distributing disinfectant to the hands. The different steps of the routine, detailed in WHO guidelines, were given names and represent the chorus of the song. The chorus lasts $30 \mathrm{~s}$, and, if memorized, will give guidance for the right steps and duration for correct hand rub. The launch of the video was carefully planned for World Hand Hygiene Day (May, $5^{\text {th }}$ 2021) both on the hospitals intranet page and on the hospitals social media sites. It was also released on music streaming platforms.

Results: The video gained immediate attention and passed 100.000 hits on social media within two days. The media reach was wide for a small country as Norway with, currently (May, $25^{\text {th }}$ ), about 236.000 views and 3700 shares on Facebook and almost 40.000 views on YouTube. It was also referred to in local and national media. Additionally, it also caught attention in Sweden through a popular Instragram account (@dyngbaggegalan) which generated 400.000 views. Through commentary we learned that the song was especially well received by health care workers and among primary school children, although we do not have data to confirm this in detail.

Conclusion: Our song and video promoting the standard WHO hand hygiene routine in a fun and memorisable way gained wide attention and traction both among health care workers and the general public.

Disclosure of Interest: None declared

\section{$\mathrm{CO2}$}

Clean hands, save lives international children's painting festival M. H. Aelami i', E. Zerehsaz ${ }^{2}$, S. Khajehnejad ${ }^{2}$, B. Bonyadi ${ }^{1}$, H. Aelami ${ }^{2}$, P. Ghahremani Nejad ${ }^{2}$, A. Mohajeran ${ }^{3, *}$

${ }^{1}$ Hand Hygiene and Infection Control Research Center Imam Reza Hospital, MASHHAD UNIVERSITY OF MEDICAL SCIENCES, ${ }^{2}$ The institute for the intellectual development of children and young adults of Khorasan Razavi, ${ }^{3}$ Hand Hygiene and Infection Control Research Center Imam Reza Hospital, MASHHAD UNIVERSITY OF MEDICAL SCIENCES, Mashhad, Iran, Islamic Republic Of

Correspondence: A. Mohajeran

Antimicrobial Resistance \& Infection Control 10(1): C02

Abstract video clip description: Hand hygiene is the most important ways for prevention of infections especialy during COVID-19 pandemic. Painting is the universal language and very popular among children. We announced an international painting competition about clean hands save lives from March 15 till July 15 2020. We received 8452 paintings from 29 countries of different continents. Selected paintings of this festival act in this clip to show importance of hand hygiene and role of health care workers in COVID-19 pandemic.

Disclosure of Interest: None declared

$\mathrm{CO3}$

Combating antimicrobial resistance through research and community engagement in Nigeria

I. Yusuf ${ }^{1, *}$, S. Umar ${ }^{2}$

${ }^{1}$ Microbiology, Bayero University, Kano, Kano, ${ }^{2}$ Chemistry, Federal University Dutsimma, Dutsimma, Katsina, Nigeria

Correspondence: I. Yusuf

Antimicrobial Resistance \& Infection Control 10(1): C03

Abstract video clip description: Dr Yusuf and his colleagues have conducted scientific surveys revealing the appearance and spread of disease-causing antibiotic-resistant bacteria in local communities with Kano State, Nigeria through a new initiative. The initiative developed new model to sensitize and engage students on antimicrobial resistance research and communicating the research to the needed community. Key to this initiative is that students and community members are recruited to help spread the message, and educate others in the community, who may not otherwise listen.

Objective: The initiative aimed at safeguarding the efficacy of antibiotics through research and community engagement for prudent use of antibiotic.

Methodology: The initiative involves conducting community specific research and travel into the community to educate community leaders, who influence others in their towns and villages, particularly sellers and hawkers of illicit or fake antibiotics. It also involve travelling to remote farming communities, speaking directly with farmers who either give antibiotics to their livestock, or take antibiotics wrongly believing they may act as painkillers.

1. Local/specific researches on drivers of AMR in human, animal and environments 
2. Community engagements on prudent use of antibiotics based on research findings

3. Dissemination of AMR research findings through various channels such as seminar, conferences, symposium, clubs, debates in secondary schools

Results: The initiative has achieved the following results in the last 6 months

1. Direct engagement of literate and illiterate persons in sharing of AMR news in plain language

2. Development of skills of students in AMR research and findings dissemination

3. Development of student's presentation and engagement skills

4. Involvement of non science students of university and secondary schools in AMR knowledge and sharing

5. Communicating AMR knowledge to the grass root community members that abuse antibiotics most

Conclusion: Communicating data of abuse and resistance to the grass root community members in language they understand is yielding positive result in fighting antibiotic misuse and resistance development

Disclosure of Interest: None declared

\section{CO4}

An awareness about antimicrobial intake and antimicrobial resistance among geriatric population in Chennai city

R. $\mathrm{C}^{1, *}$

${ }^{1}$ public health dentistry, ragas dental college and hospital, chennai, India Correspondence: R. C

Antimicrobial Resistance \& Infection Control 10(1): C04

Abstract video clip description: The devastating threat of antimicrobial resistance (AMR) to global human health is unquestionable. The difficult-to-treat AMR infections require longer hospital stays and more costly treatment, increase morbidity and mortality, and reduce productivity; therefore, increasing the economic burden of the countries. The major contributing factors of AMR are known to be overuse and misuse of antimicrobials. Antibiotics are commonly prescribed among older adults, and inappropriate use of antibiotics has been noted. Antibiotic consumption by the elderly population was found to be between 11 to $45 \%$, with a significant rise over the past decade. Healthcare providers face challenges in adequately diagnosing and managing infections in this vulnerable population because of the altered drug metabolism in this particular group. Nursing homes or long term care facilities were found to be a reservoir for AMR pathogens due to excessive, inappropriate and prolonged broad-spectrum antimicrobial use, inadequate infection control and increased risk of pathogen colonization. It is therefore important that both healthcare professionals and the public know the threat antimicrobial resistance poses and the individual actions they can take to combat antimicrobial resistance.

The objective of this video is to assess the awareness about antimicrobial intake and antimicrobial resistance among geriatric population in Chennai city. A questionaire was adapted from validated surveys that were previously used and tailored to suit the local population and assure its applicability and modifications were made as necessary so that the questionnaire was simple to understand and answer, yet gave accurate data. Informed consent was obtained from all participants.

In the present video, widespread use of antibiotics was reported, most of the antibiotics were being accessed without prescription. Respondents displayed poor knowledge particularly in regard to the role of antibiotics in minor viral illness. Participants had poor attitude toward the use of antibiotics for cold and sore throat. There were also significant malpractices such as failing to take full dose, purchasing antibiotics without prescription. Hence, educational interventions on antibiotics use and its association with drug resistance are needed to promote judicious use of antibiotic.

Disclosure of Interest: None declared
$\mathrm{C05}$

Role of infection control in a tertiary teaching hospital

S. Z. Mohamad Razali ${ }^{1,}$, , S. Sri La Sri Ponnampalavanar ${ }^{1,2,3}$, I. N. Ismail ${ }^{1}$

${ }^{1}$ Infection Control, University Malaya Medical Centre, ${ }^{2}$ Medicine, Univer-

sity of Malaya, ${ }^{3}$ Infectious Disease Unit, University Malaya Medical Centre,

Lembah Pantai, Malaysia

Correspondence: S. Z. Mohamad Razali

Antimicrobial Resistance \& Infection Control 10(1): C05

Abstract video clip description: Infection prevention and control (IPC) is a practical, evidence-based approach which prevents patients and healthcare workers (HCW) from being harmed by avoidable infection. The role of tertiary university hospital is to strengthen the IPC among all category of HCW, students and public using a multimodal approach such as education and training, competency assessment, self-directed learning via online module, video demonstrations and campaigns. This video is a compilation of the IPC measures done in University Malaya Medical Centre to promote IPC measures and patient safety to reduce the risk of acquiring and transmitting healthcare associated infections (HAl). Among the activities done are surveillance for multidrug resistant organisms (MDROs), environment and cleaning, $\mathrm{HCAl}$, hand hygiene, operation theatre audit, surgical site infections (SSIs), reprocessing of endoscopes, and blood stream infections. There is also continuous education and training provided for all category of HCW to educate and increase awareness of the burden of HCAI on the healthcare system. UMMC has won the APSIC Safe Surgery Award 2018 for the SSIs quality improvement project. We also have been selected as a finalist for 2021 Hand Hygiene Excellence Award. The role of antimicrobial stewardship program also plays an important role in reducing the burden of HCAI. To increase the awareness among HCWs, we organized Antibiotic Awareness Week campaign and had a soft launching of UMMC Antibiotic Guideline Edition 2020 which can be accessed online via the staff portal. Apart from that, video demonstrations related to personal protective equipment and hand hygiene are also made available online as a reference for the HCWs.

Disclosure of Interest: None declared

C06

Infection prevention and control: the role of insourcing

O. Orlova ${ }^{1,}$, O. Karpov ${ }^{1}$, N. Yumtsunova ${ }^{1}$, A. Zotova ${ }^{1}$

${ }^{1}$ Pirogov National Medical and Surgical Center, Moscow, Russian Federation

Correspondence: O. Orlova

Antimicrobial Resistance \& Infection Control 10(1): C06

\section{Abstract video clip description}

Introduction: Complex of disinfection measures which includes routine and general cleaning, plays an important role in interrupting the direct transmission of infection diseases pathogens which are often present on the environmental surfaces surrounding patients.

Objective: Determine the role of insourcing in the prevention of infectious diseases.

Methods: The analysis of the insourcing multi-modal program results has been carried out in a large multidisciplinary medical center with 602 beds in the following units with high epidemiological risk: hematology unit, intensive care unit, operation rooms. Moreover, the microbiological contamination of highly sensitive objects (HTO) in the hospital environment, which are a source of contamination for personnel and their hands, was assessed. The thoroughness of cleaning was also monitored using fluorescent labels. The costs on purchasing disinfectants have been analyzed. Results: With the usual bucket method cleaning (i.e. 2015 - 2017 years), the level of microbial contamination was $8,4 \pm 8,1 \%$. The consumption of disinfectant solution (per day) was $1169 \mathrm{~L}$. At the beginning the baseline level result of cleaning the fluorescent marks in operation rooms was $65,90 \pm 29,75 \%$. After one month of the program implementation and training of the hospital staff, the second audit showed that the level of cleaning improved to $83,63 \pm 20,83 \%(p=0,02)$. As a result of the 
multi-modal program implementation, the consumption of disinfectant solution decreased to $223,8 \mathrm{~L}$ (per day). The average monthly consumption of disinfectant solution was reduced by 5,22 times to $6714 \mathrm{~L}$ per month $(p=0,18)$. The proportion of microbial contamination of environmental surfaces in the units with high epidemiological risk decreased to $6,7 \pm 5,4 \%$ in 2018 - 2020 years.

Conclusion. The usage of the proposed non-bucket cleaning system allows not only faster and better to execute planned measures for disinfection of the environmental surfaces in hospitals, to improve clinical, operational and financial metrics, but also to monitor quality of hospital staff's work and discipline.metrics, but also to monitor quality of hospital staff's work and discipline.

Disclosure of Interest: None declared

\section{C07}

Applying the who multimodal strategy for successful infection prevention improvements in healthcare!

Y. F. Lee ${ }^{1, *}$, H. U. Ngian ${ }^{1}$, Z. H. Chin ${ }^{2}$, H. H. Chual', S. Sri La

Ponnampalavanar ${ }^{3}$, T. S. Chow ${ }^{4}$, J. A. K. Lam ${ }^{5}$, K. S. Chai ${ }^{5}$, S. Amir Husin ${ }^{6}$

${ }^{1}$ Sarawak General Hospital, ${ }^{2}$ Sarawak State Health Department, Kuching,

${ }^{3}$ Universiti Malaya, Kuala Lumpur, ${ }^{4}$ Hospital Pulau Pinang, Penang, ${ }^{5}$ Malaysia Red Crescent Society, Sarawak, ${ }^{6}$ Infection Prevention \& Control Unit, $\mathrm{MoH}$, Putrajaya, Malaysia

Correspondence: $Y$. F. Lee

Antimicrobial Resistance \& Infection Control 10(1): C07

Abstract video clip description: Healthcare-associated infections have affected hundreds of millions of individuals worldwide, leading to significant mortality, particularly in resource-limited countries. This video demonstrates the application of the World Health Organization multimodal strategy in hand hygiene for successful infection prevention improvement in healthcare settings, by providing examplary action plans on the five key collaborative strategies, namely by, (1) 'Building it' with System Change, (2) 'Teaching it' with Training and Education, (3) 'Checking it' with Evaluation and Feedback, (4) 'Selling it' with Reminers in Workplace, and (5) 'Living it' for Instititutional Safety Climate, to improve and sustain infection prevention and control across different cultures and geographic regions.

Disclosure of Interest: None declared

\section{C08}

How to COVID-19 pandemic infection control and prevention in Cho Ray hospital, Vietnam

T. M. Phung Manh ${ }^{1, *},{ }^{\prime}$, Nguyen Tri ${ }^{2}$

${ }^{1}$ Infection Control, ${ }^{2}$ Arrhythmia, CHO RAY HOSPITAL, Ho Chi Minh city,

Viet Nam

Correspondence: T. M. Phung Manh

Antimicrobial Resistance \& Infection Control 10(1): C08
Abstract video clip description: This video Clip demonstrated hand hygiene compaign for patients and their relatives in Cho Ray hospital. Patients recieved treatment in Vietnam and Cho Ray hospital usually take part in not only healthcare worker but also a lot of people from their families. Therefore, improvement of knowledge hand hygiene for patients and their families are very important in Vietnam and Cho Ray hospital to reduce healthcare associated infection. This Clip is really effect to enhace hand hygiene compliance in Cho Ray hospital up to $81 \%$ in recent year.

Disclosure of Interest: None declared

\section{C09}

\section{COVID-19 prevention im your wokplace}

R. S. Al Azmi 1 ${ }^{*}$, S. Al Zayani ${ }^{2}$, H. saleh², A. Ahmed ${ }^{2}$, A. Nasser ${ }^{2}$, T. khairallah², T. $t^{2}, M . M^{2}$

${ }^{1}$ Infection Prevention \& Control, ${ }^{2}$ Media Center, Eastern Health Cluster,

dammam, Saudi Arabia

Correspondence: R. S. Al Azmi

Antimicrobial Resistance \& Infection Control 10(1): C09

Abstract video clip description: Neglecting the infection control measure during COVID-19 pandemic at workplace from the employee like not disclose having respiratory symptoms before showing to work, sharing personal items, not wearing the mask all time or follow cough etiquette and social distances. lead to transmit the infection among the healthcare workers, result to be infected and expose to an infected case.

Objective: Motivate the awareness of COVID-19 Prevention measures at Workplace

Disclosure of Interest: None declared

\section{Publisher's Note}

Springer Nature remains neutral with regard to jurisdictional claims in published maps and institutional affiliations. 